\title{
PROCEEDINGS OF THE WORKSHOP ON
}

\author{
" \\ "PHYSICS WITH FAST
}

MOLECULAR-ION BEAMS"

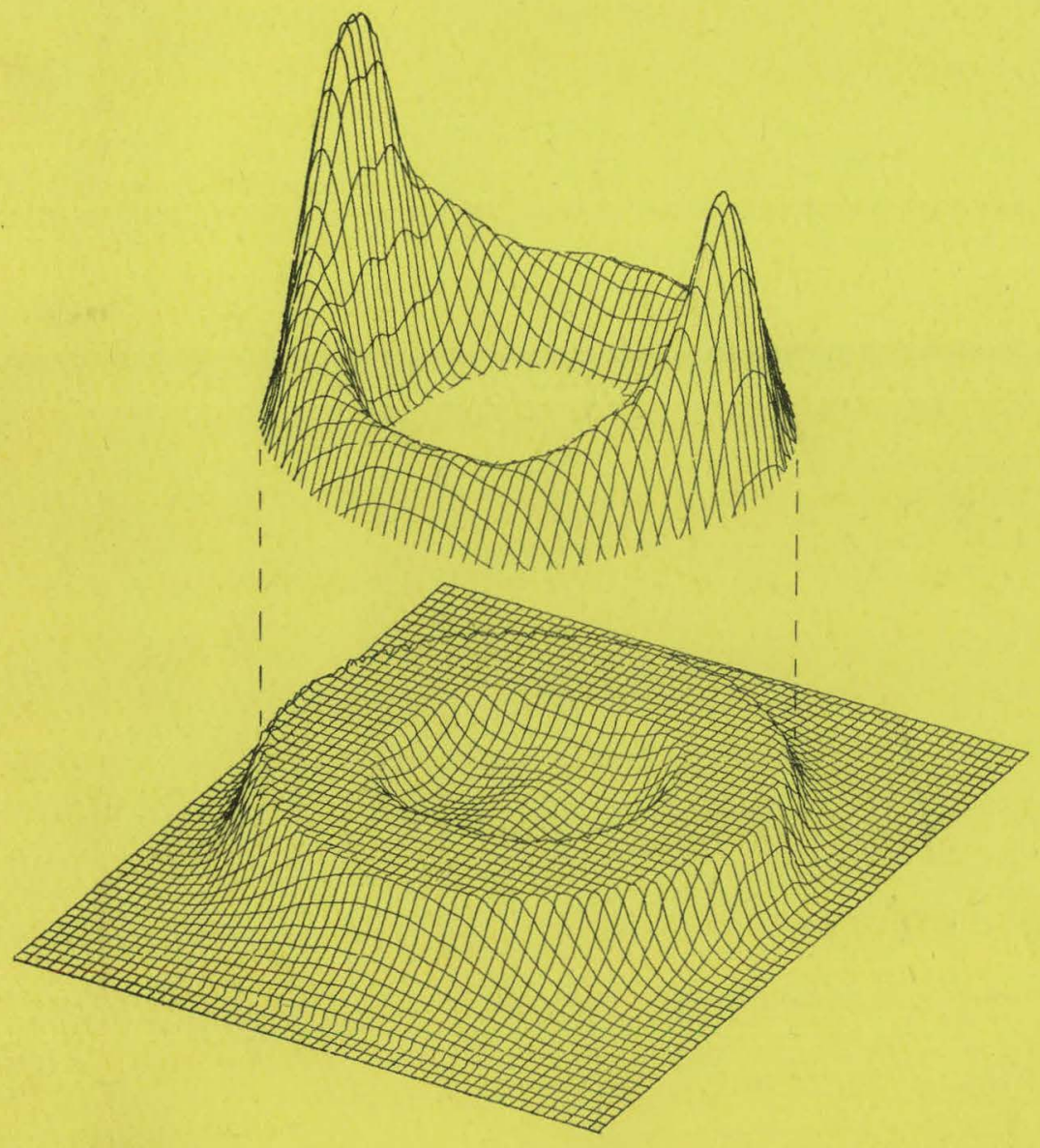

AUG. 20-2I, 1979

\author{
EDITOR: \\ D.S. GEMMELL
}

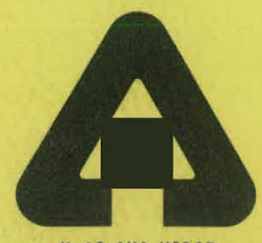

ARGONNE NATIONAL LABORATORY, ARGONNE, ILLINOIS

Operated under Contract W-31-109-Eng-38 for the

U. S. DEPARTMENT OF ENERGY

\section{MASTER}




\section{DISCLAIMER}

This report was prepared as an account of work sponsored by an agency of the United States Government. Neither the United States Government nor any agency Thereof, nor any of their employees, makes any warranty, express or implied, or assumes any legal liability or responsibility for the accuracy, completeness, or usefulness of any information, apparatus, product, or process disclosed, or represents that its use would not infringe privately owned rights. Reference herein to any specific commercial product, process, or service by trade name, trademark, manufacturer, or otherwise does not necessarily constitute or imply its endorsement, recommendation, or favoring by the United States Government or any agency thereof. The views and opinions of authors expressed herein do not necessarily state or reflect those of the United States Government or any agency thereof. 


\section{DISCLAIMER}

Portions of this document may be illegible in electronic image products. Images are produced from the best available original document. 
The facilities of Argonne National Laboratory are owned by the United States Government. Under the terms of a contract (W-31-109-Eng-38) among the U. S. Department of Energy, Argonne Universities Association and The University of Chicago, the University employs the staff and operates the Laboratory in accordance with policies and programs formulated, approved and reviewed by the Association.

\section{MEMBERS OF ARGONNE UNIVERSITIES ASSOCIATION}

The University of Arizona

Carnegie-Mellon University

Case Western Reserve University

The University of Chicago

University of Cincinnati

Illinois Institute of Technology

University of Illinois

Indiana University

The University of Iowa

Iowa State University
The University of Kansas

Kansas State University

Loyola University of Chicago

Marquette University

The University of Michigan

Michigan State University

University of Minnesota

University of Missouri

Northwestern University

University of Notre Dame
The Ohio State University

Ohio University

The Pennsylvania State University

Purdue University

Saint Louis University

Southern Illinois University

The University of Texas at Austin

Washington University

Wayne State University

The University of Wisconsin-Madison

\section{NOTICE}

This report was prepared as an account of work sponsored by an agency of the United States Government. Neither the United States nor any agency thereof, nor any of their employees, makes any warranty, expressed or implied, or assumes any legal liability or responsibility for any third party's use or the results of such use of any information, apparatus, product or process disclosed in this report, or represents that its use by such third party would not infringe privately owned rights. Mention of commercial products, their manufacturers, or their suppliers in this publication does not imply or connote approval or disapproval of the product by Argunue National Laboratory or the United States Government. 
WORKSHOP ON

\section{PHYSICS WITH FAST MOLECULAR ION BEAMS}

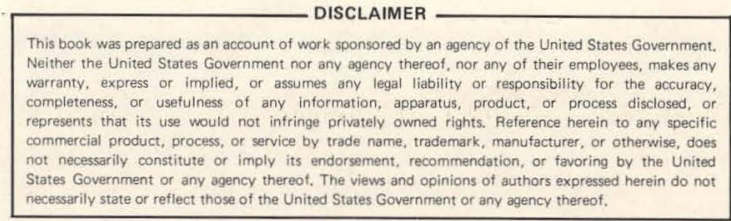

Held at Argonne National Laboratory Argonne, Illinois 60439

August $20-21,1979$

Editor, D. S. Gemmell

Cover picture: "Ring pattern" for $\mathrm{N}^{4+}$ fragments arising from $3-\mathrm{MeV} \mathrm{N}_{2}^{+}$ projectiles dissociating in a $75-\AA$ thick carbon foil. (D. S. Gemme1l, I. Plesser and B. J. Zabransky, Argonne National Laboratory, 1979) 

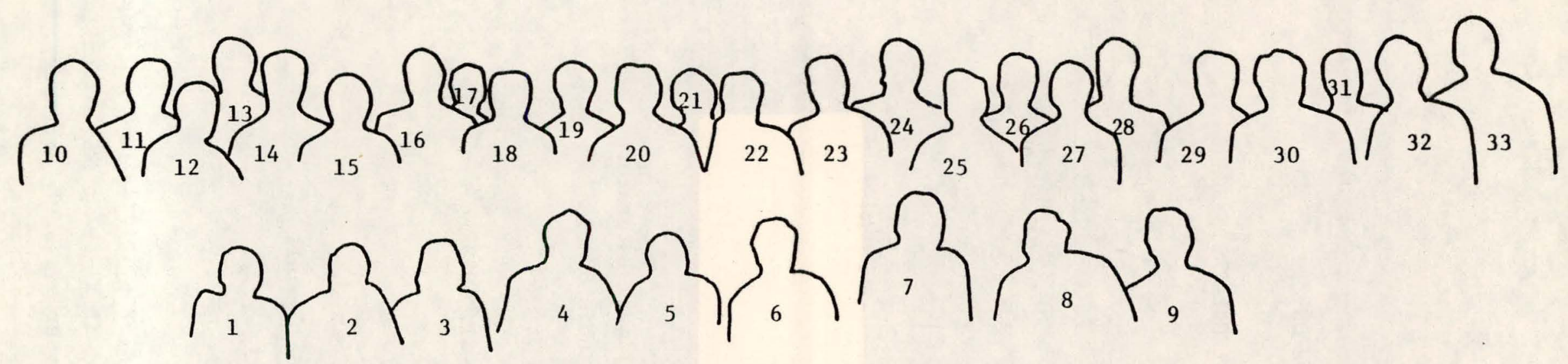

1. N. Cue

2. A. K. Edwards

3. M. Kitagawa

4. F. Fujimoto

5. E. P. Kanter

6. D. S. Gemmel1

7. P. J. Cooney

8. B. J. Zabransky

9. W. H. Escovitz

10. A. Faibis

11. M. Gaillard
12. J. Remillieux

13. H. E. Wegner

14. H. G. Berry

15. Y. H. Ohtsuki

16. M. W. Lucas

17. R. Kaim

18. R. Laubert

19. R. H. Ritchie

20. P. M. Echenique

21. R. A. Baragiola

22. W. Brandt
23. Z. Vager

24. W. Pietsch

25. R. DeSerio

26. P. B. Treacy

27. R. M. Schectman

28. D. G. E11is

29. S. Levit

30. R. Levi-Setti

31. P. Thieberger

32. T. R. Fox

33. T. J. Gay 


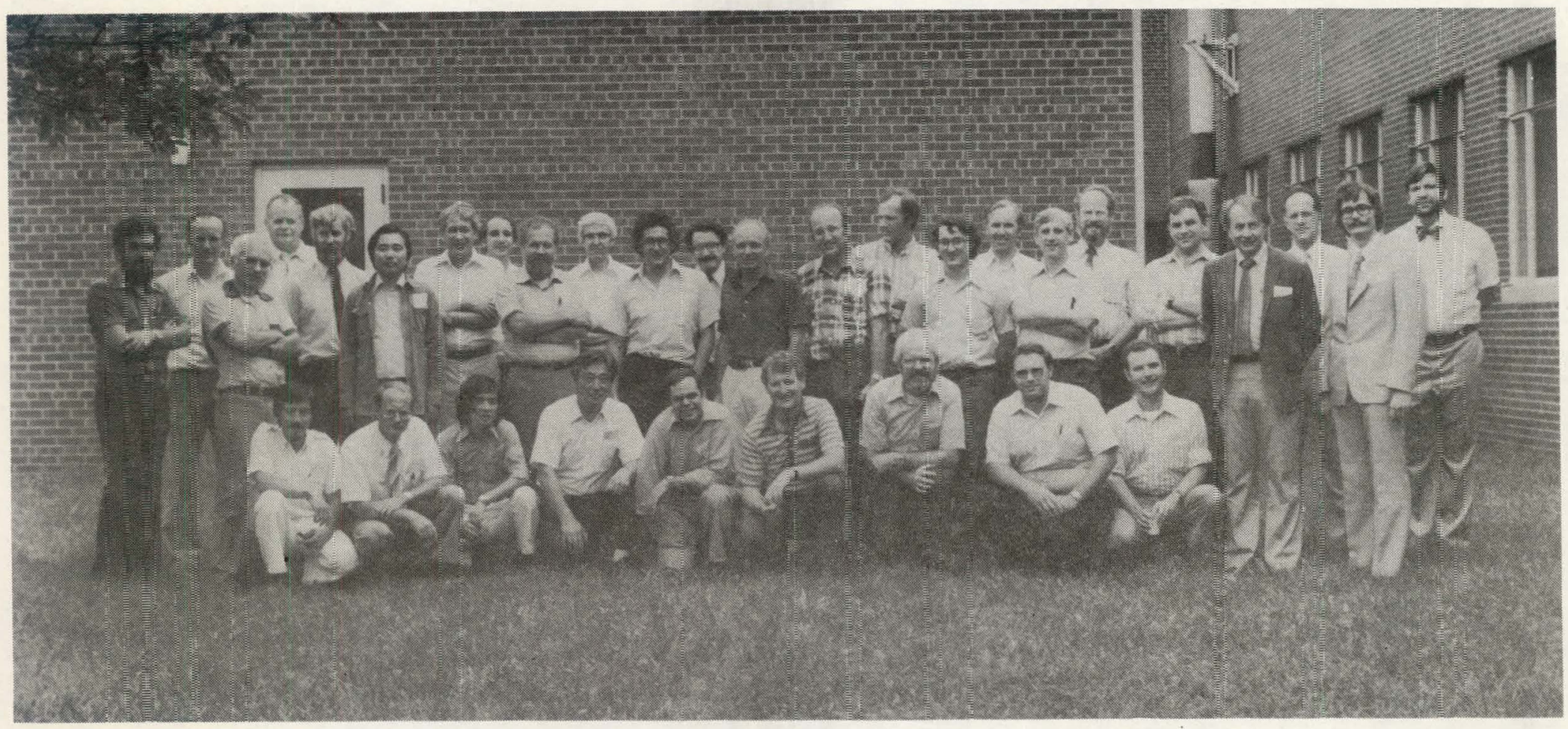


The Workshop on "Physics with Fast Molecular-Ion Beams" was held in the Physics Division, Argonne National Laboratory on August 20 and 21, 1979. The meeting brought together representatives from several groups studying the interactions of fast (MeV) molecular-ion beams with matter. This area of physics has seen a rapid development in the past few years and it was therefore considered appropriate to arrange an informal meeting bringing together many of the leading practitioners of the art. Several participants attended following the 8th International Conference on Atomic Collisions in Solids held in Hamilton, Ontario, August 13-17, 1979. This permitted the Argonne Workshop to acquire quite an international flavor-there were 35 attendees from 9 different countries.

By keeping the Workshop program sharply focussed on current work related to the interactions of fast molecular ions, it was made possible for the participants to engage in vigorous and detailed discussions concerning such specialized topics as molecular-ion dissociation and transmission, "wake" effects, ionic charge states, cluster stopping powers, beam-foil spectroscopy and electron-emission studies with molecular-ion beams, molecular-ion structure determinations, etc.

The program was arranged so as to have a series of informal

presentations from the various groups represented at the Workshop. It was left up to the members of these groups to organize their presentations and to choose their spokesmen.

The proceedings are a "minimum effort" proceedings. That is to say almost no effort has been invested in editing. The participants were asked to bring with them to the Workshop camera-ready text and diagrams (or copies of their slides and "Vugraph" transparencies). This has allowed us to put together in a short time a collection of contributions which represent the essence of the Workshop. We hope and believe that the non-uniformity of these contributions (ranging from sets of Vugraph copies to finished papers complete with references, figure captions, etc.) will be more than offset by the benefit to the community of interested scientists in receiving these proceedings rapidly.
D. S. Gemme11
P. J. Cooney
E. P. Kanter
I. Plesser
B. J. Zabransky
October 1979 


\section{Workshop on "Physics with Fast Molecular Ion Beams"}

\section{PROGRAM}

Monday, August 20

Morning Session Chairman: W. Brandt

8:30 - 8:45 Welcome and Introduction

8:45 - 9:45 Weizmann Institute (Faibis, Kaim, Levit, Plesser, Vager)

9:45 - 10:15. Brookhaven (Thieberger, Wegner)

10:15 - 10:45 Coffee

10:45 - 11:45 Argonne (Cooney, Gemme11, Kanter, Pietsch, Vager, Zabransky)

11:45 - 12:15 Tour of Dynamitron Facilities

12:15 - 1:15 LUNCH

$1: 15-1: 30 \quad$ Group Photograph

Afternoon Session Chairman: F. Fujimoto

1:30 - 2:30 University of Lyon (Cue, Gaillard, Remillieux)

2:30 - 3:10 Bariloche (Baragiola)

$3: 10-3: 30 \quad$ Coffee

3:30 - 4:30. New York University and East Carolina University (Brandt, Lauburt)

4:30 - 5:00 Bonn-Cologne-Argonne (Pietsch, Gemmell)

6:00 - 10:00. Cocktails and Dinner in Building 600 .

Tuesday, August 21

\begin{tabular}{|c|c|}
\hline Morning Session & Chairman: Z. Vager \\
\hline $8: 30-9: 30$ & Brandt, Echenique and Ritchie \\
\hline $9: 30-10: 10$ & Kitagawa and Ohtsuki \\
\hline $10: 10-10: 30$ & Coffee \\
\hline $10: 30-11: 00$ & University of Georgia (Edwards) \\
\hline $11: 00-11: 30$ & Australian National University (Treac) \\
\hline $11: 30-12: 00$ & Tour of Tandem-Linac Facilities \\
\hline $12: 00-1: 00$ & Lu \\
\hline
\end{tabular}

Afternoon Session Chairman: R. Baragiola

1:00 - 2:00 "Photon and Electron Emission" (Berry, Ellis, Gay, Lucas, Schectman)

2:00 - 2:30 University of Chicago (Levi-Setti)

2:30 - 3:15 Summary (Remillieux)

$3: 15-3: 30 \quad$ Coffee 
TABLE OF CONTENTS

Page

A. Morning Session, 20 August 1979

1

1. MEASUREMENTS OF THE DISSOCIATION OF $11.2-\mathrm{MeV} \mathrm{OH}{ }^{+}$ AND THE STUDY OF THE INDUCED POLARIZATION

IN SOLIDS

A. Breskin, A. Faibis, G. Goldring, $M$. Hass, R. Kaim, I. Plesser, 2. Vager, and N. Zwang

2. $\mathrm{C}_{3,4}^{-}$MOLECULAR ION STRUCTURE DETERMINATION

G. Goldring, Y. Eisen, P. Thieberger and $H . E$. Wegner.

3. CLUSTER EFFECTS ON CHARGE STATE DISTRIBUTIONS

FOR HEAVY ION MOLECULAR BEAMS

P. Thieherger

4. RECENT WORK WITH FAST MOLECULAR-ION BEAMS AT ARGONNE NATIONAL LABORATORY

P. J. Cooney, D. S. Gemmell, K.-O. Groeneveld,

E. P. Kanter, W. J. Pietsch, Z. Vager, and

B. J. Zabransky

B. Afternoon Session, 20 August 1979

1. TRANSMISSION OF MOLECULES AND NEUTRAL PARTICLES USING HYDROGEN AND HELIUM MOLECULAR BEAMS

J. Remilzieux et al.

2. STOPPING OF LOW ENERGY MOLECULAR IONS

J. C. Eckhardt, G. H. Lantschner, N. R. Arista and R. A. Baragiola

3. ENERGY LOSS OF CLUSTERS

R. Laubert 
4. THE POTENTIAL ESTABLISHED IN THE VICINITY OF SWIFT IONS IN SOLIDS

- R. Laubert

5. $\mathrm{H}_{2}^{+}$CENTRAL PEAKS

W. Brandt

6. PROBING THE WAKE OF A SWIFT ION IN MATTER

D. S. Gemmezl, G. Krösing, G. Kumbartzki, and W. J. Pietsch

C. Morning Session, 21 August 1979

1. THE WAKE

W. Brandt

2. WAKE EFFECT EXPLANATION TO BELL AND BETZ EXPERIMENT

P. M. Echenique

3. WAKE FLUCTUATIONS

R. H. Ritchie

4. SKIPPING MOTION OF IONS AT THE SURFACE

Y. H. Ohtsuki and M. Yamamura

5. SOLITON SOLUTION IN THE WAKE INTERACTION

M. Kitagawa and $Y$. H. Ohtsuki

6. MOLECULAR DISSOCIATION PRODUCED BY FAST HEAVY ION BOMBARDMENT

A. K. Edwards, R. M. Wood, and M. F. Steuer

7. LOCALISATION OF ELECTRON CONTINUUM STATES FOLLOWING CHARGE EXCHANGE

P. B. Treacy 
D. Afternoon Session, 21 August 1979

1. OPTICAL MEASUREMENTS OF MOLECULAR DISSOCIATION BY THIN FOILS

H. G. Berry

2. FOIL INDUCED DISSOCIATION OF FAST $\mathrm{H}_{2}{ }^{+}$MOLECULAR 269 IONS STUDIED BY QUANTUM BEAT SPECTROSCOPY

R. M. Schectman and D. G. ElZis

3. OPTICAL OBSERVATIONS OF MOLECULAR DISSOCIATION IN THIN FOILS

T. J. Gay and H. G. Berry

4. ELECTRON EMISSION

M. W. Lucas

5. HYDROGEN ION TRAVERSAL OF THIN SOLID FOILS: NEW DATA AT LOW ENERGY AND AN OVERVIEW

W.H. Escovitz, T. R. Fox and R. Levi-Setti 
MORNING SESSION

20 August 1979

W. Brandt, CHAIRMAN 
Measurements of the dissociation of $11.2-\mathrm{MeV} \mathrm{OH}^{+}$ and the study of the induced polarization in solids

A. Breskin, A. Faibis, G. Goldring, M. Hass, R. Kaim, I. Plesser, Z. Vager and N. Zwang

Department of Physics, Weizmann Institute of Science, Rehovot, Israel.

\section{ABSTRACT}

Combined energy and angle distributions of protons resulting from Coulomb axplosion of 11.2 MeV Cut in zatbon foils liave been measured in simultaneous coincidence with different emerging oxygen charge-states. The measurements were made with targets of thickness ranging from $80 \AA$ to $740 \AA$. The proton distributions show strong asymnetry effects due to the electronic wake produced by oxygen atoms in the solid, and there is also a marked dependence of the shape of the distributions on both the target thickness and the oxygen chargestate downstream from the target.

The interpretation of molecular ion interaction with solid through conventional stopping power theories is criticised. Comparison of experimental data to simulations of proton velocity distributions after $11.2 \mathrm{MeV} \mathrm{OH}^{+}$dissociation in carbon foils supports this criticism. A different description of the average potential around a moving ion in solid due to electronic polarization is given. 
The polarization of the Fermi sea of electrons is described by the coulomb distortion of the single electron wave functions due to scattering on the moving ion. The use of such potentials for the simulation of experiments agrees favourably with the measured data.

\section{1. . INTRODUCTION}

It is now well established that when a fast molecular ion is incident on a thin foil target, the distributions in energy and angle of break-up products detected downstream from the target are sensitive to wake effects inside the solid(1)(2)(3). Here we report results of an experiment in which measurements of distributions of protons from the break-up of $\mathrm{OH}^{+}$were used as a means to probe the electronic charge-density in the vicinity or the oxygen atoms as they pass through the target. Measurements in coincidence with emergent oxygen charge states, and use of a range of target thicknesses, allow the effects of oxygen-proton interaction inside and outside the target to be differentiated. The experiment is therefore specifically designed to provide a stringent test for the validity of models used to describe the effects of electron wakes in the target.

\section{EXPERIMENTAL}

A high-energy molecular $\mathrm{OH}^{+}$beam was produced in a Pelletron 14 UD accelerator by stripping off $\mathrm{OH}^{-}$in nitrogen gas at the terminal. A collimated beam of $\mathrm{OH}^{+}$at an energy of $11.2 \mathrm{MeV}$ was incident on a carbon target, and fragments emerging from the target entered a magnetic spectrometer 
(see Fig. 1). A horizontal spectrometer entrance slit was set with an opening of $\pm 0.8 \mathrm{mrad}$, which restricted entrance to the spectrometer to only those protons whose trajectories were nearly in a horizontal plane parallel to the magnetic field. Oxygen ions emerged from the target with much smaller divergence than the protons and were therefore not affected by the slit.

The detection system consisted of two independent gas detectors: a detector for protons placed in the focal plane of the spectrometer and a detector for the oxygen ions inserted between the pole pieces of the magnet. The oxygen detector was a position-sensitive proportional counter comprising an anode wire and parallel delay line. The position of an incident oxygen ion was obtained from the time difference between signals from the wire and from one of the edges of lise ielay-iine. The $1 \mathrm{~mm}$ fwhtM resolution of the counter was insufficient to distinguish energy changes due to the Coulomb explosion, but quite adequate to resolve different charge-states of the oxygen ions.

The proton detector (described in detail in ref. 4) is composed of two independent parts mcunted in a common vessel: a drift chamber which provides a bidimensional measure of the position of the incident particle and a paraliel plate avalanche counter which provides a fast timing signal. The drift time of electrons produced by an incident proton gives a measure of its position along the spectrometer focal plane, and hence of its energy $\left(E_{p}\right)$. The orthogonal position, obtained by extracting delayed induced signals from a delay-line, is proportional to the angle $\left(\theta_{p}\right)$ of the proton 
trajectory in the plane defined by the entrance slit to the spectrometer. The resolution of the drift chamber was about $0.5 \mathrm{~mm}$ FWH in both dimensions. The total experimental angle and energy resolution are (FWHM) $0.35 \mathrm{mrad}$ and $0.5 \mathrm{keV}$.

The bidimensional counter was calibrated with respect to both angle and energy in a separate measurement using a beam of $0^{+}$from the accelerator. Once the energy dimension had been calibrated, the thickness of carbon targets could be determined by measuring the energy loss of oxygen ions in each target. The energy loss was converted to target thickness using the stopping power data of Booth and Grant ${ }^{(5)}$ for oxygen in carbon.

\section{RESULTS.}

Fig. 2 shows an oxygen charge-state spectrum obtained with the position sensitive proportional counter, demonstrating that different emergent charge-states were well resolved from one another. The angle and energy of each proton on the bidimensional focal-plane counter could therefore be measured in coincidence with the corresponding oxygen ion in its emergent charge-state. In Fig. 3 we show angle/energy spectra of protons in coincidence with four different oxygen charge-states and for a target thickness of $80 \AA$, while in Fig. 4 spectra of protons in coincidence with $0^{6+}$ are compared for different target thickness. 
In addition to the measured quantities $\Delta \mathrm{E}_{\mathrm{p}}$ (the change in energy due to the Coulomb explosion) and $\theta_{p}$ (the angle of trajectory relative to the beam direction), the axes in Figs. 3 and 4 have been labelled according to the components of the final velocity of the protons in the rest frame of the center of mass of the molecules.

A striking feature of the data in Figs. 3 and 4 is the very pronounced backward peak (bäkwaird direction in the-Cri-frame) in the spectra, and the almost complete absence of a forward peak.

It can be seen in Fig. 3 that the total size of the ring in velocity space, i.e. the total magnitude of the Coulomb explosion, increases with increasing oxygen charge-state $q$. This dependence can be represented by plotting, for different targets, the energy difference $\delta \mathrm{E}_{\mathrm{p}}$ between the forward and backward peaks $\left(\theta_{\mathrm{p}} \sim 0^{0}\right)$ as a function of $q$. Such a plot is shown in Fi $\mathbf{F}_{\bar{\sigma}}, 5$ and, as expected. the dependence of $\delta \mathrm{E}_{\mathrm{p}}$ on $\mathrm{q}$ is weaker for thicker targets since in this case most of the explosion occurs inside the target and the oxygen ion acquires a well defined average charge. For thicker targets there is reduction in both the magnitude of $\delta E_{p}$ and its dependence on emergent charge

- states which we succeeded to reproduce in simulation calculations. This former reduction is mainly due to läger multiple scattering angles in thicker targets. An interesting aspect of the proton spectra in Fig. 3 is the dependence on $q$ of the intensity of the backward peak relative to the "sides" of the distribution $\left(\Delta \mathrm{E}_{\mathrm{p}} \sim 0\right)$ : the relative intensity of the peak is clearly less for smaller $q$. The effect is strongest for the $80 \AA$ target as shown in Fig. 3 , but it is also seen for thicker targets. It should be emphasized that what is observed here is a dependence on the charge-state outside the target of a peak which is caused by wake effects inside the target. 
The data presented here provide a detailed picture of the effect of the oxygen wake on the proton distributions. A reliable model for the wake should be able to reproduce all the main features of the data, including the dependence on target thickness and emergent oxygen charge-state.! In what follows we describe a study of the shape of the proton velocity distributions, concentrating our attention on the shape of the backward peak and its relation to the physics of the ion-solid interaction. Several models will be examined for the electronic density following the moving ions in the solid. For each model we will derive the average potential and the average forces due to the corresponding "clectronic wakc".

These forces are then used in a computer simulation of the experiment.which is compared with the data in figure 4.

4. The comneter simulation.

The computer simulation solves the classical equations of motion for the proton and the oxygen under the following assumptions on the forces and initial conditions.

I. When the molecular ion enters the foil its orientation is random. The nuclear separation is distributed as a gaussian with mean value $r_{0}=1.05 \AA$ and a standard deviation $\sigma=0.17 \AA$. The initial rotational and vibrational velocities are ignored.

II. In the foil, the proton has a unit positive charge and the oxygen has an average charge $z_{\text {eff }}$ (between 5.6 to 6.1 ). The value of $z_{\text {eff }}$ was chosen such that the calculated stopping power for a given model of the forces agrees with the experimental stopping power. The two ions repel each other with a Coulombic force and are also influenced by their own and their partners electronic wake forces. 
III. Outside the foil, the oxygen ions obtain their final

charge states and the proton-oxygen pair goes into a pure Rutherford trajectory.

....... We consider here specifically an emergent oxygen of charge state $6^{+}$.

The computed velocity distribution of the protons is smeared to account for the experimental angle and energy-resolution and for the multiple scattering in the target.

5. Potentials and forces derivable from dielectric functions.

Almost all theories of heavy ion stopping power are based on first order perturbation theories ${ }^{(6)}$ : For such theories the response of a solid medium to a perturbing projectile is linear. Thus, the polarization charge, potential and field can be formally expressed in terms of a dielectric function $\varepsilon(k, \omega)$ (ignoring anisotropies) where $\vec{k}, w$ are Fnurier variables ronjugate to space $\vec{x}$, and time $t$. The use of such fields for the interpretation of the backward peaks in the dissociation of molecular ions in solid could be erroneous. The reason is that although the electron-ion cross-section dependence on momentum transfer is well described by the Born approximation, the Born scattering wave function can deviate strongly from the true scattering wave function near the scattering center. But the polarization charges near the moving ion are determined by the square of the true wave function near the scattering center. Nevertheless, it is instructive to examine the applicability of stopping power theories for the interpretation of molecular ion dissociation in solids. The Fourier transform of a charge density of a point charge $z$ eff moving along the $z$-axis with a velocity $v$ is given by: 


$$
\rho_{\text {ext }}(\vec{k}, \omega)=z_{\text {eff }} e^{\left.\frac{\delta(\omega-k}{-V}-v\right)}
$$

The potential due to the polarization of the medium is therefore:

$$
\phi(\vec{x})=\frac{z_{e f f}}{2 \pi^{2}} \cdot \int \frac{e^{i \vec{k} \cdot \vec{x}}}{k^{2}}\left[\frac{1}{\varepsilon\left(k, k_{z} v\right)}-1\right] d^{3} k
$$

where $\vec{x}=\vec{x}^{\prime}-\vec{v} t$ is a set of coordinates which moves with the projectile. The potential $\phi(\vec{x})$ is stationary with respect to the projectile.

6. The simple plasma wake.

A comparison of theoretical considerations with detailed experimental proton distribution was carried out by the Argonne group (3). Thi proton distribution after the dissociation of $2.0 \mathrm{MeV} \mathrm{HeH}{ }^{+}$in $85 \AA$ of carbon foil was measured and simulated with the use of forces derivable from the classical plasma dielectric function.

$$
\varepsilon(\omega)=1-\omega_{p}^{2} / \omega^{2}
$$

where $\omega_{p}=\left(4 \pi n e^{2} / m\right)^{\frac{1}{2}}$ is the plasma frequency due to a density of electrons $-n$.

The expression for the potential

$$
\phi(\vec{x}):=z_{\text {eff }} \text { ek } \int \sin \left(k_{p} \zeta\right)\left[x^{2}+y^{2}+(z+\zeta)^{2}+(\hbar / m v)^{2}\right]^{-\frac{1}{2}} d \zeta
$$

$\left(k_{p}=\omega_{p} / v\right)$ is a slight modification of eq. (2) which takes into account close electronic collisions (see ref. 3). Fig. 6a shows the potential $\phi(\vec{x})$ of eq. (4). Fig. 7 shows an application of this model to simulations of the proton velocity distributions 
after $11.2-\mathrm{MeV}$ oH dissociation in carbon targets of four thicknesses.

This should be compared with the experimental results given in

fig. 4. The two thicker targets

simulations (7.(c) and 7 (d)) exhibit extra backward peaks which are very different from the observed backward peaks. It seems that a shorter range potential is needed to keep the backward particles together for the longer time required by the thicker targets.

7.

The Lindhard dielectric function

The above plasma dielectric function is a special approximation to a more general function derived by $J$. Lindhard ${ }^{(7)}$. Other approximations to the Lindhard dielectric function are known in the literature and were used for the study of stopping power of clusters ${ }^{i j}$, surf riding electrons and several other subjects which are related to the potential around moving projectile in solid. We chose to examine the original longitudinal dielectric function of Lindhard.

For a degenerate free electron gas with Fermi velocity $v_{F}$, a quantum mechanical first order perturbation treatment leads to the following dielectric function

$\varepsilon^{\ell}(u, z)=1+\frac{\chi^{2}}{z^{2}}\left[\frac{1}{2}+\frac{1}{8 z}\left\{1-(z-u)^{2}\right\} \log \frac{z-u+1}{z-u-1}+\frac{1}{8 z}\left\{1-(z+u)^{2}\right\} \log \frac{z+u+1}{z+u-1}\right]$

where $x^{2}=\frac{e^{2}}{\pi \hbar v_{F}}, \quad z=\frac{k}{2 k_{F}}$ and $u=\frac{\omega+i \delta}{k v_{F}}$.

Small positive $\delta$ ensures a retarded dielectric function. 
When $\varepsilon(k, \omega)=\varepsilon^{\ell}(u, z)$ is introduced into eq. (2) the three dimensional integration can be carried out to produce the Lindhard potential (fig. 6b) and similarly a force field can be computed. The numerical integration is carried out in cylindrical coordinates. The angle integration is straightforward and poles in the $k_{z}$ integration are treated analytically. As can be seen in fig. 6 the long range part of this potential is smaller than that of the plasma potential, yet the stopping power (the slope of the potential at the origin) is the same. This comes about because for our velocity regime a major part of the stopping is due to close collisions with. ! electrons and not due to the long range plasma excitations.

The results of the $\mathrm{OH}^{+}$dissociation simulations with this potential are shown in fig. 8 . The overall similarity to the experimental distributions is much better than for the plasma wake case. This stresses the importance of the close collisions to the potential distribution. For thin targets simulations (Figs. 8 a and 8b) the ratio of peak height to rim height is 1arger than the experimental ratios and for thick targets (Figs. $8 \mathrm{C}$ and $8 \mathrm{~d}$ ) there is still a remainder of extra backward peaks which appeared in the plasma wake simulations.

8. Electronic density from coulomb wave functions.

As was mentioned before, the molecular dissociation is dominated by the short range forces near the moving ions. It is therefore instructive to consider an extreme opposite model to the plasma wake model by ignoring the collective electronic interaction and considering only exact close collisions. Then, the electrons 
can be considered as free particles (6). distributed in velocity by a Fermi gas law. The electrons are scattered by the moving ion via a pure coulomb potential. Therefore, the average electronic charge polarization is given by

$$
\rho(\vec{x})=n\left(\left\langle\left|\psi_{c}(n, x)\right|^{2}\right\rangle_{v}-1\right) \text {. }
$$

Here $n=z_{\text {eff }} e^{2} / \hbar v,<>_{v}$ is an average over the Fermi velocity distribution and $\psi_{c}$ is the coulomb wave function

The potential due to this density is given by the Poisson equation:

$$
\nabla^{2} \phi(\vec{x})=-4 \pi \rho(\vec{x})
$$

The use of the partial wave expansion of $\psi_{c}$ makes it possible to compute the multipole expansion of $\rho(\overrightarrow{\vec{x}})$ and $\phi(\overrightarrow{\vec{x}})$ in terms of products of $F_{\ell}(k r)^{(9)}$. The density deviation $\rho(\vec{x})$ was set to zero for radii above $3 \&$ from the projectile. The potential for oxygen ions is shown in fig. $6 \mathrm{c}$. It is of much shorter range and shallower than the previously discussed potentials (fig. 6d). The simulations of the proton velocity distributions are shown in fig. 9 . The resemblance to the experimental data (fig. 4) is impressive.

9. CONCLUSIONS

We have presented detailed measurements of proton velocity distributions after dissociation of $11.2 \mathrm{MeV} \mathrm{OH}^{+}$molecules in carbon foils. The data provide sensitive criteria for the examination of different models for the electronic polarization near a moving projectile in solid. We have shown that the Coulomb scattering wave functions of electrons on a moving 
ion in solid,play an important role in the description of the polarization near the projectile. This result is important also for the considerations of cluster stopping power, surf riding electrons, onvoy electrons, $x$-ray production in solid and transient magnetic field when ions are slowed down in ferromagnets.

The role of linear stopping power theories in the description of electronic wake potentials is criticized. The experimental $\mathrm{OH}^{+}$ dissociation data rule out the plasma wake model (3) but do not rule out a potential derived from the Lindhard dielectric function $(7)$. Since for the oxygen ions involved, the Sommerfeld parameter $n$ is around 1 , it is suggested that the polarization of the electronic density is more accurately described by the coulomb wake and not by linear theories. 


\section{REFERENCES}

1. D. S. Gemmell, J. Remillieux, J.-C. Poizat, M.J. Gaillard, R. E. Holland and Z. Vager, Nucl. Instrum. Methods 132 (1976) 61.

2. Z. Vager, D. S. Gemmell and B. J. Zabransky, Phys. Rev. A14 (1976) 638 .

3. Z. Vager and D. S. Gemmell, Phys. Rev. Lett. 37 (1976) 1352.

4. A. Breskin, I. Tserruya and N. Zwang, Nucl. Instrum. Methods 148 (1978) 275 .

5. W. Booth and I. S. Grant, Nucl. Phys. 63 (1965) 481.

6. U. Fano, Ann. Rev. Nuc1. Sci. 13 (1963) 1:

?. J. Lindhard, K. Dan. Vidensk. Selsk. Mat. Fys. Medd. 28; No. 8 (1954).

8. W. Branil, A. Ratikowski and R. H. Rilcinie, Phys. Rev. Lett. 33 (1974) 1325 .

9. L. I. Schiff, Quantum Mechanics (McGraw-Hil1) 117. 


\section{FIGURE CAPTIONS}

Fig.1: $\quad$ Experimental arrangement.

Fig.2: Spectrum of emergent oxygen charge states.

Fig.3: $\quad$ Spectra of protons from $11.2 \mathrm{MeV} \mathrm{OH}^{+}$

incident on $80 \AA$ carbon. The spectra were

recorded simultaneously, each in coincidence

with axygen ions emerging with the charge

state indicated.

Fig.4: Spectra of protons in coincidence with $0^{6+}$,

for carbon targets of different thickness.

Fig.5:- Energy difference $\delta E_{p}$ between leading and

trailing protons as a function of emergent

oxygen charge state and thickness of target.

$\left(1 \mu \mathrm{g} / \mathrm{cm}^{2} \simeq 50 \&\right.$ carbon $)$.

Fig.6 : Potentials near a moving oxygen ion associated with

(a) classical plasma polarization wake, (b) Lindhard dielectric function (c) coulomb scattering of electrons and (d) comparison of the three potentials for $R=0$. The ion track is along the $z$-axis and $R$ is the radial distance.

Fig.7: Simulation of proton velocity distribution after $\mathrm{OH}^{+}$ dissociation in carbon foil of thickness (a) $80 \AA$, (b) $152 \AA$, (c) $285 \AA$ and (d) $740 \AA$. The protons are in coincidence with the $6^{+}$final charge state of the outgoing oxygen ions. Wake forces are derived from the plasma wake model (see fig. 6a). The velocity span is identical to that of fig. 4 . 
Fig.8: Same as 7 except that wake forces are derived from the Lindhard dielectric function (see fig. 6b).

Fig.9:- Same as 7 except that wake forces are derived from polarization density due to Coulomb scattering of electrons (see fig. 6c). 


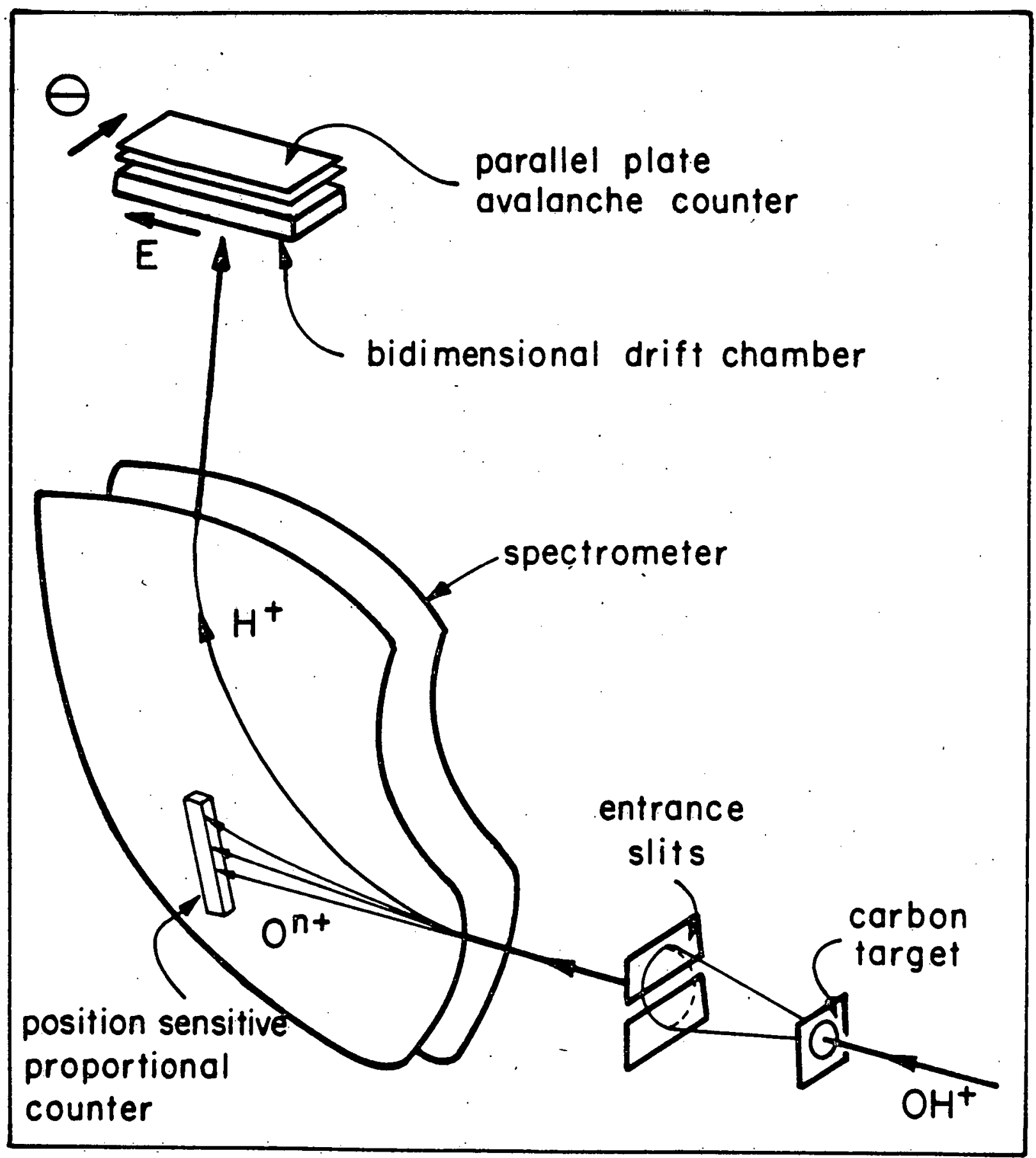




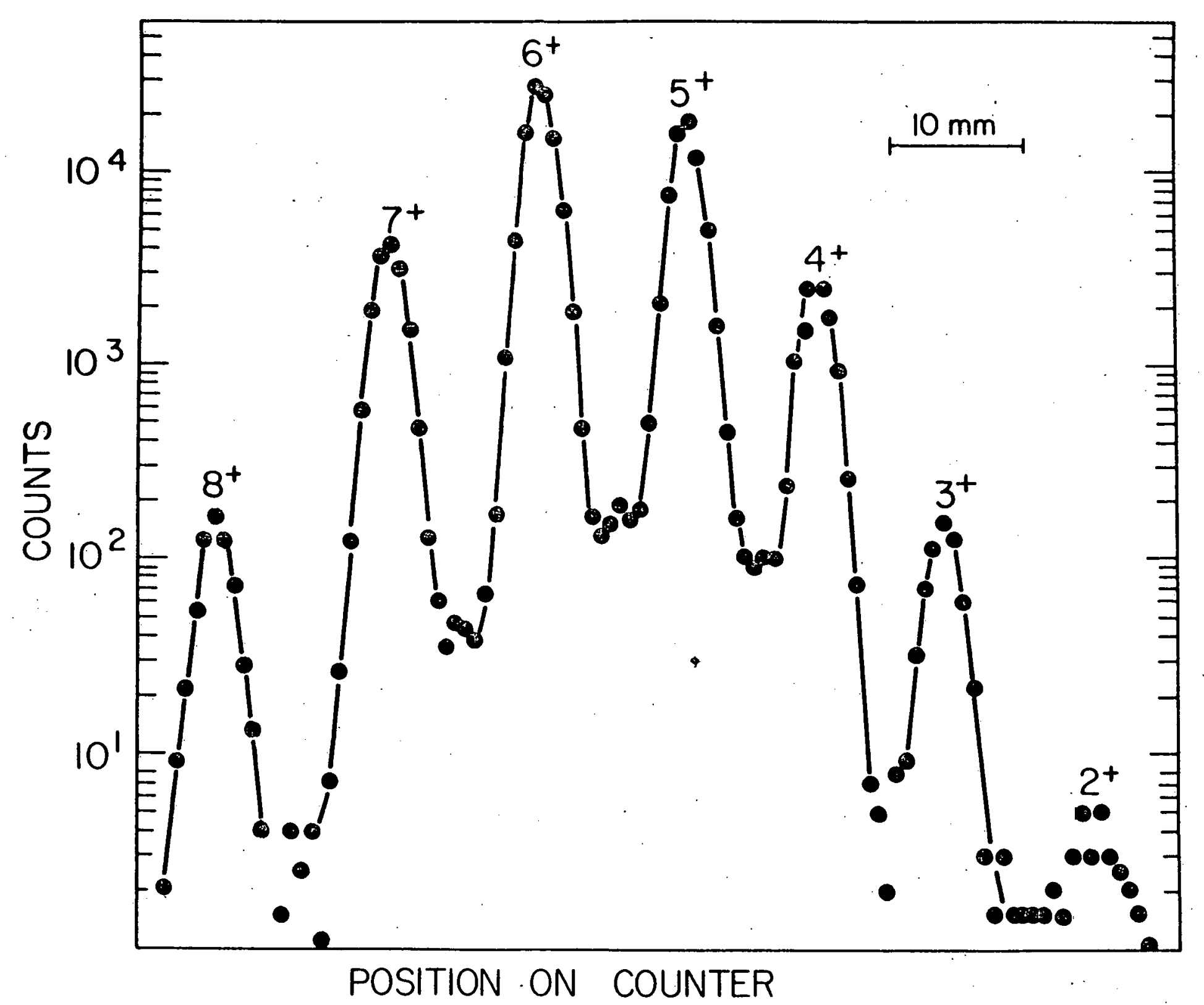

Figure 2 


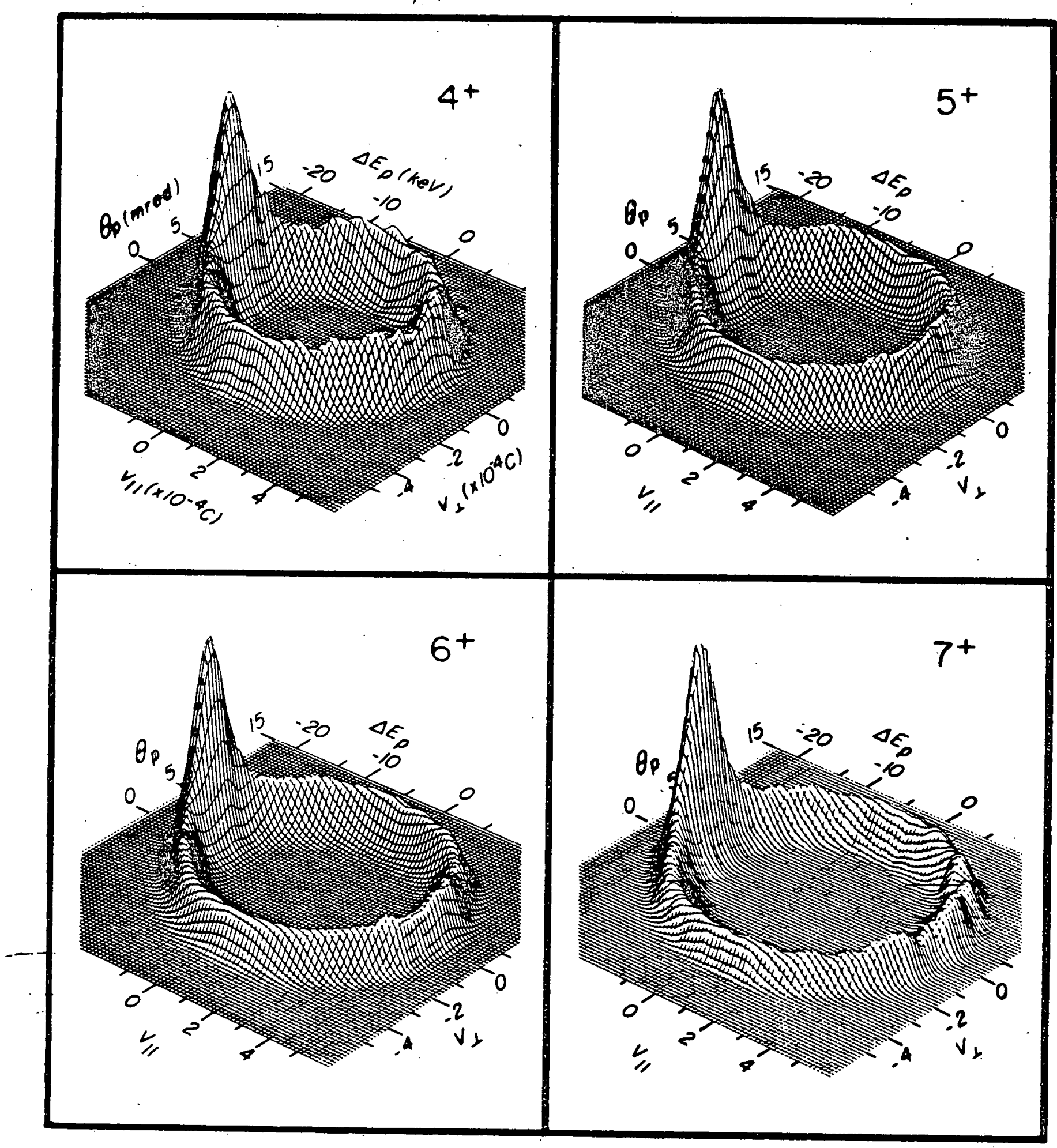

Figure 3 


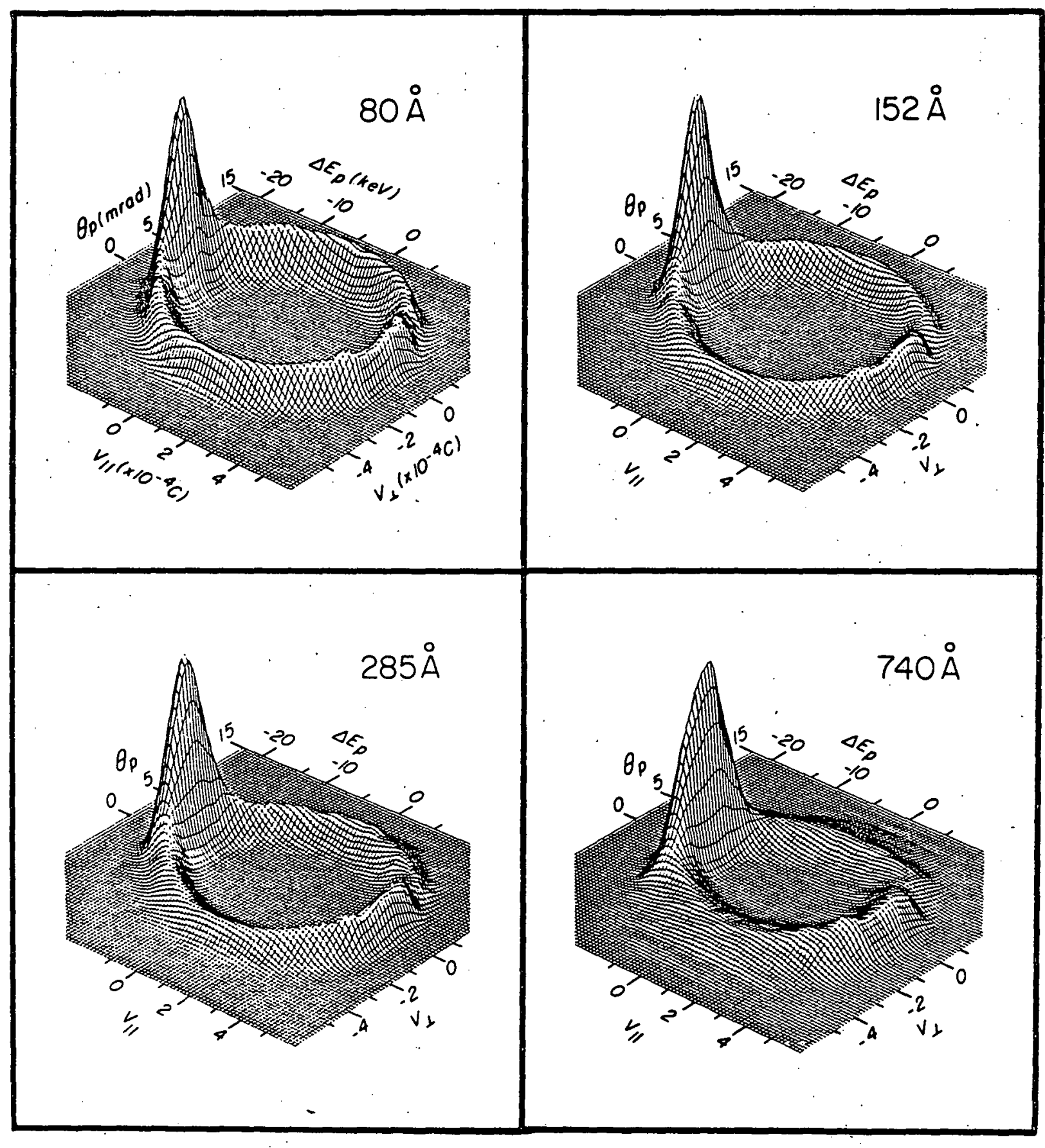

Figure 4 


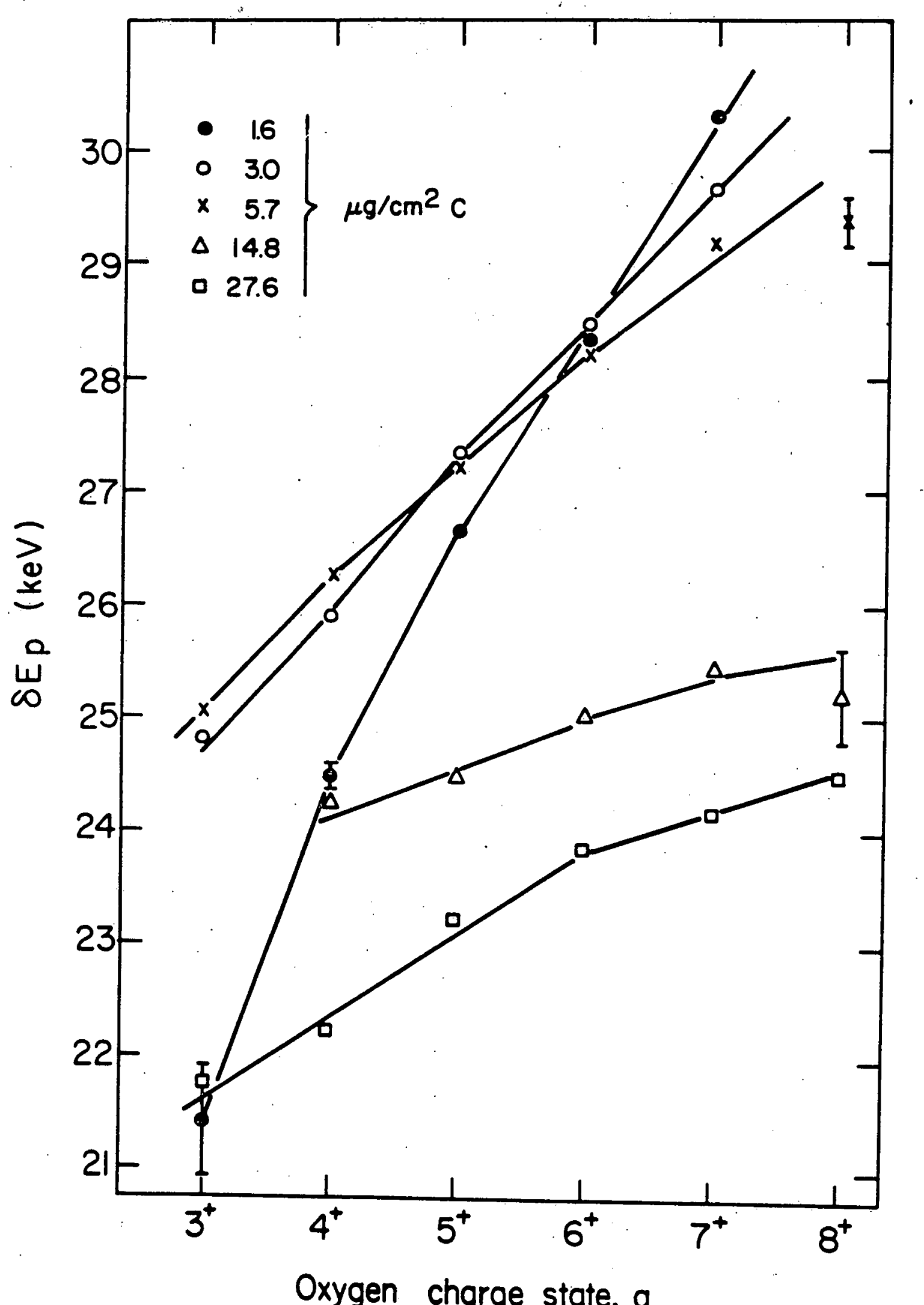

Figure 5 


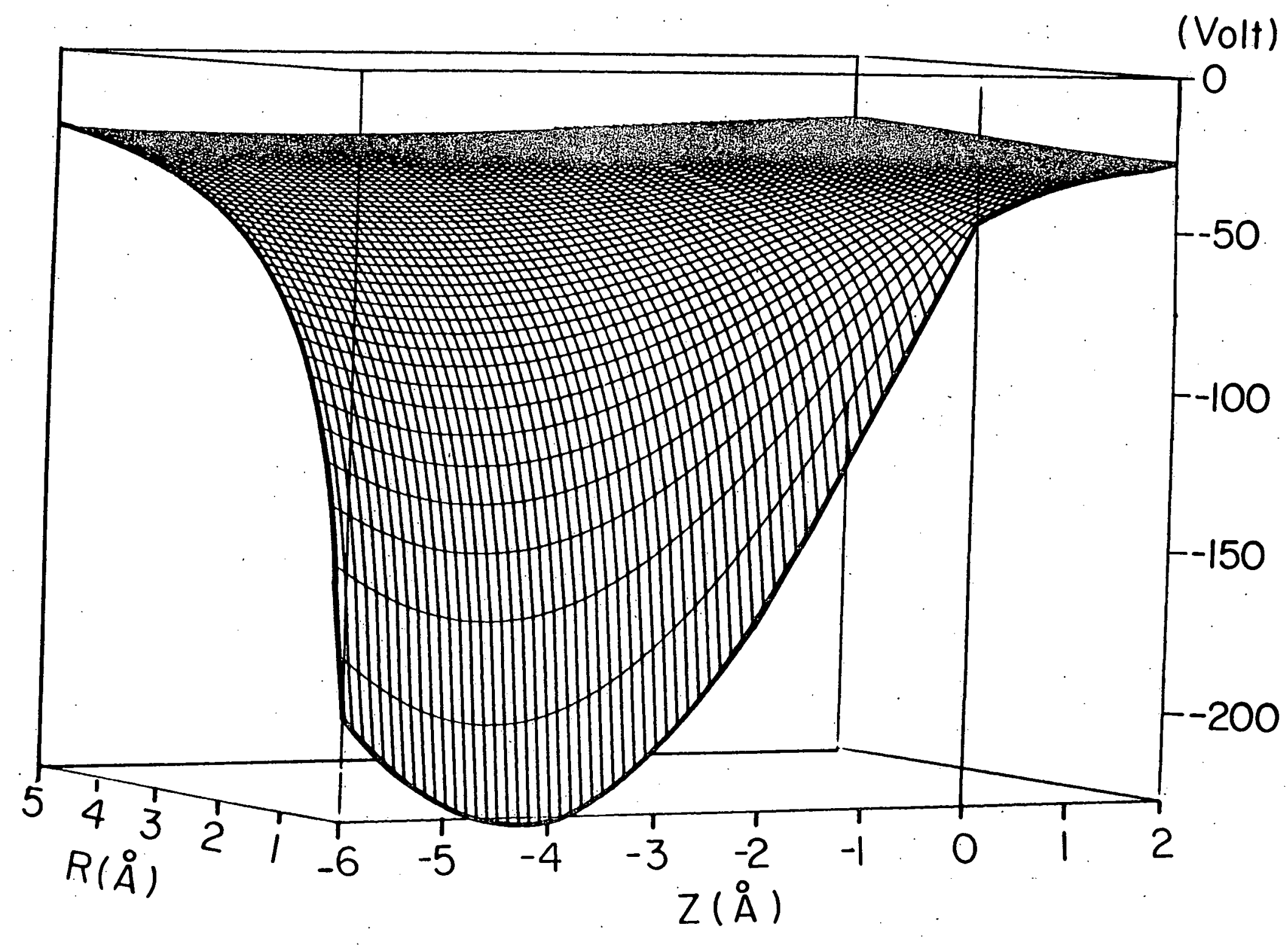


(Volt)

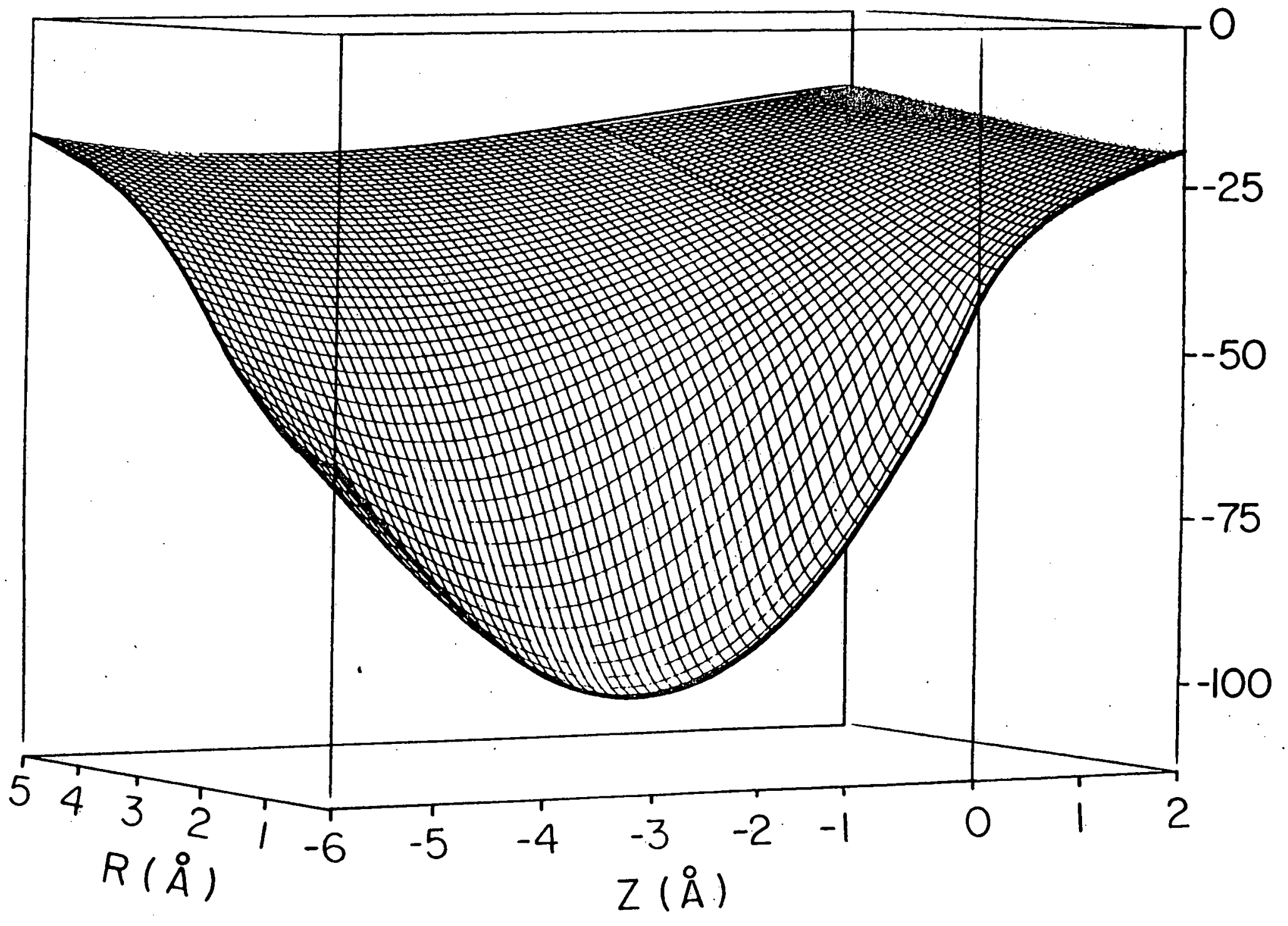

F1gure $6 b$ 
(Volt)

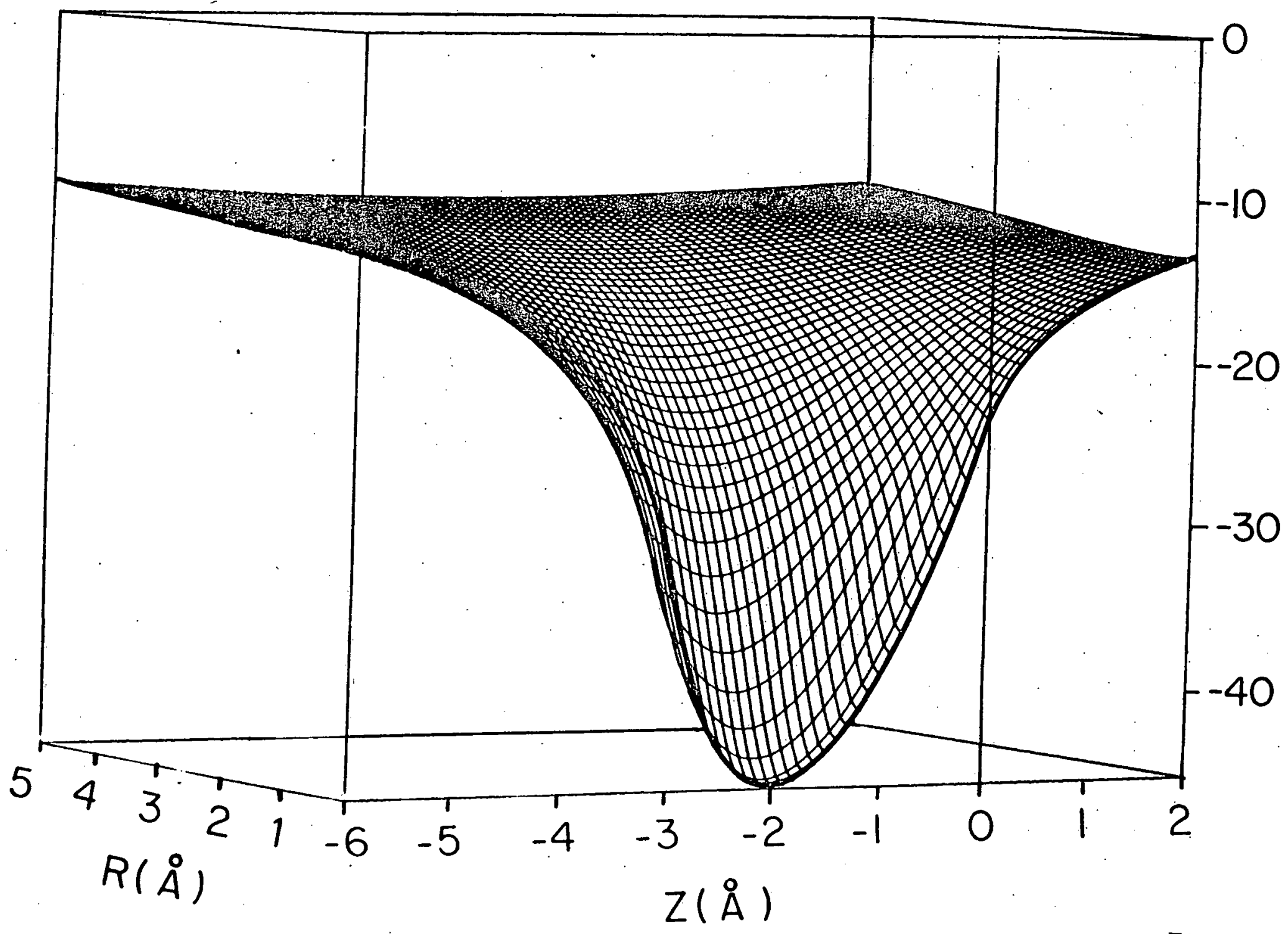





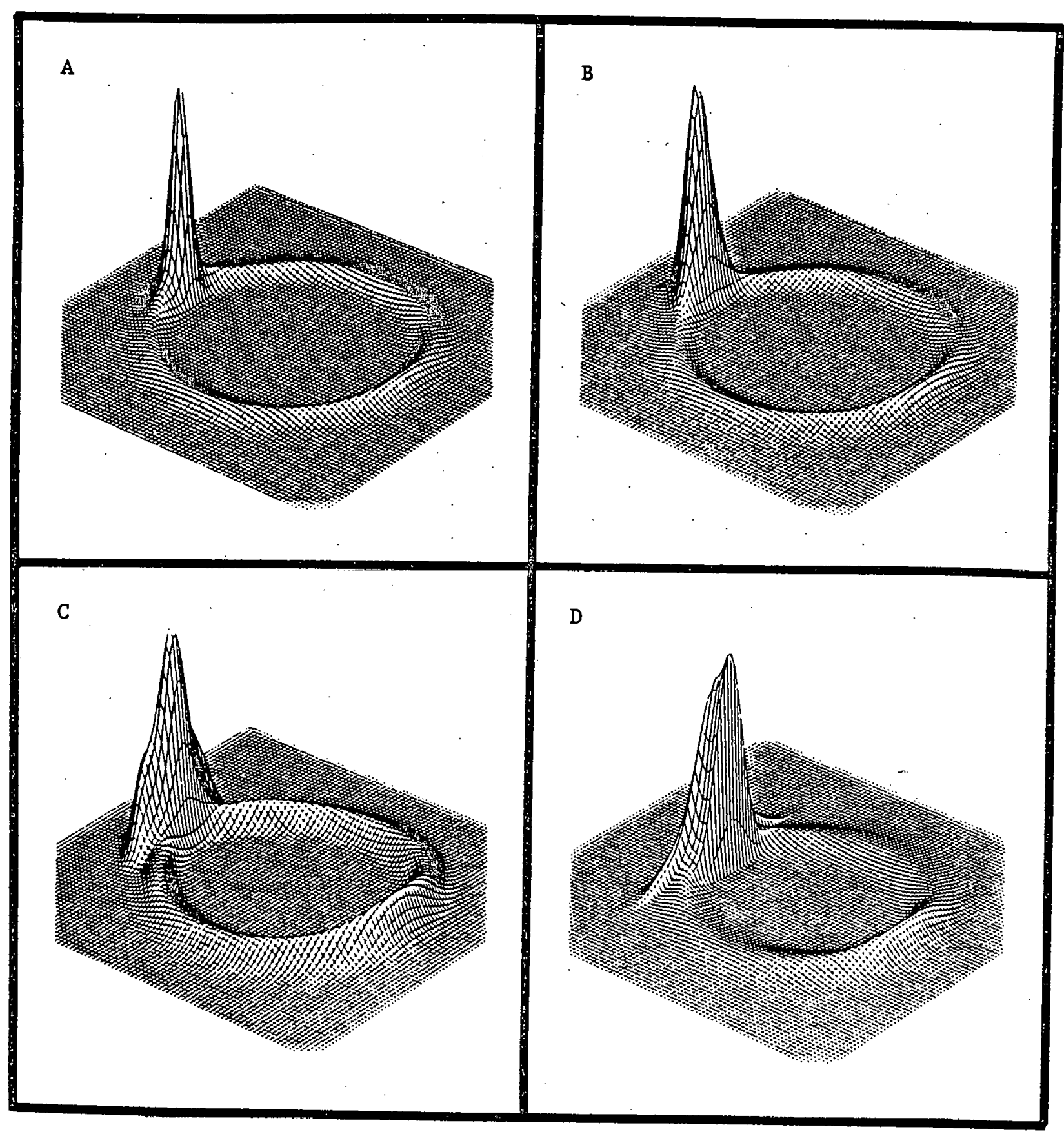

Figure 8 


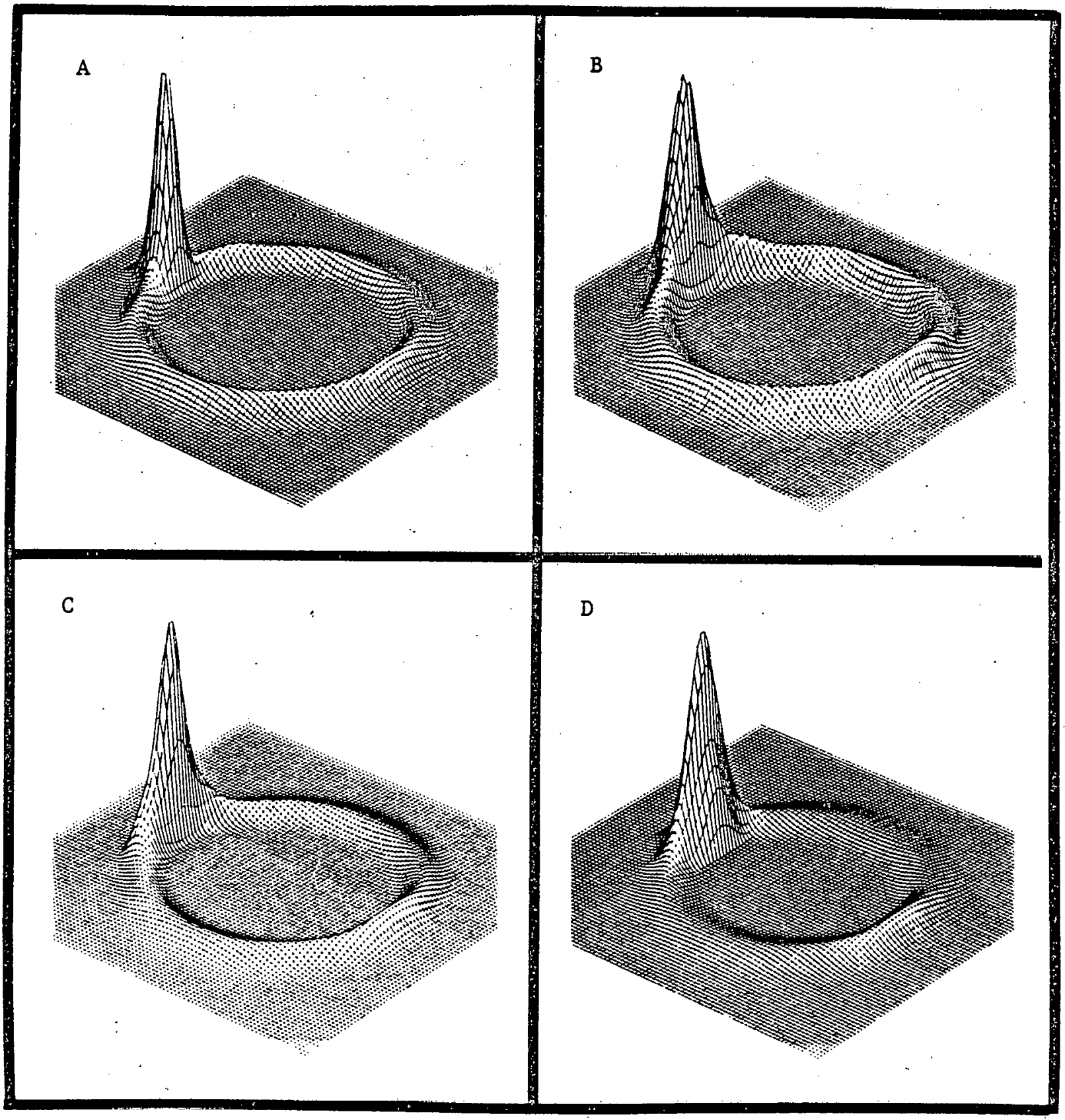




\title{
$\mathrm{C}_{3,4}^{-}$Molecular Ion Structure Determination by Coulomb Explosion G. Goldring
}

Weizmann Institute of Science, Rehovot, Israel

\author{
Y. Eisen \\ Center for Nuclear Research, Soreq, Israel \\ P. Thieberger and H. E. Wegner * \\ Brookhaven National Laboratory, Upton, N.Y. 11973
}

\section{INTRODUCTION}

The purpose. of this experiment is to acquire structural information about multiple atom carbon molecules through their Coulomb explosion in thin carbon foils. The data presented here relate to the structure of $\mathrm{C}_{3}^{-}$and $\mathrm{C}_{4}^{-}$ molecular ions where the $\mathrm{C}$ ions resulting from the Coulomb explosion were detected in polycarbonate sheets. A preliminary analysis of the $\mathrm{C}_{3}^{-}$molecular structure data has been made and is presented here; however, the $\mathrm{C}_{4}^{-}$ molecular data has not yet been analyzed. In the future, it is planned to employ electronic multidimensional detectors with computer storage and analysis to improve the present study and extend it to carbon molecules as large as $\mathrm{C}_{12}^{-}$

\section{EXPERIMENTAL PROCEDURE}

The injector MP tandem, which is the first accelerator of the Brookhaven National Laboratory three-stage Van de Graaff facility, is equipped with a sputter ion source ${ }^{1}$ in the high voltage terminal that provides negative ions for three-stage acceleration. This source produces carbon molecular ions 
ranging from $\mathrm{C}_{2}^{-}$to $\mathrm{C}_{12}^{-}$as illustrated in Fig. 1 which shows the mass spectrum from the ion source when it is being operated with a carbon cone. The data shown were taken with an external source similar to that used in the high voltage terminal and the negative carbon molecular ions were identified by accelerating them through the tandem accelerator and measuring the magnet1c rigidity of the resulting positive carbon ions.

In this experiment, the terminal ion source was used to provide accelerated $\mathrm{C}_{3}^{-}$and $\mathrm{C}_{4}^{-}$molecular ions wh1ch were then magnetically analyzed for proper mass identification followed by supercollimation as shown in Fig. 2 . The two adjustable apertures are followed by a permanent magnet and adjustable steering magnet that provide a $5^{\circ}$ deflection of the negative ions with the same mass product as the $90^{\circ}$ analyzing magnet. This final bend removes all of the negative ions that have been slit-or gas-scattered because they are then either neutral or positive and consequently removed from the beam: Horizontal and vertical cleanup slits adjusted to be close to but not touching the supercollimated beam ensure an extremely clean microbeam with sharp edges of a size varying from 0.05 to $1.0 \mathrm{~mm}$ diameter, depending on the selected aperture settings. After the beam is steered through the various apertures it is decreased in intensity to a few thousand ions per second as monitored by a solid state detector. Final steering adjustments are then made to ensure that the beam is going through the center of a $1 \mu \mathrm{g} / \mathrm{cm}^{2}$ carbon stripping foil which is mounted on a $3 \mathrm{~mm}$ diameter foil holder.

Once the final steering correction has been made, the intensity is decreased to approximately 2-5 counts/second by decreasing the aperture sizes further and closing down the object slit of the $90^{\circ}$ analyzing magnet with all quadrupoles in the beam transport turned off to aid in the attenuation and to Increase the optical quality of the beam. 
The polycarbonate plastic sheets ${ }^{2}$ are $0.37 \times 3 \times 5 \mathrm{~cm}$ in dimension and mounted as shown schematically in Fig. 2. The holder is rotated back and forth and raised and lowered manually. The holder is stepped vertically $2 \mathrm{~mm}$ before each rotary scan and a uniform exposure of $10-20$ events $/ \mathrm{cm}^{2}$ would result if the beam current were absolutely stable. Unfortunately, beam current fluctuations cause frequent event clustering. However, many single events are observed. After exposure, the plates are developed by a combined chemical and electrical etching technique and a typical exposed plate is shown in Fig. 3. A number of data in each exposure are artifacts of various kinds and can be eliminated by observation through a microscope with a magnification of $100 x$.

Figures 4 and 5 show examples of isolated three and four carbon events corresponding respectively to $\mathrm{C}_{3}^{-}$and $\mathrm{C}_{4}^{-}$ions of 4.67 and $3.50 \mathrm{MeV}$. The jagged edge spots are characteristic of heavy ion events with this etching technique. A few events show all three atoms of the $C_{3}^{-}$molecule anonymously closely. spaced as shown in the lower right hand example of Fig. 4. These events could be due to molecular disassociation by collisions with residual gas molecules in the transport system followed by subsequent stripping in the carbon foil with very little or no Coulomb explosion effect. Such events illustrate the excellent spatial resolution of this detection method which is smaller than the diameter of the spots.

The energy of the carbon atoms in the $\mathrm{C}_{4}^{-}$exposure is $875 \mathrm{keV}$; the lowest energy so far recorded by this method. The detection efficiency appears to be close to $100 \%$ even at this low energy.

\section{DISCUSSION}

Altogether $37 \mathrm{C}_{3}^{-}$events and $21 \mathrm{C}_{4}^{-}$events have been identified unambigously and recorded. A rigorous analysis of these specimens would have to take into 
account, in addition to the molecular parameters, the charge distribution of the carbon ions (somewhat different from atomic carbon ${ }^{3}$ ), multiple scattering and the projection statistics; however, the limited number of events at present does not justify such a complete and rigorous treatment. Instead, a simplified procedure was adopted for the $\mathrm{C}_{3}^{-}$events in an attempt to extract some basic structure information from the data.

The eight largest triangles, having at least one side larger than 11 mrad, were selected for this analysis and are shown in Fig. 6. I twas considered likely that the largest side of these triangles is either in the projection plane or at a small angle to it. This conclusion is also supported by the fact that the length of the largest side corresponds to a Coulomb explosion of $\mathrm{C}_{3}^{-}$ions with an internuclear spacing of about $1 \AA$. If the largest side were at a large angle to the projection plane, the internuclear separation would be unreasonably smal1. If the long leg of the triangle is coplanar as assumed, then the events shown in Fig. 6 are projections obtained by rotating the original triangle around the largest side. The eight triangles are then found to be consistent with random projections of a Coulomb exploded equilateral triangle taking into account the possible different charge states, but they are not necessarily equilateral triangles. Figure 7 shows as an example the relation of the apex angle of an isosceles triangle molecular structure of equal mass and charge particles before and after a Coulomb explosion. Although the equilateral triangle structure transforms into itself, there is also a large range of initial isosceles triangles which generate final apex angles close to $60^{\circ}$. It is found in this way that the apex angle of the $c_{3}^{-}$molecules can be as large as $140^{\circ}$ and the present data are also compatible with the carbons vibrating around an aligned configuration with a bending amplitude of at least $\pm 40^{\circ}$. 
The closest known molecules to $\mathrm{C}_{3}^{-}$are $\mathrm{C}_{3}$ and $\mathrm{CNC}$ (isoelectronic with $\mathrm{C}_{3}^{-}$). The $\mathrm{C}_{3}$ molecule is linear and "floppy", having a very low bending frequency. of $65 \mathrm{~cm}^{-1}$ while CNC is linear and quite rigid. ${ }^{4}$ we can conclude from our data that $\mathrm{C}_{3}^{-}$is unlike the rigid CNC molecule (unless the $\mathrm{C}_{3}^{-}$ions are formed preferentially in excited vibrational states) but could be similar to $\mathrm{C}_{3}$, an extremely floppy linear molecule.

*Research supported by the Department of Energy under Contract No. EY-76-C-02-0016.

\section{REFERENCES}

1. Hiconex Model $832 \mathrm{Cs}$-Ion source manufactured by the General Ionex Corp., 19 Gras Rd., Newburyport, Ma. 01950.

2. R. A. Oswald and R. V. Wheeler, 22nd Annual Meeting of the Health Physics Society, Atlanta, Ga, 1977; and $\mathrm{Y}$. Eisen, et al., to be published in Health Physics.

3. P. Thieberger, Cluster Effects on Charge State Distributions for Heavy Ion Molecular Beams, Proceedings of this Conference.

4. We are Indebted to Prof. G. Herzberg for these considerations. 
FIGURE CAPTIONS

Fig. 1 Mass spectrum of negative ions produced by a cesium sputter ion source operated with a carbon cone. The numbers indicate the molecular mass of multiple carbon ions which were positively identified.

Fig. 2 Schematic layout of the experimental arrangement for super collimation of the accelerated negative molecular lons and the mechanical arrangement for exposing polycarbonate plastic sheets to the Coulomb exploded ions. (See text for details.)

Fig. 3 Etched polycarbonate foil after exposure to stripped $4.67 \mathrm{MeV}$ $\mathrm{C}_{3}^{-}$ions.

Fig. 4 Further magnification of selected $\mathrm{C}_{3}^{-}$molecular explosion events at $4.67 \mathrm{MeV}$ ion energy:

Fig. 5 Selected $\mathrm{C}_{4}^{-}$molecular explosion events at $3.50 \mathrm{MeV}$ ion energy.

Fig. 6 Plots of largest observed $\mathrm{C}_{3}^{-}$triangles.

Fig. 7 Initial and final apex angles of isosceles triangles of three particles of equal mass and charge experiencing a Coulomb explosion. 


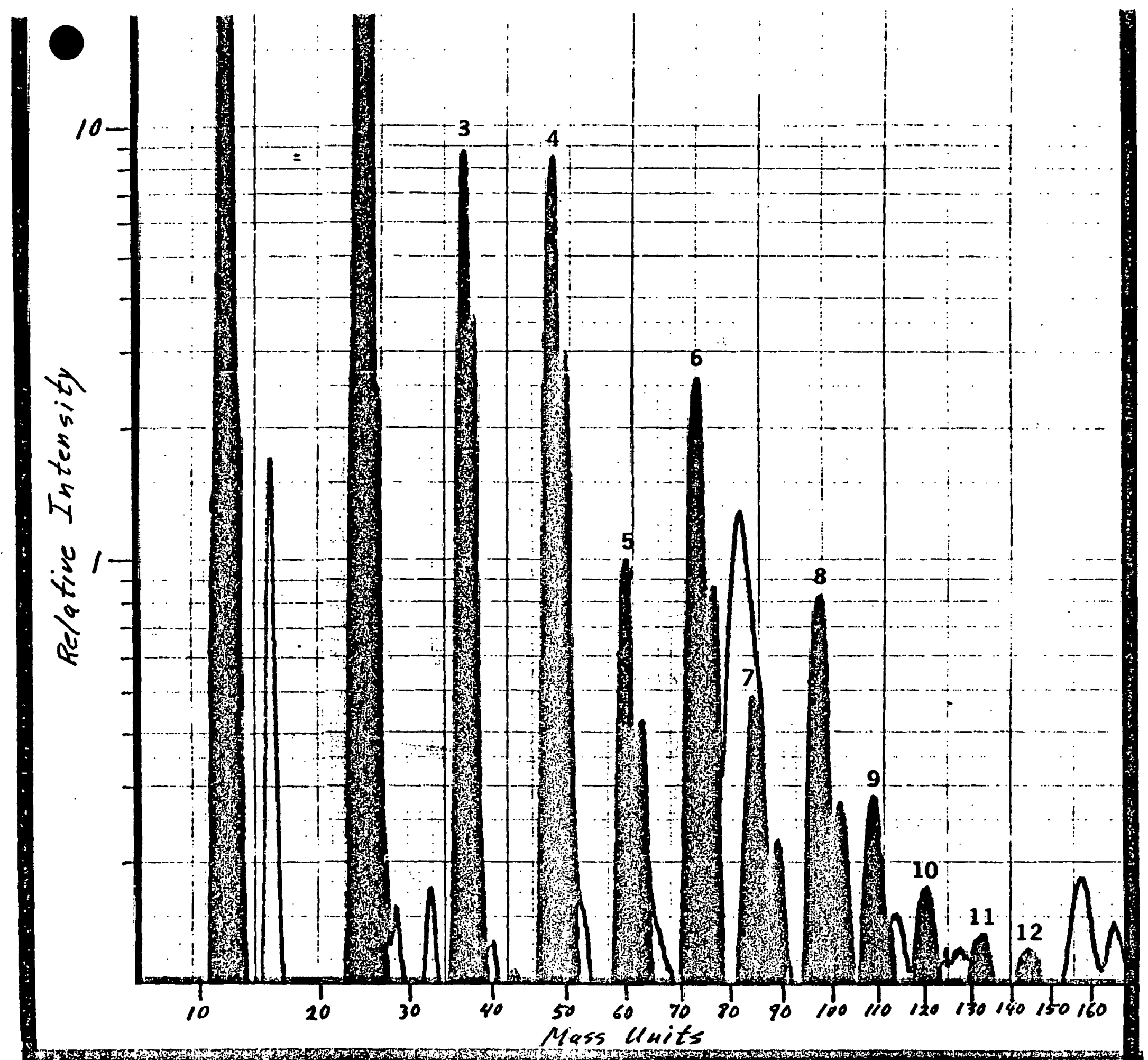

$\underset{\omega}{\omega}$

Fig 1 


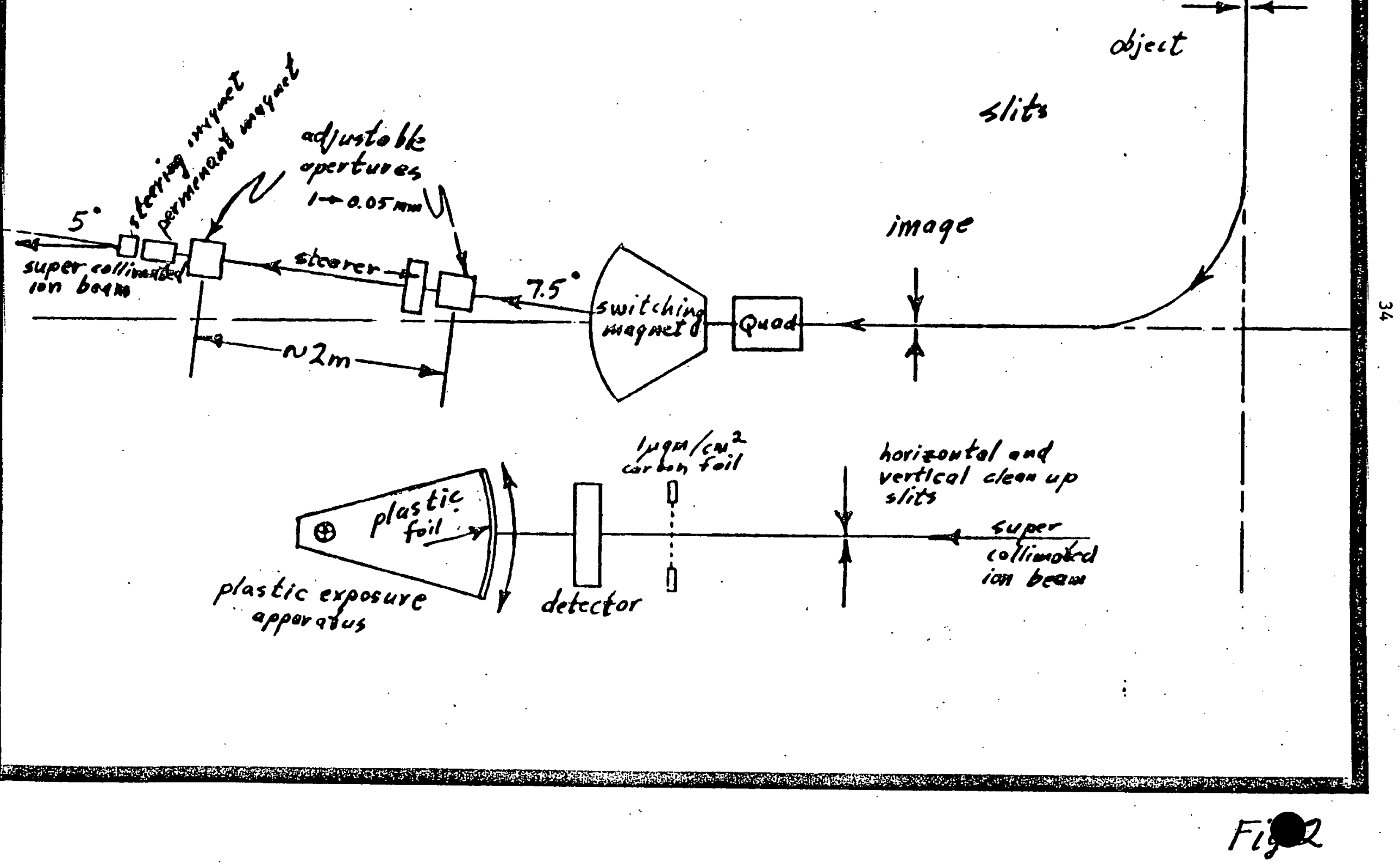




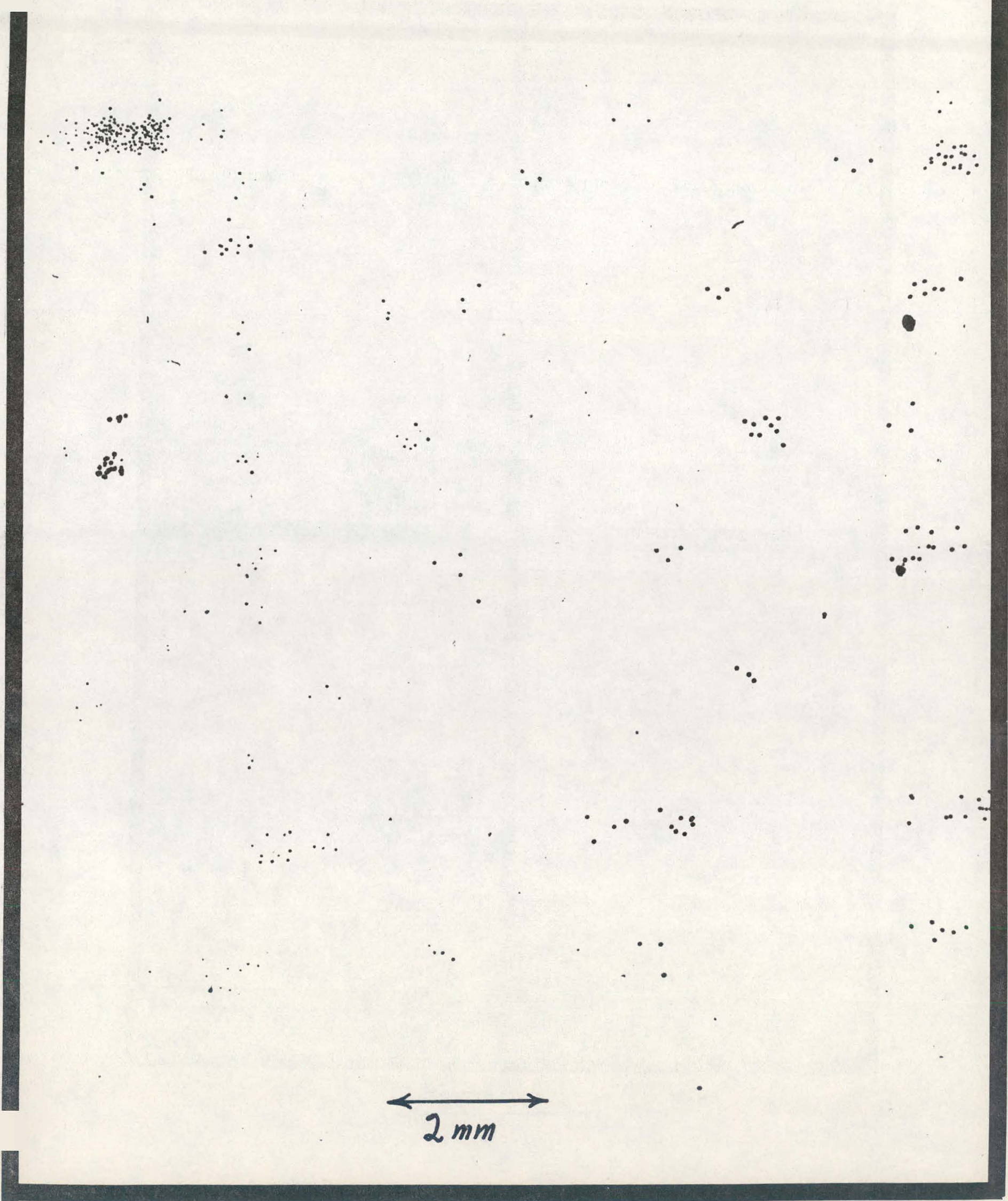

Fiq3 


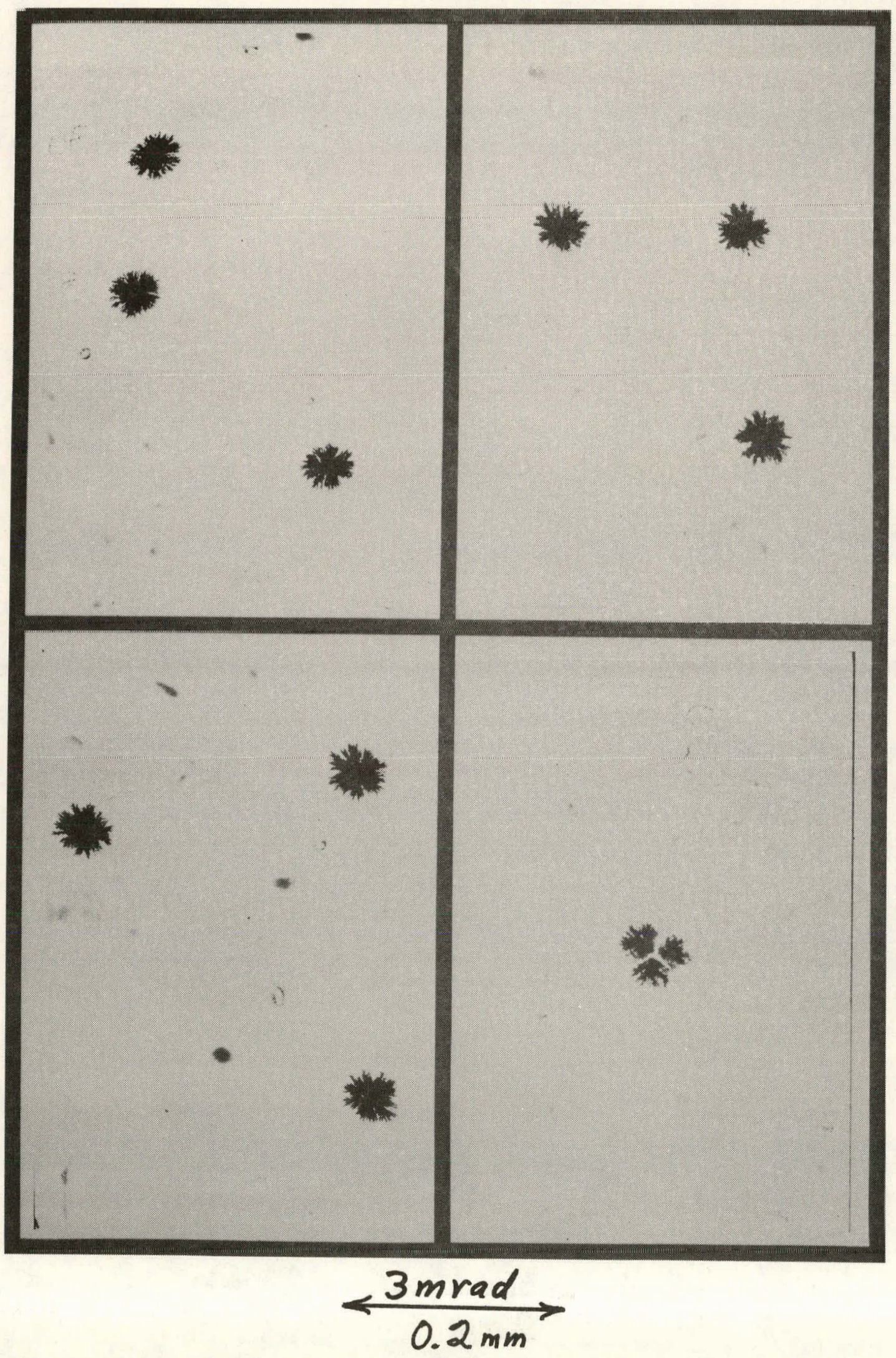




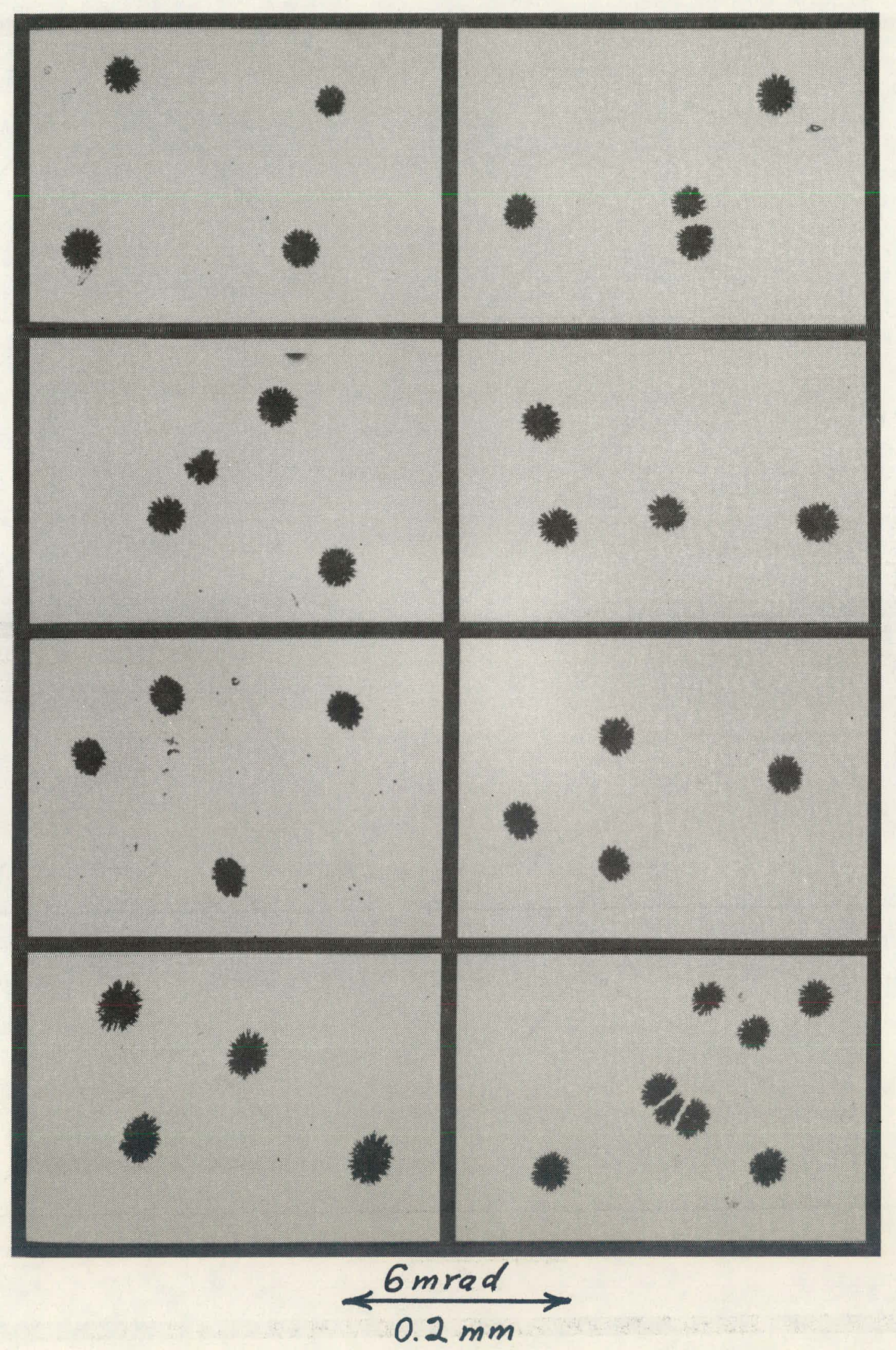



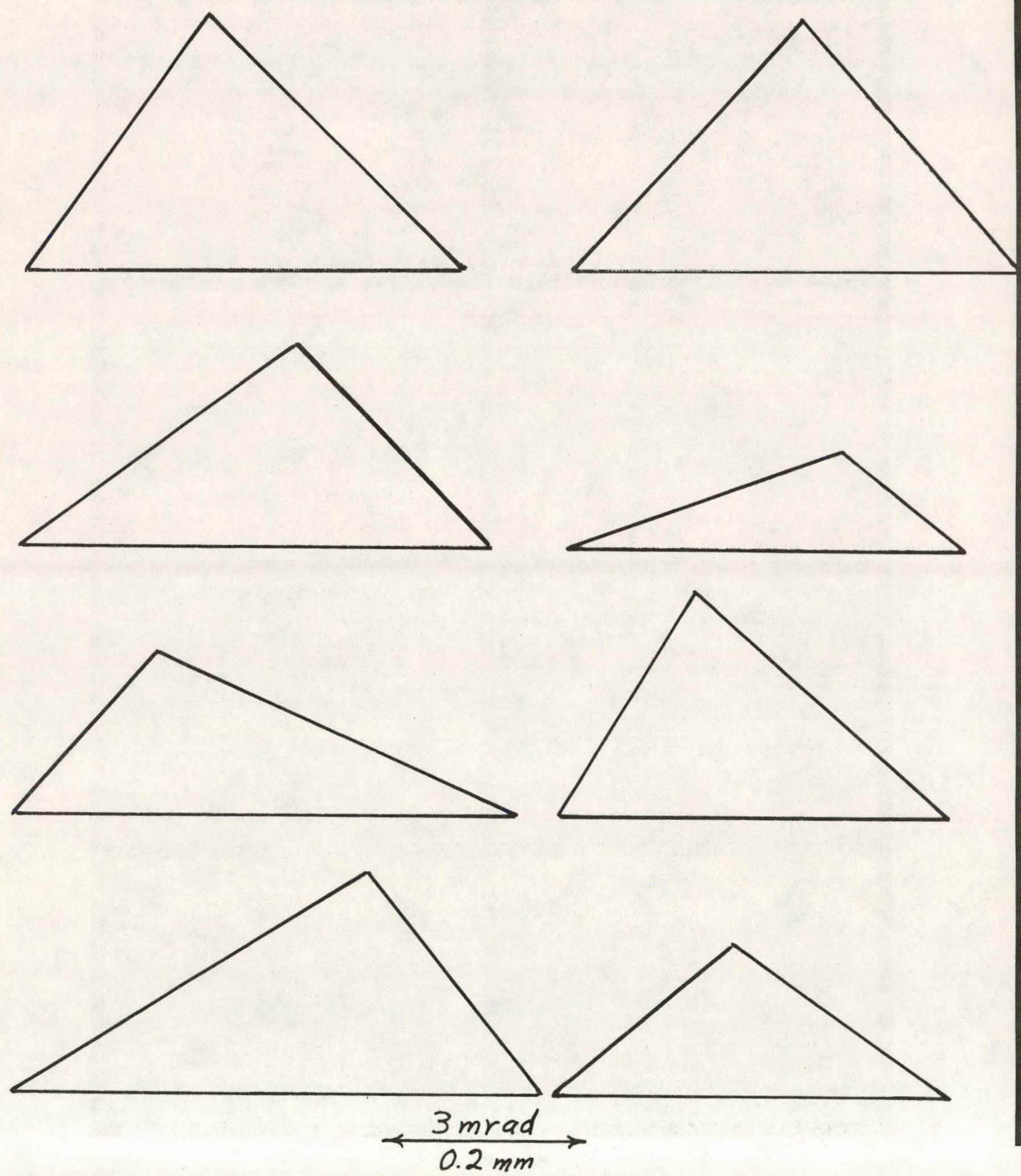

Fig 6 


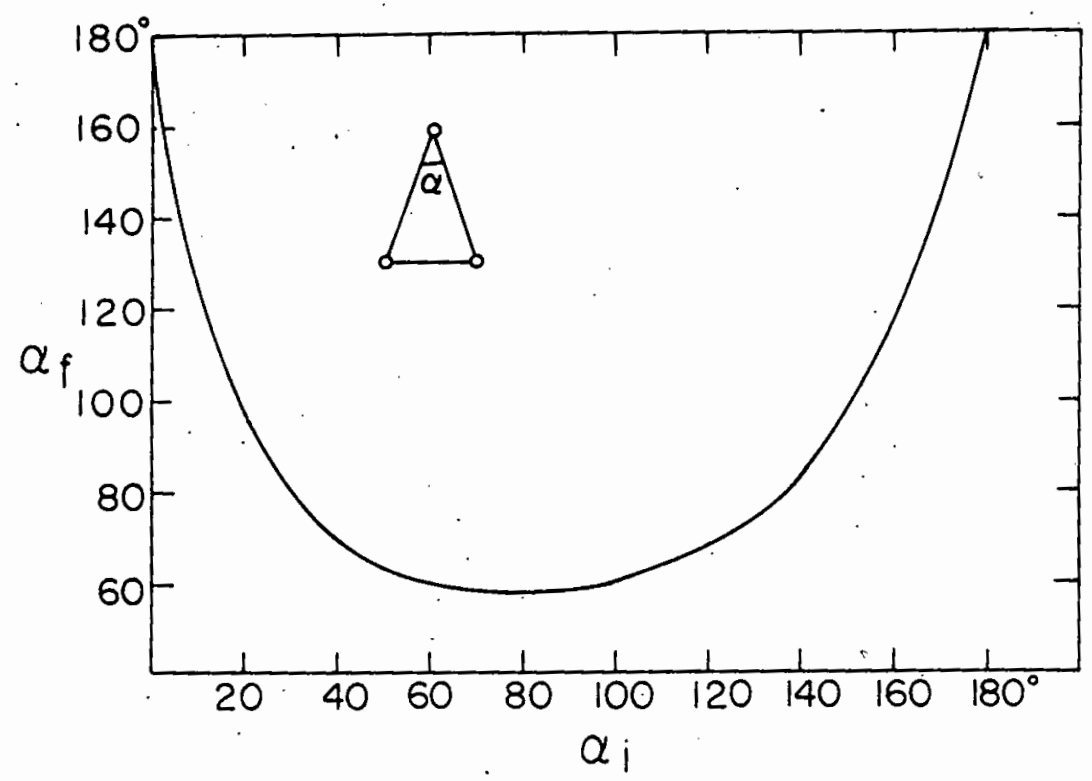

Fig. 7 
THIS PAGE

WAS INTENTIONALLY

LEFT BLANK 
Cluster Effects on Charge State Distributions for Heavy Ion Molecular Beams*

P. Thieberger

Brookhaven National Laboratory, Upton, NY 11973

The charge state distributions of ions emerging from carbon foils were compared for equal velocity monoatomic and polyatomic negative ions incident on the foil. Such comparisons were performed for $\mathrm{O}_{2}^{-}$vs $\mathrm{O}^{-}$using foil thicknesses from 1 to $55 \mu \mathrm{g} / \mathrm{cm}^{2}$ and for $\mathrm{C}_{3}^{-}$vs $\mathrm{C}^{-}$and $\mathrm{C}_{4}^{-}$vs $\mathrm{C}^{-}$using a single foil thickness of $1 \mu \mathrm{g} / \mathrm{cm}^{2}$. The experimental arrangement is shown in Fig. 1. A monitor detector close to the target and a digital current integrator used for the carbon measurements are not shown. The carbon measurements were performed in collaboration with H. E. Wegner in conjunction with the stereo-chemistry experiments reported elsewhere in these proceedings. Figures 2 and 3 show measured charge state distributions for carbon and oxygen, respectively, and Fig. 4.shows how the observed effect varies with foil thickness for the oxygen case. In ail cases, the distribution is shifted towards lower average charge states for the molecular beams and the effect persists, even though attenuated, up to and including the thickest foils used.

The most straightforward interpretation of these results is similar to the interpretation of the cluster effect for neutral hydrogen production from $\mathrm{H}_{2}{ }^{+}$ions. $^{1}$ Namely, that for each component of the cluster there are more correlated electrons available for pickup than for single ions. A comparison of these results to those of hydrogen shows that the effect is 
much larger because it clearly manifests itself in the average charge state rather than in the rare neutral fraction and because it persists much longer as a function of foil thickness or dwell time. Also, no short-dwelltime effect is observed which would have been of opposite sign to that for positive molecules.

The interatomic distance $\left(R_{A B}\right)$ distribution at the foil exit was estimated for the $\mathrm{O}_{2}^{-}$experiments. Figure 5 shows the results of a Monte Carlo-type computer simulation in which the foils were divided into thin layers and only uncorrelated multiple scattering was considered in each layer while a screened Coulomb explosion was superimposed for the curve shown in Fig. 6. (Multiple scattering is observed to play a doninant role in the thicker foils.) Figures $7 \mathrm{a}$ and $7 \mathrm{~b}$ show the fraction of pairs emerging within the atomic distances $R\left({ }_{A B}\right)$ smaller than 5 and $10 \AA$, respectively. In each case, the lower curves correspond to the calculation with Coulomb explosion included. If the average interatomic distances for the thickest foils are as large as indicated, then the persistence of the observed effect with increasing thickness seems surprising and difficult to understand.

${ }^{*}$ Research supported by the Department of Energy under. Contract No. EY-76-C-02-0016.

\section{REFERENCES}

1. M. J. Gaillard, J. C. Poizat, A. Ratkowski, J. Remillieux, and M. Auzas, Physical Review A 16, number 6, 2323 (1977). 


\section{FIGURE CAPTIONS}

Fig. 1 Schematic diagram of the experimental setup.

Fig. 2 Measured charge state distributions for $\mathrm{C}_{3}^{-}$and $\mathrm{C}_{4}^{-}$beams incident on $1 \mu \mathrm{g} / \mathrm{cm}^{2}$ carbon foils compared to distributions obtained with $\mathrm{C}^{-}$incident beams of the same velocities.

Fig. 3 Measured charge state distribution for a $3.36 \mathrm{MeV} \mathrm{O}_{2}{ }^{-}$beam incident on a $2 \mu \mathrm{g} / \mathrm{cm}^{2}$ carbon foil compared to the distribution obtained with a $\mathrm{O}^{-}$incident beam of the same velocity.

Fig. 4 Variation with foil thickness of the difference between average charge states for $\mathrm{O}_{2}^{-}$and $\mathrm{O}^{-}$incident beams for constant exit velocity. The black dot indicates the result of a test measurement in which a $10 \mu \mathrm{g} / \mathrm{cm}^{2}$ foil was replaced by two $5 \mu \mathrm{g} / \mathrm{cm}^{2}$ foils $1 \mathrm{~mm}$ apart. As expected the effect disappears.

Fig. 5 Calculated probability distributions of interatomic distances at the foil exit for three foil thicknesses with $210 \mathrm{keV} / \mathrm{amu} \mathrm{O}_{2}{ }^{-}$ incident beam. Only uncorrelated multiple scattering was considered.

Fig. 6 Calculated probability distributions of interatomic distances at the foil exit for three foil thicknesses with $210 \mathrm{keV} / \mathrm{amu} \mathrm{O}_{2}{ }^{-}$ incident beam. A screened Coulomb explosion and uncorrelated multiple scattering were considered.

Fig. 7 Calculated fractions of clusters emerging with interatomic distances smaller than $5 \AA$ and $10 \AA$ as functions of foil thickness for $210 \mathrm{keV} / \mathrm{amu}$ incident $\mathrm{O}_{2}{ }^{-}$beams. The upper curves correspond to only uncorrelated multiple scattering and for the lower ones the effect of a screened Coulomb explosion has been included. 


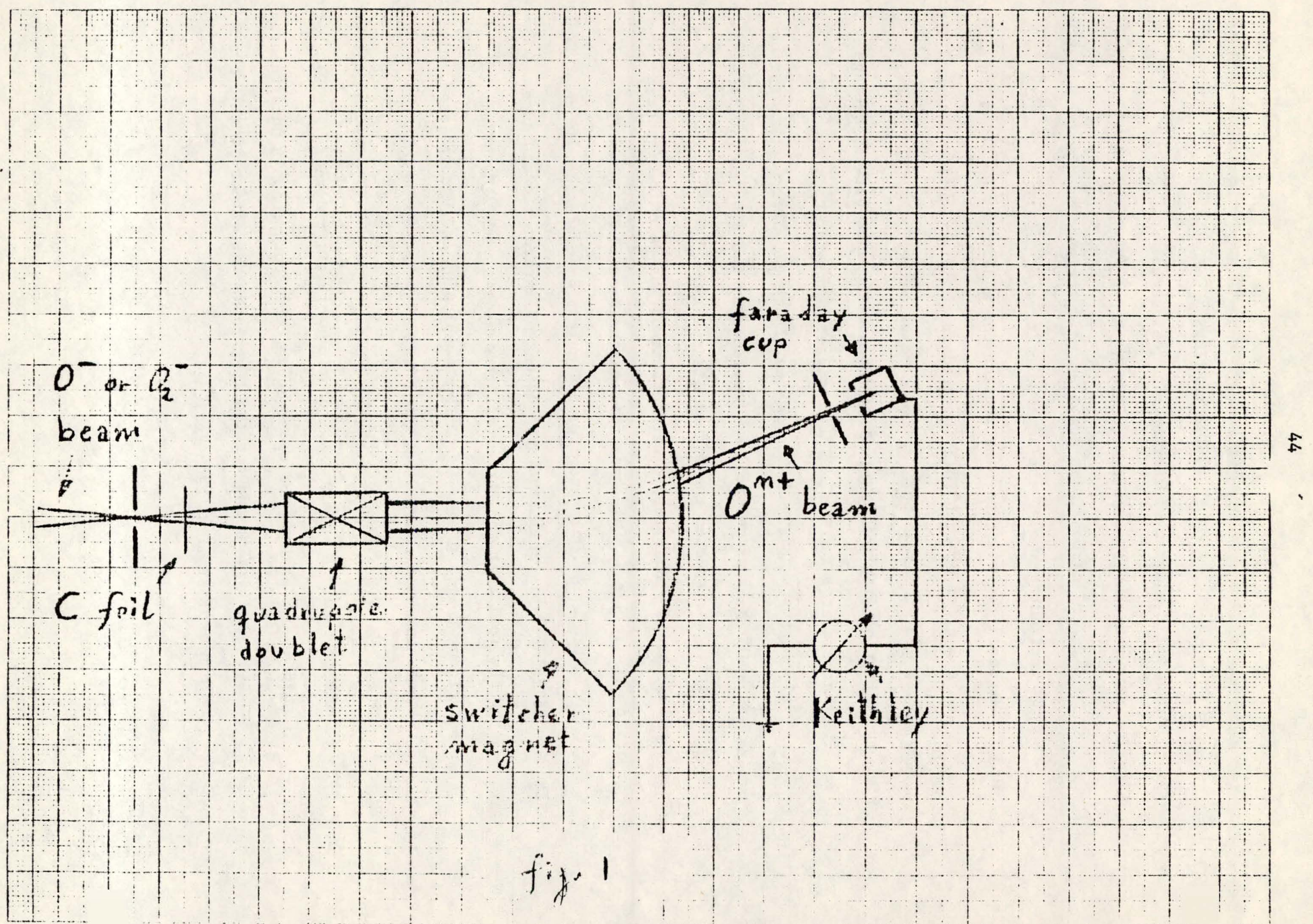




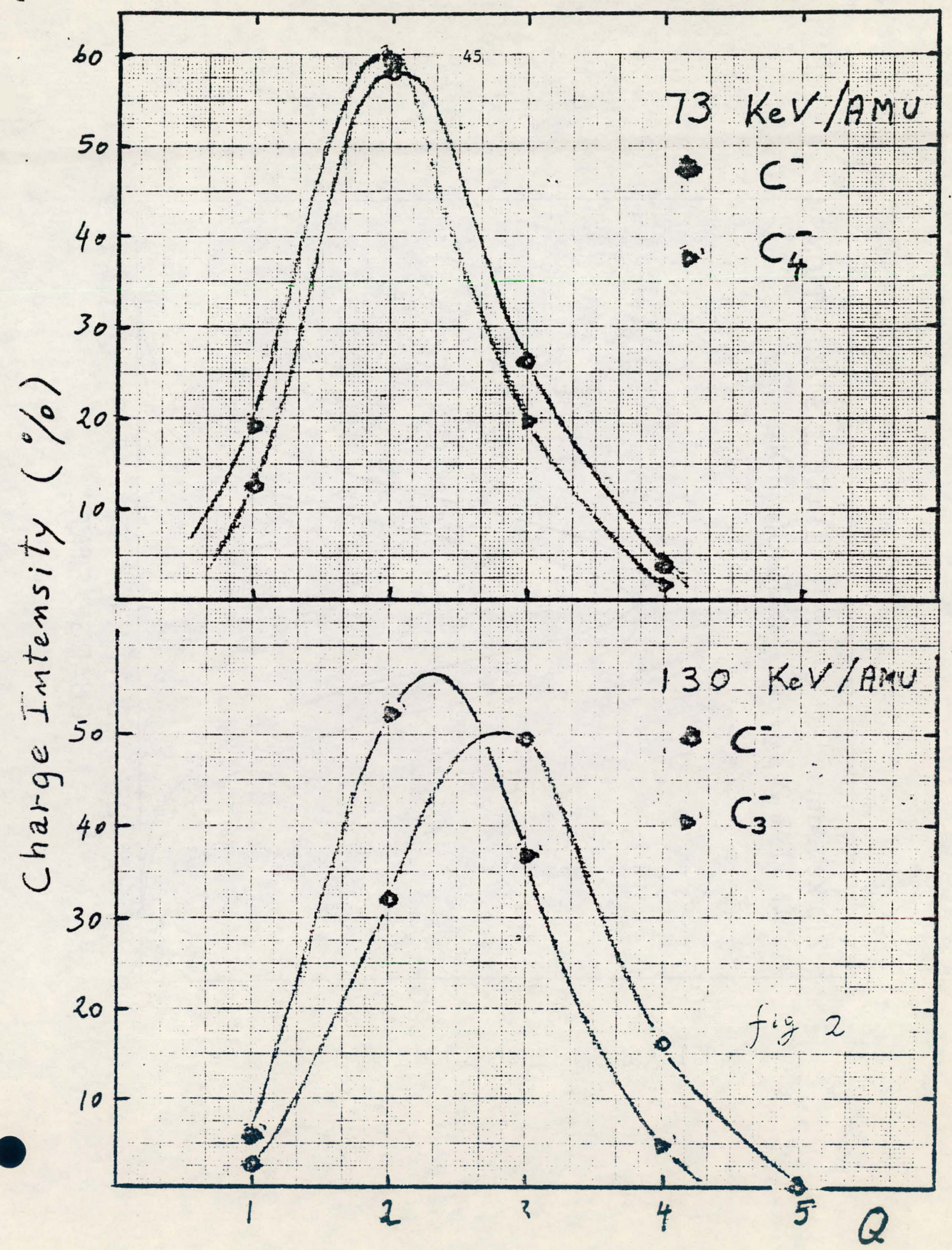




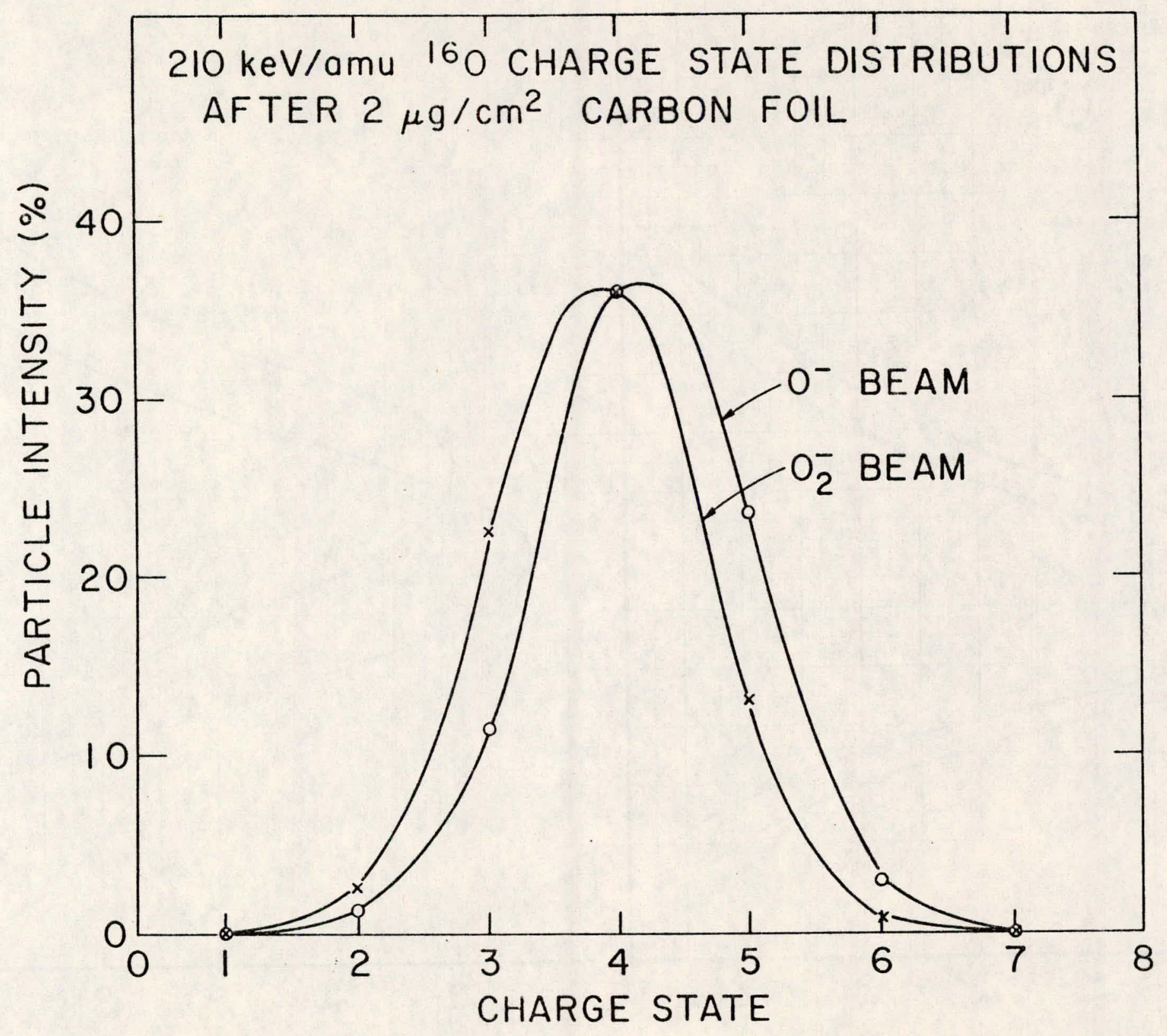




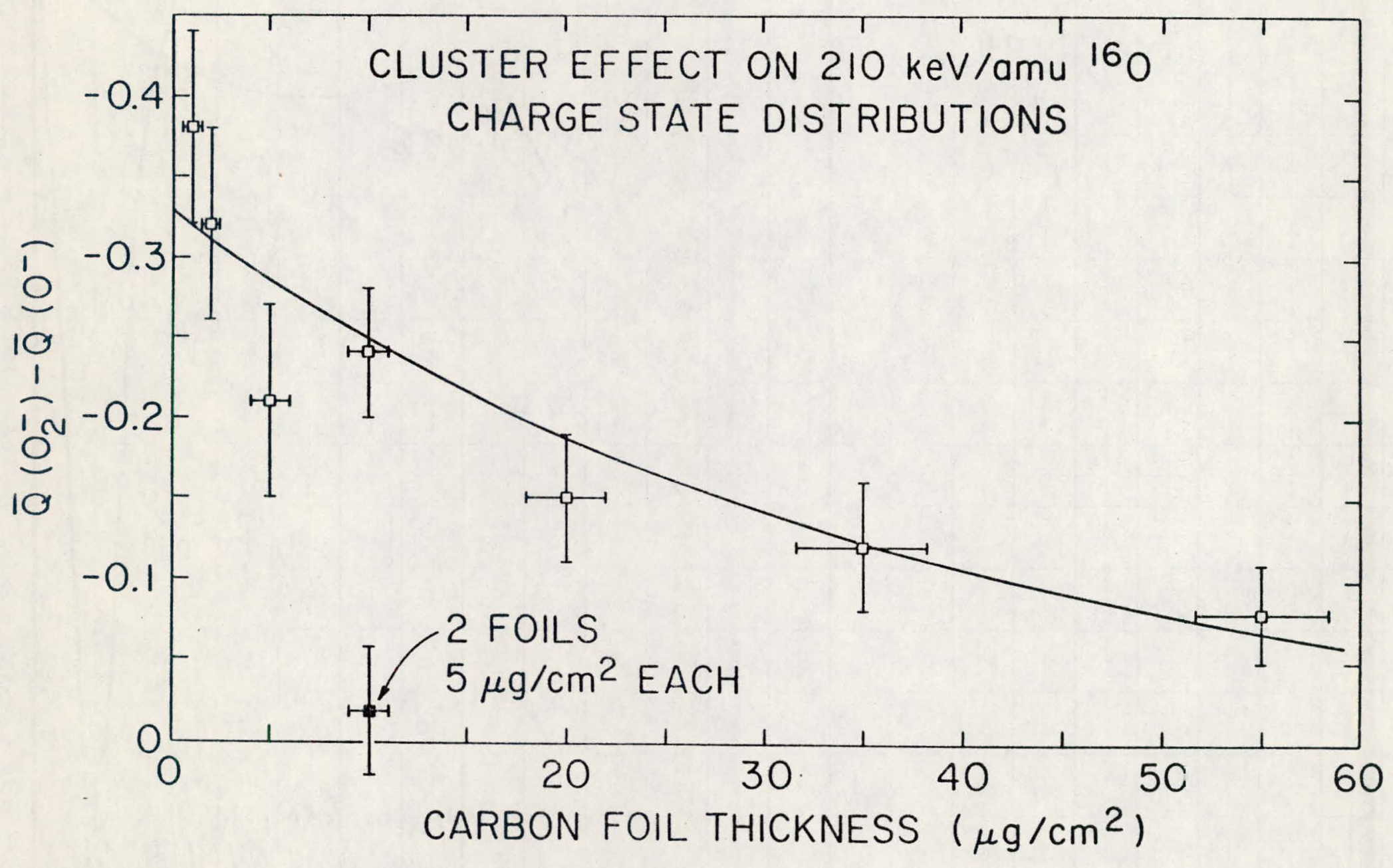




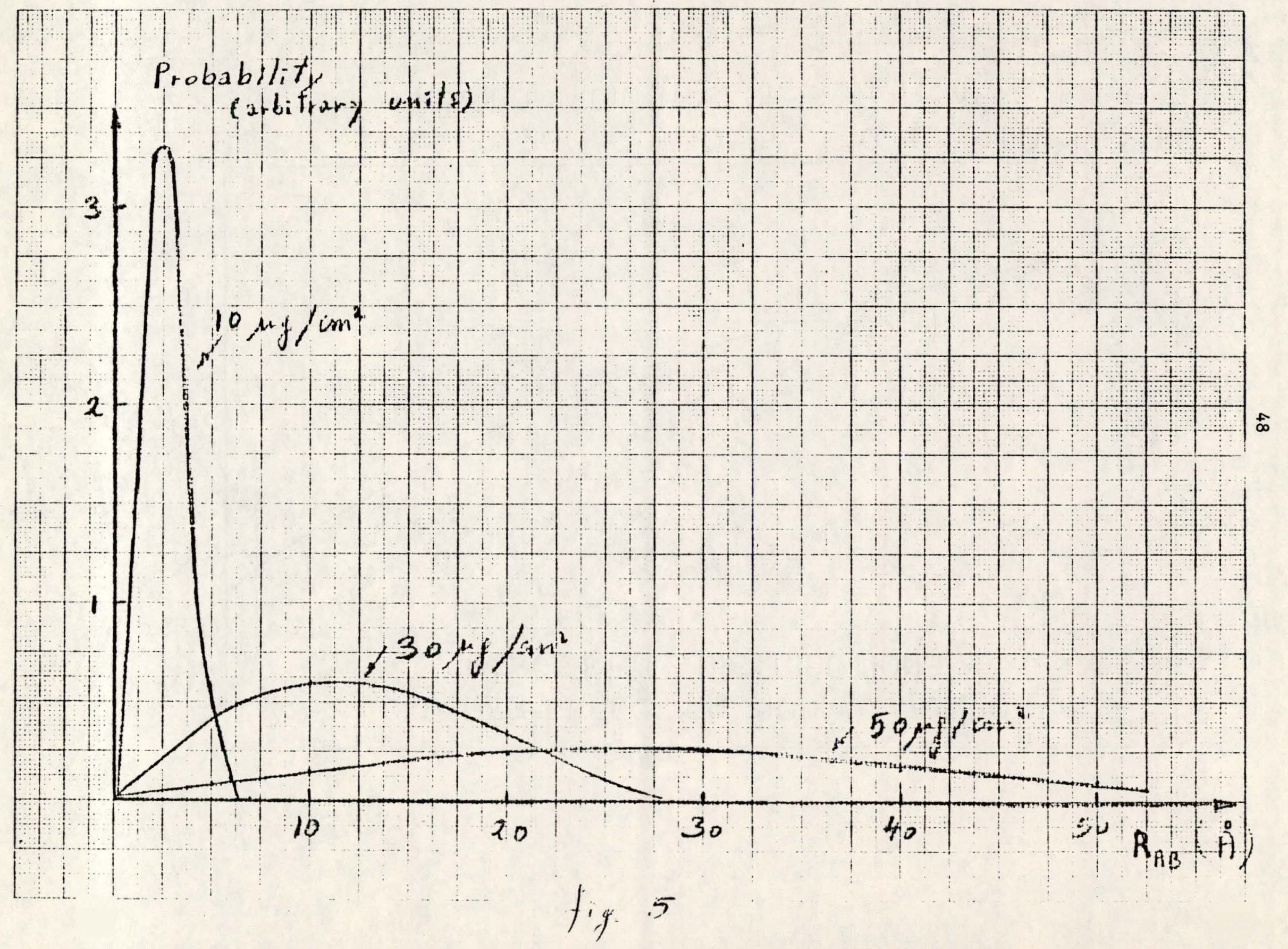


O , :

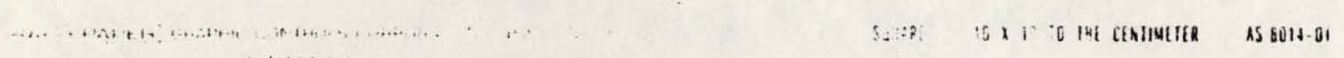

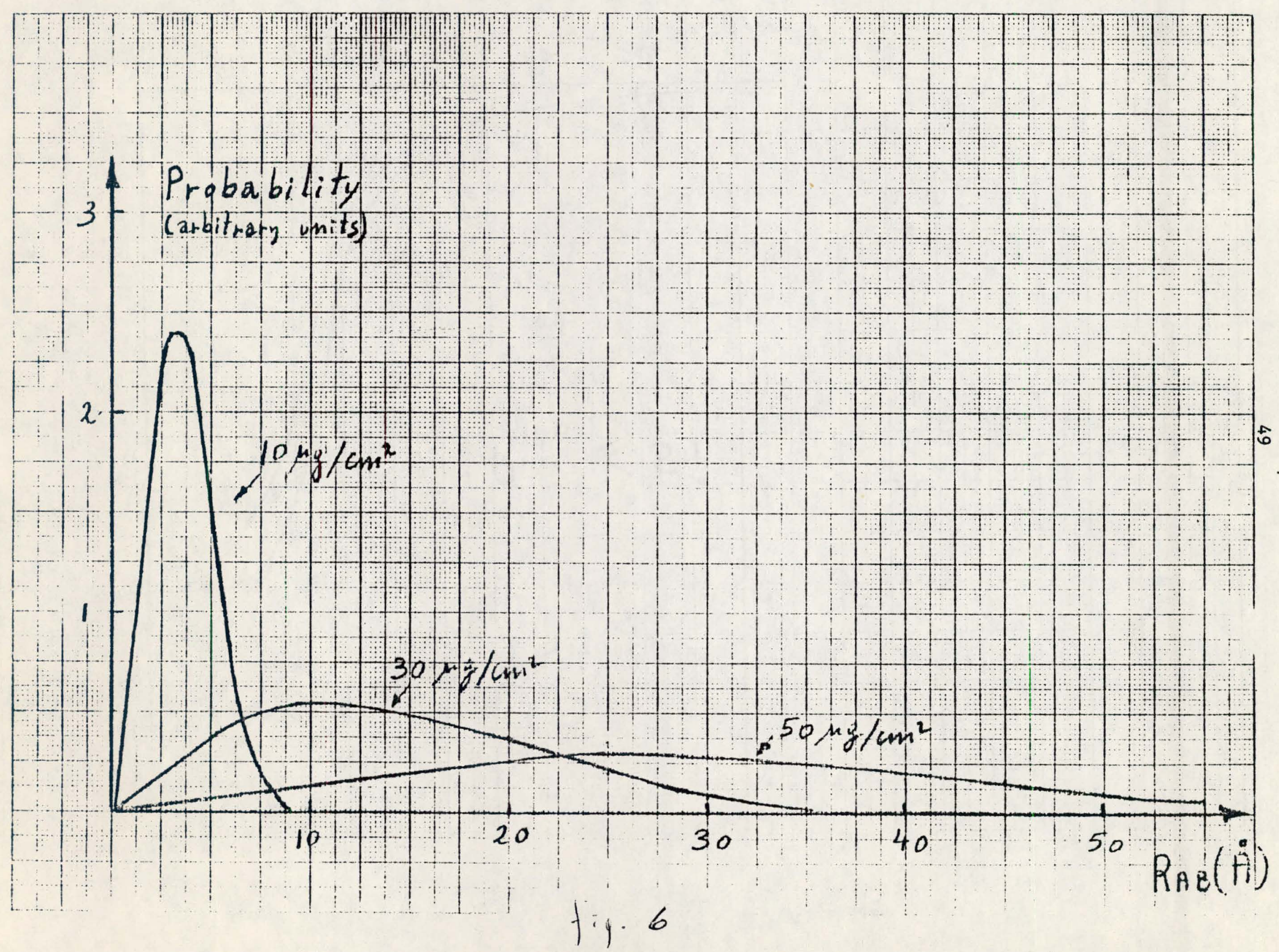




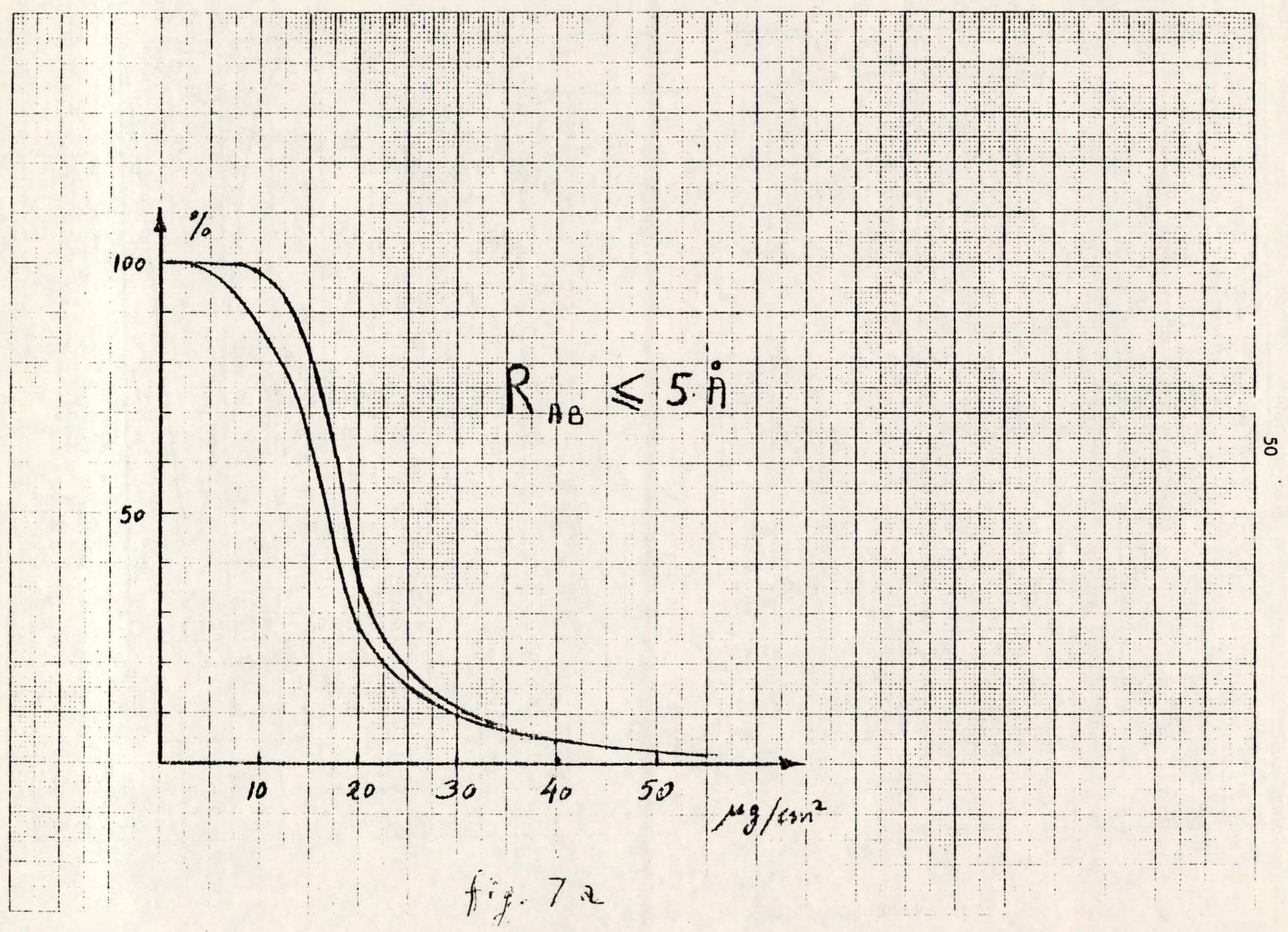




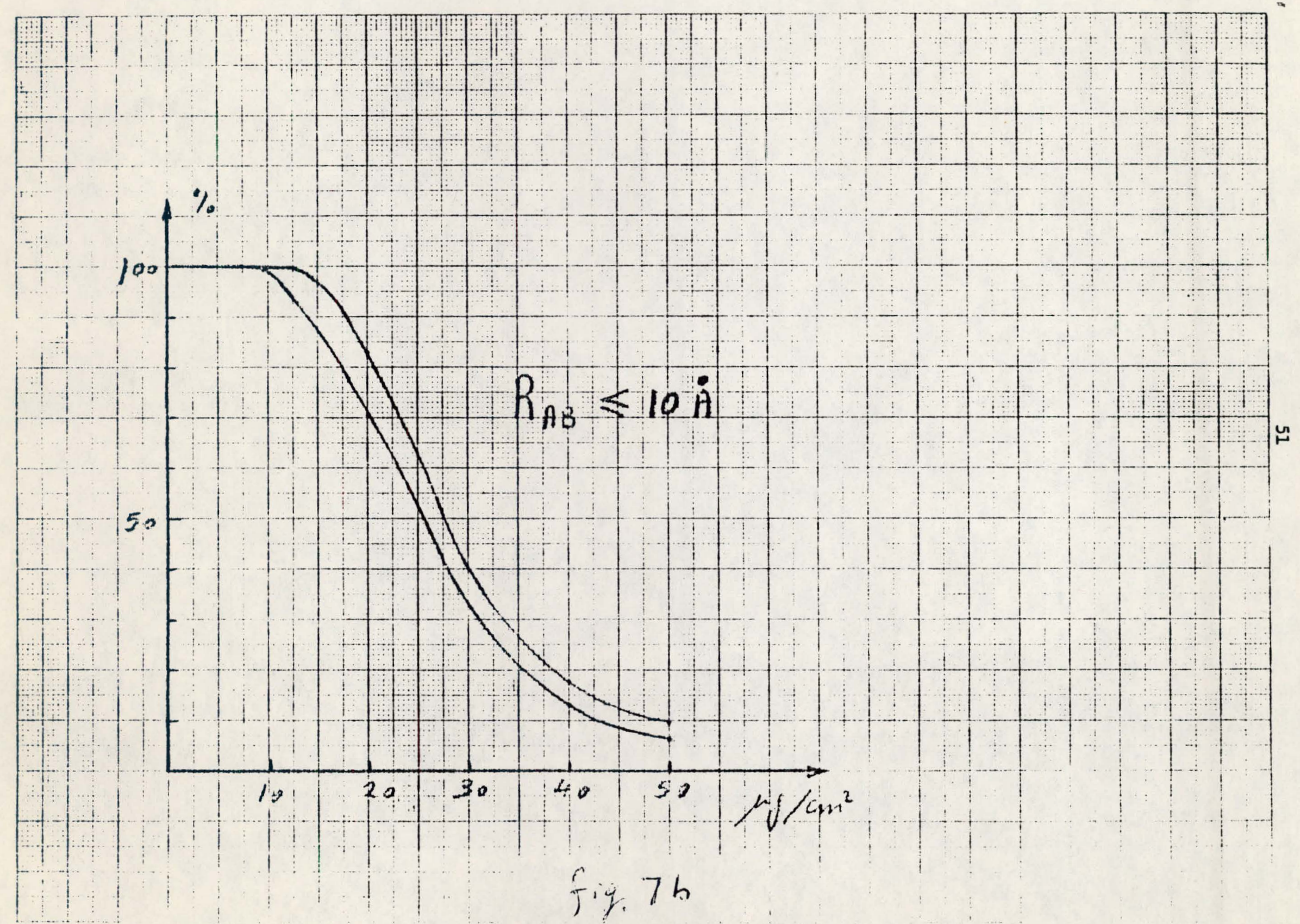


THIS PAGE

\section{WAS INTENTIONALLY \\ LEFT BLANK}


Recent Work with Fast Molecular-ion Beams at Argonne National Laboratory *

P. J. Cooney, D. S. Gemmell, K.-0. Groeneveld, E. P. Kanter, W. J. Pietsch $†$ Z. Vager $\frac{\ddagger}{2}$ and B. J. Zabransky

Physics Division, Argonne National Laboratory, Argonne, I11inois 60439

During the past year, we have invested our efforts primarily into the following areas:

a) Studies of molecular-ion dissociation in gaseous targets.

b) Developing an understanding of the origins of "central peaks" and of the two (as we believe, related) phenomena of the "transmission" of fast. molecular ions through thin foil targets and of the production of neutral fragments from collision-induced dissociation of fast molecular projectiles.

c) Studies exploring the extent to which high-resolution measurements on dissociation fragments can be used to determine the steroechemical structures of the molecular ions in the incident beam.

d) Extensive modifications to the beam-line and apparatus at the 4-MV Dynamitron so as to permit a wide variety of coincidence measurements on fragments from collision-induced molecular-ion dissociation. 
The apparatus that is currently in use at Argonne for these measurements is shown schematically in Fig. 1. Magnetically-analyzed molecular-ion beams from Argonne's 4-MV Dynamitron accelerator are collimated to have a maximum angular divergence of $\pm 0.09 \mathrm{mrad}$ at the target position. A set of "pre-deflector" plates permits electrostatic deflection of the beam incident on the target. Similarly, a set of "post-deflectors" is used to deflect charged particles emerging from the target. Charged particles entering the electrostatic analyzer are energy-analyzed with a relative resolution of $\pm 3 \times 10^{-4}$. The angular acceptance of the analyzer is $\pm 0.11 \mathrm{mrad}$. Distributions in angle and energy are made for particles emerging from the target by varying the voltages on the pre-deflector and/or post deflector in conjunction with that on the analyzer. Neutrals are energy-analyzed by first stripping them in a $2100-\AA$ thick carbon foil located just ahead of the analyzer. The overall angular resolution is $\pm 0.15 \mathrm{mrad}$ ( 0.008 degrees). Selection of the required charge state for particles leaving the target is facilitated by a suitable combination of pre- and post-deflection. The optimal combination also rejects spurious incident beams (e.g. pre-dissociated fragments arising from interactions in residual gas upstream from the target). Elaborate precautions are taken to ensure that no carbon buildup occurs on the target foils. A gas target can also be used in lieu of foils. For coincidence measurements, there are two movable (computer-controlled) detectors available in the detector chamber located upstream from the electrostatic analyzer (see Fig. 2).

Figures 3,4 and 5 show typical distributions measured for $\mathrm{H}_{2}^{+}$, $\mathrm{HeH}^{+}$, and ${ }^{3} \mathrm{He}_{2}^{+}$projectiles. 
Figure 6 illustrates the manner in which parameters determined in the LAB frame are related to those in the COM frame. In the LAB frame, we measure the yield of a dissociation fragment as a function both of its angular shift ( $\theta_{x}$ or equivalently, $\theta_{y}$ ) from the direction of the incident beam (z-direction) and of its energy shift $(\Delta E)$ from the value $E=1 / 2 \mathrm{~m}_{\Gamma} \mathrm{V}_{u}^{2}$ correspnniting to the fragment's share of the projectile's kinetic energy $\left(m_{f}\right.$ is the fragment's mass, and $\vec{V}_{0}$ is the beam velocity). These LAB variables are related in a simple way to the asymptotic COM velocity $\vec{v}$ acquired by a fragment following the dissociation of a projectile. Since the momentum transfer is very small in most of the collisions leading to dissociation at these incident energies, we can assume that the projectile COM is undeflected in the collisions. If $\vec{V}=\vec{V}_{0}+\vec{v}$ is the asymptotic LAB velocity of the fragment, then, for $v \ll v_{0}$

$$
\begin{aligned}
& \theta_{x}=v_{x} / v_{o} ; \theta_{y}=v_{y} / v_{o} ; \\
& \Delta E=1 / 2 m_{f}\left(v^{2}-v_{o}^{2}\right) \approx m_{f} v_{z} v_{o},
\end{aligned}
$$

and

$$
\Delta E / E=2 v_{z} / v_{0}
$$

The approximation in Eq. (1b) amounts to ignoring terms with relative magnitude $\sim_{v} / V_{0}$ (typically, $v / v_{0} \sim 5 \times 10^{-3}$ for the cases considered here). The (LAB) volume element in $\left(\theta_{x}, \theta_{y}, \Delta E / E\right)$ space is then

$$
\tau=\delta \theta_{x} \delta \theta_{y} \delta(\Delta E / E)=2 v_{0}^{-3} \delta v_{x} \delta v_{y} \delta v_{z}
$$


That is, this LAB volume is directly proportional to the volume element in COM velocity space. This simple and convenient relationship is a consequence of the smallness of $\mathrm{v} / \mathrm{v}_{\mathrm{o}}$; the vector $\overrightarrow{\mathrm{V}}$ is almost parallel to $\vec{v}_{0}$ (Fig. 6) and so the transformation between $L A B$ and COM frames, which in velocity space is a simple displacement of the origin by $\mathrm{V}_{0}$, also amounts (to a good approximation) to a linear transformation between the $\mathrm{LAB}\left(\theta_{x}, \theta_{y}, \Delta E / E\right)$ frame and the COM velocity frame.

For a diatomic projectile, the Coulomb potential energy immediately. after the loss of the binding electrons is $z_{1} z_{2} e^{2} / x$, where $z_{1}$ and $z_{2}$ are the effective charges on the ions as they begin to separate from an initial distance $r$. (For fast light ions and solid targets, $z_{1}$ and $z_{2}$ are very nearly equal to the atomic numbers of the fragments.) This Coulomb energy is typicanly several tens or even hundreds of $\mathrm{eV}$. In comparison, we may normally neglect the small energies of vibrational and rotational motion that the projectile may possess at the beginning of the Coulomb explosion. The COM velocity subsequently achieved by a fragment of mass $m_{f}$ is then

$$
v=\left(1 / m_{f}\right)\left(2 \mu z_{1} z_{2} e^{2} / r\right)^{1 / 2}
$$

where $\mu$ is the reduced mass. [In some cases, e.g. when treating dissociation in a gas target, it is obviously more appropriate to write Eq. (3) in terms of the proper dissociative potentials for the electronic states involved.] From the foregoing, one sees that the diameter of an observed ring pattern is approximately proportional to the geometric mean of the effective charges on the fragments and inversely proportional to the square root of the 
bond length. Experiments with several species of diatomic molecular-ion projectiles $^{1-3}$ have established that the bond lengths obtained from such . data agree well (typically within $0.01 \AA$ ) with calculated values when the effective charges $z_{1}$ and $z_{2}$ are chosen to have values that give the correct stopping power. The width of the "rim" of a ring pattern reflects the distribution $D(r)$ of the internuclear separations in the projectiles and this in turn is dependent upon the population of vibrational and electronic states in the incident beam.

Wake effects manifest themselves in slight distortions of the shape of a ring pattern and in a redistribution of the intensity around the ring. As is to be expected, the ring patterns observed for fragments arising from dissociation in gas targets show no sign of wake effects (e.g. Fig. 5). These patterns do however display a dominant central peak.

The first high-resolution measurements on foil-induced dissociation of energetic beams of $\mathrm{HeH}^{+}$revealed the presence of a small central peak in the ring pattern obtained for the resulting protons. ${ }^{4}$ The authors suggested that this peak arises from the breakup mode $\mathrm{HeH}^{+} \rightarrow \mathrm{He}^{\mathrm{O}}+\mathrm{H}^{+}$. Subsequently central peaks were reported ${ }^{1}$ in the cases of $\mathrm{H}^{+}$from $3-\mathrm{MeV} \mathrm{H}_{2}{ }^{+}$and $2.1-\mathrm{MeV} \mathrm{H}_{3}{ }^{+}$ and of ${ }^{3} \mathrm{He}^{++}$from $3-\mathrm{MeV}^{3} \mathrm{He}_{2}^{+}$(all for thin foil targets). No central peaks were observed ${ }^{1}$ for protons from $2.97-\mathrm{MeV} \mathrm{OH}_{2}{ }^{+}$and $3.25-\mathrm{MeV} \mathrm{CH}^{+}$or for ${ }^{3} \mathrm{He}^{+}$from $3-\mathrm{MeV}^{3} \mathrm{He}_{2}^{+}$. Following this work, studies on the central peak observed for $\mathrm{H}^{+}$from the foil-induced dissociation of $\mathrm{H}_{2}^{+}$have been extended to both higher ${ }^{5}$ and lower ${ }^{3,6-8}$ beam energies. Figures 7 and 8 show some of the experimental data. 
The central peak region in the cases of both gas and foil targets can be accounted for $^{3}$ in terms of those dissociations that produce one charged and one neutral fragment. For gas targets, this occurs via a collision-induced transition of the projectile to an excited electronic state which then decays dissociạtively yielding a neutral and a charged fragment. Such processes are favored in the large-impact-parameter collisions that are possible in gases. For this type of excitation the resultant COM velocity acquired by the fragments can be small $(<0.1 \mathrm{eV}$, say) if the initial internuclear separation is large [i.e. on the tail of the distribution $D(r)$ ]. Such fragments, although arising in only a small fraction of the dissociations, are detected for all initial projectile orientations and thus give rise to the large central peak. For foil targets the central peak can originate in two main ways. For very thin foils corresponding to dwell times $\delta 10^{-16} \mathrm{sec}$ a neutral fragment may originate and survive inside the foil. For thicker foils and longer dwell times a neutral and a charged fragment may be produced through capture of one or more electrons by one fragment at or near the point of exit from the foil. (Strictly speaking, the capture leads to the formation of a dissociative molecular state.) Inside the foil a normal Coulomb explosion develops. If the initial internuclear separation is large, the Coulomb explosion is weak and the fragments emerge with very low relative kinetic energy. This results in central peaks being observed in the ring patterns. The relative intensities of the central peaks are obviously sensitively dependent upon such factors as dwell time, $D(r)$, fragment charge, beam velocity, capture cross sections, etc. (for a fuller discussion, see Refs. 3, 6 and 9). 
By performing coincidence measurements between $\mathrm{H}^{+}$and $\mathrm{H}^{\circ}$ from 290-keV H${ }_{2}{ }^{+}$bombarding a $2-\mu \mathrm{g} / \mathrm{cm}^{2}$ carbon foil, Laubert and Chen ${ }^{6}$ have elegantly demonstrated the connection between the $\mathrm{H}^{+}$central peak and the associated $\mathrm{H}^{\circ}$.

A close inspection of the ring pattern for $\mathrm{H}^{+}$from foil-dissociated $\mathrm{H}_{2}^{+}$reveals ${ }^{1}$ that the central peak is actually more like a central ridge than a peak (see also Fig. 3). The ridge extends in a direction corresponding to projectile orientations (referred to here as "transverse orientations") where the internuclear axis is approximately normal to the beam direction. We believe that this reflects an orientation-dependence of the electron-capture probability of the two protons as they emerge from the foil.

Further evidence for these orientation-dependent effects is to be found in the ring patterns measured for $\mathrm{H}^{\circ}$ atoms arising from the $\mathrm{H}_{2}^{+}$and $\mathrm{HeH}^{+}$. bombardment of thin foils. These patterns (Fig. 9) may be considered. as consisting of two components: a) a central peak (a small central peak- not visible in Fig. 9- sits in the center of the pattern for $\mathrm{HeH}^{+}$), and b) a. ring that is enhanced for transverse orientations (or possibly reduced for in-line orientations). In the case of $\mathrm{HeH}^{+}$, the orientation-dependence of the ring actually creates a dip in the forward direction. The rings correspond to Coulomb exploding diatomic clusters whose explosion is truncated at the exit surface by electron capture (the dependence of the ring diameter on target thickness confirms this). The central peaks for $\mathrm{H}^{\circ}$, are attributable to projectiles that lose their binding electrons, but do not explode appreciably in the target. These are incident projectiles having large initial internuclear separations. The neutral hydrogen then arises from capture at the exit surface. [For very short dweli times $\left(₹ 10^{-16} \mathrm{sec}\right)$ there may also be a contribution from neutrals whose electrons are contained in the incident projectile.] 
If the electron capture at the exit surface takes place into a bound state of the emerging (but "non-exploding") ions a transmitted molecular ion may result. There is thus a direct correlation between the observation of central peaks and of transmission. Figure 10 shows the result of a measurement demonstrating that the internuclear separations for transmitted $\mathrm{H}_{2}^{+}$ions are indeed much larger than the average for the incident beam.

The relative smallness of the central peaks (Figs. 4 and 9) and of the transmission ${ }^{1}$ for $\mathrm{HeH}^{+}$as compared with $\mathrm{H}_{2}^{+}$, is a consequence of the narrowness of the potential well [and thus of the distribution $D(r)$ ] for $\mathrm{HeH}^{+}$as compared with $\mathrm{H}_{2}^{+}$. In addition the higher nuclear charges and smaller equilibrium internuclear separation in $\mathrm{HeH}^{+}$produce a more rapid Coulomb explosion. A further factor is the necessity for capturing two electrons in the case of $\mathrm{HeH}^{+}$transmission or in the case of a central peak in the $\mathrm{H}^{+}$ring pattern for $\mathrm{HeH}^{+}$projectiles. Similar considerations explain the absence of both central peaks and transmission when thin foils are bombarded by $\mathrm{CO}^{+}, \mathrm{O}_{2}^{+}, \mathrm{OH}_{2}^{+}, \mathrm{OH}^{+}, \mathrm{CH}^{+}$, etc. ${ }^{1}$

From an analysis of the ring patterns obtained with thin foil targets, one can determine $D(r)$, the distribution of internuclear separations in the incident projectiles. ${ }^{3}$ Figure 12 shows the population of vibrational states corresponding to the measured $D(r)$ for an $\mathrm{H}_{2}{ }^{+}$beam. It is evident that the incident beam is considerably "cooler" than would be expected, say, on the basis of a Franck-Condon distribution. This preferential population of the ground and low-lying vibrational states is characteristic of our measurements on a great variety of molecular-ion beams. Our measured $D(r)$ are not detectably dependent on ion-source parameters over the range that these can be varied. 
For dilute gas targets most collisions are much less violent than for foil targets. On the average fewer electrons are removed and there is thus a greater probability for dissociation into less highly charged fragments. One therefore expects to observe dissociation modes in which the molecular projectile, during a collision with a target atom or molecule, makes a vertical transition to an excited electronic state that then dissociates into separate fragments. (Vibrational excitation to continuum states is expected to be relatively infrequent at high projectile velocities. ${ }^{11}$. Using the (known) potential curves shown in Fig. 12, and the distributions $D(r)$ extracted from data obtained with foil targets, one can' analyze the gas target data (e.g. Fig. 5) to determine the role played by various excited electronic states of the projectiles $\mathrm{H}_{2}^{+}$and $\mathrm{HeH}^{+}$. We find that for $\mathrm{H}_{2}^{+}$about $90 \%$ of the dissociations proceed via the Coulomb (totally Ionized) state and about $10 \%$ via the $2 \mathrm{p} \pi_{\mathrm{u}}$ electronic state [leading to $\mathrm{H}^{+}$and $\mathrm{H}^{\circ} .(\mathrm{n}=2)$ ] It would be interesting to perform beam-gas spectroscopy measurements with $\mathrm{H}_{2}{ }^{+}$beams to verify that the $\mathrm{n}=2$ state in hydrogen is strongly populated. Analysis of the $\mathrm{HeH}^{+}$gas data is more complicated because a 2-electron projectile is inovlved. However the dissociation is clearly dominated by the $\mathrm{He}^{+}(1 s)+\mathrm{H}^{+}$channel resulting from formation of the 1 so electronic state of $\mathrm{HeH}^{++}$.

High-resolution studies of fragments from.the collision-induced dissociation of fast molecular-ion beams offer attractive possibilities for determining the stereochemistry of the projectiles. We have already noted ${ }^{1}$ that bond lengths for several diatomic projectiles have been measured with 
an accuracy of about $0.01 \AA$. Calculations indicate that for not-toocomplicated molecular ions containing a smali number of atoms, bond lengths and bond angles should be measurable to accuracies of about $0.01 \AA$ and $1^{\circ}$, respectively. Although this level of accuracy is poor compared with that attainable with photon techniques (when they can be applied), it is sufficiently good to be of value in resolving many conflicts between predictions from structure calculations. Furthermore, this level of accuracy should be good enough to help practitioners of the photon techniques to zero in on the frequencies of their (usually very narrow) resonances. It is therefore of considerable interest to explore the extent to which these techniques with high-energy beams may be employed to determine the geometrical structures of polyatomic molecular-ion projectiles.

Following an initial joint experiment ${ }^{12}$ with the University of Lyon and the Weizmann Institute on the structure of $\mathrm{H}_{3}^{+}$, we at Argonne have made a series of studies on somewhat more complicated molecular-ion species. Figure 13 displays energy spectra for various charged fragments from 3.5- $\mathrm{MeV} \mathrm{CO}_{2}{ }^{+}$and $\mathrm{N}_{2} \mathrm{O}^{+}$dissociating in carbon foils. ${ }^{13}$ These molecular ions in their ground and low-1ying states are known ${ }^{14}$ to be linear; but while $\mathrm{CO}_{2}^{+}$ has the symmetric form $(\mathrm{O}-\mathrm{C}-\mathrm{O}) ; \mathrm{N}_{2} \mathrm{O}^{+}$is asymmetric (N-N-O). From just a. 'cursory inspection of the spectra in Fig. 13 (merely counting the peaks) one can immediately deduce the main features of these structures (i.e. their linearity and the-order of atoms). Of course, to extract accurate bond lengths and angles, a much more detailed analysis is required. However, this example does point to the power of the technique in determining gross structures. 
Figure 14 shows again how gross structure features are readily observed in such measurements - in this instance for the series $\mathrm{CH}_{\mathrm{n}}^{+}(\mathrm{n}=0-4)$. Here one also sees clear evidence of the Jahn-Teller distorton of the methane ion.

We have performed many measurements of this type-i.e. high-resolution studies of individual fragments in particular charge states. Except for very simple and/or highly symetric ions $\left(\mathrm{H}_{3}{ }^{+}, \mathrm{C}_{3}{ }^{+}\right.$, etc.) such measurements on single fragments yield only gross structure information. For example, our measurements on $\mathrm{C}^{2+}$ fragments from $3.6-\mathrm{MeV} \mathrm{C}_{3} \mathrm{H}_{3}{ }^{+}$ions dissociating in thin foils demonstrate only that the carbons sit on the corners of an approximately equilateral triangle. [That is, we have a beam of cyclopropenyl ions and not propargyl ions (which are linear in carbons).] Similarly, our measurements on single fragments from $\mathrm{OH}_{2}{ }^{+}$show only that the protons are equivalent and that the oxygen is "in the middle". The accuracy in determining the bond length and bord angle is poor ( $0.1 \AA$ and $\sim 10^{\circ}$, respectively). For polyatomic ions, particularly those having low symmetry and/or many atomic constituents, the structures can be much more precisely determined if spatial and temporal coincidences can be recorded for two or more dissociation fragments from a given projectile. With this in mind, we recently revised the apparatus at Argonne so as to permit a wide variety of coincidence measurements (see Figs. 1 and 2). We can presently measure double or triple coincidences and record simultaneously information on fragment charge states, energies, and flight times from the target. The system has been tested with various simple diatomic and triatomic projectiles $\mathrm{(H}_{2}^{+}, \mathrm{HeH}^{+}, \mathrm{CH}^{+}, \mathrm{NH}^{+}, \mathrm{OH}^{+}, \mathrm{H}_{3}^{+}, \mathrm{CH}_{2}^{+}, \mathrm{NH}_{2}^{+}, \mathrm{OH}_{2}^{+}$, etc.). Figure 15 shows a spatial scan of the proton-proton double coincidence rate for the foil-induced dissociation of $3.6-\mathrm{MeV} \mathrm{OH}{ }_{2}^{+}$. ions. Note that a given combination of 
post-deflector and electrostatic analyzer voltage settings amounts to choosing for study a limited subset of the incoming projectile orientations. For the data shown in Fig. 15, only those $\mathrm{OH}_{2}{ }^{+}$ions in which one proton is trailing are selected. A simple analysis of these data gives the bond length to be $1.0 \pm 0.04 \AA$ and the $\mathrm{H}-0-\mathrm{H}$ bond angle to be $110 \pm 2^{\circ}$ in agreement with results obtained from optical measurements ${ }^{15}$ (the $\mathrm{OH}_{2}^{+}$ion is one of the eight polyatomic molecular ions whose structures have thus far been measured with photon technqiues).

Although foil-induced dissociation has the virtue that essentially every incident projectile dissociates violently into individual highly charged monatomic ions, there are complications in the data analysis where one must take into account wake effecț, target thickness, charge-state distributions, and multiple scattering. These problems do not arise if one uses a dilute gas target in which single collisions predominate. With gas targets however, there is the problem that most of the dissociations proceed through weak Coulomb explosions between ions having low charges. Also, the product fragments frequently include diatomic and polyatomic species. Thus the foil-induced and gas-induced dissociations tend to give complementary information and in studying any given projectile it is desirable to use both types of target. At Argonne we have recently begun triple coincidence measurements, e.g. on the pair of protons and the $\mathrm{N}^{3+}$ fragments arising from the dissociation of $3.6-\mathrm{MeV} . \mathrm{NH}_{2}{ }^{+}$ions in a dilute Ar gas jet. Although the triple coincidence counting rates are low, the data are very clean (Fig. 16 shows an example) and the analysis is greatly simplified as compared with the results obtained with foil targets. 


\section{References and Footnotes}

* Work performed under the auspices of theDivision of Basic Energy Sciences of D.O.E.

***isiting scientist 1978-78. Permanent address: Middlebury College, Middlebury, VT.

${ }^{* * *}$ Visiting scientist 1978. Permanent address: University of Frankfurt,

W. Germany.

${ }^{\dagger}$ Visiting scientist 1976-78. Permanent address: University of Cologne, W. Germany.

\# Visiting scientist 1977, 1978 and 1979. Permanent. address: Weizmann Institute of Science, Rehovoth, Israel.

I D. S. Gemme11, P. J. Cooney, W. J. Pietsch, A. J. Ratkowski, Z. Vager, B. J. Zabransky, A. Faibis, G. Goldring, and I. Levine, Proceedings of the 7 th International Conference on Atomic Collisions in Solids, Moscow, Sept. 19-23, 1977, to be published.

2 D. S. Gemmell, in Proceedings of the 6 th International Congress on Radiation Research, Tokyo, Japan, May 13-18, 1979.

3.E. P. Kanter, P. J. Cooney, D. S. Gemmell, K.-0. Groeneveld, W. J. Pietsch, A. J. Ratkowski, Z. Vager, and B. J. Zabransky, Phys. Rev. A (to be publishedSeptember 1979).

${ }^{4}$ Z. Vager, D. S. Gemmell and B. J. Zabransky, Phys. Rev. A 14 (1976) 638.

5. Pietsch, G. Krösing, G. Kumbartzki, and D. S. Gemmell (to be published).

6. Laubert and F. K. Chen, Phys. Rev. Lett. 40, 174 (1978).

7M. J. Gaillard, J.-C. Poizat, and J. Remillieux, Phys. Rev: Lett. 41, 159 (1978). 
${ }^{8}$ W. H. Escovitz, T. R. Fox and R. Levi-Setti, Proceedings of the 1978 Conference on the Applications of Small Accelerators in Research and Industry, IEEE Trans. Nucl. Sci., NS-26, 1147 (197.9).

9 Yu. Kagan, J. Phys. C (to be published).

10, von Busch and G. H. Dunn, Phys. Rev. A 5, 1726 (1972).

$11_{E}$. E. Salpeter, Proc. Phys. Soc. (London) 63A, 1295 (1950).

${ }^{12}$ M. J. Gaillard, D. S. Gemme11, G. Goldring, I. Levine, W. J. Pietsch, J.-C. Poizat, A. J. Ratkowski, J. Remillieux, Z. Vager, and B. J. Zabransky, Phys. Rev. A 17, 1797 (1978).

${ }^{13}$ D. S. Gemmell, E. P. Kanter, and W. J. Pietsch, Chem. Phys. Lett. $\underline{55}$, 331 (1978).

${ }^{14} \mathrm{G}$. Herzberg, Electronic spectra of polyatomic molecules (Van Nostrand, Princeton 1950).

${ }^{15}$ H. Lew and I. Heiber, J. Chem. Phys. $\underline{58,} 1246$ (1973). 


\section{Figure Captions}

Fig. 1. Schematic diagram of the experimental arrangement at Argonne's 4-MV Dynamitron accelerator.

Fig. 2. Schematic diagram showing a cross-sectional view of the detector chamber and movable detector systems at Argonne's 4-MV Dynamitron accelerator.

Fig. 3. (a) Joint energy-angle distribution for protons arising from the dissociation of $1.4-\mathrm{MeV} \mathrm{H}_{2}{ }^{+}$in an $88-\AA$ thick carbon foil. ${ }^{3}$ (b) The corresponding energy spectrum of those protons emerging from the target foil in the beam direction.

(c) The corresponding angular distribution of those protons emerging from the target with an energy equal to that of the small central peak in (b).

Fig. 4. Joint energy-angle distributions for a) protons from 3.0-MeV $\mathrm{HeH}^{+}$dissociating in a $195-\AA$ thick carbon foil and b) ${ }^{3} \mathrm{He}^{++}$ from $3.6-\mathrm{MeV}{ }^{3} \mathrm{He}_{2}{ }^{+}$dissociating in a $118-\AA$ thick carbon foil. ${ }^{2}$

Fig. 5. Joint energy-angle distributions for protons from $1.2-\mathrm{MeV} \mathrm{H}_{2}^{+}$ and $3.0-\mathrm{MeV} \mathrm{HeH}{ }^{+}$dissociating in gaseous $\mathrm{Ar}(7.8 \mathrm{mTorr})$ and He ( 9.5 mTorr), respectively. ${ }^{3}$

Fig. 6. Kinematical relationships between the parameters $(E, \theta)$ measured for the outgoing molecular-ion fragment of mass $m_{f}$ and its velocity $\vec{v}$ in the center-of-mass. (COM) system moving along with the incident projectile. ${ }^{3}$ 
Fig. 7. Representative energy spectra of fragments from foil-induced Coulomb explosions. 1,4 (a) $3-\mathrm{MeV} \mathrm{H}_{2}^{+} \rightarrow \mathrm{H}^{+}$; (b) $3.5-\mathrm{MeV} \mathrm{HeH}^{+} \rightarrow \mathrm{H}^{+}$; (c) $3-\mathrm{MeV}^{3} \mathrm{He}_{2}^{+}+{ }^{3} \mathrm{He}^{++}$; (d) $3-\mathrm{MeV}^{3} \mathrm{He}_{2}^{+}+{ }^{3} \mathrm{He}^{+}$.

Fig. 8. Energy spectrum for protons emerging in the forward direction from a $4.2-\mu g / \mathrm{cm}^{2}$ carbon foil bombarded by a $27.55-\mathrm{MeV} \mathrm{H}_{2}{ }^{+}$ beam. 5 The dwell time of the fons in the target is $0.4 \mathrm{fsec}$. Fig. 9. Ring patterns for (a) $3.0-\mathrm{MeV} \mathrm{H}_{2}{ }^{+} \rightarrow \mathrm{H}^{\circ}$ in a $132-\AA$ carbon foil, and (b) $3.63-\mathrm{MeV} \mathrm{HeH}+\mathrm{H}^{\circ}$ in a $144-\AA$ carbon foil. ${ }^{3}$

Fig. 10. Energy spectra of protons from $1-\mathrm{MeV} \mathrm{H}_{2}^{+} \rightarrow \mathrm{H}^{+}$. The incident $\mathrm{H}_{2}^{+}$were first prepared by passage through a $330 \AA$ carbon foil.

Fig. 11. Population distribution for the vibrational states of $\mathrm{H}_{2}^{+}$ions (shaded distribution) as deduced from the foil-induced dissociation of a $3.0-\mathrm{MeV} \mathrm{H}_{2}^{+}$beam. For comparison, the distributions expected from a Franck-Condon model and deduced from photo-dissociation experiments ${ }^{10}$ are also shown.

Fig. 12 Interaction potentials used in fitting the c.m. velocity distributions of $\mathrm{H}^{+}$from (a) $\mathrm{H}_{2}^{+}$and (b) $\mathrm{HeH}^{+}$ion beams. 3

Fig. 13. Zero-angle energy spectra for (a) $\mathrm{O}^{4+}$ and (b) $\mathrm{c}^{2+}$ resulting from 3.5-MeV CO${ }_{2}^{+}$bombarding a $133 \AA$-thick C foil, and for (c) $0^{4+}$ and (d) $\mathrm{N}^{3+}$ resulting from $3.5-\mathrm{MeV} \mathrm{N} \mathrm{N}^{+}$bombarding a $160-\AA$ thick. C foil. 13 
Fig. 14. Energy widths measured for $\mathrm{C}^{4+}$ ions emerging at $0^{\circ}$ from a 125- $\AA$ thick carbon foil bombarded by beams of $\mathrm{CH}_{n}^{+}$for $\mathbf{n}=0-4$. The beams all have the same velocity (corresponding to $194 \mathrm{keV} / \mathrm{amu})$.

Fig. 15. Coincidence counting rate for protons from $3.6-\mathrm{MeV} \mathrm{OH}_{2}^{+}$dissociating in a $80-\AA$ thick carbon foil. The rate is plotted as a function of the angle between the electrostatic analyzer (set on the low-energy, $0^{\circ}$, proton group) and one of the movable surface-barrier detectors (see Figs. 1 and 2).

Fig. 16. Top: $\mathrm{H}^{+}-\mathrm{N}^{3+}-\mathrm{H}^{+}$triple coincidence counting rate for $3.6-\mathrm{MeV}$ $\mathrm{NH}_{2}^{+}$ions dissociating in an Ar gas-jet target. $\mathrm{N}^{3+}$ ions are detected at $0^{\circ}$ in the electrostatic analyzer (Fig. 1). Protons are detected in the two movable detectors (Fig. 2). The coincidence rate is plotted as a function of the angle between the (symmetrically placed) proton detectors and the beam direction. Bottom: Same, but double coincidences $\left(\mathrm{N}^{3+}-\mathrm{H}^{+}\right)$obtained with a $70-\mathrm{A}^{\circ}$ carbon foil target. In both figures the total (energysummed) proton-singles rates in the movable detectors are shown as dashed curves. In the top figure, the numbers $(0,1,2,3)$ on the dashed curve refer to the corresponding nitrogen-ion charge state. 


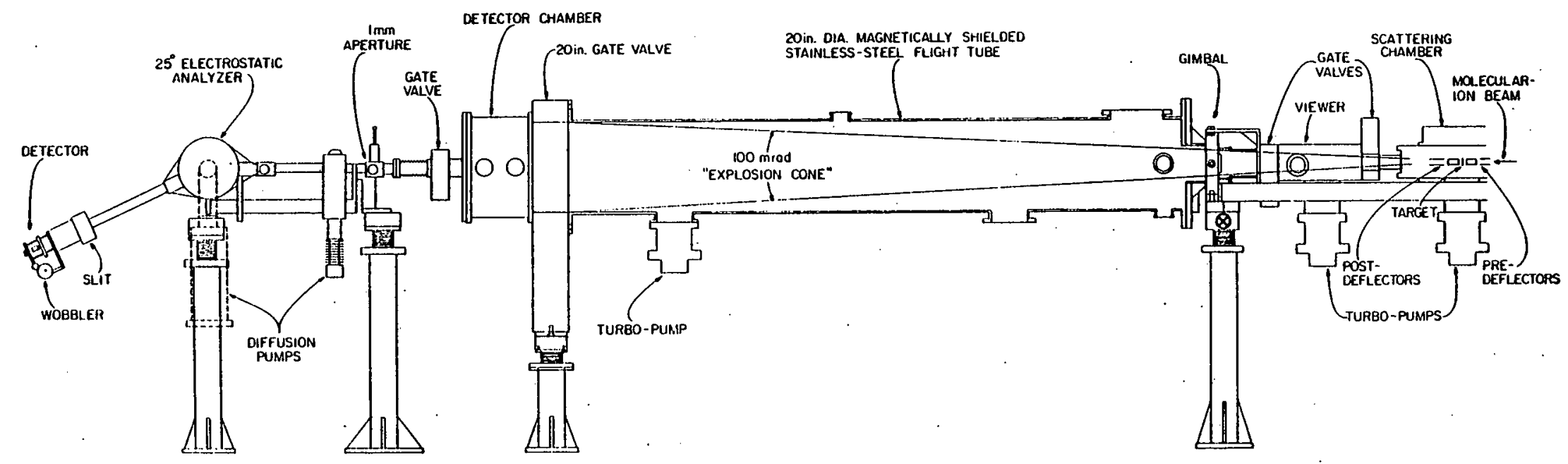




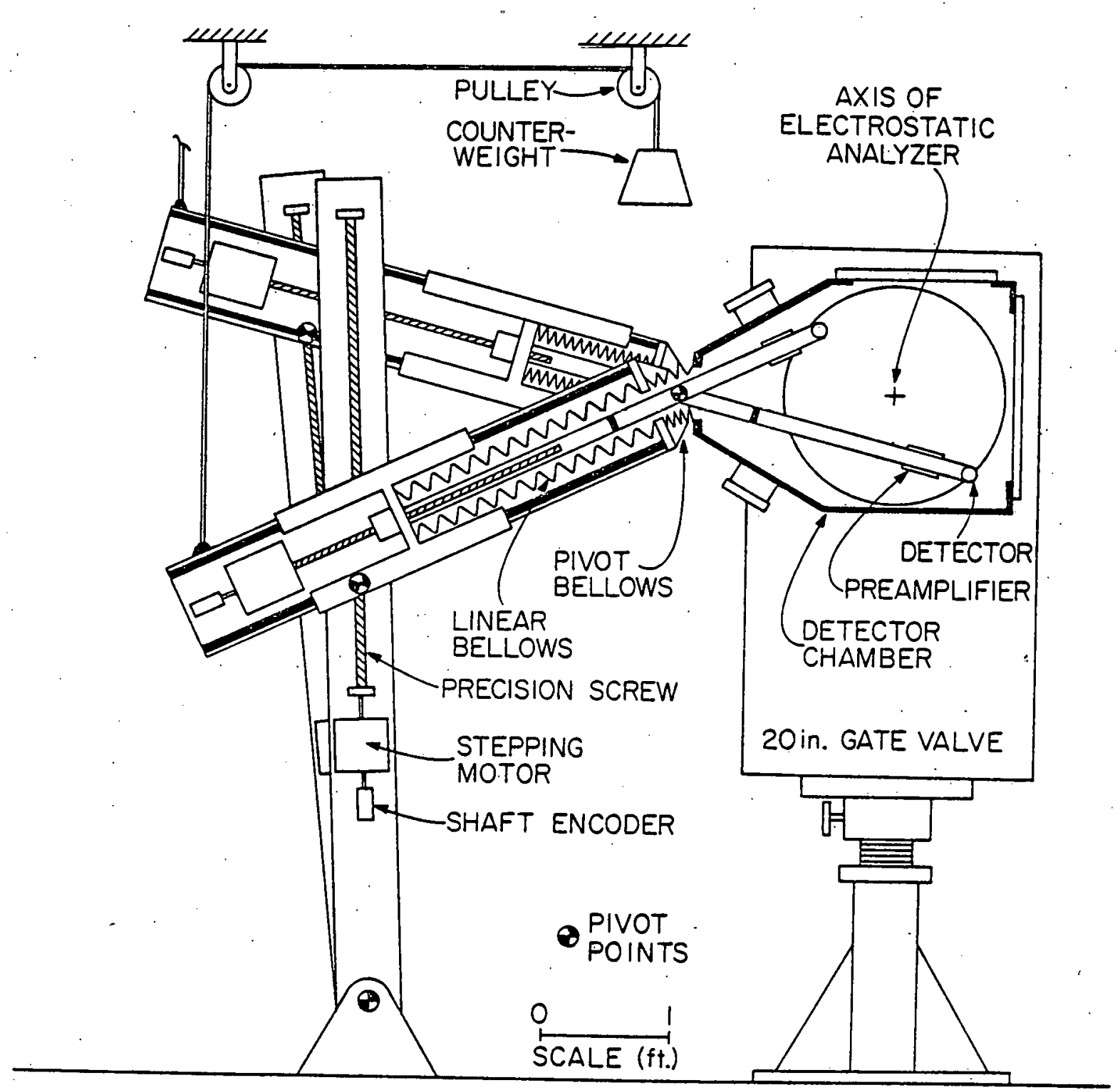

Fig. 2 


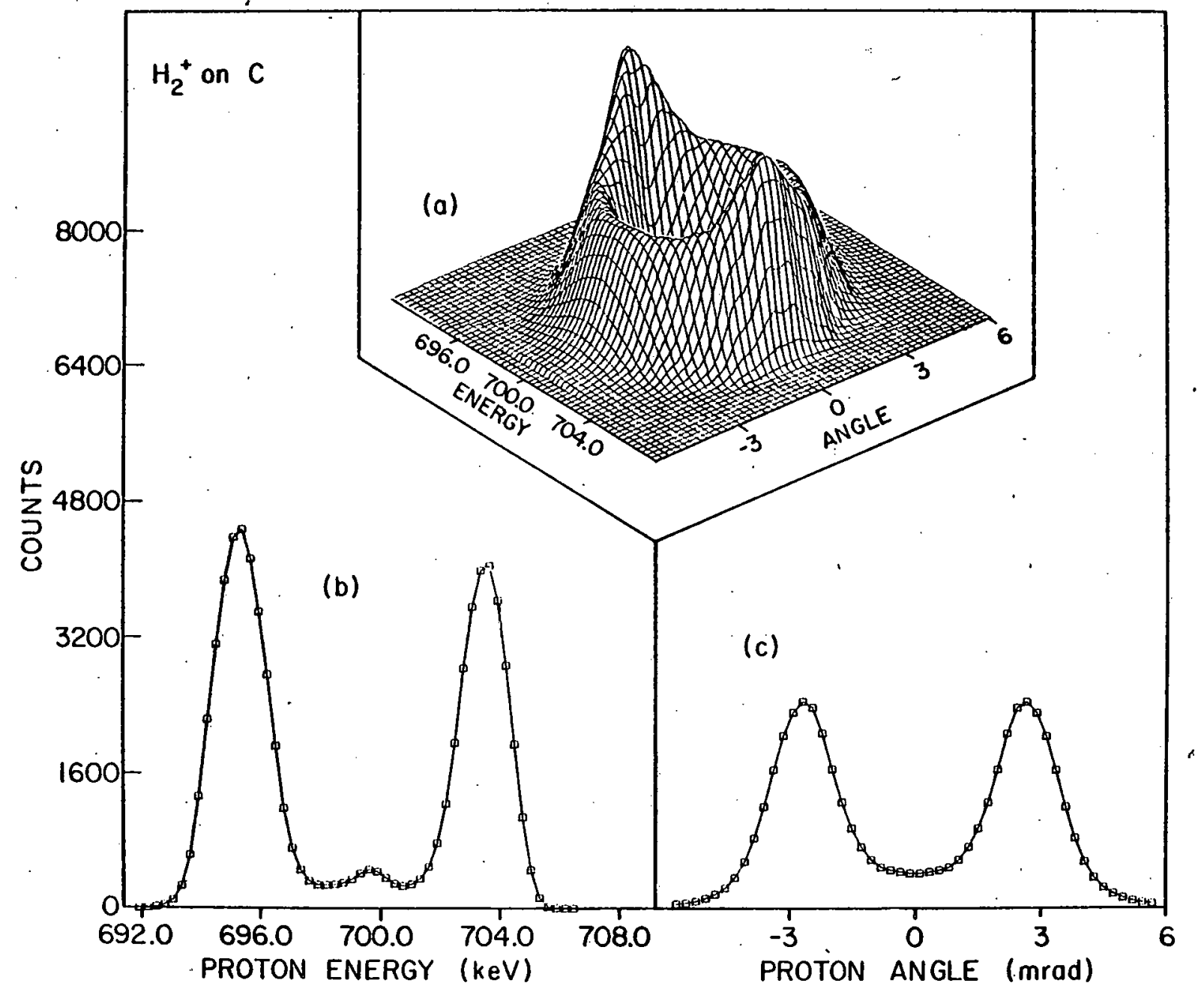

Fig. 3 


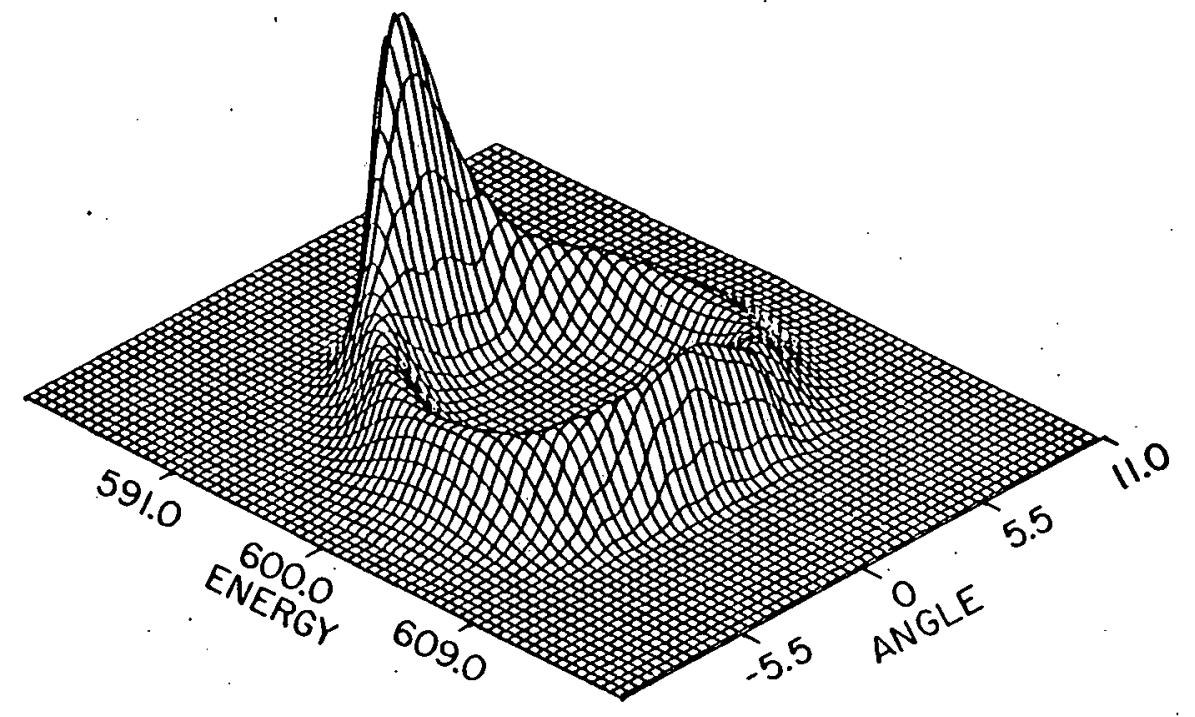

$a$

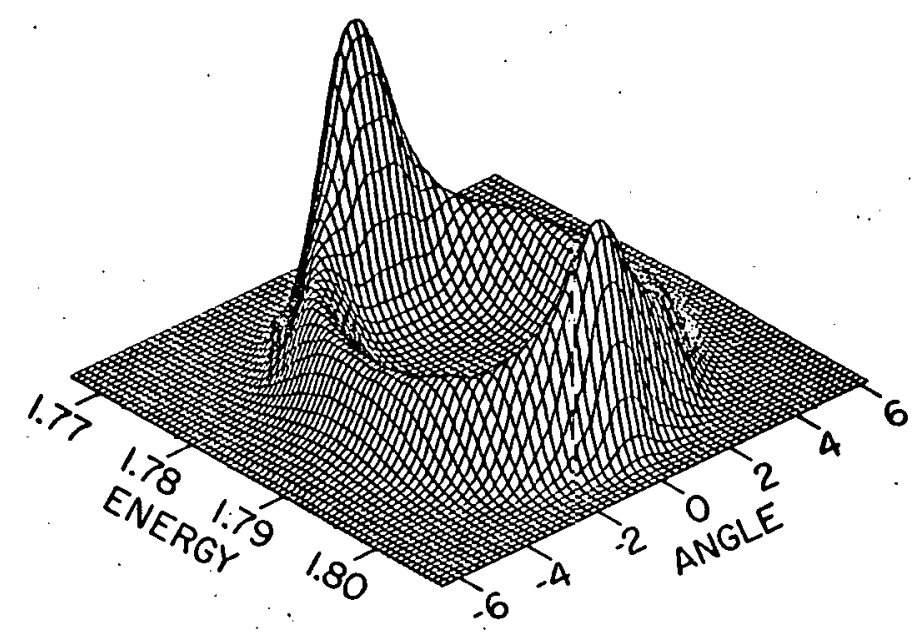

6

Fig. 4 

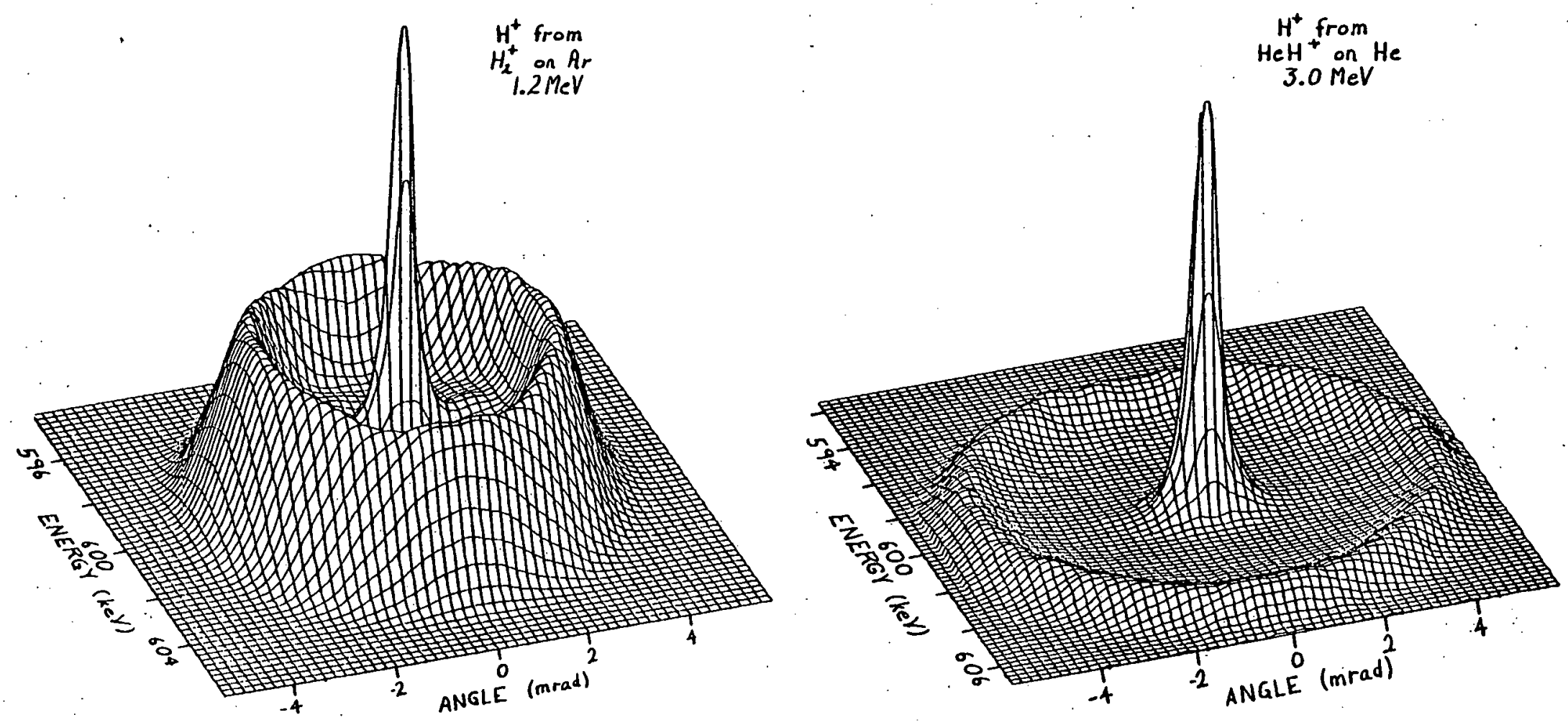


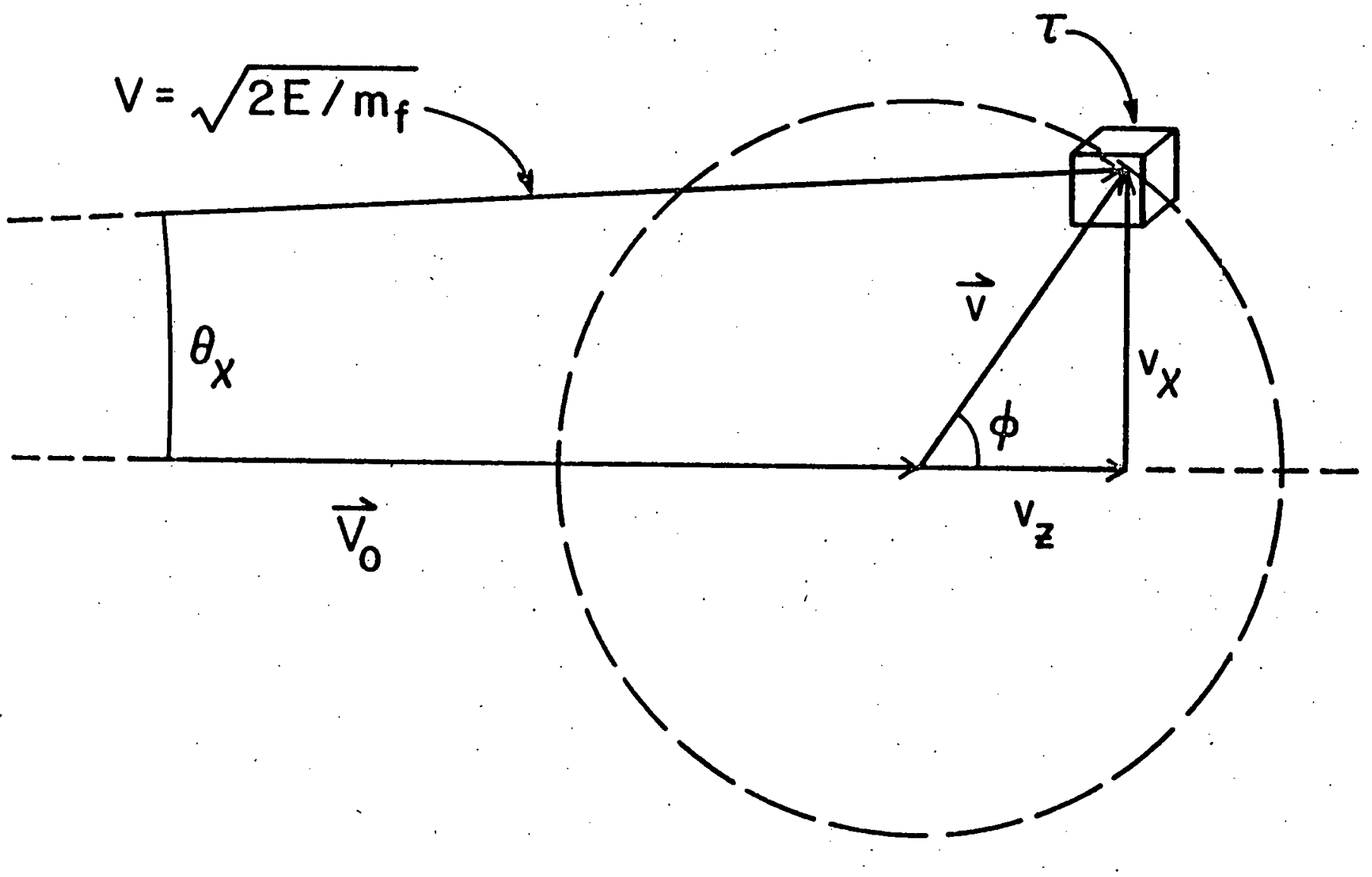




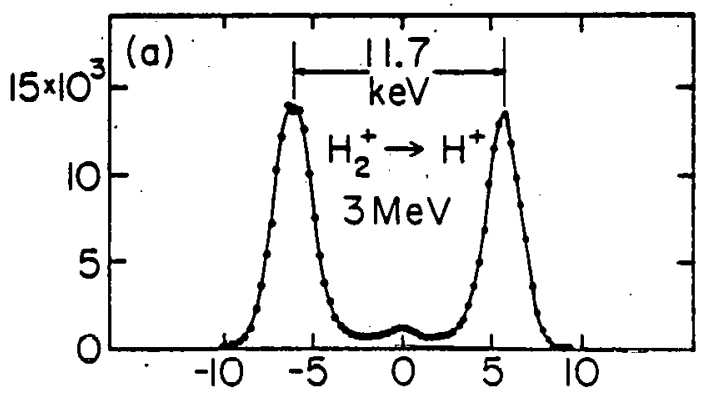

RELATIVE PROTON ENERGY (keV)
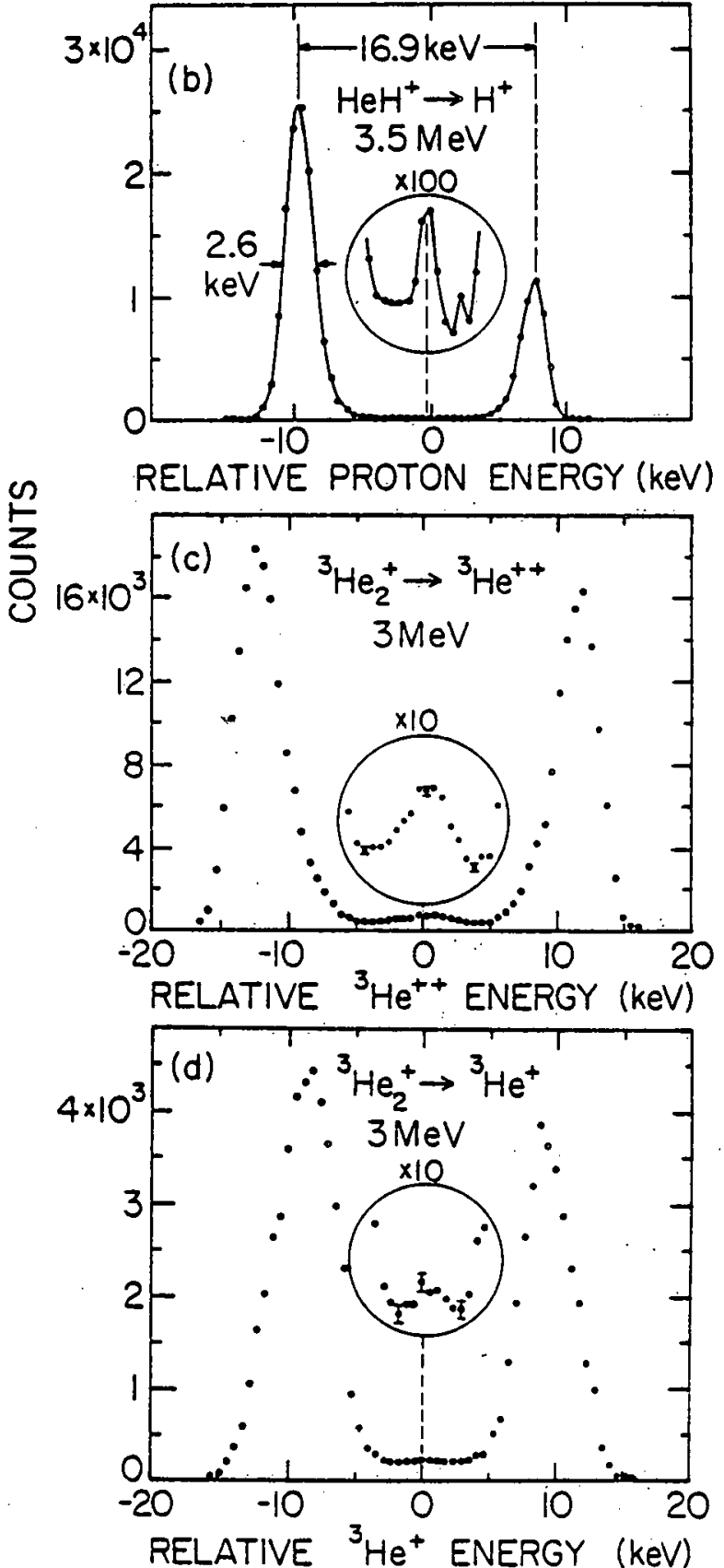

Fig. 7 


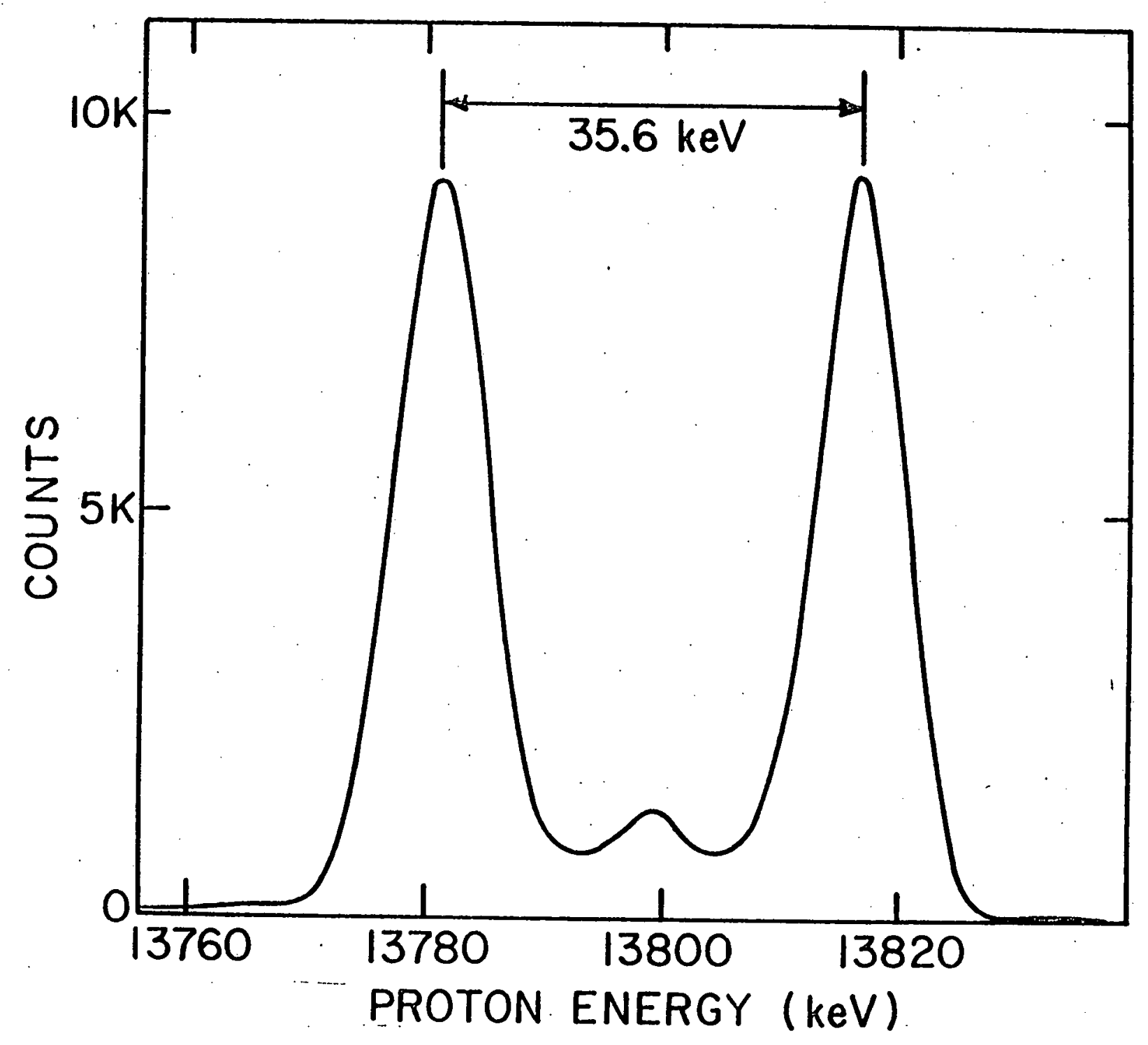


(a) $\mathrm{H}_{2}^{+} \rightarrow \mathrm{H}^{\circ}$

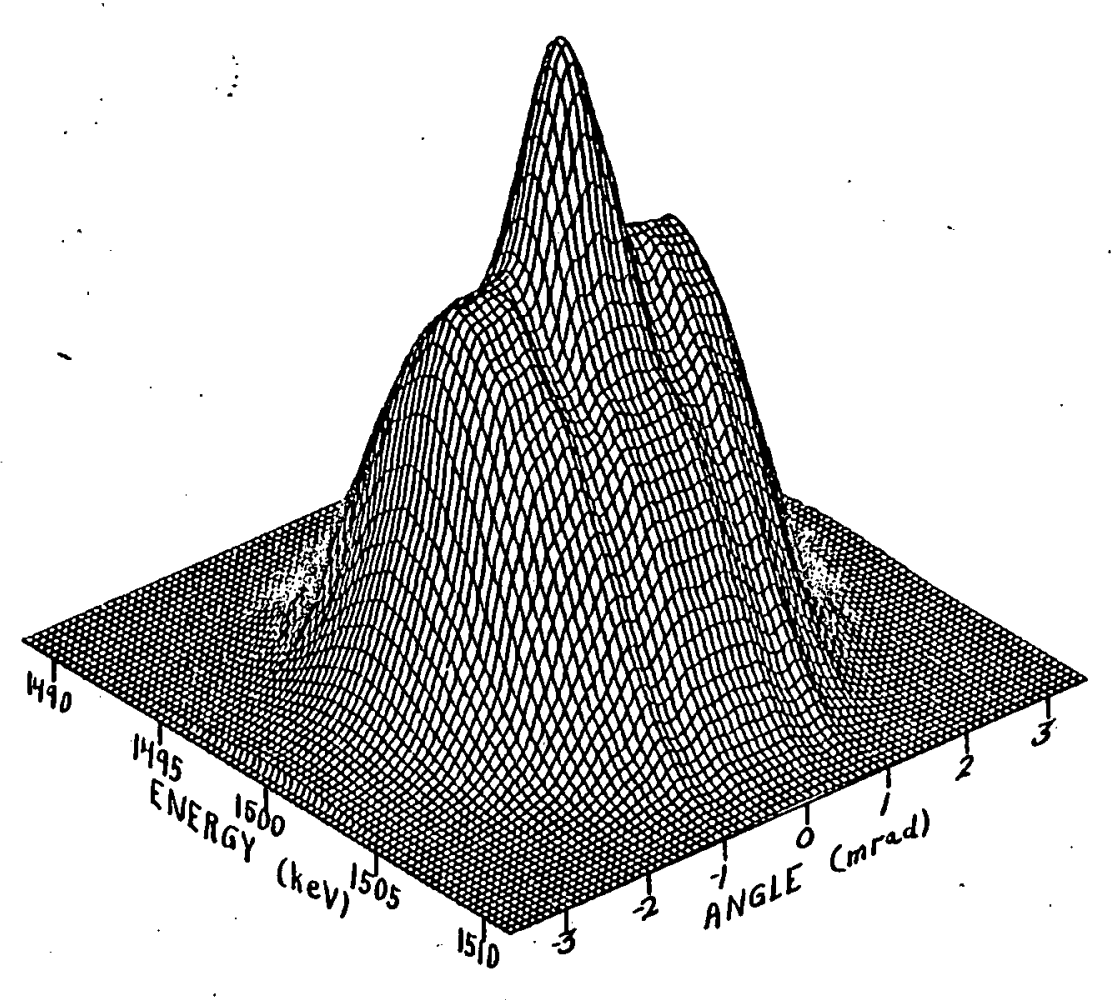

(b) $\mathrm{HeH}^{+} \rightarrow \mathrm{H}^{0}$

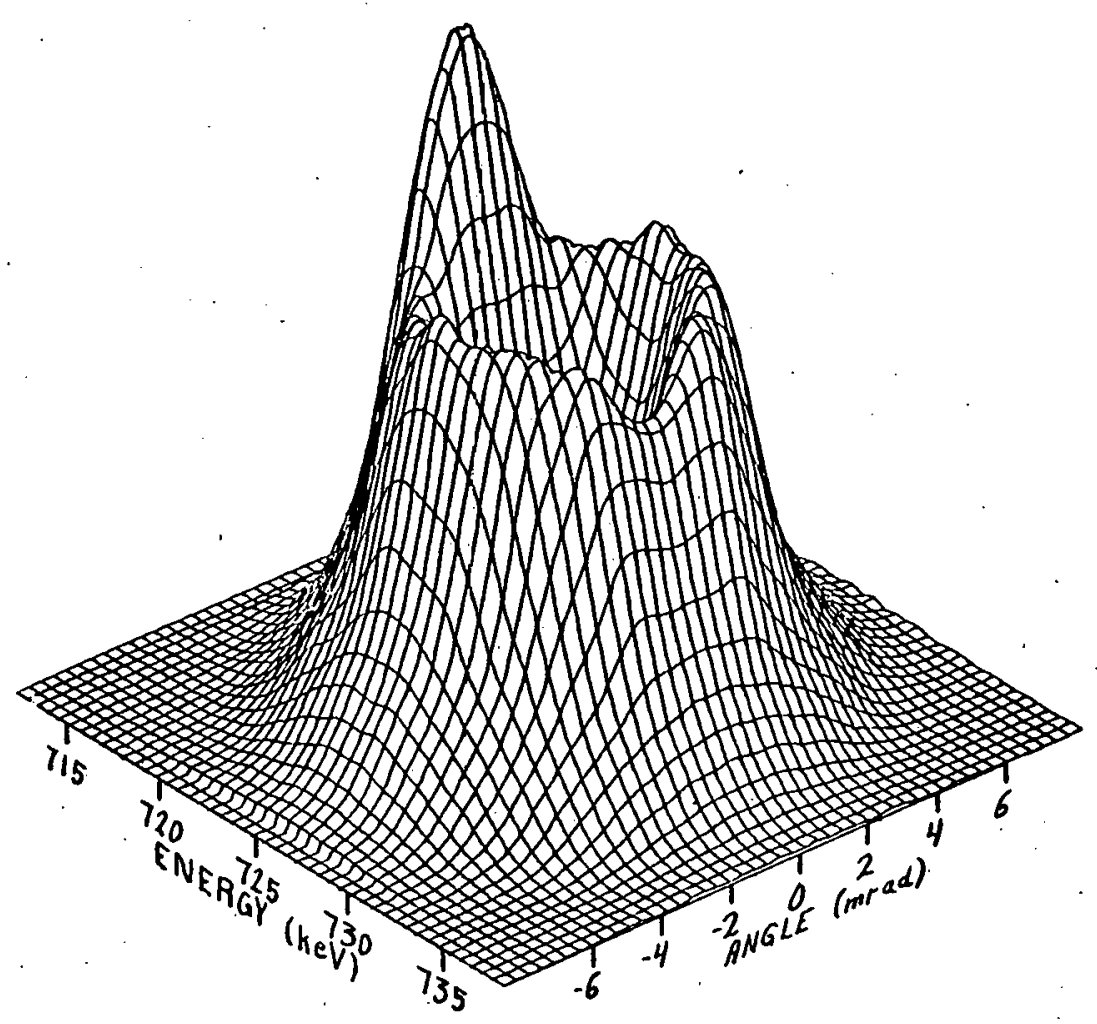




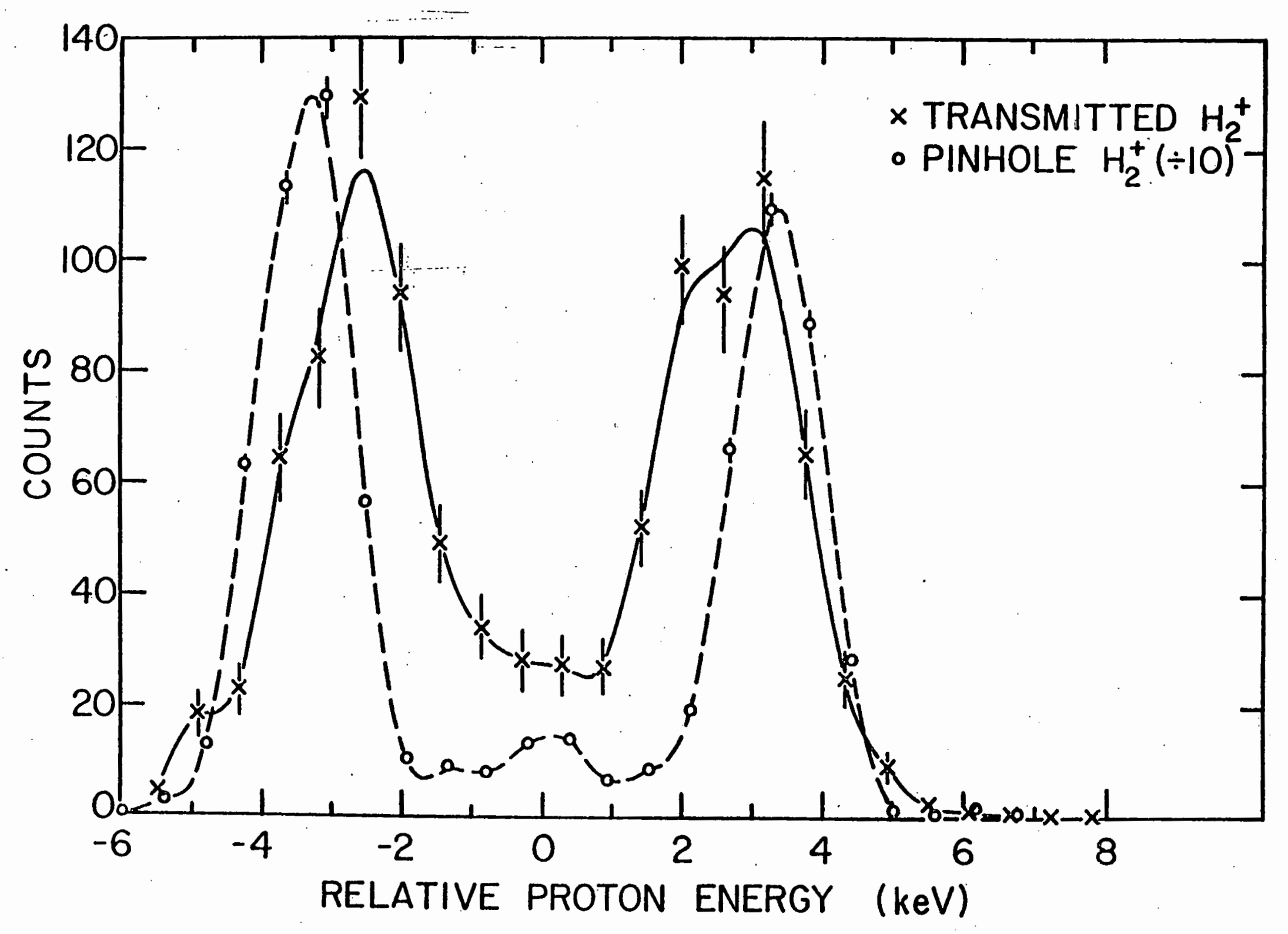




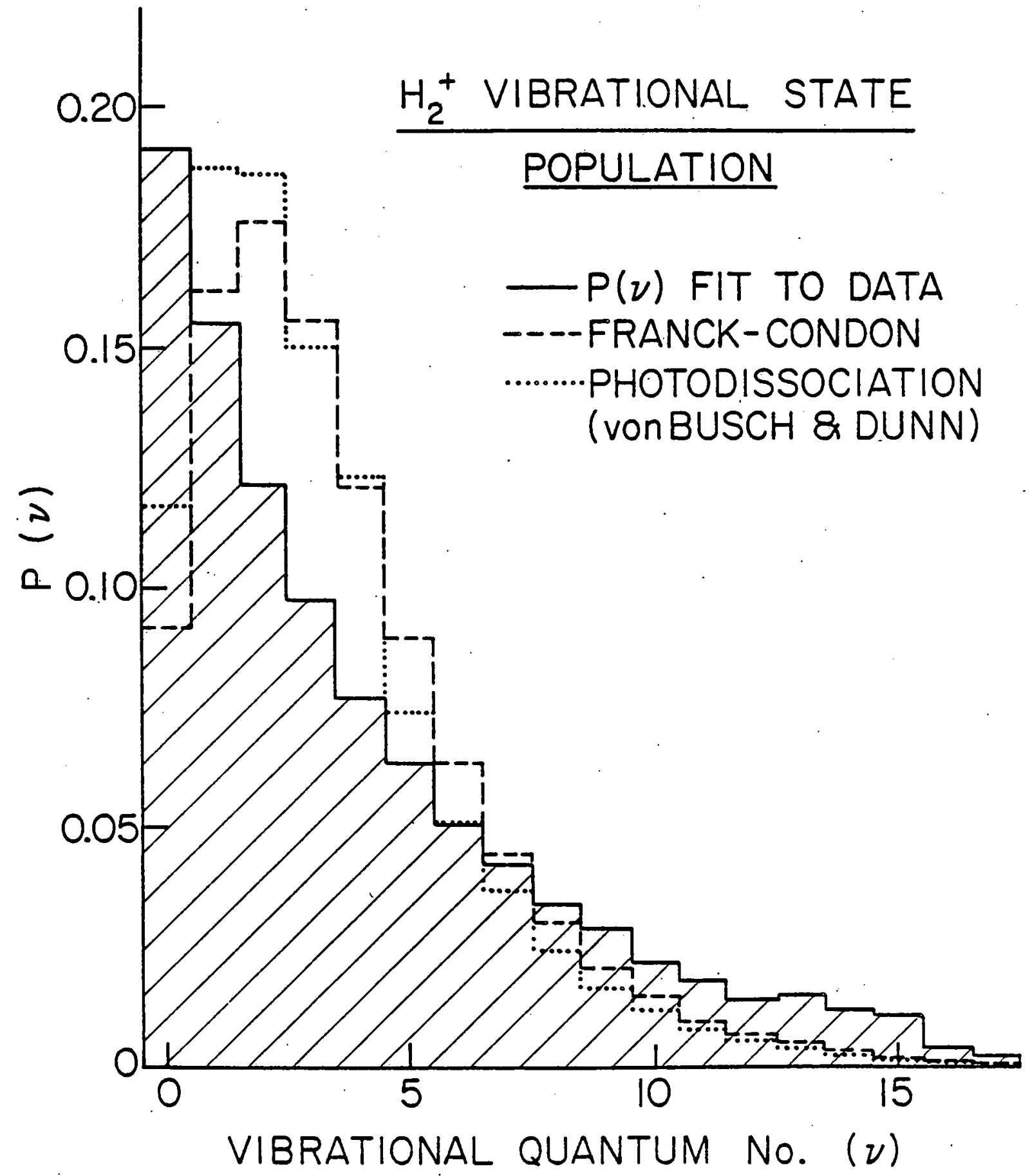

Fig. 11 


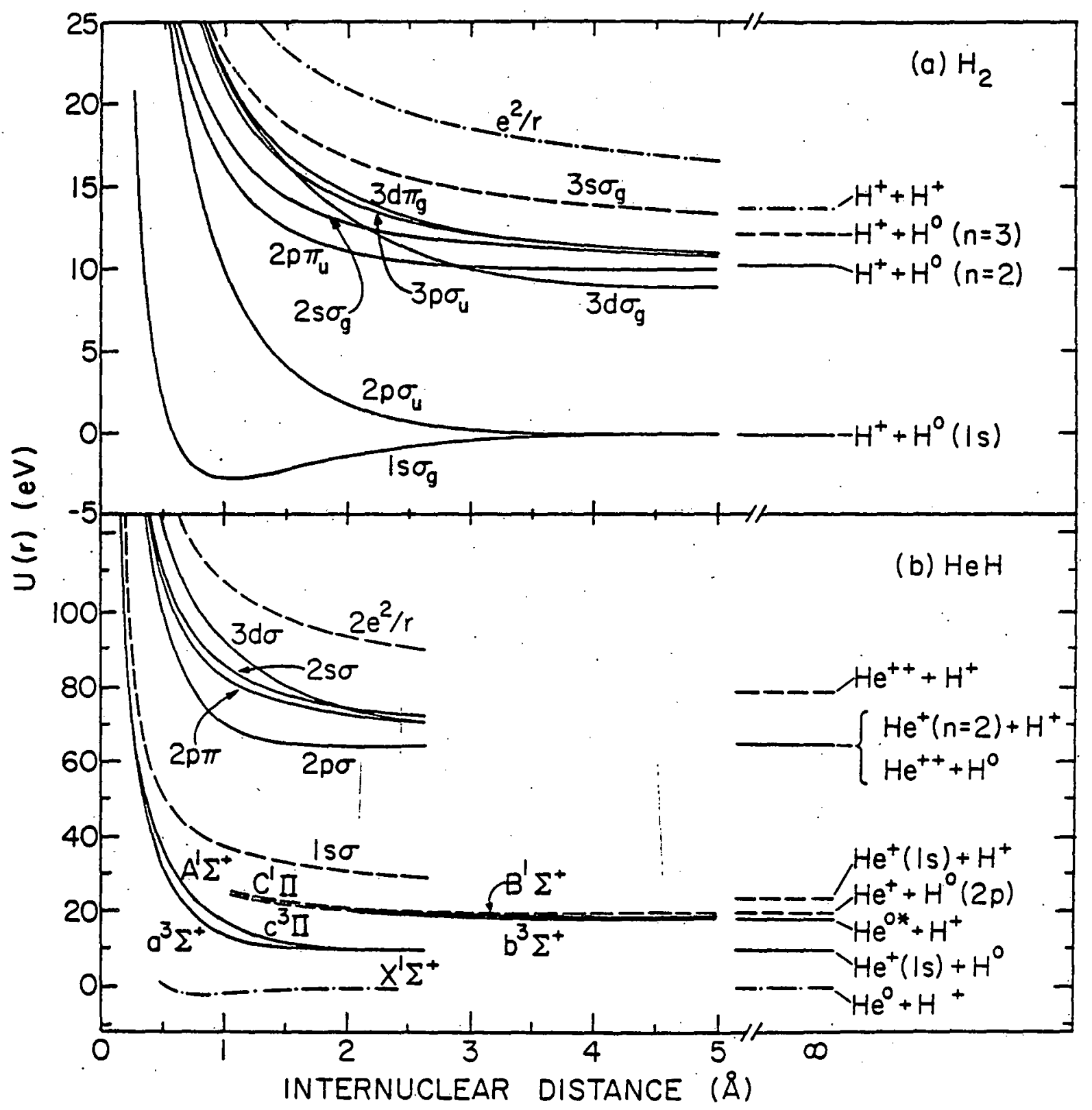

Fig. 12 


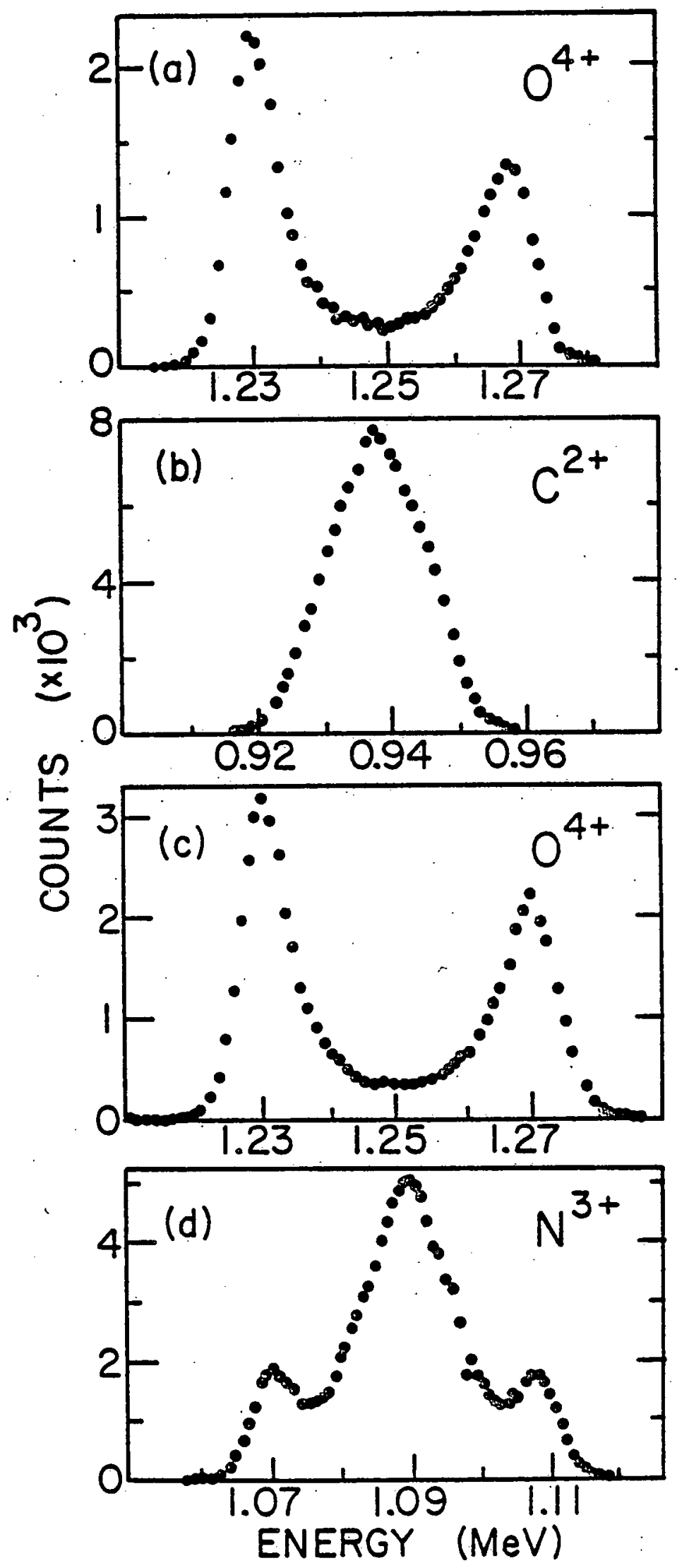

Fig. 13 


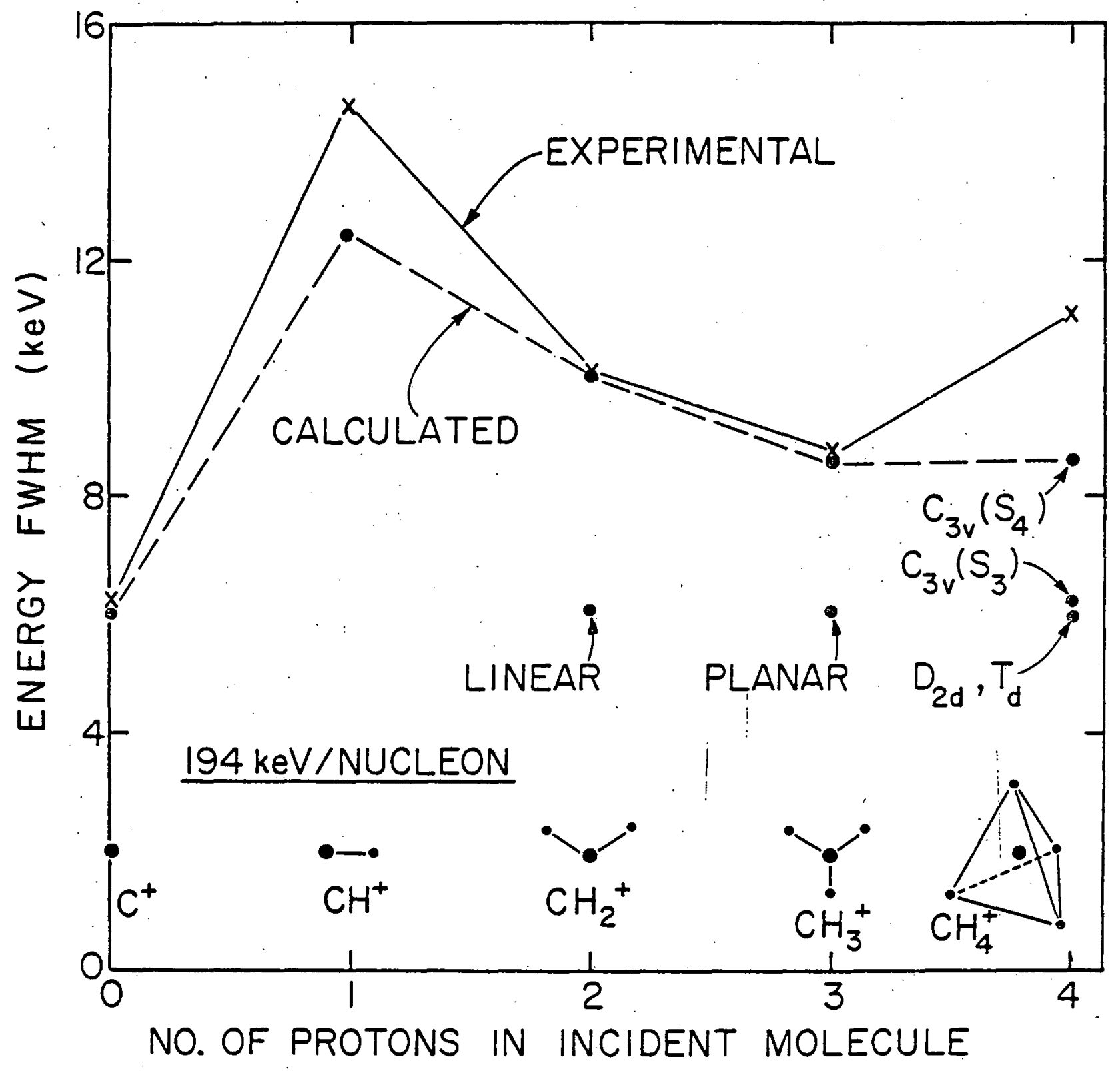

Fig. 14 


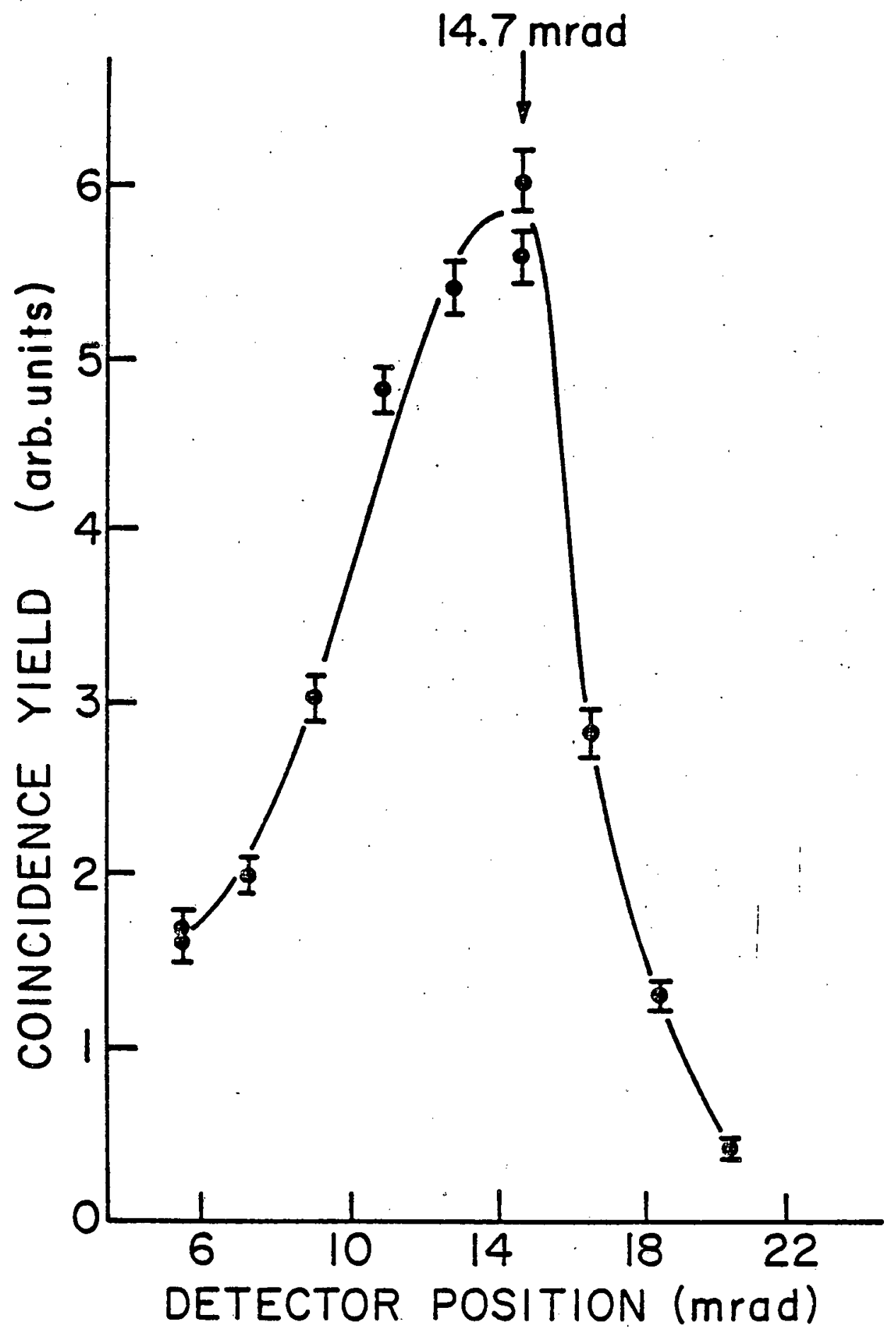

FIg. 15 


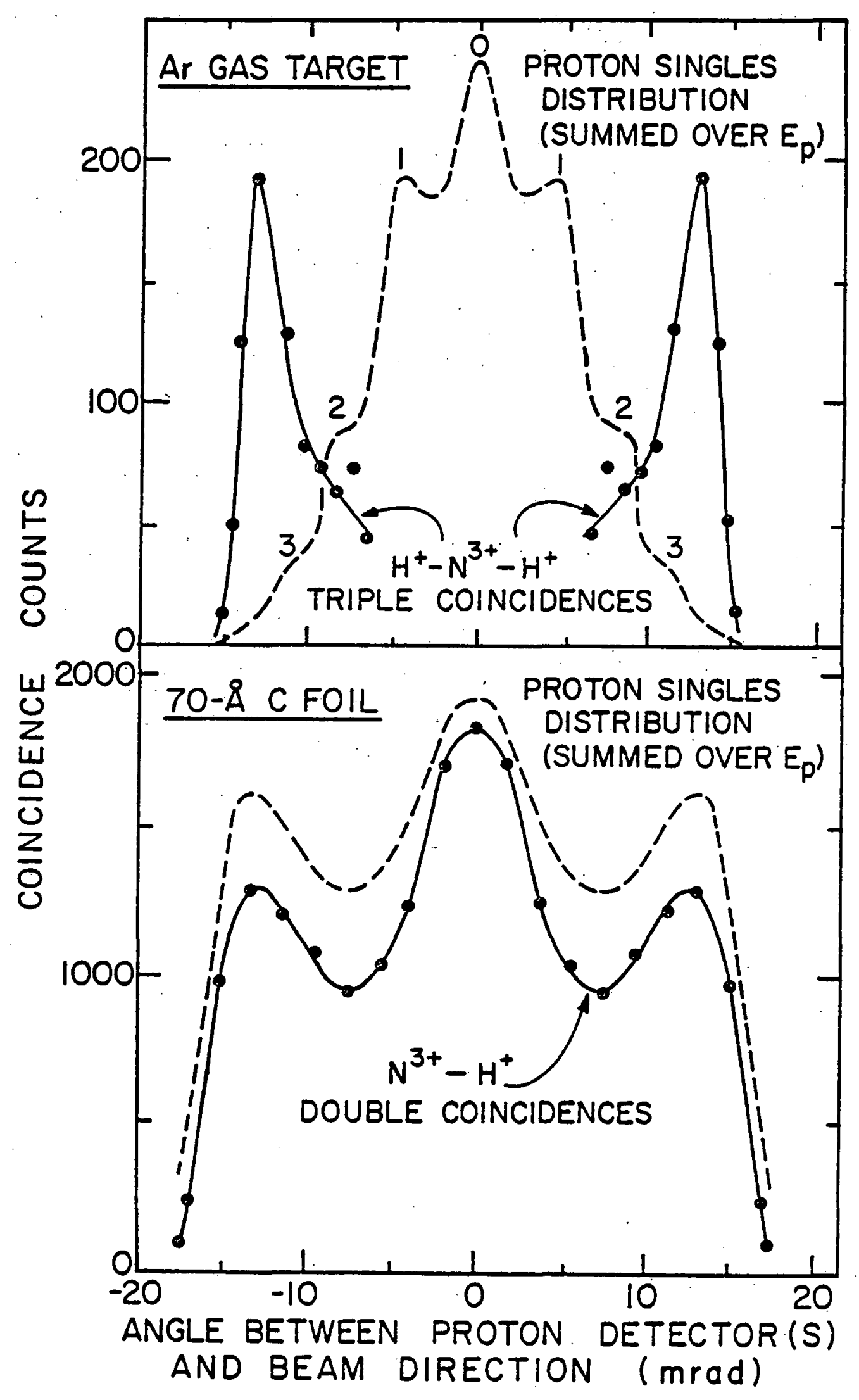

Fig. 16 
AFTERNOON SESSION

20 August 2979

F. Fujimulo, CHAIRMAN 
TRANSMISSION OF MOLECULES AND NEUTRAL PARTICLES

USING INDROGEN AND HELLIUM MOLECULAR BEAMS

J. Remillieux et al. 
The LYON Gromp

88

Nelson CUE (SUNH/ABBAY)

Nelison IE CASTRO FARIA LPUC Re Rio de JAAEI

Michel-Jean GAILLARD

Jean-Clande PollzaII

Joseph Reminue UH

Robert KIRSCH

Projectites: $H$ and 14 spesias $\left(v>v_{0}\right)$

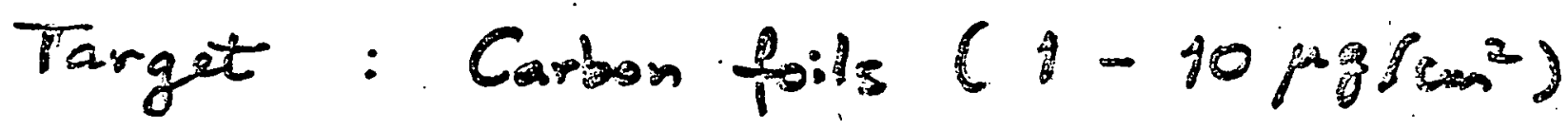

$(60-600 \AA)$

Measurzments: Total giviles

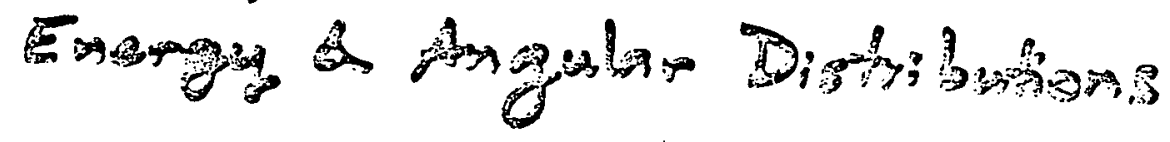




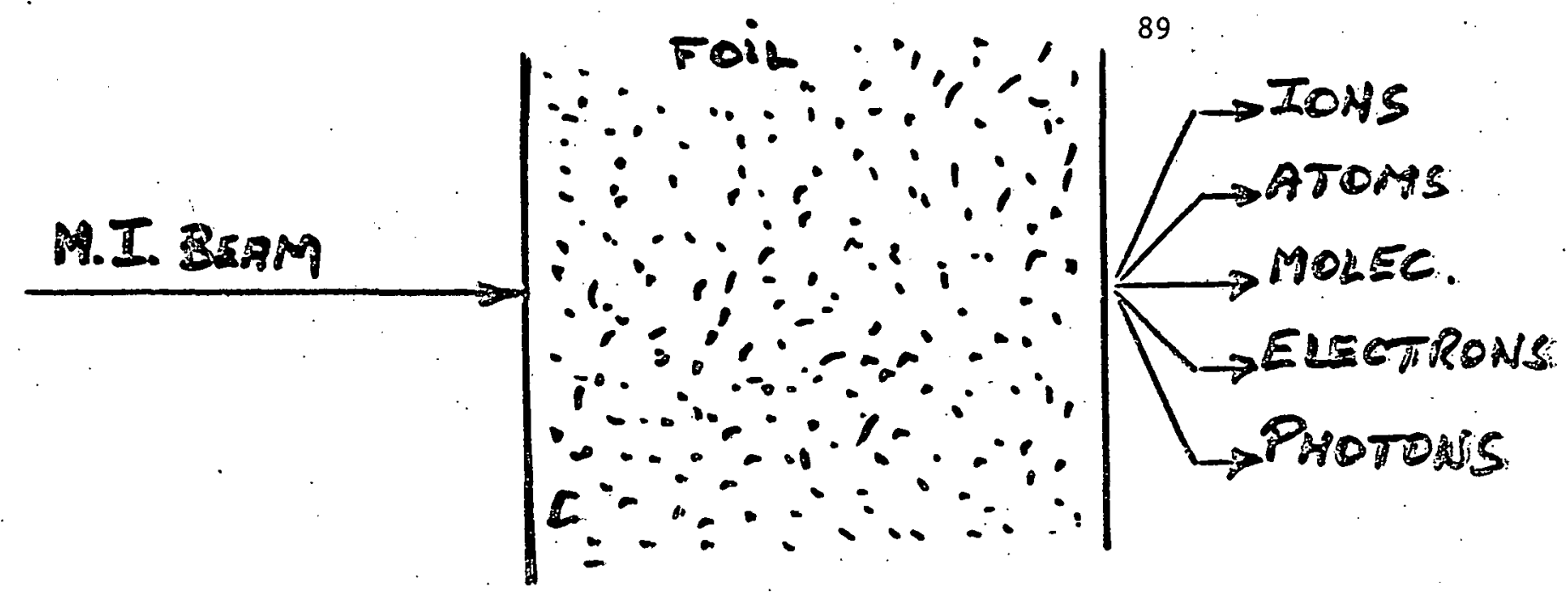

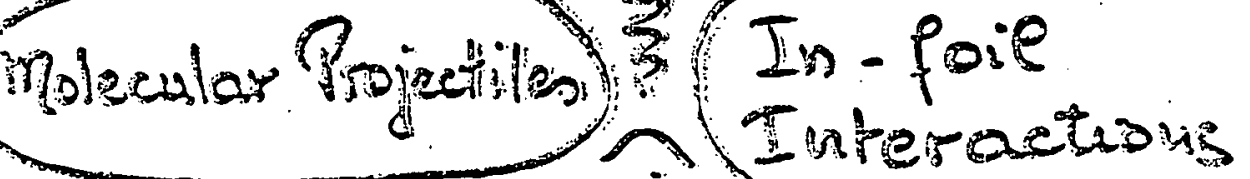

Post-pois eppers

s. I. production Coulourls Poree 3

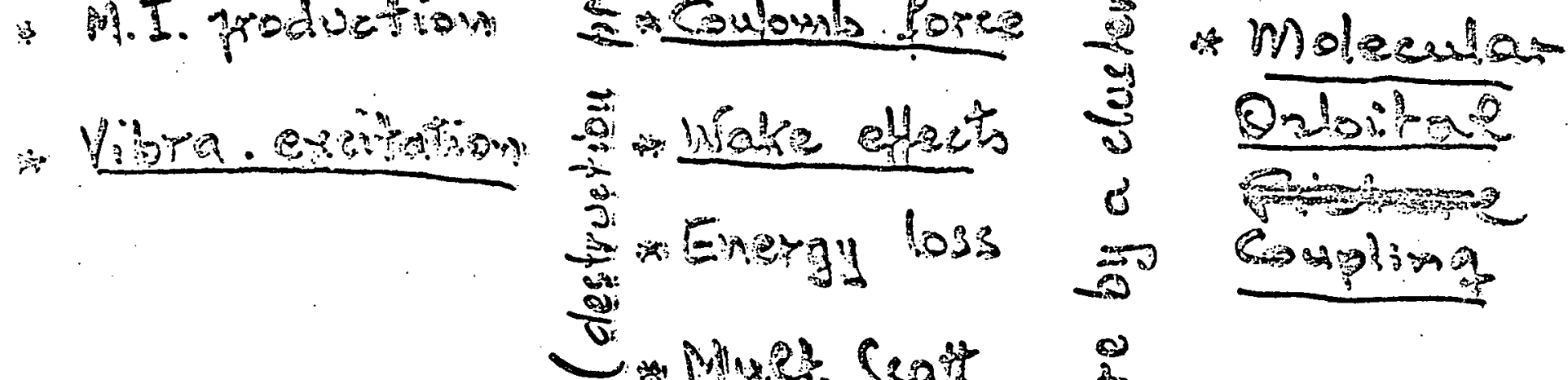
5realing

3

\#

3

2 
90

FOUR PARTS OF THE TALK

1. Cross sections for projectile $e^{-}$loss (atomic and molecular species) and target election captive (atomicispecies) from transmisswin measconements.

2... Central peak and intending asymmetry of explosion peaks in the $0^{\circ}$ energy specter

3. A quanhtature model for the yields of $\mathrm{H}_{2}{ }^{+}$and $\mathrm{H}^{\circ}$. from incident $\mathrm{H}_{2}{ }^{+}$

4. change exchange between supanculingy fragments. 
91

1

ELECTRONIC STATE OF SIMPLE MOAS \& MOLECULES

115 - SOLID

MEASUREMENT OF $\sigma_{L}$ AND $\sigma_{c}$

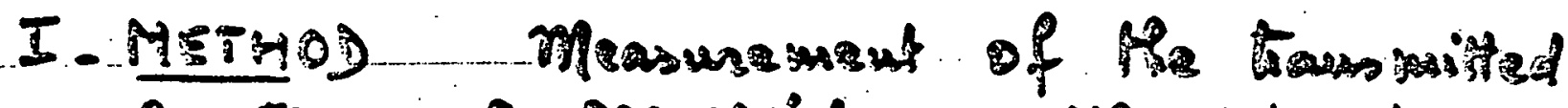

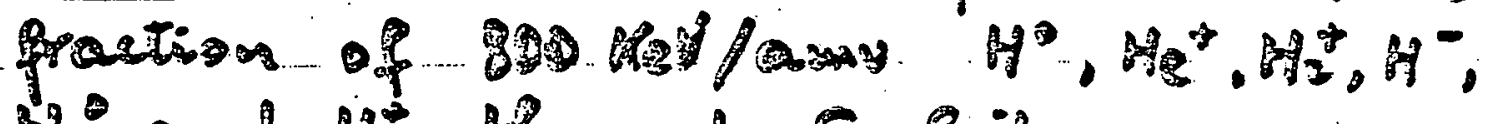

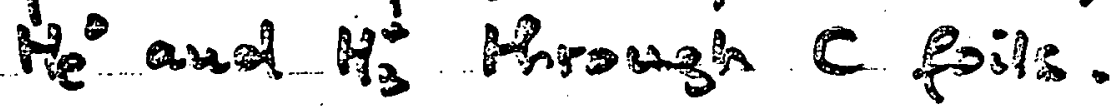

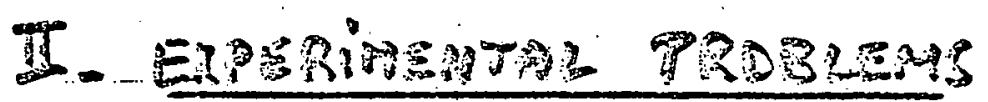

- $4^{\circ}, \mathrm{H}^{3}, \mathrm{H}^{-}$Beams, use of a shifts Pail jos gas feature in th a bears ling. H-

target pails, pinhole elf last elsyaing

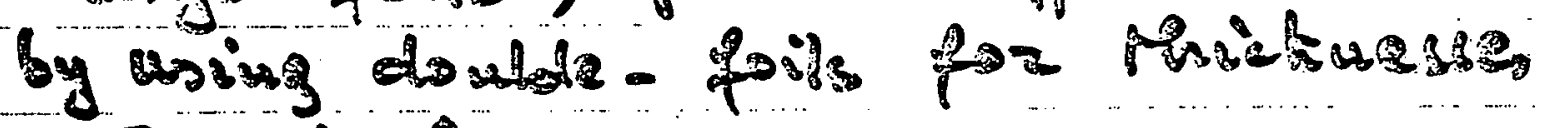
$73 \mathrm{Ng} / \mathrm{Ca}^{2}$.

III- RESULTS $(9 \% 301)$

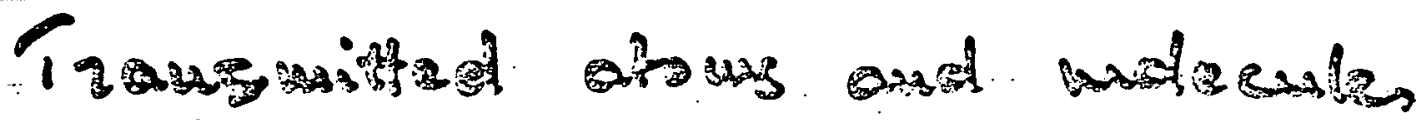
cos bs

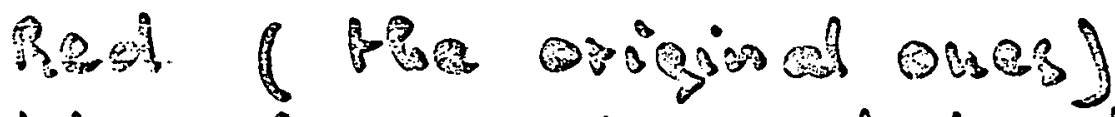

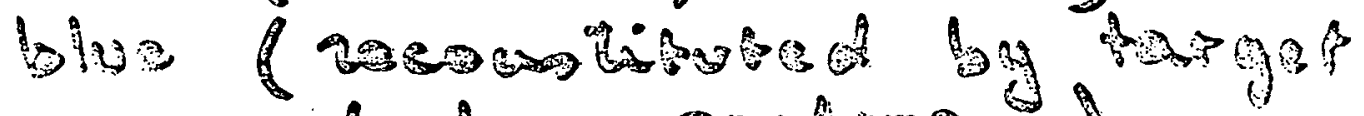

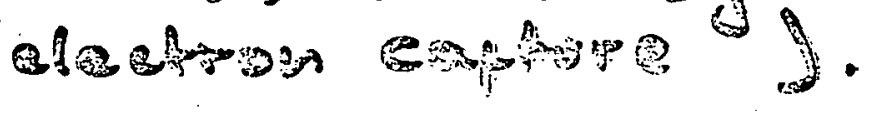




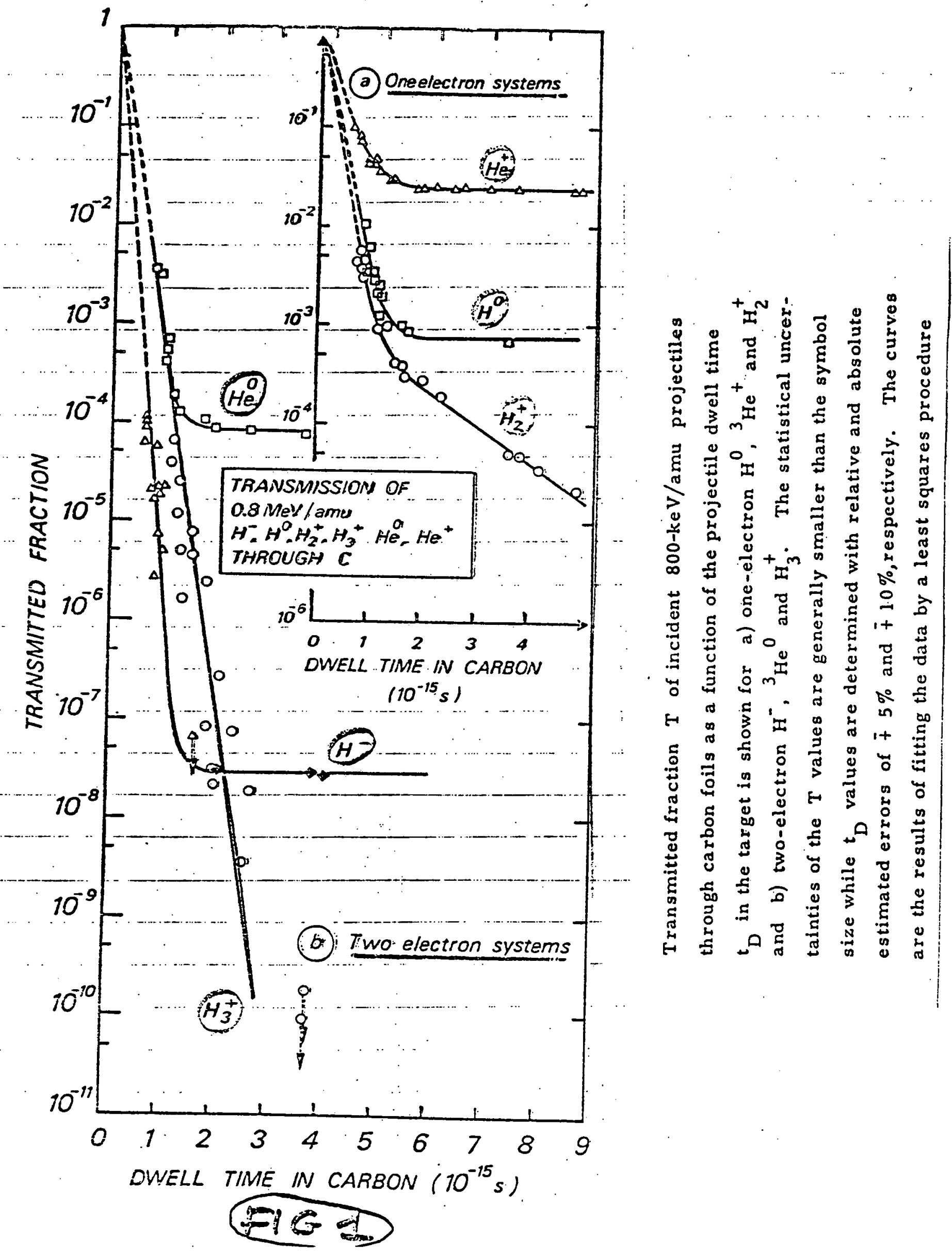


2

Trua Trassanission Regizass
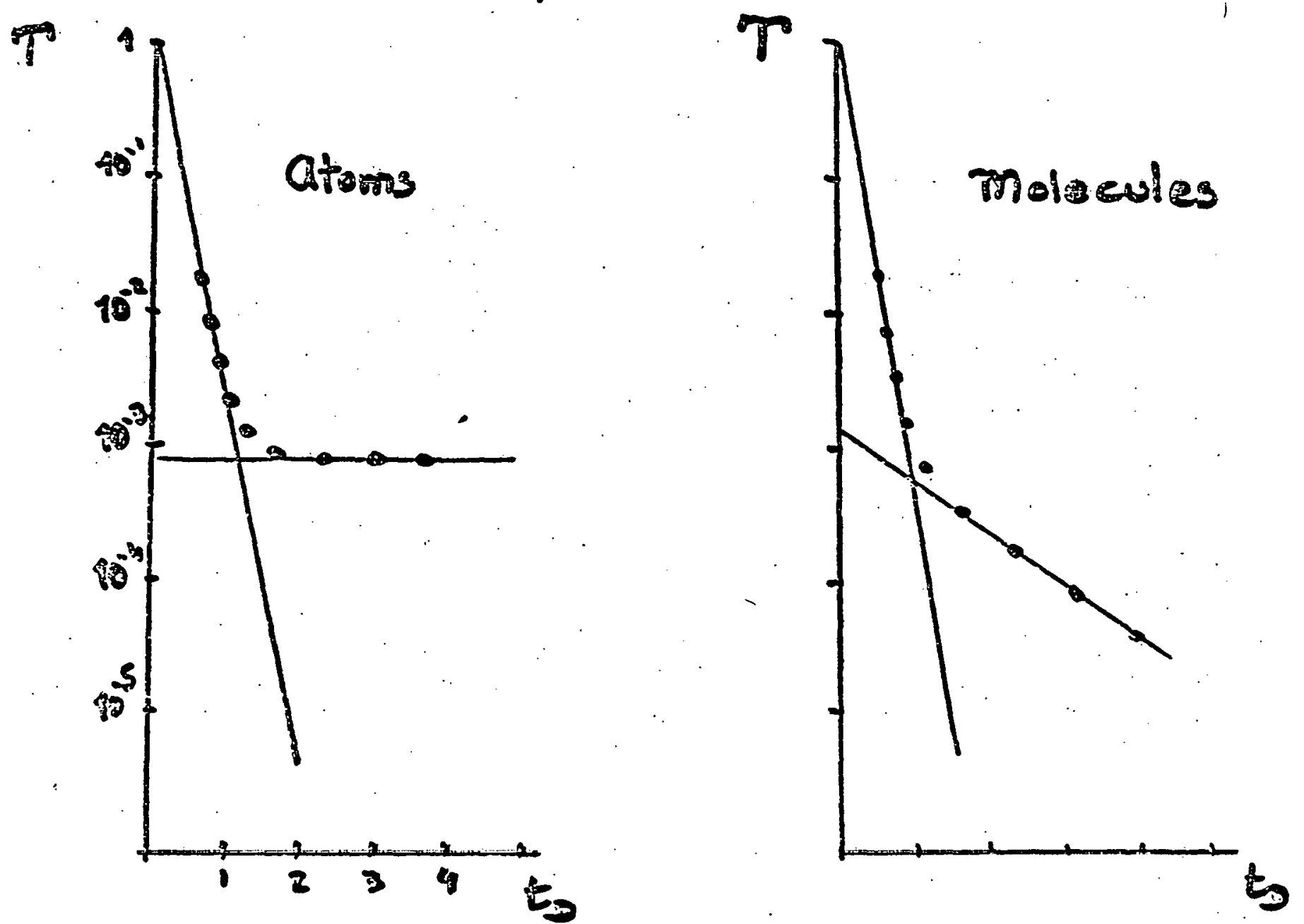

RDD ATOMS

RED MOLECU1SS

$T=e^{-t D / 5}$

$T=e^{-t o / 2}$

BQ⿱一土㇒ ATOMS

BUE MOLECULSS

$T=\cos \theta \%$

(F⿻二丨 2

$T=f(\infty)$

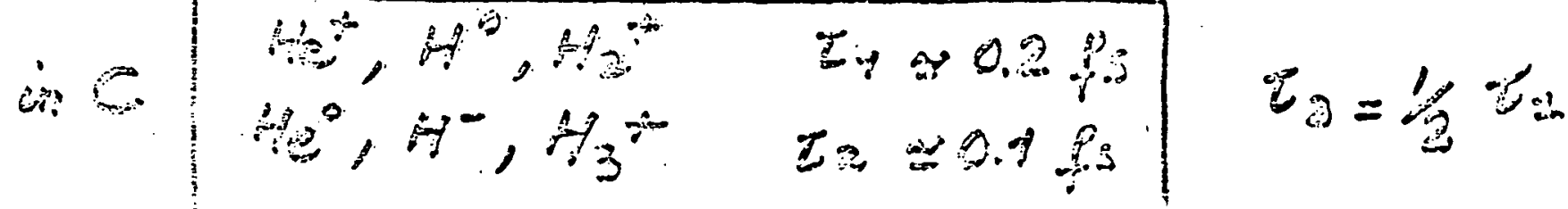


$3 j$

94

II DEstruEtion cross section

Transmisation yired

$$
T=(1-B) \exp \left(-\frac{t a}{\tau}\right)+B \exp \left(-b t_{0}\right)
$$

- for atous: $b=0, B=\phi_{i \infty}$

- for $\mathrm{H}_{3}^{+}: 3=0$

Desturstion crass section

$$
\sigma_{p}=\frac{1}{M+v_{1}}
$$

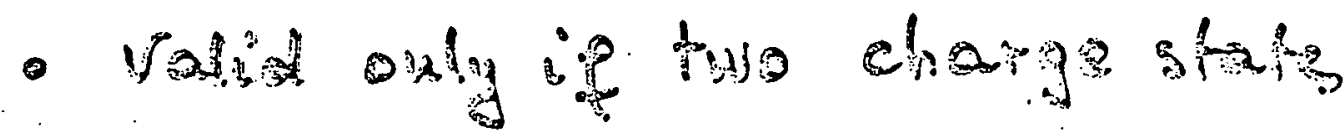
ars iaragiat

Tlols prayed by othes change otats

- masmarmant of Ho ores

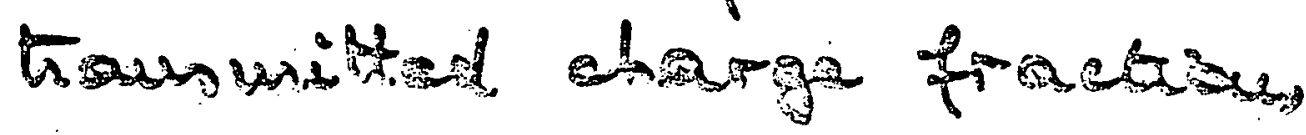

- Par insidass $\mathrm{H}^{-} \cos \mathrm{H}^{\circ}$ $(\sin 3)$

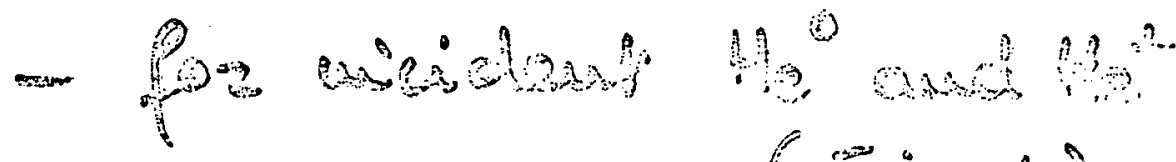
$(8,6)$ 


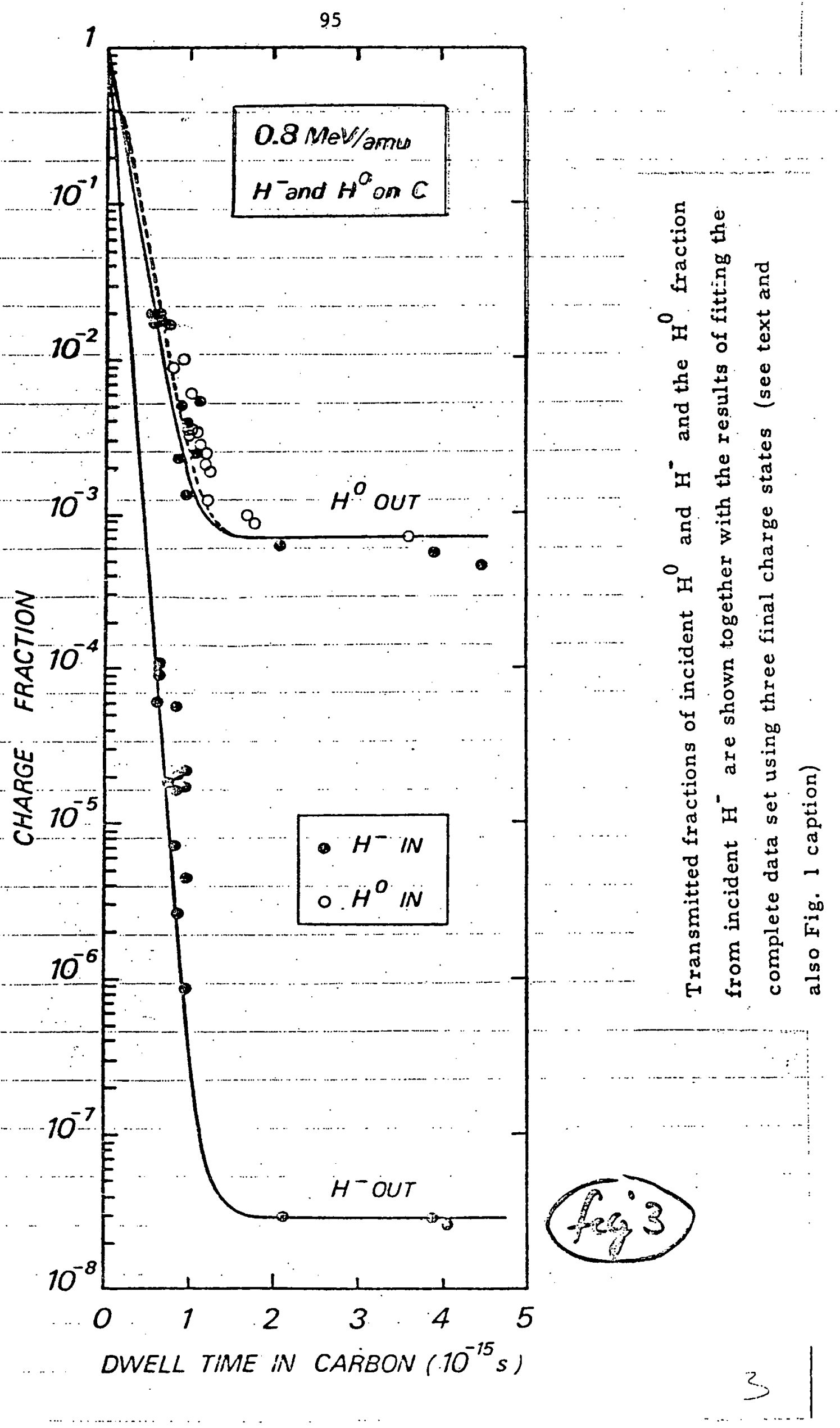




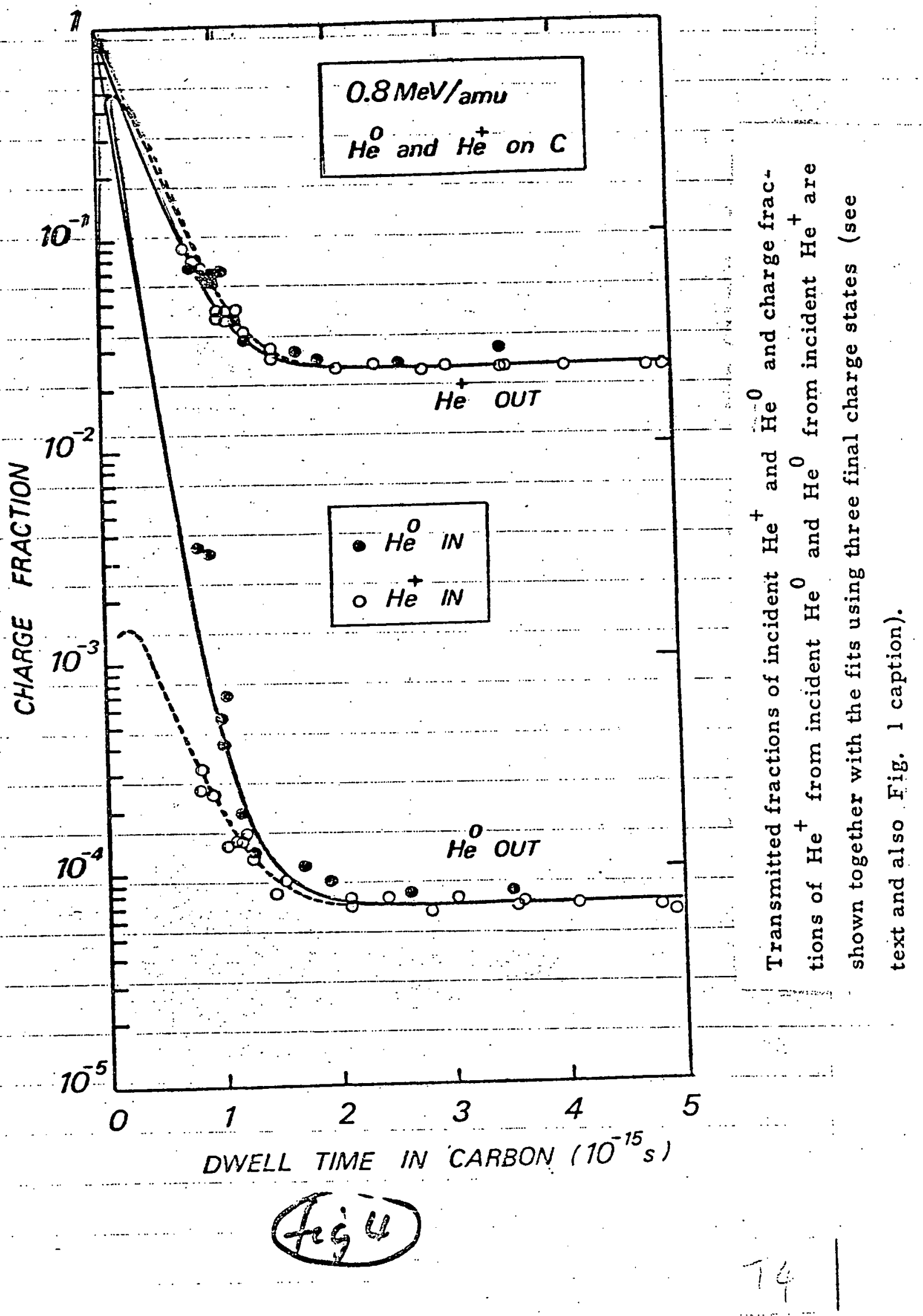


4

.97

$\sigma_{e}=f\left(z_{e} f f\right)$ and $\sigma_{e}=f\left(z_{e} p\right)$ Q a fit of the trangmision yized for atows in the 2-chang-otats esue aud the 3-chenge-obets caney give, the chom section for tor or

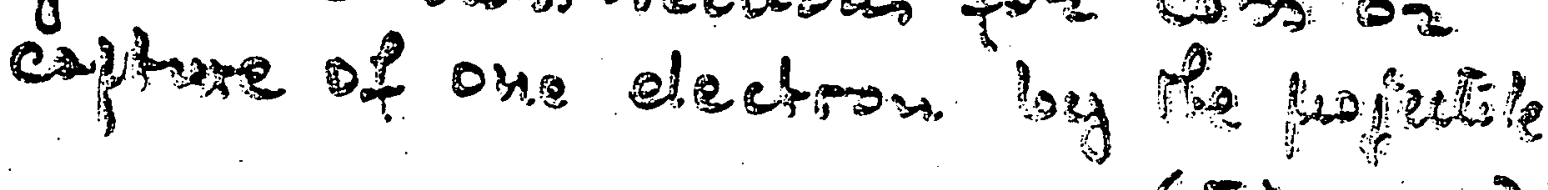

- Zem $(5.9 .5-6)$

- Pos absu

$$
\begin{array}{cccc}
H^{-} & 4^{\circ} & \mathrm{H}^{2} & H^{2} \\
0.3 & 1 & 1.9 & 2
\end{array}
$$

- Pon ond

$$
\begin{aligned}
& \mathrm{H}^{2} \mathrm{H}_{3}^{2} \mathrm{Her} \\
& 1.11 .5 \quad 1.2
\end{aligned}
$$

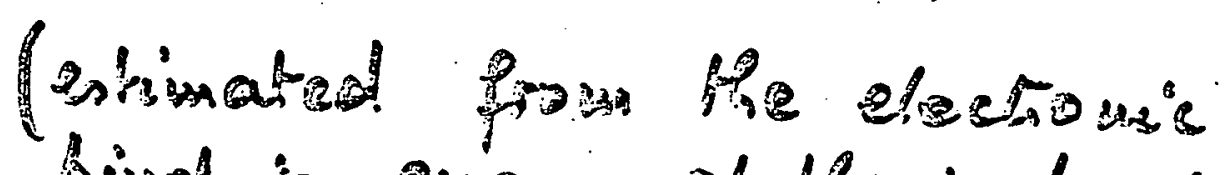

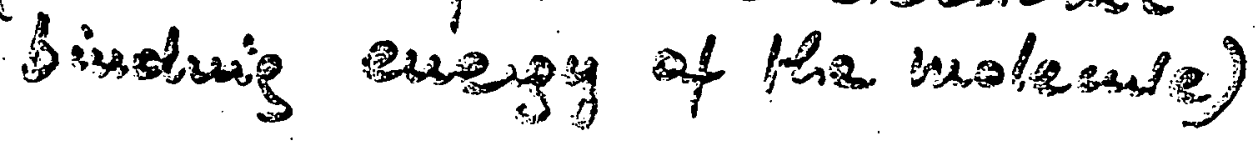

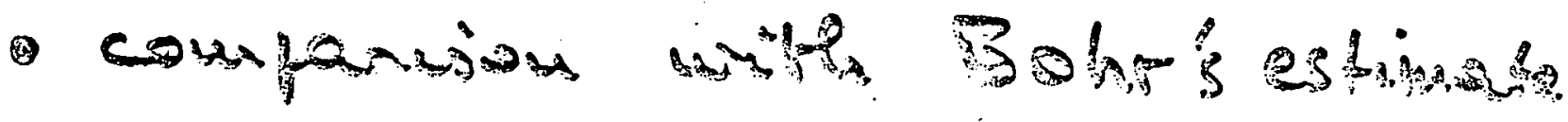




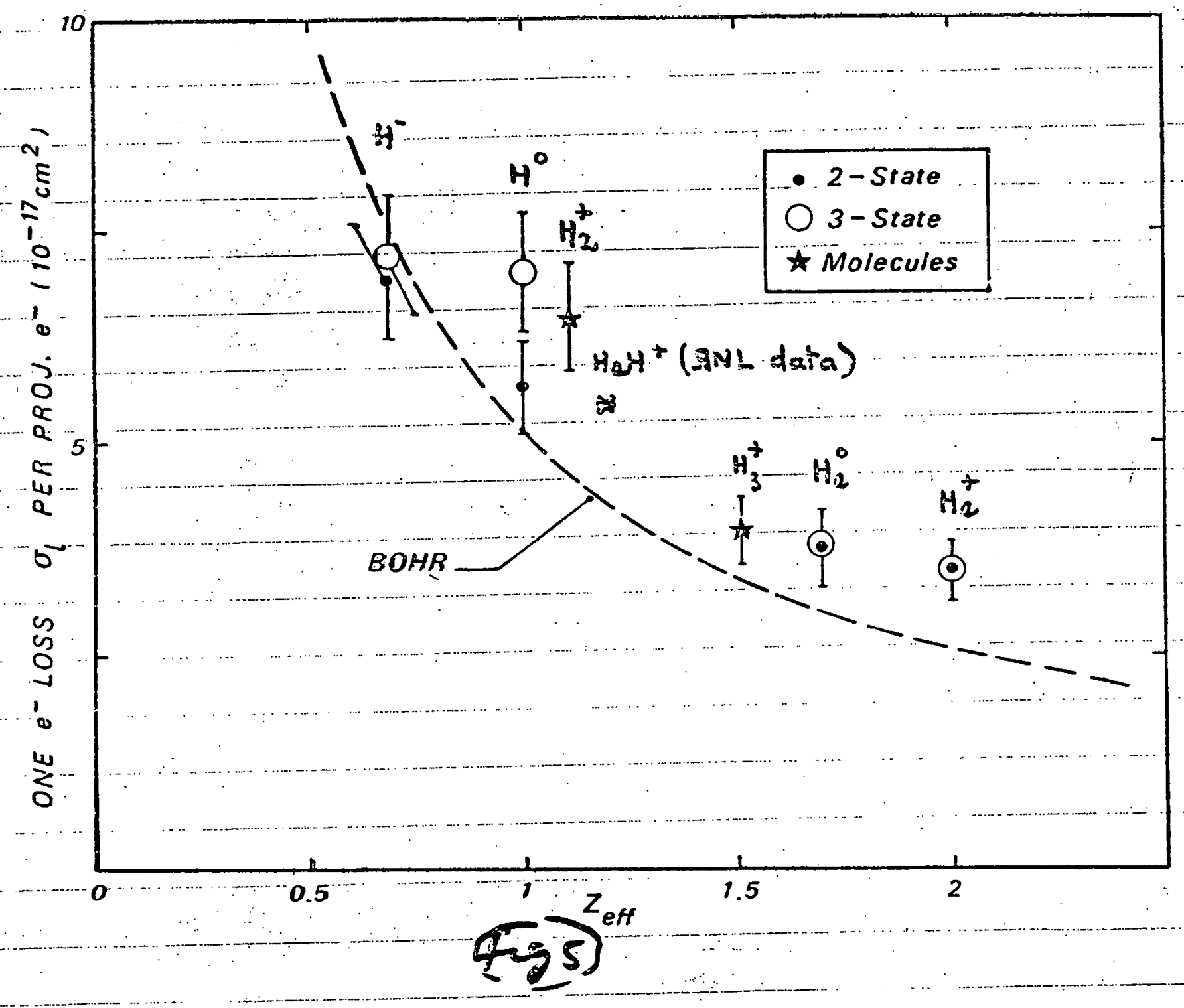

One-electron loss cross sections $\sigma_{\ell}$ deduced from the data in Figs. $1-3$ are displayed according to the $Z_{\text {eff }}$ of the incident projectiles. These $\sigma_{\ell}$ have been divided by the number of electrons in the projectile. The errors were assigned on the basis of the uncertainty in the target thickness measurements. The curve represents the corresponding predictions of Bohr 


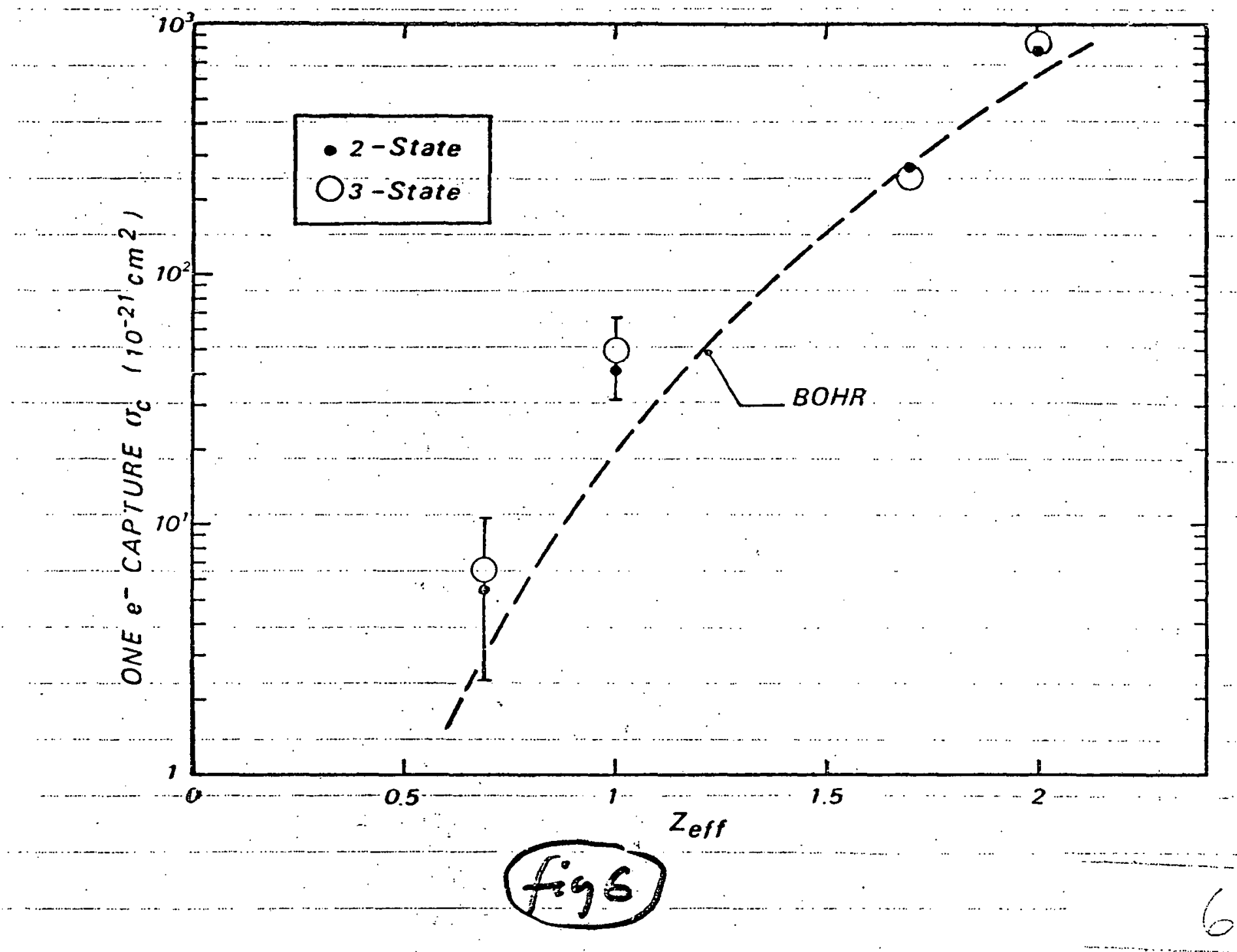

Experimental one-electron capture cross sections $\sigma_{c}$ and the corresponding theoretical predictions of Bohr (curve). Here the $Z_{\text {eff }}$ refers to the final state (see text and also Fig. 4 caption). 
from Genunell et al.

100

$\mathrm{HeH}^{+}$transmission

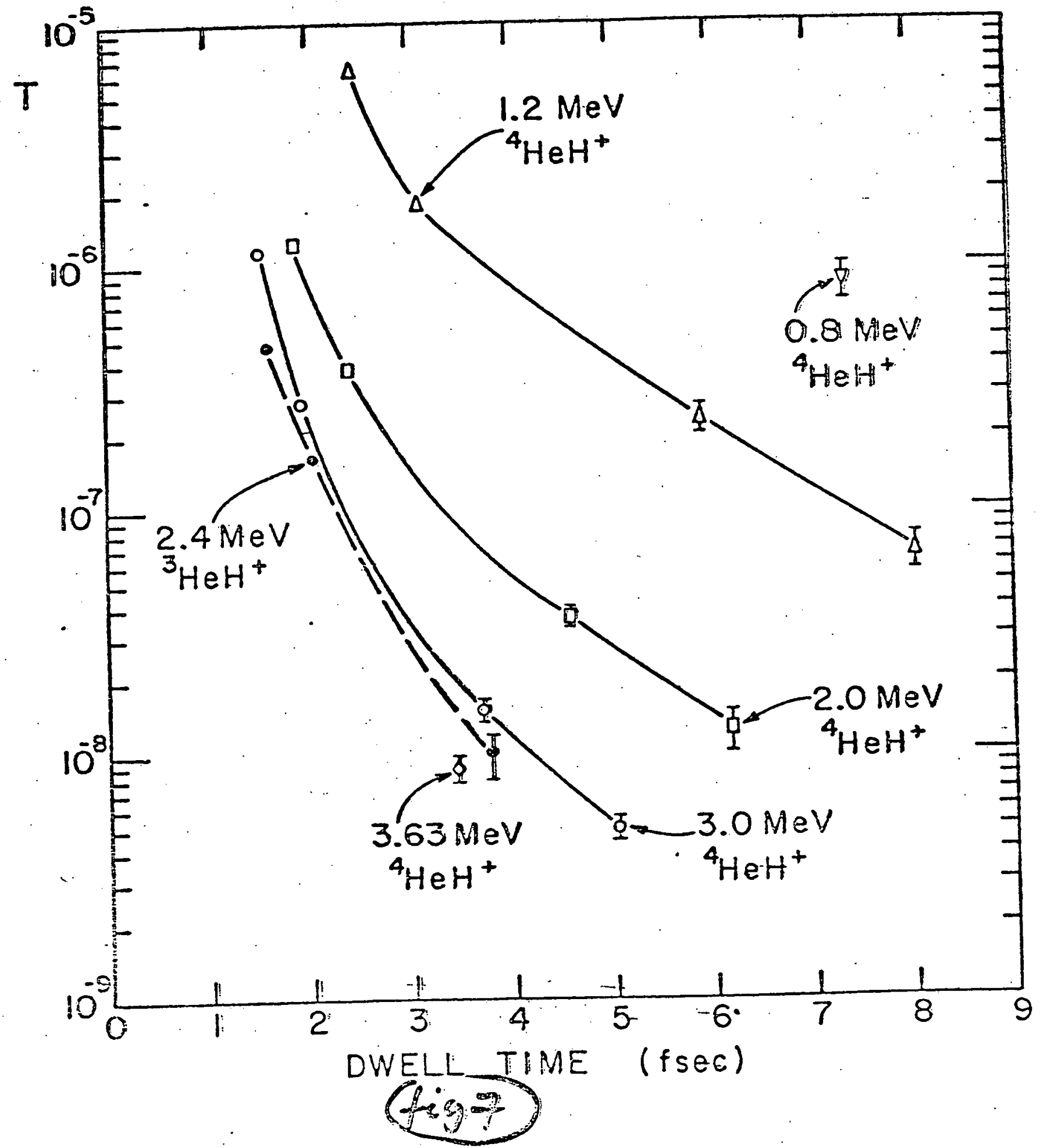


$\equiv 1$

101.

Ellectrrenic starte in salial.

- change exzhrase cross section ui gos and is $\rightarrow$ oliog. for $H^{\circ}$ (Fib.8)

- urear fra fath of zed eoud frez $e^{-}$of the saur ver-

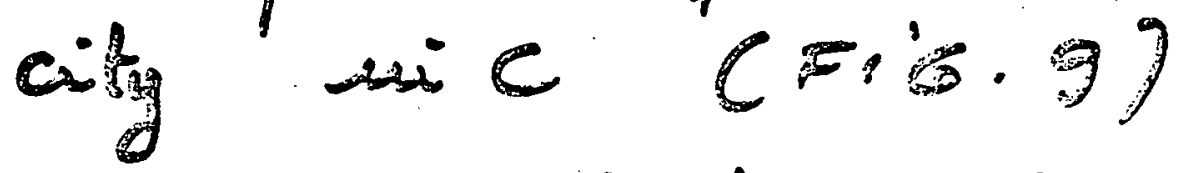

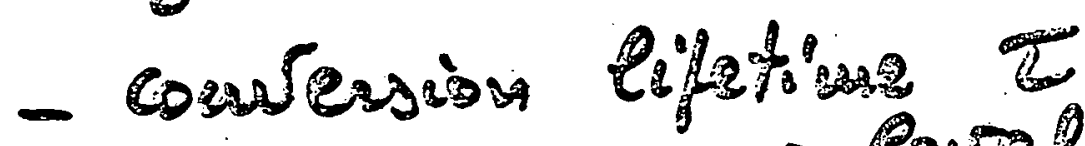

$\rightarrow$ Pans widts

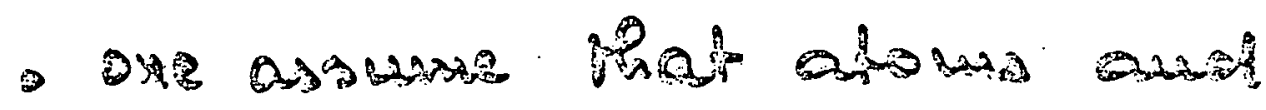

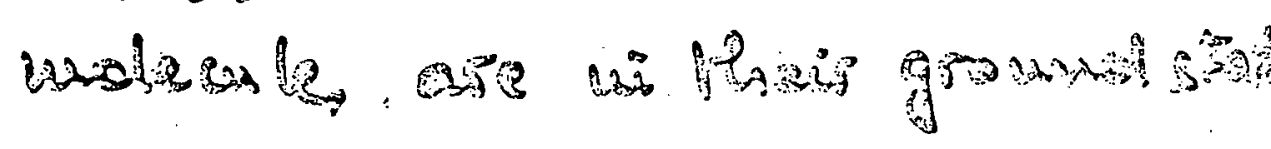

- $P=P($ destrastion angrga $D)$

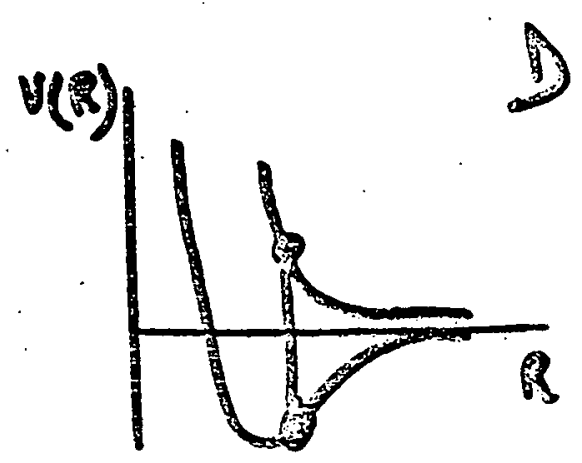

D $\rightarrow$ Eb for atoun

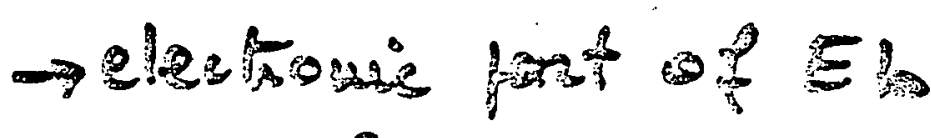

Pon male exts

$(516.10)$

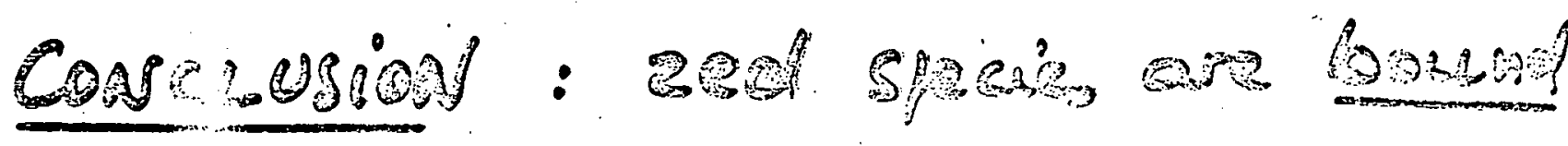

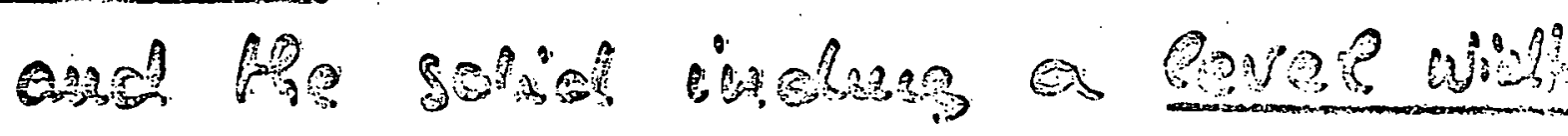

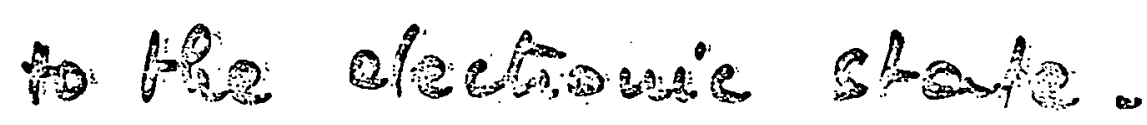




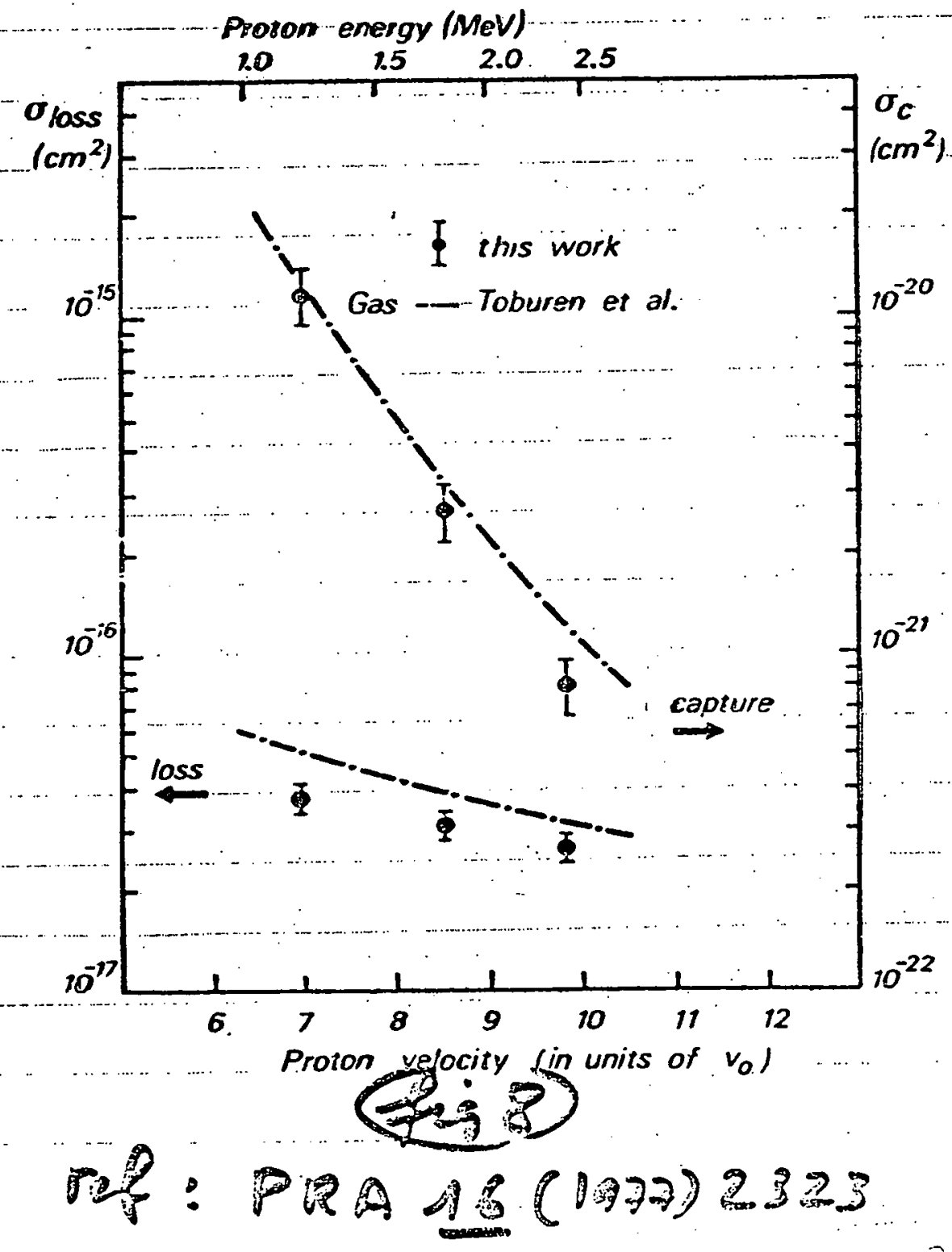



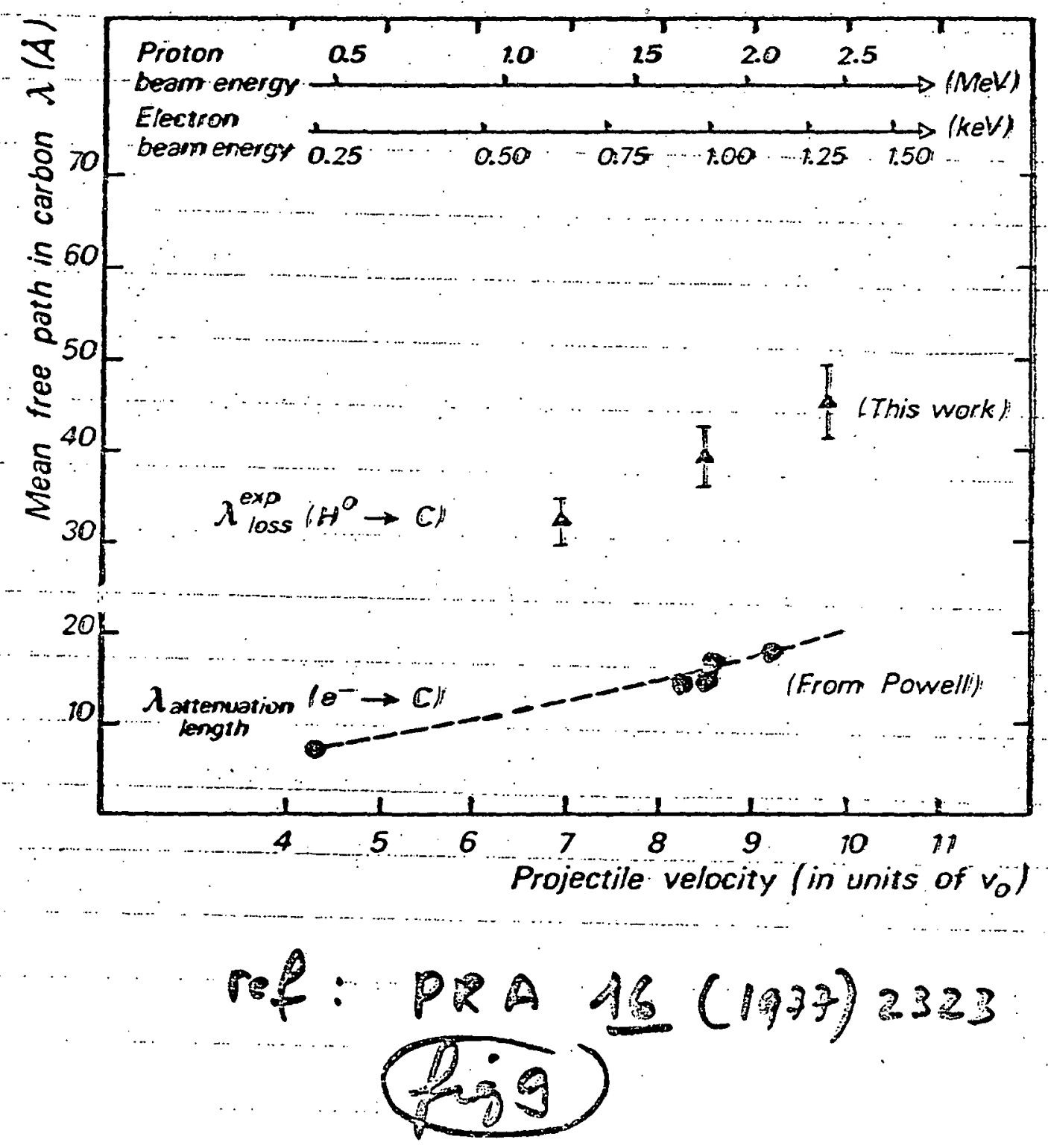

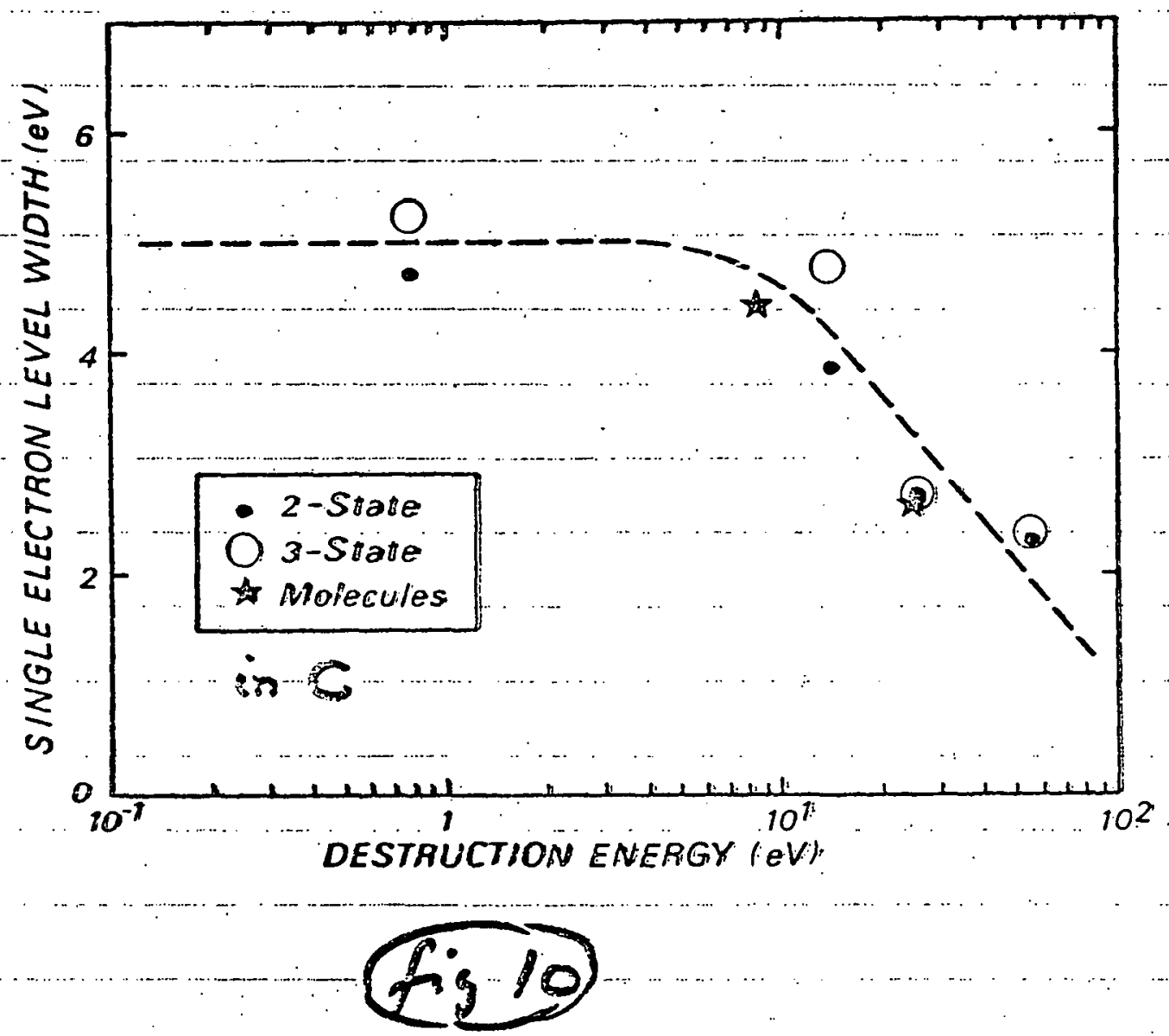

Single-electron level widths $\Gamma$ deduced from the corresponding $\sigma_{2}$ in Fig. 4 are displayed according to the energy required for the destruction. of the incident projectile (see text fir details). The curve drawn is only for the purpose of indicating the general trend. 
$2 \mid$ HO "CEMTIRAR PEARs"

AND "RED REGIOJES"

$\rightarrow$ Experinsutat sot up (Fis.

$$
\rightarrow\left[\mathrm{H}^{+} \rightarrow \mathrm{H}^{0}+\mathrm{H}^{+}\right] \quad(\mathrm{Fig})
$$

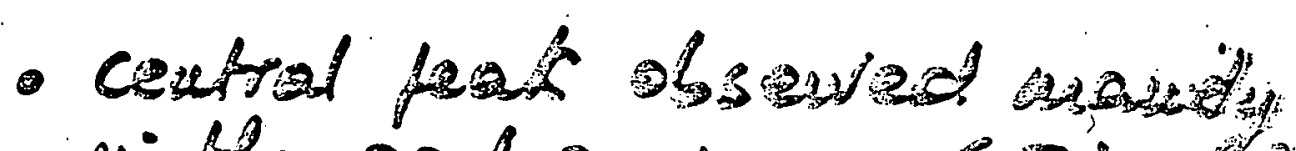

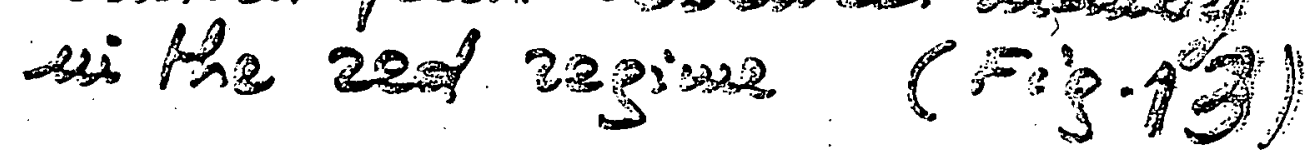

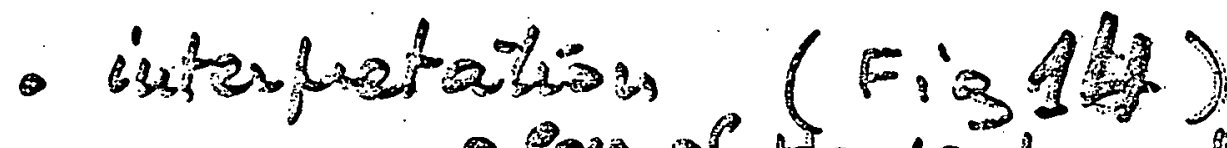

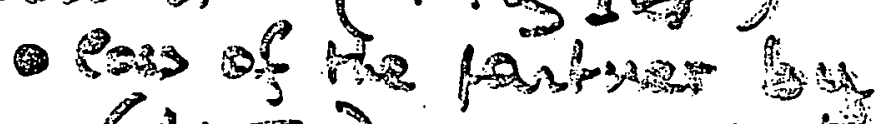

- $\left.\frac{(A+3)}{6}\right)_{0}$

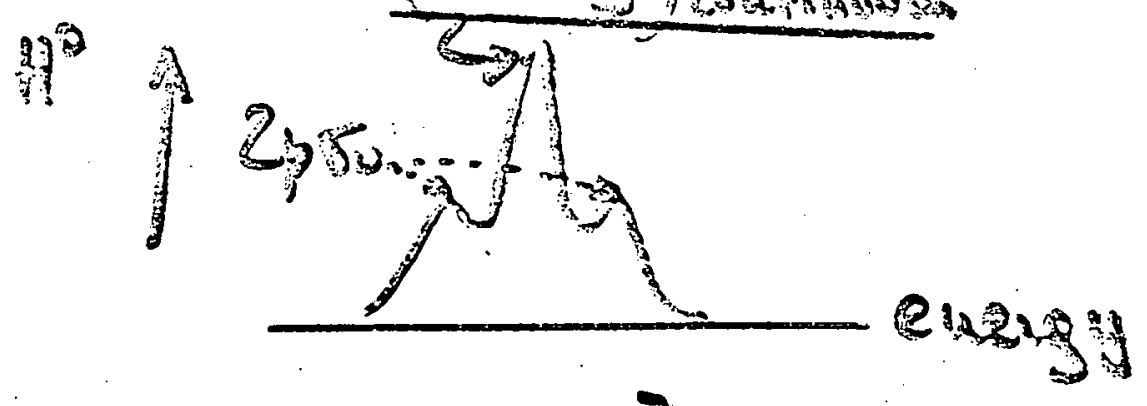

$$
\begin{aligned}
& \rightarrow\left[\mathrm{H}_{3}^{+} \rightarrow \mathrm{H}_{4}^{0} \mathrm{H}^{+}+\mathrm{H}^{+}\right] \quad\left(\mathrm{F}_{3} \cdot 3\right)
\end{aligned}
$$

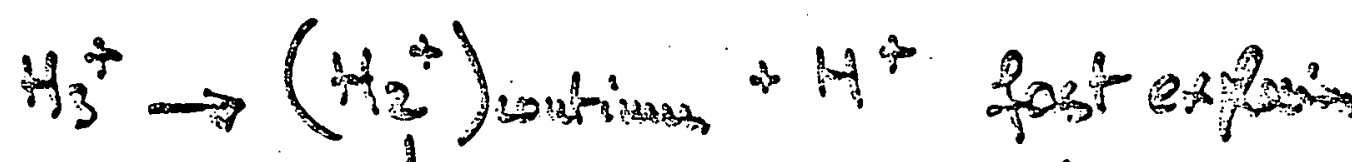

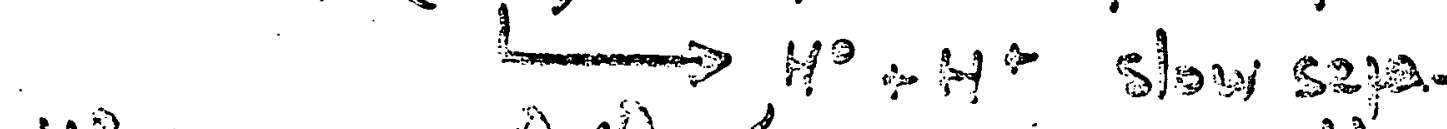

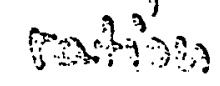


106

Detectors

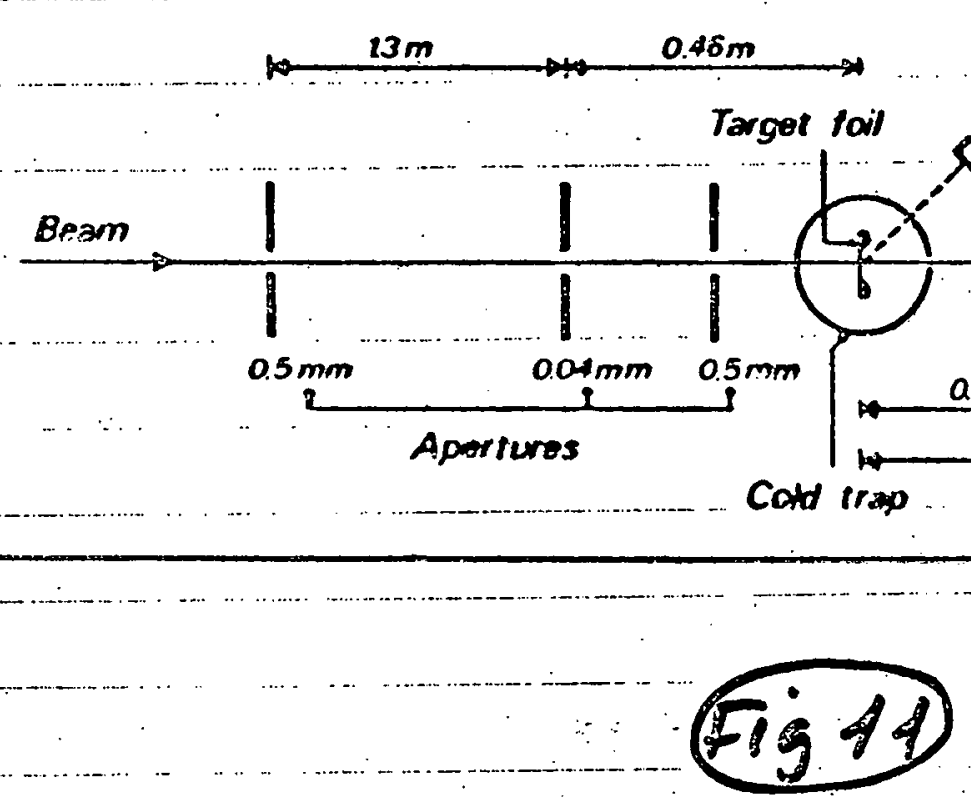


107
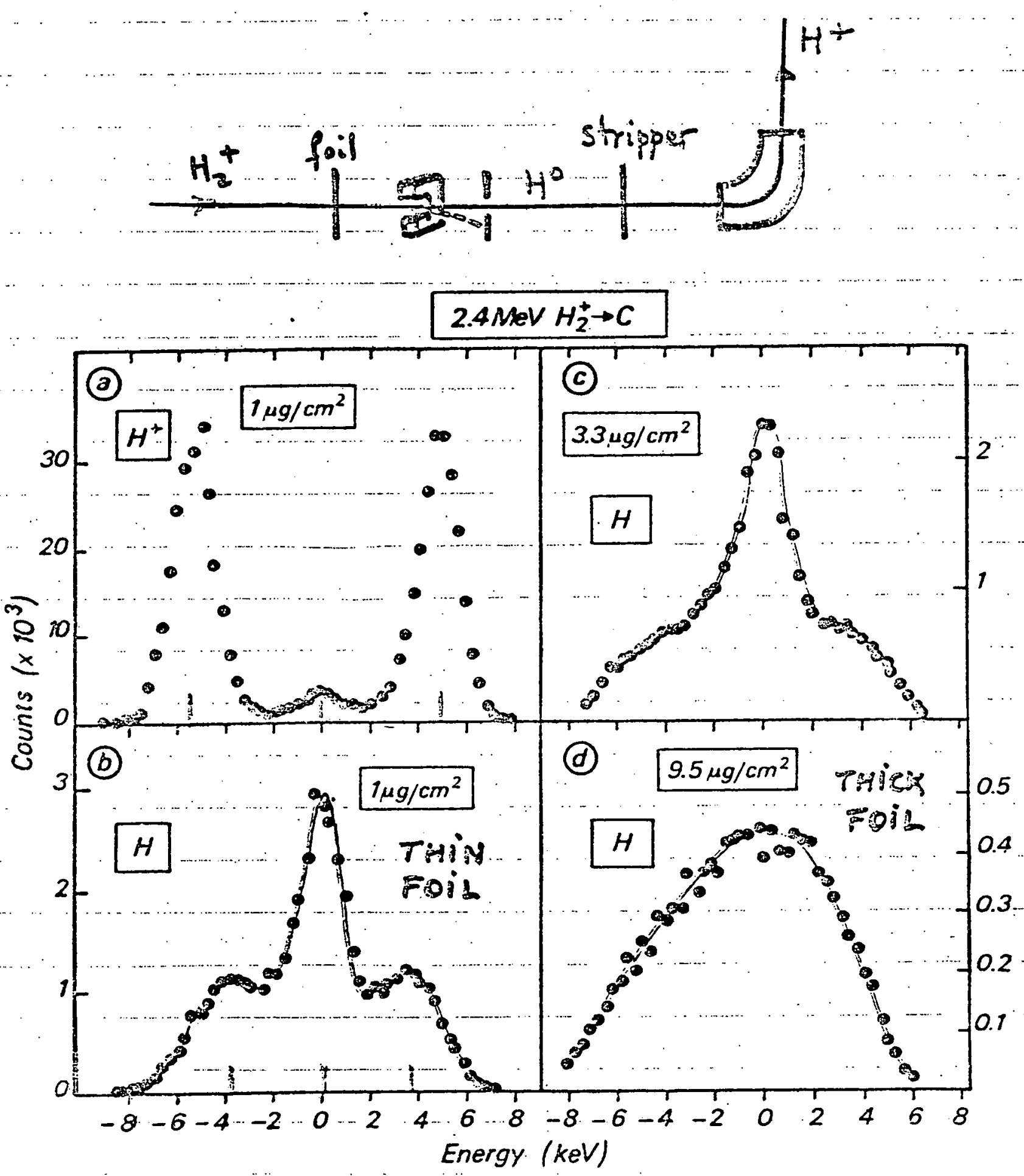

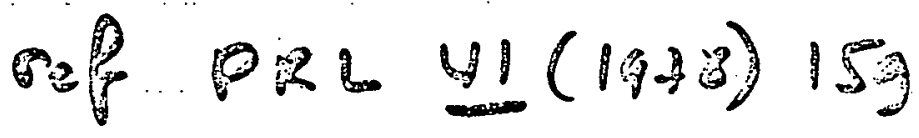

$f i g / 2$ 
108

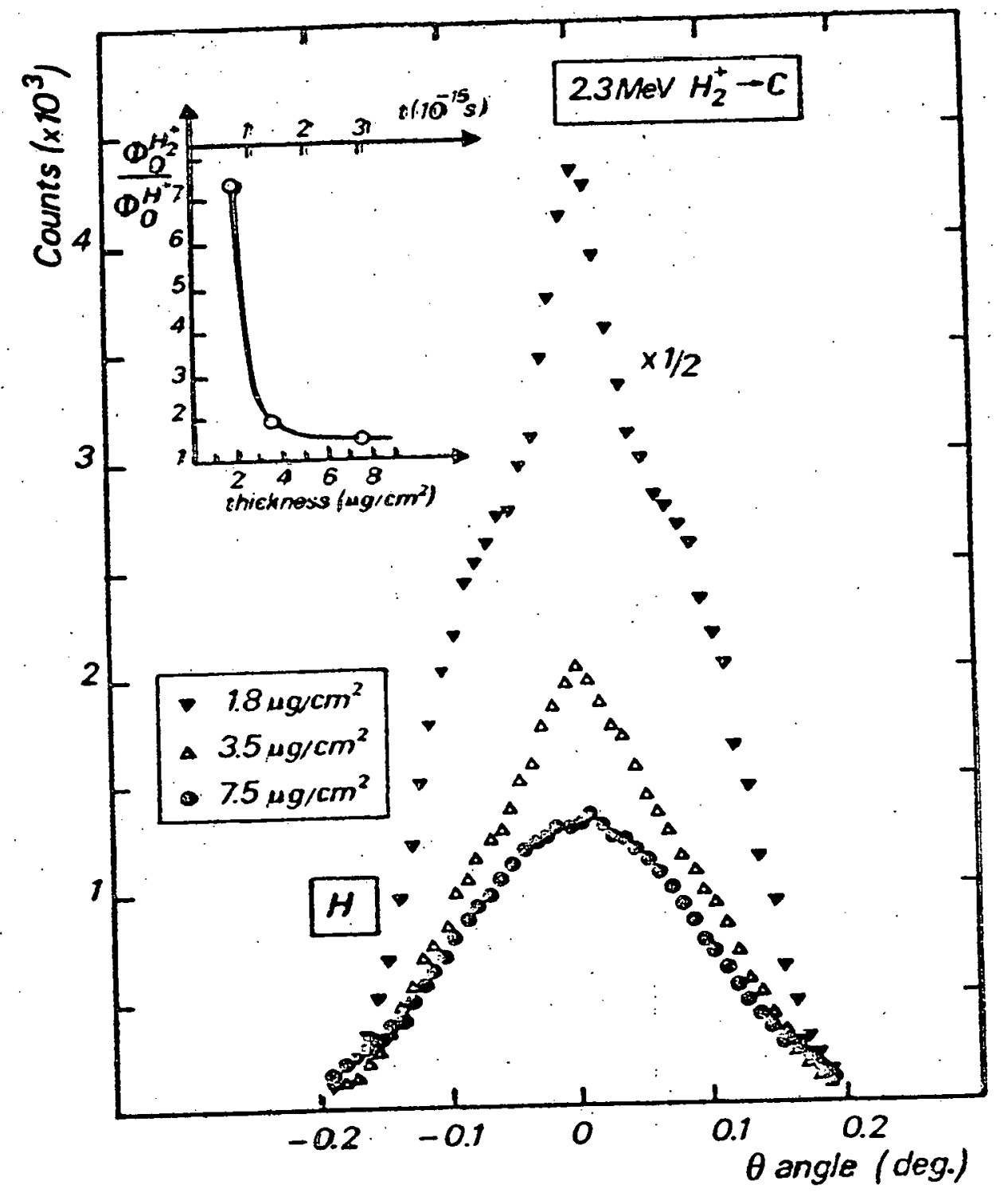

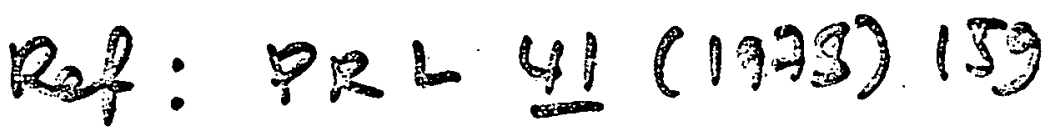

(ig) 


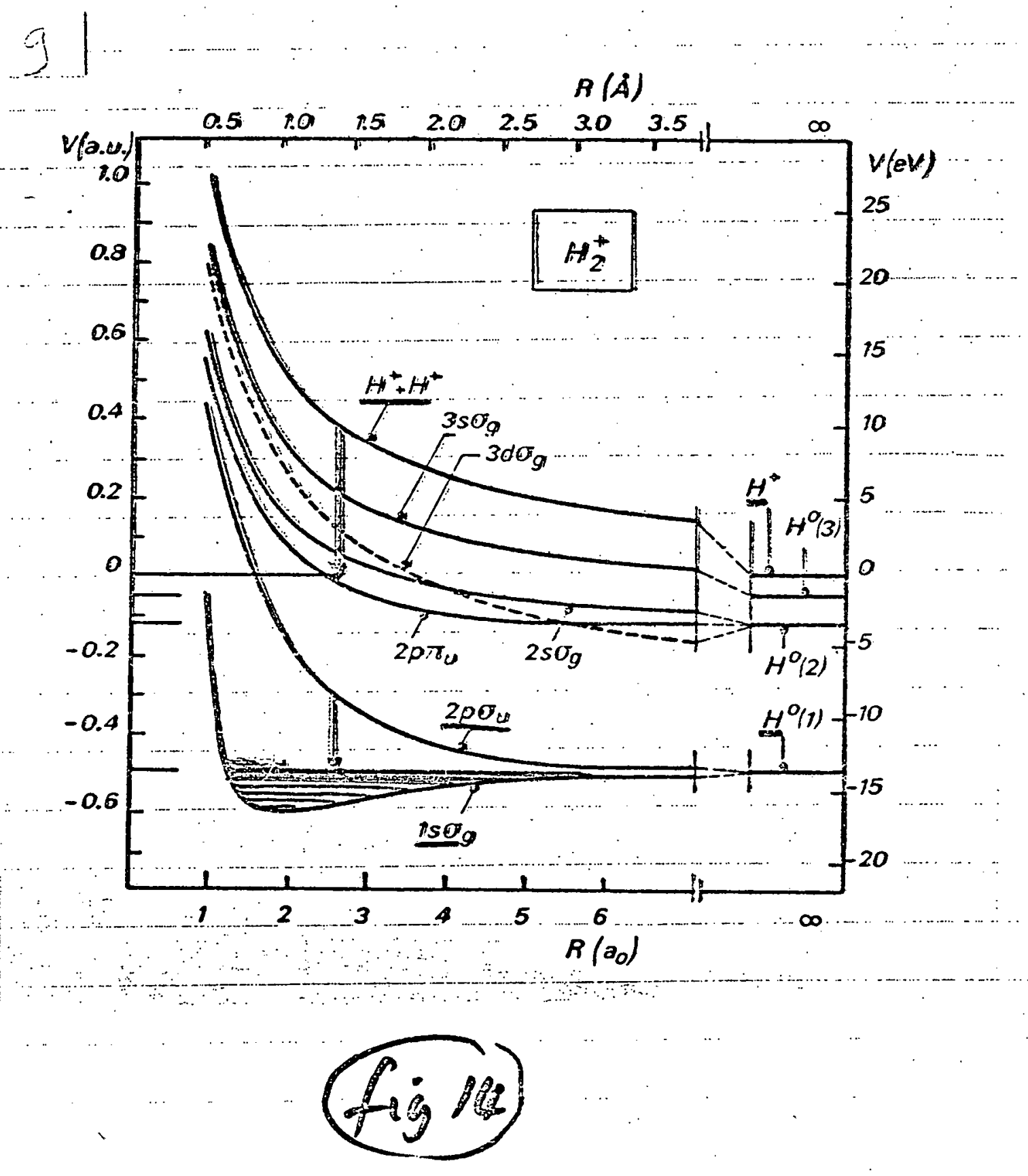



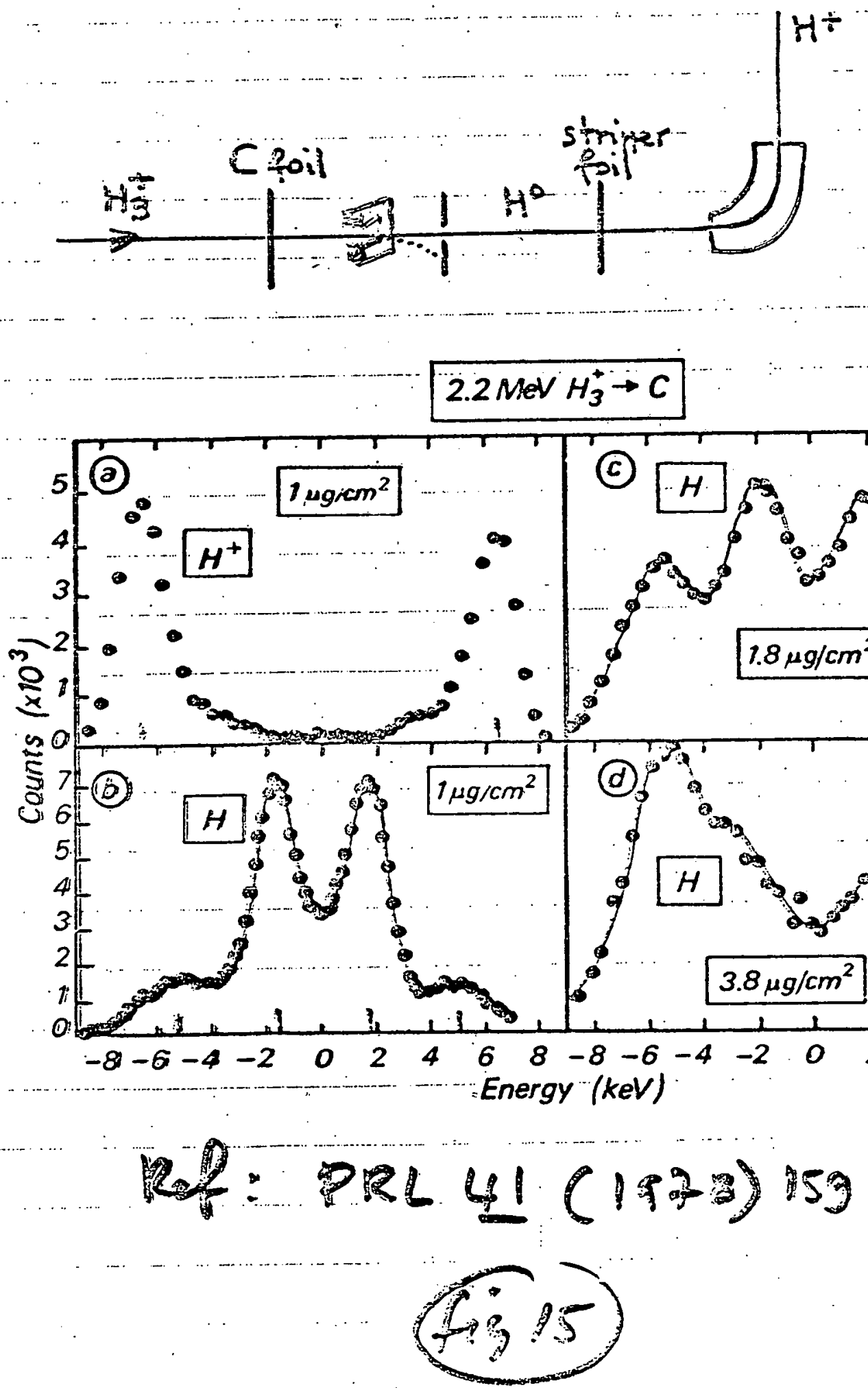


$$
\left.\rightarrow\left[H_{3}^{+} \rightarrow H_{4}^{+}+H^{0}\right] \text { (Fig. } 16\right)
$$

$\frac{2}{6}\left[\begin{array}{c}\text { ELETTROA } \\ \text { IA THE HA }\end{array}\right.$

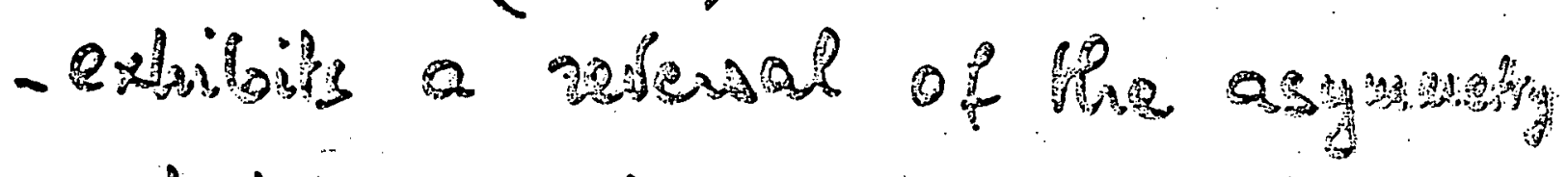

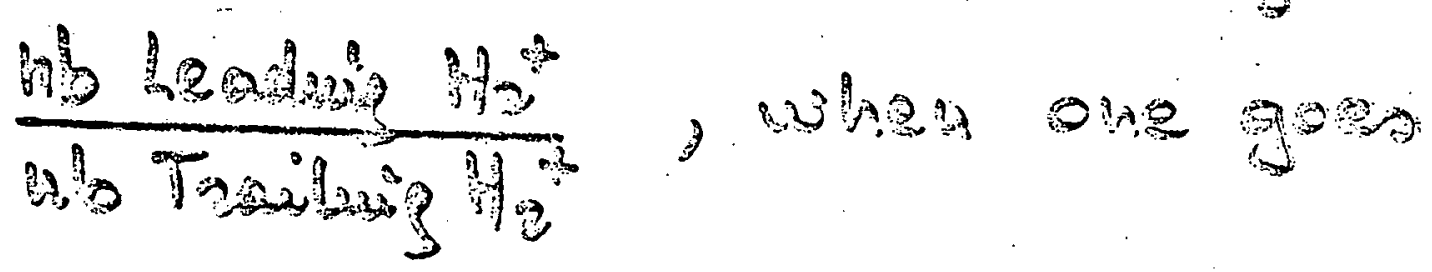

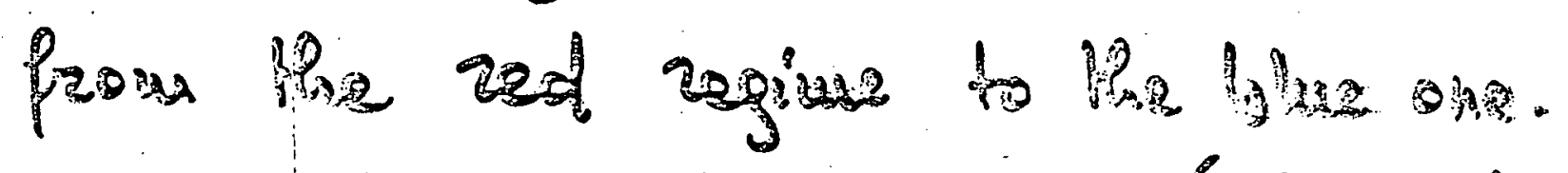
(

Interfatation (Fig.48)

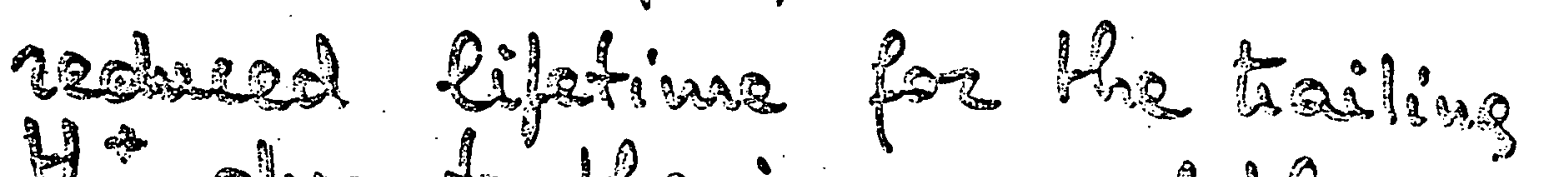

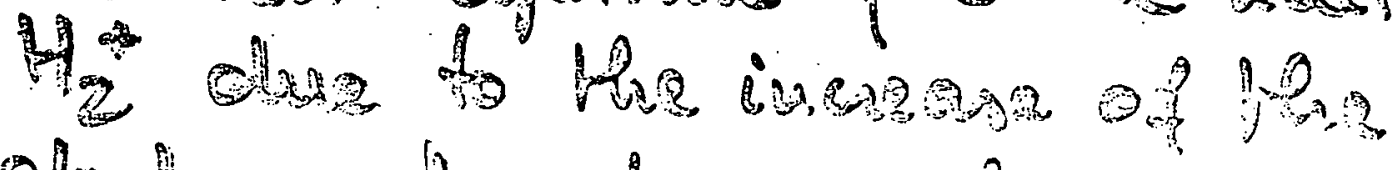

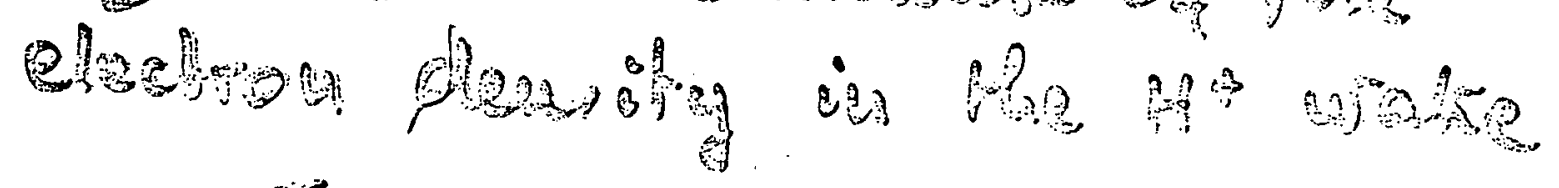

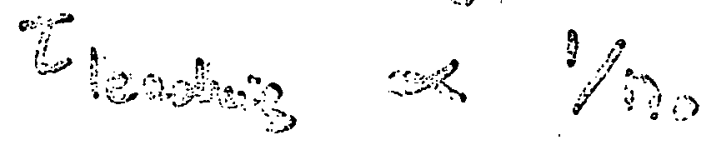

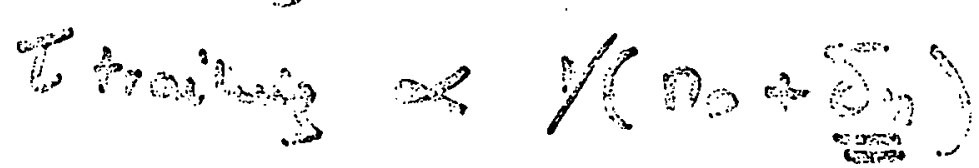


112

2.2. $\mathrm{MeN}_{3}^{+} \mathrm{C}$ o
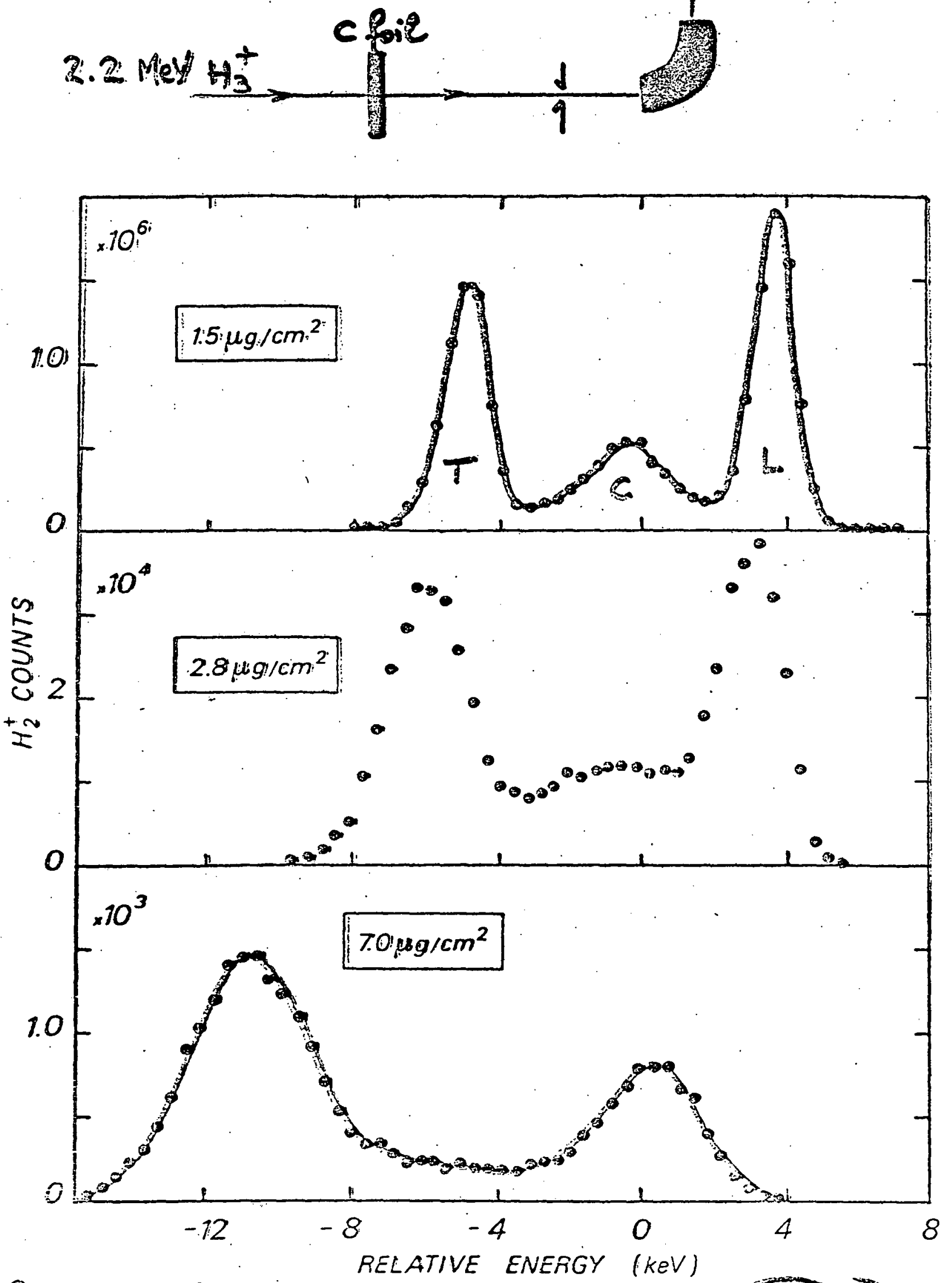

50: 9 9

$$
H_{3}^{+} \rightarrow H_{3}^{*}+H^{\circ}
$$




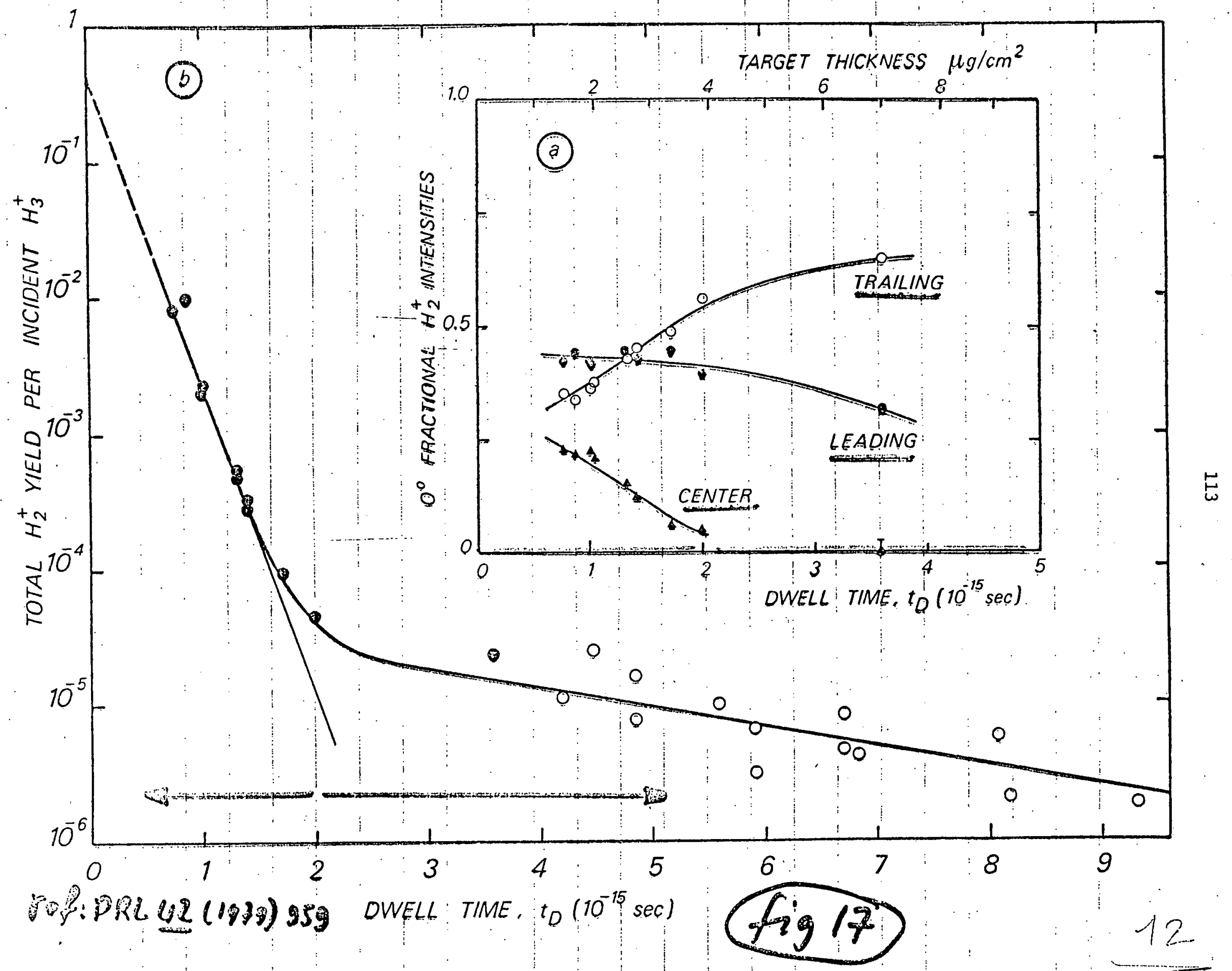


114

insident $4_{3}^{x}$

$\rightarrow \frac{2}{6}$

fी $\rightarrow$

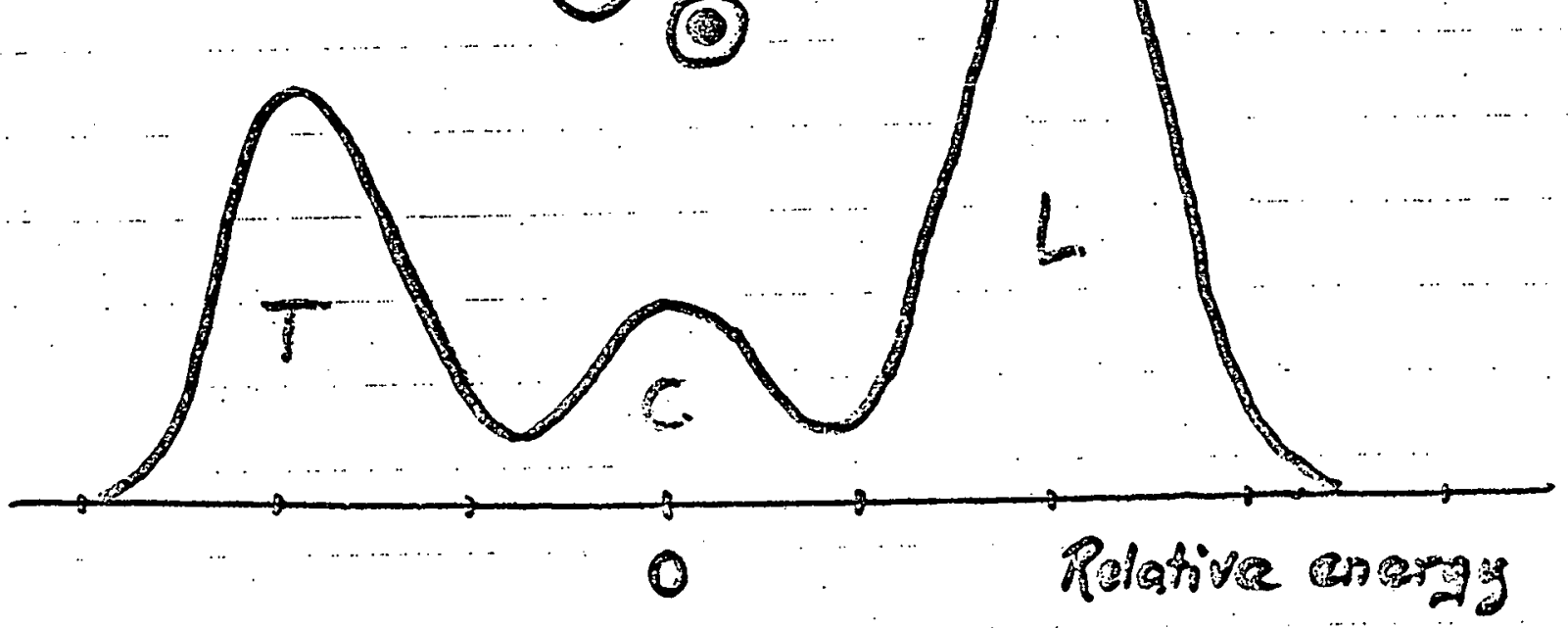

Fig

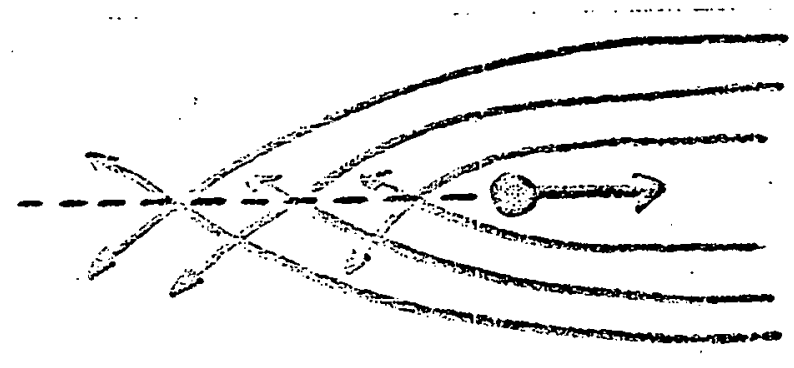

forrsing o 18 . tange electrong 


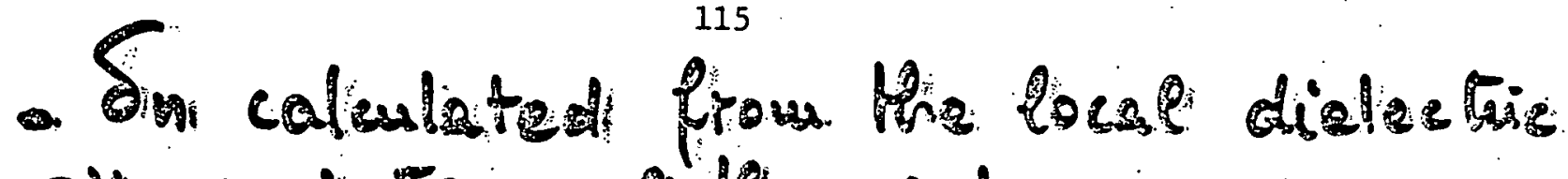

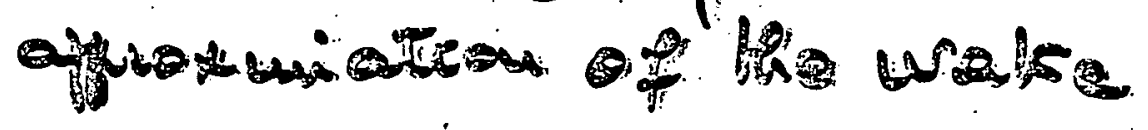

too sungl to account for the eflest

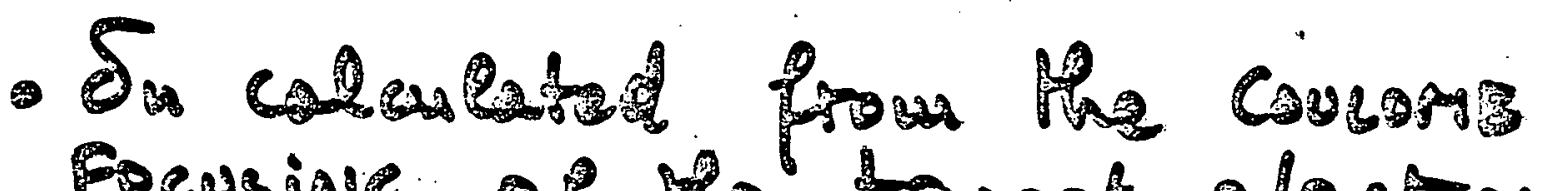
Focusine of ha tange clactous a clasied celculatiou

$$
\text { gives } \frac{\delta_{u}}{n_{0}} \text { as } 8 \%
$$

in rother gaod

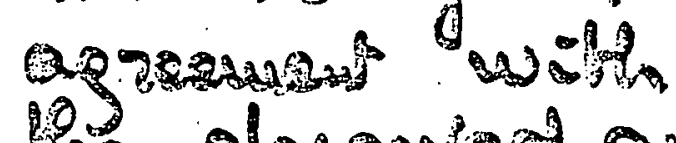

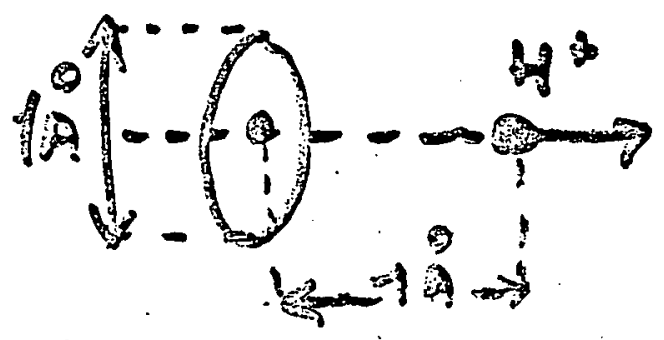

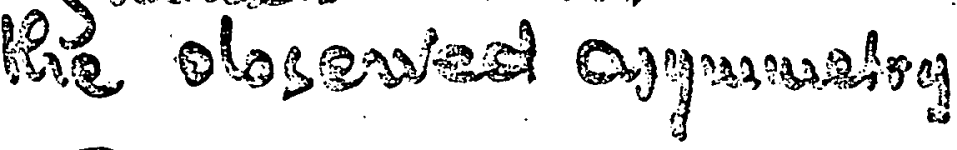

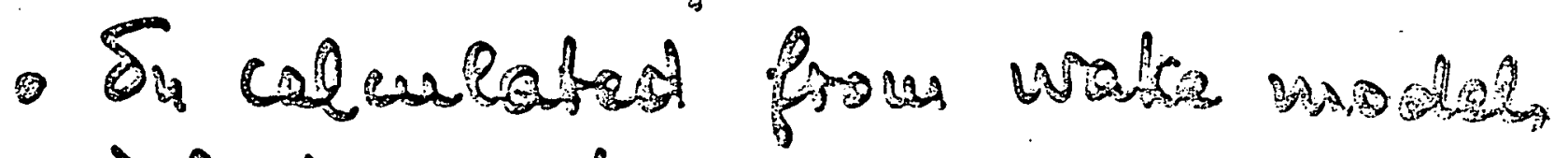

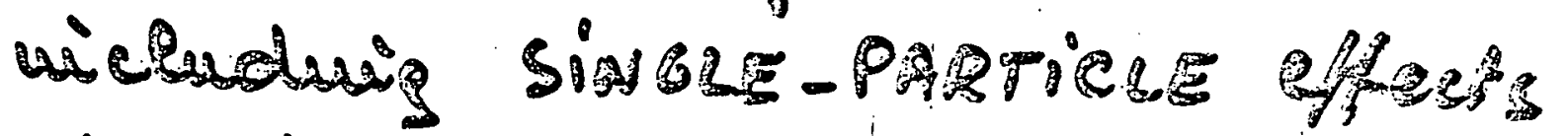
gives here a valus whish bi glome to the furdiction of He Coulareto

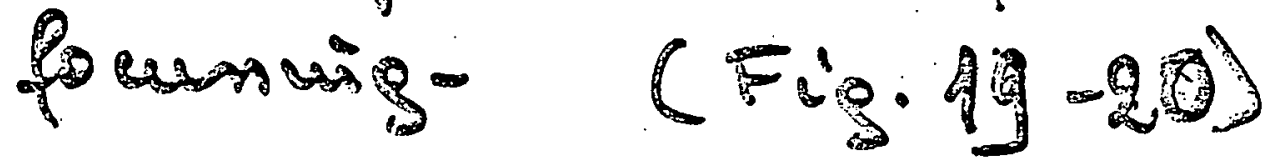

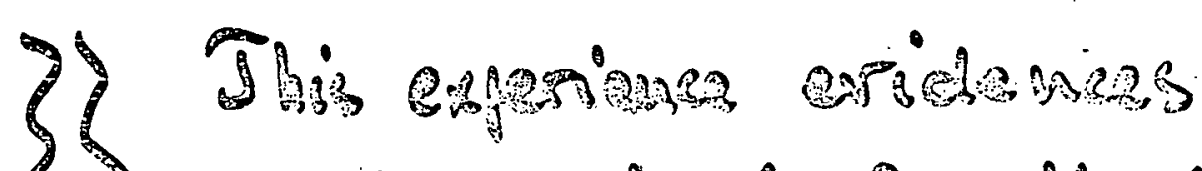

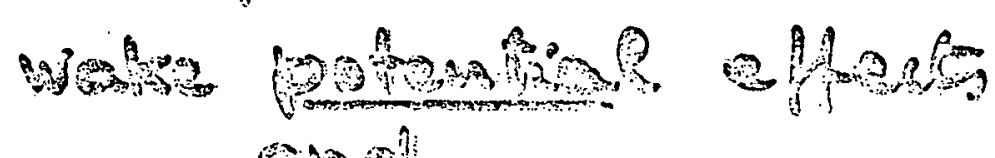
ans:

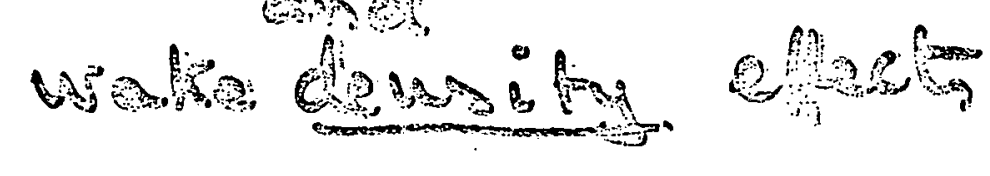


Prow Echenique, Ritechie, Brandt

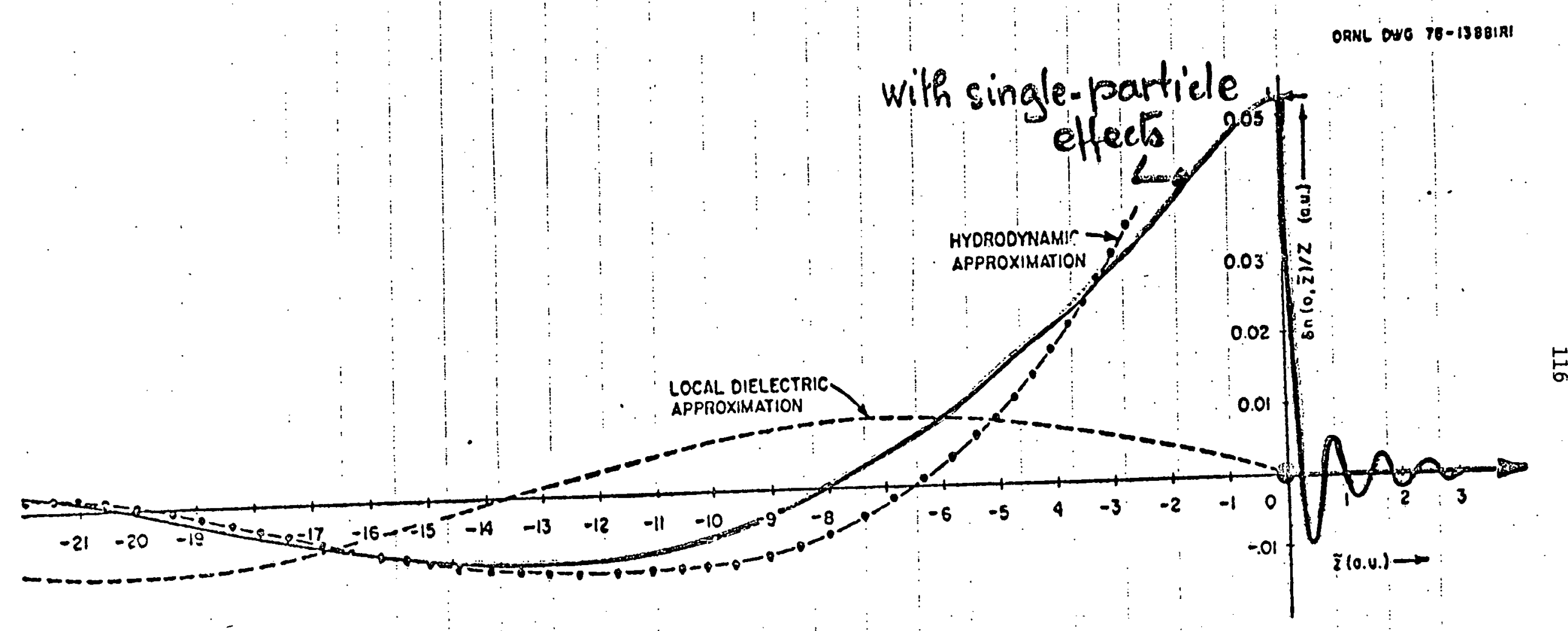

Electron density fluctuations on the ion track

fig 19

挜 
117

Paos Echanique, Ritehie, Brandt

ORNL DWG 79-8393R2

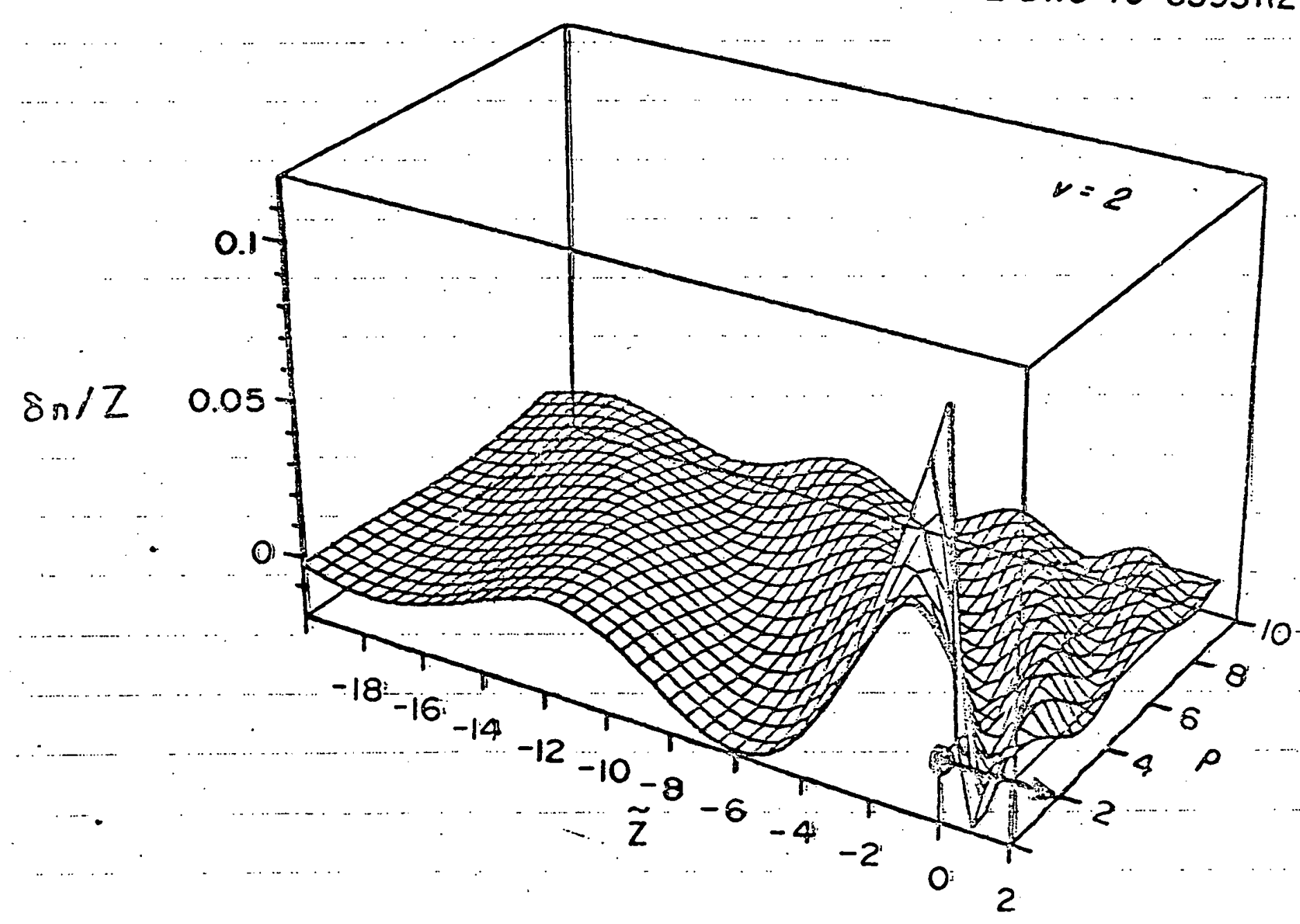

(4920

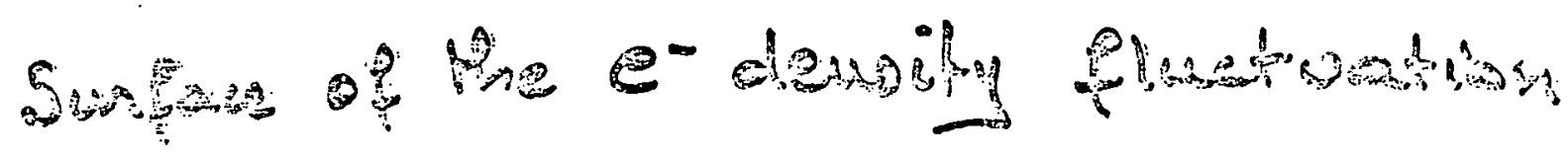

158 
118

3 A Quantitative Model of $\mathrm{H}^{\circ}$ and $\mathrm{H}_{2}^{+}$Yields in tes Passage of $\mathrm{H}_{2}^{+}$through Carbon Foils

Blue resime

Moded (Fig. 2ip)

- Electron in incident $H_{2}^{+}$removed at forl entrance

- Digrotion separate due to comlomb explosion and miltiple seatkering (incricrent)

- Targer election cathe near foil ejit into $18 \sigma_{g}$ and $2 p \sigma_{u}$.

- Captin into lo $\sigma_{g}$ will lead to blue $H_{2}^{+}$if $\in \leq 0$, athexwise ore gets $H^{\circ}$.

- Cugturse into apoes leses to $H^{\circ}(\sin )$.

- Conjiar cuos pections charateriged in torms of effectimen Lerge which degends on to internuclear separatsion (Fig. S3)

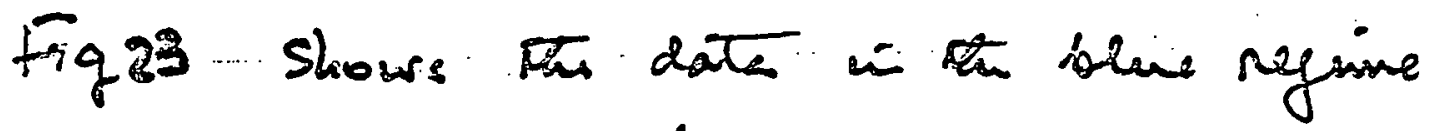
and calcatertions. Solie canne considers

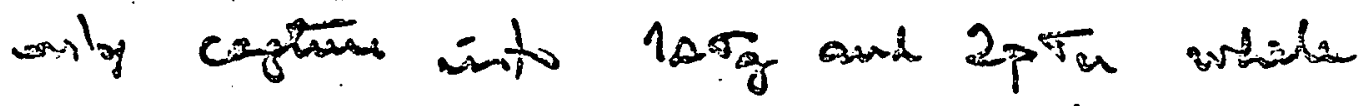

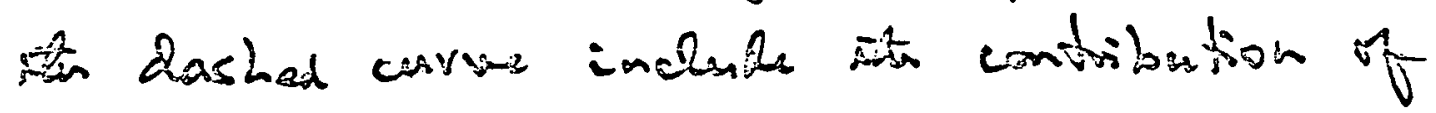
capizane into o $2 p \pi_{u}$ and $3 d \sigma_{g}$.

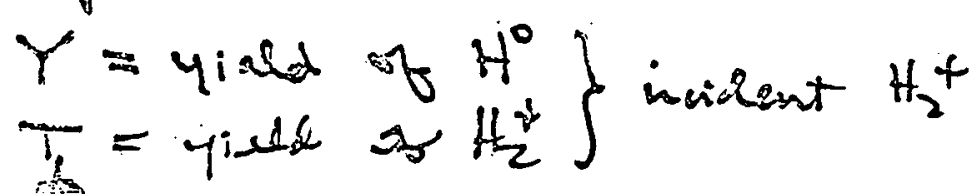

$T_{0}=4:$ ild of $H^{\circ}$ from incident $f^{*}$ of ters same val. 


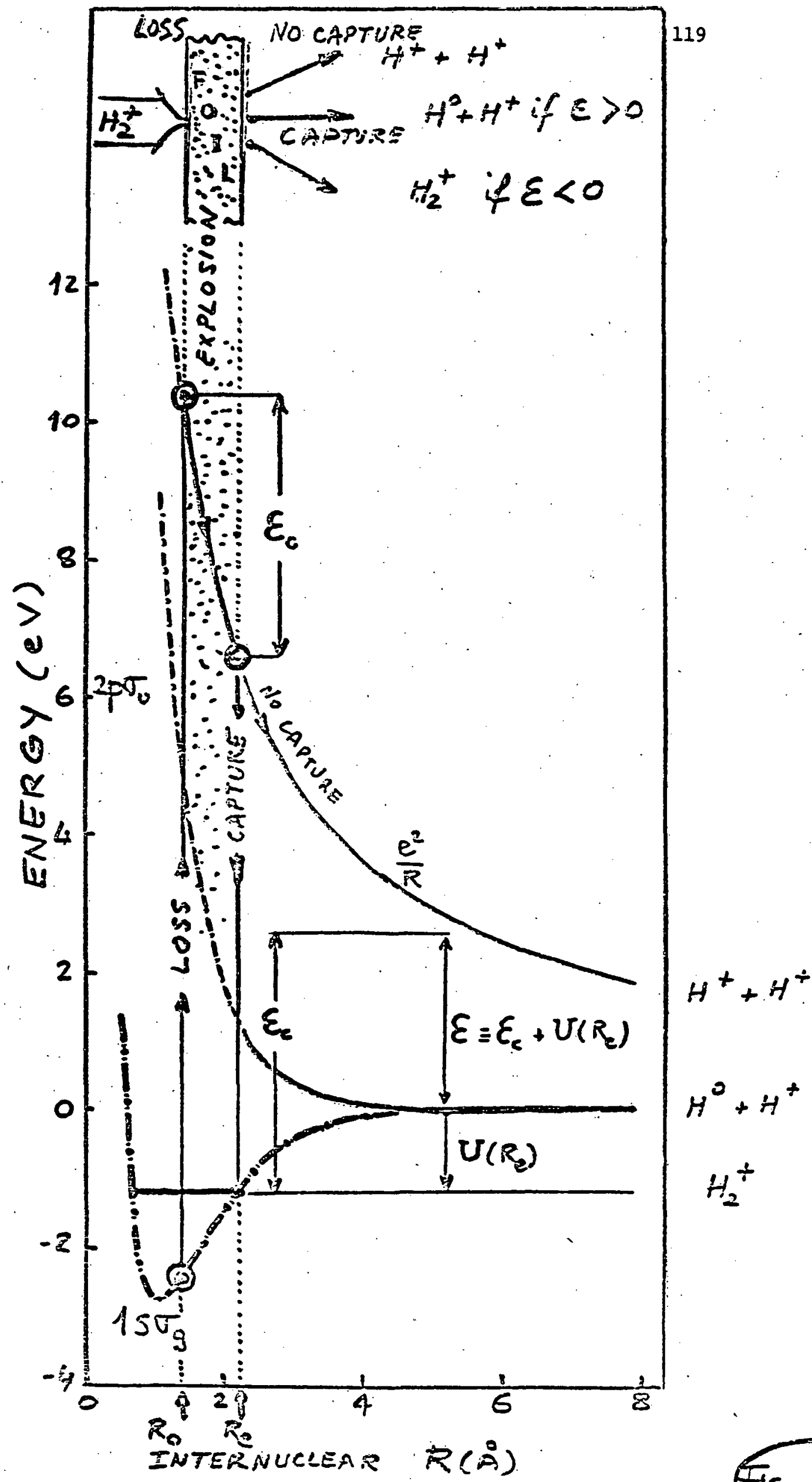


Me Carroll, Piacentini \& Salin, J. Phys. 8 3,137 (1970) Dalgarno \& Poots, Proc. Phys. Soc A 67, 343 (1954).
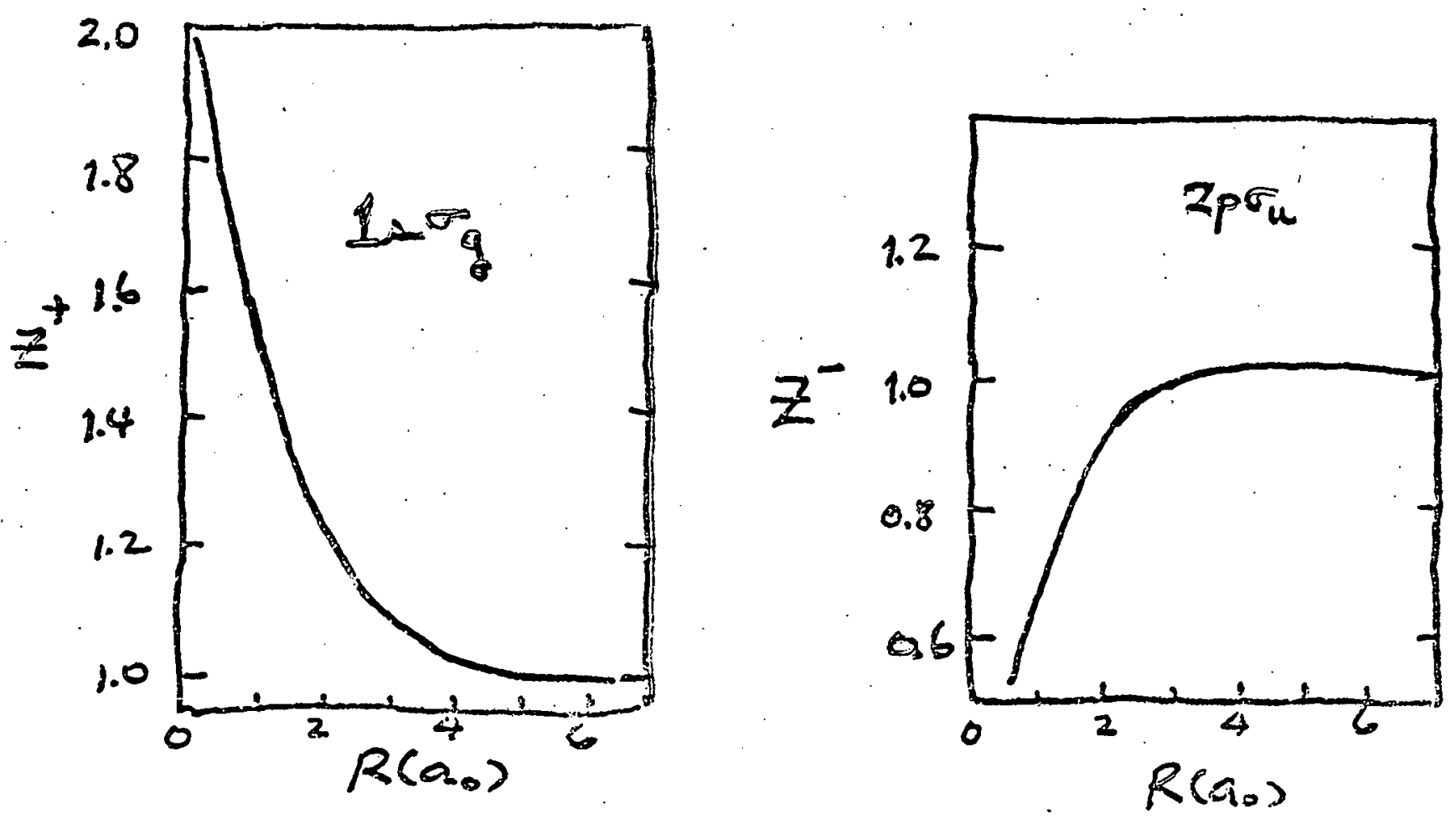

Viviationally optionigzed wor. (2SAO)

$$
\begin{aligned}
& x^{ \pm}=\frac{1}{\sqrt{3}}\left(\frac{z^{2}}{\pi}\right)^{1 / 3}\left[2 x_{p}\left(-z^{2} r_{A}\right) \pm 2 x_{p}\left(-z^{ \pm} r_{13}\right)\right] \\
& \sigma_{s}\left(1-\sigma_{3}\right)=\left(z^{+}\right)^{5} \sigma_{s}(1+)
\end{aligned}
$$

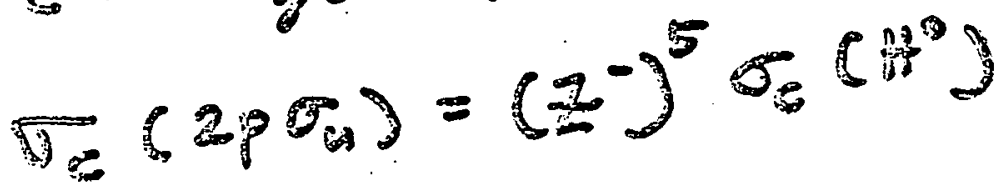

iig. 22 


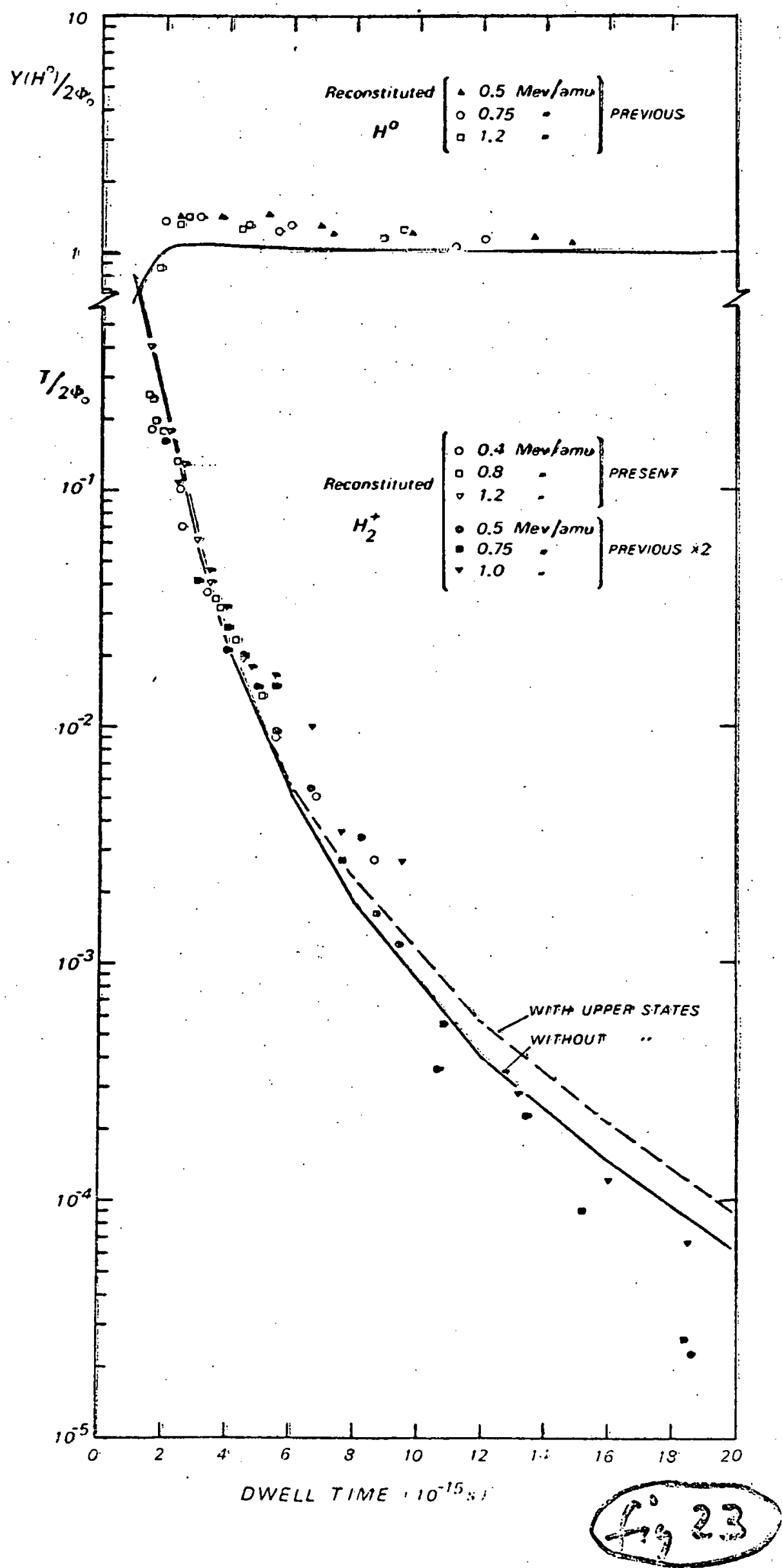




\section{INHIBITED H' PRODUCTTON IN THE FOIL BREAKUP OF EAST H.}

I. PURPOSE

- Examine charge exchange processes between $\mathrm{H}^{-}$and a nearby $\mathrm{H}^{+}$.

II. BACKGROUND (figuregig)

- The lifetime $\tau$ of $\sim 1 \mathrm{MeV} / \mathrm{a} \mathrm{mu} \mathrm{H}_{2}^{+}$in $\mathrm{C}$ has been measured to be $\tau \sim 0.2 \mathrm{fs}$

- We expect for a typical case of dwelltime in the foil $t_{D} \geq 4 T$

(1) the $e^{-}$to be stripped from $\mathrm{H}_{2}^{+}$for $t<t_{D}$

(2) the cluster then explodes

(3) target $\mathrm{e}^{-}$capture at ${ }^{t_{D}}$ into the $\mathrm{MO}$ of $\mathrm{H}_{2}$ correlated with the $\mathrm{H}^{-}+\mathrm{H}^{+}$to lead to the detected $\mathrm{H}^{-}$

(4) this $\mathrm{H}^{-}$yield to be sensitive to the processes outside of the foil.

- Eventhough these are $\sim 1 \mathrm{MeV} / \mathrm{a} \mathrm{m}$ u clusters, the $\mathrm{H}^{-}$separates from $\mathrm{H}^{+}$with a relative $\mathrm{KE} \sim 5 \mathrm{eV}$ only. The processes sampled are thus the same as those governing the mutual neutralization phenomenon encountered in the $\mathrm{H}^{-}+\mathrm{H}^{+}$ collisions at low energy. 


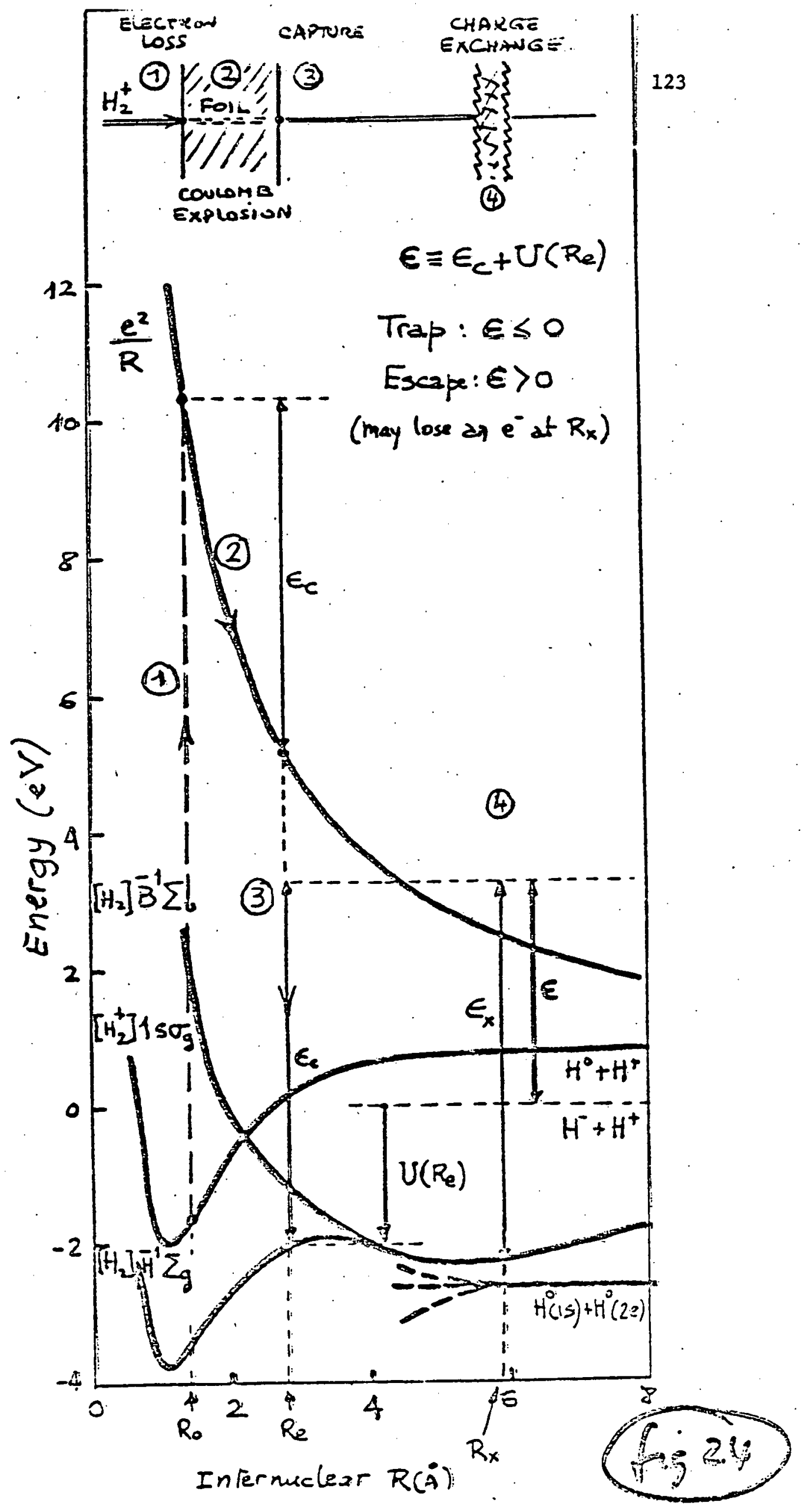


iiI. MEASUREMENTS (figure 28 )

- Yields of $\mathrm{H}^{-}$from $0.4-, 0.8$ - and $1.2 \mathrm{MeV} / \mathrm{a} \mathrm{mu} \mathrm{H}_{2}^{+}$incident on $\mathrm{C}$ $\left(1-8 \mu \mathrm{g} / \mathrm{cm}^{2}\right)$ and from the same velocities $\mathrm{H}^{+}$.

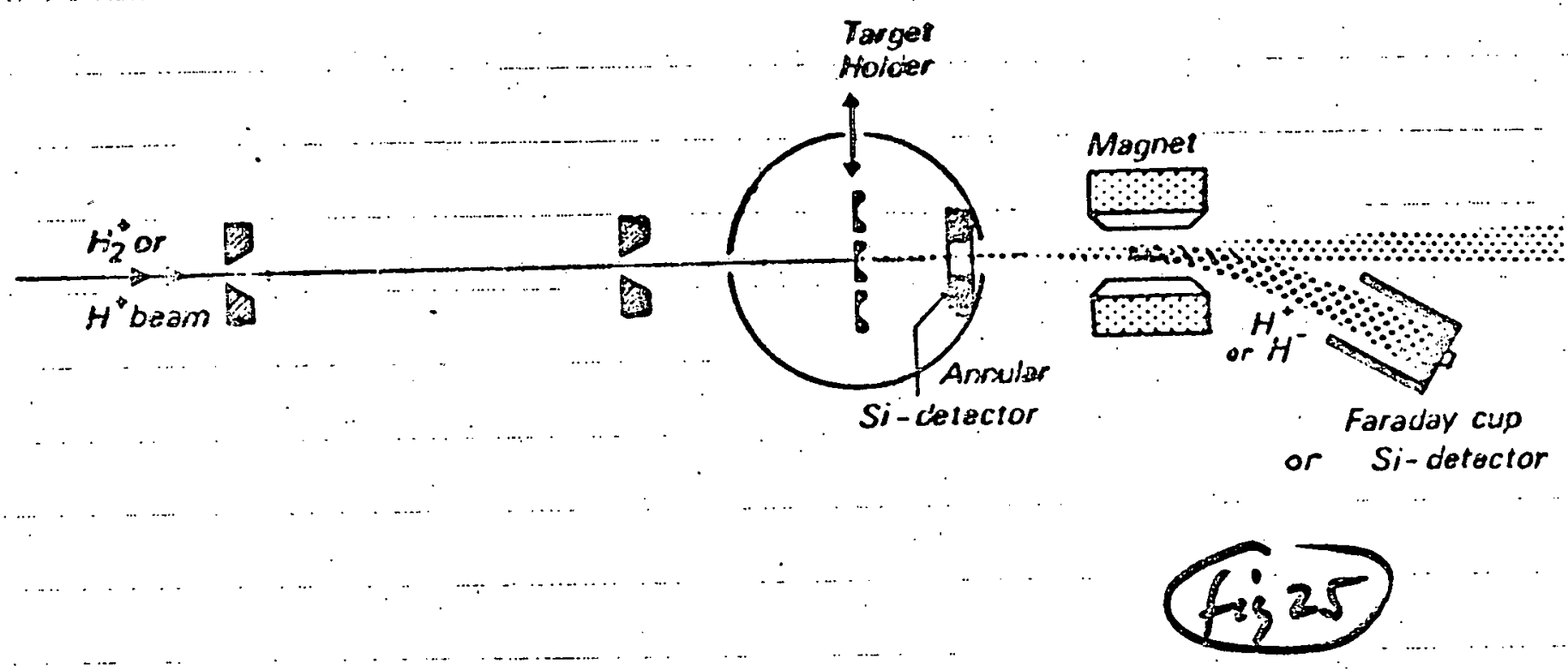

IV. RESULTS (figures 26 and 32 ?

- Figure 3 displays the yield $\Phi \frac{M}{1}$ of $\mathrm{H}^{-}$per proton for incident $\mathrm{H}_{2}^{+}$.

- Figure 4 displays the correspondin: yield $\Phi \frac{A}{l}$ for incident $H^{+}$. 

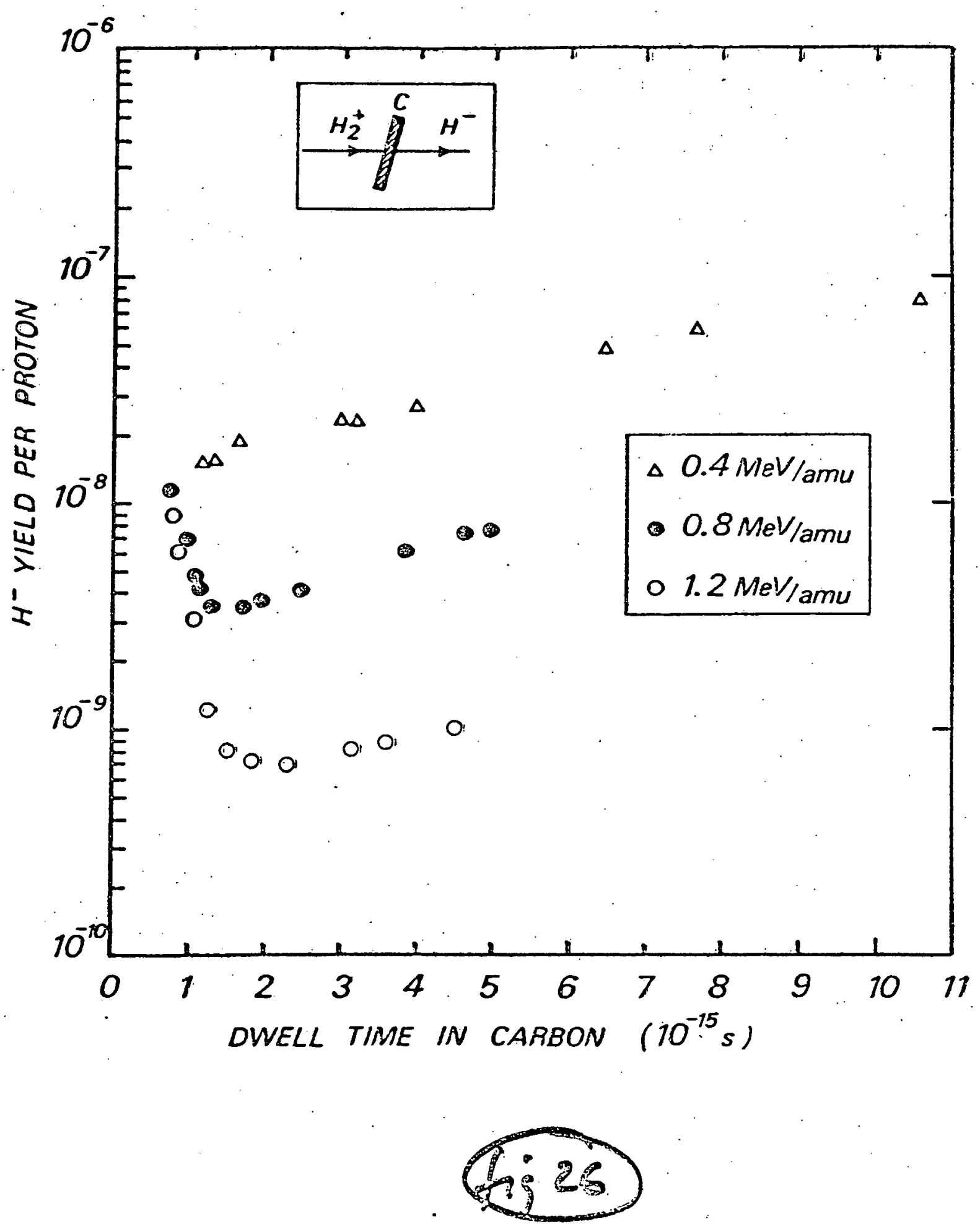
126

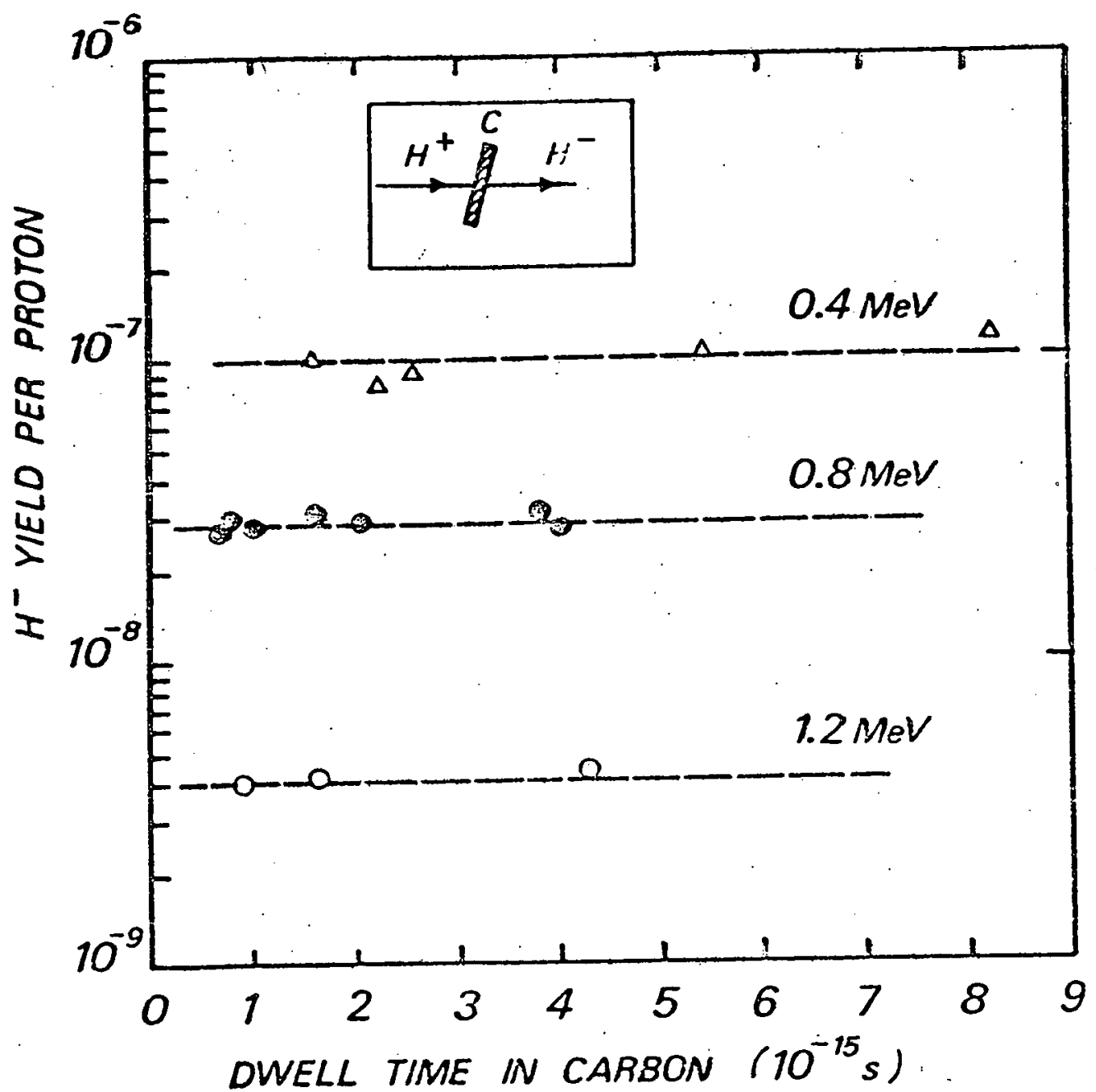

f.9 27 
v. DISCUSSION (figures 28 and 23

- Molecular effects are important because the ratios $\Phi \frac{M}{1} / \Phi \frac{A}{1}$ deviate significantly from unity as shown by symbols in figure 23

- To get $\mathrm{H}^{-}$from $\mathrm{H}_{2}^{+}$requires the capture of at least one target $\mathrm{e}^{-}$, and we may expect the capture probability by a diproton cluster to be enhanced (per proton) over that by a single proton, as is observed in the case for $\mathrm{H}^{\circ}$ from $\mathrm{H}_{2}^{+}$.

- Since capture occurs near the foil exit, the large observed inhibition for $\mathrm{H}^{-}$car: only be reasonably understood in terms of charge exchange outside of the foil.

- The inhibition effect can be expressed in term of the $\mathrm{H}^{-}$survival probability

$$
S \equiv \Phi \frac{M}{I} / \varphi \frac{M}{1}
$$

where $\varphi \frac{M}{1}$ is the corresponding yield expected from the capture process alone. Estimates of $\varphi \frac{M}{1}$ are shown as curves in figure 39 With these $\varphi \frac{M}{1}$, we obtained $S$ shown in figure $g^{2}$ These $S$ are seen to define a nearly universal trend independent of the incident velocity.

- We interpret $S$ to be

$$
S=S_{\text {tr }} S_{\text {ex }}
$$

where $S_{t r}$ is the probability for surviving the trapping into the attractive well of the $\mathrm{MO}\left(\mathrm{H}^{-}\right)$in $\mathrm{H}_{2}$ (see figure 24 ), and $\mathrm{S}$ ex is that escaping the charge exchange region without losing an $e^{-}$.

- Estimates of $S_{t r}$ (dashed curve in figure show that the trapping effect is comparatively small.

- Within the Landau-Zener model for charge exchange, we may write

$$
\begin{aligned}
S_{\text {ex }} & =\exp \quad\left[-\frac{2 \pi}{h v}\left|H_{x}\right|^{2} R_{x}^{2}\right], & R\left(t_{D}\right) & \leq R_{x} \\
& =1 & & R\left(t_{D}\right)>R_{x}
\end{aligned}
$$

- Estimates using the $H_{x}$ and $R_{x}$ values of Bates and Lewis (BL) yrield the curve BL.

- By reducing the $\mathrm{H}_{x}$ value of $\mathrm{BL}$ by $1 / 3$, we obtained the curve $\mathrm{S}_{\text {ex }}$ which, when combined with $S_{t r}$, yield the curve $S$. These theoretical $S$ are seen to reproduce the data rather well. 


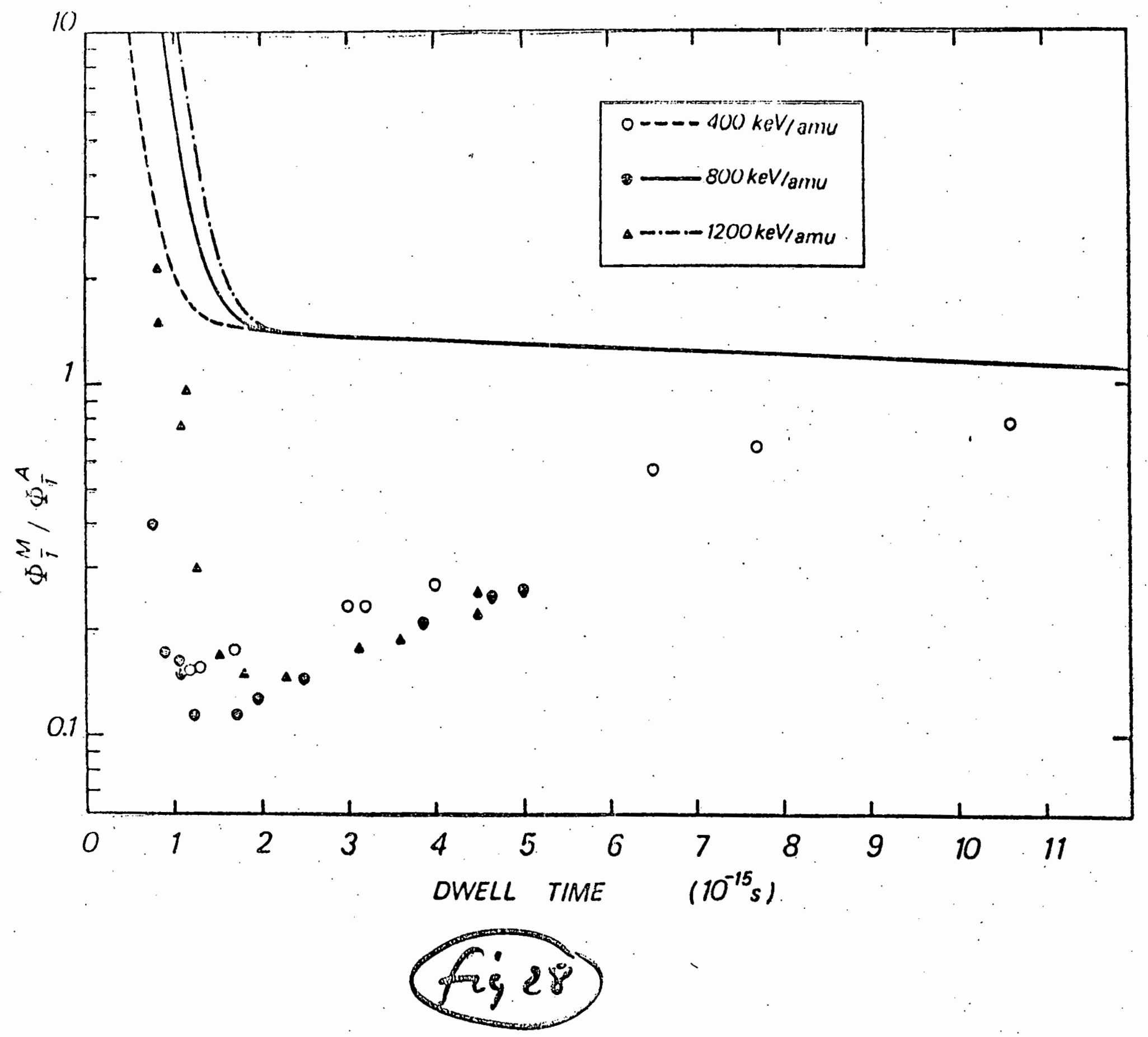




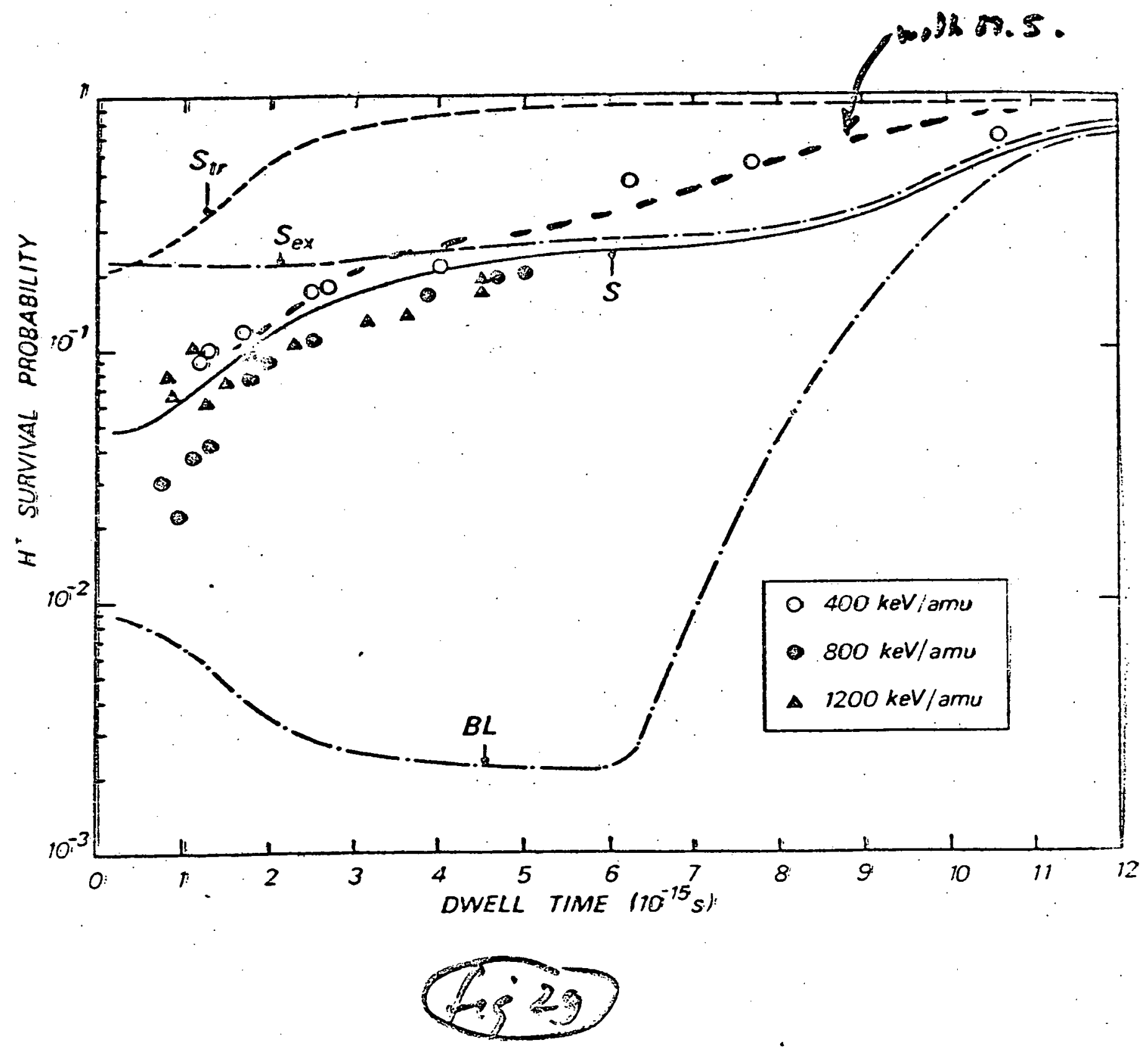


VI. CONCLUSIONS.

- Foil breakup of fast diatomic molecules provides a unique view of the charge transfer processes between slowly separating atoms because of the "one-passage". conditica. ए530

- In the present case, the Landau-Zener model provides an adequate description of the charge exchange processes. 


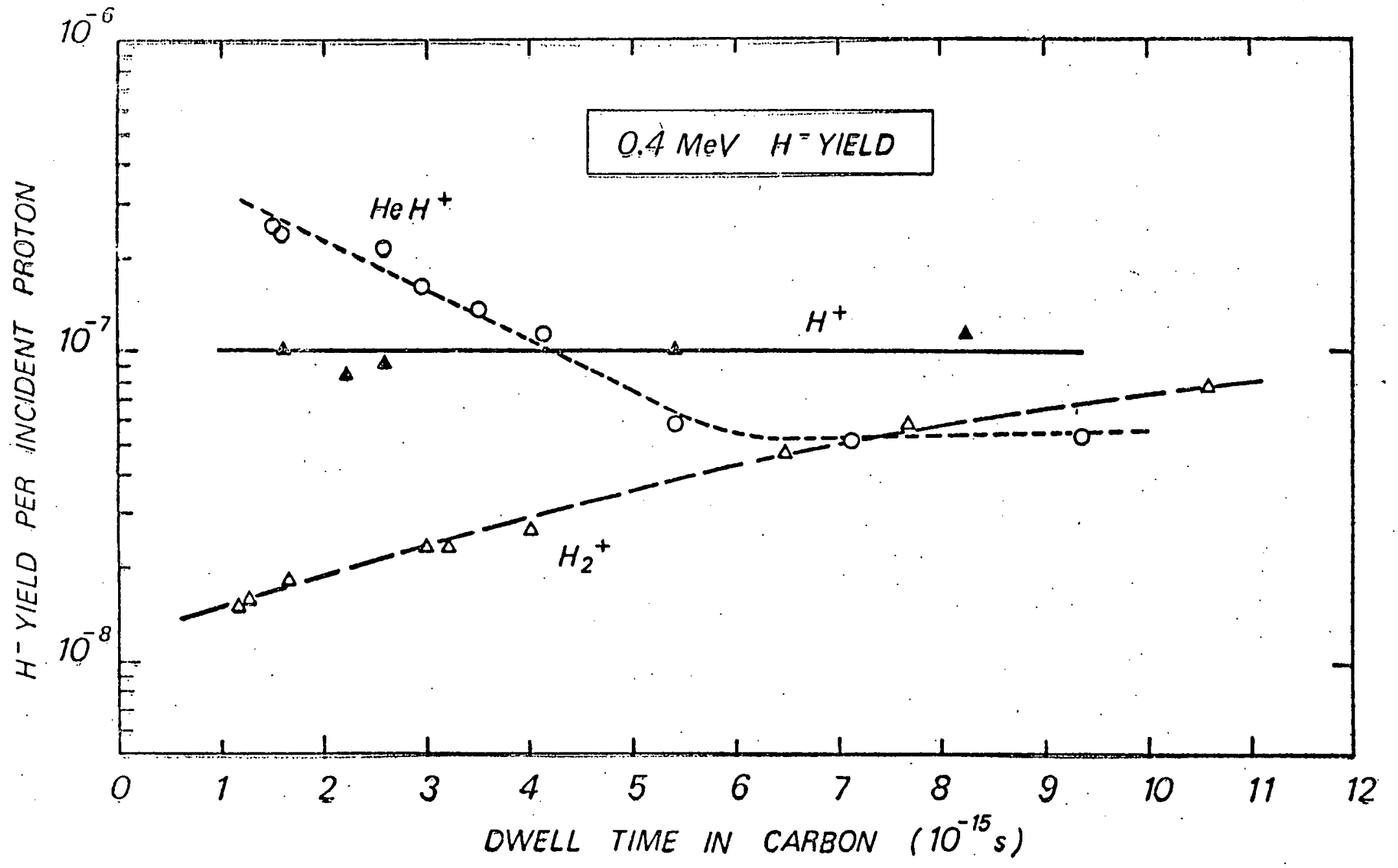

(5) 30 
THIS PAGE

\section{WAS INTENTIONALLY \\ LEFT BLANK}


Stopping of Low Energy Molecular Ions J.C.Eckardt, G.H.Lantschner, N.R.Arista and R.A.Baragiola Centro Atómico Bariloche ${ }^{*}$ - Instituto Balseiro ${ }^{\dagger}$ 8400 - S.C. de Bariloche - RN - Argentina

We have studied the energy loss of $12,5-130 \mathrm{keV} \mathrm{H}^{+}$and $\mathrm{H}_{2}^{+}$in thin foils. The experiments were described in the literature ${ }^{1}$ but they appear to have passed unnoticed in later work ${ }^{2,3}$ so we will repeat the essence here correcting some errors made by the journal.

The ion beams were produced in the Bariloche Kevatron accelerator and sorted in mass by a $90^{\circ}$ magnetic analyzer. The beam energy was stable to $\pm 2 \times 10^{-4}$ during each set of energy loss measurements. The energy losses were determined by measuring alternatively the energy of the beam without and with a thin foil placed in its path. The $90^{\circ}$ electrostatic analyzer with its slits set to an energy resolution of $0.4 \%$ accepted particles transmitted through the foils within vertical and horizontal angles of 0.75 and $6.3 \mathrm{mrad}$ centered around the beam axis. Two liquid-nitrogen-filled coaxial traps were located inmediately before and after the foils to keep the build-up of contaminant layers from being discernible. More experimental details can be found elsewhere ${ }^{4,5}$.

Fig. 1 shows normalized energy spectra obtained for $267 \mathrm{keV} \mathrm{H}_{2}^{+}$incident on a $\sim 200 \AA$ Aluminum foil.Spectra were obtained also faster using the dynamic scan mode ${ }^{4,5}$ which allowed also more accurate determinations of the energy losses. Typical times for each energy loss measurements (several scans of the peaks) were of the order of a few minutes, thus minimizing problems caused by instabilities in the beam energy.

\footnotetext{
*Comisión Nacional de Energía Atómica

†Universidad Nacional de Cuyo
} 
Using the notation:

$$
\begin{aligned}
& E_{0} \text { - Energy of the incident } \mathrm{H}_{2}^{+} \\
& \mathrm{E}_{1} \text { - Energy of } \mathrm{H}^{+} \text {in transmitted beam } \\
& \mathrm{E}_{2} \text { - Energy of } \mathrm{H}_{2}^{+} \text {in transmitted beam }
\end{aligned}
$$

we can define

$$
\begin{aligned}
\Delta \mathrm{E}\left(\mathrm{H}_{2}^{+}\right)_{1}= & \frac{1}{2} \mathrm{E}_{\mathrm{O}}-\mathrm{E}_{1} \\
\Delta \mathrm{E}\left(\mathrm{H}_{2}^{+}\right)_{2}= & \frac{1}{2}\left(\mathrm{E}_{\mathrm{O}}-\mathrm{E}_{2}\right) \\
\Delta \mathrm{E}\left(\mathrm{H}^{+}\right)= & \text {energy loss for protons at the same } \\
& \text { velocity. }
\end{aligned}
$$

and the ratios

$$
\begin{aligned}
& \mathrm{R}_{1}=\Delta \mathrm{E}\left(\mathrm{H}_{2}^{+}\right)_{1} / \Delta \mathrm{E}\left(\mathrm{H}^{+}\right) \\
& \mathrm{R}_{2}=\Delta \mathrm{E}\left(\mathrm{H}_{2}^{+}\right)_{2} / \Delta \mathrm{E}\left(\mathrm{H}^{+}\right)
\end{aligned}
$$

which should be one if no molecular or interference effects were present.

Fig. 2 shows $R_{1}$ and $R_{2}$ for $\sim 150 \AA$ Carbon (average of 3 foils) and $\sim 200 \AA$ Aluminum (average of 5 foils) as a function of ion velocity. Interference effects can be seen to persist down to the lowest velocities, and that at low velocities, $R_{2}<1$ and $R_{2}<R_{1}$. The error bars in the results of Fig.2 are substantially smaller than those of later reports ${ }^{2,3}$ of low-energy experiments, presumably due to our high beam energy stability during our short measuring times and our averaging over tens of measurements at each point in Fig. 2.

The results of Fig.2, particularly $R \neq 1$ at low velocities, confirm the prediction of Arista ${ }^{6}$ that interference effects should occur in single particle excitations and can explain at least in part the ear.., experimental evidence of Makarov and Petrov ${ }^{7}$ who found that low velo- 
city molecular hydrogen ions have somewhat larger ranges than atomic hydrogen ions at the same velocities. The results are also in agreement with the experimental observation that low energy $\mathrm{H}_{2}^{+}$ions produce less electron emission per nucleon from clean metals than $\mathrm{H}^{+}$at the same velocity ${ }^{8}$.

A possible explanation for $R_{2}<R_{1}$ and $R_{2}<1$ at low velocities in terms of Arista's theory was given before ${ }^{6}$. Figure 3 shows calculated ratios $\mathrm{R}$ for di-protons in an electron gas with dielectric constant appropiate for $A 1$, as a function of cluster separation, orientation and velocity. Plasmom damping is not included in the calculations and this causes sharp changes close to $v=1.24$ a.u., the threshold for plasmon excitation.

The low values of $R_{2}$ for $A l$ at our lowest velocities can be explained if we assume that $\mathrm{H}_{2}^{+}$in the transmitted beam result from di-protons which have travelled inside the foil with their internuclear axis aligned close to the beam direction. The alignment force can be provided by the anisotropy in the polarization cloud around the moving ions. Transmitted protons, on the other hand, will probalbly result from di-protons for which multiple scaterring and energy stragging have overruled the alignment force and for which the stopping ratio should be better described by the orientation-averaged $<\mathrm{R}>$.

A more detailed comparison between experiment and theory must await knowledge of the variation for the internuclear separation with transit time in the foil which requires in turn knowledge of correlation effects in multiple scattering and of the internuclear potential of the protons in the solid. The importance of the multiple scattering and target dielectric properties can be illustrated by our observations that the relative yield of $\mathrm{H}_{2}^{+}$to $\mathrm{H}^{+}$in the transmitted beam was a 
factor of more than 1.00: for Al than for C, much smaller for $250 \AA \mathrm{G \epsilon}$ than for $C$ and not observable within our sensitivity $\left(\sim 10^{-3}\right)$ for $200 \AA$ Ag foils. Quantitative data on these relative yields is the goal of present experiments.

\section{$\underline{\text { References }}$}

1) J.C.Eckardt, G.Lantschner, N.R.Arista and R.A.Baragiola, J.Phys. C 11 , L851 (1978)

2) R.Laubert, IEEE NS-26, 1020 (1979)

3) W.H.Escovitz, T.R.Fox and R.Levi-Setti, IEEE NS-26, 1147 (1979)

4) A.Valenzuela, W.Meckbach, A.J.Kestelman and J.C.Eckardt, Phys. Rev. B $\underline{6}, 95$ (1972)

5) J.C.Eckardt, Phys.Rev. A 18, 426 (1978)

6) N.R.Arista, Phys.Rev B 18,1 (1978)

7) V.V.Makarov and N.N.Petrov, Sov.Phys.Sol. State $\underline{8}, 2993$ (1967)

8) R.A.Baragiola, E.V.Alonso, O.Auciello, J.Ferrón, G.Lantschner and A.0liva-Florio, Phys. Letters 67 A, 211 (1978). 


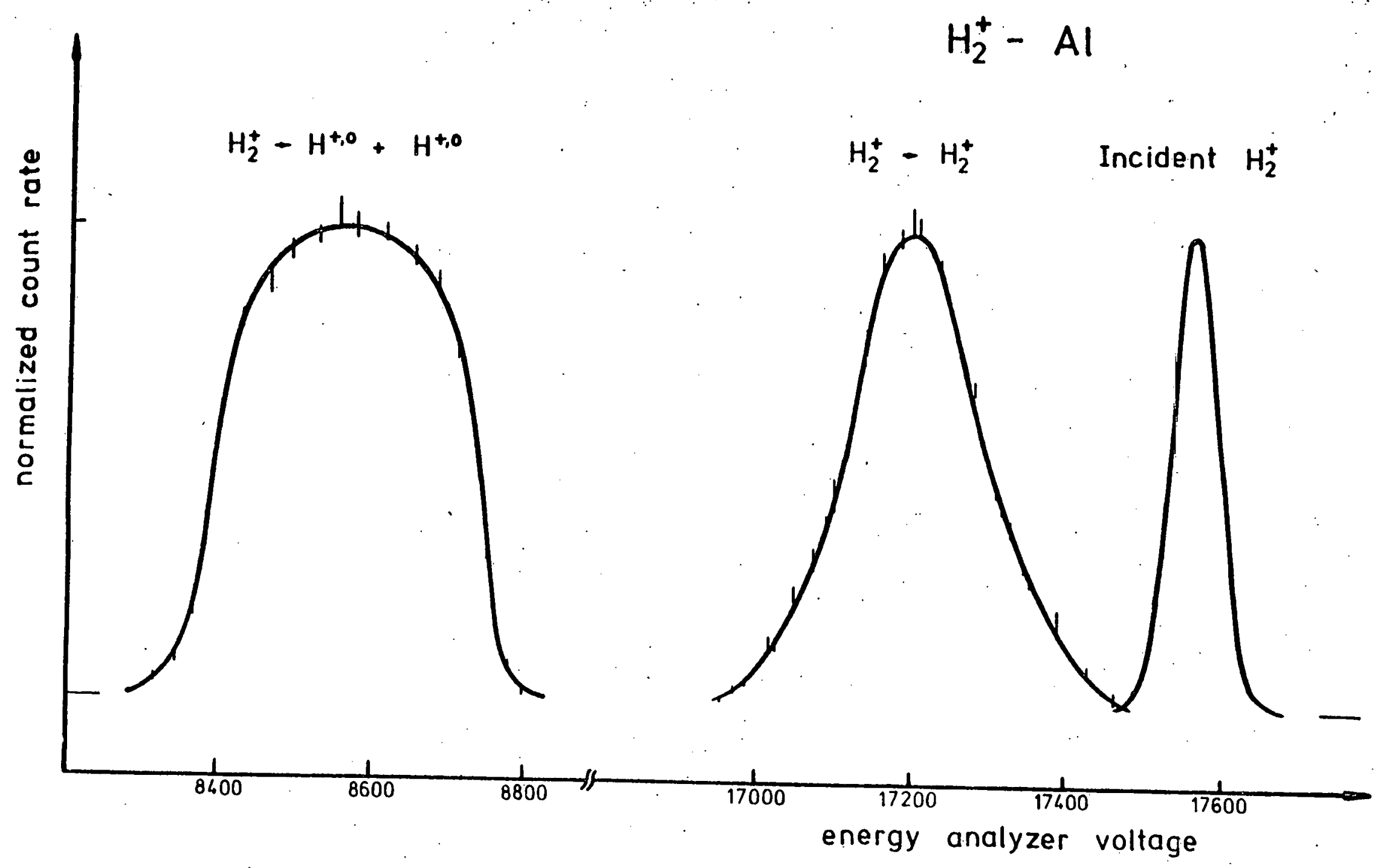

Figure 1: Normalized energy spectra for $267 \mathrm{keV} \mathrm{H}{ }_{2}^{+}$ions on a $\sim 200 \AA$ Aluminum foil. The analyzer constant is 15.21 . The peak labelled "Incident $\mathrm{H}_{2}^{+}$" was obtained without foil. 
$\mathrm{R}$

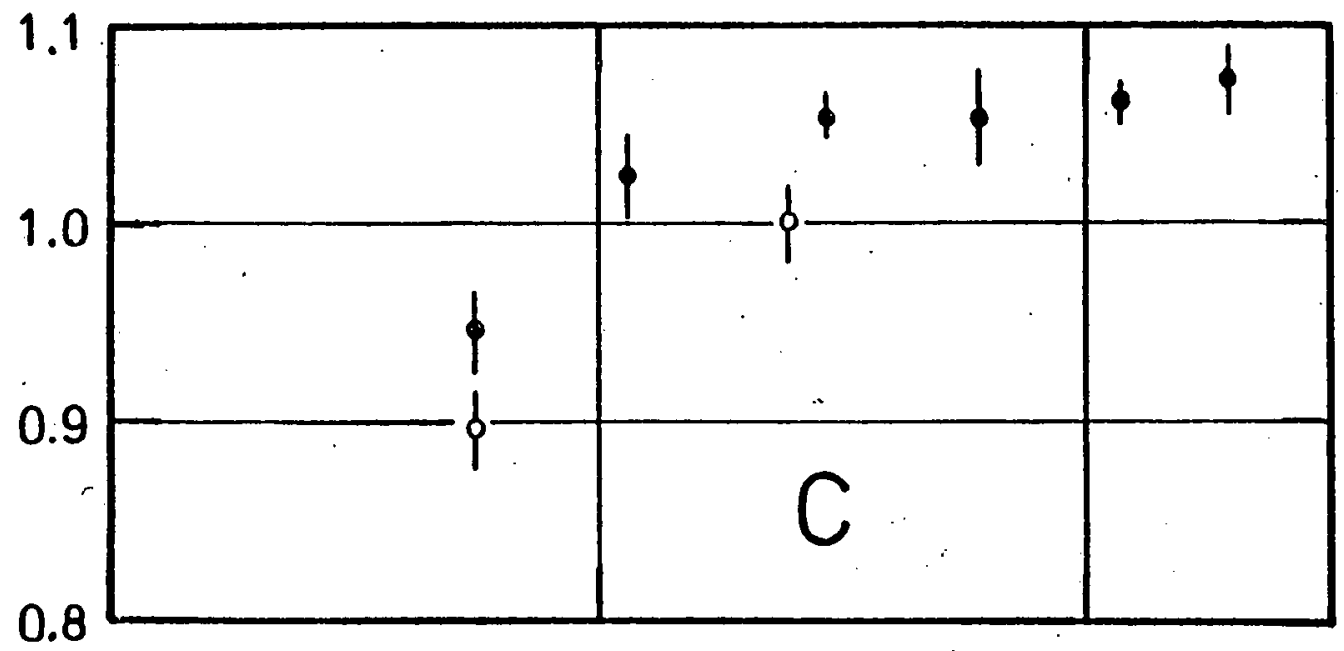

Figure 2:

Ratios $R$ of stopping powers per nucleon for incident $\mathrm{H}_{2}^{+}$and $\mathrm{H}^{+}$ ions on $\sim 150 \mathrm{~A}$ carbon and $i 200$ $\AA$ Aluminum foils. Open and

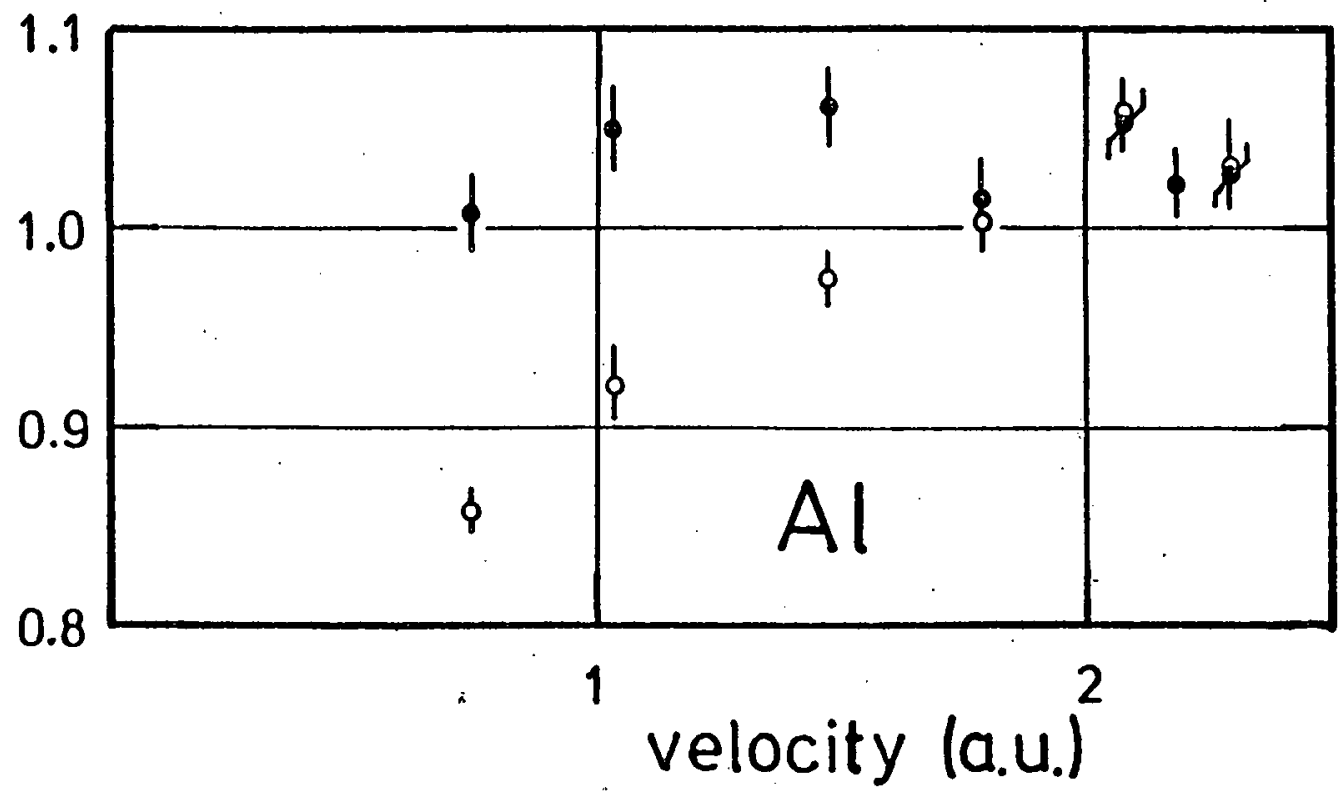

closed circles refer to measurements performed on transmitted $\mathrm{H}_{2}^{+}\left(\mathrm{R}_{2}\right)$ and $\mathrm{H}^{+}\left(\mathrm{R}_{1}\right)$ respectively 

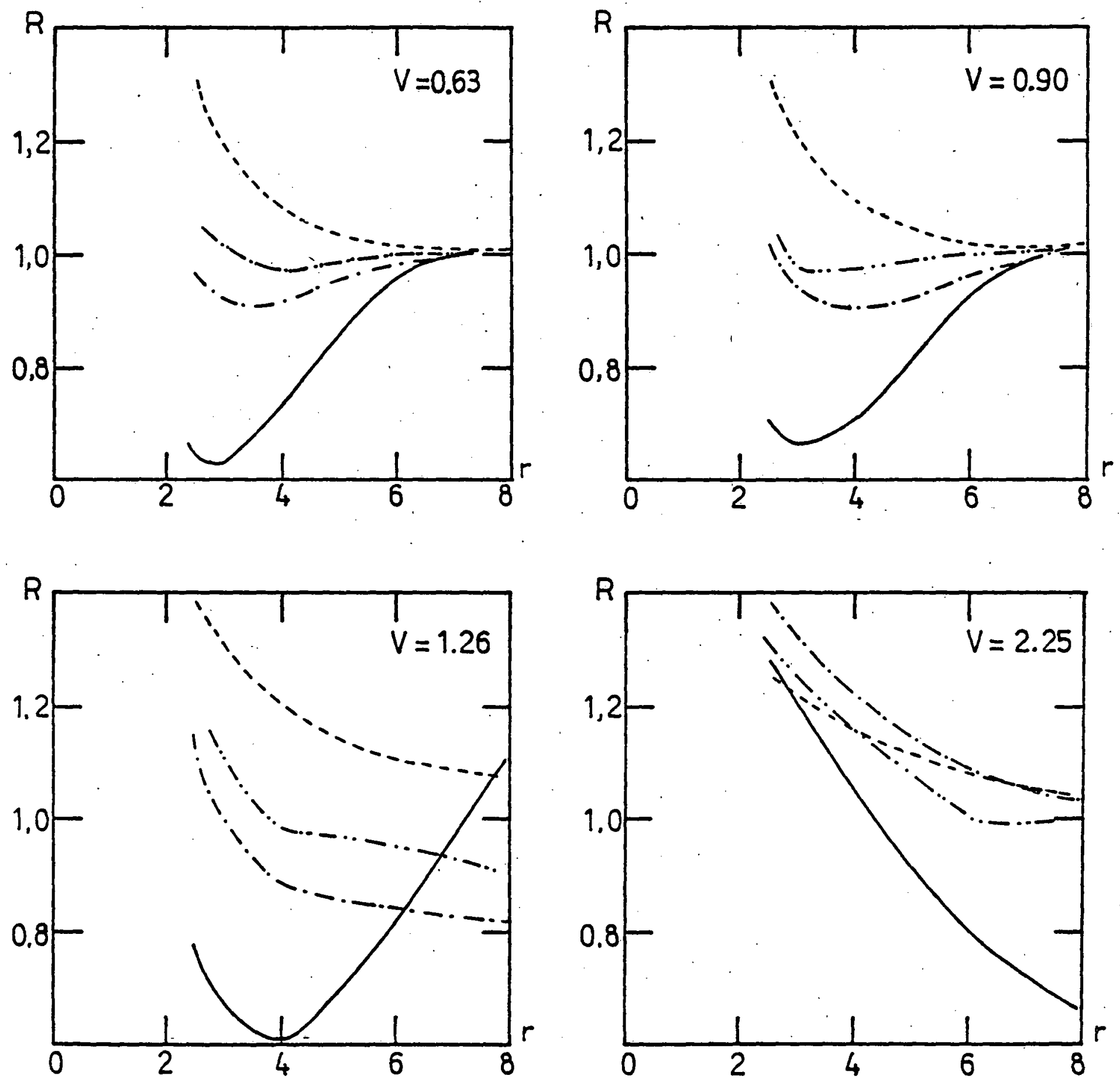

Figure 3: Calculated values of $R(\theta)$ versus internuclear separation $r$ (in a.u.) for different values of the velocity $v$ (in (a.u.) - - < < $>$ averaged over all angles $\theta$ between the internuclear axis and the direction of motion;$R\left(\theta=0^{\circ}\right)$; - - $R\left(\theta=45^{\circ}\right) ; \cdots R\left(\theta=90^{\circ}\right)$. The threshold velocity for plasmon excitation is $v=1.24$ a.u. 
THIS PAGE

\section{WAS INTENTIONALLY \\ LEFT BLANK}




\section{ENERGY LOSS OF CLUSTERS}

\section{R. Laubert}

East Carolina University, Greenville, North Carolina 27834

Figure 1--The fact that molecules or clusters have greater energy loss than the corresponding atomic particles was shown by W. Brandt et al. (Phys. Rev. Lett. 33, 1325(1974)). The original theoretical formulation involved the material dependent partition function between close and distant collisions suggesting the possibility that the stopping power ratio is a way to evaluate this constant. (W. Brandt and R. H. Ritchie, Nuc1. Inst. Meth. 132, $43(1976))$.

Figure 2--The theoretical energy loss ratio as a function of cluster velocity and inter-cluster separation. The decreased and increased stopping of clusters result from an interference of the wakes of the Individual atoms of the cluster. (N. Arlsta, Phys. Rev. B18, 1 (1978)) The stopping power ratio is also dependent on the orientation of the cluster (In file clusters have a lower energy loss) however experiemental vertfication of this requires targets of $\leq i \mu g / \mathrm{cm}^{2}$ and particle energies of $\mathrm{E}<50 \mathrm{keV} / \mathrm{amu}$.

Flgure 3 shows the experimental arrangement employed to measure the stopping power ratio of clusters to atoms. The fon beam $\left(10^{-3} \mathrm{~cm}\right.$ dia and $1-5 \times 10^{-11} \mathrm{~A}$ intensity) is collimatted to $\pm 0.1 / \mathrm{mrad}$. by a pair of slits. The target is surrounded by a cold tran to reduce carbon depositron to $-3 \AA /$ hour. The energy distribution of the exiting beam Is measured by a magnetic spectrometer (MII) which has an energy resolution of 1-5 $\times 10-4$. Post target slits permit measurements of the angular distribution. Not shown are a pair of pre and post target electric plates which permitted one to measure the energy and angular distribution of neutral particles. 
Figure 4--The angular distribution for $150 \mathrm{keV}$ protons incident on $5 \mu \mathrm{g} / \mathrm{cm}^{2}$ carbon target. The distribution is independent of the exiting particle or of the geometric orientation: The solid line is the expected distribution calculated according to the prescription of Meyer (L. Meyer, Phys. Status. Sol1di, 44, 253(1971).

Figure 5--The mean energy loss (as determined by the centroid of the energy distribution) as a function of the scattering angle for $150 \mathrm{keV} \mathrm{H}^{+}$ on $5.3 \mu \mathrm{g} / \mathrm{cm}^{2}$ carbon. The slight increase in the energy loss with increasing angle is from target non-uniformity and possible contribution from nuclear energy losses. The constancy of the energy loss as a function of scattering angle permits one to determine $\Delta E$ by measuring the energy distribution in the $\theta=0$ direction.

Figure 6--The energy and angular distribution when $300 \mathrm{keV} \mathrm{H}_{2}^{+}$ particles are incident on $2 \mu \mathrm{g} / \mathrm{cm}^{2}$ carbon target. For comparison the cuss of equal veloctty protons is shown in Part D. For exiting charged particles (Fig. A), a three peak distribution is obtained that Is considerably wider in energy and angle than the proton distribution. Figure B shows the distribution for exiting neutral and negative particles and for protons in colncidence with a neutral particle. The mean energy loss is independent of the scattering angle and of the exiting particle. This means that a measurement of the energy distribution in the $\theta=0$ direction is sufficient to determine the energy loss of the clusters. The distribution for exiting charged molecules $\left(\mathrm{H}_{2}^{+}, \mathrm{H}_{2}^{+}\right)$is shown in Fig. C. Here the angular distribution is narrower $(\sqrt{2})$ while the energy distribution is wider $(\approx \sqrt{2})$ than the corresponding atom $\left(\mathrm{H}^{+}, \mathrm{H}^{+}\right)$ case. In Fig. A\& B, the point marked $E_{0}$ is determined by gaseous dissociation. 
Figure 7--The Angular distribution of the various exiting particles when $300 \mathrm{keV} \mathrm{H} \mathrm{H}_{2}^{+}$is incident on $2 \mu \mathrm{g} / \mathrm{cm}^{2}$ carbon.

Figure 8--The angular distribution as a function of the incident $\mathrm{H}_{2}^{+}$energy for a $2 \mu \mathrm{g} / \mathrm{cm}^{2}$ carbon target. The solld line for the atom case $\left(\mathrm{H}^{+}, \mathrm{H}^{+}\right)$is calculated according to Meyer (L. Meyer, Phys. Status. Sol1d1. 44, 253 (1971). The other solld lines are to guide the eye.

Figure 9--The energy loss ratio of clusters to atoms for a $2 \mu g / \mathrm{cm}^{2}$ carbon target as a function of the incident energy. The measurements are performed in atom-cluster-atom sequence to insure reproduclbility. The scatter of the data reflects the difficulty of Insuring use of the same target spot for atoms and molecules. The error bar result from the uncertainty of determining the four centroids and the small energy loss involved $(-1.0-1.5 \mathrm{keV}$ total). The solid line is the calculation of N. Arista (Phys. Rev. B18, I (1978)). Considering the errors involved the agreement between experiment and theory is satisfactory.

Figure 10--The energy loss ratio of cluster to atomic particles for a $5 \mu \mathrm{g} / \mathrm{cm}^{2}$ carbon target. The ratio is closer to unity indicating larger average internuclear separation. The square point is from A. R. Nyalesh et al. J. Phys. C11, 2917 (1978). The solid line is from N. Arlsta. Similar results have been reported by Eckardt et al. (J. Phys. C11, L851 (1978)).

The energy loss ratio of exiting molecules for $5 \mu \mathrm{g} / \mathrm{cm}^{2}$ carbon target. The solid line is from (N. Arista Phys. Rev., B18, I (1978)) and is the same one as in the previous figure. Clearly, the energy loss of particles from clusters that end up as molecules is less than the energy loss of particles that end up as individual atoms. This 
could result from a lock step mechanism of wake riding or that the molecules are preferentlally formed from clusters that traverse

thinner parts of the target (the yleld of $\mathrm{H}_{2}^{+}$varies $-t^{-3}$ ). At the present time we cannot distinquish between the two mechanisms. 


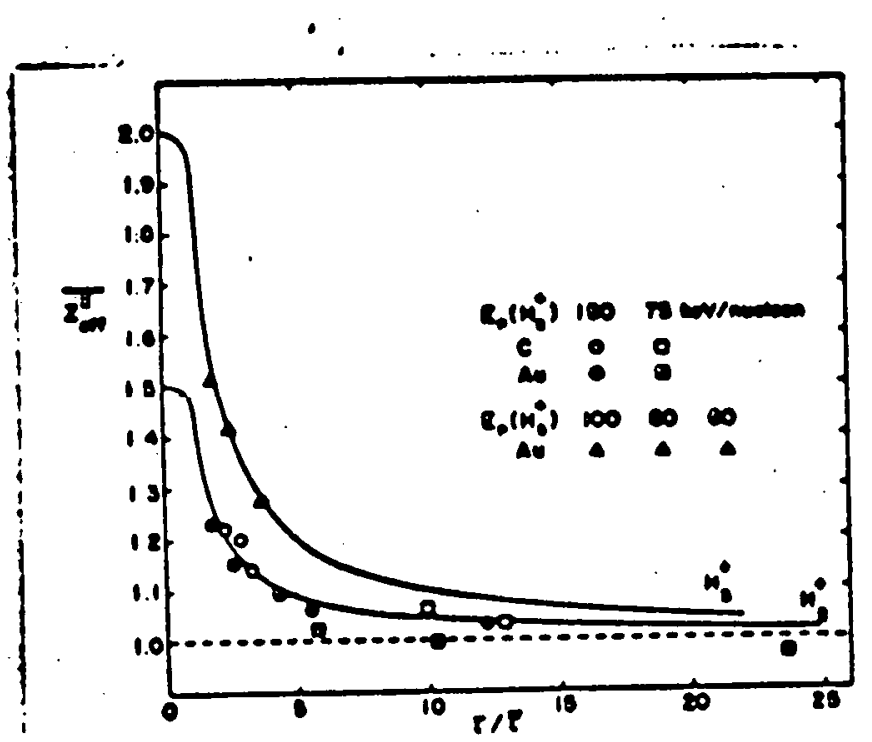

Fie 12. Effective equared proton charge deduced from cluster stoppins power meusurements. The colid curves repreent the vicinage function calculated in the approximation of the equipartition rule $\left.(C-0)^{\circ}\right)$.

Brandt tal Phys. Rer. Lett. 33, $1325(1974)$

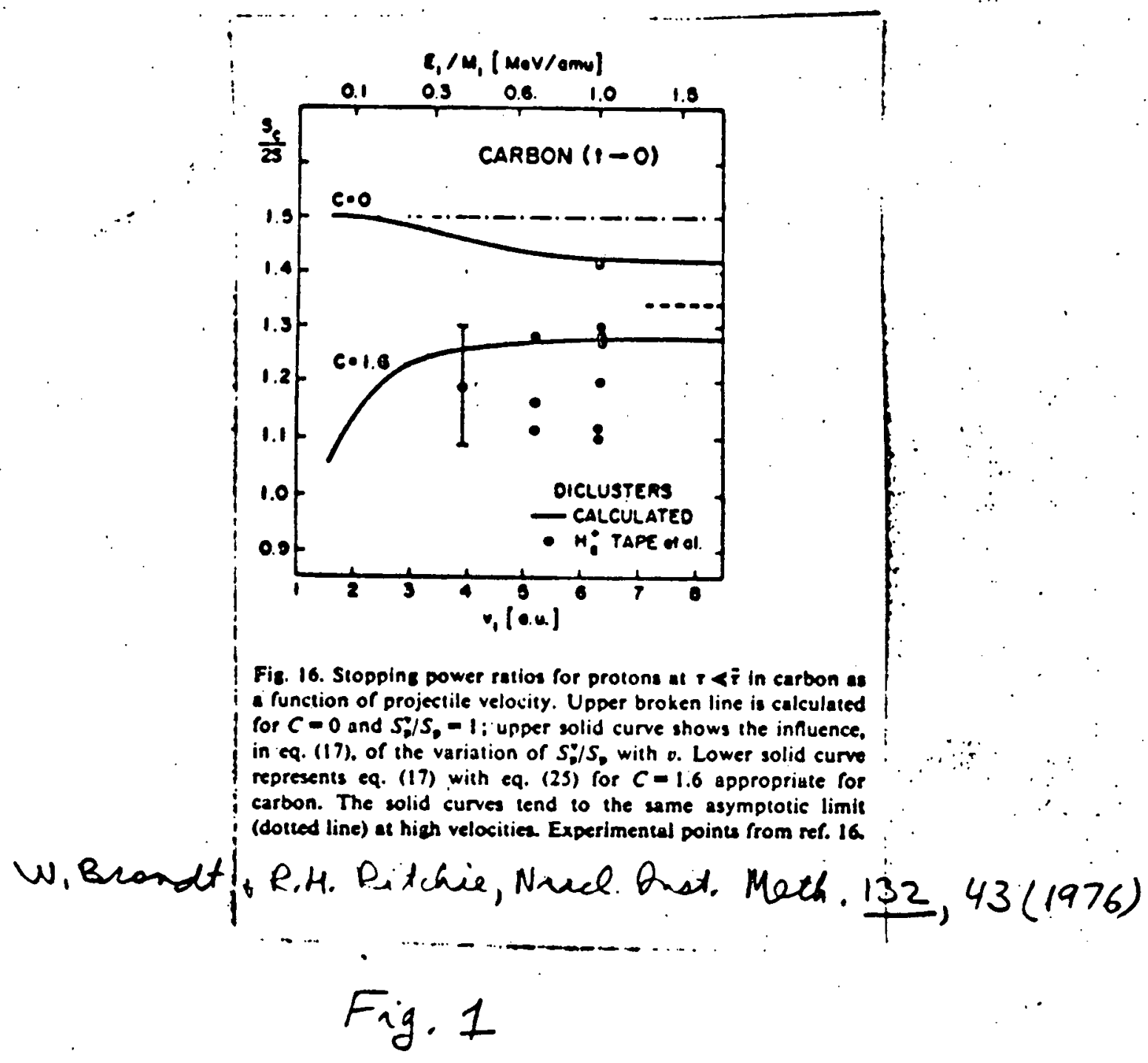




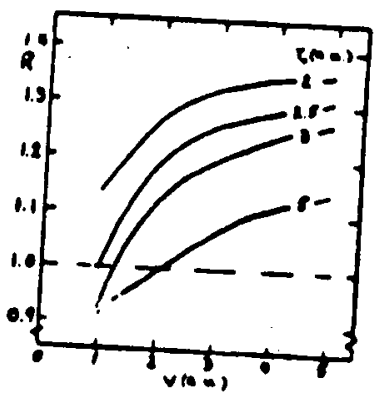

The theoretical energy loss ratlo, R. as

internuclear the text; for varlous average

are from ref. 11.

Fig. 2 


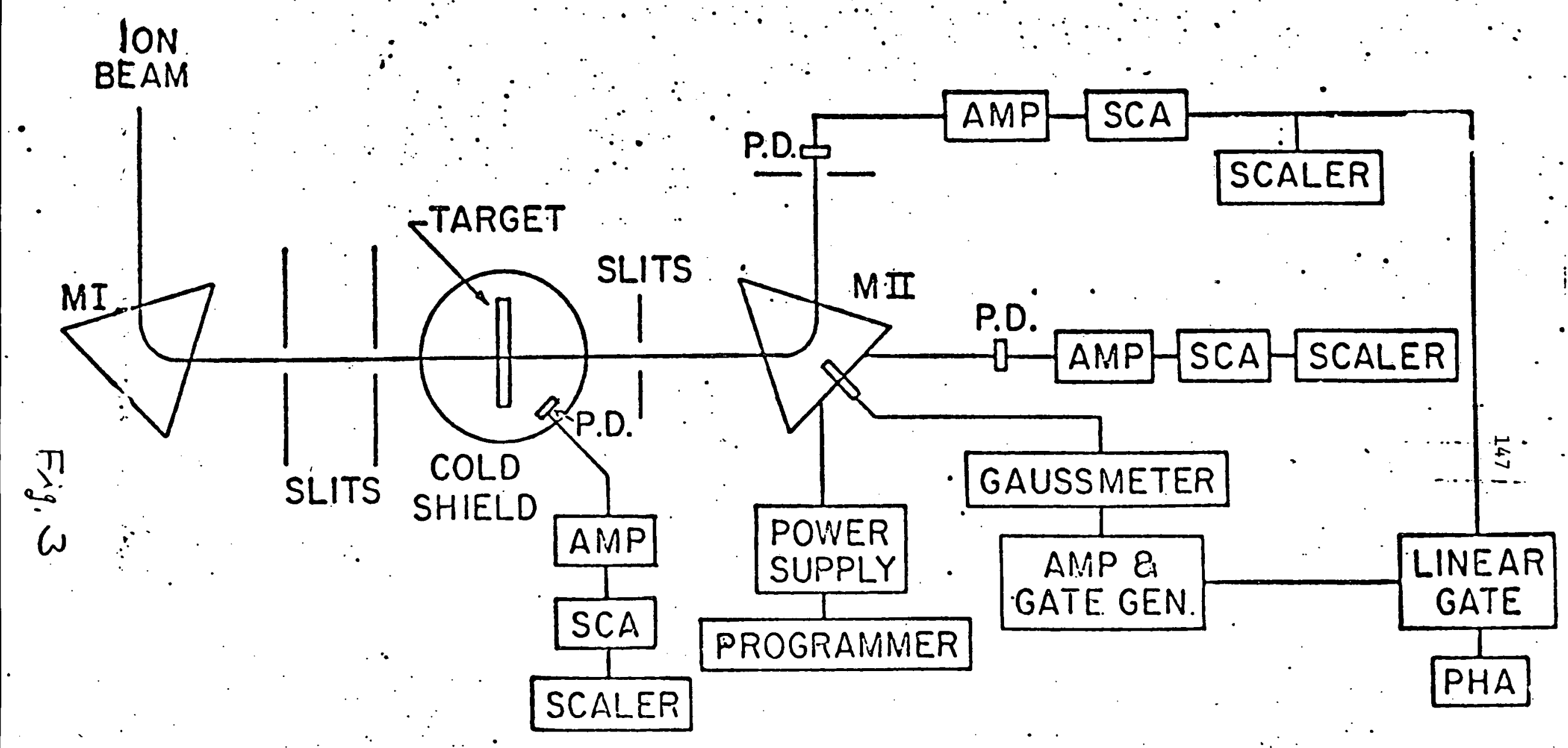



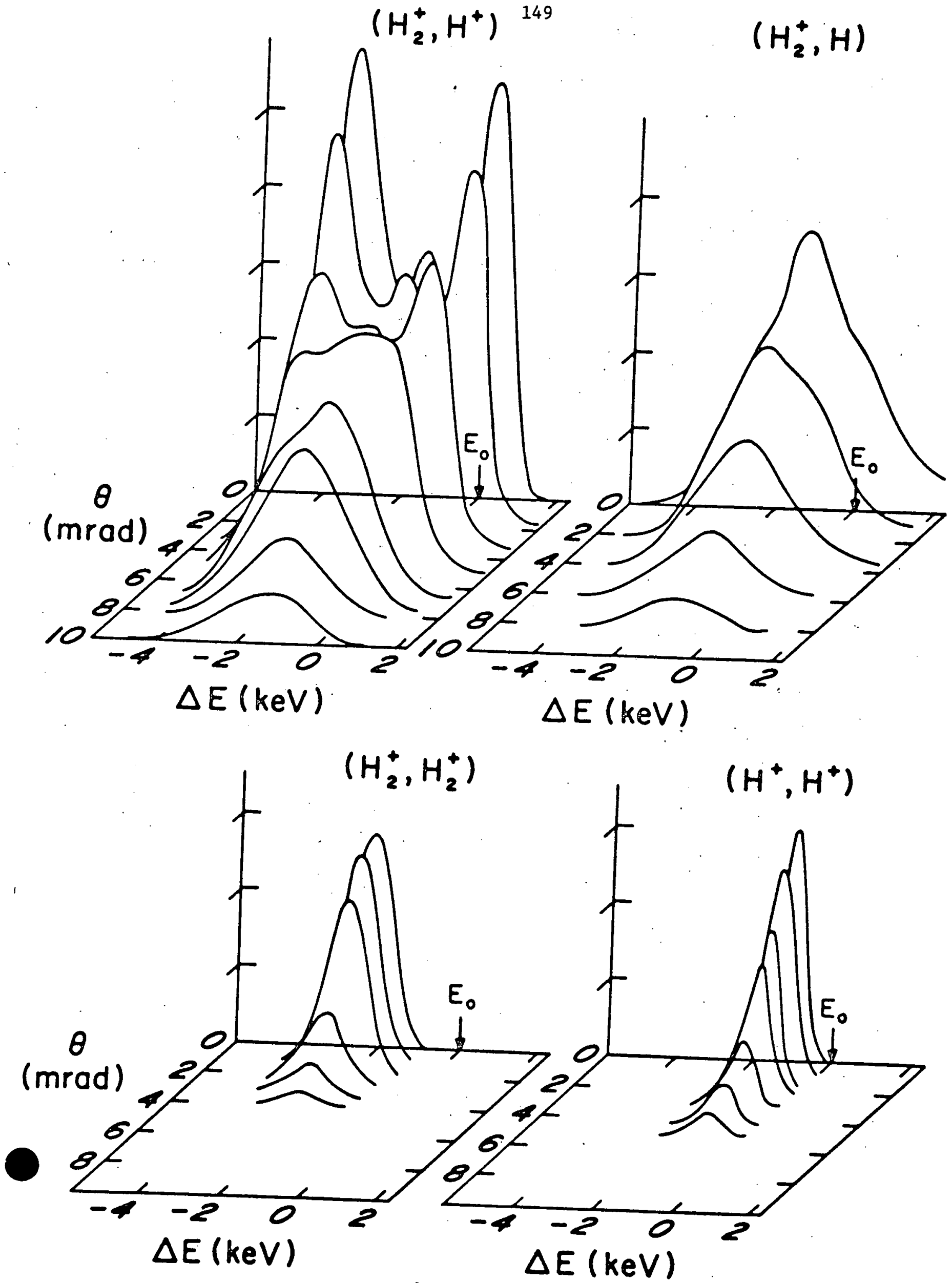

$F_{i g .6}$ 


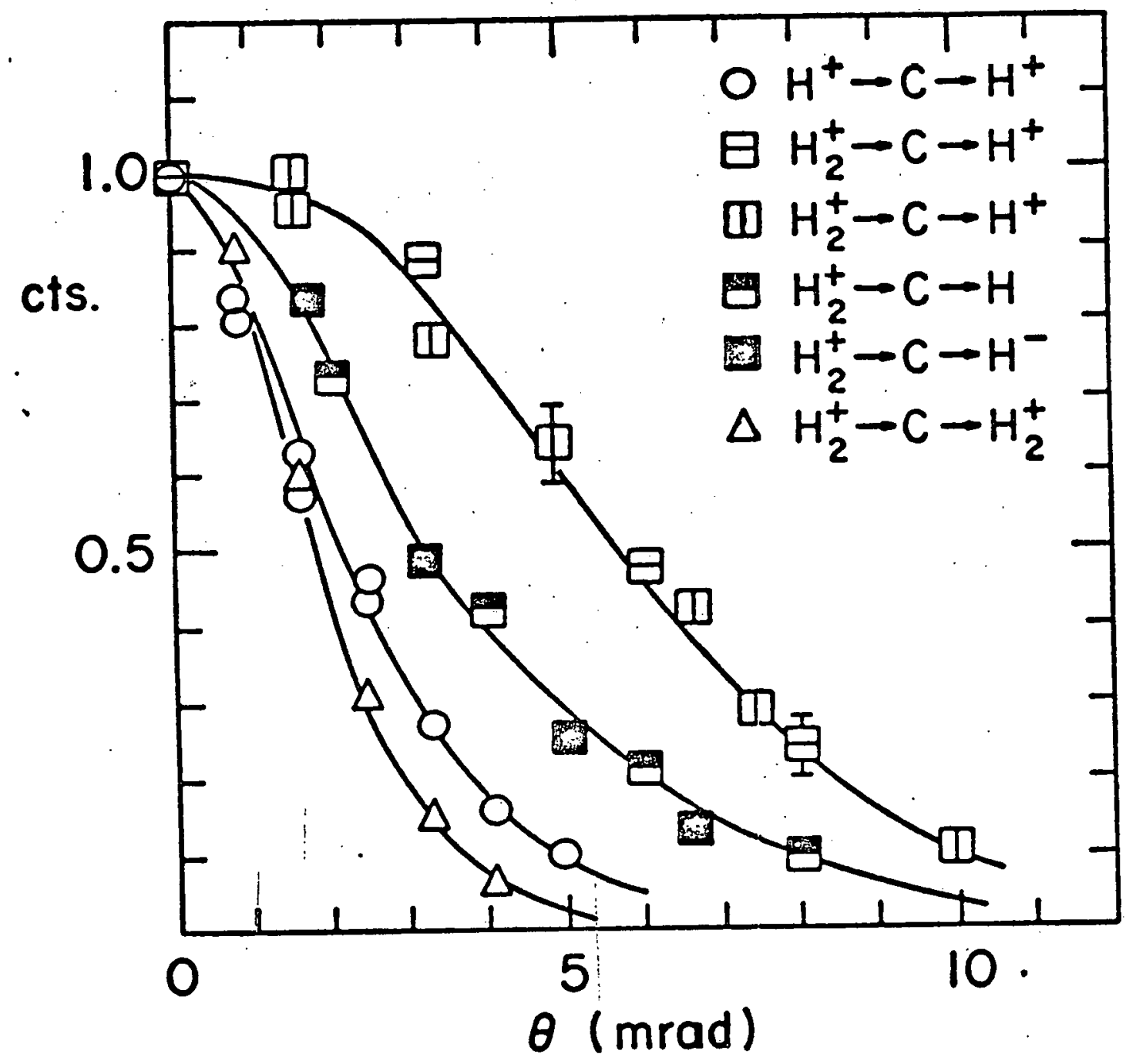

Fig. .7 


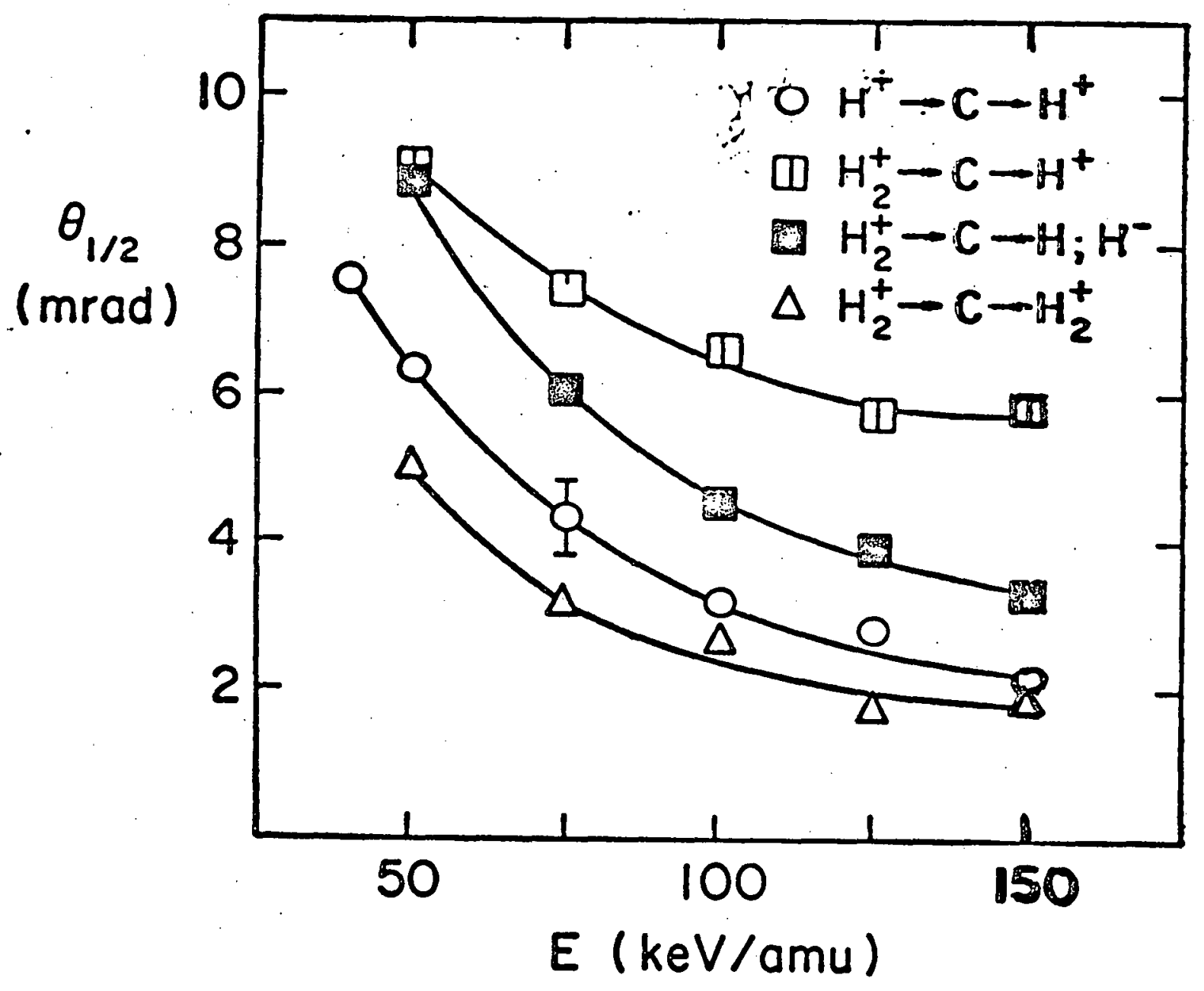

Fig. 8 


$$
t=2 \mu / \mathrm{cm}^{2} \mathrm{C}
$$

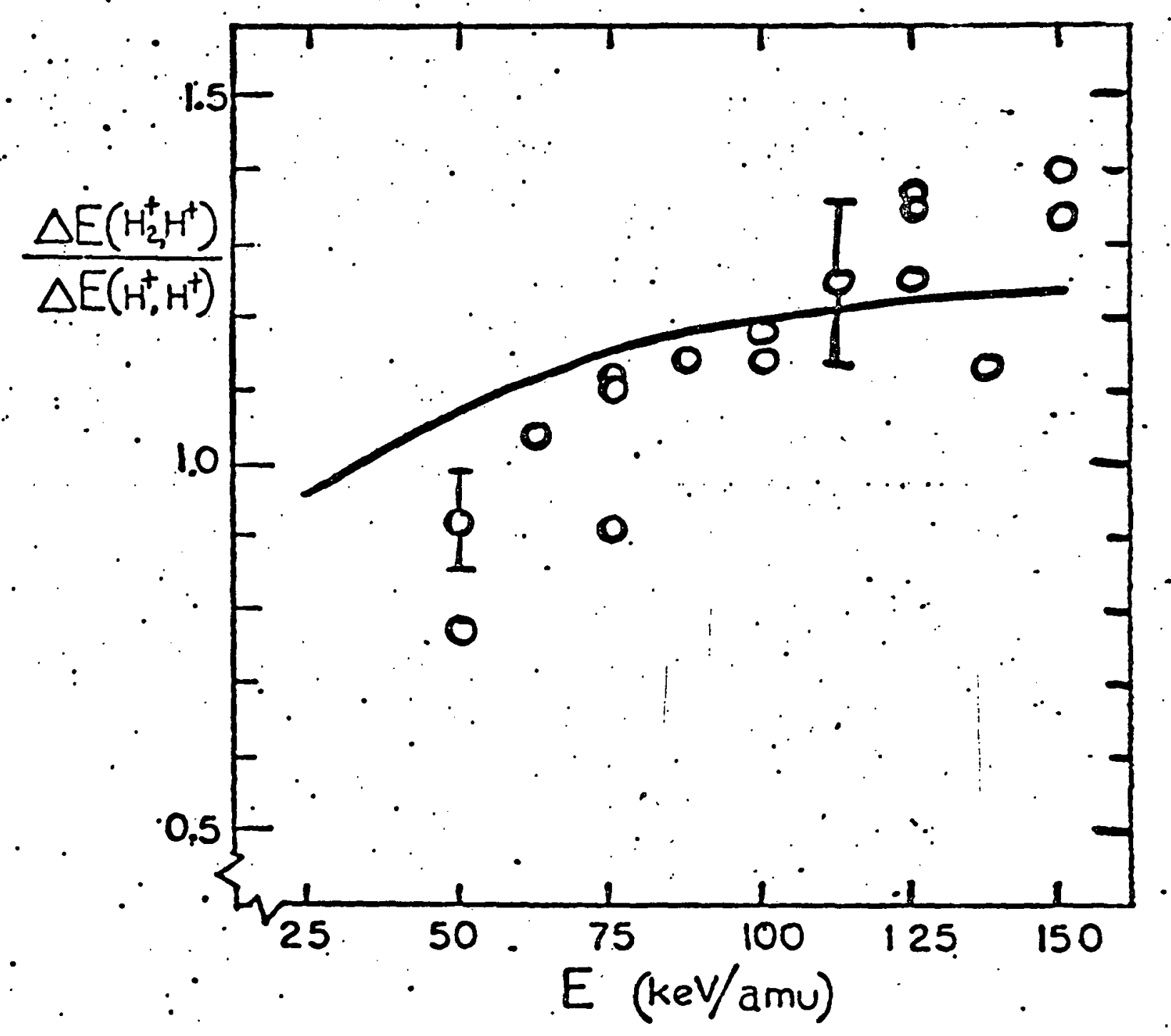

Fig. 9 
153

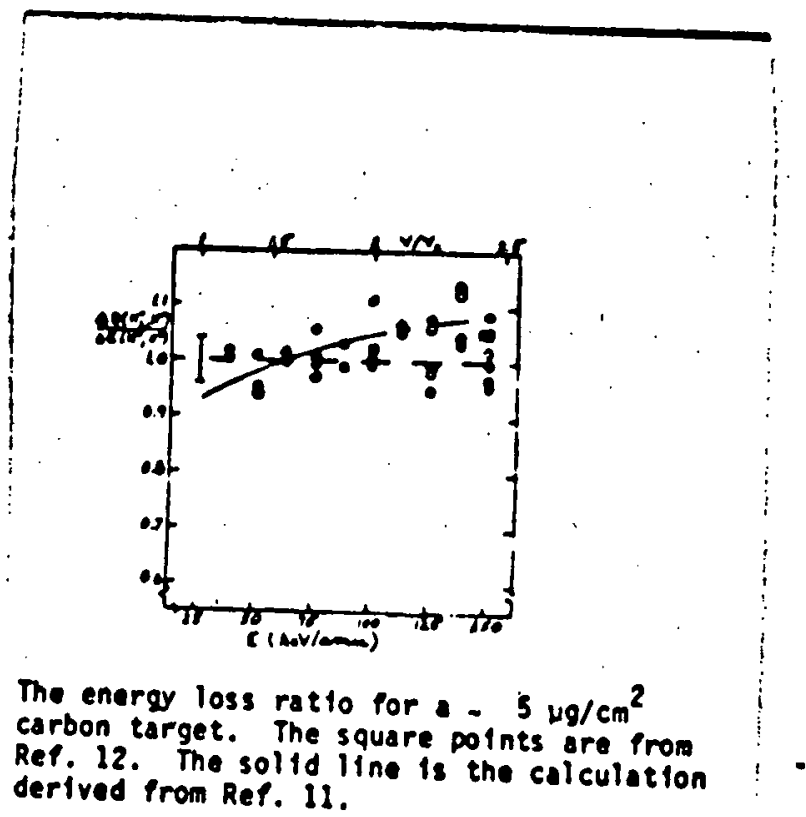
The energy loss rat to for a $5 \mathrm{ug} / \mathrm{cm}^{2}$
carbon target. The square points ore from
Ref. 12. The solid line is the calculation
derived from Ref. Il.

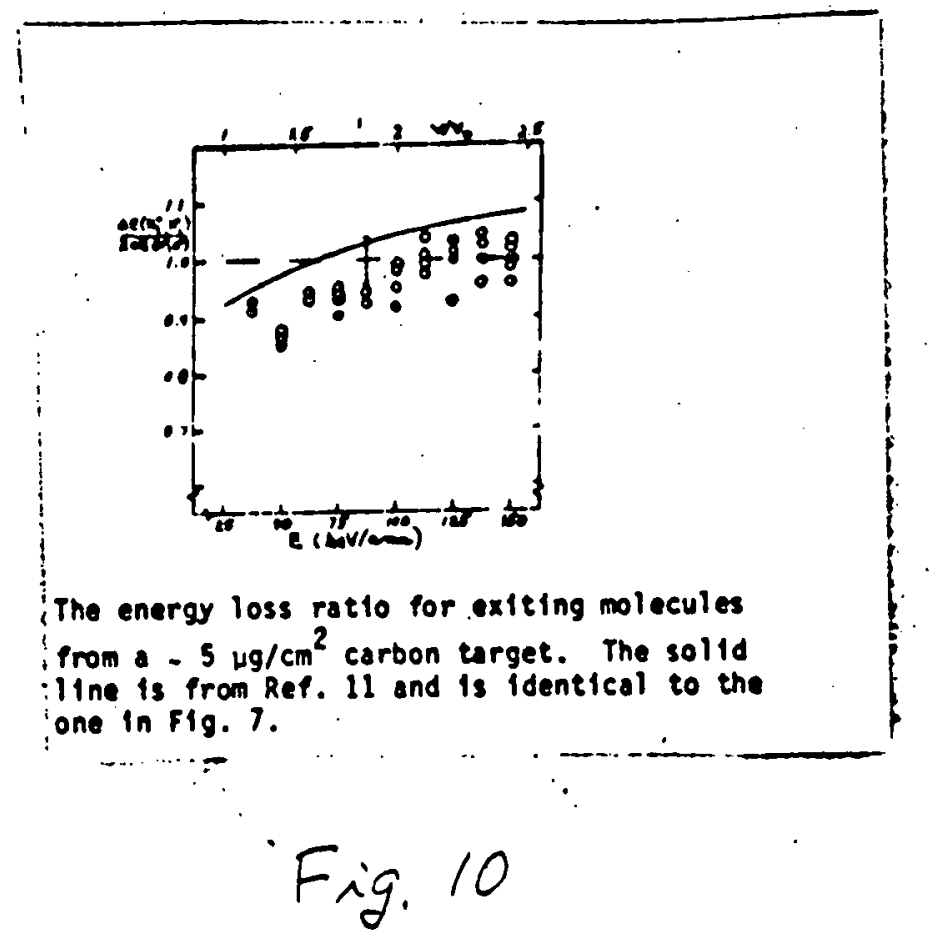


THIS PAGE

\section{WAS INTENTIONALLY LEFT BLANK}


THE POTENTIAL ESTABLISHED IN THE VICINITY OF SWIFT IONS IN SOLIDS

R. Laubert

East Carolina University, Greenville, North Carolina 27834

F1gure 1--The energy and angular distributions for $300 \mathrm{keV} \mathrm{H}{ }_{2}^{+}$on $2 \mu \mathrm{g} / \mathrm{cm}$ carbon.

Figure 2--The energy and angular distributions for $300 \mathrm{keV} \mathrm{HeH}^{+}$on 2. $\mu \mathrm{g} / \mathrm{cm}^{2}$ carbon.

Figure 3--The energy distribution at $\theta=0$ for $250 \mathrm{HeD}^{+}$on $1.7 \mathrm{\mu g} /$ $\mathrm{cm}^{2}$ carbon. Note the three peak distribution of $\mathrm{D}^{+}$and the different asymetry of the leading and trailing particle in the neutral $D$ distribution.

Figure 4-Tbe energy distribution at $\theta=0$ for $290 \mathrm{keV} \mathrm{HeH}^{+}$on $1.4 \mu \mathrm{g} / \mathrm{cm}^{2}$ carbon. The energy distribution of the positive and neutral hydrogen as well as the proton in colncidence with a neutral helium 1s shown. Note the difference of this distribution to the $\mathrm{H}_{2}^{+}$case (F1g. 1) and in particular the absence of a three peak distribution for the $\mathrm{H}^{\circ}$ spectrum. (See R. Laubert, IEEE Trans. Nuc1. Sc. N9-26, $1020(1979))$.

It 18 our ascertion that the two peak distribution observed for $\left.\mathrm{HeH}^{+}, \mathrm{H}\right)$ and $\left(\mathrm{HeH}^{+}, \mathrm{H}^{-}\right)$is Inconsistent with the viewpolnt that moving protons in solids have bound states. The observed energy distribution can be obtalned if one assumes that moving protons do not have bound states in solids while allowing for bound.states for the projectiles, or conversly that the projectiles do not have bound states while protons do. Given these alternatives one chooses the former, 1.e., that using explosion inside a solid as an operational criteria, the majority of protons do not exhlbit bound states in solids while moving at velocities $v_{1} \geq v_{0}$. If this is the case, what is the origin of the central peak of the three peak distribution observed In Fig. 1a, b, and Fig. 2? 
A possible explanation for the central peak can be put forward by considering electron capture by the cluster upon exiting from the target. Clearly, if not electrons are captured the nuclei are in a Coulombic potential and experience post-target explosion. If the exiting cluster captures one electron then for diprotons, the post target potential established is that of an $\mathrm{H}_{2}^{+}$molecule, which in the ground state $15 \sigma_{8}$ has negative values for internuclear distances that are greater than the equilibrium internuclear distance. This will tend to Inhibit explosion and hence decrease the final energy separation. Th1s can result in the formation of the central peak. If the internuclear velocity $v_{1}<\left(\frac{2 \varepsilon}{M}\right)^{\frac{1}{2}}$, where $\varepsilon$ is the potential of 1S $\sigma$ state of $\mathrm{H}_{2}^{+}$at the exiting internuclear separation, then a $\mathrm{H}_{2}^{+}$ molecule will be formed. For $2 \mu \mathrm{g} / \mathrm{cm}^{2}$ targets and velocities considered here typlcally $10 \%$ of the incldent particles will satisfy. this condition. The mafority of clusters will have an internuclear velocity greater than this amount and the molecule wi11 dissoctate, with equal probability, into a charged and neutral hydrogen. Hence one would expect the neutral, proton, and coincident distributions to reflect the central peak. This is what is observed in Fig. 1.

If the potential established by the exiting cluster is that of an excited $\mathrm{H}_{2}^{+}$molecule $\left(\mathrm{e} .8\right.$. , the $2 \rho \sigma_{\mathrm{u}}$ or $2 \rho \pi_{\mathrm{u}}$ states) that atoms of the cluster will dissociate and gain kinetic energy associated with that particular state. Therefore it is not surprising to observe a three peaks in the neutral and coincldent energy distributions shown in Figure $1 \mathrm{~b}$.

For an $\mathrm{HeH}^{+}$Incident cluster hydrogen can be observed from the followlng reactions: 


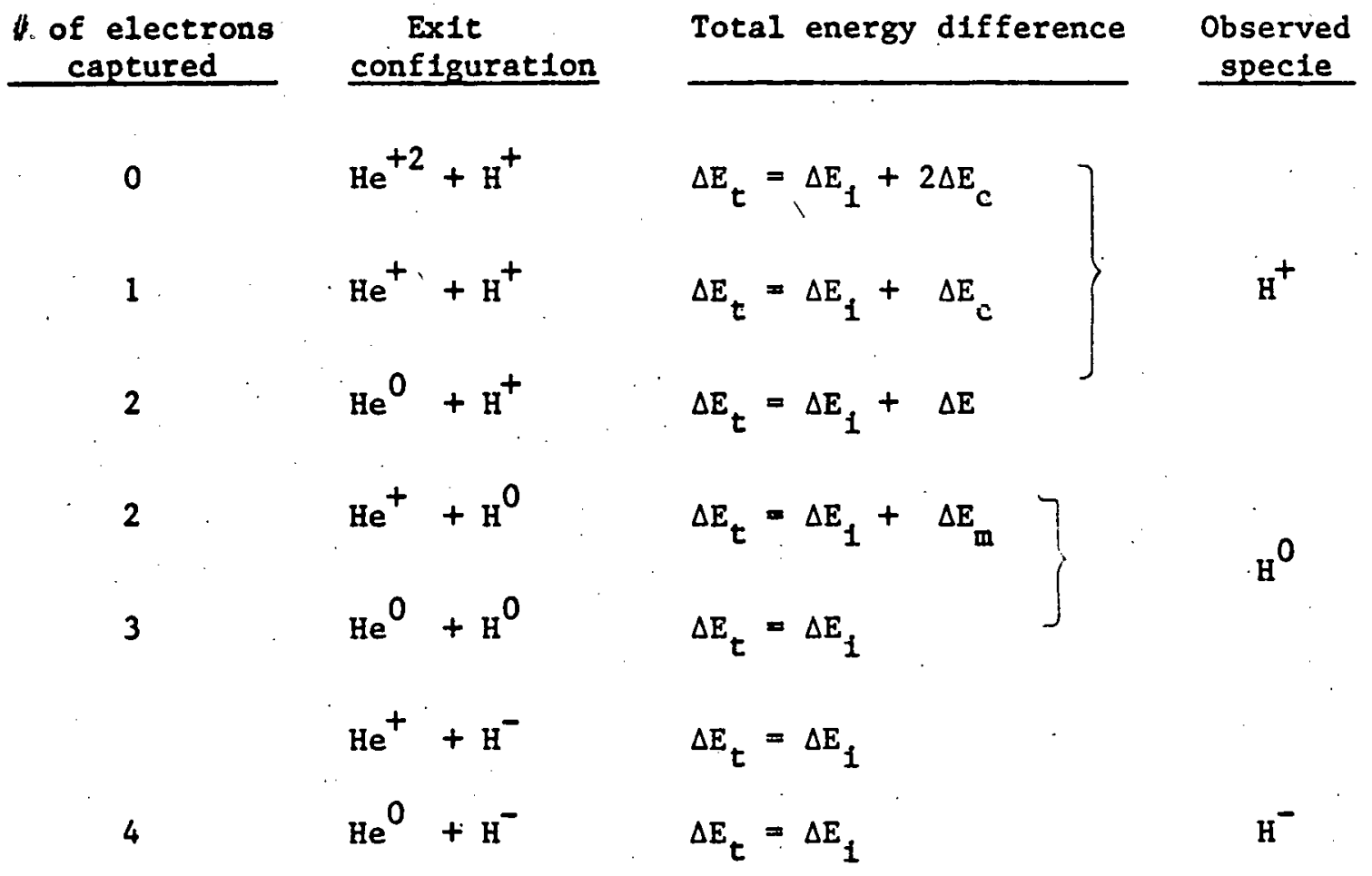

where $\Delta E_{t}$ is the total energy difference of the emerging particles and is the sum of the energy gained inside the target, $\Delta E_{i}$, the post target Coulombic explosion, $\Delta E_{c}$, and the energy change from the molecular potential, $\Delta \mathrm{E}_{\mathrm{m}}$, at the exit internuclear separation.

At the present velocities the one and two electron capture processes will dominate the final proton energy distribution. The central peak in Fig: $8 a$ is accounted for by the two electron process and the resulting implosion, while the side peaks result from the one electron process with the additional post target explosion. At higher Incident velocities the two-electron capture probability decreases relative to the 1 or 0 electron capture probability resulting in the diminution, and eventual disappearance, of the central peak.

When we measure the neutral hydrogen energy distribution only the three-electron capture process is important since an estimate of the yleld of neutral hydrogen from the two electron capture process is 
$-1 / 5-1 / 10$ of the yleld from the three electron capture process. Th1s was checked experimentally by measuring a ratio -10 for the yleld of charged to neutral hydrogen when fast $\mathrm{HeH}^{+}$molecules dissociate in a gas. Hence the exfting neutral and negative hydrogen energy distributions when $\mathrm{HeH}^{+}$is incident on sollds measures the separation occurring Inside the solid. The $\left(\mathrm{HeH}^{+}, \mathrm{H}^{+}\right)$and all of the final energy distributions observed with $\mathrm{H}_{2}^{+}$incident projectiles are altered by the formation of short lived molecular states at the exit surface of the target. Using a retarding electric field technique we were able to establish $10^{-9}$ sec as an upper Iimit to the lifetime of these molecules.

The present experimental results lead us to the conclusion that, using explosion inside a solid as an operational criteria, the majority of protons do not exhibit a bound state while moving in solids at velocities $v_{1} \geq v_{0}$. The observed central peak in the three peak distribution can result from the formation of a short lived molecule at the exit of the solid which subsequently dissoclates. To observe this central peak stringent requirements have to be met. The incident particle energy and target thickness have to be such that the explosion energy In the laboratory system is greater than the straggling and smallangle scattering energy, and the probability of electron capture has to be sufficiently large to detect a reasonable fraction of the total particles. To map the potential distribution of lons in solids by measuring the dissociation energy of clusters we must choose a system whose energy distribution is not altered (or can be easily corrected) by molecule formation or post target explosion. In addition the transit 
time of the cluster in the target must be sufficient for the particle to Interact with the potential and yet the target must be thin enough so that energy straggling and small angle scattering do not obscure the results. From this it is clear that ones experiments must be chosen carefully. 

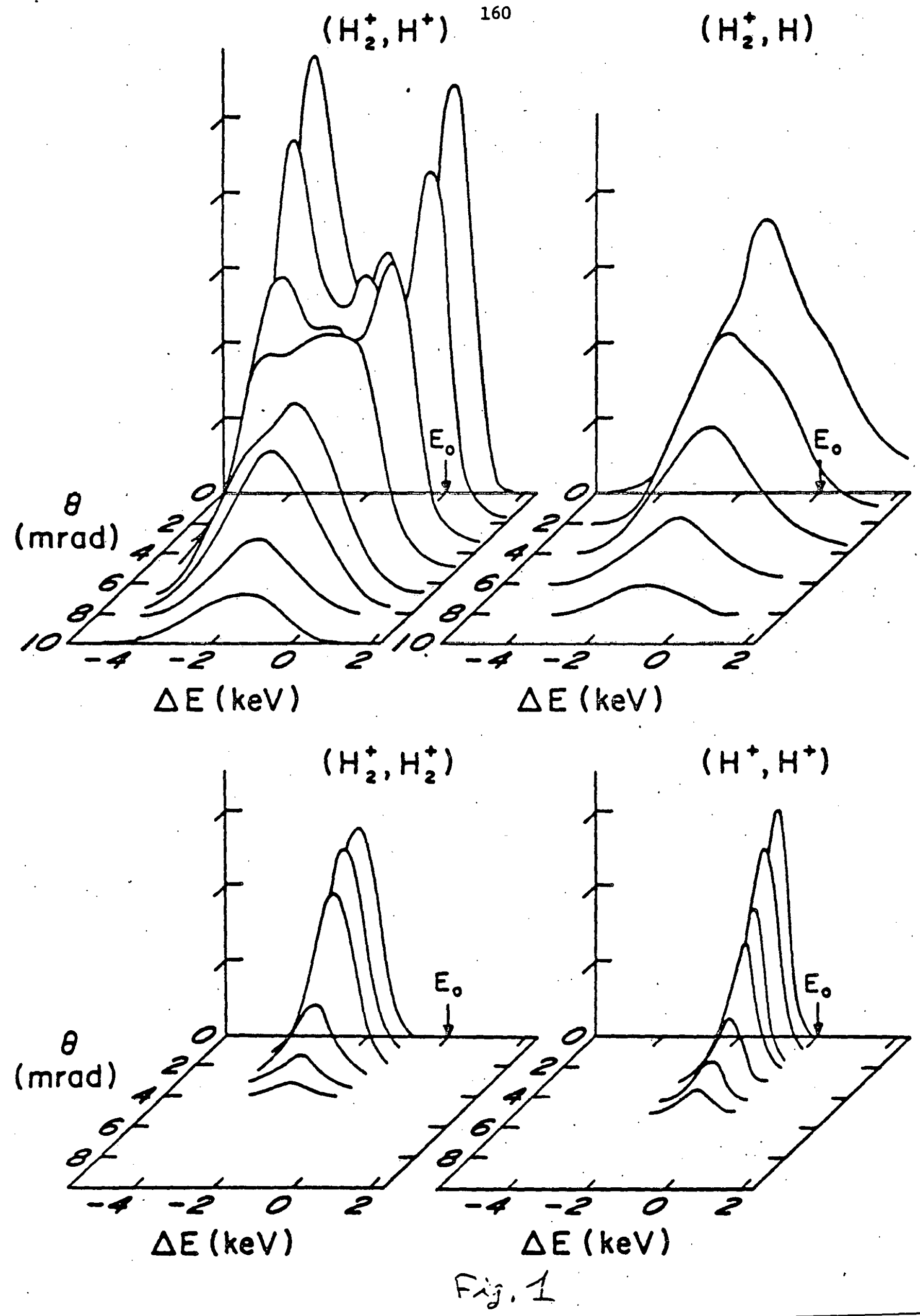

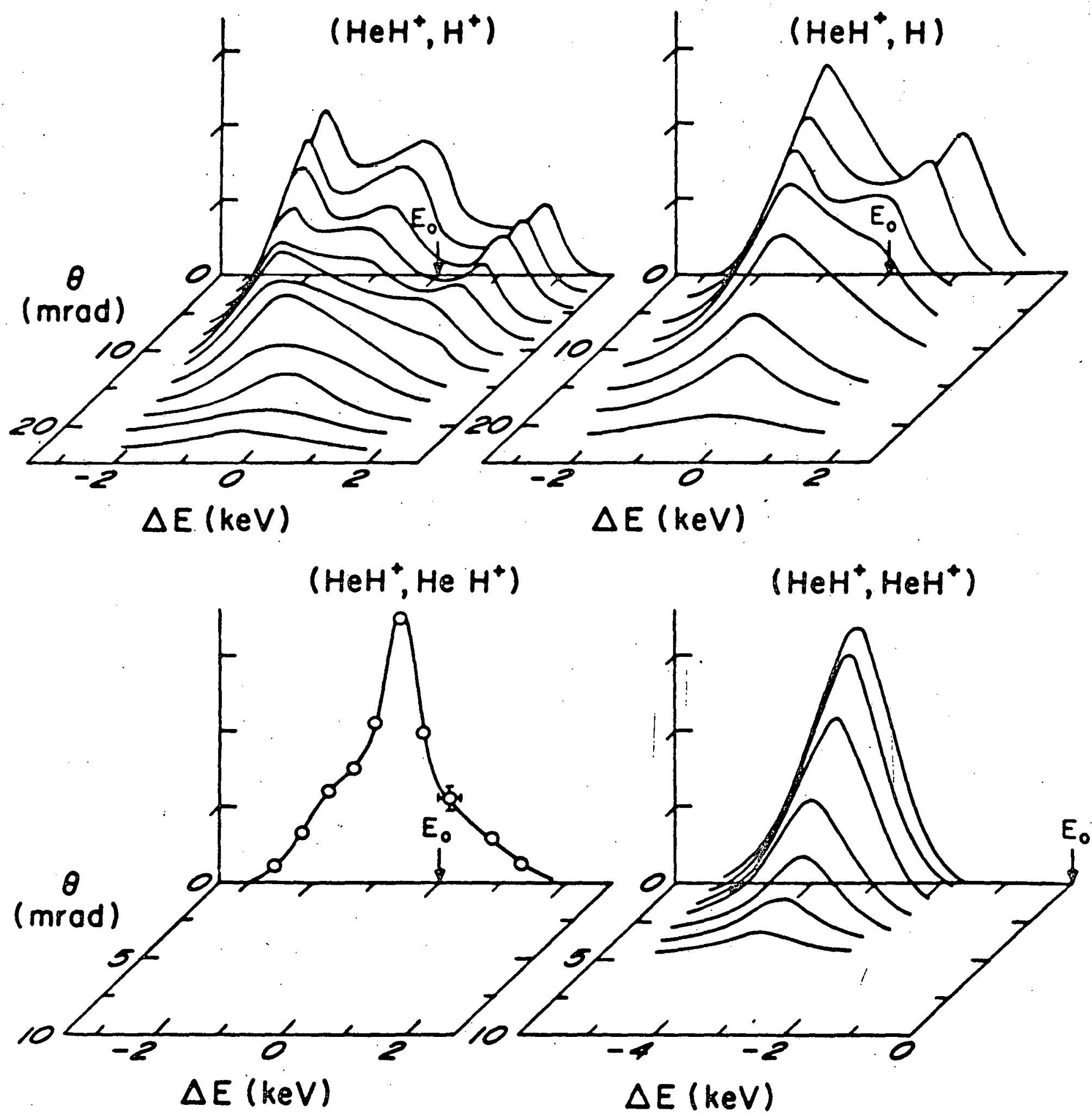

Fig. 2 
162

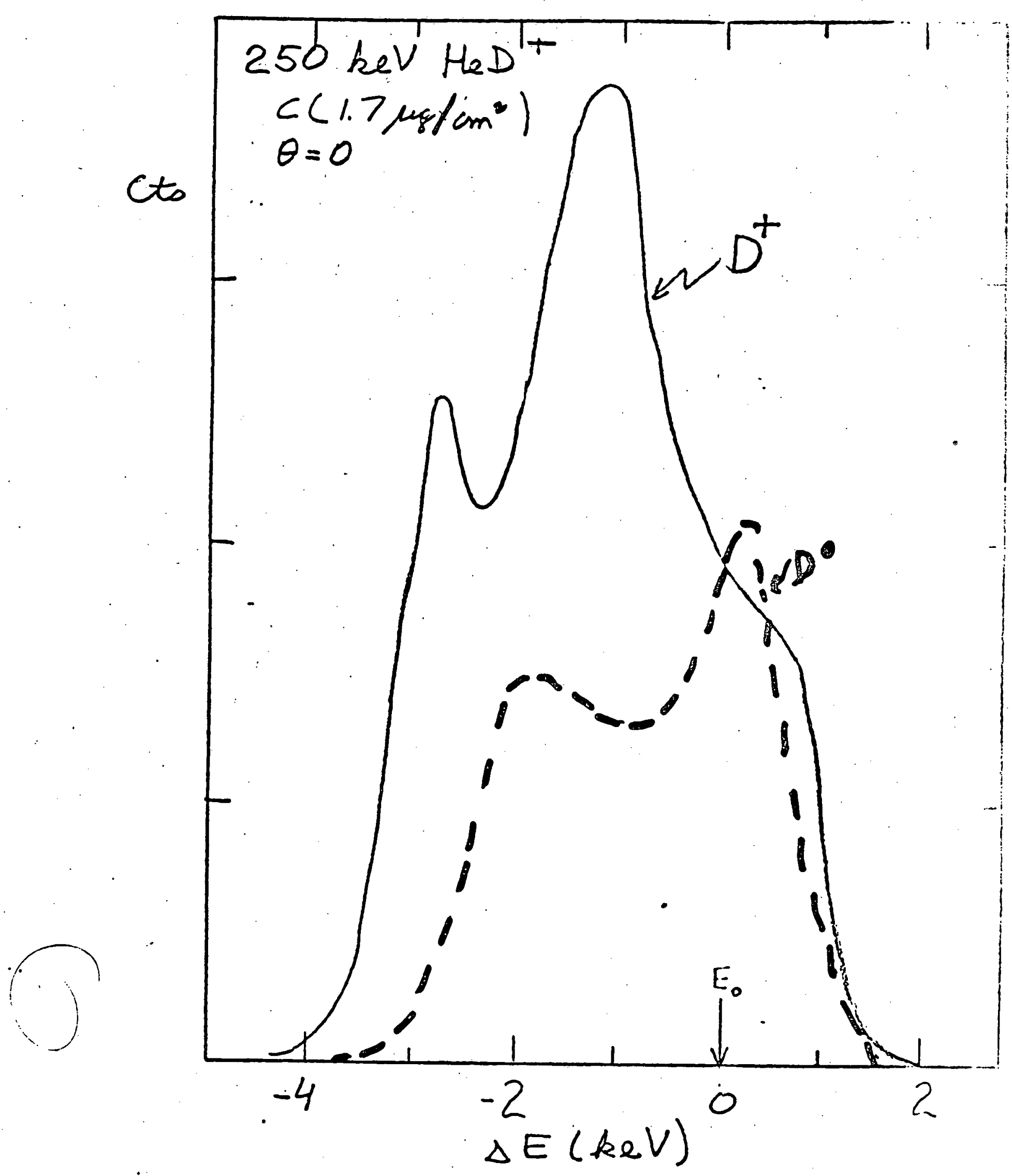

Fig. 3 
163

$$
290 \mathrm{keV} \mathrm{HeH}^{+} \rightarrow \mathrm{C}\left(1.4 \mathrm{mp} / \mathrm{cm}^{2}\right)
$$

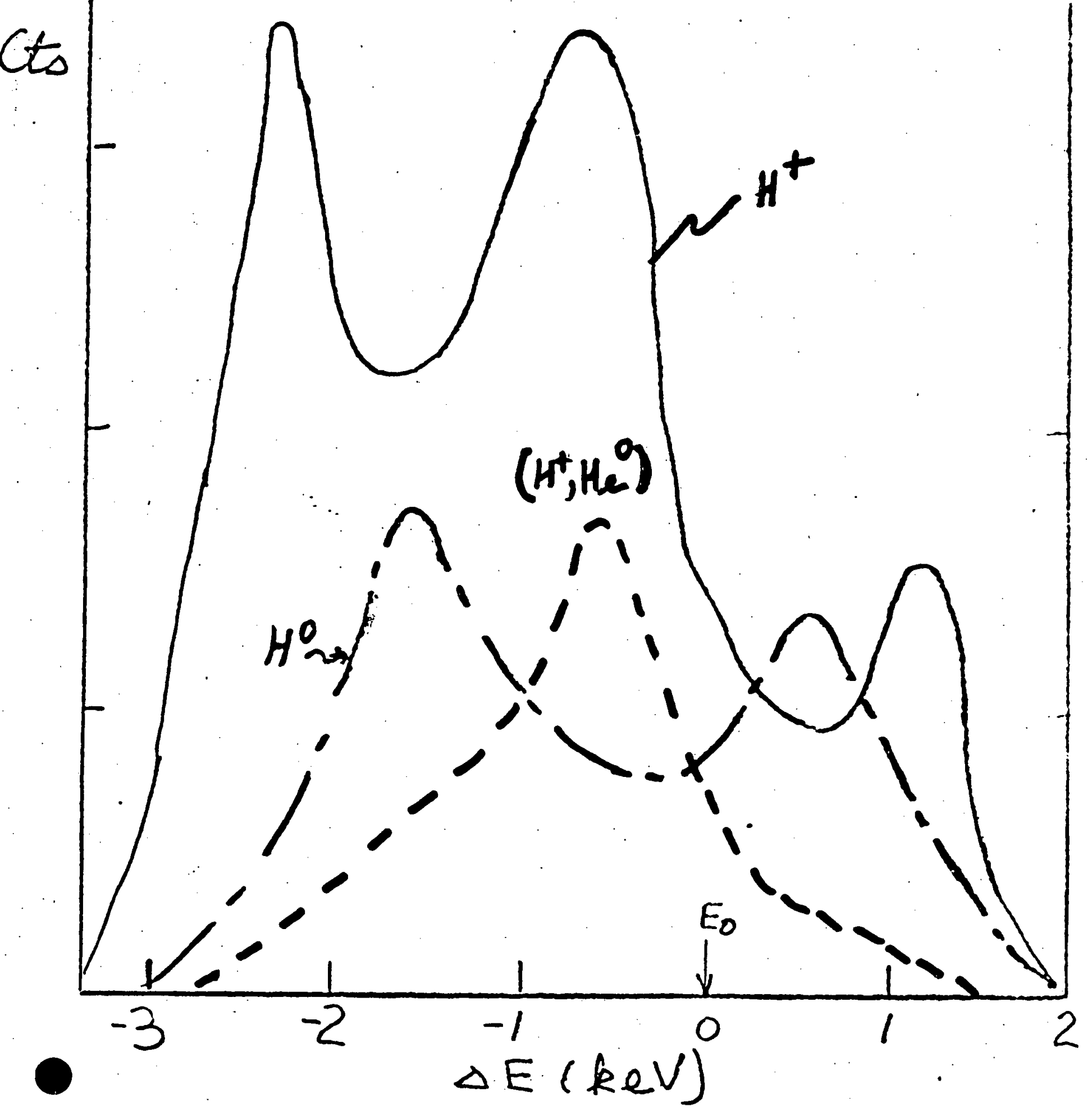

Fig. 4 
THIS PAGE

\section{WAS INTENTIONALLY LEFT BLANK}


$\mathrm{H}_{2}^{+}$Central Peaks.

W. Brandt

New York University, N.Y. 10003

R. Lauberst, F.K.Cheu, P.RL. 40,174 (1978)

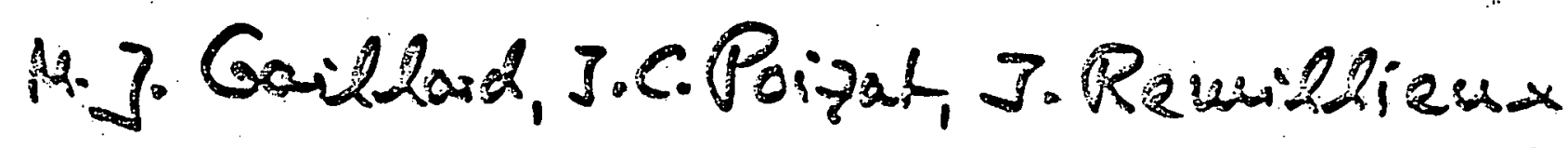

P.R.L \& 159 (10.79)

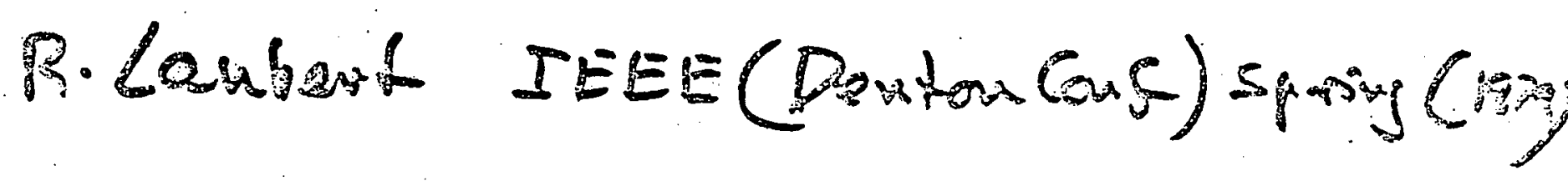

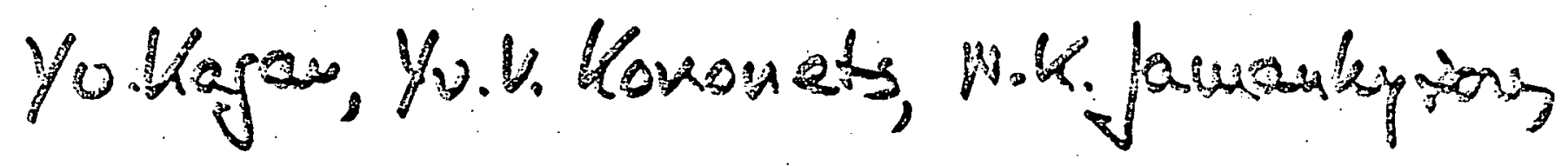

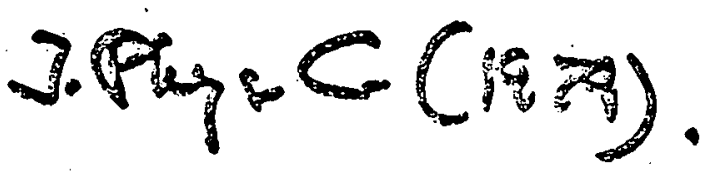

165 
166

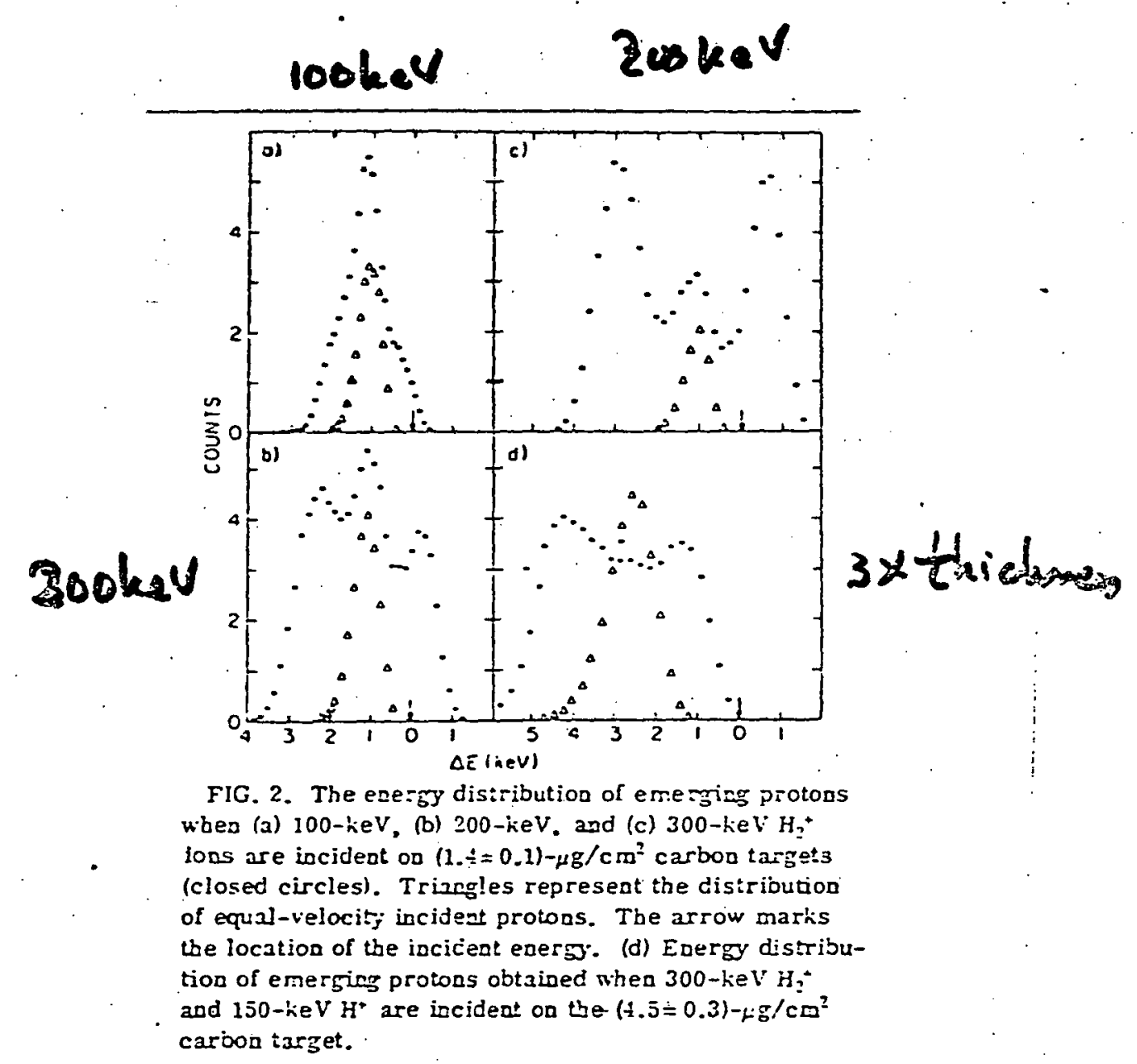

Central peat decreases cost $E$

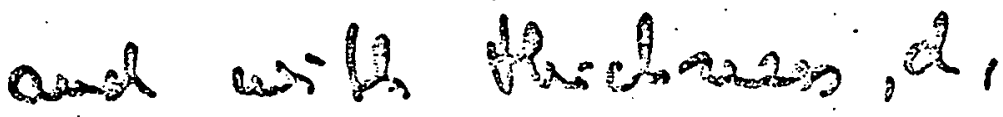

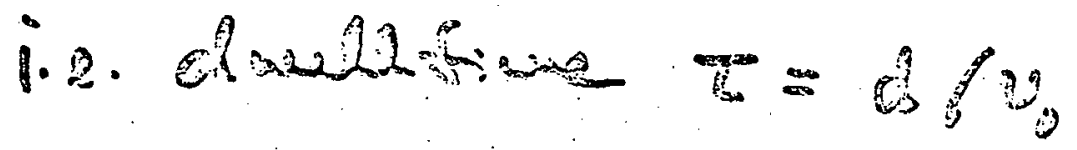




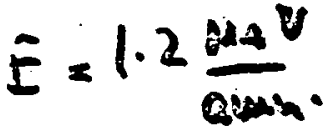

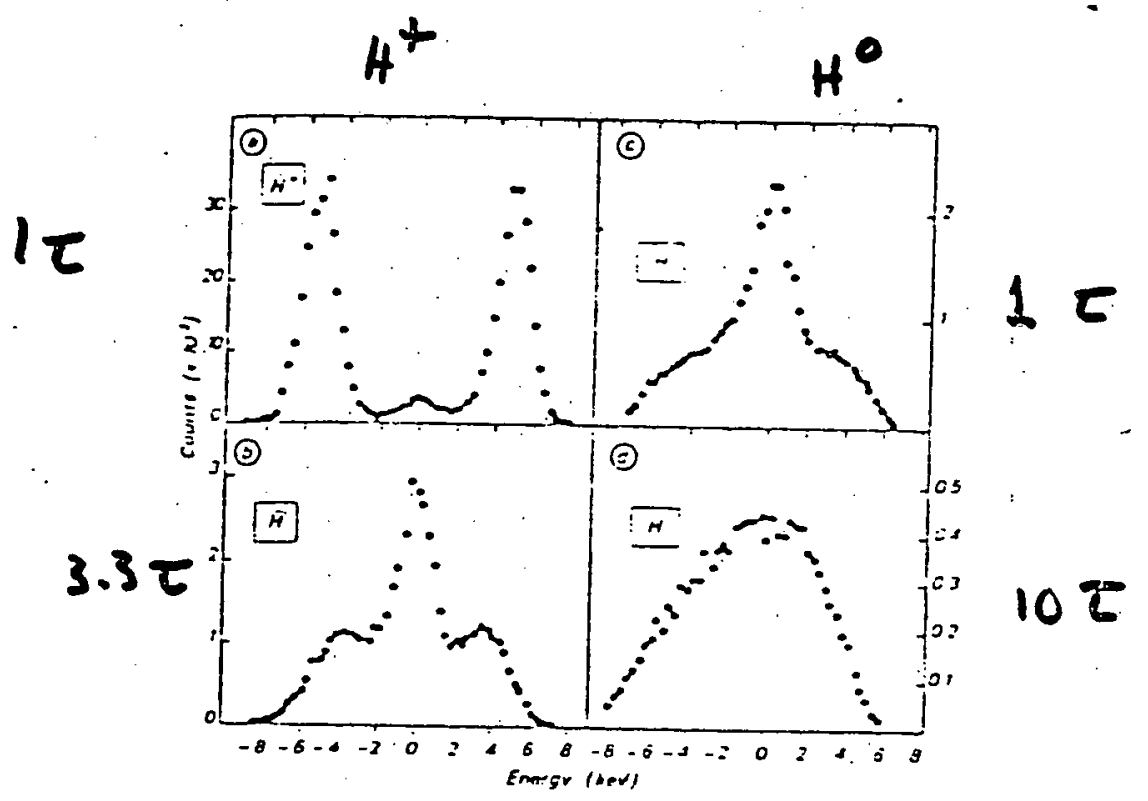

FIG. 2. The eaers distribution of :a! protons and

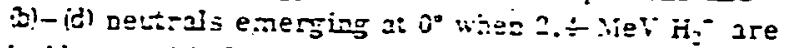
iccideat on (a), (b) $1-$, (c) $3.3-$, a ad (d) $2.5-j 5$ / $\mathrm{cm}^{2}$ carios foils. 
168.

2

Centool reale: No Contomb exp2osion?

1. Assume on inside So2

$\mathrm{H}_{3}^{+}$or $\mathrm{H}^{2} \mathrm{H}^{\circ}$ are trausurithed

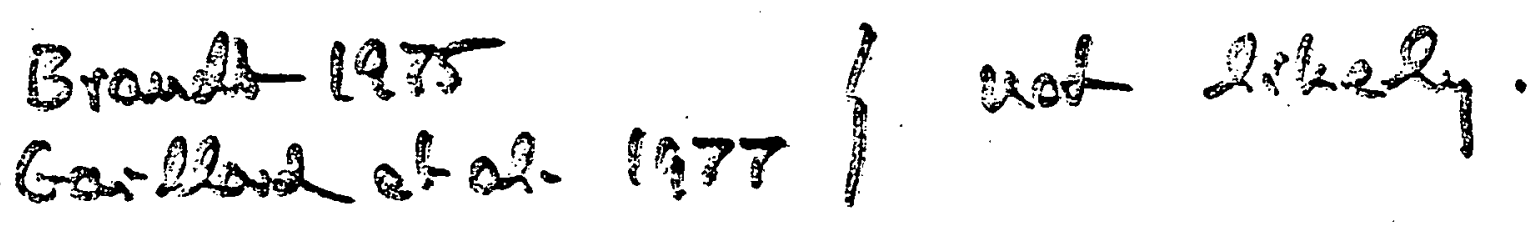

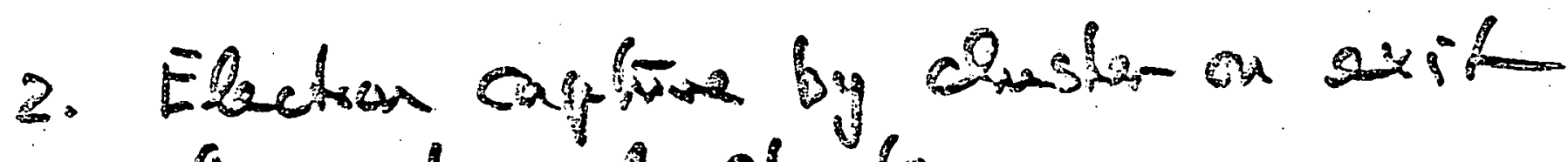

- Ove boviz stale

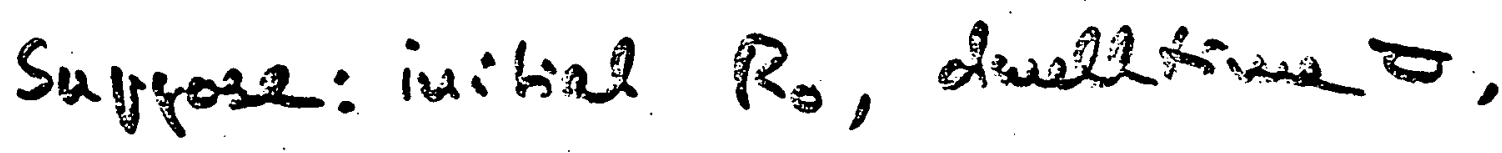

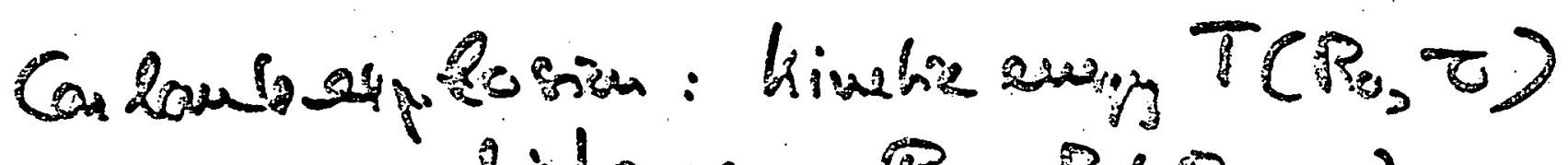
aistanes $R=R\left(R_{3,}, 5\right)$

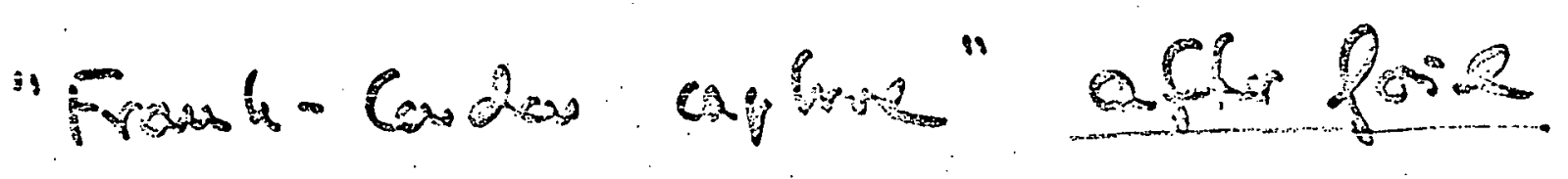




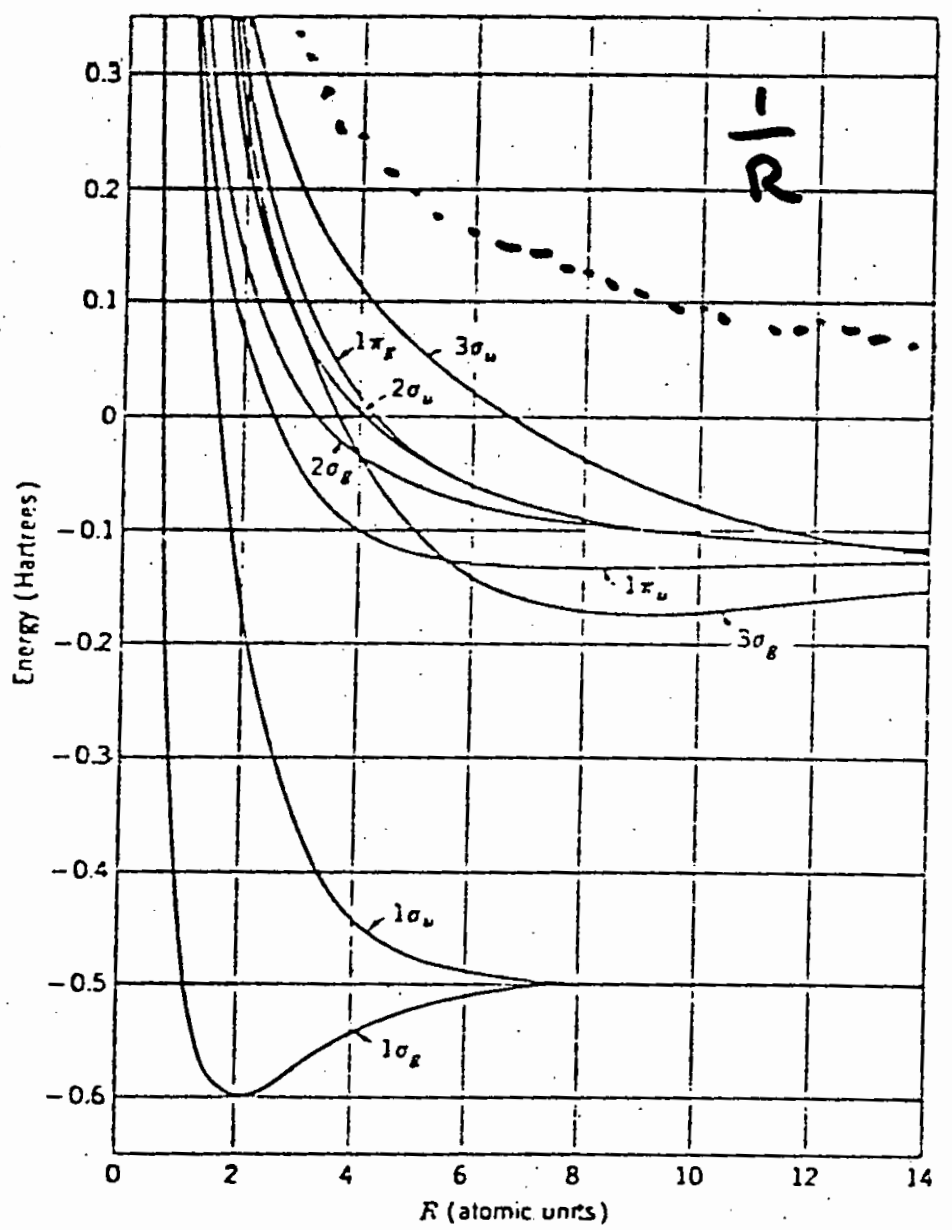

Fig. 19-5. Lowrst cherge levels of $\mathrm{H}^{-}$as function of in:e?nuriegr disisnre, iscluding internurles: repulsive enerar. 
170

3

On 2uranguna:

Enougy

$$
E\left(R_{0}, \tau\right)=T\left(R_{0}, \tau\right)+C(R)
$$

culare $C(R)=$ extraws and at R.

$\therefore$ Astuen captare ints grosingstale

$$
1 s \sigma_{g} \text { of } H_{2} t \text {. }
$$

if $\in\left(R_{0} \tau\right)<0 \quad H_{2}^{+}$boumed in

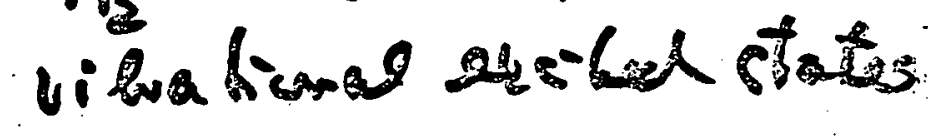

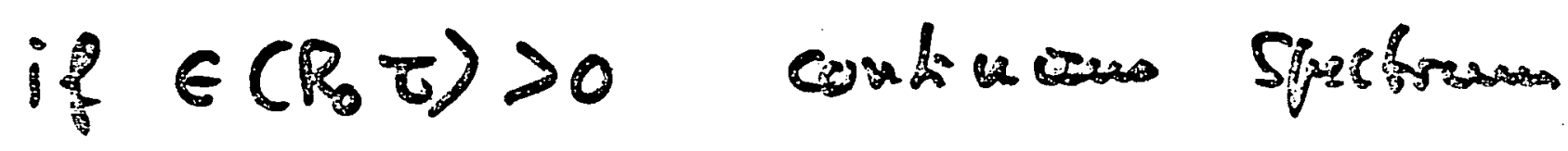

$$
\mathrm{H}_{2}^{+2} \text { Qreangs infs } \mathrm{H}^{*}+4^{\circ}
$$

D.

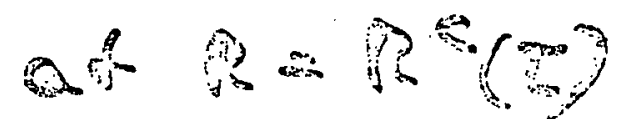




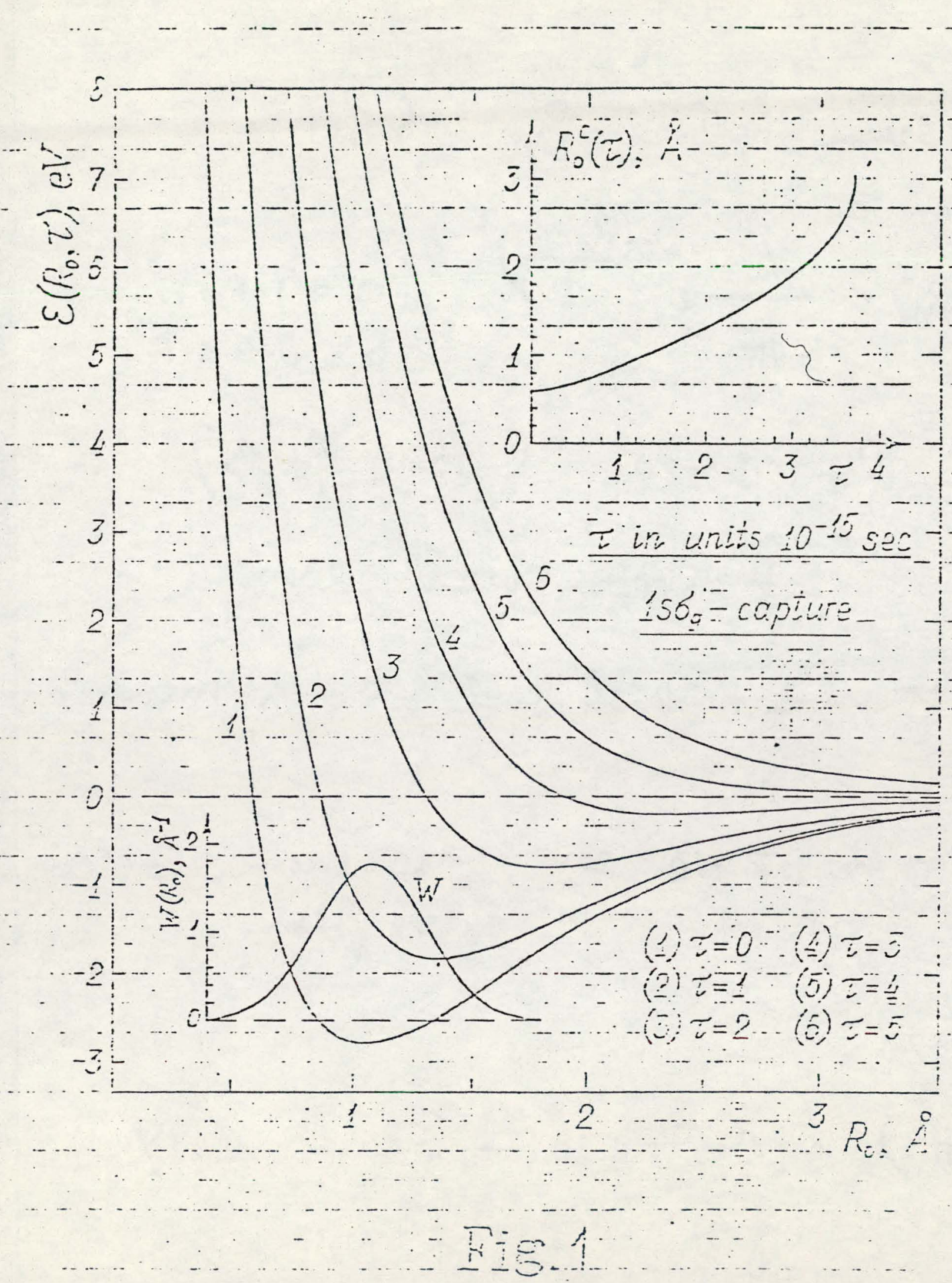


172

Asscever inition $R_{0}$ distination

$$
\text { WV }\left(R_{0}\right)=\frac{1}{b \sqrt{\pi}} e^{-\left(\frac{B_{0}-a}{b}\right)^{2}} \quad \begin{aligned}
a & =1.080^{\circ} \\
b & =0.32 A^{\circ}
\end{aligned}
$$

inar for $\tau \rightarrow 0$ (tiviv Peres)

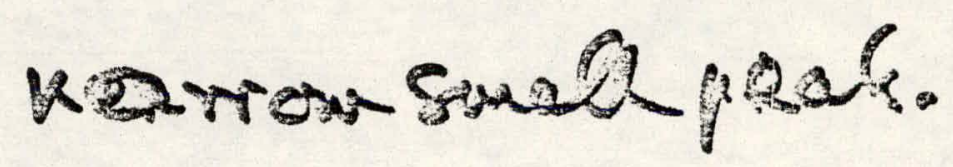

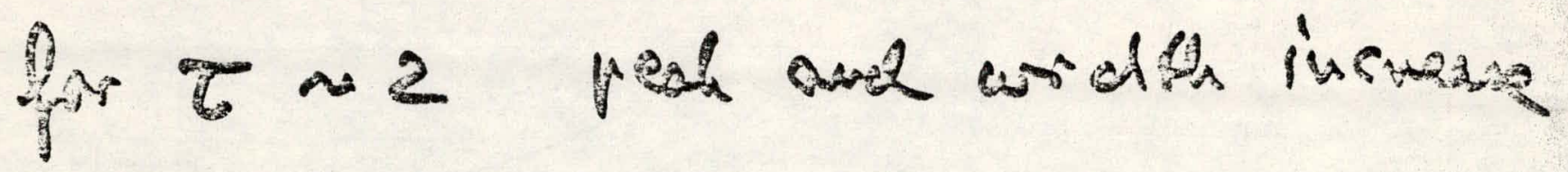

Ger $\tau \propto 3.7 \cdot 10^{-15} \mathrm{~s}$. mo ware $\epsilon=0$. ungar.

in 2028

$y(H 0) \sim 0.5$ at sobar (nyy)

a $10^{-29}$ of $1.22895(28902)$ 


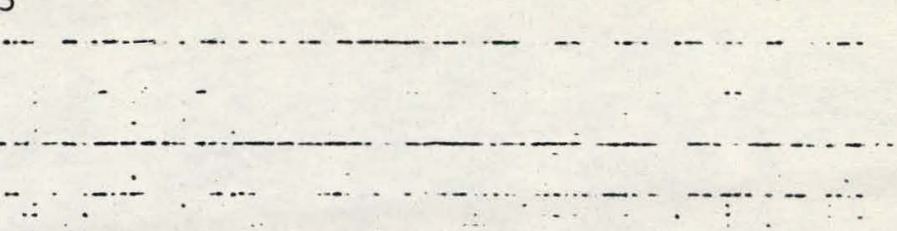

-

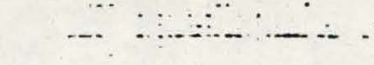

$-\cdots$

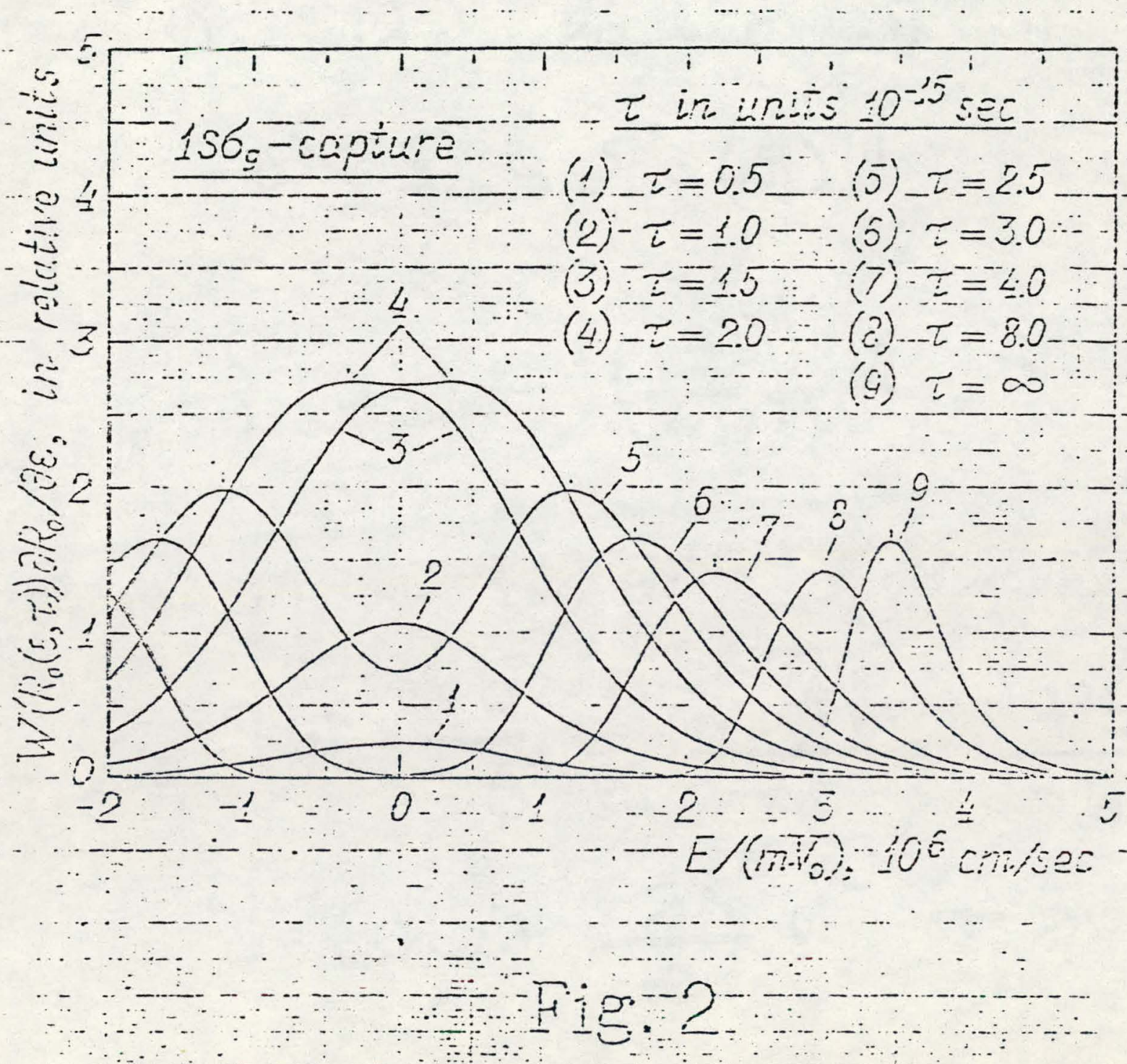


174

Distribution in angle.

iv $R_{0}$ andonizablian $\theta$ li $\left(R_{0}\right) d R_{0} \alpha \Omega / 4 \pi$

Nex

$$
E=M\left\{\left(\frac{E}{Y V_{\theta}}\right)^{3}+\left(U_{0} \theta\right)^{2}\right\} \quad x=\text { prod }
$$

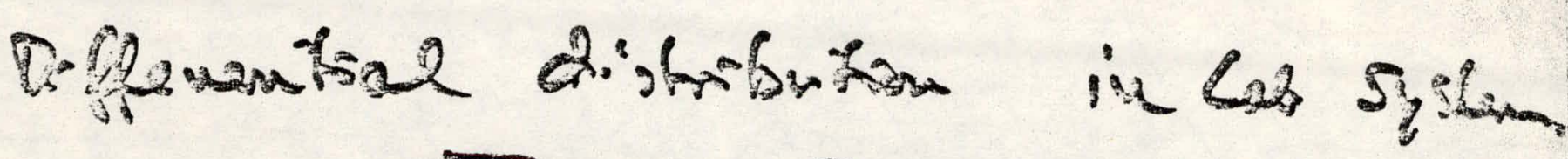

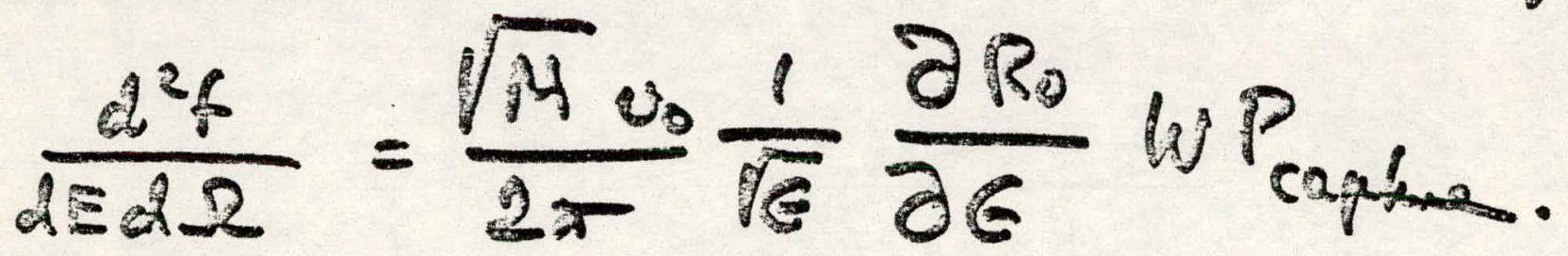
$a t s \rightarrow$ b) $\frac{\partial R}{\partial G}$ finile

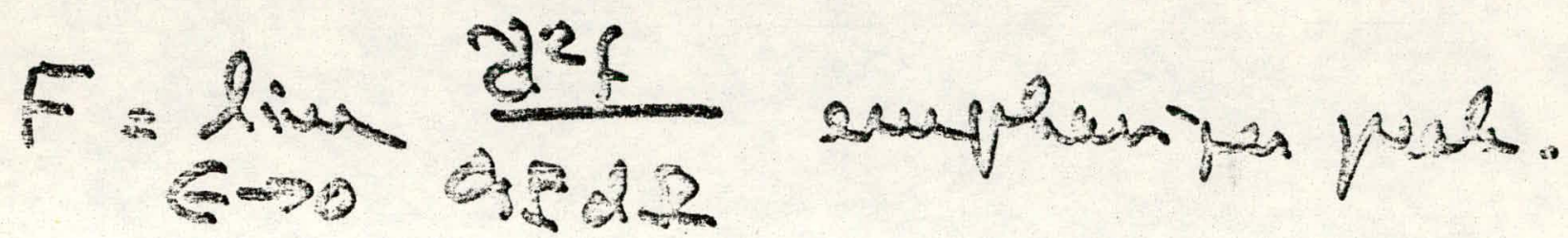




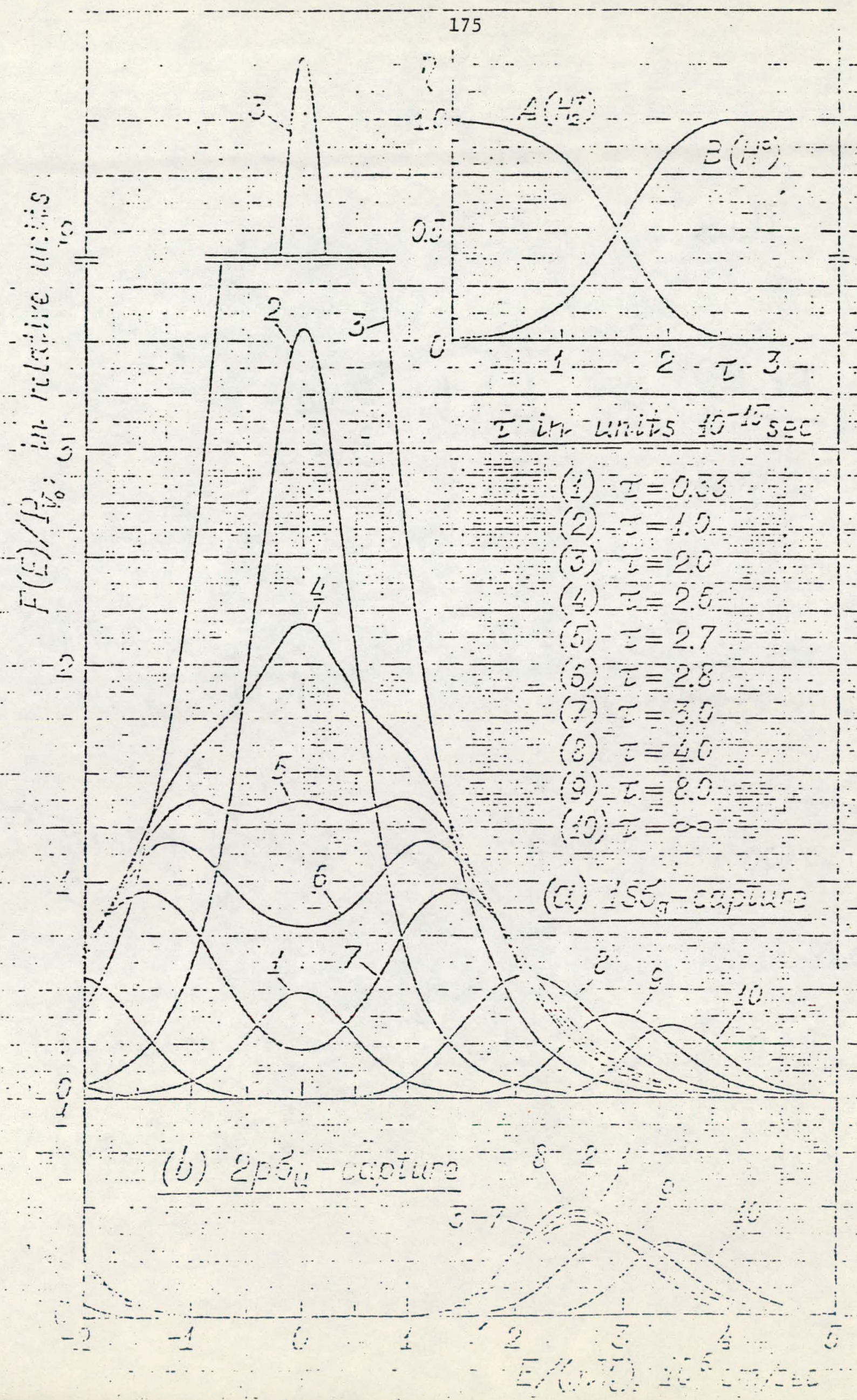


176

Thens

- $\frac{F(E)}{P_{\text {cagt. }}} \sim \frac{1}{\sqrt{E}} W \frac{\partial P_{0}}{\partial t}$

suuphessi ias pesh.

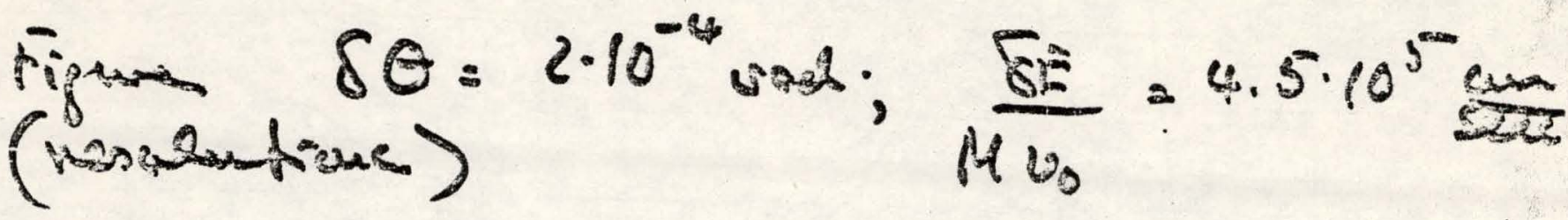

Gores

Growth $\tau=0$ b $\tau_{\text {mas }}=1.8 \cdot 10^{-15} \mathrm{se}$

2230 resse

$$
F\left(\tau_{\text {mex }}\right) \sim 20 F(\infty) \text { ! }
$$

Guxhel pash dinagigas of $5 \times 3 \cdot 10^{-15}$ e.

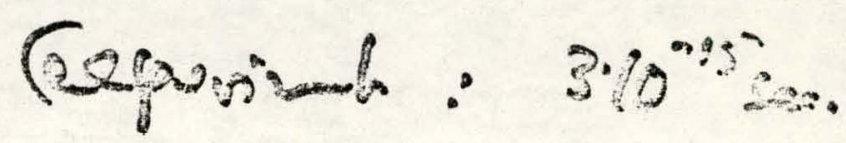


177

Cenclurions on $\mathrm{H}_{2}{ }^{\top}$

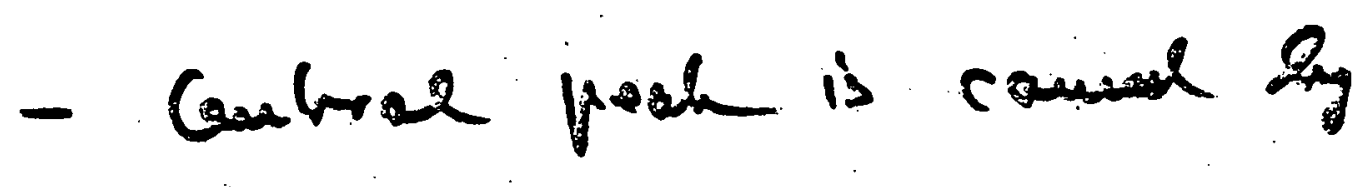
post gore captre

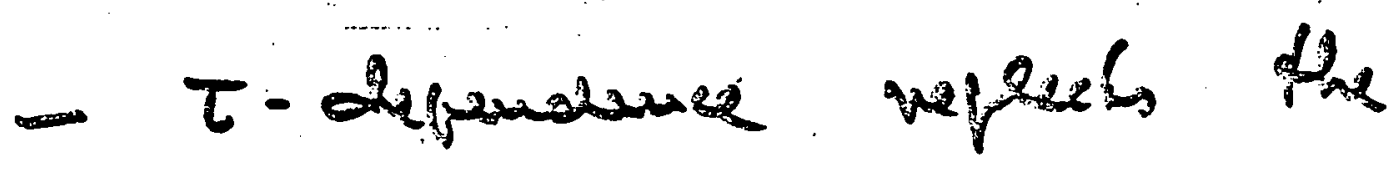
Poliy of W(Ro) wis aR/Oso

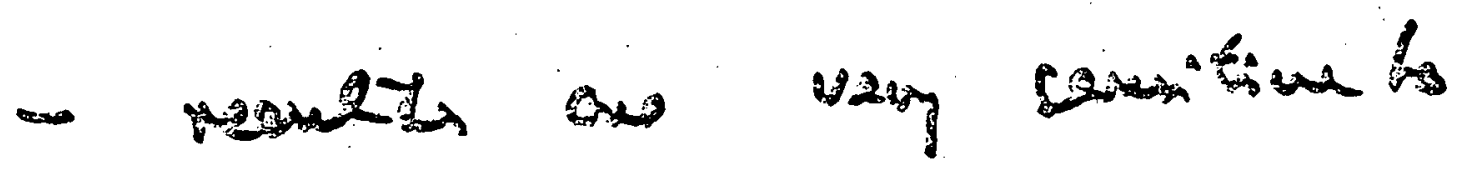

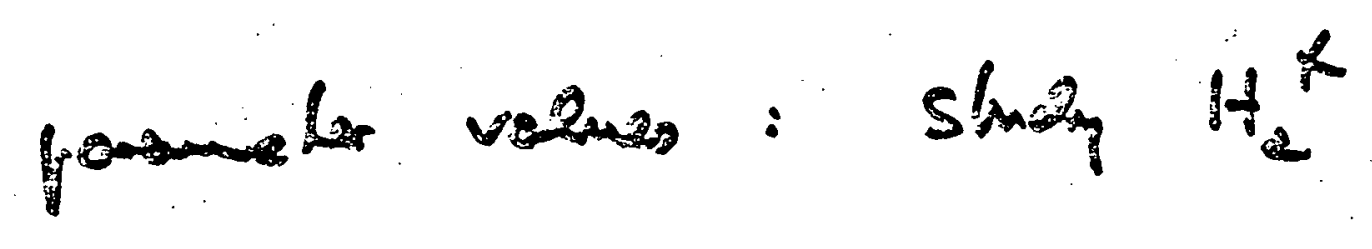
$D_{2}^{*}$ and $\tau=2 / 2$.

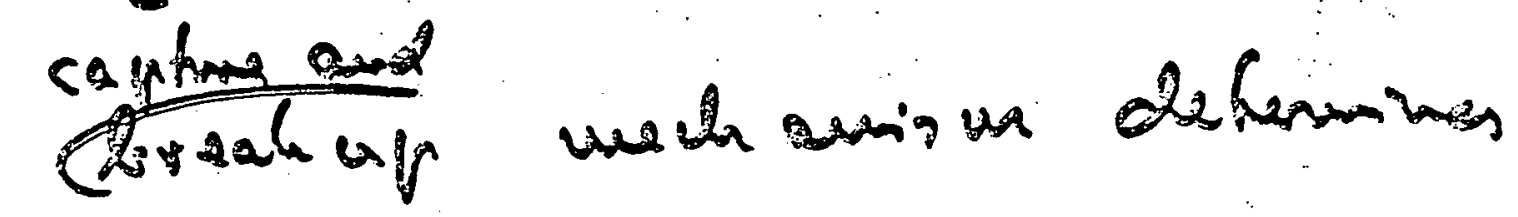

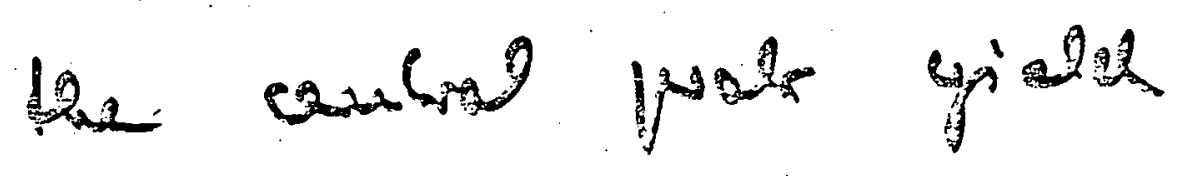

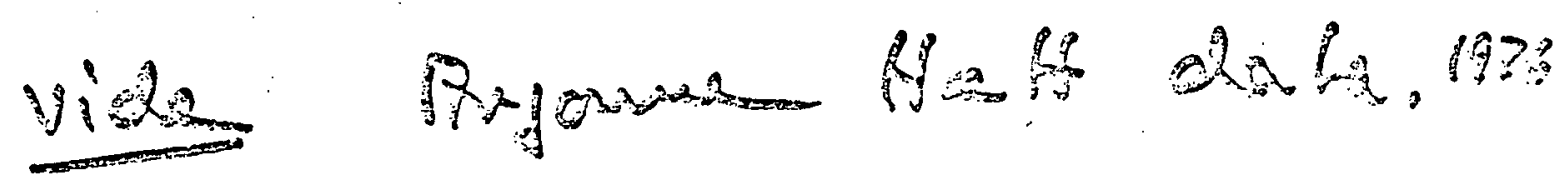




\section{THIS PAGE}

\section{WAS INTENTIONALLY LEFT BLANK}




\title{
Probing the Wake of a Swift Ion in Matter
}

\author{
W. J. Pietsch
}

Erstes Physikalisches Institut, University of Cologne, Federal Republic of Germany and

G. Krösing and G. Kumbartzki

Institut fur Strahlen- und Kernphystk, University of Bonn, Federal Republic

\section{of Germany}

and

D. S. Gemmell

Physics Division, Argonne National Laboratory, Argonne, I11inois 60439, U.S.A. . 
The concept of a polarization "wake" induced by a fast charged particle traversing a solid was introduced by $N$. Bohr ${ }^{1}$ in 1948 . Since that time the concept has been discussed and developed by other authors, $2-8$. most notably by R. H. Ritchie and coworkers. The wake represents the collective response of the electrons in the solid to the passage of the projectile. Theoretical treatments characterize the solid as an electron gas and the wake consists of electron density oscillations that trail behind the charged projectile. The wavelength of the wake is the distance moved by the projectile in one plasma oscillation period:

$$
\lambda=2 \pi v / \omega_{p}
$$

where $v$ is the projectile velocity (here assumed to be non-relativistic, but large compared with the Fermi velocity of the electron gas), and $\omega_{p}$ is the volume plasma frequency. The wake concept provides an interesting alternative to the more usual descriptions of stopping power (the net attractive force between the projectile and the electrons in its wake acts as a brake on the projectile).

Wake effects have recently achieved considerable attention as a result of suggestions that they play a vital role in the interactions of fast molecular ions with solid targets. $4,6,7,9-15$ Thus, for example, it has been postulated ${ }^{4,6}$ that "wake-riding" (in which a second particle becomes trapped in a potential minimun of the wake of a projectile leading it) may account for the phenomenon of transmission of molecular ions through foils. 16 
Similarly, it has been predicted ${ }^{17}$ that target electrons may be trapped into wakebound states trailing a fast ion traversing a solid, and that these ("convoy") electrons may be the main component of the characteristic cusp-shaped velocity distribution ${ }^{18}$ of the electrons emerging from the solid (the distribution exhibits a peak at the beam velocity). It must be admitted that the experimental evidence for wake effects playing an important role in the above two phenomena is far from definitive (see, for example, references 6, 11, 13, 15, 19 and 20). Perhaps the most convincing experimental evidence for wake effects lies in the results of high-resolution measurements of the energy and angle distributions for dissoclation fragments produced when fast molecular-ion beams strike thin foil targets. $7,10,14,15,21$ A wake model ${ }^{7}$ has been successful in accounting quantitatively for these distributions which exhibit asymetries attributable to the influence of wake forces. Even here, however, the wake potential is tested only over a distance of one or two Angstroms (the internuclear separations of the fragments while they are inside the foil), whereas the wake wavelengths are calculated to be typically several tens of Angstroms. Using a very thick foil target (to increase the inter-fragment separation achieved in the foil) is precluded because of the blurring effects of multiple scattering. Multiple scattering can be greatly reduced by channeling the incident molecular ions in a monocrystaline target foil. 10 However the added complication of channeled trajectories makes the analysis of such measurements difficult for thick targets. 
With the above considerations in mind we have performed the experiment reported here, designed to demonstrate the reality of a wake potential existing over large distances (several wake wavelengths).

The measurement employed a beam of $28-\mathrm{MeV} \mathrm{H}_{2}{ }^{+}$lons fiom the University of Bonn cyclotron. Figure $1 \cdots$ shows the experimental arrangement schematically. The beam spot at the target position was $0.2 \mathrm{~mm}$ diam. The angular divergence of the beam was 1 ess than $\pm 0.1 \mathrm{mrad}$. and its energy Instability was less than $\pm 2 \mathrm{keV}$. The target consisted of two thin foils stretched flat, parallel to one another, and mounted perpendicular to the bean direction (Figs. 2 and 3). The separation between the foils was adjustable (with a plezoelectric element) in fine steps from about a micron up to several tens of microns. Protons emerging from the target and lying within $\pm 0.1 \mathrm{mrad}$ of the beam direction were momentum analyzed with a magnetic spectrograph having a relative resolution of $3.4 \times 10^{-5}$.

An example of a proton energy spectrum obtained with this arrangement (but with only one target foil) is shown in Fig. 4. The two. peaks in the spectrum correspond to trailing (low-energy peak) and leading (high-energy peak) protons arising from incident molecular ions whose internuclear axes 1 ie close to the incident beam direction. (These molecular orientations are selected by the stringent angular collimation in the experimental arrangement.). The slight asymetry of the energy spectrum-there are more counts in the low-energy than in the high-energy peak- is attributable to the influence of wake forces $7,10,14,15$ which for small internuclear separations ( $\sim 1 \AA$ ), tend to pull the trailing protons in towards the beam direction. For internuclear separations 1 ying between $\lambda / 2$ and $\lambda$ (where $\lambda$ is 
the wake wavelength), one would expect this asymmetry to reverse, since the wake forces would then tend to push trailing protons away from the beam direction.

The double-foil target arrangement permits exploration of the wake force over several wavelengths. The first foil encountered by a beam projectile is $4-\mu g / \mathrm{cm}^{2}$ ( $200-\AA$ thick) carbun. This foil serves to remove the binding electron from each $\mathrm{H}_{2}{ }^{+}$ion and thus to inftiate the "Coulomb explosion" of the resultant two protons. In the short dwell time $\left(4 \times 10^{-16}\right.$ sec. $)$ in this first foil, the net effect of wake forces is negligibly small. In the time it takes the two protons to traverse the gap between foils, their separation increases from an initial value $r_{0}$ to a value $r$ given by

$$
r=(2 d / v)\left(e^{2} / M_{0}\right)^{172}
$$

where $d$ is the gap width and $M$ is the proton mass. [The initial COM velocities of the protons due to molecular vibrations and rotations are small compared with the COM velocity acquired in the Coulomb explosion and are neglected here. Equation (2) is based on the assumption that $d / v$ is large compared with the characteristic time $\left(210^{-15} \mathrm{sec}.\right)$ for the Coulomb explosion to develop.] The second foil is much thicker ( 1000 to 10000 \&) and is chosen to be of a material (e.g. Be, Al, etc.) with low atomic number (to reduce multiple scattering). and with a well defined volume plasma oscillation (to maximize wake effects over several oscillation periods). Since the experimental arrangement selects 
only those incident $\mathrm{H}_{2}^{+}$ions whose interproton vectors lie close to the beam direction, the phase with which a trailing proton encounters the wake generated in the second foil by its partner preceding it is given by

$$
\phi=2 \pi r / \lambda
$$

Combining Eqs. (1)-(3), we see that varying the gap width by

$$
d_{\lambda}=\left(\pi v^{2} / \omega_{p}\right)\left(M r_{0} / e^{2}\right)^{1 / 2}
$$

moves this phase through $2 \pi$.

Figure 5 shows how the asymetry in population of the two peaks in the proton energy spectrum changes as the gap between the foils is varied. The solid line is a fit to the data in the form of an exponentially decaying sine wave. If we identify the wavelength of this sine wave with the value $\mathrm{d}_{\lambda}$ in Eq. (4), we find $\mathrm{d}_{\lambda}=11.0 \pm 0.1$ microns. Other measurements ${ }^{21}$ have shown the most probable initial internuclear separation to be $r_{0}=1.17 \AA$. Using these values: in Eq. (4), we derive a value for the volume plasmon energy in aluminum of $f i \omega_{p}=14.7 \pm 0.2 \mathrm{eV}$ in agreement with the value $\hbar_{\mathrm{p}}=14.92 \pm 0.06 \mathrm{eV}$ determined from electron energy-loss measurements. 22 The fitted curve in Fig. 5 gives a decay to $1 /$ e after about 3 oscillations. The corresponding value in the electron energy-loss experiments is 6 oscillations. 22 (The faster decay seen here is probably due to the fact that the range of values of $\dot{\mathrm{r}}_{0}$ in the incident projectiles tends to increase the rate of decay of the asymmetry in in proton energy spectra.) 
185

The measurements reported here although not yet conclusive, constitute the first experimental indication that wakes actually do persist behind fast ions traversing solids. These results can be expected to lend added credence to the many suggestions that have been made concerning the role of wakes in ion-solid interactions.

We wish to thank G, E. Thomas, Jr. for his help in preparing the foil targets. One of us (D.S.G.) gratefully acknowledges the receipt of a NATO/Minna-James-Heineman award in support of the work reported here. This work was supported jointly by the Bundesministerium fur Forschung and Technologie, by the U. S. Department of Energy, Division of Basic Energy Sciences, and by the Minna-James-Heineman-Stiftung of Hannover. 


\section{References}

${ }^{1}$. Bohr, K. Dan. Vidensk. Selsk. Mat.-Fys. Medd. 18, 1 (1948).

2D. Pines, Phys. Rev. 92,626 (1953).

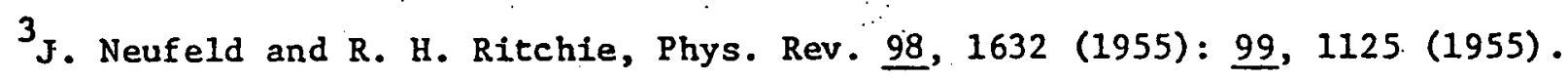

4v. N. Neelavathi, R. H. Ritchie, and W. Brandt, Phys. Rev. Lett. 33,302 (1974).

5. H. Day, Phys. Rev. B12, 514 (1975).

${ }^{6}$ W. Brandt and R.H. Ritchie, Nucl. Instrum. Methods 132, 43 (1976).

7Z. Vager and D. S. Gemmel1, Phys. Rev. Lett. 37, 1352 (1976).

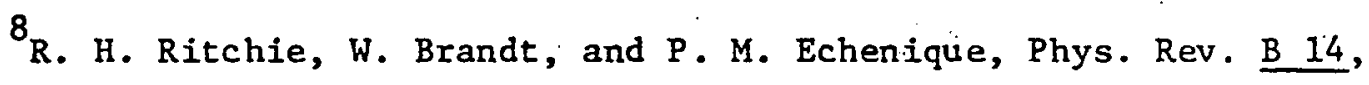
4804 (1976).

9. Brandt, A. Ratkowski and R. H. Ritchie, Phys. Rev. Lett. 33; 1325 (1974).

10D. S. Gemmell, J. Remillieux, J.-C. Poizat, M. J. Gaillard, R. E. Holland, and $Z$. Vager; Phys. Rev. Lett. 34, 1420 (1975).

${ }^{11} \mathrm{~J}$. Remillieux, from Proceedings of the Fifth International Congress of Radiation Research, Academic Press, New York (1975), p. 302.

${ }^{12}$ N. R. Arista and V. H. Ponce, J. Phys. C 8, L188 (1975).

13W. Brandt, R. Laubert, and A. Ratkowski, Nucl. Instrum. Methods 132,

57 (1976).

14, Vager, D. S. Gemmell, and B. J. Zabransky, Phys. Rev. A14, 638 (1976). 
15 D. S. Gemmell, P. J. Cooney, W. J. Pletsch, A. J. Ratkowski, Z. Vager, B. J. Zabransky, A. Faibis, G. Goldring and I. Levine, Proceedings of the 7th International Conference on Atomic Collisions in Solids, Moscow, September 19-23, 1977, to be published.

$16 \mathrm{~J} . \mathrm{C}$ : Polzar and J. Remillieux, Phys. Lett. 34A, 53 (1971).

${ }^{17}$ W. Brandt and R. H. Ritchie, Phys. Lett. 62A, 374 (1977).

${ }^{18}$ H. K. G. Harrison and M. W. Lucas, Phys. Lett. 33A, 142 (1970).

${ }^{19}$ W. Meckbach, N. Arista and W. Brandt, Phys. Lett. 65A, 113 (1978).

20 . Laubert, I. A. Sellin, C. R. Vane; M. Suter, S. B. Elston, G. D. Alton, and R. S. Thoe, Phys. Rev. Lett. 41,712 (1978).

${ }^{21}$ E. P. Kanter, P. J. Cooney, D. S. Gemmell, K:-0. Groeneveld, W. J. Pietsch, A. J. Ratkowski, Z. Vager and B. J. Zabransky, Phys. Rev. A (to be published). ${ }^{22}$ H. Raether, Solid State Excitation by Electrons, Springer Tracts in Modern Physics $\underline{38}, 84$ (1965). 
TARGET

SY1S

SX15

XY SLITS

$0,1 \times 0,1 \mathrm{~mm}$

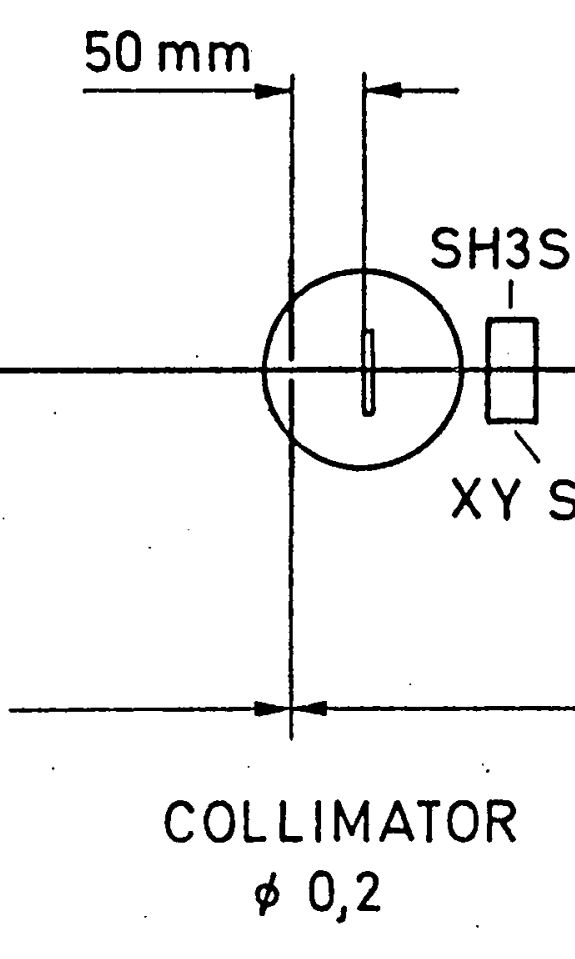

$\delta \theta \approx 0.1 \mathrm{mrad}$ 


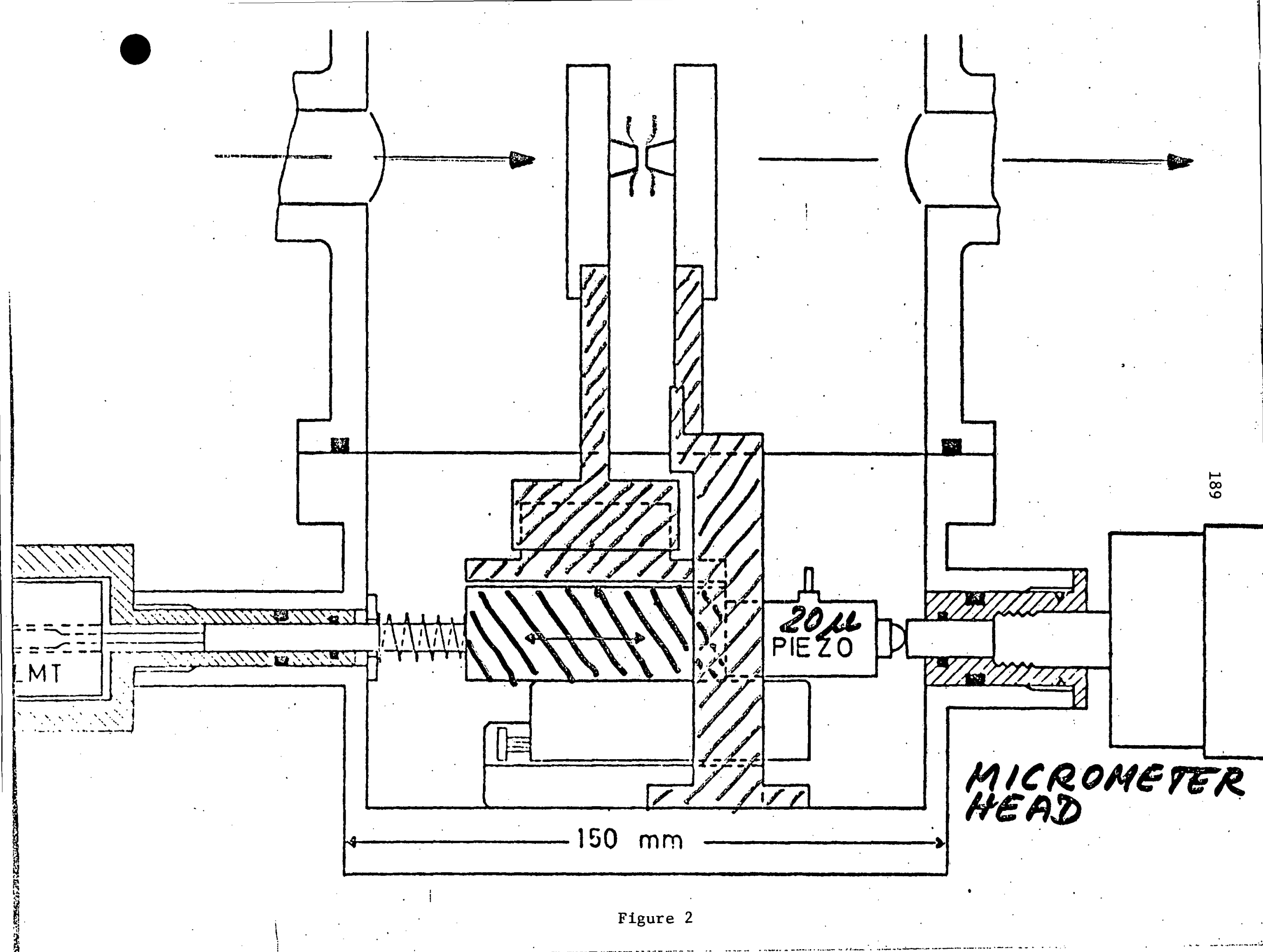




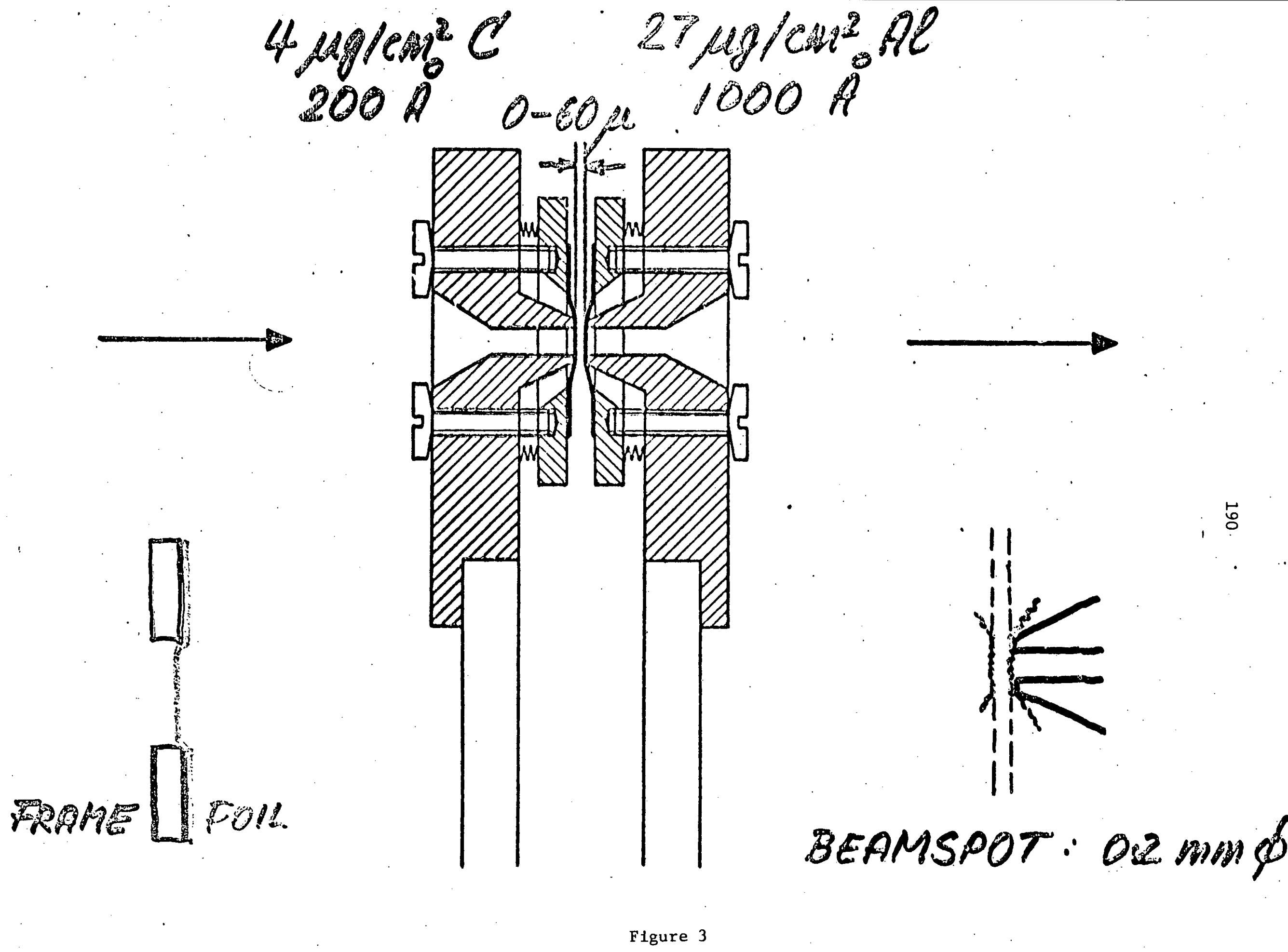




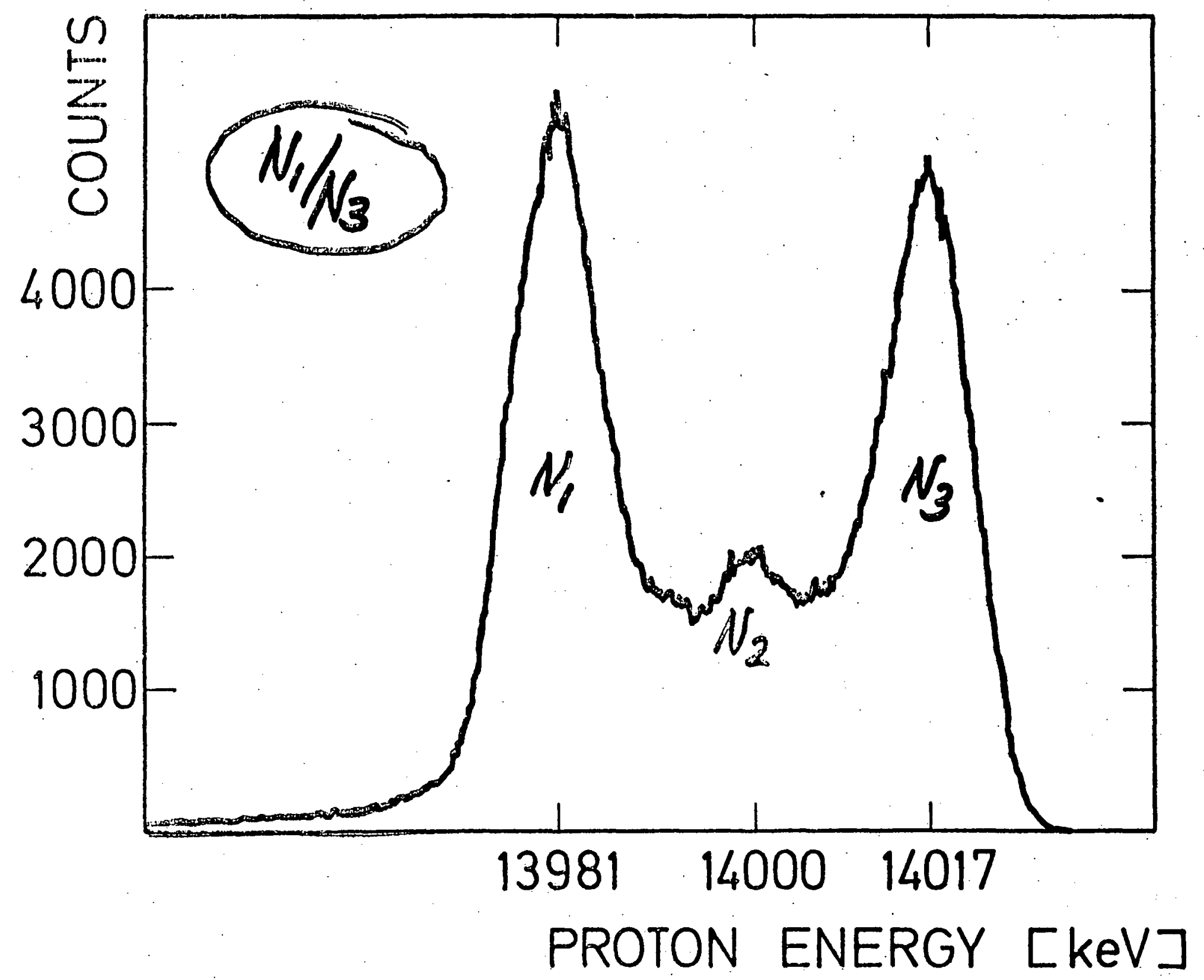


MORNING SESSION

21 August 1979

z. Vager, CHAIRMAN 
THE WRKE

W. Brandt

New York University, New York, N.Y. 10003

Electron-density fluctuations fores a wale.

$\therefore$ Discussion of wake structinta with increasring sophistication of descristion of dectrai vesyoure.

I. bake structive

I. The 2imit of RPA-tests of walke propartias

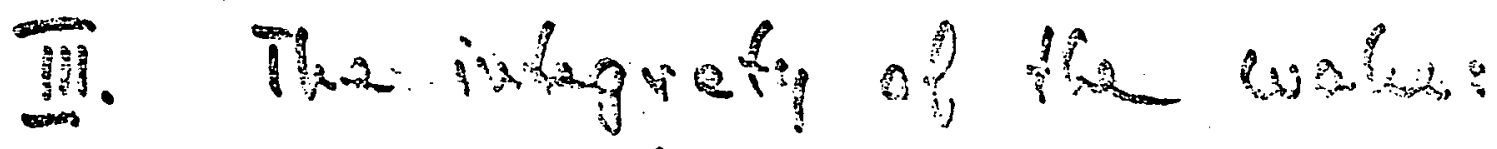
fenetratiens

193 
194

Scazar electrie wake poteucisal in $\rho, \tilde{j}$

$$
\begin{aligned}
& \Phi(\rho, \tilde{\zeta})=\frac{z_{e}}{\pi} \int_{0}^{\infty} x J_{0}(\rho x) d x \int_{-\infty}^{+\infty} d \omega e^{\frac{i \omega \tilde{\xi}}{v} / k^{2}} \in(k \omega) \\
& e=\left(x^{2}+y^{2}\right)^{1 / 3} ; \\
& \tilde{z}=z-u t ; \\
& k=\left(x^{2}+w^{2} / v^{2}\right)^{1 / 2}
\end{aligned}
$$

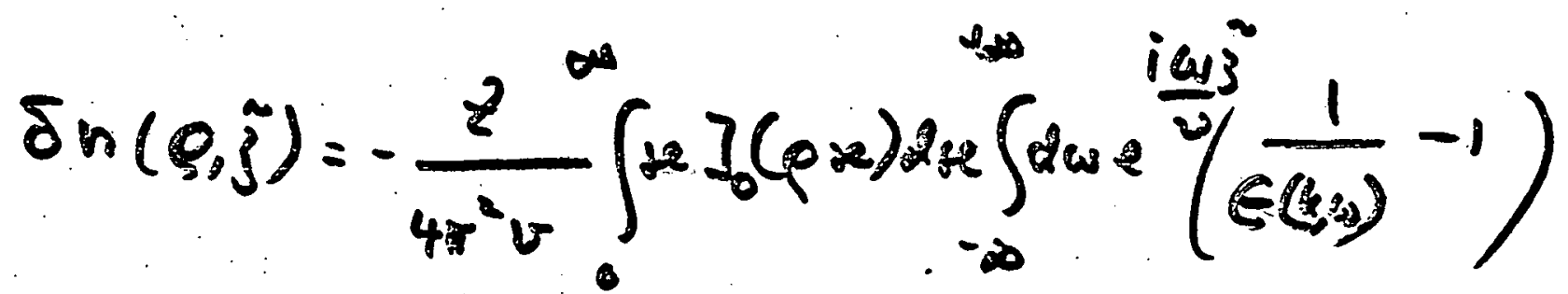

I. Local afpatimation

$$
\epsilon(k, \omega) \cdot 1-\frac{\omega_{p}^{2}}{\omega(\omega+i v)} \Theta\left(k_{c}-k\right)
$$

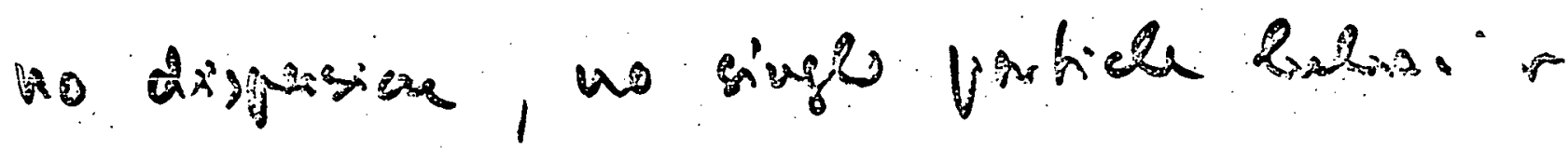


195

I local (cont'd $)$

$$
\begin{aligned}
& \Phi=z_{e} l(\rho, \tilde{j})+2 \frac{z_{e c o s}}{v} \sin \left(\frac{\omega_{s} \tilde{3}}{v}\right), l_{0}\left(\frac{\omega_{i} \rho}{v}, \frac{v}{v_{E}}\right) \\
& x e^{\frac{x 3^{2}}{22}} \theta(-\dot{\xi}) \\
& f(y)=\int_{0}^{y} \frac{y y_{0}(s, y)}{1+y^{2}} d y \text {. } \\
& \delta_{n}=-\frac{z \omega_{\mu}{ }^{2}}{2 \pi v v_{F} \rho} J_{1}\left(\frac{\omega_{\varphi} \varphi}{v}\right) \sin \left(\frac{\omega_{p} \tilde{z}}{v}\right) e^{\frac{\gamma \tilde{s}}{2 v}} \theta\left(-\xi^{2}\right) \\
& \lim _{R \rightarrow 0}\left(\phi-\frac{z_{0}}{R}\right)=-\frac{\pi Z_{e} \operatorname{ton}_{p}}{20}
\end{aligned}
$$

I. Plaswen dis pusiest

$$
\begin{aligned}
& \in\left(\xi_{1} \omega\right)=1+\frac{\omega_{p}^{2}}{\beta^{2} \beta^{2}-\omega(\omega+n)} \\
& \beta=\left(\frac{3}{5}\right)^{3}
\end{aligned}
$$


196

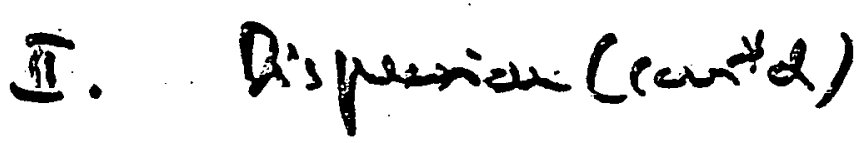

$$
\delta n=\frac{z \omega_{p}^{2}}{2 \pi \beta\left(v^{2}-\beta^{2}\right)^{4}} \frac{\cos \left(\omega_{p} \Lambda / \beta\right)}{\Lambda} \theta\left(\Lambda^{2}\right)
$$

III Singe patiob wate coutriantion

$$
\begin{aligned}
& E(k, \omega)=1+\frac{\omega_{\beta}^{2}}{\omega_{g}^{2}+\beta^{2} k^{2}+\frac{\hbar^{2} k_{k}^{4}}{k \omega^{2}}-\omega\left(\omega+i_{j}^{\prime}\right)} \\
& \Phi_{\omega}=\phi_{0}+\phi_{1}+\phi_{2} \\
& \phi_{0}=-\gamma_{e}\left[h(\rho, \tilde{\xi})-\frac{1}{R}\right] \\
& \text { uon assogisaty } \\
& \text { sireavy. } \\
& d=\text { osergation } \\
& \text { coses: fint }
\end{aligned}
$$

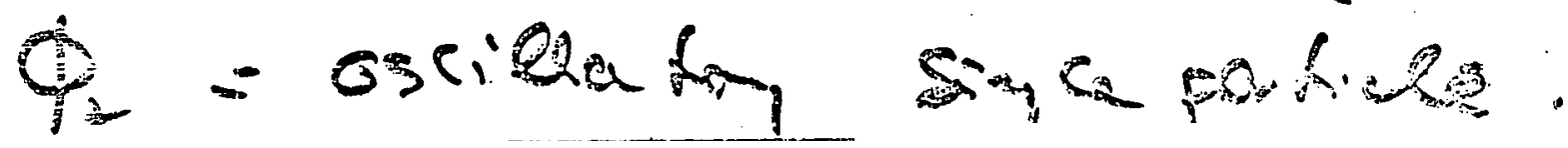




\section{ORNL DWG: 79-8395R2}

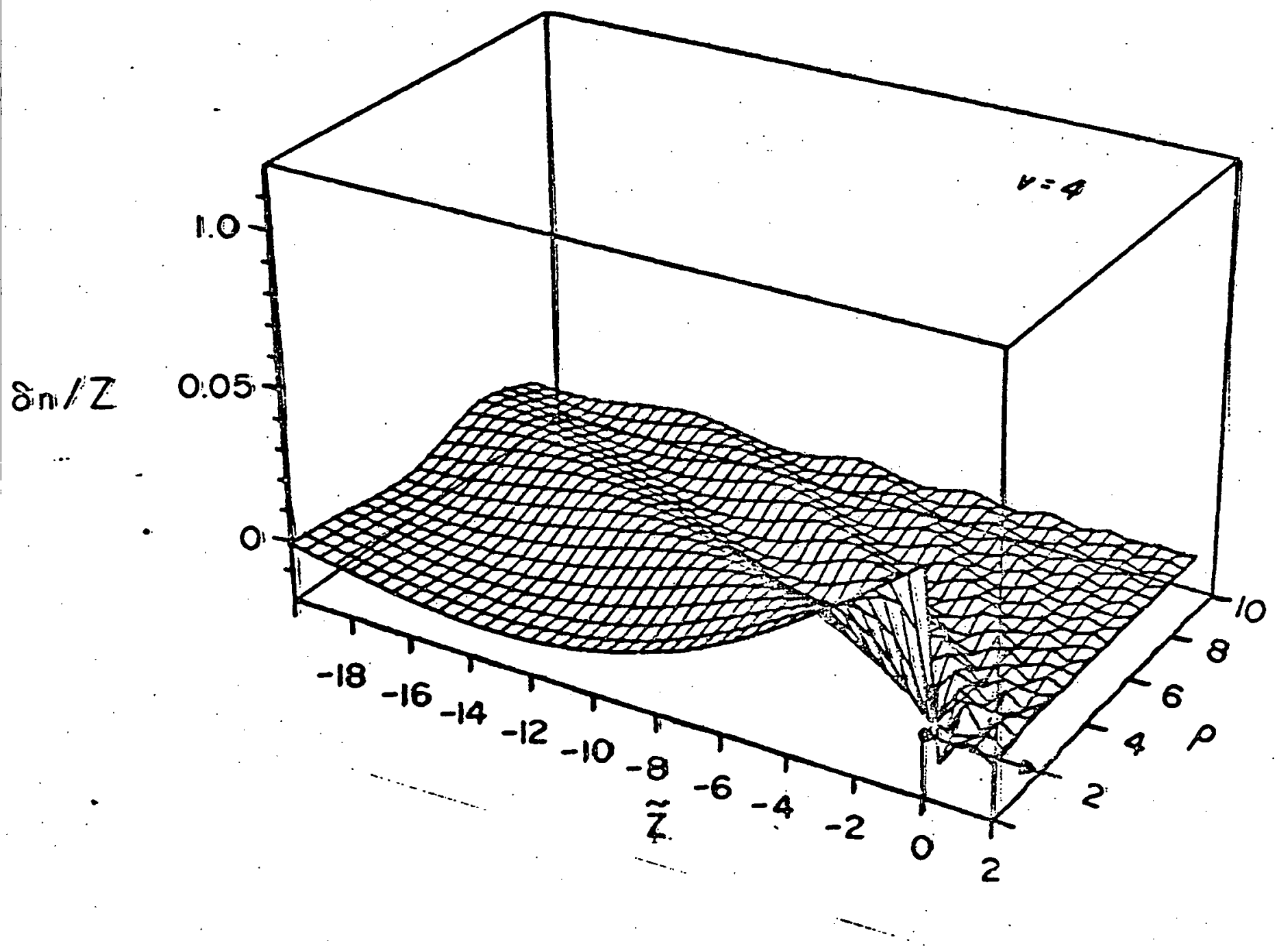


ORNL DWG 79-8394R2

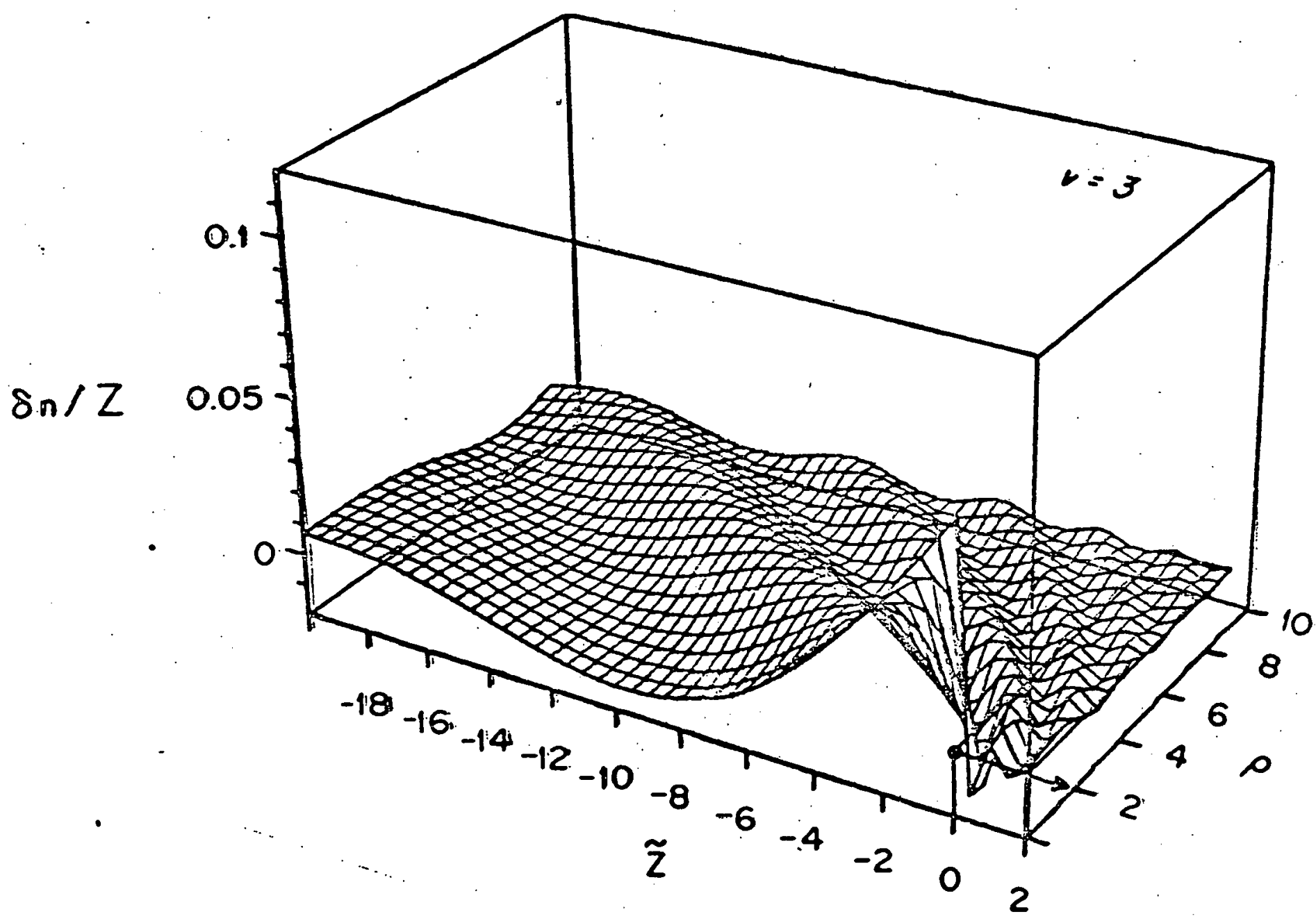


WAKE EFFECLI EXPLANATION TO BELL AND BETZ EXPERIMENT

P. M. Echenique

University of Barcelona, Spain 
Problems

(1) Destruction of the wake at the surface Sudden approximation(?) $\quad t_{D} \sim \frac{v}{\omega_{p}}$

Robles Break up of symmetry:'

$$
\varepsilon(\underline{q}, \omega)(?]
$$

(2) Ions

electrons : feet velocity dependent

image potentials.

Oscillations, Superposed. Maize detectable experimentally.

Asymmetry Pungy contrary to CTC

(3) Experiments. W. Pietsch.

Probes long distance from ion Dispersion of plasmon tied! 
201

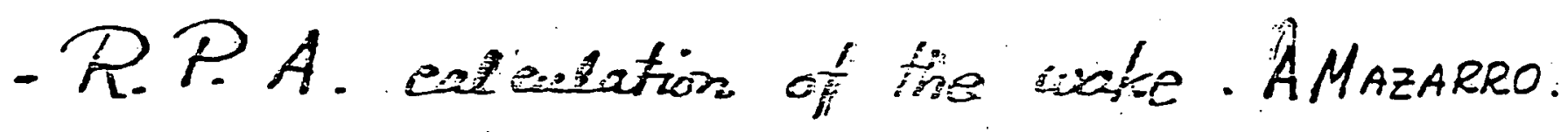

- Bell and Retre experiment. St-pery eneagies of multiplet

- Sigface

3. Transitions. J. Tejäda P. Pascual

P.P.A.

- Comporison with PP. ( Lundqaist)

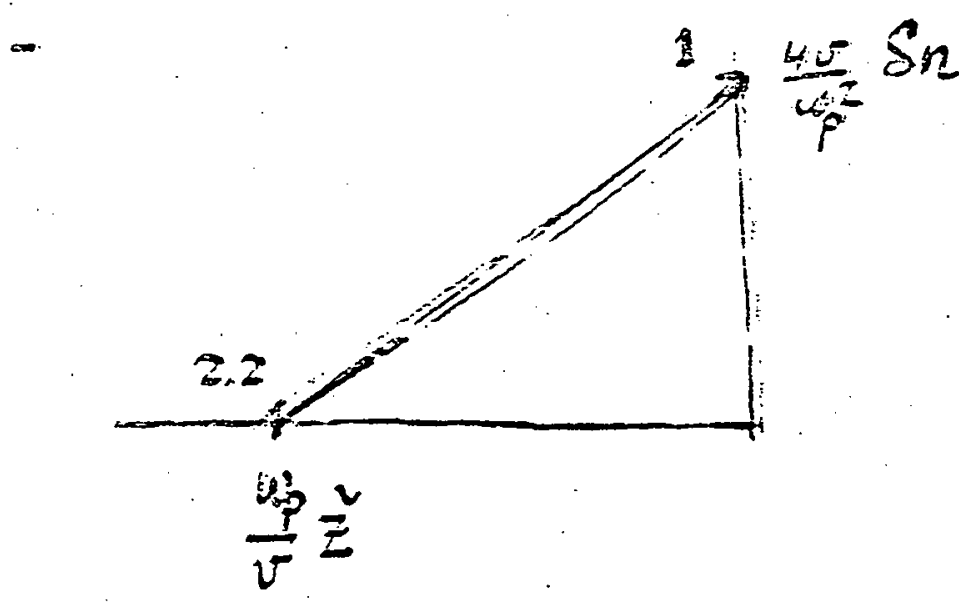

- or depontence!; Wakes at low irelueties. 


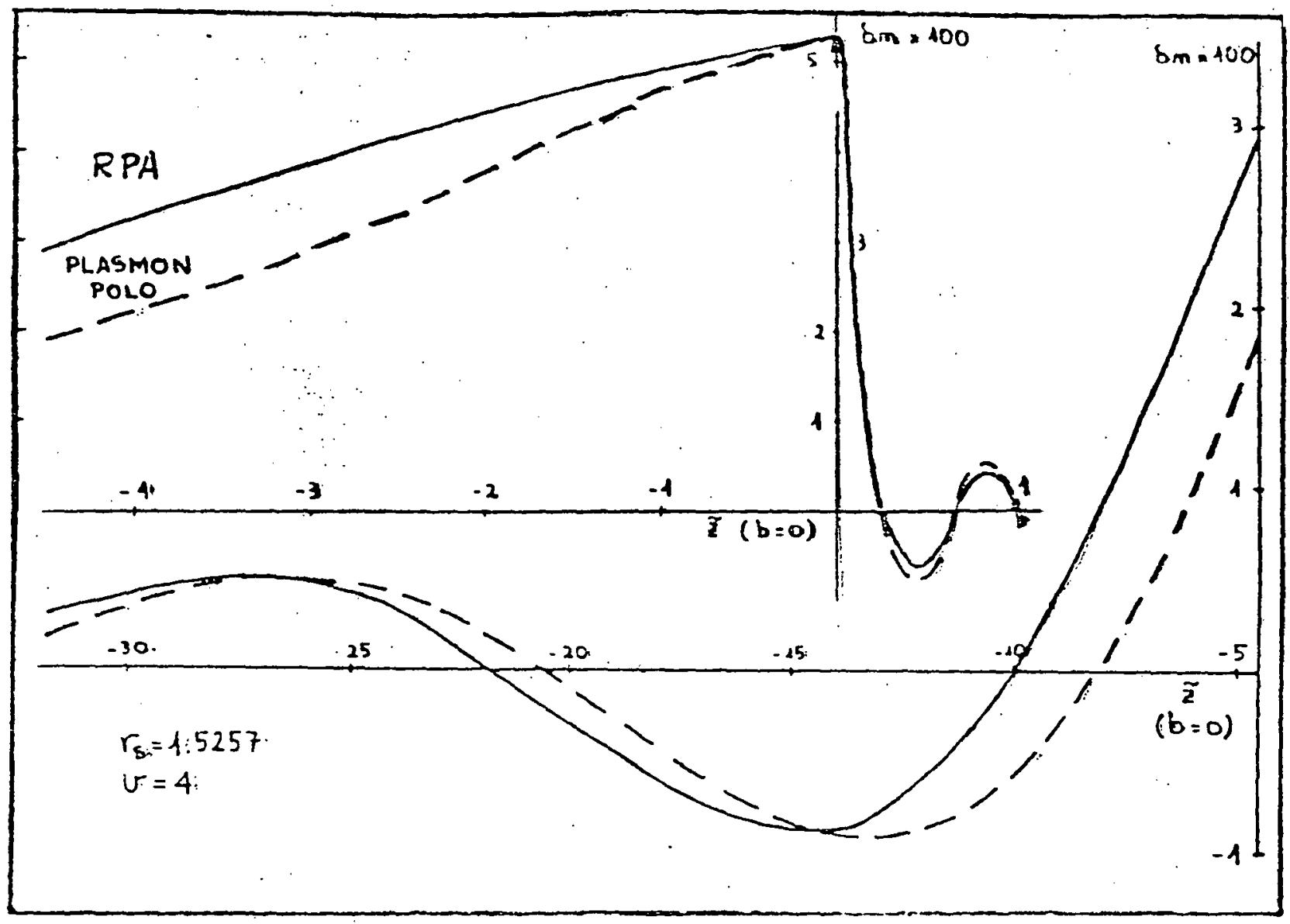

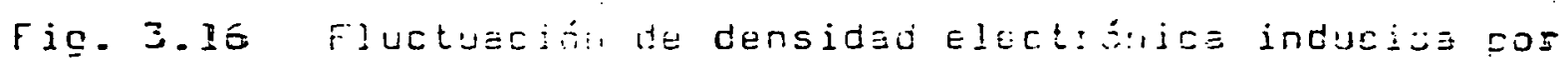

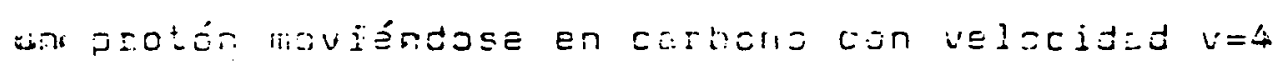

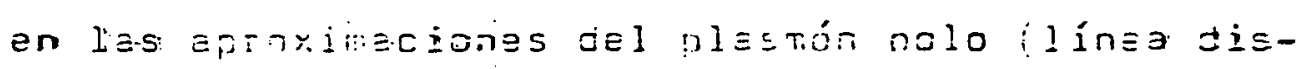

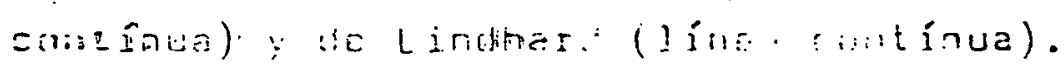




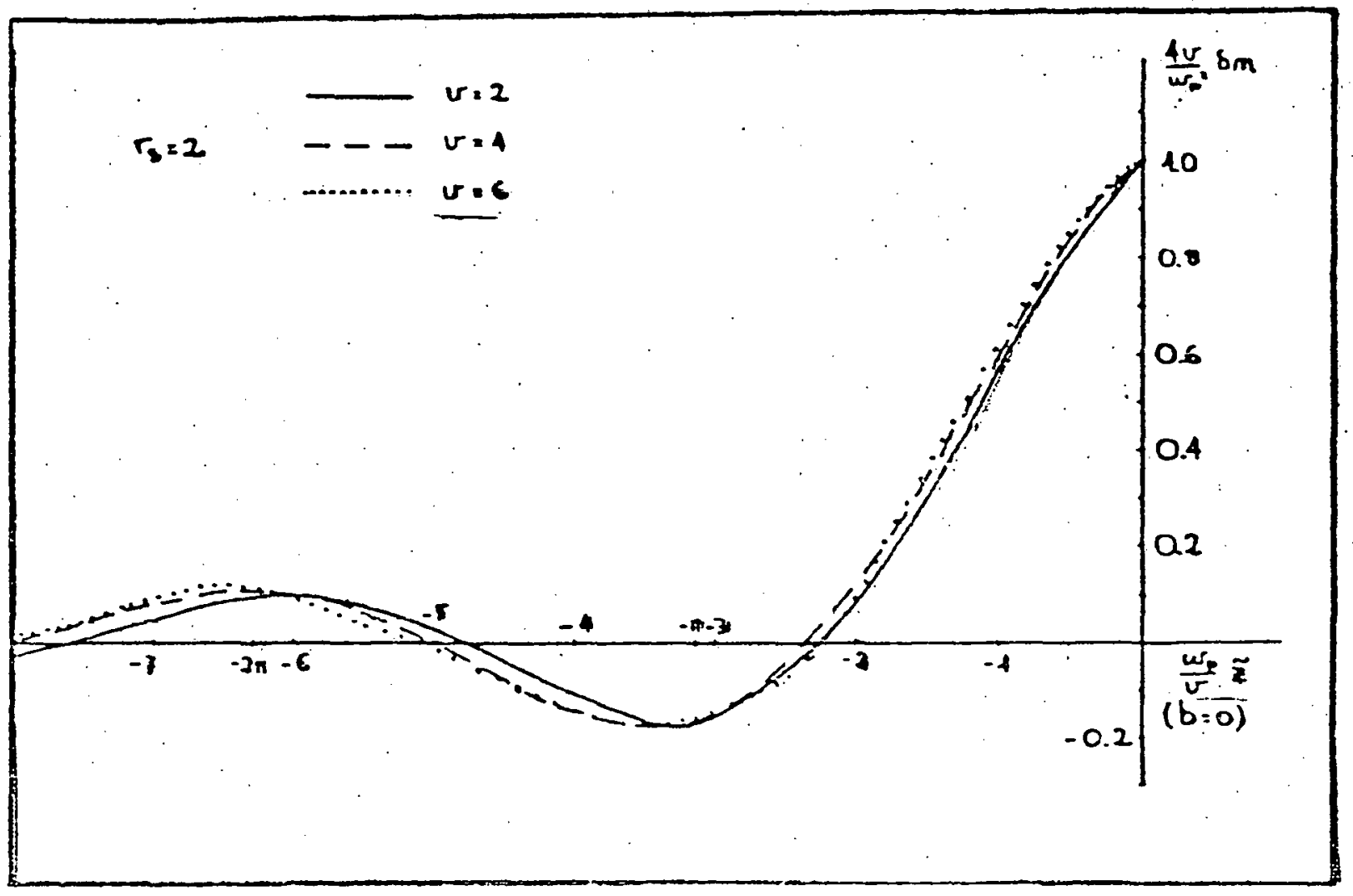

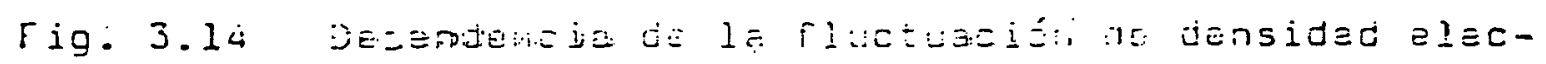

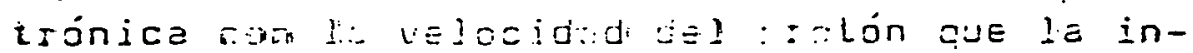

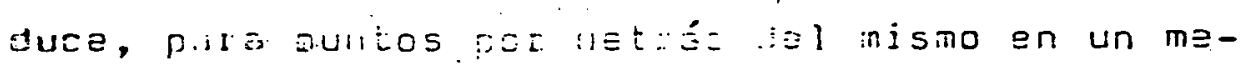

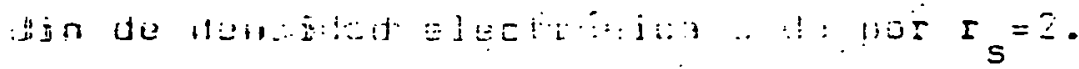


204

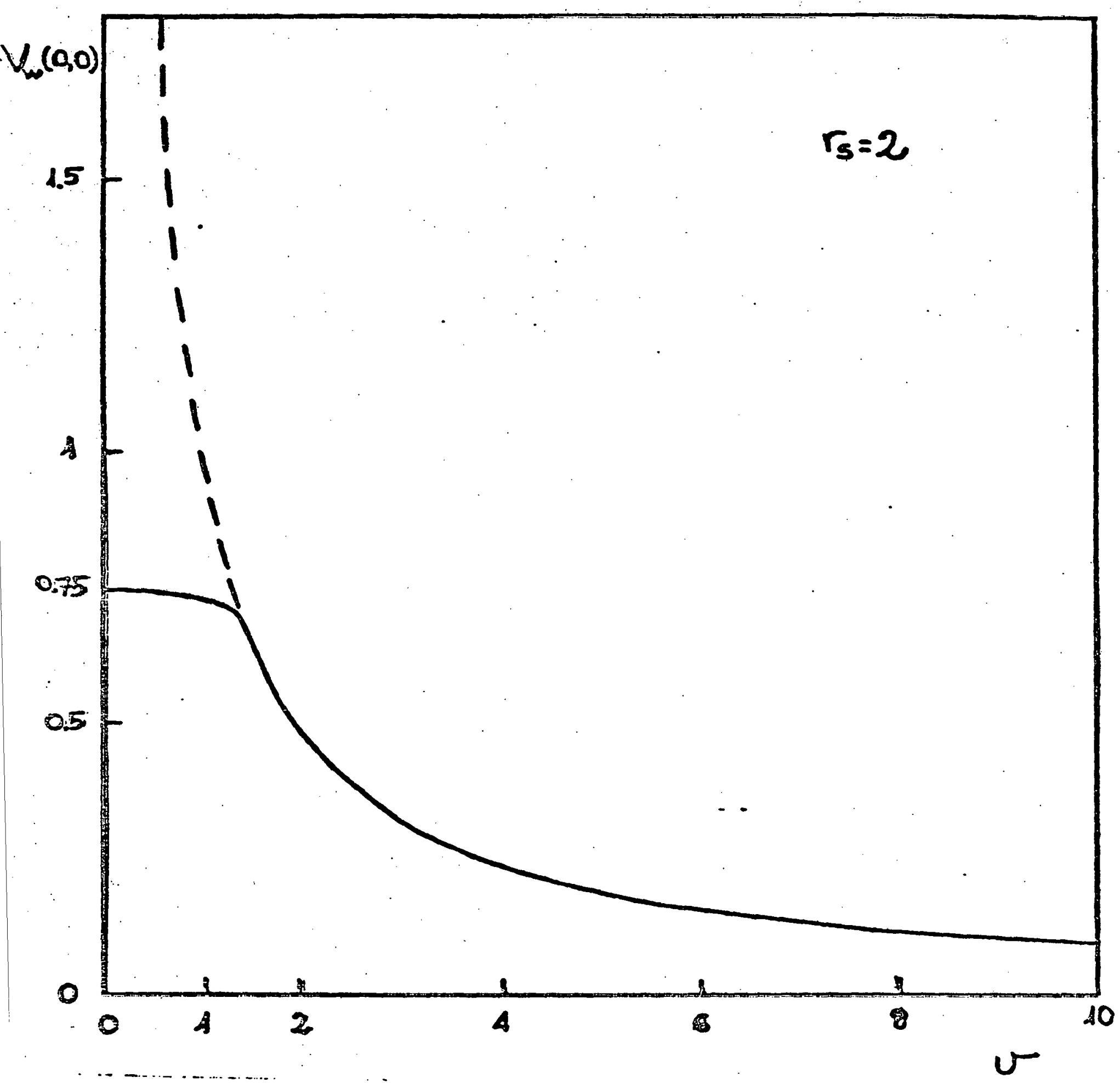


205
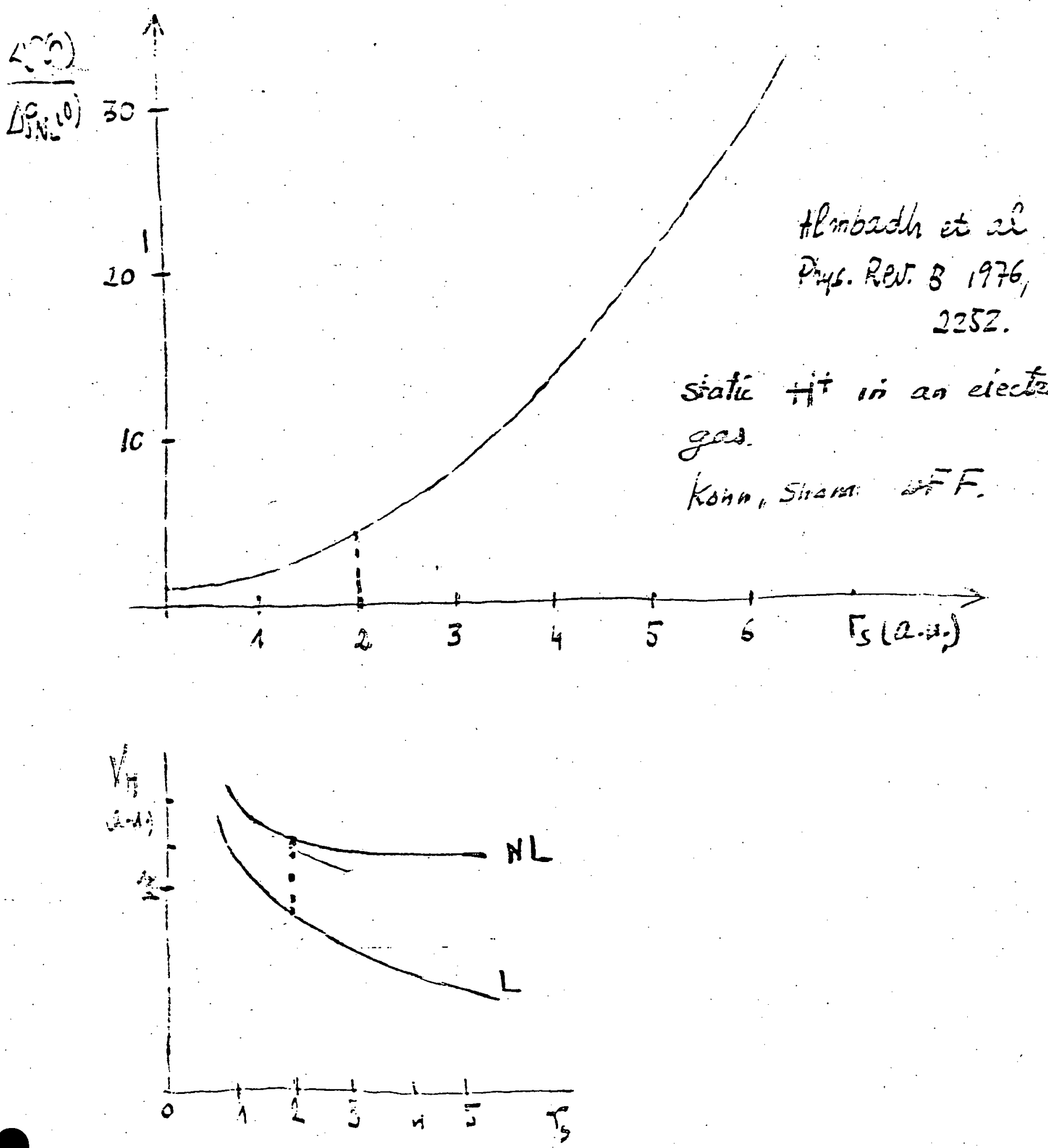
3.

206

The B.B. experiment

F. Bell, H-D Bet, H Panke and W staling J Phys BQ, 1976

$$
\text { pg } 1-443
$$

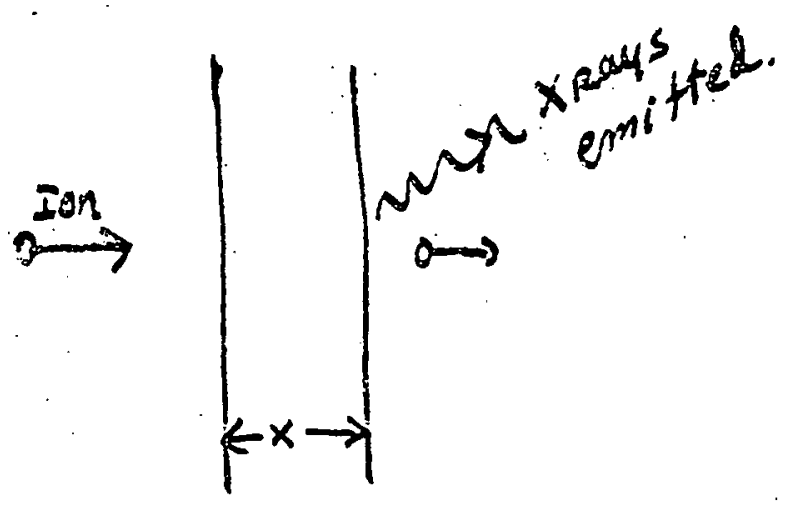

rigeatsare X -ray energies of musaplet transitions in helium -

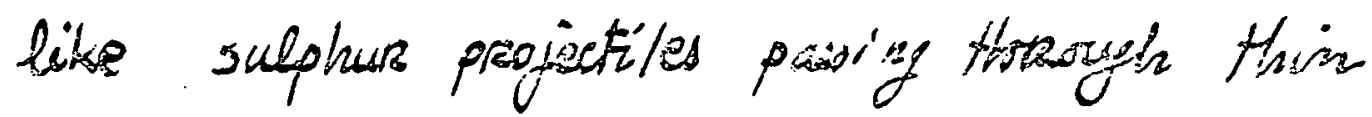
Aluminum fils

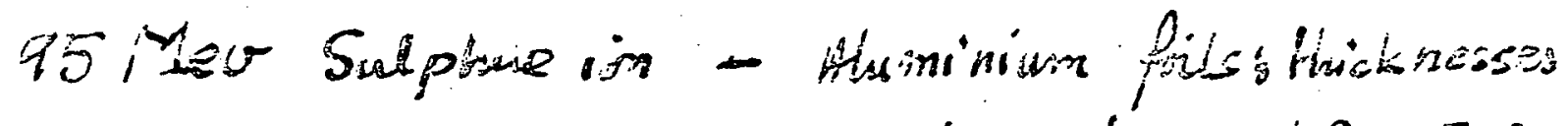

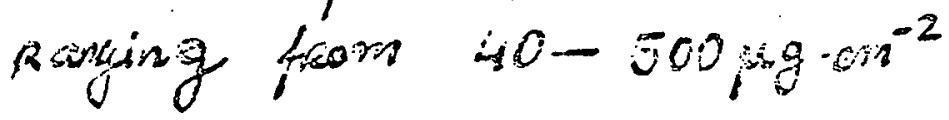

$D$
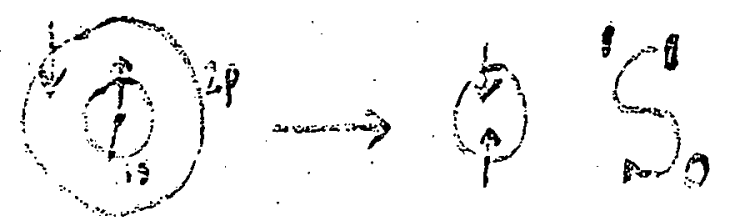

${ }^{3} P_{1}$ target thickness

Variation of $\frac{\downarrow}{x}$ affects the degree of dynamical iso screening for the ' $P_{1}$ and not for the ${ }^{3} P_{1}-' S_{0}$. 
207

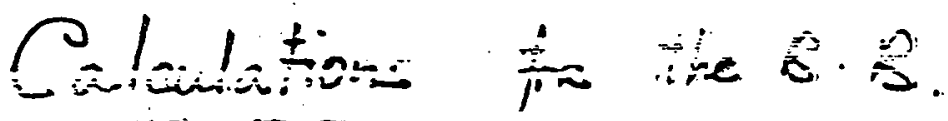

- Self -energy formalism

- Variationally determines 2-i wave functions

- Plasmon-pole approximation to treat the electron-gas.

$$
\Delta E_{1}=0.99 \mathrm{eV}
$$

Experiment gives $(1 \pm 0.2) \mathrm{eJ}$.

However : More experiments needed

Important both : density dependence

$\therefore$ velocity dependence 


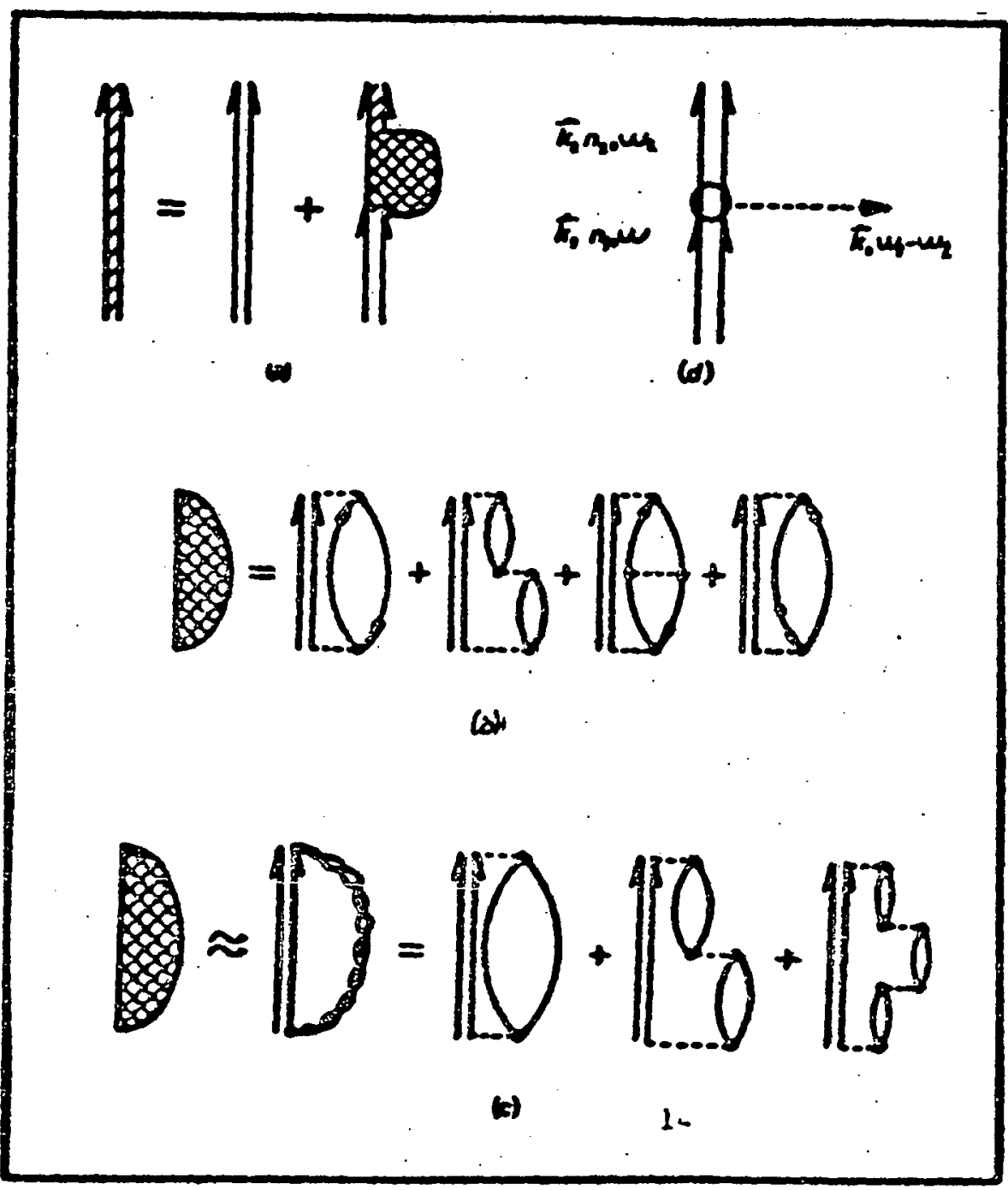

Lohen urre

WIx́n: Kana

non $r_{0} \tan r_{0} R$.

non iotolaktro berdintrek emi

non Kk loi-aiaki eroiaran ots ic ohaktroisron ir $\rightarrow$ 3i tomir A $>>1$ dola

$$
\begin{array}{r}
\dot{w} x^{\circ} n \\
-j
\end{array}
$$

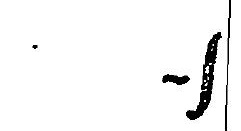

\subsection{Inveia}

Faynman-on diegramak, ioi lasterron ots ingunvix.s sioktroian artako oibarrokintas indikatuz. Ixus azalpans textuan.

viciaran ab 


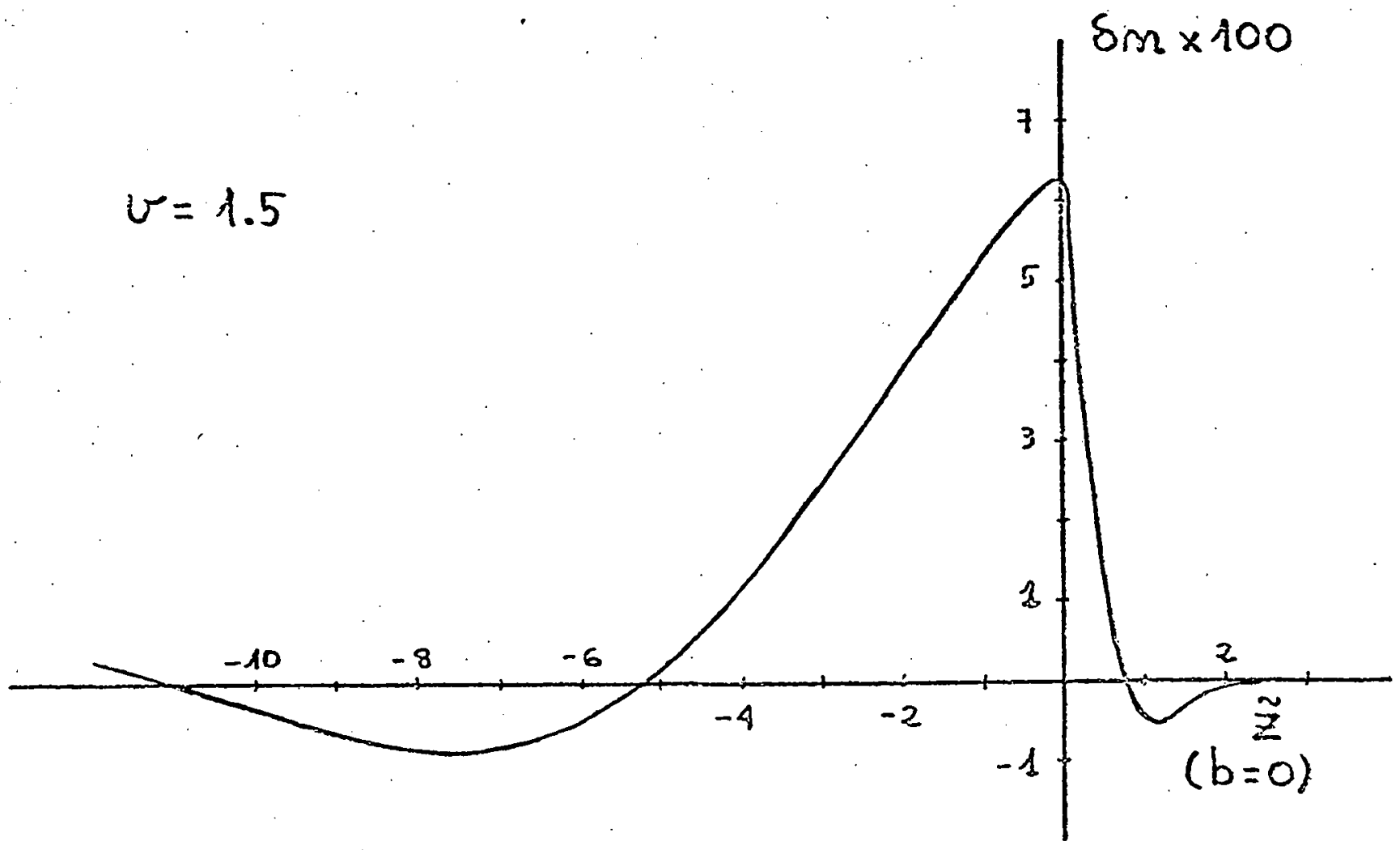

$v=2$

$\delta_{m \times 100}$

$=$

$-10$

$-8$ 


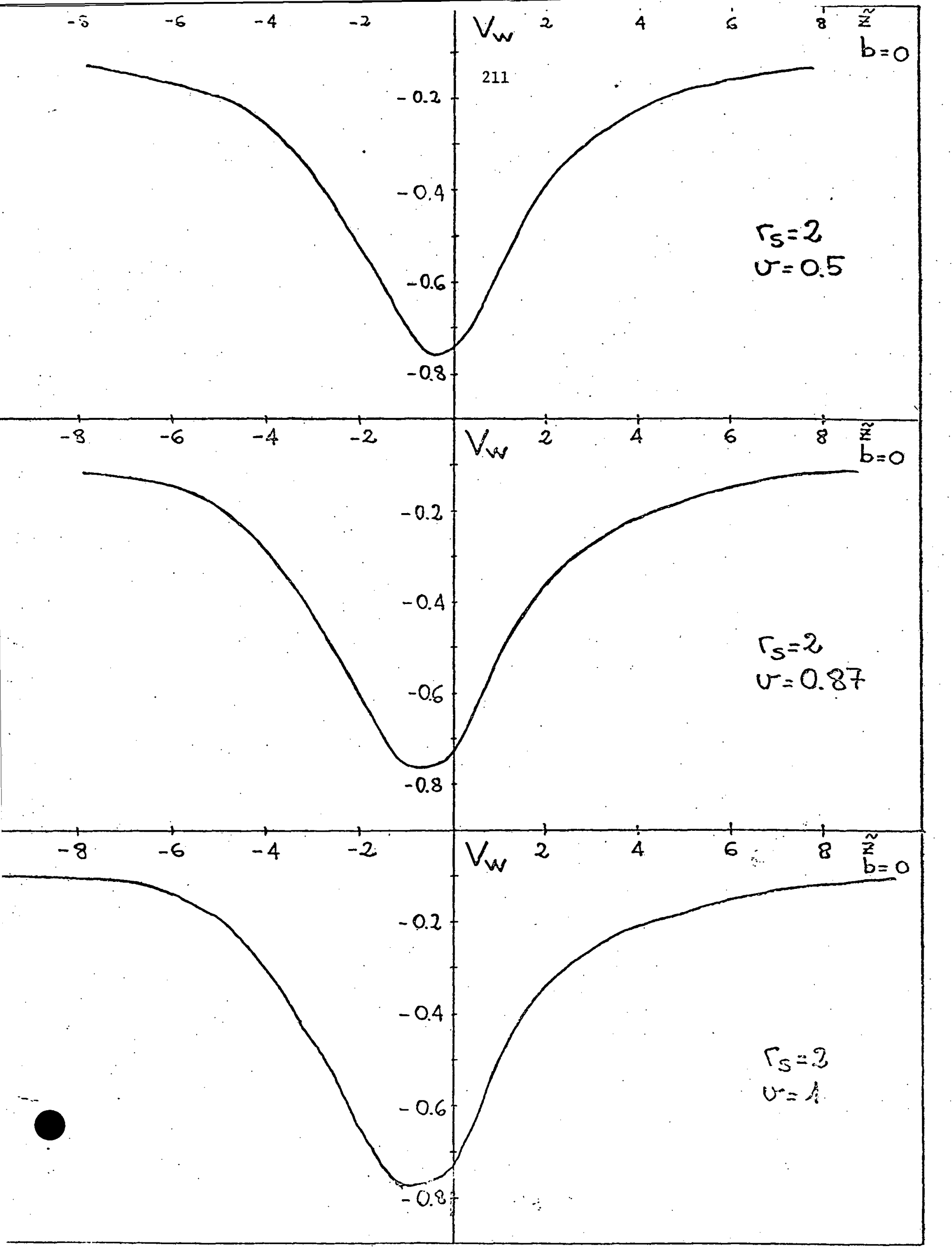




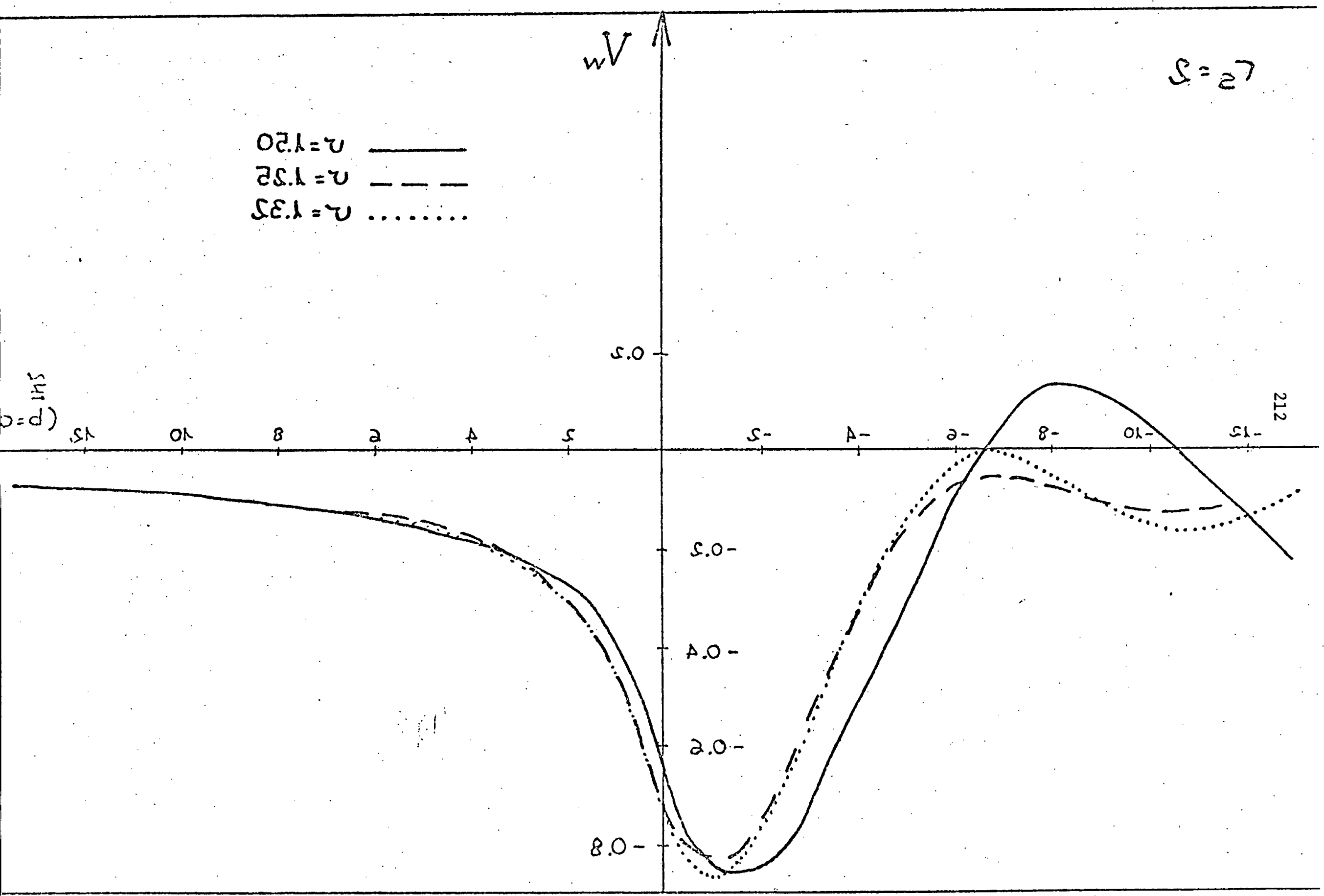




\section{Wake Fluctuations*}

R. H. Ritchie

Health and Safety Research Divisicn

Oak Ridge Hational Laboratory

Oak Ridge, Tennessee 373.30

The wake potential function just discussed by my colleagues Nerner Brandt and Pedro Echenique represents a statistical average of a quantity that is subject to quantal fluctuations. Some workers have suggested that these fluctuations may be so large as to vitiate the concept of the wake as a quantity which may be used, e.g., in calculations of wake shifts in atomic states on channeled ions and in estimating electron binding in the wake of swift ions.

To examine the magnitude of such fluctuations, we have studied a simple model system consisting of a swift projectile and a charge $Q$ moving with the same velocity and at specified distance from the projectile. It is relevant to compare straggling in energy loss by the test charge due to the wake of the leading ion with that due to direct interactions with the undisturbed medium. For completeness we also compare energy losses originating from these two separate sources.

We have show ${ }^{1)}$ that the straggling, $\Omega^{2}(\rho, \tilde{z})$ in energy loss by the test charge may be written

$$
\Omega^{2}(\rho, \tilde{j})=\left\langle(d E)^{2}\right\rangle-\langle d E\rangle^{2}=\left|Q Z e \Omega_{w}^{2}(\rho, \tilde{z})+Q^{2} \Omega_{0}^{2}\right|^{2}
$$

where

$$
\Omega_{w}^{2}(\rho, \tilde{z})=d R \cdot \frac{2 \hbar}{\pi v^{2}} \int_{0}^{\infty} k d k \int_{0}^{\infty} \frac{\omega^{2} d \omega}{k^{2}} J_{0}(k \rho) q u\left\{\frac{-e^{\lambda \omega \tilde{\xi} / v}}{\epsilon(k, \omega)}\right\}
$$

is the straggling in energy loss of the test charge due to the wake of the leading ion and

* Research sponsored by the Office of Health and Environmental Research, U.S. Department of Energy, under contract $W-7405-e n g-26$ with the Union Carbide Corporation. 
$\Omega_{0}^{2}=d R \cdot \frac{2 \hbar}{T v^{2}} \int_{0}^{\infty} k d x \int_{0}^{\infty} \frac{\omega^{2} d \omega}{k^{2}} \sin \left\{\frac{-1}{\epsilon(k, \omega)}\right\}$

is that due to the direct interactions of the test charge with the medium. In the above equations $\vec{v}$ is the common velocity of the leading projectile and the test charge, $d R$ is the path length of the charges in the medium, $\varepsilon(k, w)$ is the dielectric response function of the medium, $J_{0}(x)$ is the Bessel function and $k^{2}=k^{2}+\omega^{2} / v^{2}$. The test charge is taken to be located at lateral distance $\rho$ from the track of the leading projectile and trailing at distance- $-\tilde{z}$ behind the projectile. Similar expressions may be written for the energy loss of the test particle in traversing the same path length.

Figure (1a) shows the results of calculations of $\Omega^{2} / d R$ vs $\tilde{z}$ for a typical case where $v=3$ atomic units $(225 \mathrm{keV} / \mathrm{amu}), Z=1$ and $Q=e$. We have assumed that the medium in which the projectiles travel is an electron gas with a plasma energy of $25 \mathrm{eV}$ and have taken $\rho=0$, i.e., the test charge is taken to be on the track of the leading projectile. The dashed curve of Fig. (1a) shows $\Omega^{2} d d R$, the straggling which would obtain if the test charge were far away from the leading charge. One sees that at separations $|z| \geq 2.5$ au, representative of those existing in molecular ion systems, $\Omega^{2}$ is augmented by only about $10 \%$ due to its proximity with the leading ion. Since the width of the energy loss distribution curve is $\sim \Omega$, this implies only $\sim 5 \%$ increase in the actual energy width. Figure ( $1 b$ ) shows the resuits of a calculation of the total energy loss per unit path of the test charge as it depends upon its separation from the leading charge. The energy loss of the isolated test charge is shown as the dashed curve. 
We conclude from the calculation of $\Omega^{2}$ that under conditions of experimental interest, energy fluctuations in the wake of a leading ion have only a relatively small effect on ions moving in the wake of the leading ion. This is to be expected since the trailing part of the wake consists mainly in small displacements of a relatively large number of electrons and, hence, carries rather small momenta. The leading ion plows its way through the medium leaving only gentle perturbations that persist in the neighborhood of its track. These perturbations may affect the trailing ion appreciably but are expected to give rise to minimal fluctuations in its motion compared with the fluctuations it experiences in direct interaction with the undisturbed medium.

Figure 2 shows some experimental data on resonant coherent excitation of atomic states of channeled ions (the Okorokov effect) taken by workers at Oak Ridge. (2) The broad dips in the curves of the number of nitrogen $6^{+}$ions plotted as a function of ion velocity are interpreted as due to the excitation of single electrons from is states on the projectile to $n=2$ hydrogenic states by the fluctuating electric field of the lattice atoms. Resonance occurs at some harmonic of the fundamental frequency $2 \pi v / a$, where $v$ is the ion velocity and $\underline{a}$ is the lattice spacing. The doublet structure in these curves is thought to be due to wake-splitting of the degenerate $n=2$ states. The theory of wake splitting ${ }^{(3)}$ is couched in terms of a wake potential evaluated over the charge cloud of the atomic electron. Fluctuations in this wake potential may be regarded as responsible for the transfer of energy to a bound electron and, hence, lead to excitation and ionization of the electron. Calculations ${ }^{(3)}$ of the rates of such transitions give energy broadening of the split $n=2$ 
levels in general agreement with the observed widths of the doublet: structures shown in Fig. 2. We conclude that both experiment and theory bear out the idea that quantal filuctuations in the wake of an ion are not so large as to invalidate the use of statistically averaged expressions for the wake potential in computing wake splitting of atomic energy levels on swift ions.

In addition, it may be shown ${ }^{(4)}$ that lifetime broadening of wakebound electrons is primarily due to direct interaction of the electron with the medium ${ }^{(5)}$ and that contributions of lifetime broadening from fluctuations in the wake of the leading ion are of secondary importance in comparison.

In conclusion, under many conditions of importance in experiment, fluctuations in the wake of a leading ion in condensed matter are relatively unimportant compared to fluctuations accompanying direct interaction with the medium. The reason is that the wake of a leading ion is composed in the main of relatively small perturbations of large numbers of electrons. These perturbations may add to yield a large average force but, because they carry small momenta, give rise to rather small fluctuations of the force about its mean value. 
217

References

1. P. M. Echenique, R. H. Ritchie and W. Brandt, to be published in Phys. Rev. B.

2. S. Datz, C. D. Moak, O. H. Crawford, H. I. Krause, P. F. Dittner, J. Gomez del Campo, J. A. Biggerstaff, P. D. Miller, P. Hvelplund and H. Knudsen, Phys. Rev. Lett. 40,843 (1978).

3. 0. H. Crawford and R. H. Ritchie, to be published in The Physical Review.

4. P. M. Echenique and R. H. Ritchie, to be published.

5. R. H. Richie, W. Brandt and P. M. Echenique, Phys. Rev. B. 14, 4808 (1976). 
ORNL DWG 78-21544
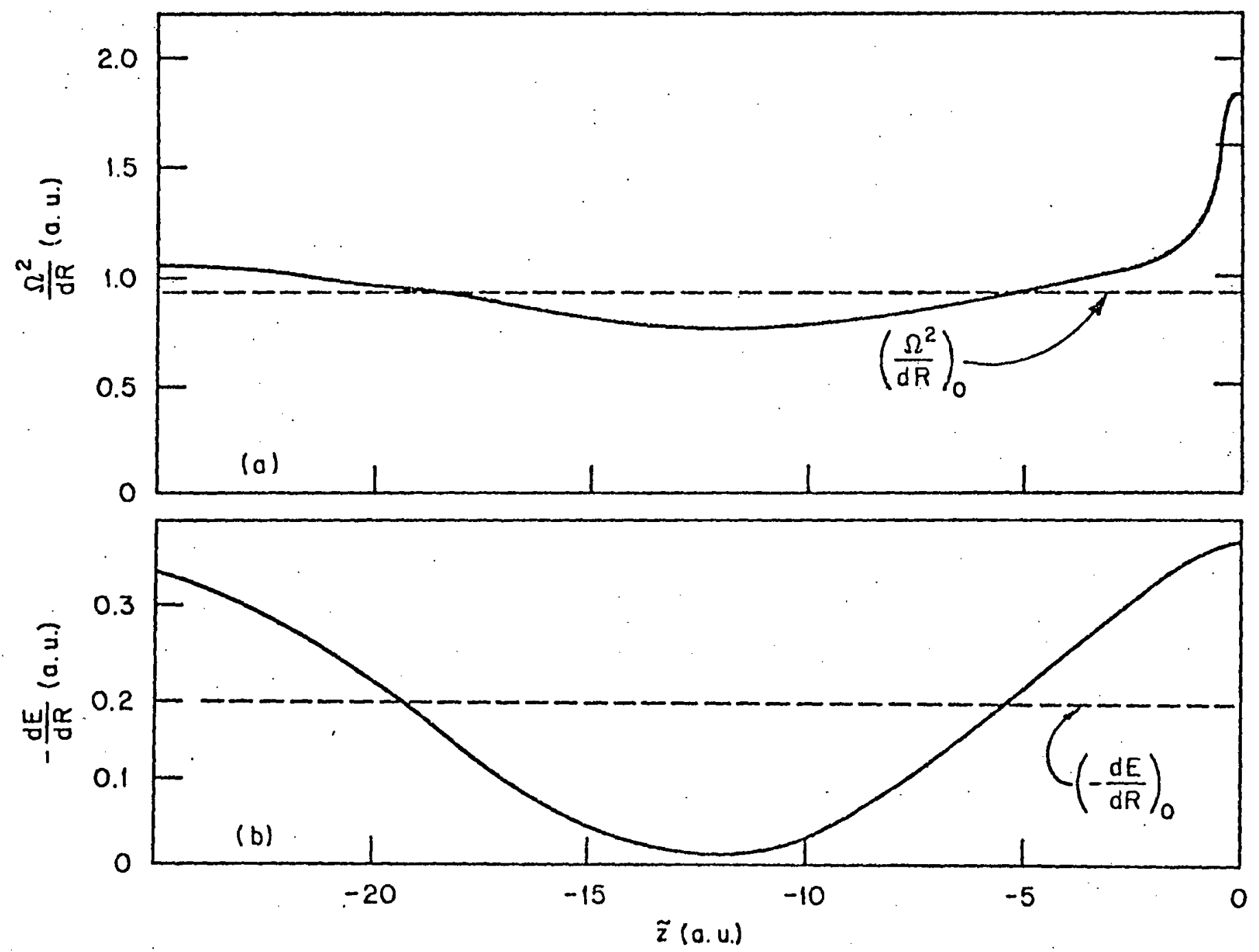


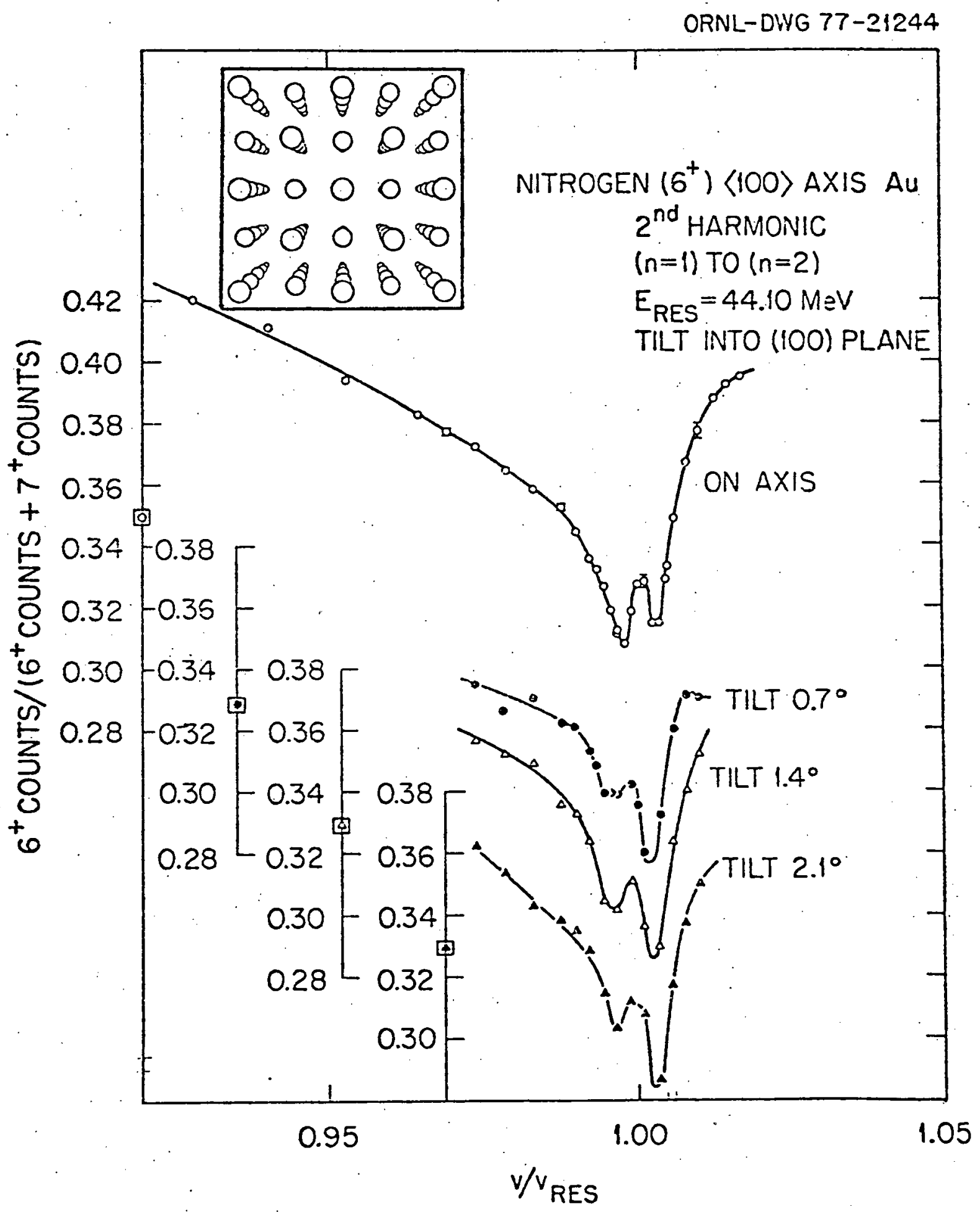


THIS PAGE

WAS INTENTIONALLY

LEFT BLANK 
Skipping Motion of Ions at the Surface*

Y.H. OHTSUKI (Tokyo)

M. Yamamura (okayama)

1. Introduction

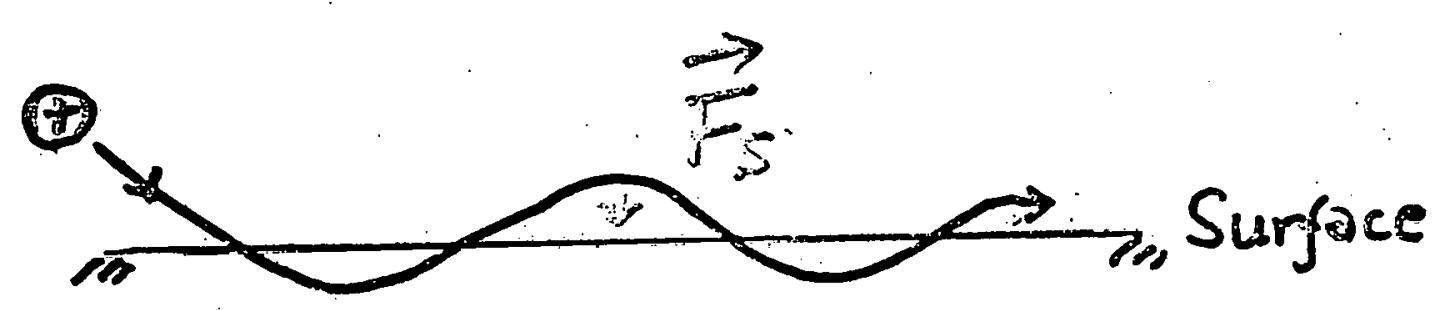

$$
\vec{F}_{S} \rightarrow \varphi_{s} \sim 3 \mathrm{eV} \text { for } \mathrm{Ni}_{i}
$$

* Phys. Rev. B in press

221 
222

2. Surface Potential

$$
\begin{aligned}
& \varphi_{S}\left(\vec{r}_{1}, t_{1}\right)=\int W(12) \rho^{\text {ext }}(2) d(2) \\
& W(12)=v(12)+\int w(13) P(34) v(42) d(34) \\
& 1 \approx 2+\underbrace{}_{3}= \\
& \begin{aligned}
P(34) \rightarrow P\left(\vec{q}_{3}, \vec{q}_{4} ; w\right) \\
=-\frac{\vec{q}_{1} \vec{q}_{2}}{m \omega^{2}} n\left(\vec{q}_{1}+\vec{q}_{3}\right)
\end{aligned} \\
& n(r)=\left\{\begin{array}{ll}
n_{0} & (z<0 \text { inside }) \\
0 & (z>0
\end{array}\right)
\end{aligned}
$$

Sh 
223

Suzuki, Kitagava and Oltsuki : Ply. Stat. sol. ('ro)

$$
\begin{aligned}
& \varphi_{B}\left(\vec{r}_{1}, t_{1}\right)=Z_{1} e \int_{0}^{\infty} d k \frac{\left(\omega_{p} / v\right)^{2}}{k^{2}+\left(\omega_{p} / v\right)^{2}} \\
& \times\left(J_{0}\left(k \rho_{-}\right) e^{-k\left|v t_{1}+\xi_{-}\right|} \theta\left(-t_{1}\right)+J_{0}\left(k \rho_{t}\right) e^{-k v t_{1}-\xi_{+} l} \theta\left(t_{1}\right)\right) \\
& +2 z_{1}+\frac{\omega_{p}}{v}\left(\sin \frac{\omega_{p}}{v}\left(v t_{1}+z_{-}\right)\right) \pi_{0}\left(\frac{\omega_{p}}{v} \rho_{-}\right)\left(\theta\left(v t_{1}+z_{-}\right) \theta\left(-t_{1}\right)_{-}\right) \\
& +\cdots \\
& \varphi_{S}\left(\vec{r}_{1}, t_{1}\right)=-z_{1} e \int_{0}^{\infty} d k \frac{\frac{1}{2}\left(\omega_{p} / v\right)^{2}}{k^{2}+\frac{1}{2}\left(\omega_{p} / v\right)^{2}}
\end{aligned}
$$

sity

$$
\begin{aligned}
& \text { iming }\left\{\times\left(J_{0}\left(k \rho_{ \pm}\right) e^{-k\left|v t_{1} \pm \zeta_{I}\right|} \theta\left(t_{1}\right)+\left(t_{1} \rightarrow-t_{1}\right)\right)\right. \\
& -2 z_{1} e \frac{\omega_{p}}{v} \frac{1}{\sqrt{2}} \sin \frac{u_{p}}{\sqrt{2} v}\left(v t_{1} \pm \xi ;\right) K_{0}\left(\frac{w_{p}}{\sqrt{2 v}} \rho_{\tau}\right) \\
& \times\left(\theta\left(v t_{1} \pm \xi_{F}\right) \theta\left(-t_{1}\right)+\theta\left(\xi_{F}\right) \theta\left(t_{1}\right)\right) \\
& -2 z_{1} e \frac{w_{p}}{v} \frac{1}{\sqrt{2}} \sin \frac{\omega_{p}}{\sqrt{2} v}\left(v t_{1} \mp \xi_{ \pm}\right) K_{0}\left(\frac{w_{p}}{\sqrt{3} \rho_{2}} \rho_{ \pm}\right)
\end{aligned}
$$

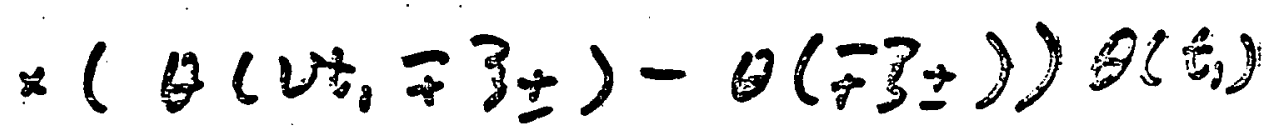




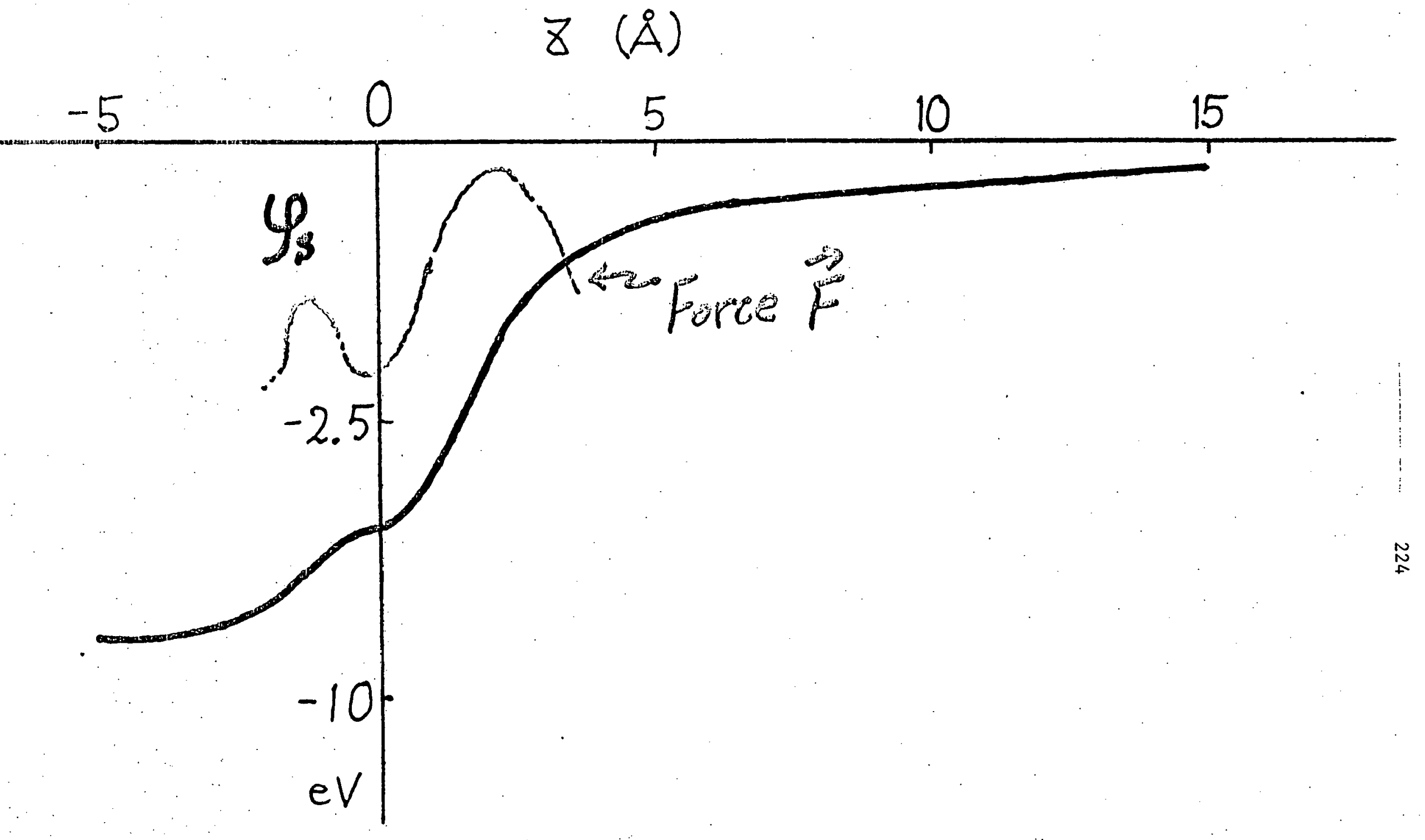

$$
30 \mathrm{KeV} \mathrm{H} \rightarrow \mathrm{Au}
$$


Surface Potential

$$
\varphi_{S}\left(t_{1}\right) \sim \frac{\pi\left(z_{1} e\right)^{2} \omega_{s}}{4 v}\left\{1-\frac{\omega_{s} t_{1}}{\pi}-\frac{4}{\pi} \alpha^{2} \omega_{s} t, \log \ldots\right\}
$$

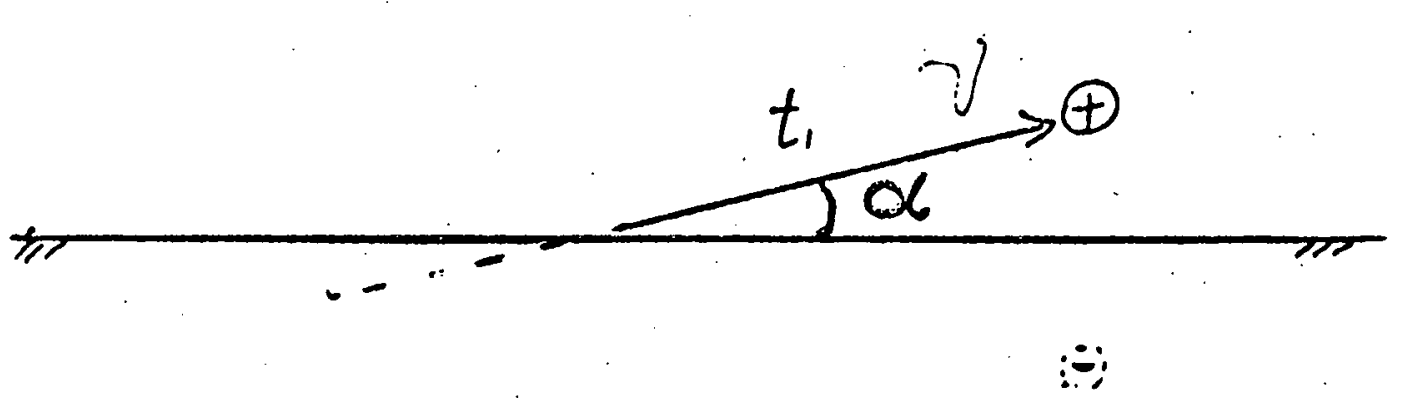

品 


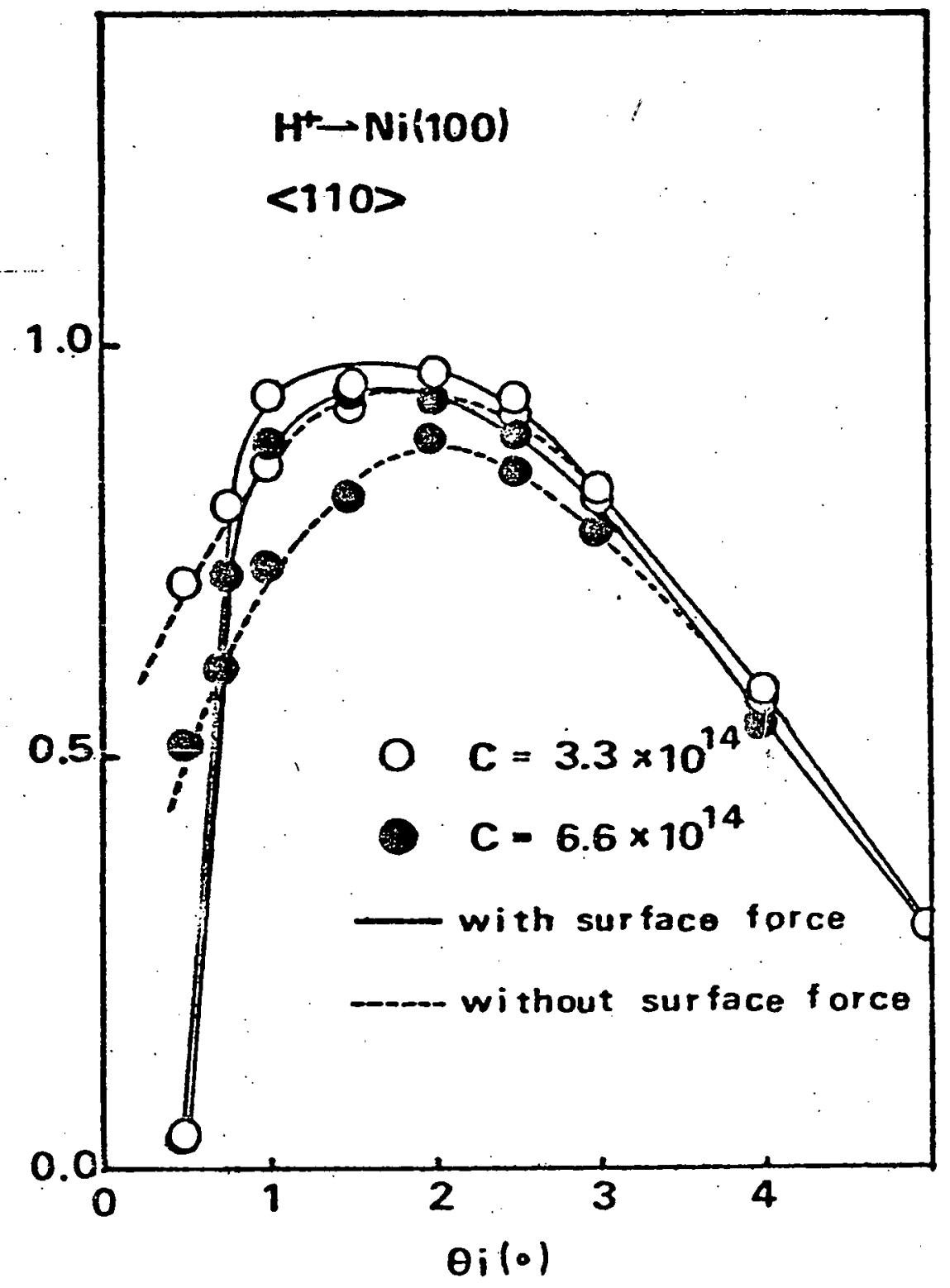


227

3. Computor Simulation.

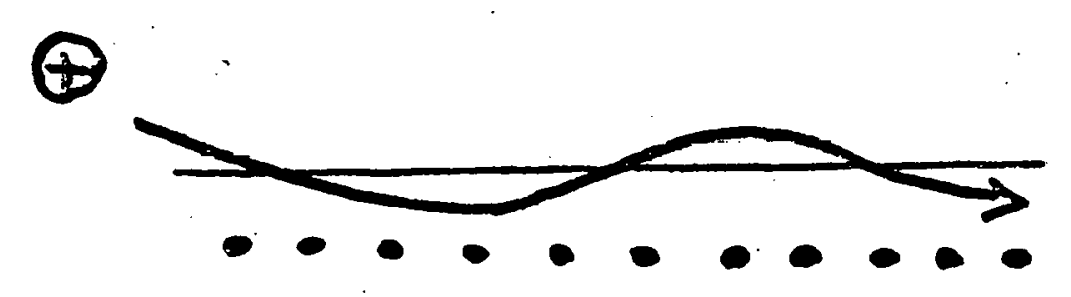

(1) Scattering By Atoms

- Rutheeford Scatt.

(2) Latrice Vibration

- included

(3) Neutratization

$$
\begin{array}{r}
P(s)=C\left\{e^{-c s}+\left(A s^{2}+B s+d\right)\right. \\
\left.x e^{-\cdots}\right\}
\end{array}
$$

(4) Energy Loss

- electronic excitation 

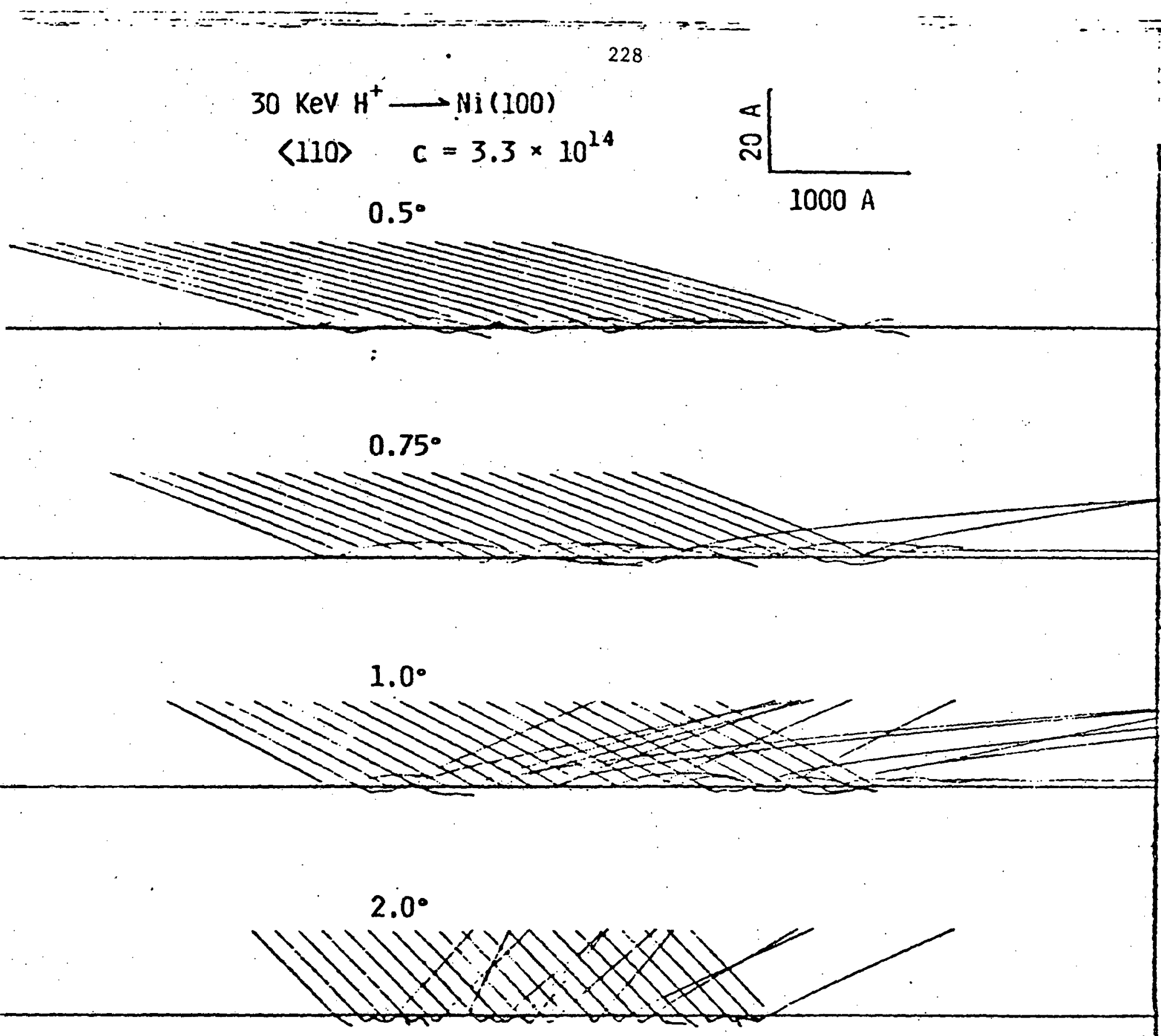

$5.0^{\circ}$

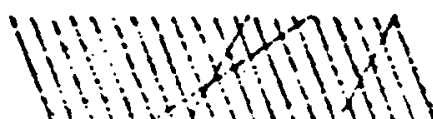



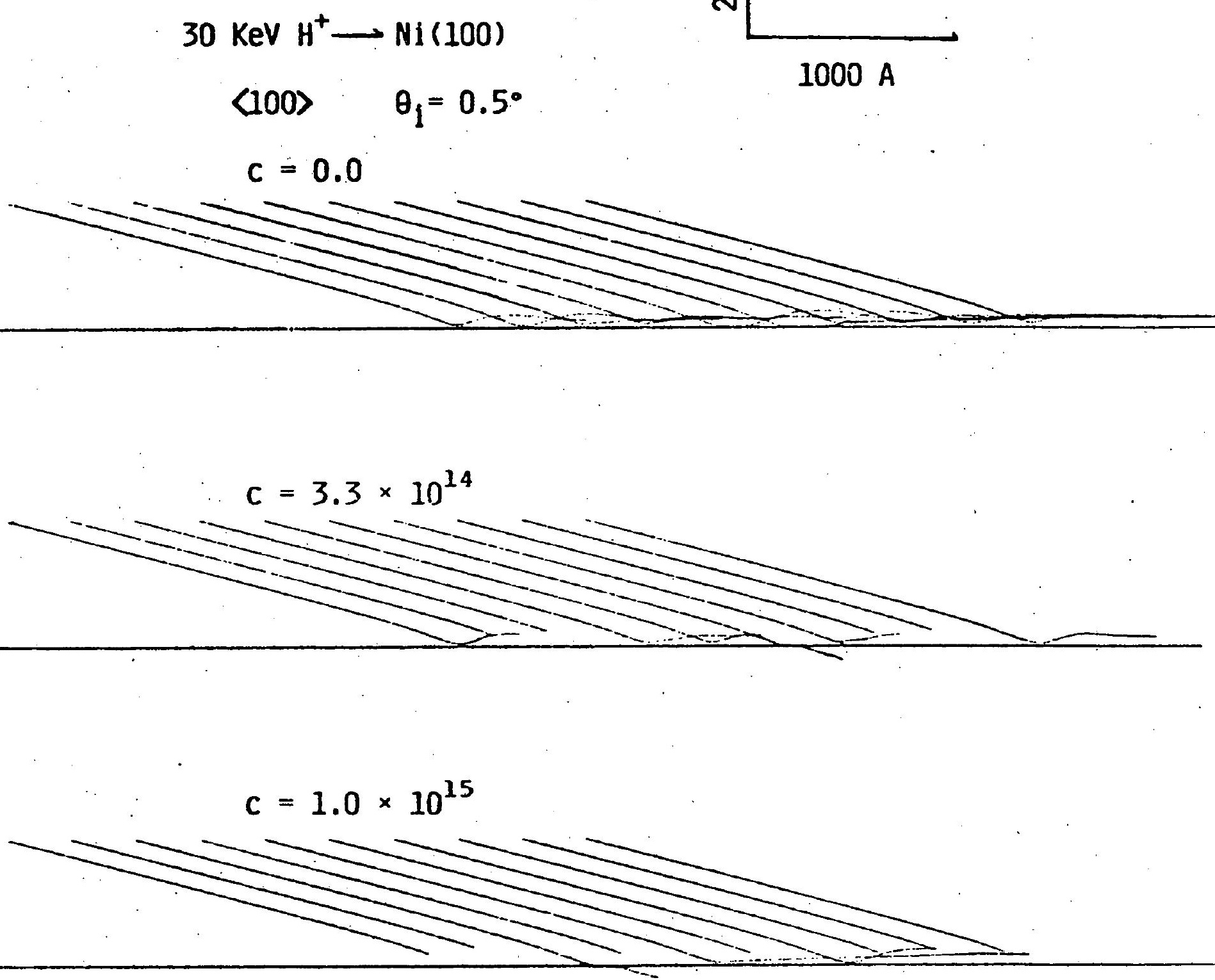


$$
\langle 110\rangle
$$

$30 \mathrm{KeV}$

$\theta_{i}=1^{\circ}$

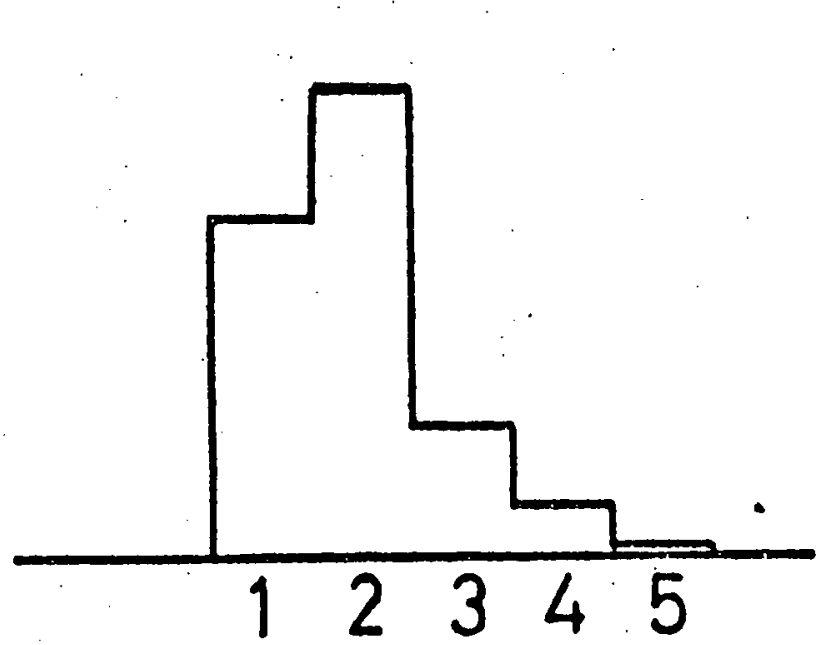

$100 \mathrm{KeV}$

$\theta_{i}=0.6^{\circ}$

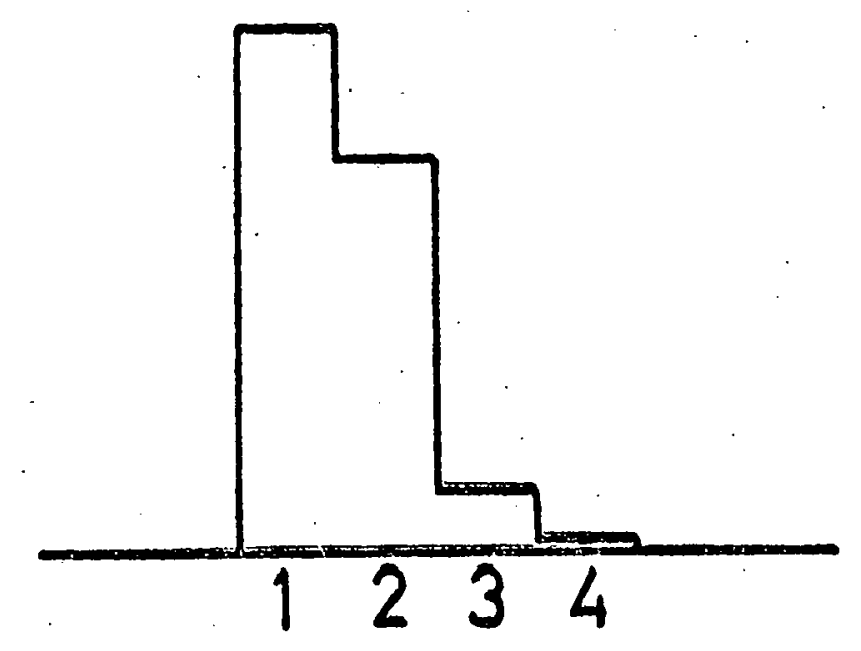

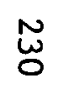

Fig. 5 Henki, Yamamura, Koyman 

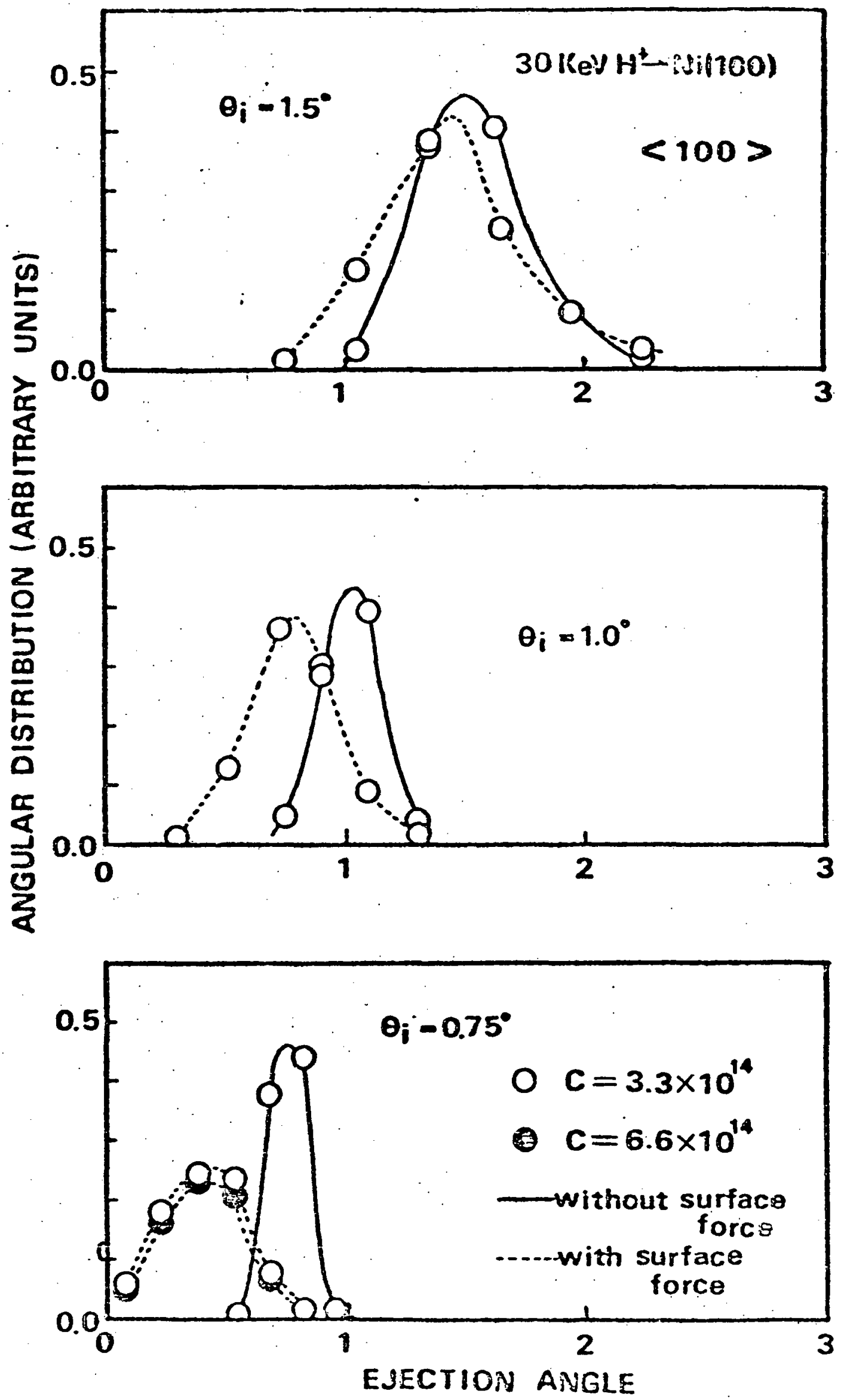


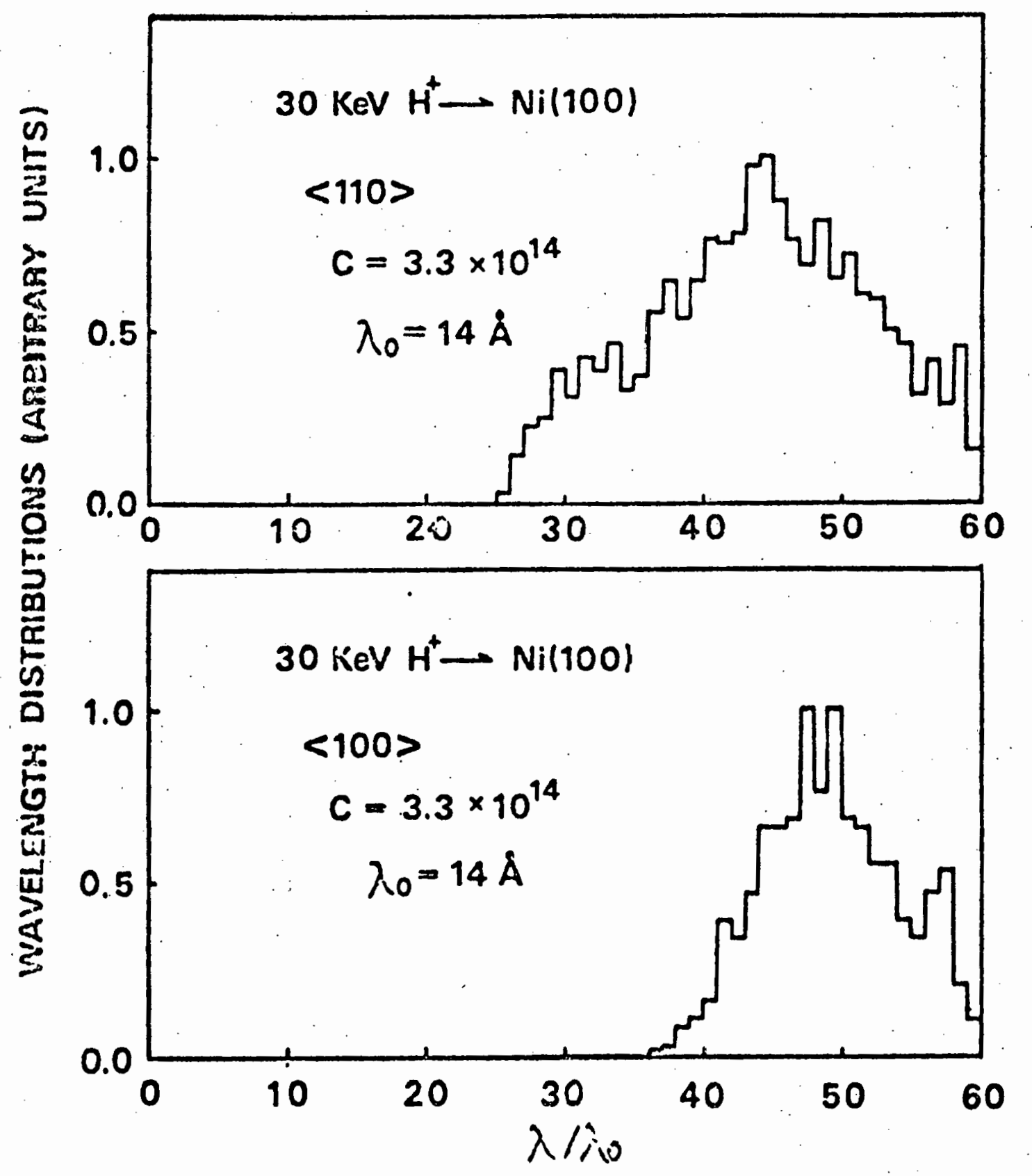




\section{- M. Kitagawa}

Department of Electronics, North Shore College, Atsugi 243, Japan

.2012

Y.H. Ohtsuki

Department of Physics, Waseda University, 4-170, Nishi-Ohkubo Shinjuku-ku, Tokyo 160, Japan

The soliton solution in a wake interaction of an ion due to virtual plasmons exchange is calculated in the region where the propagating velocity of the ion is less than the mean velocity of electrons in metals. We show that a $\operatorname{sech}^{2} x$-type soliton produces an attractive running potential in a plasmon field. The dispersion relation between the energy and the velocity, and the binding energy, of the ion in a soliton state are also calculated.

\section{Introduction}

An energetic ion, which is incident into metals, or semiconductors with a higher velocity than the phase velocity of plasmons, produces a dynamical polarization potential accompanied with virtual plasmons exchange behind it. . Such a potential is called as a wake potential, and gives directional influences to the motions of ion-ion, or ion-electron pairs. Recently, in order to detect the dynamical effect of the wake 
potential, Gemmell et al. I) have performed experiments to measure the energy and angular distributions of ions transmitted through thin solids. The experiments to detect the wake-riding states of ions, or electrons have been also performed by measuring the mass-dependence in yields of transmitted molecular ions, their effective charges, and the energy and angular distributions of secondary electrons $\left.{ }^{2}, 3\right)$.

On the other hand, in the region where the ion velocity becomes the same order of magnitude with the phase velocity of plasmons, the amplitude of the oscillatory potential decreases. In the region where the ion velocity is less than the mean velocity of electrons in metals, we have the same type of coupled differential equations with one which is known as soliton solutions in particle model 4,5 ) by taking into account of the coupling between the dynamical potential and the motion of the ion in it. We develop the calculations to get the coupled wave equations in Sec.PI. The soliton solution and the various properties of the soliton are calculated in Sec.III.

II. Dynamically Screened Potential Produced by Ions

The dynamically screened potential produced by ions, which are incident in electron gas, is represented in terms of a dielectric function as follows ${ }^{6}$ )

$\varepsilon, \omega ; \rho$,

$$
\phi(\vec{k}, \omega)=\nabla(k) \varepsilon^{-1}(\vec{k}, \omega) \rho(\vec{k}, \omega) .
$$

criexk

Here, $v(k)=4 \pi Z_{1} e / k^{2}, \varepsilon^{-I}(\vec{k}, \omega)$ and $\rho(\vec{k}, \omega)$ are Coulomb potential, the dielectric function in $\vec{k}-\omega$ representation, and the number 
density of the ion, respectively. $Z_{I} e(=G)$ is the charge of the ion. As concerms the interaction between the ion and the many electrons system, we take into account of plasmon excitations in order to calculate the wake effect. The real part of the dielectric function for plasmon excitations, $\varepsilon_{p l}^{-1}(\vec{k}, \omega)$, has a simple form and is given approximatelly ${ }^{7}$,

$\varepsilon, \omega$

$\phi, \rho$ :

$$
\varepsilon_{\mathrm{p} 1}^{-1}(\vec{k}, \omega)=\left(1-\frac{\omega_{k}^{2}}{\omega^{2}}\right)^{-1} \text {. }
$$

Greik

In Eq. (2), $\omega_{k}^{2} \fallingdotseq \omega_{p}^{2}+V_{A V}^{2} k^{2}$ indicates the dispersion relation of the plasmon frequency, and $V_{A V}$ is the mean velocity of electrons in metals. From Eqs.(1) and (2) and performing the Fourier transformation to $\vec{r}-t$ space, we have

$$
\left.\begin{array}{l}
-\frac{\partial^{2} \phi(\vec{r}, t)}{\partial t^{2}}+v_{A V}^{2} \nabla^{2} \phi(\vec{r}, t)-\omega_{p}^{2} \phi(\vec{r}, t)=4 \pi G R(\vec{r}, t) \\
\nabla^{2} R(\vec{r}, t)=\frac{\partial^{2}}{\partial t^{2}} \rho(\vec{r}, t) \quad\left(v_{A V} \fallingdotseq(3 / 5)^{1 / 2} V_{F}\right)
\end{array}\right\} .
$$

In the above, $V_{F}$ is the Fermi velocity. We calculate the following one which has the form of the separation variable in the solutions of Eq.(3),

$$
\left.\begin{array}{l}
R(\vec{r}, t)=R_{\perp}\left(r_{\perp}\right) R_{z}\left(z-V_{z} t\right) \\
\rho(\vec{r}, t)=P_{\perp}\left(r_{\perp}\right) \rho_{z}\left(z-V_{z} t\right) \\
\phi(\vec{r}, t)=f_{\perp}\left(r_{\perp}\right) \phi_{z}\left(z-V_{z} t\right)
\end{array}\right\}
$$

In Eq.(4), $\mathrm{V}_{z}$ is the propagating velocity of the ion and the plasmon field along z-axis. Substituting Eq.(4) into Eq.(3), we have the equations which include the contants of separation variable, $C_{0}, C_{1}, D_{0}$ and $D_{1}$, 
ф. $\omega$

$\rho, \Omega$ $\epsilon$ :

$$
\begin{aligned}
& -\frac{\partial^{2} \phi_{z}}{\partial t^{2}}+v_{A V}^{2} \frac{\partial^{2} \phi_{z}}{\partial z^{2}}-\left(\omega_{p}^{2}-D_{0}\right) \phi_{z}= \\
& \frac{\partial^{2} R_{z}}{\partial z^{2}}+C_{0} R_{z}=C_{1} \frac{\partial^{2} \rho_{z}}{\partial t^{2}} \\
& \nabla_{\perp}^{2} f_{\perp}=C_{0} f_{\perp}, \quad \rho_{\perp}=C_{1} R_{\perp}=C_{1} D_{\perp} f_{\perp}
\end{aligned}
$$

$f_{\perp}, P_{\perp}$ and each constant of separation variable are determired from $P_{\perp}\left(r_{\perp}\right)$ (the number-density of the $i$ in in transversal plane) $>0$, and the normalization of $\rho_{1}\left(r_{1}\right)$, then we have

$$
\begin{aligned}
& P_{\perp}\left(r_{\perp}\right)=\frac{b^{2}}{2 \pi} K_{0}\left(b r_{\perp}\right), \quad f_{\perp}\left(r_{\perp}\right)=K_{0}\left(b r_{\perp}\right) \\
& C_{0}=2 \pi C_{1} D_{1}=b^{2}=\Omega^{2} / V_{A V}^{2}, \quad D_{0}=\Omega^{2}
\end{aligned}
$$

where $\mathbb{K}_{0}\left(b r_{1}\right)$ is the modified Bessel function of the second kind, and $\nabla_{\perp}^{2} f_{\perp}\left(r_{\perp}\right)=b^{2} f_{\perp}\left(r_{\perp}\right)=2 \pi \cdot \rho_{\perp}\left(r_{\perp}\right)$ means the Poisson equation in the two-dimensionally cylindrical space. $\Omega^{2}$ is determined from the normalization of $\rho_{z}$. The differential equations for $\phi_{z}(=$ $\phi_{z}^{0}+\phi_{z}^{L}$ ) and $\rho_{z}$ through $R_{z}$ are also represented as a differential-integral form by use of the Green's function method, and the results are

$$
\begin{aligned}
& -\frac{\partial^{2} \phi_{z}^{I}}{\partial t^{2}}+v_{A V}^{2} \frac{\partial^{2} \phi_{z}^{I}}{\partial z^{2}}-\left(\omega_{p}^{2}-\Omega^{2}\right) \phi_{z}^{I}=2 G b^{2} v_{z}^{2} \rho_{z} \\
& -\frac{\partial^{2} \phi_{z}^{0}}{\partial t^{2}}+v_{A V}^{2} \frac{\partial^{2} \phi_{z}^{0}}{\partial z^{2}}-\left(\omega_{p}^{2}-\Omega^{2}\right) \phi_{z}^{0} \\
& =-2 G b^{3} v_{z}^{2} \int_{-\infty}^{\infty} d z^{\prime} \rho_{z}\left(z^{\prime}\right) \sin \left(b\left(z^{\prime}-\left(z-V_{z} t\right)\right)\right) \theta\left(z^{\prime}-\left(z-V_{z} t\right)\right)
\end{aligned}
$$

where $\theta\left(z^{\prime}-\left(z-V_{z} t\right)\right)$ is the Heaviside step function. $\rho_{z}\left(z-V_{z} t\right)$ is the number density of the ion. $\phi_{z}^{I}\left(z-V_{z} t\right)$ and $\phi_{z}^{0}\left(z-V_{z} t\right)$ are the localized and oscillatory parts of plasmon field, respecti$1 y$. 
III. Soliton-type Solution

Eq.(7-1) is the Klein-Gordon type wave equation produced by $\rho_{z}$. Now, we assume the variation of $\phi_{z}^{0}$ is small within the region of the localized length of $\rho_{z}$. By taking into account of the cou-

$\rho, \phi$, $\psi, \omega$, $\therefore, \nu$ : Greek pling between the dynarical potential produced by the ion, and the motion of the ion in it, we calculate the structure of the ion wave function. The coupling between $\phi_{z}^{I}$ and $\rho_{z}=\left|\psi_{z}\right|^{2}$ given by Eq. $(7-I)$ and the Schrödinger equation, gives the same-type equations with the coupled equations which are known as the soliton solution in particle model. Then we have,

$$
\left.\begin{array}{l}
-\frac{\partial^{2} \phi_{z}^{L}}{\partial t^{2}}+V_{A V}^{2} \frac{\partial^{2} \phi_{z}^{I}}{\partial z^{2}}-\left(\omega_{p}^{2}-\Omega^{2}\right) \phi_{z}^{I}=2 G b^{2} V_{z}^{2}\left|\psi_{z}\right|^{2} \\
i \hbar \frac{\partial \psi_{z}}{\partial t}=-\frac{\hbar^{2}}{2 M} \frac{\partial^{2} \psi_{z}}{\partial z^{2}}+G \phi_{z}^{I} \psi_{z}
\end{array}\right\} .
$$

The non-linear effect appears through the coupling of differential equations, and then we obtain the soliton solution. From Eq. (8), we derive the following-type solutions, in which $\psi_{z}$ has a plane wave term in phase,

$$
\left.\begin{array}{l}
\psi_{z}=\psi_{0}(h) \exp (i k), \quad \phi_{z}^{I}=\phi_{0}(h) \\
h=\nu_{1} z-\omega_{1} t, \quad k=\nu_{2} z-\omega_{2} t
\end{array}\right\} .
$$

Thẹn we have,

$$
\begin{aligned}
& \left(\nu_{1}^{2} v_{A V}^{2}-\omega_{1}^{2}\right) \frac{d^{2} \phi_{0}}{d h^{2}}-\Omega_{p} \phi_{0}=2 G b^{2} v_{z}^{2} \psi_{0}^{2} \\
& \frac{\hbar^{2} \nu_{1}^{2}}{2 M} \frac{d^{2} \psi_{0}}{d h^{2}}-\left(\frac{\hbar^{2} \nu_{2}^{2}}{2 M}-\hbar \omega_{2}\right) \psi_{0}=G \phi_{0} \psi_{0} \\
& \left(\frac{\hbar^{2} \nu_{1} \nu_{2}}{M}-\hbar \omega_{1}\right) \frac{d \psi_{0}}{d h}=0 \quad\left(\Omega_{p}=\omega_{p}^{2}-\Omega^{2}\right)
\end{aligned}
$$


From Eq. (10-3), we obtain $\nu_{2}=\mathbb{M} \omega_{1} / \hbar \nu_{1}=\mathbb{M N} / \frac{1}{2}$, where $\omega_{1} / \nu_{1}$ $=v_{z}$, and $d \psi_{0} / d h=0$ gives the trivial solution that $\psi_{0}$ is constant. In the region where $\omega_{1}^{2} / \nu_{1}^{2}=v_{z}^{2} \neq v_{A V}^{2}$, we have the $\phi_{0}=$ $\gamma \psi_{0}$ type solition solution from Eqs.(10-1) and $(10-2)$, where $\gamma$ i., i, is a constant

$\psi, \phi$

$Y, \Omega$, Grak

$$
\begin{aligned}
& \frac{d^{2} \phi_{0}}{d h^{2}}-\frac{\Omega_{p}}{\nu_{I}^{2} v_{A V}^{2}-\omega_{I}^{2}} \phi_{0}-\frac{2 M}{\hbar^{2} \nu_{I}^{2}} G \phi_{0}^{2}=0 . \\
& \pi \omega_{2}=\frac{1}{2} \mathrm{MV}_{\mathrm{z}}^{2}+\mathrm{E}_{\mathrm{B}} \quad\left(\mathrm{E}_{\mathrm{B}}=-\frac{\hbar^{2} \mathrm{R} p}{2 \mathrm{M}\left(\mathrm{V}_{\mathrm{AV}}^{2}-\mathrm{V}_{\mathrm{z}}^{2}\right)}\right) \\
& \frac{1}{y^{2}}=\frac{M\left(V_{A V}^{2}-V_{z}^{2}\right)}{\hbar^{2} b^{2} V_{z}^{2}}>0 ; \quad\left(V_{A V}^{2}>V_{z}^{2}\right)
\end{aligned}
$$

In the above, Eq. (II-2) gives a dispersion relation between the velocity and energy of the ion, which appears in the phase term of the ion wave function. Eq.(II-I) has the solution which is given in terms of the Jacobian Elliptic Function, that is given

$$
\left.\begin{array}{l}
\frac{d^{2} F}{d X^{2}}-4 B^{2}\left(K^{4}-K^{2}+I\right)^{I / 2} F-6 B ; \frac{2 K^{2}}{A} F^{2}=0 \\
F=A \operatorname{sn}^{2}(B X)+C, \quad C=A /\left(\left(K^{4}-K^{2}+I\right)^{1 / 2}-\left(K^{2}+1\right)\right)
\end{array}\right\} .
$$

Here $K$ is the modulus of the Jacobian Elliptic Function. In series solutions given in terms of $K\left(0 \leqq K^{2} \leqq 1\right)$, the Bell-type soliton solution, which becomes zero at $z-V_{z} t \rightarrow \pm \infty$, is obtained at $\mathrm{K}^{2}=1$. By use of Eqs. $(11-1),(12)$ and $\mathrm{K}^{2}=1$, we have

$$
\left.\begin{array}{l}
\phi_{0}=-A \operatorname{sech}^{2}\left(B^{\prime}\left(z-V_{z} t\right)\right) \\
\psi_{0}= \pm A^{\prime} \operatorname{sech}^{2}\left(B^{\prime}\left(z-V_{z} t\right)\right) \\
A=3 \hbar^{2} \Omega_{p} /\left(4 \operatorname{GM}\left(V_{A V}^{2}-V_{z}^{2}\right)\right), A^{\prime}=3 \pi \Omega_{p} /\left(4 G b V_{z}\left(M\left(V_{A V}^{2}-V_{z}^{2}\right)\right)^{I / 2}\right)
\end{array}\right\}
$$


$B^{\prime}=\Omega_{\mathrm{p}}^{I / 2} /\left(2\left(V_{\mathrm{AV}}^{2}-V_{\mathrm{z}}^{2}\right)^{I / 2}\right)$

from

Futhermore, the normalization condition of $\psi_{0}^{2}$, we obtain

$$
\left(1-\alpha^{2}\right)^{3}=\frac{16 Z_{I}^{4} E_{z}^{2} E_{C}}{9 n^{2} \omega_{p}^{2} E_{A V}^{2}}\left(E_{A V}-E_{z}\right) \alpha^{4}
$$

LC, $\psi$

$\omega, \phi$,

Greek

$$
\begin{aligned}
& \left.a^{2}=\Omega^{2} / \omega_{p}^{2}, \quad E_{A V}=\frac{1}{2} M V_{A V}^{2}, \quad E_{z}=\frac{1}{2} M_{z}^{2},\right) \\
& E_{C}=\frac{1}{2} M V_{B}^{2}, \quad V_{B}=\frac{e^{2}}{2}
\end{aligned}
$$

Then $\Omega^{2}$ is determined. In Fig.(1), $\alpha^{2}$ is shown for each $E_{0}=16 Z_{1}^{4} E_{z}^{2} E_{c}\left(E_{A V}-E_{z}\right) / 9 n^{2} w_{p}^{2} E_{A V}^{2}$ in the case of $\mathrm{H}^{+}$in Au. As is obtained from Fig.(1), $\Lambda^{2}$ is much smaller than $\omega_{p}^{2}$ in the region where $E_{z}$ has the same order of magnitude with $E_{A V}$, except the region where $E_{A V}-E_{z}$ becomes the magnitude within $0.1 \mathrm{eV}$. In Eq. (13), $\phi_{0}\left(z-V_{i} t\right)$ gives a $\operatorname{sech}^{2} x$-type attractive running potential, and $E_{B}=-\hbar^{2} \Omega_{p} /\left(2 M\left(V_{A V}^{2}-V_{Z}^{2}\right)\right)$ in.Eq. (Il-2), represents the binding energy of the ion which is bound in the attractive running potential. The results are shown in Fig.(2). For the case of $\mathrm{H}^{+}$in $\mathrm{Au}$, the values of $\mathrm{E}_{\mathrm{B}}$ are the order of magnitude from a few $\mathrm{eV}$ to $10 \mathrm{eV}$ within the $30 \mathrm{eV}$ lower region than $E_{\mathrm{AV}}$ ( $\cong 6 \mathrm{keV}$ ). From the dispersion relation between the velocity $V_{z}$ and energy $E\left(\hbar \hbar \omega_{2}\right)$ of the ion, we obtain the following results. As is shown in Fig.(1) for the case of $\mathrm{H}^{+}$in $\mathrm{Au}$, the contributions of $\ell^{2}$ to $\omega_{p}^{2}$ are, about $1 / 10$ at $E_{A V}-E_{z}=0.1 \mathrm{eV}$, about $1 / 50$ at $E_{A V}-E_{z}=1 \mathrm{eV}$, and about $1 / 100$ at $E_{A V}-E_{z}=10 \mathrm{eV}$. Therefore, except the region. where $E_{A V}-E_{z}$ becomes the magnitude within $0.1 \mathrm{eV}$,

$$
E \cong E_{z}-\frac{\hbar^{2} \omega_{p}^{2}}{4} \frac{1}{\left(E_{A V}-E_{z}\right)} \quad\left(E_{z}<E_{A V}\right)
$$

is a good approximation. 
From Eq. (15), we have

$$
E_{z}-E_{A V}=\frac{1}{2}\left(\left(E-E_{A V}\right) \pm\left(\left(E-E_{A V}\right)^{2}-n^{2} \omega_{p}^{2}\right)^{1 / 2}\right)
$$

From the condition of $E_{z}-E_{A V}<0$, we have $E-E_{A V} \leqq-\hbar \omega_{p}<0$. The relation between $E-E_{A V}$ and $E_{z}-E_{A V}$ is shown in Fig. (3). E (ii) $\nu$ : $\Omega$. Girik becomes the double-values' function of $E_{z}$, and $\left|E-E_{A V}\right|<\hbar \omega_{p}$ is forbidden. At $E-E_{A V}=-n \omega_{p}$, the phase velocity of the ion $V_{p h}$, which is defined as

$$
v_{p h}=\frac{d \omega_{2}}{d \nu_{2}} \simeq v_{z}\left(I-\frac{\hbar^{2} \omega_{p}^{2}}{4} \frac{1}{\left(E_{A V}-E_{z}\right)^{2}}\right)
$$

becomes zero, and the duality between $E$ and $E_{z}$ corresponds to the pair of opposite phase-velocity states.

By taking into account of Eqs. $(7-2),(13)$ and that $\Omega^{2}$ is much smaller than $\omega_{p}^{2}$ in the region where. $v_{z}<V_{A V}$, the amplitude of the oscillatory part of the polarization potential in this region, becomes smaller than the amplitude of the wake potential in the region where $\mathrm{V}_{\mathrm{z}}>\mathrm{V}_{\mathrm{AV}}{ }^{8,9)}$.

IV. Concluding Remarks

The soliton solution in the wake interaction of the ion due to virtual plasmons exchange was calculated in the region where the propagating velocity of the ion is less than the mean velocity of electrons in metals. We showed that the soliton solution, which has the form of $\operatorname{sech}^{2}\left(z-V_{z} t\right)$ along the propagating direction, and of $K_{0}\left(b r_{\perp}\right)\left(K_{0}\right.$ is the modified Bessel function of the second kind) in the transversal space perpendicular to the propagation, produces the attractive running potential in the plasmon field. The soliton amplitude is propor- 
tional to $\left(z_{1} e M\right)^{-1}$, which is the characteristic property of the non-linear effect. We also showed that the dispersion relation between the velocity and the energy of the ion in soliton state appears in the phase factor of the ion wave function, which is interpreted that the ion is bound in the attractive running LC. potential in the plasmon field. The binding energy of the ion ireck has the order of magnitude from a few eV to $10 \mathrm{eV}$ within the 30 $\mathrm{eV}$ lower region than $\mathrm{E}_{\mathrm{AV}}(\cong 6 \mathrm{keV})$ for the case of $\mathrm{H}^{+}$. in $\mathrm{Au}$. The duality between the kinetic energy and the total energy of the ion also appeared, which corresponds to the pair of opposite phase-velocity states. The result that $\left|E-E_{A V}\right|<\hbar \omega_{p}$ is forbidden, was also obtained as a noted property derived from the duality:

\section{References}

I) D.S. GemmeIl, J.Remillieux, J.-C. Poizat, M.J. Gaillard, R.E. Holland and 2. Vager, Nucl. Instrum. Methods 132, 61 (1976).

2) W. Brandt and R.H. Ritchie, Nucl. Instrum. Methods 132, $43(1976)$.

3) M.J. Gaillard, J.-C. Poizat, A.J. Ratkowski and J. Remillieux, Nucl. Instrum. Methods 132, 69 (1976).

4) S. Kubo, M. Namiki and I. Ohba, Prog. Theor. Phys. 55, $860(1976)$.

5) M.G. Makhankov, Phys. Rep. 35, No.I (1978).

6) I. Hedin and S. Iundquist, Solid State Phys. 23 (1969). 
7) D. Pines, Elementary Excitations in Solids (Benjamin, New York, 1964).

8) V.N. Neelavathi, R.H. Ritchie and W. Brandt, Phys. Rev. Lett. 33, 302 (1974).

9). M. Kitagawa and Y.H. Ohtsuki, Phys. Rev. Bl6, 5321 (1977).

\section{Pigure Captions .}

Fig.(1): The values of $\alpha^{2}$ for $\mathrm{H}^{+}$in $\mathrm{Au}$.

Fig.(2): The binding energy $\mathrm{E}_{B}$ of $\mathrm{H}^{+}$in soliton state. for the case of $\mathrm{H}^{+}$in $\mathrm{Au}$.

Fig.(3): The duality between the kinetic energy $E_{z}$ and the total energy $\mathrm{E}$ of $\mathrm{H}^{+}$in soliton state for the case of $\mathrm{H}^{+}$in Au. 


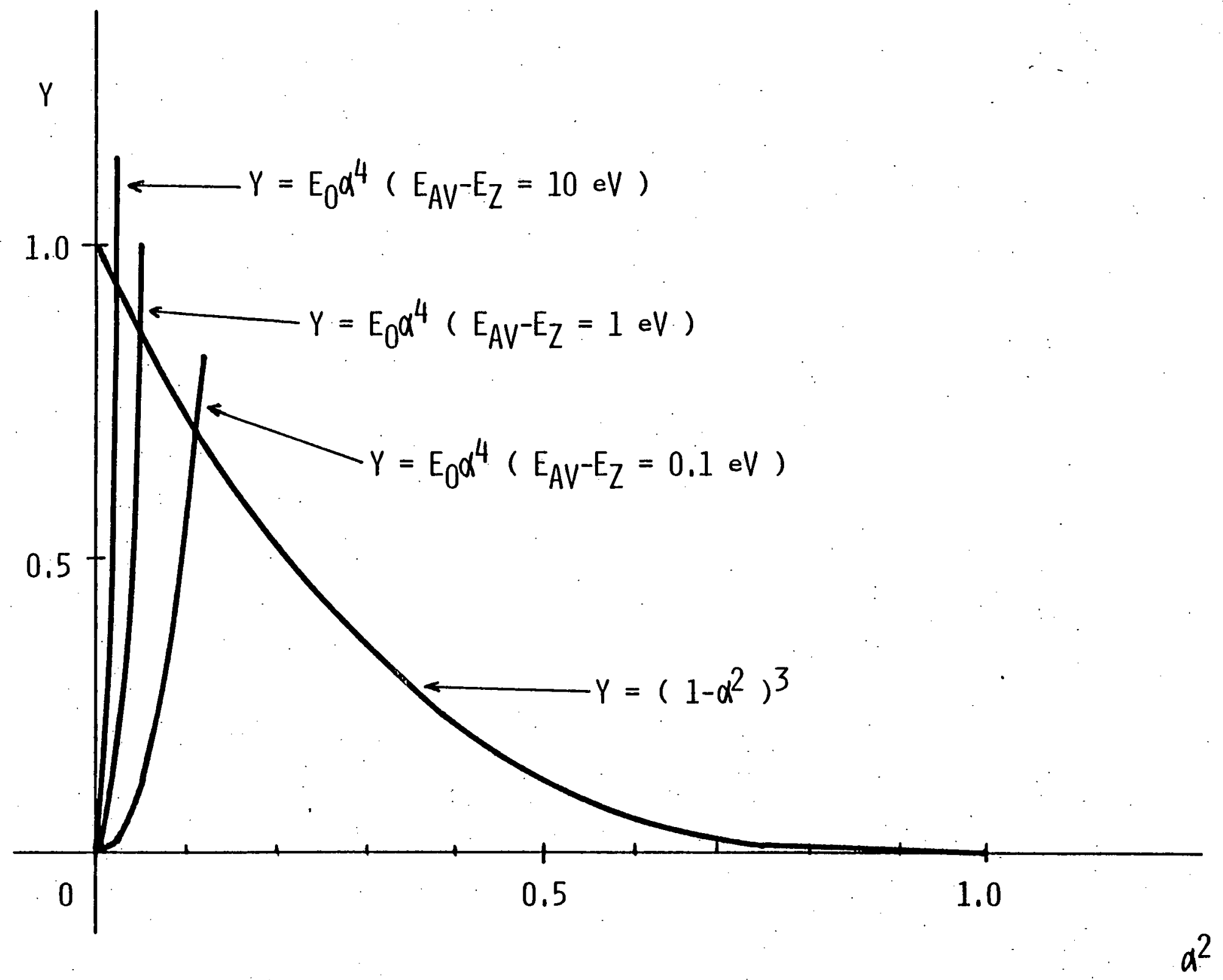


Fig. (2)

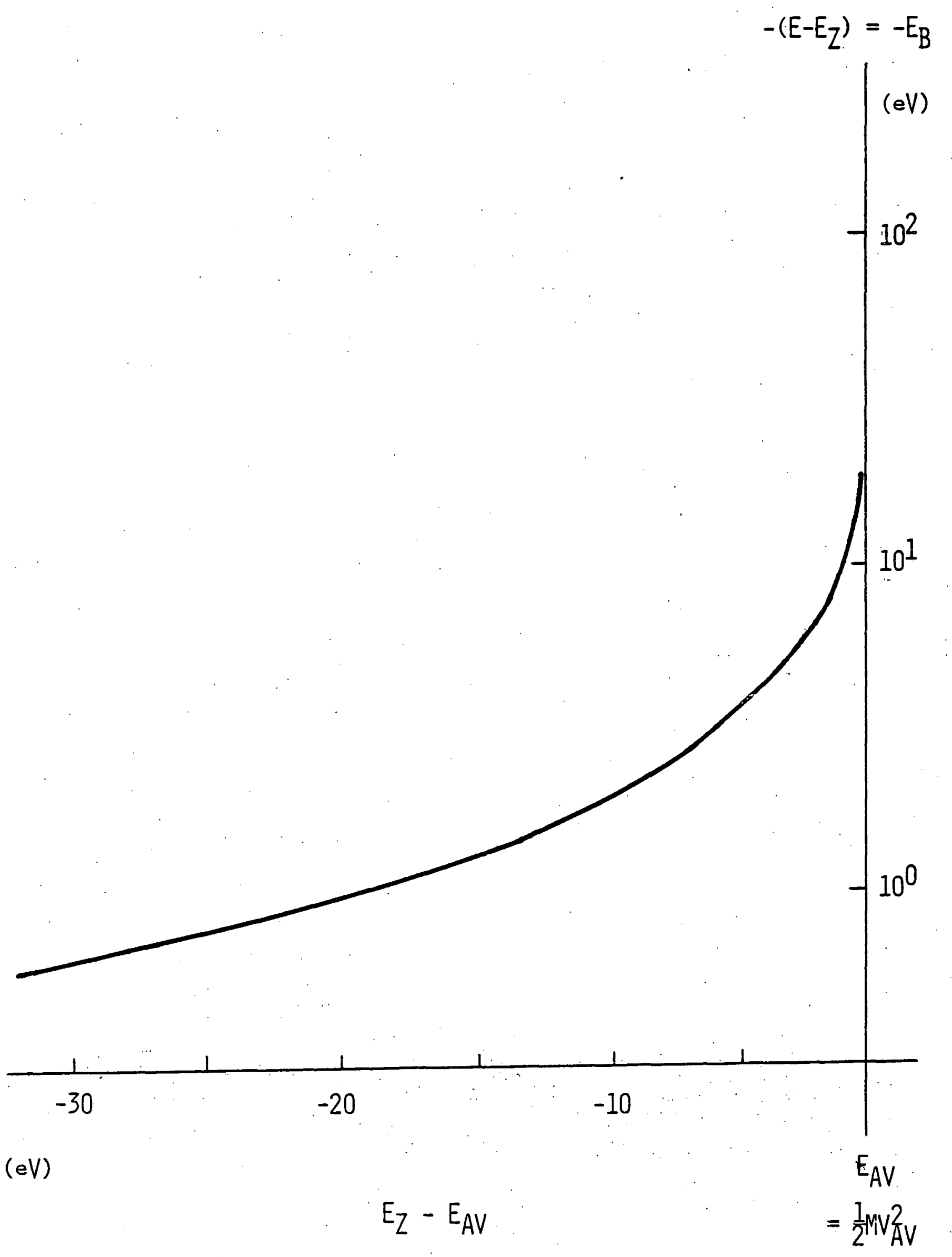


Fig. (3)

\section{$E-E_{A V}(e V)$}

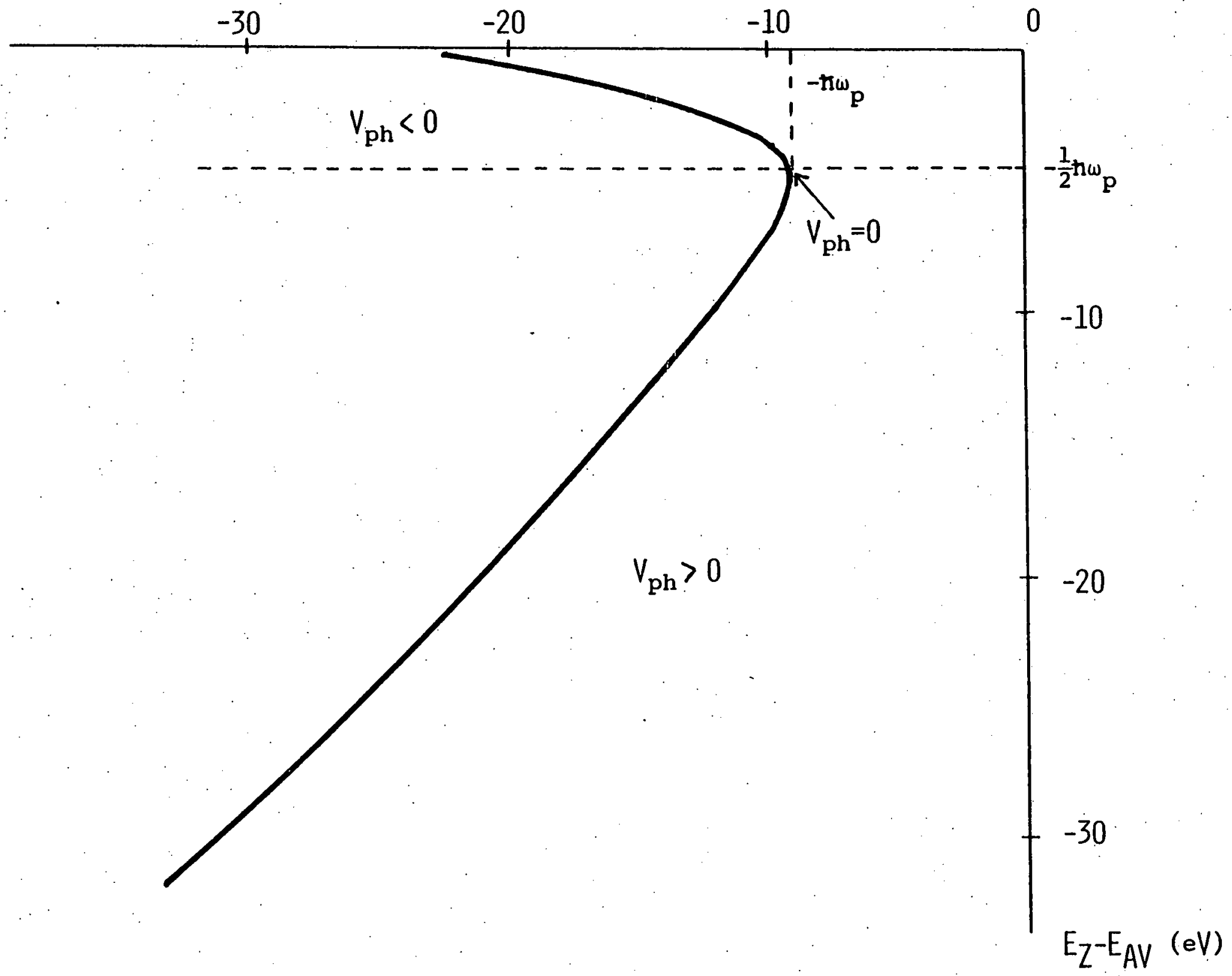


THIS PAGE

\section{WAS INTENTIONALLY \\ LEFT BLANK}


MOLECULAR DISSOCIATION PRODUCED BY FAST HEAVY ION BOMBARDMENT

A. K. Edwards, R. M. Wood, and M. F. Steuer

Department of Physics and Astronomy, University of Georgia, Athens, GA 30602

\section{Abstract}

A technique has been devloped to study the dissociative ionization of molecules by fast, heavy ions. A pulsed beam of projectiles is focused into a differentially-pumped chamber containing a gaseous, molecular target. The fragment ions from the molecular target are investigated by measuring their kinetic energy and their time-of-flight (TOF) from the collision region to a detector. The simultaneous measurement of kinetic energy and TOF separates the fragments according to their mass-to-charge ratios and yields the kinetic energy spectrum of each. This time-energy spectroscopy method has been applied to several diatomic molecules and simple hydrocarbons. Dissociative states of molecules and ionizing collisions by different projectiles have been investigated.

\section{INTRODUCTION}

A technique has been devloped ${ }^{l}$ in our laboratory to study the dissociation of molecules that have been ionized by fast, heavy ions. This method separates the fragment ions of the dissociation process according to their mass-to-charge ratio (it does mass spectrometry) and yields the kinetic energy spectra of each fragment.

The structure observed in the kinetic-energy spectra is found to be different for different bombarding projectiles. The projectiles used most are $\mathrm{H}^{+}, \mathrm{He}^{+}$, and $\mathrm{O}^{+}$in the energy range of 0.5 to $4 \mathrm{MeV}$. The kineticenergy spectra produced by $\mathrm{H}^{+}$projectiles are similar to those observed in low-energy electron bombardment experiments indicating that single ionization of the target molecule dominates.

When $\mathrm{He}^{+}$and $\mathrm{O}^{+}$ions are used as projectiles, additional structure is found in the kinetic-energy spectra of the singly ionized fragments, plus higher charge states of the fragments are produced with far more intensity. The $\mathrm{O}^{+}$projectile is the more efficient ionizer of the target molecule than the $\mathrm{He}^{+}$projectile. 
Experimental Procedure

The experimental method involves measuring the time-of-flight (TOF) of the fragments produced by dissociation plus the fragments kinetic energy. Figure 1 is a schematic diagram of the apparatus and electronics used in this time-energy spectroscopy technique. Beam pulses from the University of Georgia Van de Graaff accelerator, typically 100 nsec wide, pass through a differentially-pumped collision chamber containing the target gas. The fragments formed in the dissociative processes and ejected at $90^{\circ}$ from the beam direction pass through a focusing element and a parallel-plate electrostatic analyzer. A channeltron detector is positioned at the exit of the analyzer. Both the kinetic energy of a fragment and its TOF from the collision region to the detector are measured.

Timing signals are generated by the beam pulses passing through a capacitive pick-off unit placed before the collision region. The beampick-off (BPO) signal is used to stop a time-to-amplitude converter (TAC) which is started by a channeltron pulse; The TAC output is a signal whose amplitude is linearly related to the TOF of the ion. It is recorded on the $y$ axis of a $64 \times 64$ array of a multi-channel analyzer (MCA).

The $x$ coordinate is the voltage on the back plate of the electrostatic analyzer and corresponds to the particle's energy at the time of its production in the collision region: The back plate is swept continuously by a triangular wave generator. Not shown in Fig. 1 are delays and other circuit elements included to insure that the signal the to $x$ and $y$ axes of the MCA arrive simultaneously for proper storage in the $64 \times 64$ array.

For a species of ion with a fixed mass-to-charge ratio, the locus of possible $\mathrm{T}$ and $\mathrm{E}$ coordinates is described by the following equation:

$$
\mathrm{T}=\mathrm{K}_{1}-\mathrm{K}_{2}(\mathrm{~m} / \mathrm{q})^{1 / 2} \mathrm{E}^{-1 / 2}-\mathrm{K}_{3}(\mathrm{~m} / \mathrm{q})^{1 / 2} \text {. }
$$

In the equation, $T$ is the time recorded by the TAC. The energy, mass, and charge of the fragment ion are $\mathrm{E}, \mathrm{m}$, and $\mathrm{q}$, respectively. The constant $\mathrm{K}_{1}$ is determined by the adjustable delay in the time pickoff signal. The constant $\mathrm{K}_{2}$ is determined by the length of the flight path of the ion before it is accelerated by any of the applied voltages. The constant $\mathrm{K}_{3}$ is determined by the analyzer voltage, focus voltage, and the geometry of the analyzer system. 
As Eq. (1) indicates, ions with a specific $\mathrm{m} / \mathrm{q}$ ratio are represented by $a$ unique curve in the two-parameter data array. An on-line computer is used to find the energy spectrum for each of the separated ions curves. In addition, the computer is programmed to determine the constants, $\mathrm{K}_{I^{\prime}}$ $\mathrm{K}_{2}, \mathrm{~K}_{3}$ in Eq. (1) by fitting data corresponding to known $\mathrm{m} / \mathrm{q}$ ratios.. Another program is then employed to determine the $\mathrm{m} / \mathrm{q}$ value for data corresponding to an unknown ion.

Figure 2. is a typical contour plot of the new raw data obtained when $\mathrm{CO}$ is bombarded by $1-\mathrm{MeV} \mathrm{He}{ }^{+}$projectiles. Different, clearly separated curves are observed for $\mathrm{C}^{+}, \mathrm{O}^{+}, \mathrm{C}^{2+}, \mathrm{O}^{2+}$ fragment ions. The discontinuties in the $\mathrm{C}^{+}$and $\mathrm{O}^{+}$curves are due to the choice of projectile pulse repetition rate and timing adjustments.

\section{Results}

$\underline{\mathrm{H}_{2}}$

Study of the $\mathrm{H}_{2}$ molecule is unique in that the potential curves of $\mathrm{H}_{2}^{+}$are well known. ${ }^{2,3}$ This allows us to predict a kinetic energy spectrum for each dissociative state and then to fit these to the measured spectra. The energy distribution of the $\mathrm{H}^{+}$states are predicted by the reflection approximation ${ }^{4}$ and a least square fitting routine ${ }^{5}$ is used to obtain the fraction that each state contributes to the total observed spectrum. Figure 3 shows the $\mathrm{H}^{+}$and $\mathrm{D}^{+}$spectra produced by $0.5-4 \mathrm{MeV} \mathrm{He}{ }^{+}$projectiles. The smooth lines are calculated fits to the data.

The $\mathrm{H}_{2}^{+}$states used in the fitting routine are $1 \mathrm{~s} \sigma_{\mathrm{g}^{\prime}} 2 \mathrm{p \sigma}{ }_{\mathrm{u}}, 2 \mathrm{p}{ }_{\mathrm{u}} \mathrm{u}^{\prime}$ and $2 \mathrm{~s} \sigma_{\mathrm{g}^{\prime}}$ plus a doubly-ionized state consisting of the $\mathrm{H}^{+}{ }^{\prime}-\mathrm{H}^{+}$Coulomb repulsion curve. Attempts to fit the data ${ }^{6}$ with these five curves were unsuccessful at the low energy end $(<5 \mathrm{eV})$ of the spectra. It was necessary to predict an unknown autoionizing state of $\mathrm{H}_{2}$. in order to. explain this discrepancy.

An investigation ${ }^{7}$ was made of the relative contribution that each dissociative state made to the observed spectra as a function of projectiles $\mathrm{H}^{+}, \mathrm{H}_{2}^{+}, \mathrm{He}^{+}$, and $\mathrm{O}^{+}$. Of the $\mathrm{H}^{+}$fragments that originated from an $\mathrm{H}^{+}$ dissociative state and had kinetic energy greater than $2 \mathrm{eV}$ the $2 \mathrm{p} \sigma_{\mathrm{u}}$ and $2 \mathrm{p} \pi_{\mathrm{u}}$ states contributed 85-90\% of the total. Also, for single ionization 
of the $\mathrm{H}_{2}$ target, the projectiles $\mathrm{He}^{+}$and $\mathrm{H}_{2}^{+}$exhibit the same cross section as a function of projectile velocity. However, these two projectiles behave very differently when producing double ionization. The $\mathrm{H}_{2}^{+}$ projectile produces practically no double ionization while the $\mathrm{He}^{+}$projectile shows a measurable amount of double ionization (about 29\% of the total ionization of $1 \mathrm{MeV}$ ) that decreases nearly exponentially with projectile velocity.

\section{Other Diatomic Molecules}

The other diatomic molecules that we have studied, $\mathrm{N}_{2},{ }^{8} \mathrm{O}_{2},{ }^{9} \mathrm{Co}, 10$ and $\mathrm{NO}^{10}$ do not offer the luxury of having known potential curves for the dissociative states of the molecular ions. The bound states are well known from molecular spectroscopy but not so with the dissociative states. The kinetic energy spectra of the fragment ions show a multitude of structure that is difficult to unravel. For example, the $0^{+}$- fragment energy distribution from $\mathrm{O}_{2}$ molecules can arise from dissociative states of $\mathrm{O}_{2}^{+}$, $\mathrm{O}_{2}^{2+}$, etc. Presently, the other fragment from the dissociation process is not detected and, without the aid of known potential curves, it is not possible to determine unambiguously the initial charge state of the dissociating molecular ion.

For heteronuclear molecules, such as NO and $\mathrm{CO}$, an indication of the. initial charge state is possible. The conservation of momentum and energy in the dissociation process gives a relationship between the energies of the two fragments. For example, a $\mathrm{CO}^{2+}$ molecular state that dissociates into $\mathrm{a} \mathrm{C}^{+}$and an $\mathrm{O}^{+}$ion would produce a peak in the $\mathrm{C}^{+}$kinetic energy spectrum that correlates to a peak in the $\mathrm{O}^{+}$kinetic energy spectrum according to the dictates of the conservation of momentum. On the other hand, a $\mathrm{C}^{+}$peak arising from a $\mathrm{CO}^{+}$dissociation would not show a counterpart in the $0^{+}$spectrum.

This analysis has worked very well for $\mathrm{CO}$ and NO and the results of $\mathrm{CO}$ are shown in Fig. 4. The dashed lines are the $\mathrm{C}^{+}$spectra and the smooth lines are the $0^{+}$spectra. The yields are plotted as a function of dissociation energy allowing comparison of both peak positions and intensities. When $\mathrm{H}^{+}$projectiles are used to ionize the $\mathrm{CO}$ it appears that single ionization dominates. The $\mathrm{C}^{+}$and $\mathrm{O}^{+}$fragments come from potential curves of $\mathrm{CO}^{+}$with different dissociation energies. When $\mathrm{He}^{+}$and $\mathrm{O}^{+}$projectiles are used there are two peaks that can be correlated indicating that two 
$\mathrm{CO}^{2+}$ states are excited that decay to produce $\mathrm{c}^{+}$and $\mathrm{O}^{+}$fragments. Also, for the $\mathrm{O}^{+}$projectile it appears that a peak in the $\mathrm{O}^{+}$spectrum correlates to a peak in the $c^{2+}$ spectrum (dot-dash curve) indicating that a $\mathrm{co}^{3+}$ state decays into $\mathrm{O}^{+}$and $\mathrm{C}^{2+}$ eragments.

Hydrocarbons

An investigation has begun into the molecular dissociation of simple hydrocarbons that are bombarded by fast heavy ions. Parts of the kineticenergy spectra of the various fragments ${ }^{11}$ consist of sharp peaks, indicating the dissociation of the molecule into two parts along a potential surface. Parts of the spectra.consist of smoothly varying energy distributions indicating possible multibody breakup with the dissociation energy being shared by the several fragments.

\section{References}

1. R. M. Wood, A. K. Edwards, and M. F. Steuer, Rev. Sci. Instrum. 47, 1471 (1976).

2. D. R. Bates, K. Ledsham, and A. L. Stewart, Phil. Trans. Roy. Soc. (London) A246., 215 (1953).

3. T. E. Sharp, At. Data 2, 119. (1971).

4. A. S. Coolidge, H. M. James, and R. D. Present, J. Chem. Phys. 4, 193 (1936).

5. P. R. Bevington, Data Reduction and Error Analysis for the Physical Sciences (McGraw-Hill, New York, 1969).

6. R. M. Wood, A. K. Edwards and M. F. Steuer, Phys. Rev. A 15, 1433 (1977).

7. A. K. Edwards, R. M. Wood, and M. F. Steuer, Phys. Rev. A 16, 1385 (1977).

8. A. K. Edwards, R. M. Wood and M. F. Steuer, Phys. Rev. A 15, 48. (1977).

9. M. F. Steuer, R. M. Wood and A. K. Edwards, Phys. Rev. A 16, 1873 (1977).

10. R. M. Wood, M. F. Steuer, and A. K. Edwards, J. Chem. Phys. 69, 4487 (1978).

11. A. K. Edwards, J. E. Graves, R. M. Wood, and M. F. Steuer, J. Chem. Phys. 69, 1985 (1978). 


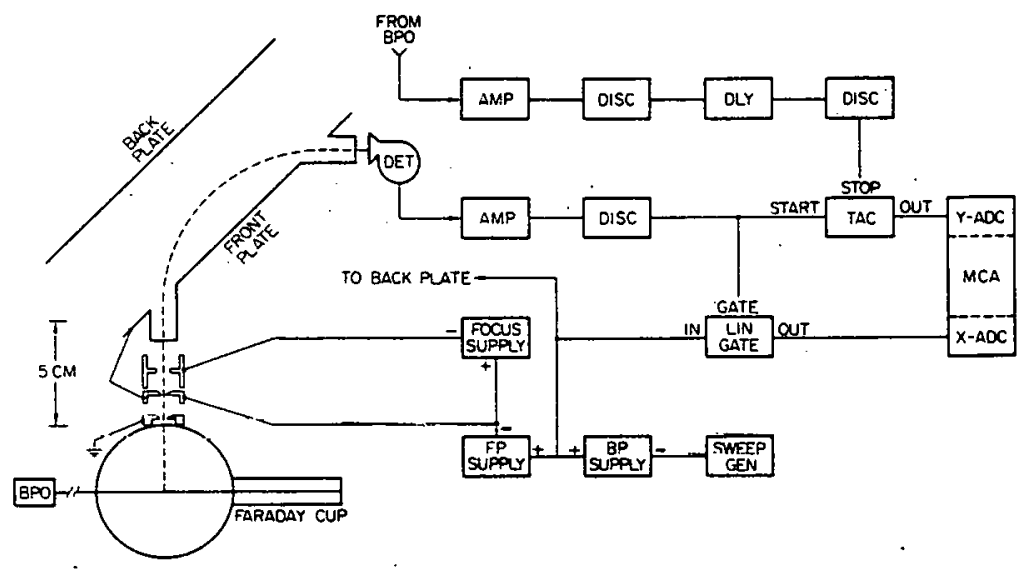

Fig. 1. Schematic of the apparatus and electronics used to obtain the kinetic energy spectra of fragment ions.

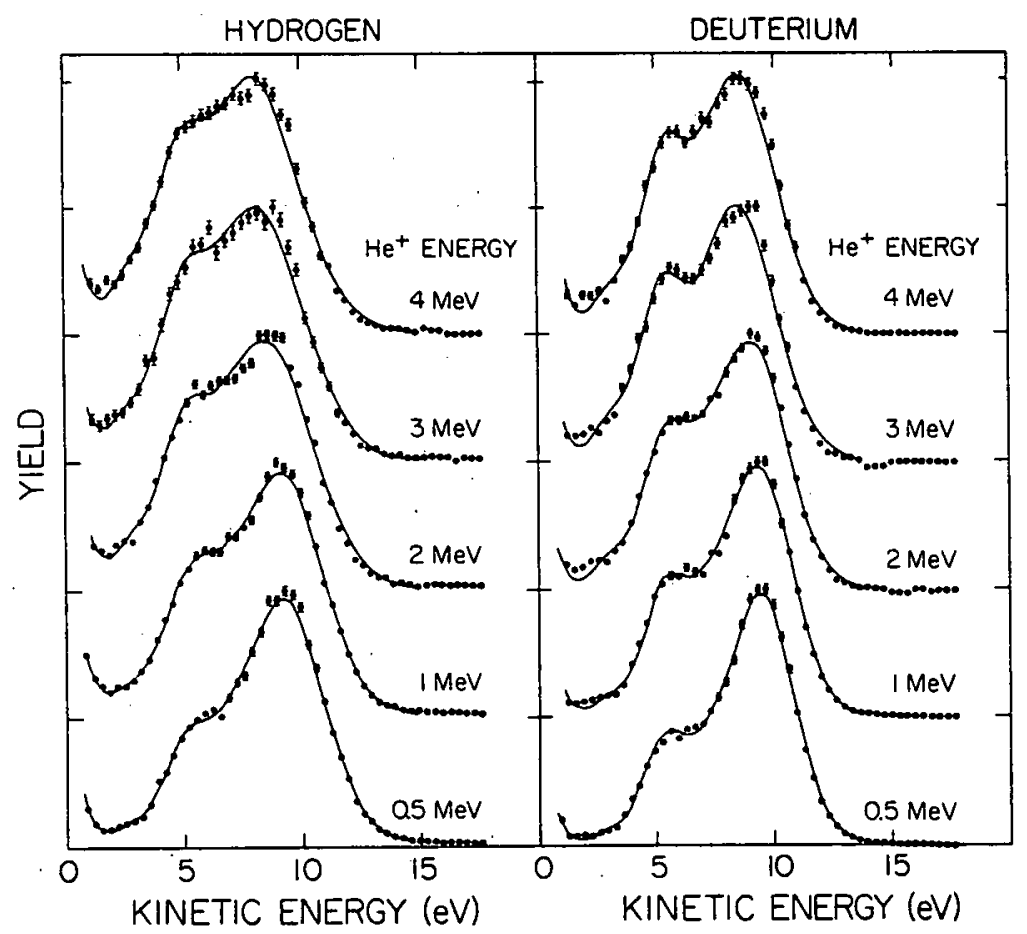

Fig. 3. $\mathrm{H}^{+}$and $\mathrm{D}^{+}$yields as a function of kinetic energy for 0.5 - to $4-\mathrm{MeV} \mathrm{He}{ }^{+}$incident on $\mathrm{H}_{2}$ and $\mathrm{D}_{2}$. The spectra are normalized to the same height. The solid curves are calculated fits to the data.

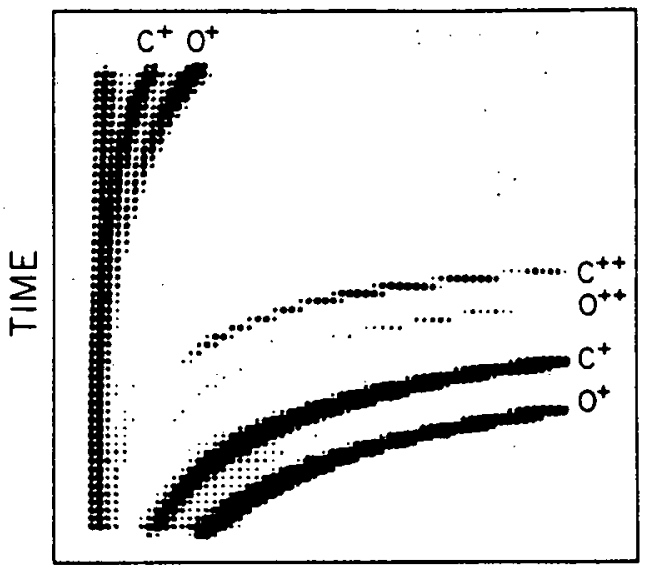

ENERGY

Fig. 2. A photograph of the raw data obtained from the dissociation of $\mathrm{CO}$.

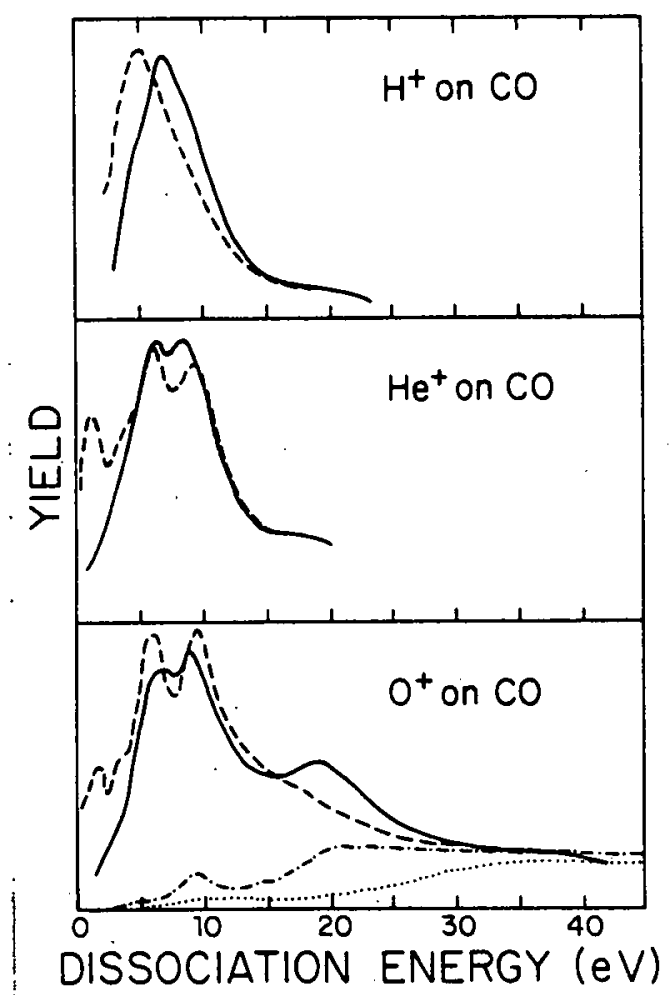

Fig. 4. Yield per unit dissociation energy as a function of dissociation energy. Dashed lines represent $\mathrm{C}^{+}$. Solid lines represent $o^{+}$. The dot-dash line represents $\dot{C}^{2+}$. The dotted line represents $\mathrm{o}^{2+}$. 
P.B. Treacy

Australian National University, Canberra.

Electrons produced by charge-exchange reactions, such as

$$
p+(\mathrm{C}+\mathrm{e}) \rightarrow(\mathrm{p}+\mathrm{e})+\mathrm{C}
$$

are known to have forward velocity spectra with sharp peaks at the projectile (p) velocity $1,2,3)$.

Such iunlilluum states are presumed to be formed near the exit surface of a solid target. The purpose of the present note is to explore the physical localisation of such states, and perhaps thus infer their stability within solid targets.

Theories based on Born approximation predict well the peak shapes of such electron velocity spectra ${ }^{2)}$. In general, it appears that a second Born term is necessary ${ }^{4}$ ). A true three-body treatment ${ }^{5)}$ based on Faddeev theory is equally successful and suggests also the origin of a background term in the velocity spectrum. Fig.l shows a typical spectrum ${ }^{6}$ ) taken after transmission of a $1.5 \mathrm{MeV}$ proton beam through a carbon foil target.

The process (1) may be considered as a pick-up process. We will now explore a treatment based on the well-known $R$-matrix theory of nuclear reactions. In this approach, known two-body interacting states of the final 3-body system are used to construct a density-of-final-states function ${ }^{7,8)}$. The simplest example of such a reaction is illustrated by the nuclear reaction ${ }^{9}$ )

$$
\mathrm{p}+{ }^{9} \mathrm{Be} \rightarrow \mathrm{d}+{ }^{8} \mathrm{Be}
$$

which feeds particle-unstable states of the Be nucleus, chiefly the ground state at $0.096 \mathrm{MeV}$ above the $(\alpha+\alpha)$ state. The energy spectrum of deuterons, or of decay products, may be described by the function

$$
p(E)=\frac{1_{2} \Gamma(E)}{\left(E_{r}-E\right)^{2}+\left({ }_{2} \Gamma(E)\right)^{2}}
$$

where $\Gamma$ is the $\left({ }^{8} \mathrm{Be}\right)$ width and $E_{r}=0.093 \mathrm{MeV}$. Experimental data with a fit using 2 final states of ${ }^{8} \mathrm{Be}$, are shown in Fig.2, taken with a $40 \mathrm{MeV}$ proton beam at Michigan State University ${ }^{10}$ ). We note that the final-states density depends only on the known states of the system: resonant and bound (if present), but not "scattering" states. (Note the structure near channel 50 in Fig.2.)

We turn to the reaction (1). It is clear that there are no resonant states of the $(\mathrm{p}+\mathrm{e})$ system. However, a density-of-states function must include bound states. We saw in Fig. 2 the nearly-bound ground state of ${ }^{8} \mathrm{Be}$, and 
there was a "tail" to higher energy. This is a ghost ${ }^{8}$, predicted by eq. (3) by the strong energy-dependence of $\Gamma(E)$. A straightforward computation may similarly be made of the density of final states of the proton-electron. system in terms of its bound states, and their ghosts: these states occur at energies $-R_{y} / n^{2}$, with orbital angular momentum 0 to $n-1$. The furthest from threshold is at $E_{r}=-13.6 \mathrm{eV}$, and there is no obvious reason to assume any explicit $n$-dependence of feeding of the terms in $p$ (eq.3). A many-level R-matrix generalisation of (3) ${ }^{11}$, has been used to calculate the density-ofstates function of $(p+e)$. In the R-matrix method ${ }^{12)}$, one must choose a channel radius which best fits the data, and impose a constant boundary condition on the eigenstates at that radius. Referring to Fig.3, the radial s-states of the $H$ atom all have their first maximum near $a_{0}=0.529 \AA$, the Bohr radius, thus forming an acceptable set of $R$-matrix states. A calculated density-of-states function at channel radius ${ }{ }_{0}$ was calculated and is shown by the dashed curve in Fig.1 (that the $2 \mathrm{~s}$, 3s... states extend beyond $a_{0}$ does not violate the $R$-matrix requirement that there be no polarising interaction beyond the channel radius, for this one-channel, fixed-potential case). The is (ground) state is a dominant component of the fit.

Thus an R-matrix ghost state, comprising mainly the "tail" of the ground state, is consistent with the velocity spectrum of electrons from the $(p+e)$ system. It follows that such a state, of size $a_{0}$, must have some stability within a solid target. In an amorphous target one may expect it to survive a thickness $t$, where

$$
\mathrm{N}_{\mathrm{t}} \pi \mathrm{a}_{\mathrm{o}}^{2} \approx \mathrm{l}
$$

with $N$ the atom density of the target. Eq. 4 implies a value in carbon of $t=10 \AA$, i.e. a surface density of $0.2 \mu \mathrm{g} / \mathrm{cm}^{2}$. It could also be expected to be channeled in a single-crystal target. Thus continuum charge states must be assumed to exist well back from the last surface of a foil target, and their properties must be allowed for alongside those of others such as "wake-riding" states.

\section{REFERENCES}

1) Crooks \& Rudd, Phys.Rev.Lett.25 (1970)1599.

2) Dettmann, Harrison \& Lucas, J.Phys.B.7(1974) 269.

3) Vane et al. Phys.Rev. Lett. 40 (1978) $10 \overline{2} 0$.

4) Shakeshaft \& Spruch, Phys.Rev.Lett. 41(1978)1037.

5) Macek, Phys.Rev. A.1 (1970) 235.

6) Lucas \& Steckelmachēr, to be published.

7) Beckner, Jones \& Phillips, Phys.Rev. 123(1961)255.

8) Barker \& Treacy, Nucl.Phys. 38(1962) 33 .

9) Hay et al. Austral.J.Phys.20(1967) 59.

10) Barker et al. Austral.J.Phys.29(1976) 245.

11) Treacy, Nucl.Phys. A193(1972) $\overline{97}$.

12) Lane \& Thomas, Rev.Mod.Phys. $30(1958) 257$. 
FIGURE CAPTIONS

Fig.1. Electron forward velocity spectrum from charge-exchange of $1.5 \mathrm{MeV}$ protons in carbon. The dashed curve is a calculated fit.

Fig. 2 Deuteron energy spectrum from the reaction ${ }^{9} \mathrm{Be}(\mathrm{p}, \mathrm{d}){ }^{8} \mathrm{Be}$ at a proton energy of $39.91 \mathrm{MeV}$.

Fig. 3 Illustration of hydrogenic wave functions (from Condon \& Shatley, Theory of Atomic Spectra, CUP, 1935) 


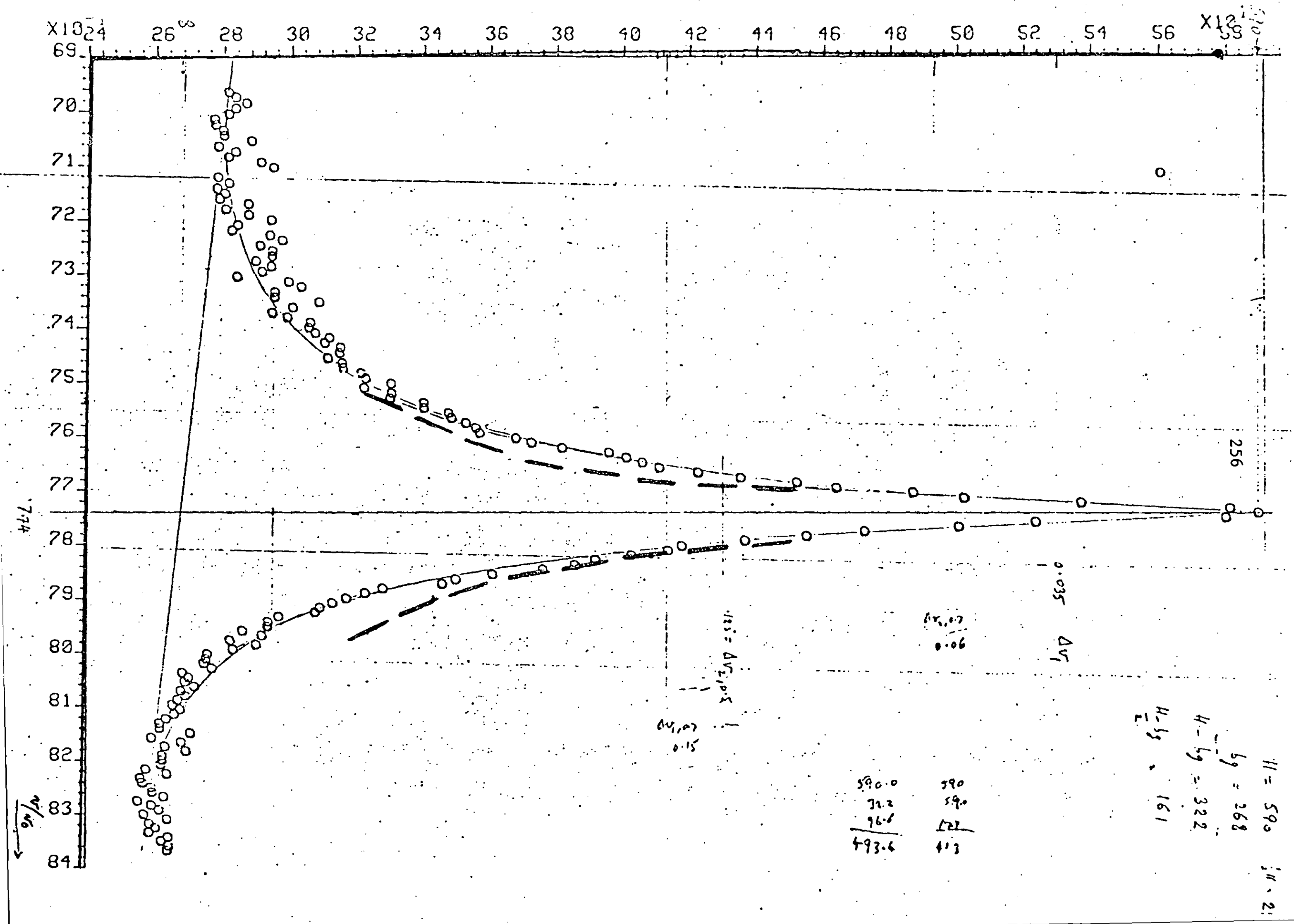




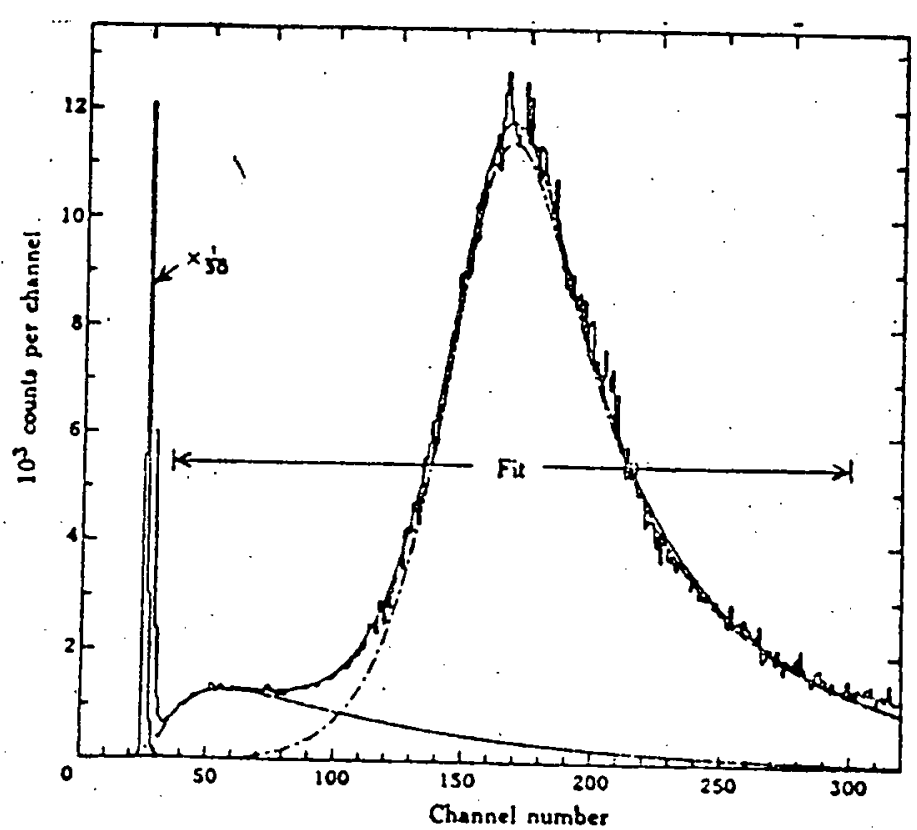

- Calculated (solid curve; and measured (histogram) spectra of deuterons at $10^{\circ}$ from the reactiols ${ }^{9} \mathrm{Bc}(\mathrm{p}, \mathrm{d})^{3} \mathrm{Be}$ with a proton bombarding energy of $39.91 \mathrm{MeV}$. The $l=0$ and 2 components of the calculated eurve are plotted as dashes and dot-dashes respectively. For channel numbers less than 30 or greater than 110. the counts per channel are renormalized to $100 \mu \mathrm{C}$ of coltected charge. The data in channcls 35 to 300 are used in the fitting procedure, with the $l=0$ component notmalized to the ground state main peak. 


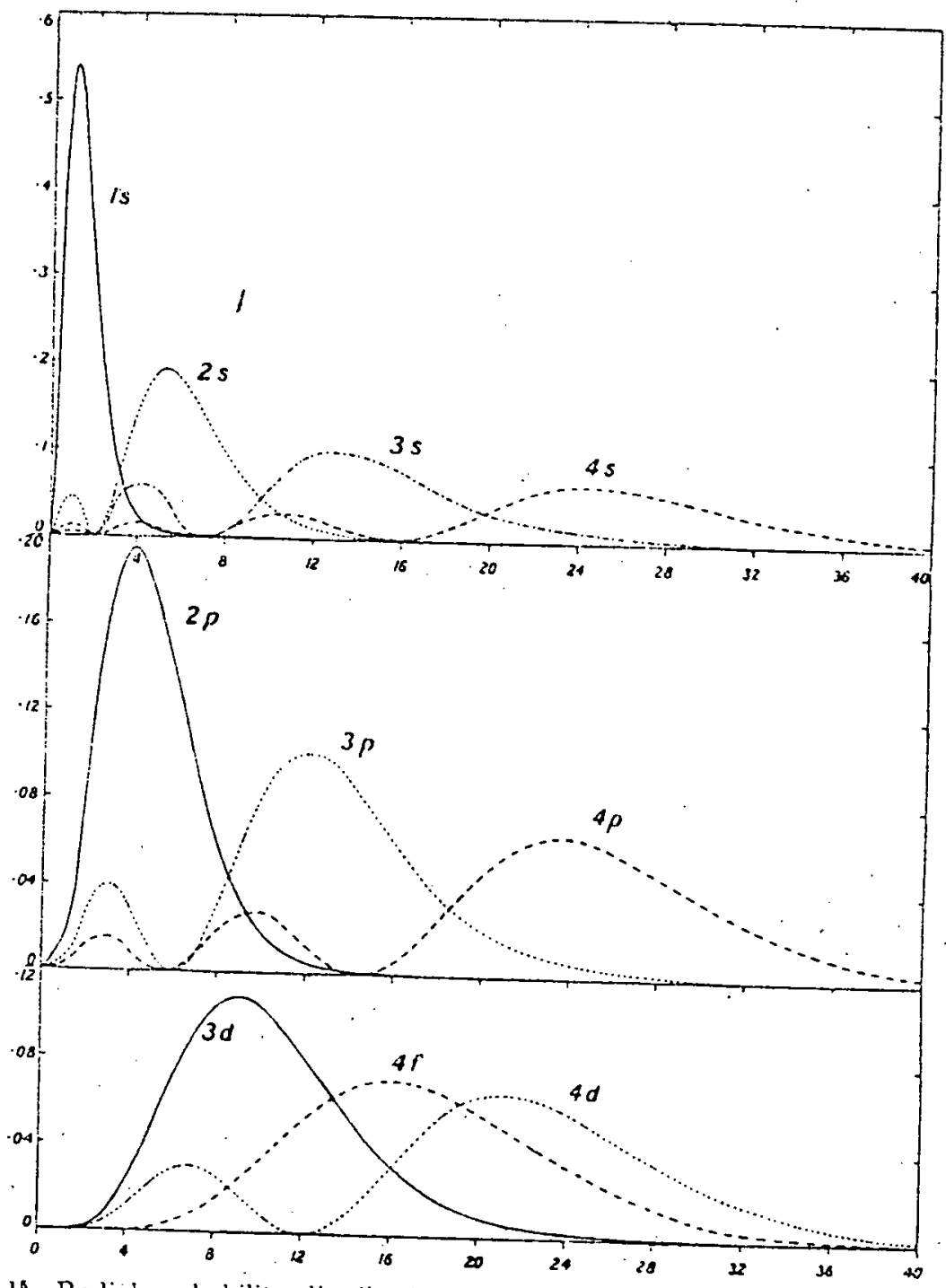

Fig. $1^{5}$. Radial probubility distribution a $R^{2}(n l)$ for severnl of the lowest levels in hydrugen. (Alscisisn is the nulius in atomic units.)

In order to normalize the rndial functions we need the result

$$
\int_{0}^{\infty} \rho^{21+2} c^{-\rho}\left[L_{n+l}^{2 l+1}(\rho)\right]^{2} d \rho=\frac{2 n[(n+l) !]^{3}}{(n-l-1) !},
$$

which is readily obtaned from the gencrnting function or from Eckart's 
AFTERNOON SESSION

21 August 1979

R. Baragiolia, CHAIRMAN 
OPTICAL MEASUREMENTS OF MOLECULAR DISSOCIATION BY THIN FOILS

\author{
H. G. Berry
}

Argonne National Laboratory, Argonne, Illinois 60439

\author{
ABSTRACT \\ This is a brief review of the types of \\ optical measurements and their results.
}


Figure 1 compares schematically the two experiments of observing atomic radiation from an excited atom or ion after a thin solid foil in the first case using an incident atomic ion, and in the second case, using an incident molecular ion. We can assume that the major effects in producing the atomic excited state occur near the final surface of the foil (within the limits of the dotted line shown in Fig. 1). Hence differences in the atomic radiation, which is observed far from this surface interaction region, will be due mainly to differences in the two cases within this surface interaction region. As most molecular ion dissociation experiments use particle detection, it is useful to compare the advantages and disadvantages of. photon detection: the principal advantage is that specific excited states of a given ion can be detected (through the photon emission). Thus, neutrals as well as charged ions can equally well be measured. Several particle experiments have qualitatively shown the sensitivity of the type of molecular state (excited or ground state) to the dissociation within the solid or gas target, to the transmission probability of molecular ions through solids, to the energy, angle ring patterns of the dissociated products, etc., and to the "central peak" parameters. It has become apparent that for improved descriptions of these dissociation processes, we need to follow the time development of the excited molecular energy levels as a function of internuclear separation. Various groups, e.g. Cue, Remillieux et al, Gemmell et al, and LeviCetti et al, have already began these approaches. However, such molecular term diagrams are largely unknown. Possibly only for the lowest states of the simplest molecular ions, $\mathrm{H}_{2}^{+}$and $\mathrm{HeH}^{+}$, have these diagrams been calculated. The optical measurements can provide measurements on unique excited states: both atomic states, which couple only to a few molecular orbitals at large internuclear separations, and molecular states in the probably rare cases of excited but bound state molecules being transmitted through the targets. 
Three principal types of photon measurements have been made thus far. These are total light yield, doppler profiles and optical polarization. Doppler profiles have been measured ${ }^{1}$ to give directly the explosion kinetics for various molecular projectiles such as $\mathrm{OH}^{+}, \mathrm{OH}_{2}^{+}, \mathrm{OH}_{3}^{+}$as functions of the final excited state of a neutral hydrogen atom. No dependence or excited state was observed. Such measurements are not as precise as the direct particle measurements (which average over all excited states) both from statistics (lack of photons) and from doppler broadening in the detection system. Note that these measurements depend on the total interaction process (Fig. 1), not just the surface excitation.

Total light yields have been compared for the various excited neutral helium states when using $\mathrm{He}^{+}$or $\mathrm{HeH}^{+}$projectiles (see T. J. Gay et al, this conference). I just note here that the yields depend sensitively on the atomic state being observed, showing that the molecular orbital picture must be invoked. The yields in general depend on the proximity of the partner ion of the original molecule in the surface interaction region. This proximity length must be of the same order as the length of the surface interaction region, and hence this type of measurement helps in analyzing the surface interaction of single ions. Our measured length appears to be in the region of 10 to $50 \AA$.

Finally, the polarization measurements provide an analysis of the alignment of the ejected excited ions. This alignment can be expected to be sensitive to the field of the partner ion as it passes through the surface interaction region. We show (see Gay et al, this conference) that the alignment, also depends sensitively on the internuclear separation of the ions at the final surface, and on. the excited states. The results for the transitions observed (Fig. 2) must be interpreted on the molecular energy diagrams for the $\mathrm{HeH}^{+}$molecule. 
The $n=2$ and $n=3$ states of neutral hydrogen have also been measured through the emission of Lyman $\alpha(n=1-2)$ by Gay et al, and of Balmer $\alpha(n=2-3)$ by Schectman et al (see Fig. 3). These measurements are sensitive to the mixing of states of different orbital angular momentum ( $s, p$ and $d$ states). Consequently, thelr interpretation in terms of molecular energy diagrams is more difficult. The close degeneracies cause many level crossings at large internuclear separations.

Work supported by the Division of Basic Energy Sciences of the U. S. Department of Energy. 
REFERENCES

1. G. Berry, A. E. Livingston and G. Gabrielse, Phys. Letts. 64A, 68 (1977). 


\section{FIGURE CAPTIONS}

Figure 1. Geometry of fast atomic ions (above) and fast molecular ions (below) incident on a thin foil. Atomic radiation (hv) is observed downbeam from the foil at a large distance relative to the final surface interaction region (within the dotted line).

Figure 2. Energy level diagram for neutral helium, showing the $\dot{n}=2-3$ transitions. The individual transitions shown can be spectrally resolved using either interference filters or a monochromator. The transitions marked have been analyzed in detail for foil dissociated $\mathrm{HeH}^{+}$ions.

Figure 3. Energy level diagram for neutral hydrogen. Balmer $\alpha$ radiation $(n=2-3)$ is a partially coherent mixing of the $2 s-3 p, 2 p-3 d$ and $2 \mathrm{p}-3 \mathrm{~s}$ transitions. 
FOIL

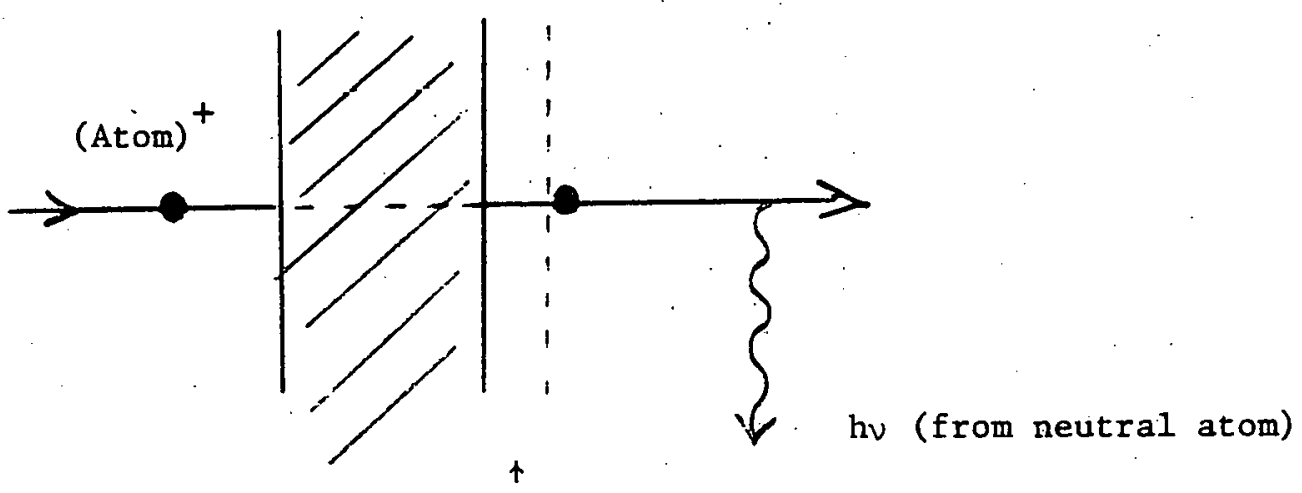

surface

layer

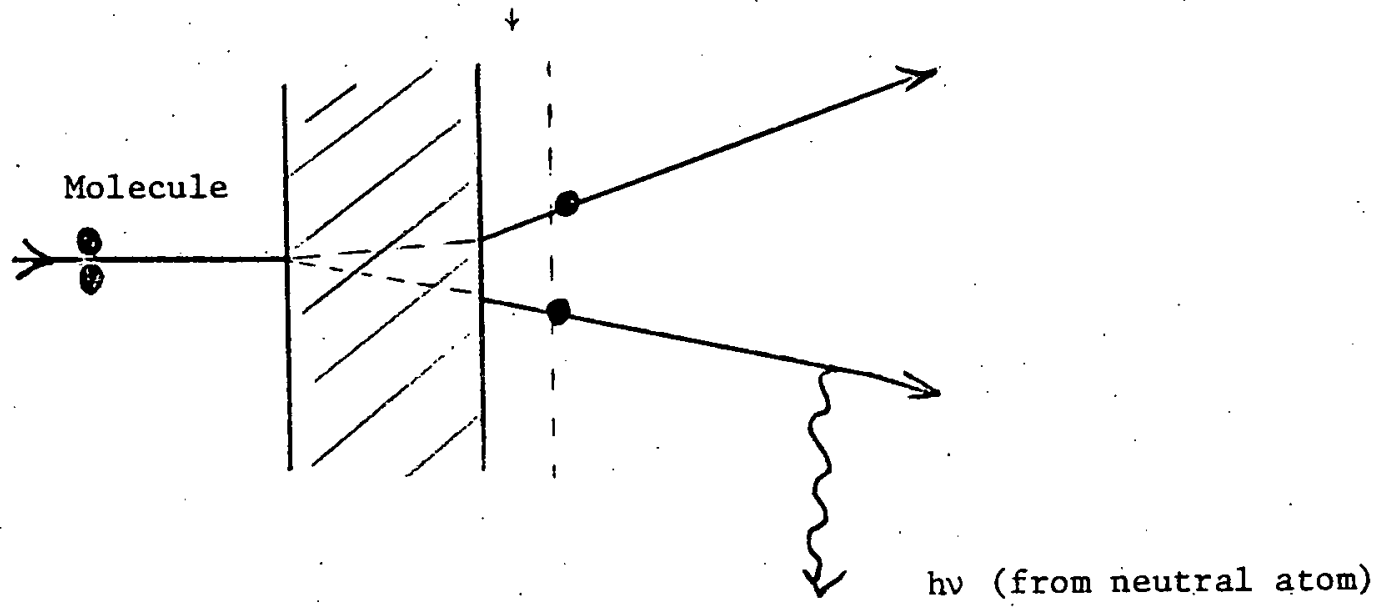

Figure 1 


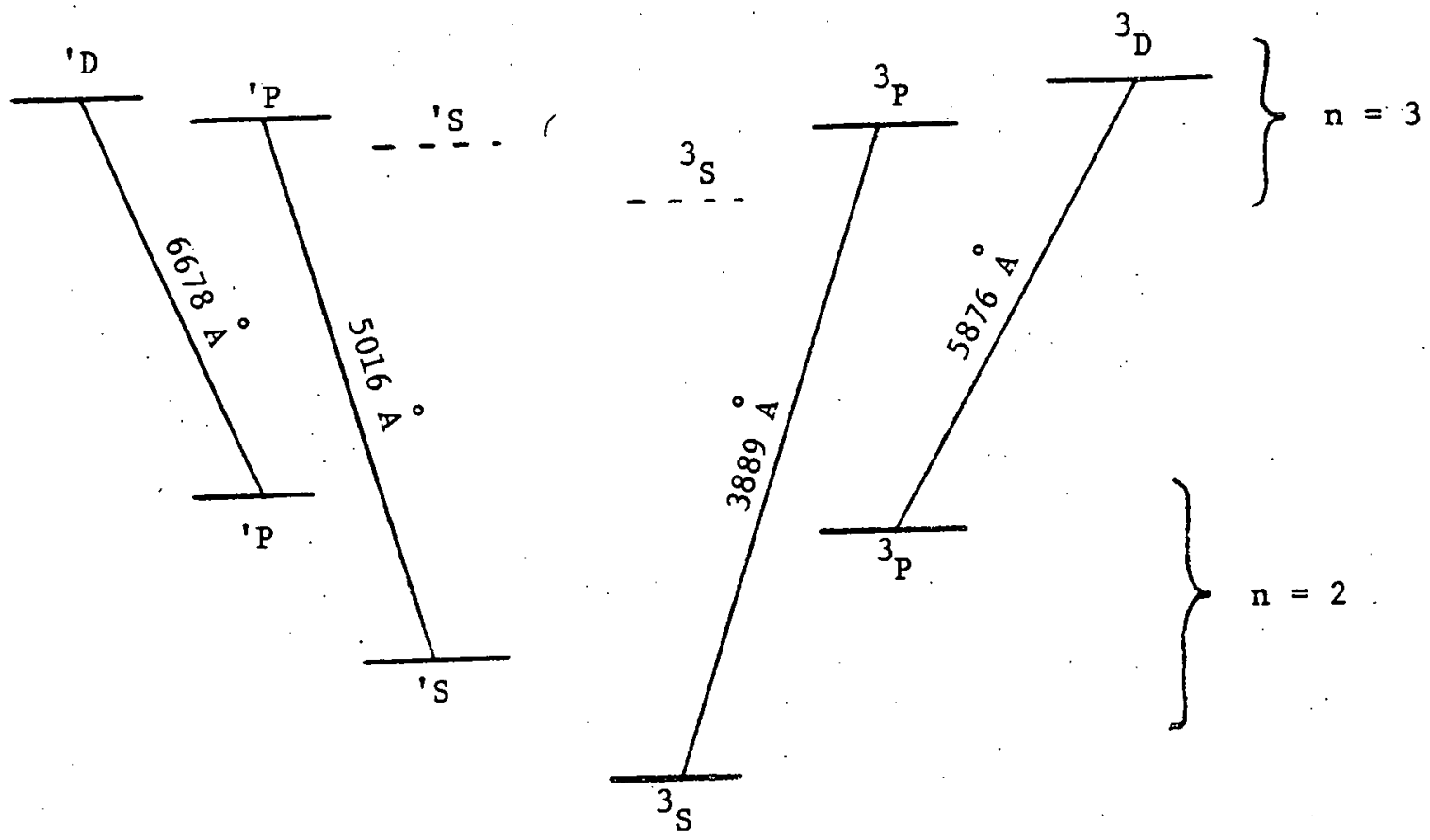

HELIUM

Figure 2 


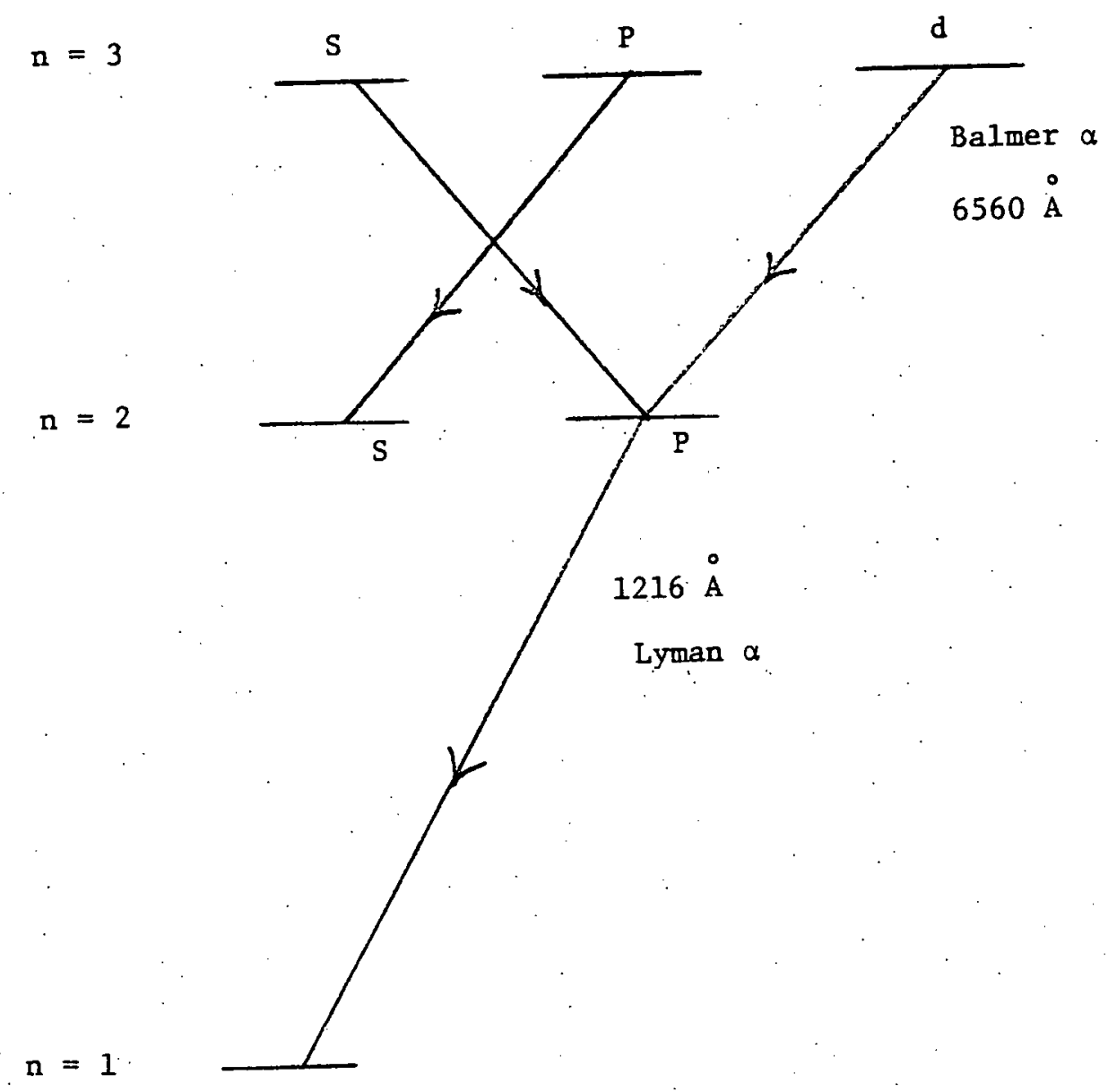

HYPROGEN

Figure 3 


\section{THIS PAGE}

\section{WAS INTENTIONALLY \\ LEFT BLANK}




\section{FOIL INDUCED DISSOCIATION OF FAST $\mathrm{H}_{2}^{+}$MOLECULAR IONS}

\section{STUDIED BY RUANTUM BEAT SPECTROSCOPY*}

R. M. Schectman and D. G. Ellis, THE UNIVERSITY OF TOLEDO

We have employed quantum beat spectroscopy to study the dissociation of fast $\mathrm{H}_{2}{ }^{+}$molecular ions leading to excited hydrogen atoms in the $\mathrm{n}=3$ quantum states. The complexity of the final state due to the near degeneracy of the levels makes it convenient to supplement conventional optical spectroscopy techniques with those of quantum beat spectroscopy and allows a wealth of detailed information concerning the excitation process to be obtained.

Fast $\mathrm{H}_{2}{ }^{+}$ions $(280-350 \mathrm{KeV}-\mathrm{v} \approx 5 \mathrm{~mm} / \mathrm{ns})$ impinge upon carbon foils of thickness $t_{F}=2-25 \mu \mathrm{g} / \mathrm{cm}^{2}$; at a distance $x$ downstream of the foit, $H_{\alpha}$ radiation from neutral $\mathrm{H}$ atoms formed in the dissociation of the $\mathrm{H}_{2}{ }^{+}$is detected by a low-noise red-sensitive photomultiplier tube and a narrrow band filter, and the intensity of the $H_{\alpha}$ radiation polarized parallel to the beam $\left(I_{11}\right)$ and polarized perpendicular to the beam $\left(I_{\perp}\right)$ is measured as a function of $\mathrm{x}$; quantum beats are observed and the seven quantities:

the excitation amplitudes

$$
\begin{aligned}
& \sigma_{s}=\sigma_{s 0} \\
& \sigma_{p}=\sigma_{p 0}+2 \sigma_{p l} \\
& \sigma_{d}=\sigma_{d 0}+2 \sigma_{d 1}+2 \sigma_{d 2},
\end{aligned}
$$

the alignments

$$
\begin{aligned}
& A_{p}=\sigma_{p 0}-\sigma_{p l} \\
& A_{d}=\sigma_{d 0}+\sigma_{d l}-2 \sigma_{d 2} .
\end{aligned}
$$

and the complex s-d mixing amplitude with magnitude $\sigma_{s d}$ and phase $\delta$

*Work supported in part by the U.S. National Science Foundation.
Where the $\sigma^{\prime} s$ are elements of the $H(n=3)$ density matrix in the $\ell_{\ell}$ representation. 
are extracted from the data. Thus detailed information concerning the magnetic substate populations of each set of $\ell$ levels is obtained. Our long range program is to determine these quantities as a function of the foil thickness $t_{F^{--a n d}}$ thereby, as a function of the interproton separation upon emergence from the foil (presumably the separation when an electron is captured forming $\mathrm{H}_{2}{ }^{+}$in a dissociating state); in addition, these results are to be compared with the $H(n=3)$ density matrix parameters for incident $\mathrm{H}^{+}$ions of the same velocity, where molecular formation cannot occur.

\section{Analysis}

Figure (1) shows the optical transitions selected by the interference filter and the quantum beats which may be observed. (For pedagogic purposes, the hyperfine structure is not displayed here.) For the case of excitation of atomic hydrogen with the beam providing the only definable spatial direction, it is easy to show that for spin independent production

$$
I_{11}+2 I_{\perp}=\kappa\left\{C_{s} \sigma_{s} e^{-\Gamma_{s}\left(t-t_{0}\right)}+C_{p} \sigma_{p} e^{-\Gamma_{p}\left(t-t_{0}\right)}+C_{d} \sigma_{d} e^{-\Gamma_{d}\left(t-t_{0}\right)}\right\}
$$

where $k$ is the overall detection efficiency, the $\Gamma^{\prime} s$ are the known decay constants for $H(n=3)$, the $C^{\prime}$ s are also known constants and $\left(t-t_{0}\right)$ is the time after excitation; thus, a least squares fit of $I_{\|}+2 I_{\perp}$ to Eq. (1.) provides the excitation amplitudes $\sigma_{s}, \sigma_{p}$ and $\sigma_{d}$; similarly

$$
\begin{aligned}
& I_{11}-I_{\perp}=k\left\{C _ { p } { } ^ { \prime } A _ { p } e ^ { - \Gamma _ { p } ( t - t _ { 0 } ) } \left(1+C_{p}{ }^{\prime \prime} \cos \omega_{p}\left(t-t_{0}\right)\right.\right. \\
& +c_{d}{ }^{\prime} A_{d} e^{-\Gamma_{d}\left(t-t_{0}\right)}\left(1+c_{D}^{\prime \prime} \cos \omega_{d}\left(t-t_{0}\right)\right. \\
& +\sum_{d_{3 / 5}}^{d_{5 / 2}} c^{\prime \prime}{ }_{s d}\left|\sigma_{s d}\right| e^{-\Gamma s d}\left(t-t_{0}\right) \cos \omega_{d s}\left(t-t_{0}+\delta\right)
\end{aligned}
$$

where $\omega_{p}$ and $\omega_{d}$ represent the known $p$ and $d$ fine structure splitting frequencies and $\omega_{\text {ds }}$ the energy differences between the $d_{5 / 2}$ and $d_{3 / 2}$ levels and the $s_{1 / 2}$ level; again the $C^{\prime} s$ and $C^{\prime \prime} s$ are known coefficients (Equation (2) is 
modified somewhat by hyperfine structure and this is taken into account in the actual analysis); a fit of $I_{12}-I_{1}$ to Eq. (2)--or rather the exact equation in the presence of HFS--gives rise to the alignments $A_{p}$ and $A_{d}$ and the sd mixing term $\sigma_{s d}$, $\delta$.

For the case of incident molecules, the interproton direction furnishes a second definable spatial direction which must be considered and which must subsequently be averaged over. If one assumes that the incident molecules lose their bonding electrons shortly after striking the foil, the protons then separate due to coulomb explosion and multiple coulomb scattering. This experiment studies those proton pairs which capture an electron somewhere near the exit surface and form an $\mathrm{H}_{2}^{+}$molecule in a state which dissociates to a proton and a hydrogen atom in an $n=3$ state. This molecular formation occurs in the field of the foil surface, and the states formed are a result of the competition between the intermolecular forces and the surface field. The purpose of this experiment is to study this competition (and, therefore, the surface field) as a function of interproton separation. The result of this competition, it can be argued, is to maintain the form of Eq. (1) and Eq. (2); however the coefficients $C^{\prime \prime}{ }_{p} C^{\prime \prime}{ }_{d}$ and $C^{\prime \prime}$ sd no longer can be predicted simply by angular momentum algebra but depend also upon the details of the molecular formation process. In fact, it is easy to argue that--after averaging over interproton orientations relative to the beam--the $C^{\prime \prime} s$ are likely to vanish--although, experimentally, this is not the case. For the purpose of phenomenologically describing observations, it is useful to define beat attenuation coefficients for $p$ and $d$ FS beats as

$$
\alpha \equiv \frac{C^{\prime \prime} \text { OBS-MOL }}{C^{\prime \prime} \text { THEOR-AT }} .
$$


Preliminary Data

Figure (2) shows the beat curve observed when $170 \mathrm{KeV}$ protons traverse $6 \mu \mathrm{g} / \mathrm{cm}^{2}$ carbon foils (dwell time $t_{D}=6.4 \mathrm{fs} *$ ). The dashed curve is the fit to theory given by Eq. (2) extended to include HFS. Since the quality of the fit is quite good, the data can be well represented by the fit curve alone and this is shown--for comparison with the molecular measurements--in Figure (3a). Major features to be noticed here include:

a) the high frequency ( $p$ level FS) beat which decays in amplitude with the p Fifetime of 5.4 ns

b) the lower frequency (d level FS) beat which decays in amplitude with the d lifetime of 15.6 ns

c) the corresponding double exponential decay of the mean value of $I_{11}-I_{\perp}$ with coefficients corresponding to both $A_{p}$ and $A_{d}$ being negative.

When $340 \mathrm{KeV} \mathrm{H}_{2}^{+}$strike a $6 \mu \mathrm{g} / \mathrm{cm}^{2}$ foil, the observations are quite different. The beat data cannot be fit by Eq. (2) without introducing the attenuation coefficients defined in Eq. (3). When this is done, the fit to the experiment is quite satisfactory and is shown in Figure (3b). Here it is clear that

a) the high frequency beat is greatly attenuated, $\alpha_{p} \approx .05$,

b) the low frequency beat is--perhaps surprisingly--only slightly decreased in amplitude, $\alpha_{d} \sim 0.5-0.6$, and

c) the $p$ alignment has changed sign.

For reference it should be noted that in traversing a $6 \mu \mathrm{g} / \mathrm{cm}^{2}$ foil, the mean separation due to coulomb explosion along would be approximately $2.8 \AA$ while, due to multiple scattering, the expected mean separation is increased to about $6.5 \AA$. Of course, a spread of separations--due to both the spread of initial separations and the statistical nature of the multiple

* Well in the "blue" region of Remillieux and Brandt. 
scattering-results. Figure (3c) shows similar results for $15 \mu \mathrm{g} / \mathrm{cm}^{2}$ foils, where $t_{D}=16$ fs and a mean separation upon emergence of nearly $30 \AA$ is predicted, mostly a result of multiple scattering. It should be noted that, while the beat pattern is approaching that observed for incident protons, significant differences remain even for these large interproton separations.

Since the Bohr radius of a $H$ atom in the $n=3$ state is roughly $4.5 \AA$, it is clear that production of dissociating molecular states must be considered. To further study the influence of the foil surface upon the states produced, measurements with a variety of foil thicknesses--especially those smaller than $6 \mu \mathrm{g} / \mathrm{cm}^{2}$, but also for some larger than $15 \mu \mathrm{g} / \mathrm{cm}^{2}$--are underway. In addition, models of the excitation--phrased in terms of superpositions of molecular orbitals--are being constructed. In the interim, it is interesting to note one feature of some of the relevant molecular orbitals shown in Figure (4). A possible mechanism for the persistence of the d-beat shown in Figure (3b) may lie in the occurrence of the indicated pseudocrossings between MO's which ultimately connect with the $d_{5 / 2}$ and $d_{3 / 2}$ levels. No such crossings connect with the $\mathrm{p}$ levels.. Since these crossings occur at interproton separations of some $2-15 \AA$, the measurements which will be carried out as a function of $t_{D}$ should be particularly sensitive to these effects. Alternately, it may prove necessary to postulate final state interactions to produce the observed coherence between close-lying molecular orbitals. 
Figure (1). Schematic diagram (not to scale) of the optical transitions selected by the narrow band interference filter employed in this experiment. Circles indicate beating transitions and approximate beat frequencies. For simplicity, hyperfine : structure is not displayed; it is, however, accounted for in the analys is procedure employed.

Figure (2). Quantum Beat Curve $\left(I_{11}-I_{\perp}\right)$ for $170 \mathrm{KeV}$ protons incident upon $6 \mu \mathrm{g} / \mathrm{cm}^{2}$ carbon foils $\left(t_{D}=6.4 \mathrm{fs}\right)$. The dashed curve is the fit to the theory--Eq. (2)--extended to include HFS.

Figure (3). Observed Molecular Effects: Comparison of beat curves for $170 \mathrm{KeV}$ protons incident upon $6 \mu \mathrm{g} / \mathrm{cm}^{2}$ carbon foils (3a) with that of $340 \mathrm{KeV} \mathrm{H}_{2}^{+}$ions incident upon foils of similar thickness (3b) and upon foils of thickness $15 \mu \mathrm{g} / \mathrm{cm}^{2}(3 c)$. The mean interproton separations upon exit from the foil in (3b) is predicted to be about $6.5 \AA$, while that in Figure $(3 c)$ is nearly $30 \AA$.

Figure (4). Schematic diagram of some of the relevant molecular orbital levels which ultimately dissociate, leading to $H$ atoms in the observed FS states. MO's connected by $\mathrm{O}-\mathrm{O}$ ' 's display pseudn crossings in the region $2-15 \AA$. 
HYDROGEN ${ }^{H_{Q}} \quad 6563.8 . \AA$ FINE STRUCTURE ENERGY LEVELS
(NOT TO SCALE)

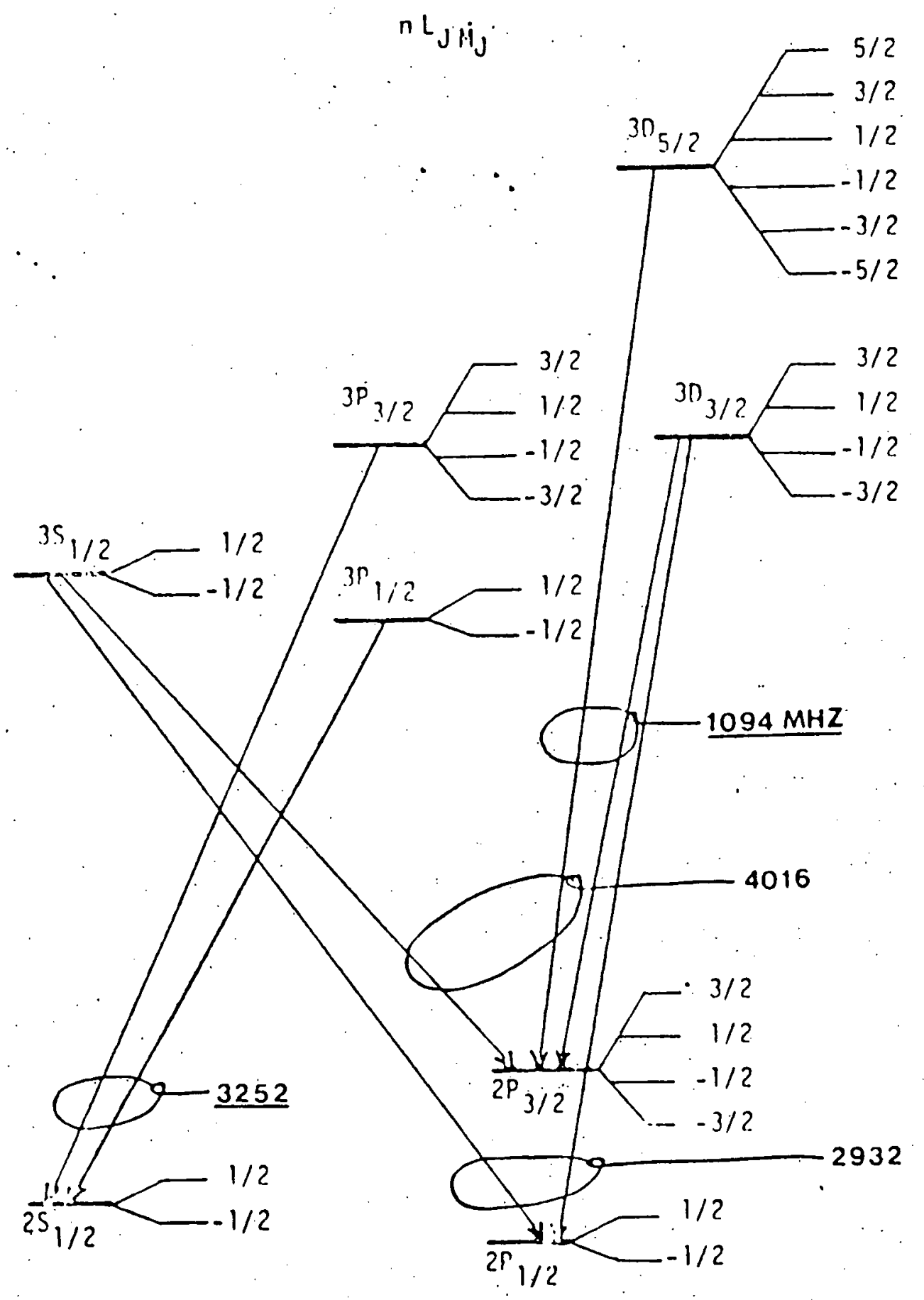

Figure 1 


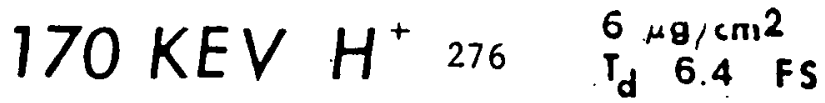

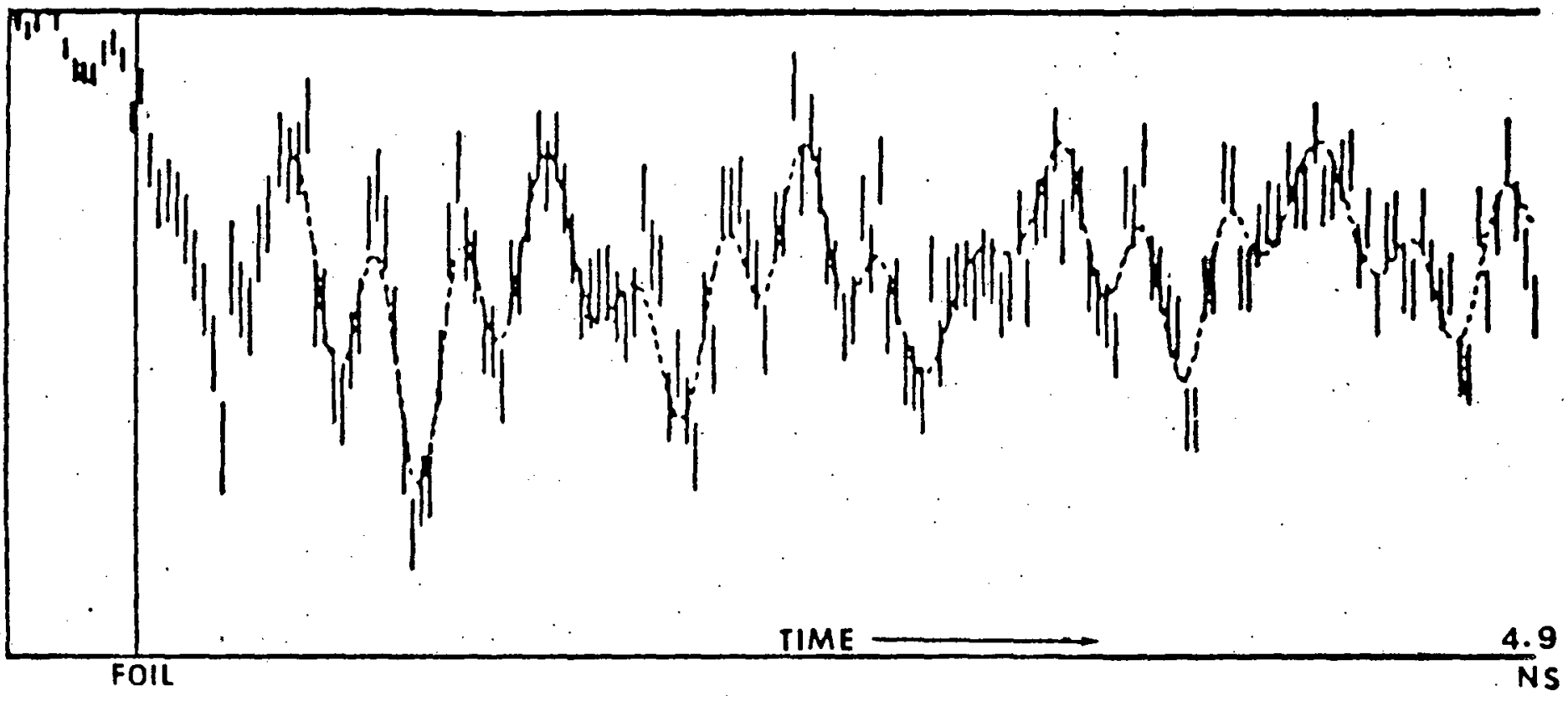

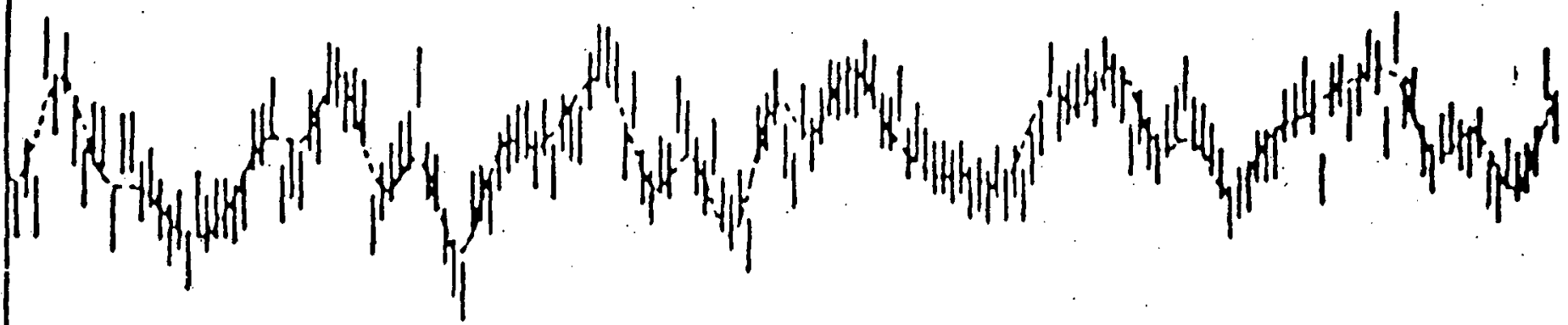

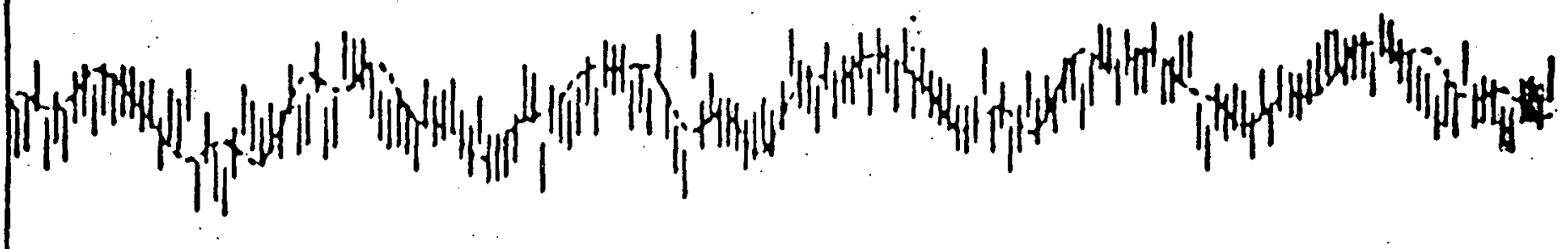

Figure 2 
MOLECULAR EFFECTS $277 \quad 170 \mathrm{KEV} / \mathrm{P}$
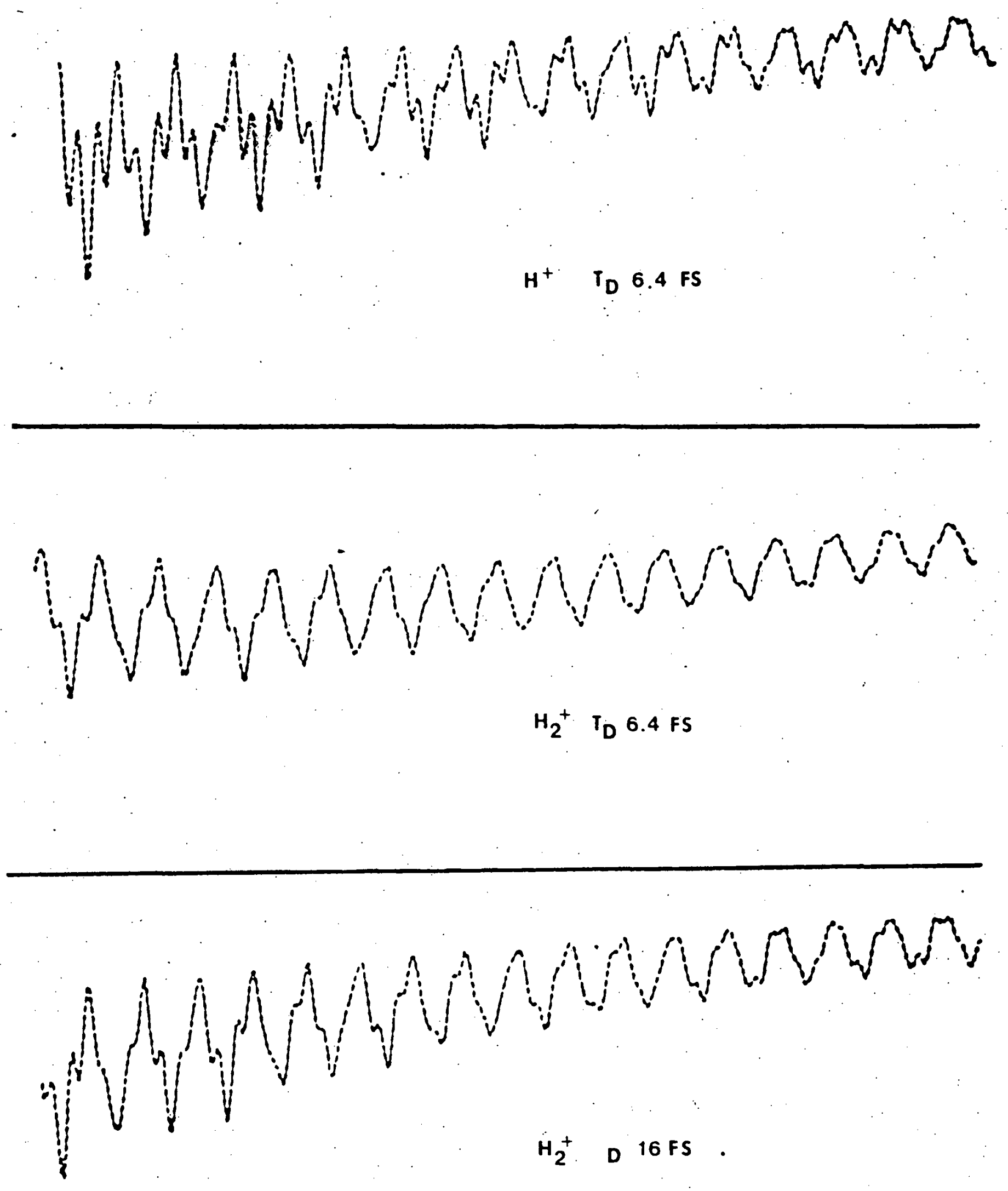

Figure 3 


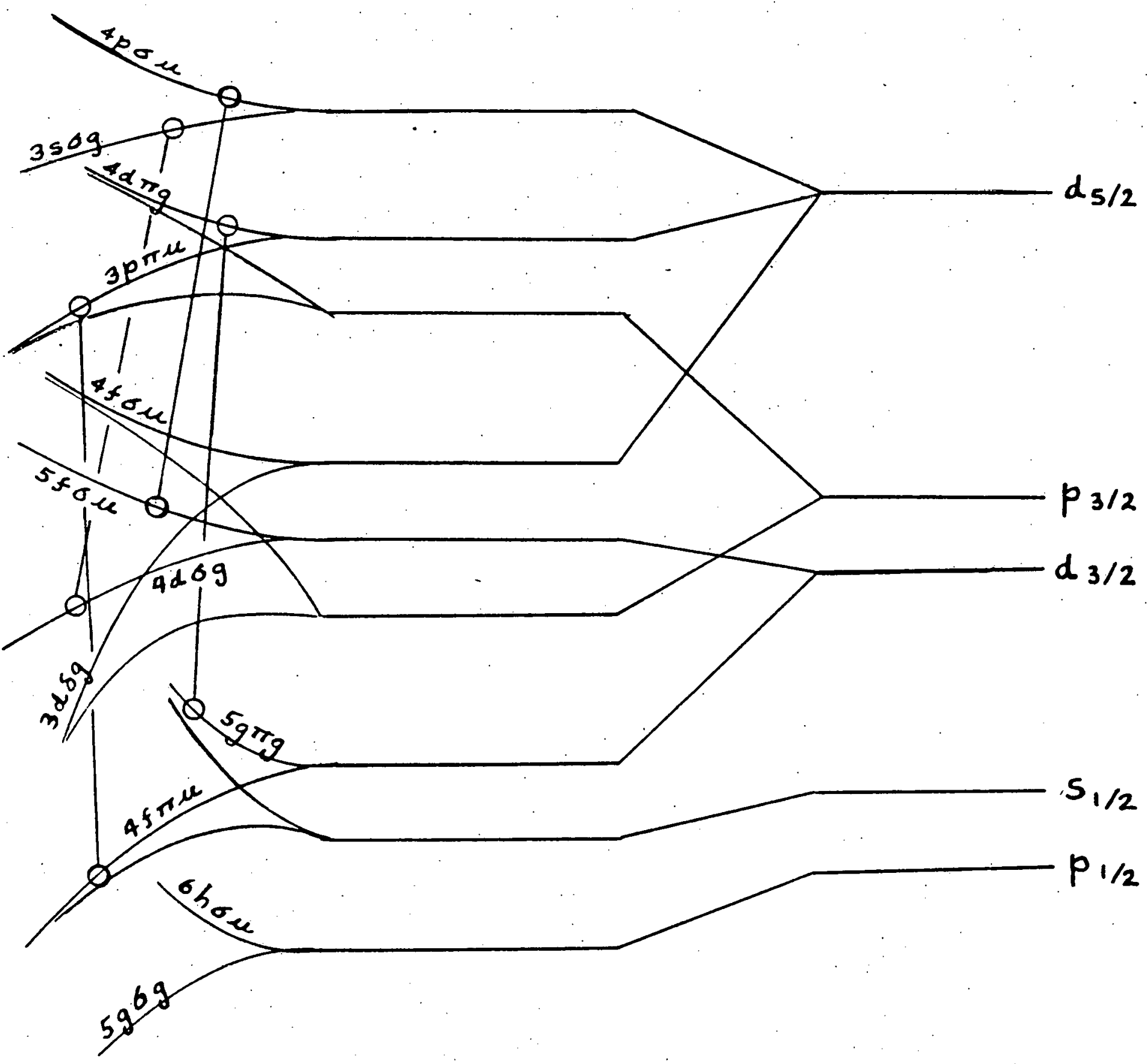

M.O. STARK»FS

$N=3 \quad F S$

Figure 4 
OPTICAL OBSERVATIONS OF MOLECULAR DISSOCIATION IN THIN FOILS

T. J. Gay * and H. G. Berry

Argonne National Laboratory, Argonne, Illinois 60439

\begin{abstract}
We present measurements of the intensity and polarization of light emitted from several excited states of neutral helium, after break-up of the $\mathrm{HeH}^{+}$ molecule in thin foils. We vary the foil thickness to study their dependence on the internuclear separation of the He and proton at the final foil surface and postulate that the variations observed are sensitive to the molecular orbitals of the dissociating $\mathrm{HeH}^{+}$molecule.
\end{abstract}


The main production of excited states in fast ions having traversed thin foils is generally accepted to take place at the exit surface of the foil. The process occurs partially through electron pick-up or electron excitation processes in the 1 to $100 \AA$ distance from the foil surface. We have previously shown that these interactions are sensitive to the foil surface temperature and have suggested that this is due directly to changes in the secondary electron distribution as the ion leaves the surface. ${ }^{1}$ Specifically, higher temperatures result in fewer secondary electrons and higher polarization or atomic alignment. This model suggests that the influence of the secondary electrons is to produce a more isotropic alignment.

In order to probe the distance parameter of these surface interactions, we have used the bombarding molecular ion $\mathrm{HeH}^{+}$. We are then able to observe the influence on the alignment of neutral helium excited states of a nearby proton. The proximity of the proton is varied by the simple procedure of measuring the alignment using different foil thicknesses. In Fig. 1, we show the results of our calculations of the average He-H internuclear separation as a function of foil thickness for ions of 125 and $650 \mathrm{keV}$ energy (per He). Multiple scattering, energy loss and Coulomb explosion have been taken into account.

In Figs. 2 and 3, we show the influence of the nearby proton on the total populations of several excited states of neutral helium. We note that the proximity of the proton induces enhanced population in the $3 p 1_{P}$ and $3 d{ }^{3} \mathrm{D}$ states, but not in the $3 \mathrm{p}^{3} \mathrm{p}$ states. The enhancement is a factor of three for the thinnest foils we have used, and it dies away much faster for the ${ }^{1}$ p state than for the ${ }^{3} \mathrm{D}$ state. The intensity is reduced to $60 \%$ for foil thicknesses of $4 \mu \mathrm{g} / \mathrm{cm}^{2}$ for the ${ }^{1} \mathrm{P}$ state, but not until $10 \mu \mathrm{g} / \mathrm{cm}^{2}$ thicknesses for the ${ }^{3} \mathrm{D}$ state. We show in Fig. 3 that the control experiment with incident $\mathrm{He}^{+}$ions, shows no intensity dependence on foil thickness. 
Figures 4,5 and 6 show that the linear polarization fraction $M / I$, which is a measure of the atomic alignment, is reduced at foil thicknesses where the exciting proton is close to the excited neutral helium atom. Thus, the presence of the proton tends to make the average excitation more isotropic. This occurs for the cases when the polarization is either positive ( $3 \mathrm{p}^{1} \mathrm{P}$ and $3 \mathrm{p}^{3} \mathrm{P}$ initial states) and negative ( $3 \mathrm{~d}^{3} \mathrm{D}$ initial state). However, it is interesting to note from Figs: 4-6 that the distance of influence of the neighboring proton on the alignment is very different for the different excited states. It ranges from 20 a.u. for the $3 p{ }^{1} P$ state, $30 \mathrm{a} . \mathrm{u}$. for the $3 \mathrm{p} \cdot{ }^{3} \mathrm{P}$ state to nearly $100 \mathrm{a} \cdot \mathrm{u}$. for the $3 \mathrm{~d}^{3} \mathrm{D}$ state. : The wide variations in these internuclear distances (which must be similar to the effective range of the surface interaction in formation of these states) suggest that a molecular model should be used to explain the variations. In particular, the $3 p^{3} p$ and $3 p{ }^{1} p$ wave functions are very similar. However, the molecular orbitals of dissociating $\mathrm{HeH}^{+}$into these levels are very different. Level crossings of dissociative levels at these large internuclear separations may account for the observed differences. Tom Green ${ }^{2}$ has recently calculated the relevant $\mathrm{HeH}^{+}$molecular orbitals as a function of internuclear separation, and we are investigating this problem further.

Work supported by the Division of Basic Energy Sciences of the U. S. Department of Energy. 


\section{FOOTNOTE AND REFERENCES}

*Also at the Department of Physics, University of Chicago, Chicago, IL 60637, USA.

I. J. Gay and H. G. Berry, Phys. Rev. Al9, 952 (1979).

2 T. Green, private communication. 


\section{FIGURE CAPTIONS}

Figure 1. The HeH internuclear separation as a function of carbon foil thickness at exit energies of $125 \mathrm{keV}$ and $650 \mathrm{keV}$.

Figure 2. The light yield of the HeI transition $2 p^{3} \mathrm{P}-3 \mathrm{~d}^{3} \mathrm{D}$ at $5876 \AA$ as a function of foil thickness using $\mathrm{He}^{+}$projectiles (open circles) and $\mathrm{HeH}^{+}$projectiles (closed circles). The beam energy was adjusted to give $650 \mathrm{keV}$ exit energy for the emitting atom.

Figure 3. The light yields for the two HeI transitions $2 s{ }^{1} p-3 p{ }^{1} p$ at $5016 \AA$ and $2 s^{3} \mathrm{~S}-3 \mathrm{p}{ }^{3} \mathrm{P}$ at $3889 \AA$ as a function of foil thickness using $\mathrm{HeH}^{+}$projectiles.

Figure 4. The linear polarization fraction of $2 \mathrm{~s}^{1} \mathrm{~S}-3 \mathrm{p}{ }^{1} \mathrm{P}, 5016 \stackrel{\circ}{\mathrm{A}}$, as a function of foil thickness, using $\mathrm{HeH}^{+}$and $\mathrm{He}^{+}$projectiles at $125 \mathrm{keV}$ He exit energy.

Figure 5. The linear polarization fraction of $2 s^{3} \mathrm{~S}-3 \mathrm{p}^{3} \mathrm{p}, 3889 \AA$, as a function of foil thickness, using $\mathrm{HeH}^{+}$and $\mathrm{He}^{+}$projectiles at $500 \mathrm{keV}$ He exit energy.

Figure 6. The 1inear polarization fraction of $2 \mathrm{p}^{3} \mathrm{P}-3 \mathrm{~d}^{3} \mathrm{D}, 5876 \stackrel{\circ}{\mathrm{A}}$, as a function of foil thickness, using $\mathrm{HeH}^{+}$and $\mathrm{He}^{+}$projectiles at $650 \mathrm{keV}$ He exit energy. 


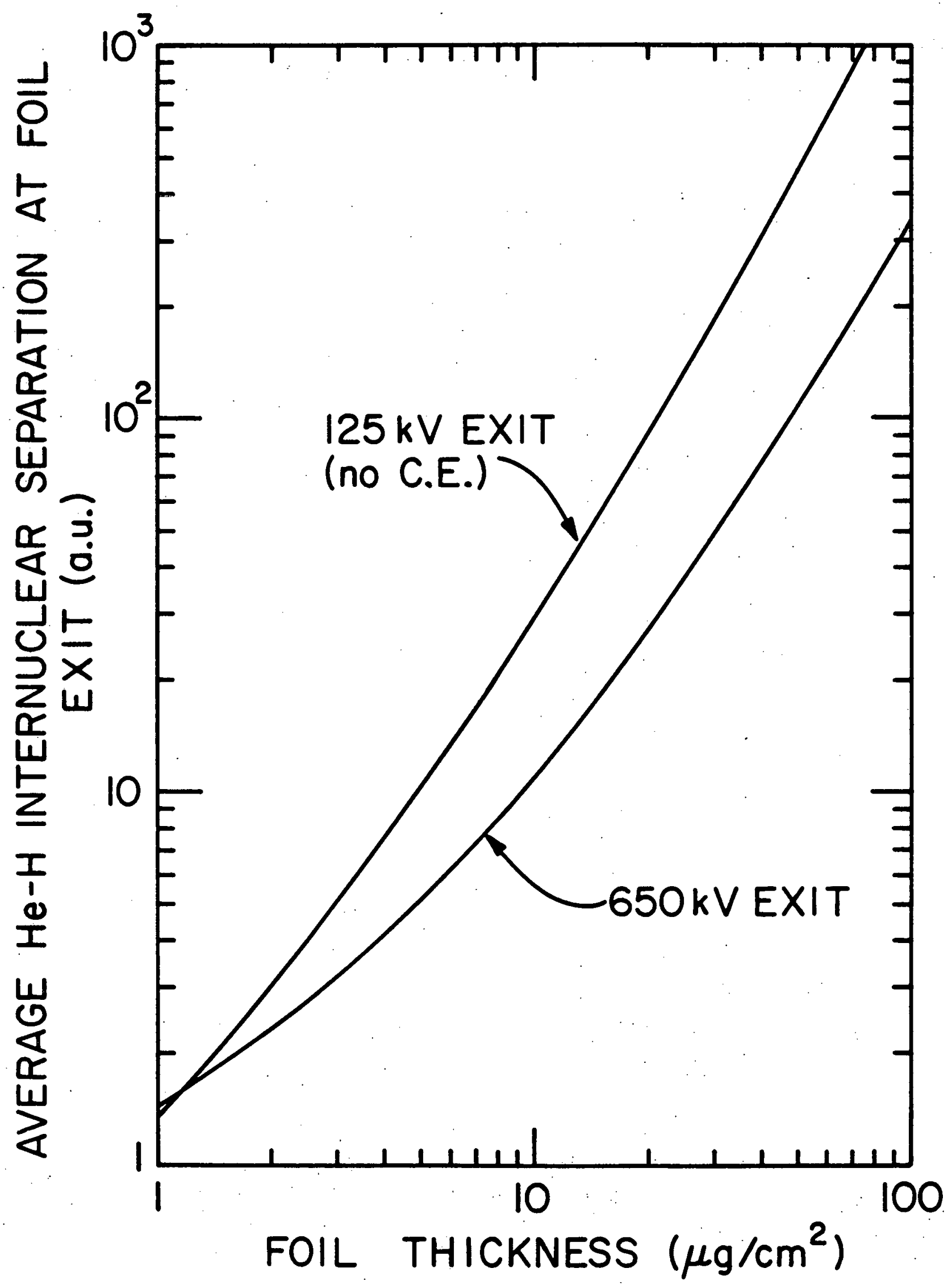

Figure 1 


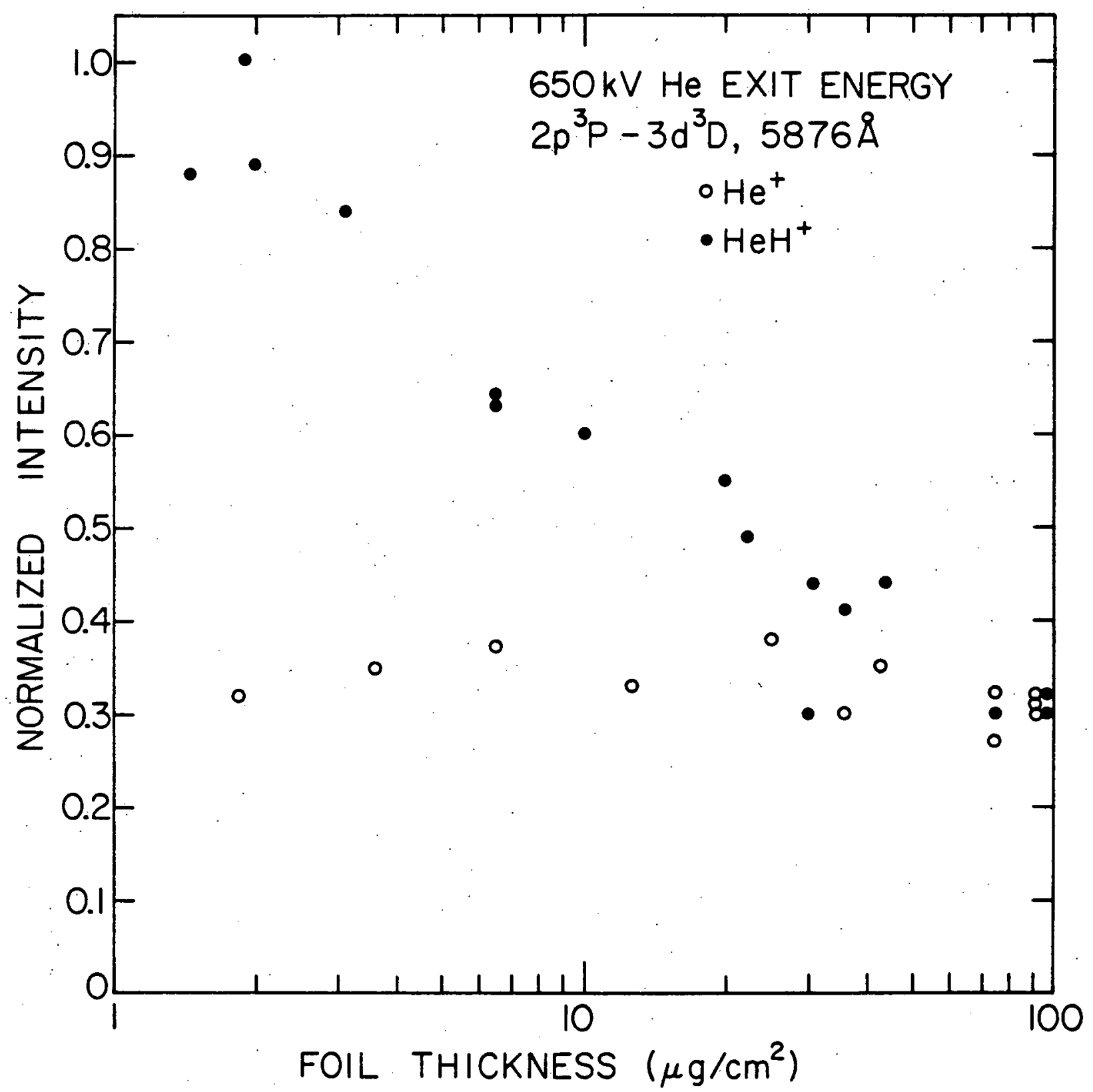

Figure 2 


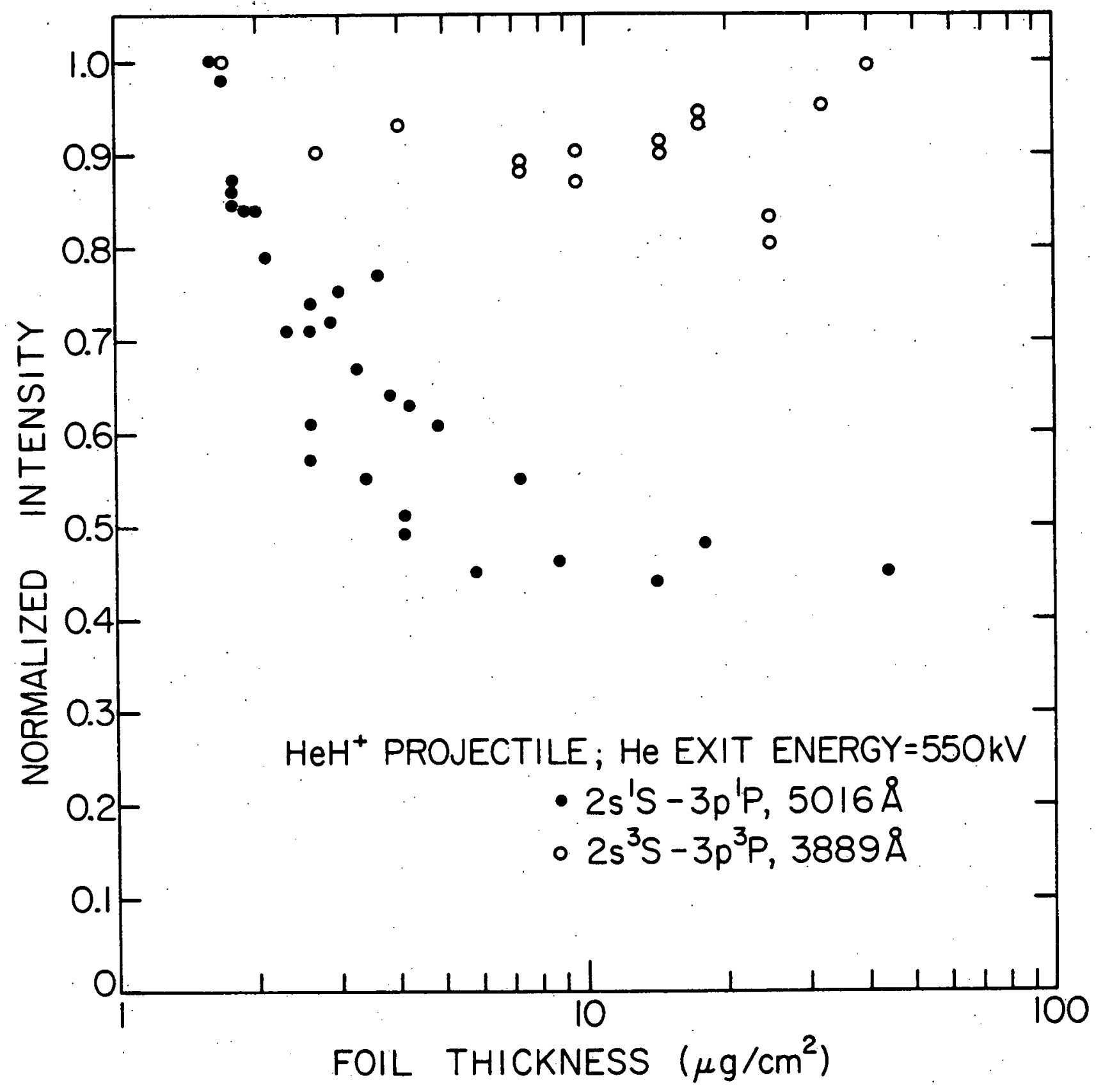

Figure 3 


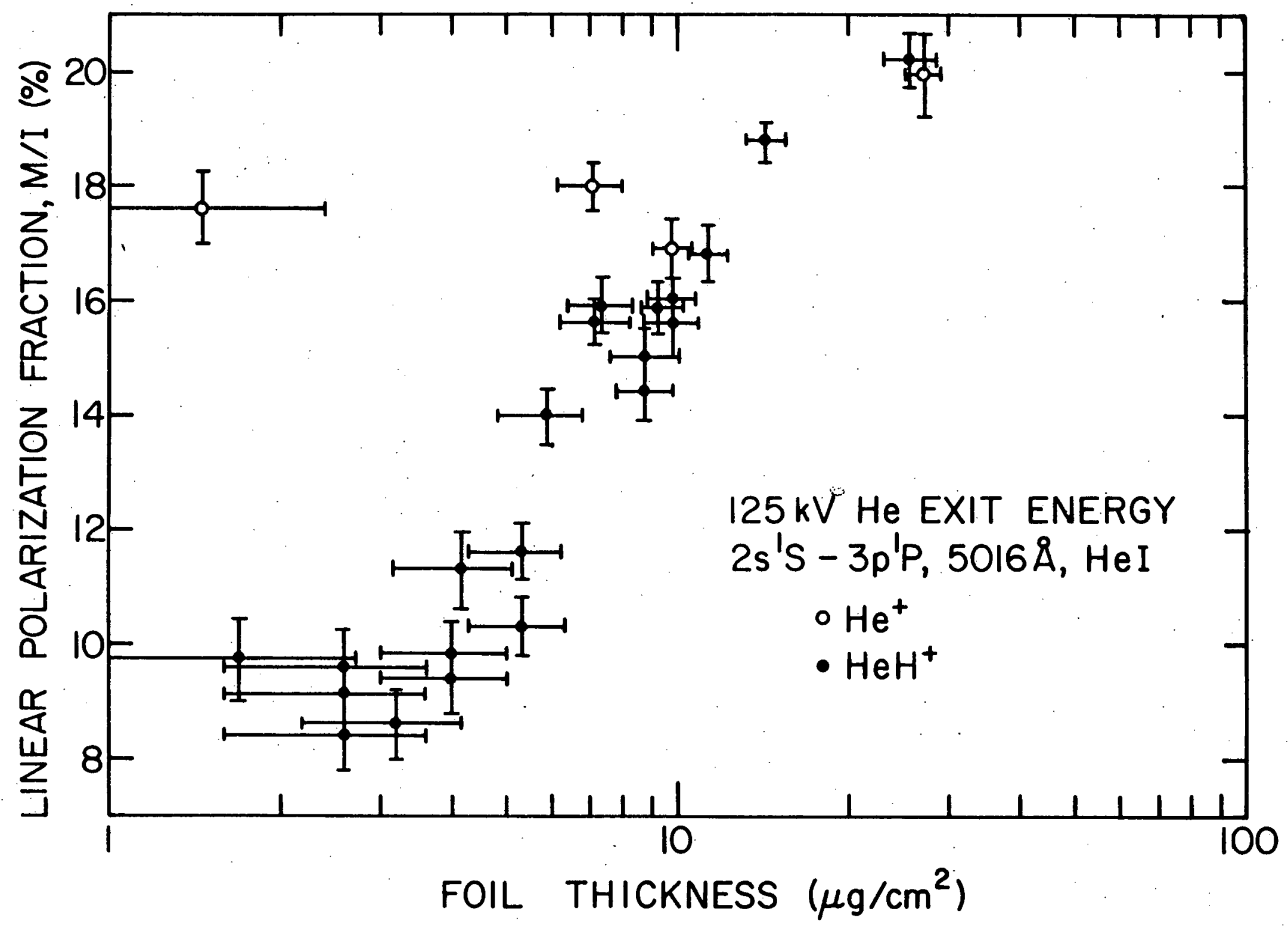




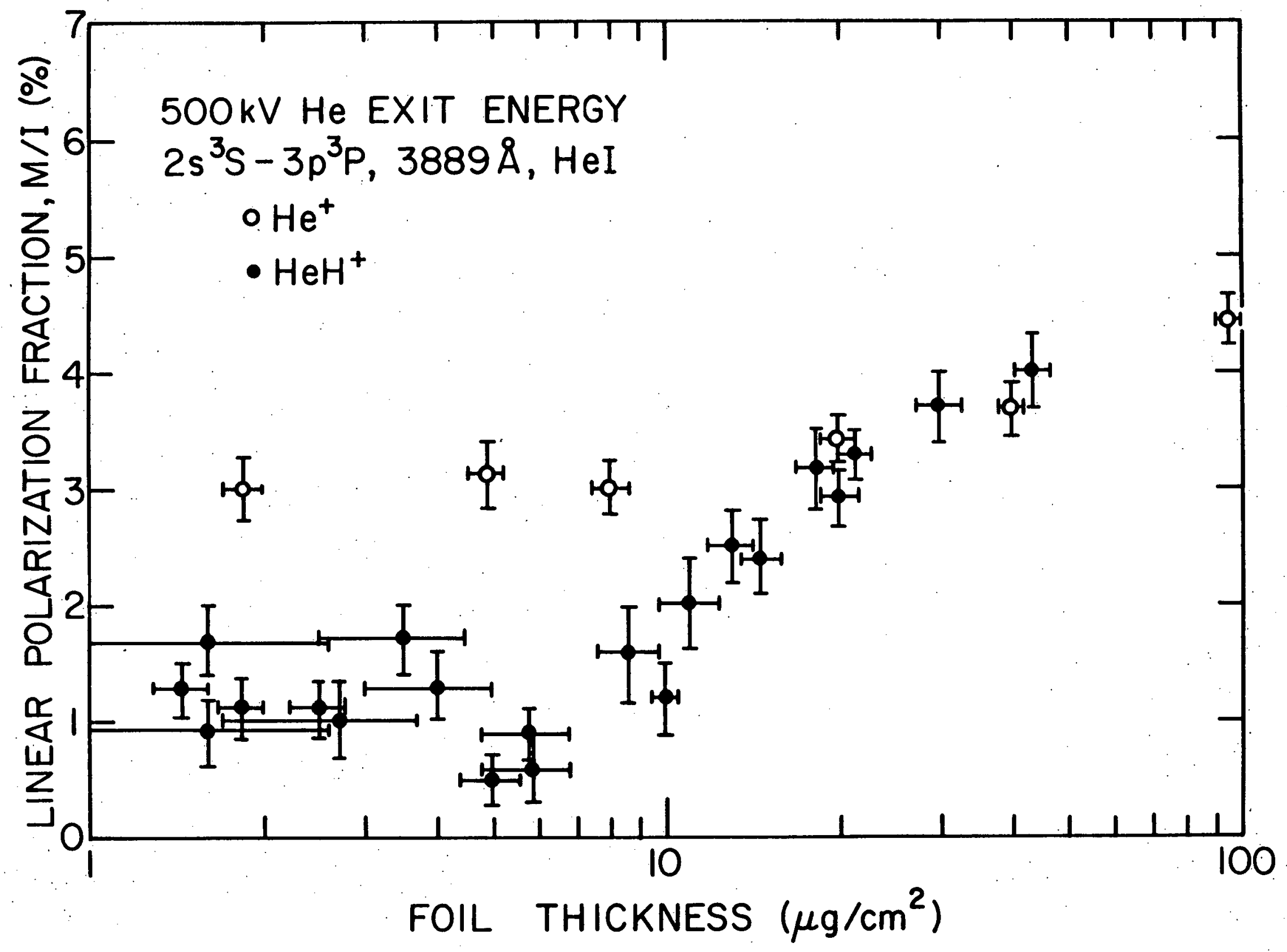




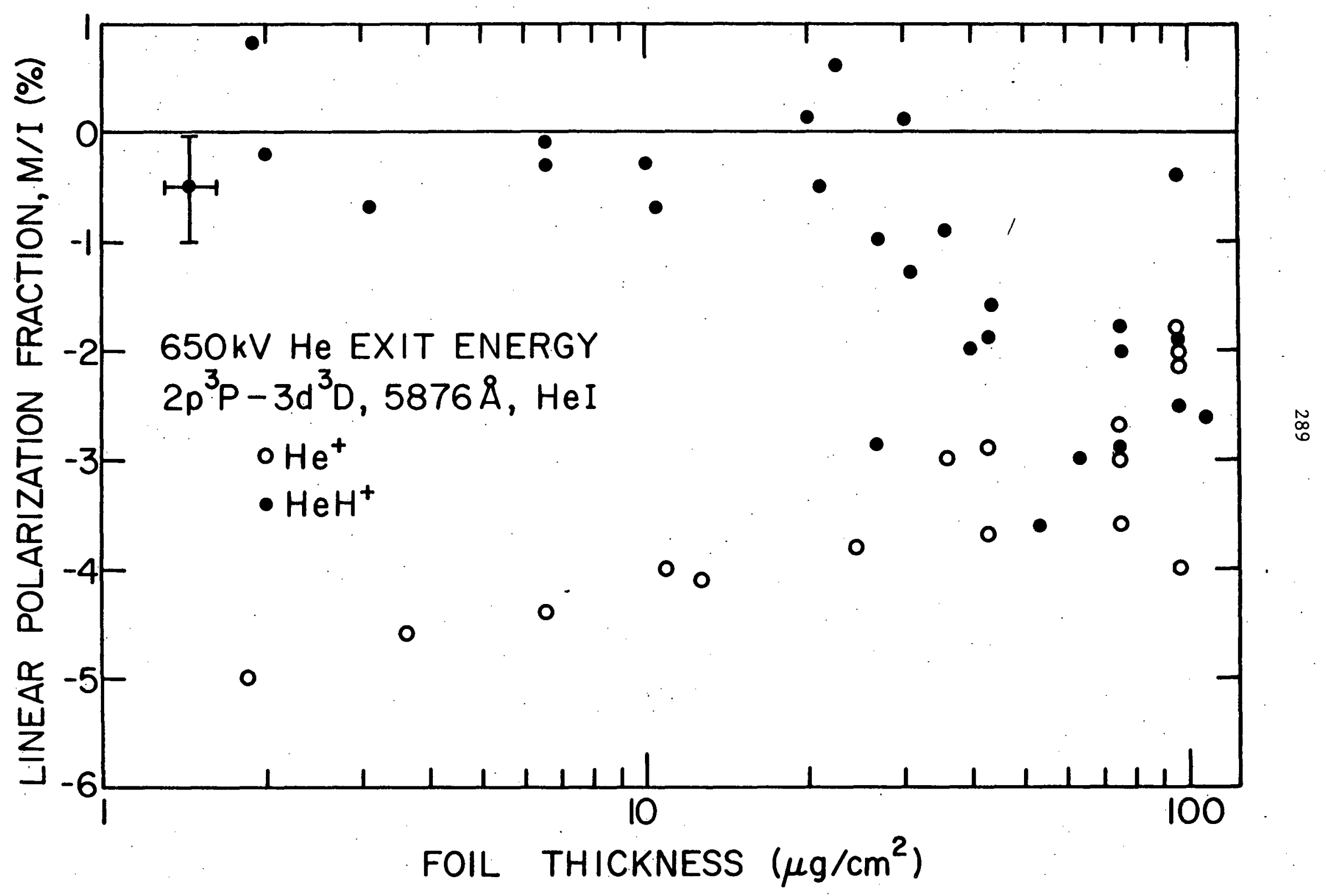




\section{THIS PAGE}

WAS INTENTIONALLY

LEFT BLANK 


\title{
Electron Emission
}

\author{
M.W. Lucas*
}

Physics Division, Oak Ridge National Laboratory

*Permanent Address: School of Mathematical and Physical Sciences University of Sussex

Brighton BNT $9 Q \mathrm{H}$ ENGLAND

*Research sponsored by the Division of Physical Research, U.S. Department of Energy, under contract W-7405-eng-26 with Union Carbide Corporation. 
I want to discuss three aspects of electron emission from ion bombarded targets.

I. The cusp. observed near $\vec{v}_{\text {electron }}=\vec{v}_{i o n} ;$ its shape, its magnitude as a function of $v_{i o n}$, differences between gaseous and solid targets and differences when molecular ions rather than monatomic ions are used as projectiles.

II. Possible interference effects associated with this cusp. These are predicted by the theoretical approach adopted by Macek(1970) but seem to be very difficult to observe.

III. The electron velocity distribution for $180^{\circ}$ ejection and its relation to the wave function of an electron moving with an ion inside a solid. This I shall call the "Back bounce Experiment" and it may help to elucidate whether such an electron is captured into an atomic or wake-bound state of the ion.

I anticipate many of the audience will be familiar with at least one of these topics but wish to bring them all together in an effort to achieve some overall assessment of our understanding of secondary electron emission from both gases and solids and its relationship to theories of Charge Exchange to the Continuum (CEC) and Wake Riding(WR).

\section{I. (a) Peak Position}

All the early CEC and WR theories agree that the cusp in the forward electron velocity spectrum occurs at $v_{\text {electron }}=v_{\text {ion }}$. To the best of my knowledge, this is confirmed in all the experimental data (to within at least $1 \%$ ) no matter what type of ion, atomic or molecular, high $Z$ or low $Z$, or what type of target is used. However, a very recent second 
order Born CEC theory of Shakeshaft and Spruch (1978) predicts that $v_{e}$ at the cusp is marginally smaller than $v_{i}$ but this has not been tested experimentally.

(b) The Dettmann theory predicts that the cusp is symetric about $v_{e}=v_{i}$ and that at a fractional height $(1-r)$ measured from the base wee expect a half-width:

$$
(\Delta v)_{H,(1-r i, H}=\frac{r}{2}\left(+\frac{1}{1-r}\right) v 0_{0}
$$

for an analyser collecting electrons in a cone of semi-angle $\theta_{0}$. For $r=1 / 2$ we obtain the much quoted relationstip

$$
(\Delta v)_{\text {Hwhin }}=\frac{3}{4} v i c
$$

though in principle $\Delta v_{e}$ may be examined at any height, although the theory is expected to be most accurate near the top of thie cusp, i.e. for small $r$. The interpretation of this result has caused some confusion in that the cusp typically sits on a smoothly decreasing signal of ejected electrons. It is important to realize that thic is not a "noise" signal to be subtracted off the experimental data but a signal incorporated within the theory. Hence the measured widths must correspond to a height taken to the base line. The way in which CEC theory describes all of the electron. ejection very close to the peak is best seen by reference to the three body coltision of figure 1 . 
Fig. I The three body collision

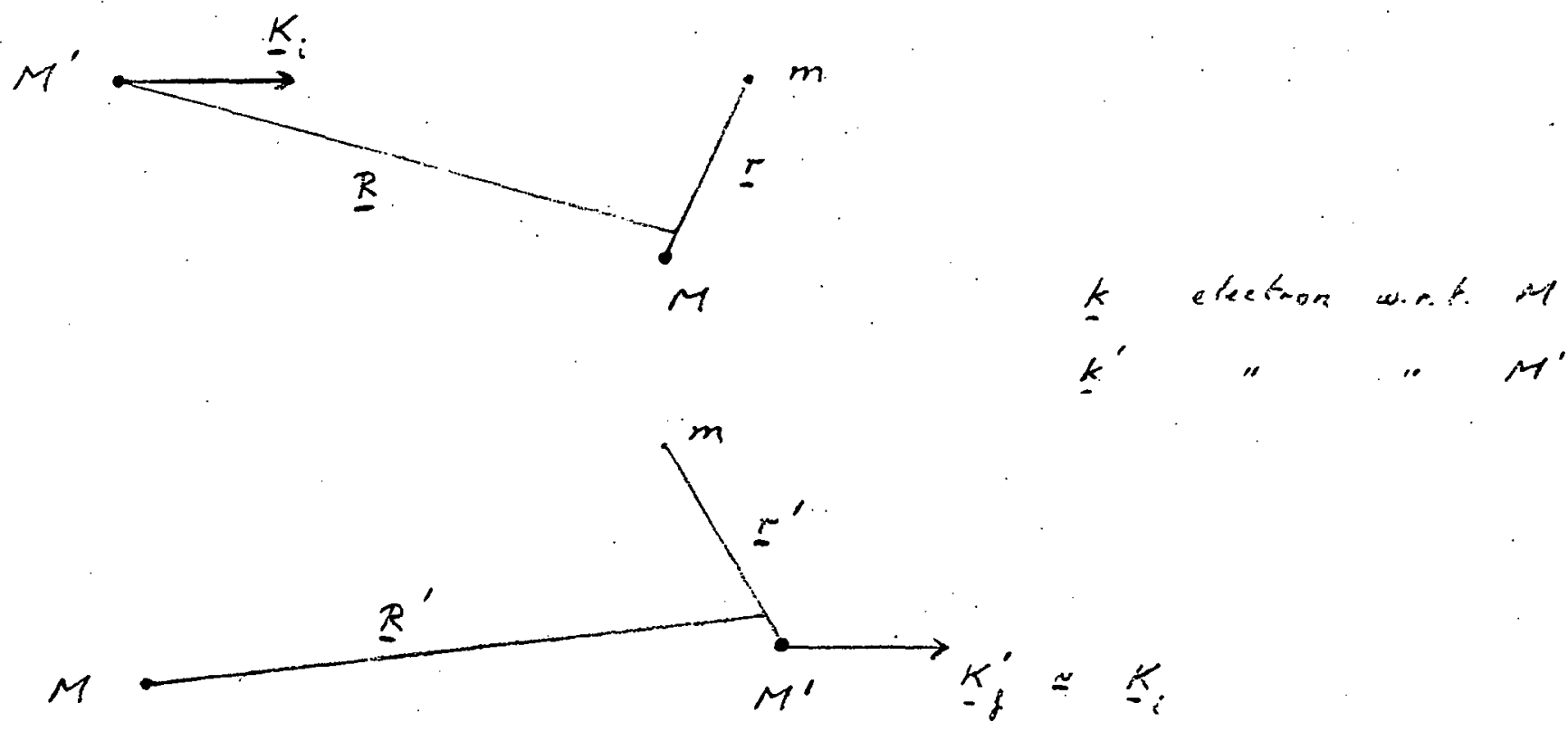

We have to find the scattering amplitude for an electron in the field of two Coulomb centres, one stationary, one moving, for all velocities of the electron relative to each centre. Once we have this amplitude the derivation of the cross section follows rigorously from the resultant $T$ matrix. The central difficulty is that we cannot write down a single wavefunction which will represent the electron for all possible situations. We have to construct separate amplitudes for the situations (a) when the interaction between the electron and target is strong and (b) when the interaction between the electron and projectile is strong.

Having done this we have counted twice--there is only one electron-so we must introduce a counter term so arranged as to cancel (a) when (b) is dominant and vice versa. These three terms are respectively $a_{23}, a_{12}$ and a of Macek's equation (16). Since the Born approximation may be more familiar we write them out below in the notation of Dettmann et al (1974) (DHL), 
restricting ourselves to first order since that is sufficient to make the point.

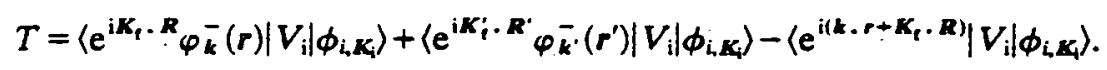

Now the first term can represent target ionisation: that is the electron ' $m$ ' moving slowly away from the target $M$, and $M$ ' moving rapidly away from $M$ and $m$. Hence if $P_{a}^{-}(r)$ is a Coulomb wave centred on $M$ this term is the usual Born amplitude and we call it 'direct ionisation'. The second term is CEC with $\varphi_{k^{\prime}}^{\prime}\left(r^{\prime}\right)$ the Coulomb wave about $M^{\prime}$, which represents $M^{\prime}$ and $m$ moving slowly away from one another, but both moving rapidly away from $M$. We could call this 'rearranged ionisation'. though strictly such a term implies a two-step process and the use of a secondorder approximation. The third term is the counter term in the limit of all three particles separating quickly. Notice how it works. If CEC dominates (second term) the electron has large $k$ with respect to the target. The coulomb wave $\varphi_{k}^{-}(r)$ becomes $e^{i k \cdot r}$ and the first and third terms cancel. There is no other source of electrons to provide a 'background' which has to be called 'direct ionisation' and subtracted. As we move into the wings the cancellation from the counter term becomes progressively imperfect so that it is necessary to calculate all three terms to predict the whole velocity spectrum. This of course is what Macek has done. We see here the great similarity between the Dettmann and Macek approaches. Macek has only a first-order approximation but can compute the whole spectrum, Dettmann retains an analytic approach and to second order but can only describe the neighbourhood of the peak in the spectrum. Now my argument concerning the cancellation of the 
first and third terms is not quite exact for the typical $v_{j}$ of from one to ten atomic units used in most experiments. Robin Shakeshaft has kindly made numerical calculations for me which show that $\underline{k}$ really does have to be rather large to get cancellation of the two terms to within say $10 \%$. However we see in figure 2 of Steckelmacher et al (1978) that for protons on carbon the half width of the peak certainly does increase with $v_{j}$ just as CEC theory predicts and is not substantially independent of $v_{j}$ as in the WR model.

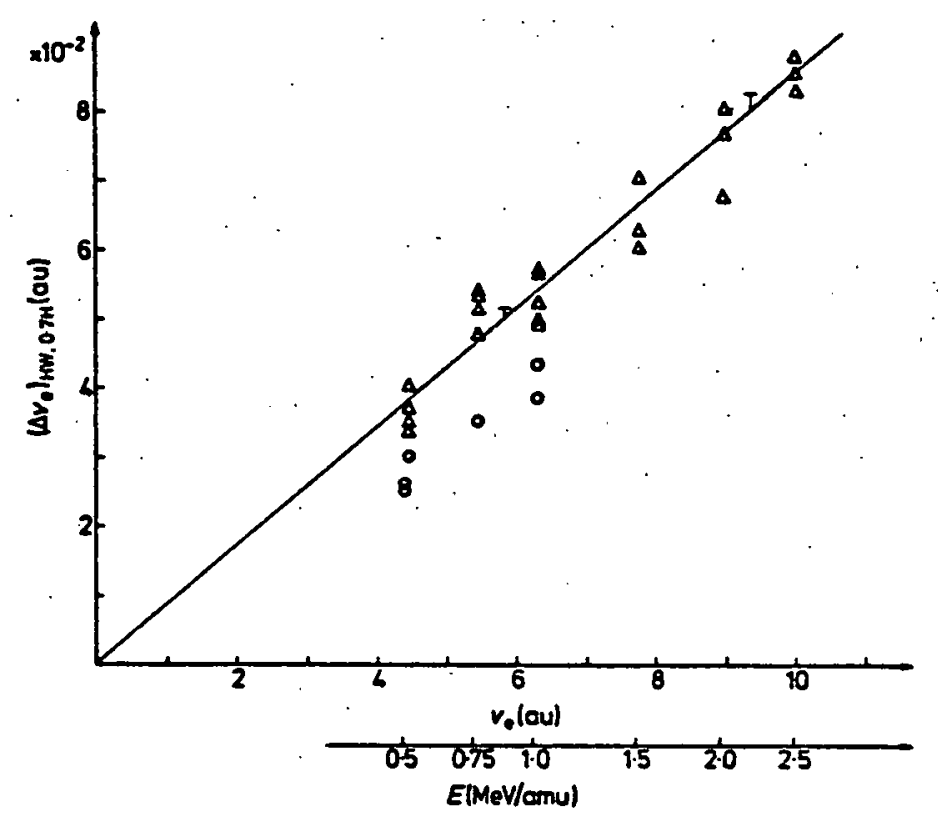

Figure 2 The half-width of the cusp at $70 \%$ of the full peat height as a function of the corresponding $\mathrm{D}_{1}$ for protons and $\mathrm{H}_{2}^{+}$ions incident on self-supporting $\left(10-15 \mu \mathrm{g} \mathrm{cm}{ }^{-2}\right)$ carbon foils. The measured values are from a number of spectra (and diferent foils) for incident energies $\left(E_{\mathrm{i}}\right)$ from $0-5-2.5 \mathrm{MeV}$ amu $^{-1} . \Delta$ protons; $\mathrm{O}, \mathrm{H}_{2}$ ions; continuous line, CzC theory $\left(\theta_{0}=1.35^{\circ}\right)$; $\mathrm{T}$, systematic error.

$$
\text { (trom stectelmacher et al 1978) }
$$


Some new results concerning peak shape are of great interest and are beginning to show consistent differences between gaseous and solid targets. I think it is correct to say that all these results indicate the peak is narrower on the high velocity side, an asymmetry particularly apparent with the high $Z$ projectiles used by Sellin's group and one that may just be seen in some of our results using even protons on gases (Cranage and Lucas 1976). For solid targets the asymmetry is less marked. Shakeshaft and Spruch (1978) have found a mistake in our algebra which qualitatively explains this. We had originally thought that the predicted peak shape was independent of whether the calculation was made in the first or second Born approximation but this now appears untrue as is shown in figure 2 of Shakeshaft and Spruch's paper.

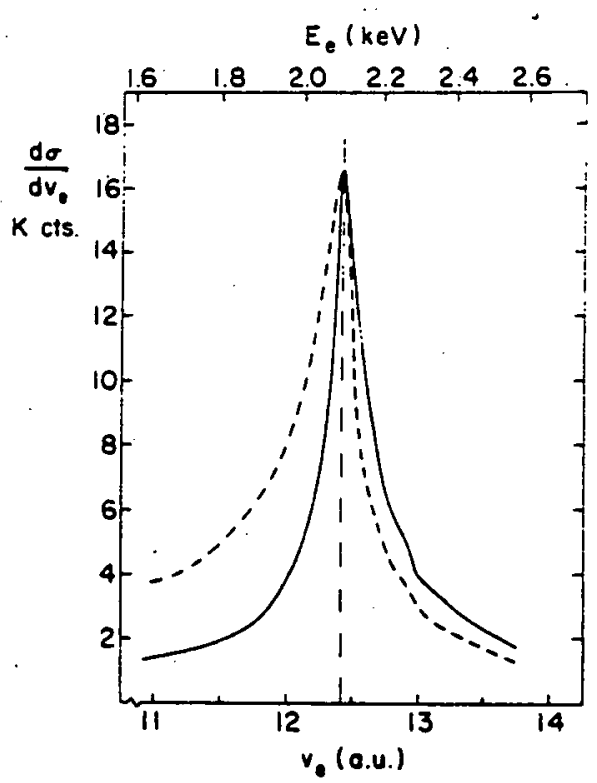

FIG. 1. The differential cross section $d \sigma / d v_{\text {a }}$ as a function of the electron velocity, $v_{\theta}$, of convoy electrons emerging at $0^{\circ}$ with respect to the lon beam for 108-MeV Si ${ }^{+19}$ (dashed curve) on 30-mTorr Ar gas and $\mathrm{Sl}^{+}$(solld curve) on $100-\mu \mathrm{g} / \mathrm{cm}^{2}$ Au foll. The electron energy $E_{e}$ is marked across the top of the figure.

$$
\text { from Laubert it al }(1979)
$$

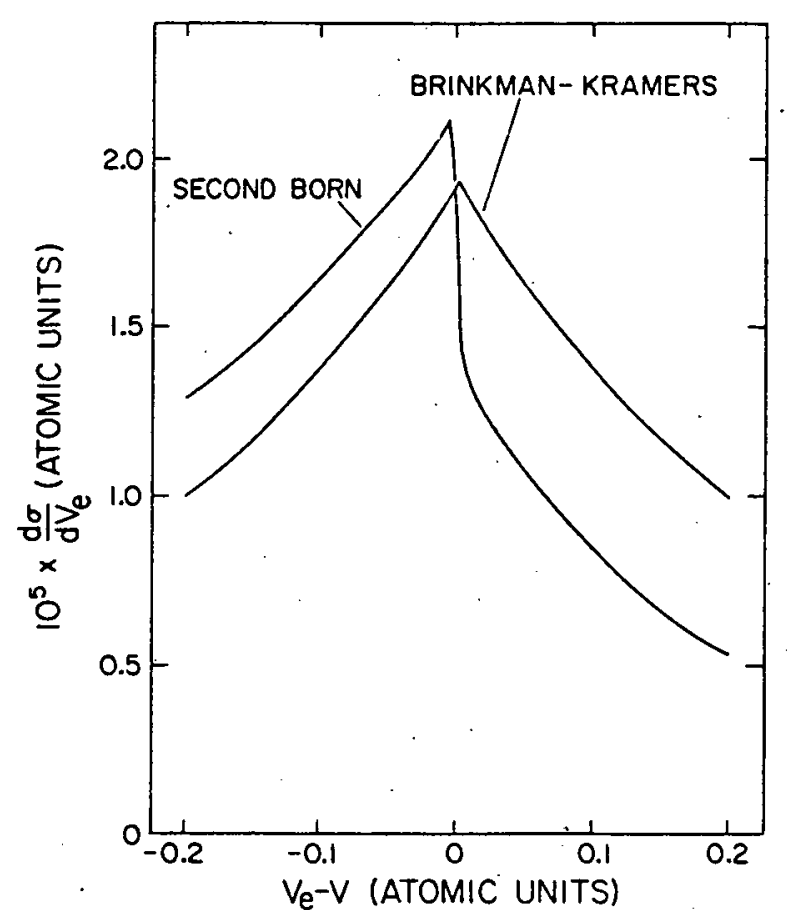

FIG. 2. The singly differential cross section for continuum electron capture from the is state of a hydrogen atom by a bare ion of atomic number $Z_{B}=6$ incident with an energy of $2 \mathrm{MeV}$ per nucleon in the lab frame. The electron emerges into a cone of semiangle $\theta_{0}$ $=1.85^{\circ}$. The curve marked "Brinkman-Kramers" is the contribution to the differential cross section from the Brinkman-Kramers amplitude, while the curve marked "second Born" is the contribution from the sum of the first- and second-order Born terms. $d \sigma / d v_{e}$ is in units of $a_{0}{ }^{2}\left(e^{2} / \hbar\right)^{-1}$.

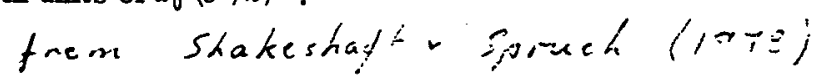


The predicted skew is obviously quite dramatic but as yet neither the calculation nor the experiment can be performed for quite the same conditions so comparison remains qualitative.

Finally, since this workshop is predominantly about molecular effects, I should like to draw attention to the points shown on figure 2 of Steckelmacher et al. for $\mathrm{H}_{2}{ }^{+}$incident on carbon foils. These; and indeed the early results of Kevin Harrison always show peak widths a little narrower than those arising from protons of the same velocity. This may be related to the correlation existing between the two protons as they traverse the foil and in that respect a manifestation of the wake force. However I would stress that on present evidence it does not appear necessary to invoke a separate WR theory to describe the shape of the electron cusps arising from solids as opposed to gases.

\section{(c) Peak magnitude}

One can of course integrate the signal over some limited range of velocity either side of the peak maximum and so examine the yield (electrons/ion) within the peak as a function of projectile velocity. Dettmann's second Born approximation predicts that at high velocity the cross section for CEC will decrease as $E_{i o n}{ }^{-5}$. A caution is in order here in that one must be careful to use fully stripped projectiles to avoid any contribution from projectile ionisation, a process which Briggs and Drepper (1978) have shown to have a cross-section substantially independent of ion velocity. For a gas target where single collision conditions may be obtained, the yield will be directly proportional to the cross-section and Rodbro and Andersen (19.78) have shown that for protons of about $1.0 \mathrm{MeV}$ incident on light gases the dependence 
of the yield does indeed approach $E_{i o n}{ }^{-5}$.

Perhaps of most interest to us here is the direct comparison of gas and solid but there is, of course, the difficulty that single collision conditions do not obtain for the latter. My own approach has been to assume that the effective target thickness is controlled by the electron's ability to leave the solid without suffering a collision which would throw it out of the peak. For an electron captured in a continuum state inside the solid to be subsequently detected outside the solid in a spectrometer set for the forward direction it must preserve both i.ts speed and direction in spite of the strong scattering to be expected. We therefore turned (DHL) to the data and calculations which are known as the 'escape depths' of electrons from solids (Duke et al 1970, Powel1 1974, Carlson 1974). These inelastic mean free paths are obtained from Auger electron spectroscopy and x-ray photoelectron spectroscopy of solids. Where data are available the latter technique is particularly apposite since the $x$-rays generate electrons at all depths in the solid just as we believe our projectiles do. However both techniques give similar results. For $262 \mathrm{eV}$ electrons in carbon, i.e. those having the same speed as $481 \mathrm{keV}$ protons, $\lambda_{i}=7.5 \AA$ (Jacobi and Hölzl 1971). Both theory and experiment show that in the energy range 100-1500 ev, $\lambda$ is approximately proportional to $E^{1 / 2}$ (Carlson 1974, Carter et al 1974) so that for an electron of $123 \mathrm{eV}$ (225 keV proton) we expect $\lambda_{i} \simeq 5.0 \AA$. Hence our choice of one atomic layer as a rough estimate of the effective target density in the $\mathrm{OHL}$ paper. Let us now apply these arguments to the relative ion energy dependence of the yield as measured by Meckbach et al (1977). We recall that the yield (electron/ proton) is given by the product of the cross section and target density. 
They observe a yield proportional to $E^{-3.1}$ from carbon foils in an energy range where the effective target density is increasing as $E^{1 / 2}$ because of the energy dependence of $\lambda_{i}$. This therefore implies an energy dependence for the cross section of $E^{-(3.1+0.5)}$ in exact agreement with the value. $E^{-3.5+0.1}$ reported by the same group (Chiu et al 1978L using the same apparatus but a helium gas target under single collision conditions.

Strong (1977) has also measured the yield from carbion foils and converts this to a cross-section by assuming $\lambda_{i}=2$ monolayers for $275 \mathrm{KeV}$ protons (150 eV electrons at the peak) rising to 10 monolayers for $3.0 \mathrm{MeV}$ protons (1632 eV electrons:). The results are compared with the theory of Dettmann, Khan and Lucas (1976) in figure 2 . The theoretical contribution from each pair of electrons is shown separately. Hartree-Fock wavefunctions for free carbion atoms were used.

\section{Interference Effects}

In the Fadeev approach to the theory of CEC first used by Macek (1970) it is essential that the three amplitudes contributing to the T-matrix be added together before squaring to get the cross section. This is because CEC is seen as a special case of ionisation. The . doubly differential cross section so obtained can exhibit interference terms as shown in figure 1 of Duncan et al (1977). These are most pronounced where the phase of the exchange amplitude is varying rapidly owing to the coulomb distortion of the final state wave function and where the magnitude of the direct ionisation and charge exchange amplitudes are similar. 


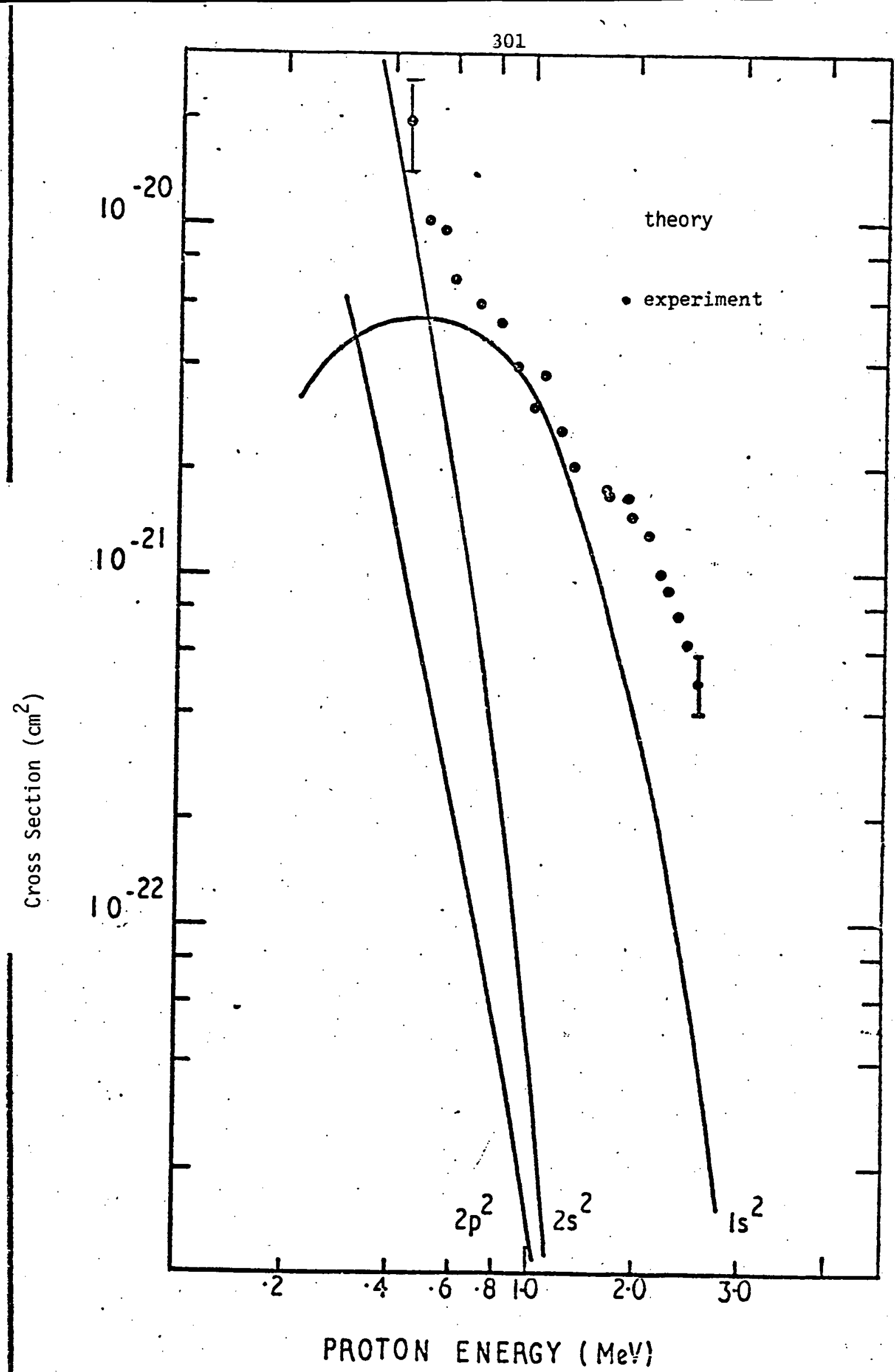

Fig. 2 The yield within the peak converted to a cross section by assuming that the effective target thickness is controlled by the electron's escape
depth. 
from: DUNCAN, MENENDiz, EISELE, AND MACEK ( $/ 9.77)$

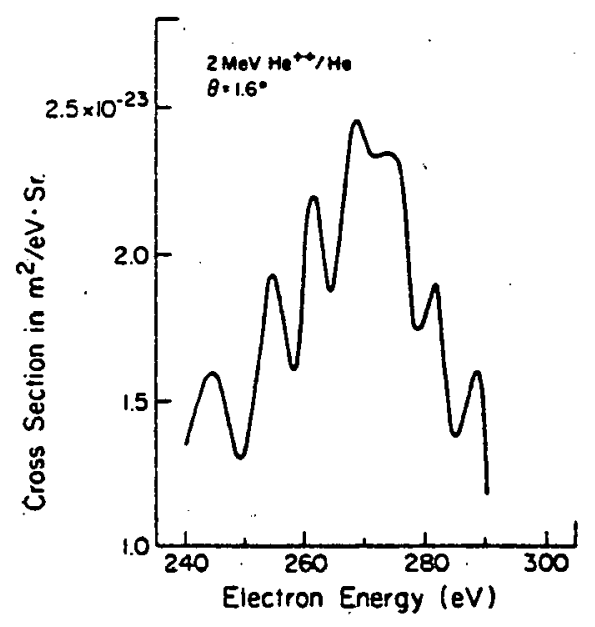

FIG. 1. A calculation of the DDCS at $1.6^{\circ}$ showing the interference structure predicted by the first approximation to Faddeev's equations.

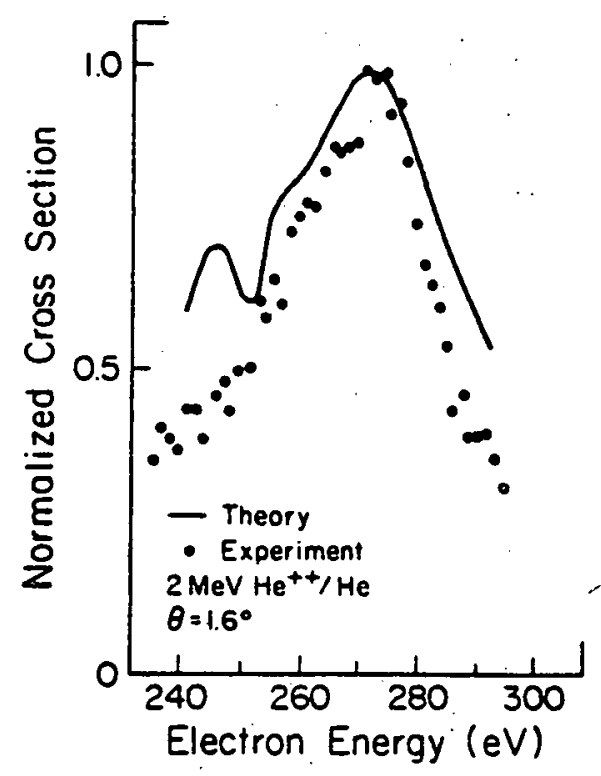

FIG. 2. DDCS of $v_{e} \sim v_{i}$ electrons normallzed to peak values of 1 . The solld curve is obtained by averaging the theoretical results over the energy and angular resolutions of the experimental apparatus. The data are shown as closed circles.

This occurs at small ejection angles, i.e. close to, but not in, the forward direction. Unfortunately the oscillations change very rapidly with angle so that the presence of a reasonable collecting angle for the electron spectrometer tends to average them out. Duncan et al (1977) worked with approximately $=0.3^{\circ}$ and at $1.6^{\circ}$ to tile forward direction. They used He+t on He gas but saw no clear structure. We have just started a similar search using carbon foil targets and have a preliminary result which though promising is very different from theory, figure 3 . We feel that if we can see such interference effects from solids it will provide powerful support for Macek's theory. 


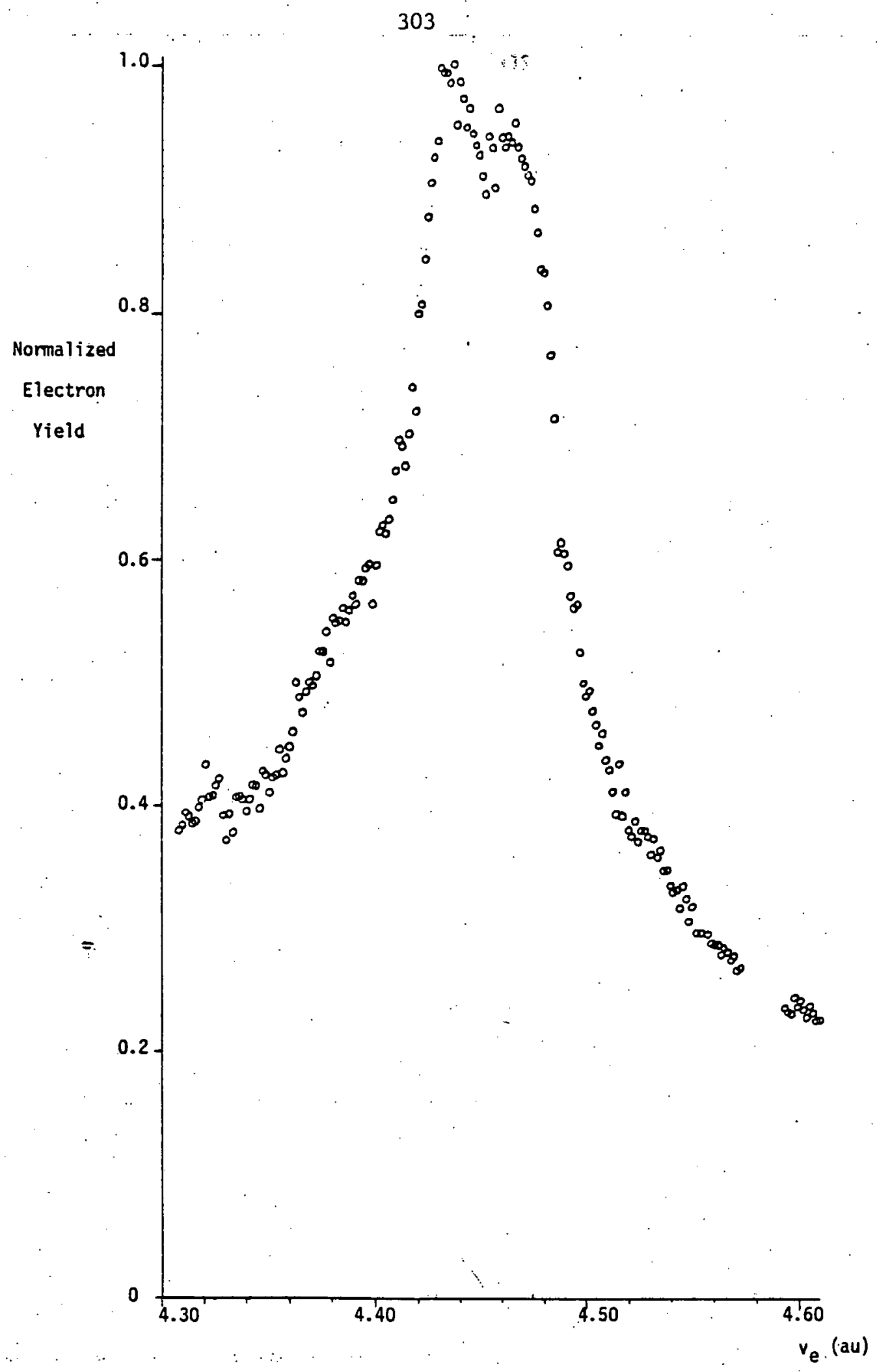

Fig. 3 Change in the shape of the CEC peak when the spectrometer is set to measure the ejection at a small angle away from the. forward direction. This example is for $0.5 \mathrm{MeV} \mathrm{H}^{+}$on a carbon foil with $\theta=0.75 \pm 0.25^{\circ}$ and an acceptance semi angle $\theta_{0}=0.13 \pm 0.13^{\circ}$

- The number of counts per channel at the top of the peak is 4900 so the statistical error on the ordinate is about three times the size of the points as drawn. 
III. "The Back Bounce Experiment".

This is an interesting experiment based on some earlier results of Burch et al (1973) which I should like to bring to your attention. A fuller discussion is given by Strong and Lucas (1977) and Strong (1977).

First imagine a monokinetic beam of free electrons, velocity $\vec{v}_{e}$ incident on a target. If we look at the $180^{\circ}$ ejection we expect a smooth backgrourid of secondary electron superimposed upon which is a del ta function from those incident electrons reflected off the target. The target atoms are heavy and nearly stationary so that the delta function appears at $-\vec{v}_{e}$. Now perform the same experiment but with the electrons carried in on say a $\mathrm{He}^{+}$ion beam. The electrons now enter the collision

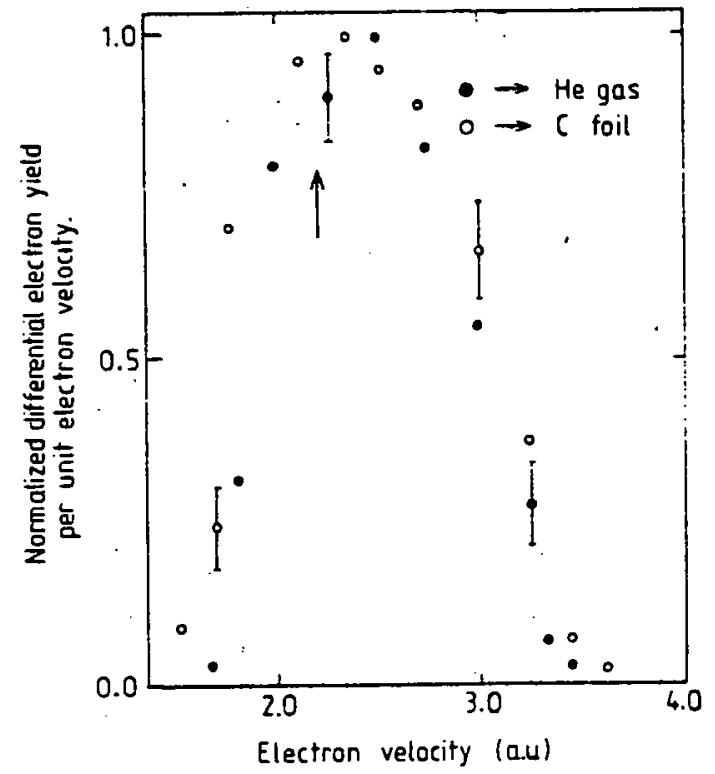

FIG. 2. Normallized background-subtracted spectra deduced from the type shown in Fig. 1. 0.8- $\mathrm{MeV}^{4} \mathrm{He}^{4}$ incident helium gas and carbon foil. The arrow marks the position of the binary-encounter peak predicted by Ref. 8.
Electron velocity scale for ${ }^{4} \mathrm{He}^{+}(\mathrm{au})$

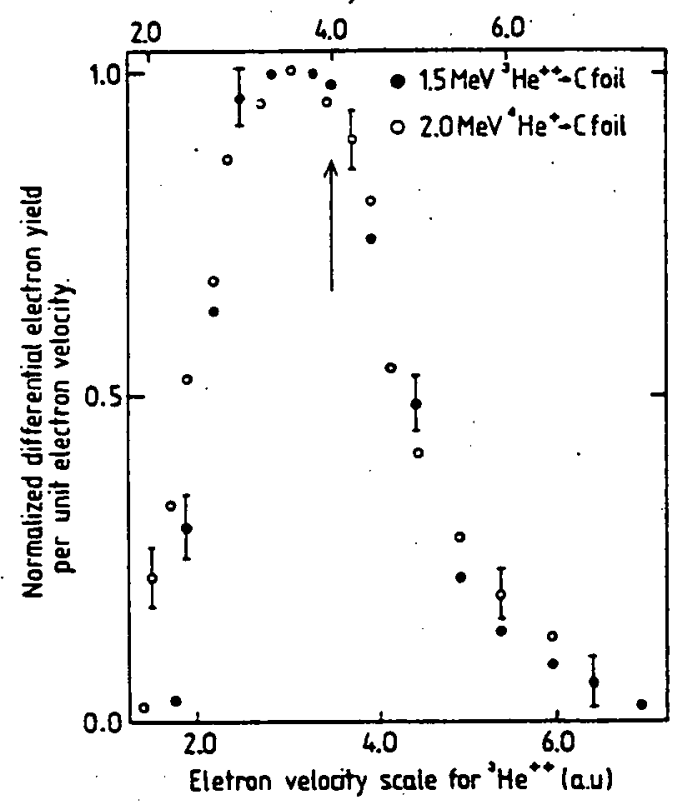

FIG. 3. Normalized background-subtracted spectra deduced from the type shown in Fig. 1. Equivelocity ${ }^{4} \mathrm{He}^{+}$and ${ }^{3} \mathrm{He}^{++}$incident on carbon foll. The arrow marks the position of the binary-encounter peak predicted by Ref. $8\left({ }^{4} \mathrm{He}^{+}\right.$scale).

$$
\text { facm Strong to idicos (ip? }
$$


with a velocity spread characteristic of the He(1s) orbital so that the velocity profile of the reflected electrons is characteristic of that orbital. This can be done for both gas and solid targets to establish that the resulting profile is nearly target independent and characteristic of the projectile, figure 2 of Strong and Lucas (1977).

Finally compare the shape of the profiles obtained from both $\mathrm{He}^{+}$ and $\mathrm{He}^{++}$incident on a solid as is done in figure 3 of Strong and Lucas (1977). $\left({ }^{3} \mathrm{He}^{++}\right.$was used merely to avoid beam contamination by $\left.\mathrm{H}_{2}^{+}\right)$. Now the $\mathrm{He}^{++}$also gives a profile but since it did not carry an electron into the solid it must have picked it up inside the solid and lost it in a subsequent collision. Now the two profiles are of the same shape implying that the electron picked up by the $\mathrm{He}^{++}$entered an orbital characteristic of the $\mathrm{He}^{+}$ion, that is, an atomic orbital. A wake type orbital, for which the binding energy is thought to be much less, would be expected to have a much narrower profile. The only difference between the $\mathrm{He}^{+}$and $\mathrm{He}^{++}$seems to be the shift in velocity scale which we view as energy lost by the electron as it travels back through the solid following ejection in the second collision.

I am indebted to many members of ORNL for their hospitality during the past year and should like particularly to thank Charlie Moak, Rufus Ritchie and Ivan Sellin and his group for their lively discussions of these and related problems with me. 
References

Briggs, J.S. and Drepper, F. (1978) J. Phys. B1], 4033.

Burch D., Wiemann H., Ingalls W.B. (1973) Phys Rev Lett $\underline{30,} 823$

Carlson, T.A. (1976) Photoelectron and Auger Spectroscopy (New York:Plenum)

Carter, W.J., Schweitzer, K.G., Carlson, T.A. (1974) J. Electron Spectroscopy and $\operatorname{Rel}$ Phenom $\underline{5}, 827$

Chiu, K.C.R., McGowan, J.W. Mitchell J.B.A., (1978) J.Phys B $11 L 117$

Cranage, R.W. and Lucas, M.W. (1978) J Phys B9,445

Dettmann, K. Harrison, K. Lucas, M.W. (1974) J Phys B7, 269

Dettmann, K. Khan, M.N. Lucas, M.W. (1978) J Phys B9, 1879

Duke, C.B. Anderson, J.R. Tucker, C.W. (1970) Surface Sci 19117

Duncan, M.M. Menendez, M.G. Eisele, F.L. Macek, J. (1977) Phys Rev A15,1785

Jacobi, K. and Hölzl, J. (1971) Surface. Sci 26, 54

Laubert, R. Sellin, I.A. Vane, C.R. Suter, M. Elston, S.B. Alton, G.D.

Thoe, R.S. (1978) Phys Rev Lett 41,712

Macek, J. (1970) Phys Rev A1, 235

Meckback, W. Chiu, K.C.R. Brongersma H.H. McGowan, J.W. (1977) J Phys B10, 3255

Powel1, C.J. (197.6) Surface Sci 44,29

Rødbro, M. and Andersen, F.D. (1978) private communication submitted to J Phys B.

Shakeshaft, R. and Spruch, L (1978) Phys Rev Lett. 41,1037

Steckelmacher, W. Strong R. Khan, M.N. Lucas, M.W. (1978) J Phys B11, 2711

Strong, R. (1977) PhD Thesis, Sussex University

Strong, R. and Lucas M.W. (1977) Phys Rev Lett 39, 1349 
HYDROGEN ION TRAVERSAL OF THIN SOLID FOILS:

NEW DATA AT LOW ENERGY AND AN OVERVIEW *十

W. H. Escovitz, T. R. Fox and R. Levi-Setti

The Enrico Fermi Institute and Department of Physics

The University of Chicago, Chicago, I11. 60637

\footnotetext{
* Research supported by the National Science Foundation and the National Institutes of Health.

'Paper presented by R. Levi-Setti at the ANL Workshop on "Physics with Fast Molecular Ion Beams" August 20-21, 1979
} 
308

Measuring instrument : STIM

(scanning transmission ion microscope)

Ion source: Field ionization source.

Ions: $\mathrm{H}^{+}, \mathrm{H}_{2}^{+} \quad 10-50 \mathrm{keV}$ (sec Fig.2)

Molecular state popes.: Ground state ${ }^{(2)}$

Measuring instrumentation: Magnetic

sector spectrometer, $P / \Delta p \sim 1000$. acceptance semiangle $\pm 30 \mathrm{mr}$.

Detectors: Channel tron at spectrometer slit, chevron array for central, annular detectors. Coincidence detection capability

Incident beam normalization: Beam switching tanget-pinhole after target imaging. Incident beam, transmitted beam spectra recorded simultaneously.

Independent target characterization: By electron energy loss spectroscopy in STEM mode. rms thickness fluctuations $10-15 \%$. 


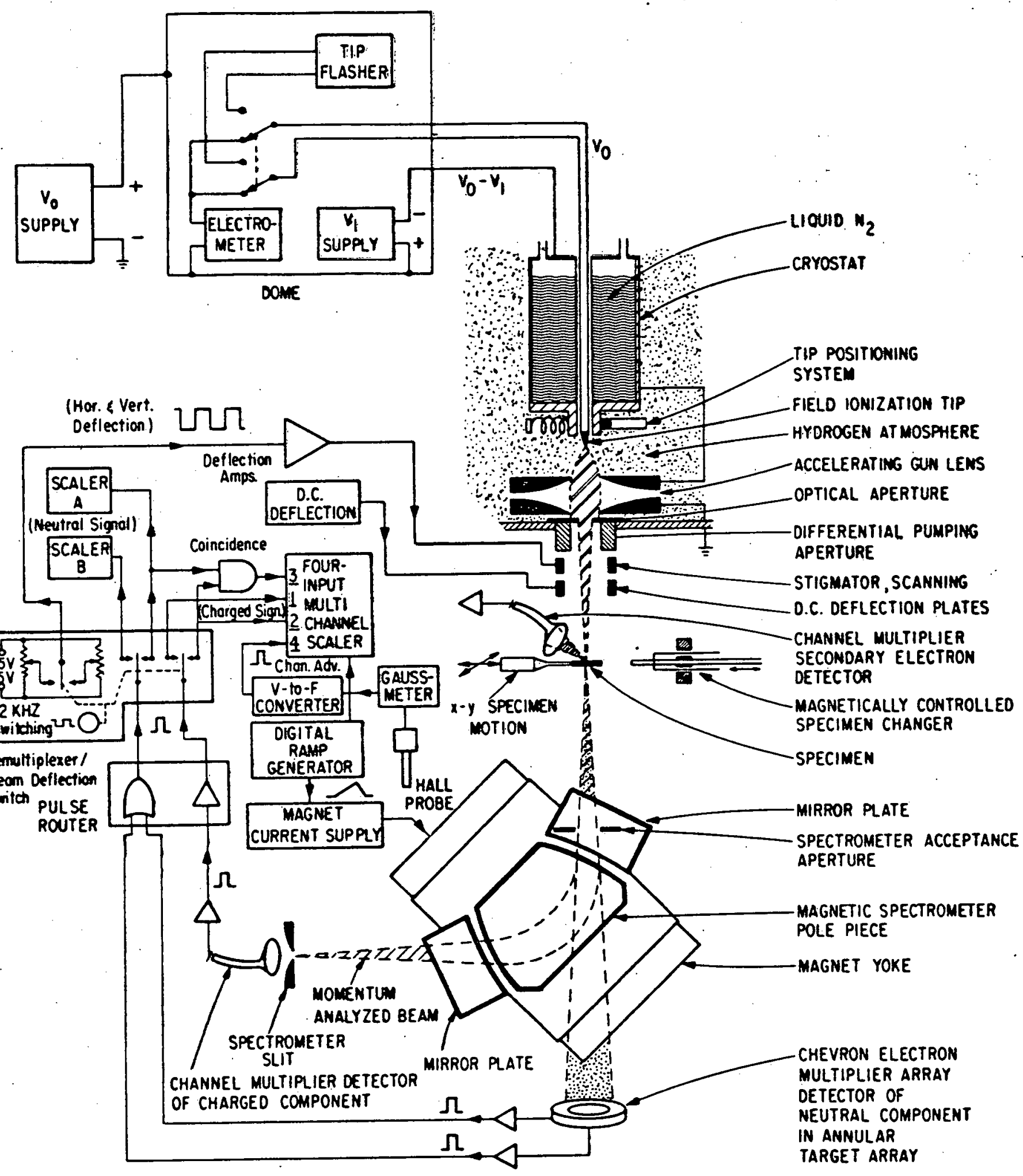

Fig.1. Schematics of the University of Chicago Scanning Trausmission Ion Microscope. 
310

Moss Composition of Beam

Extracted from. Field Ion Source

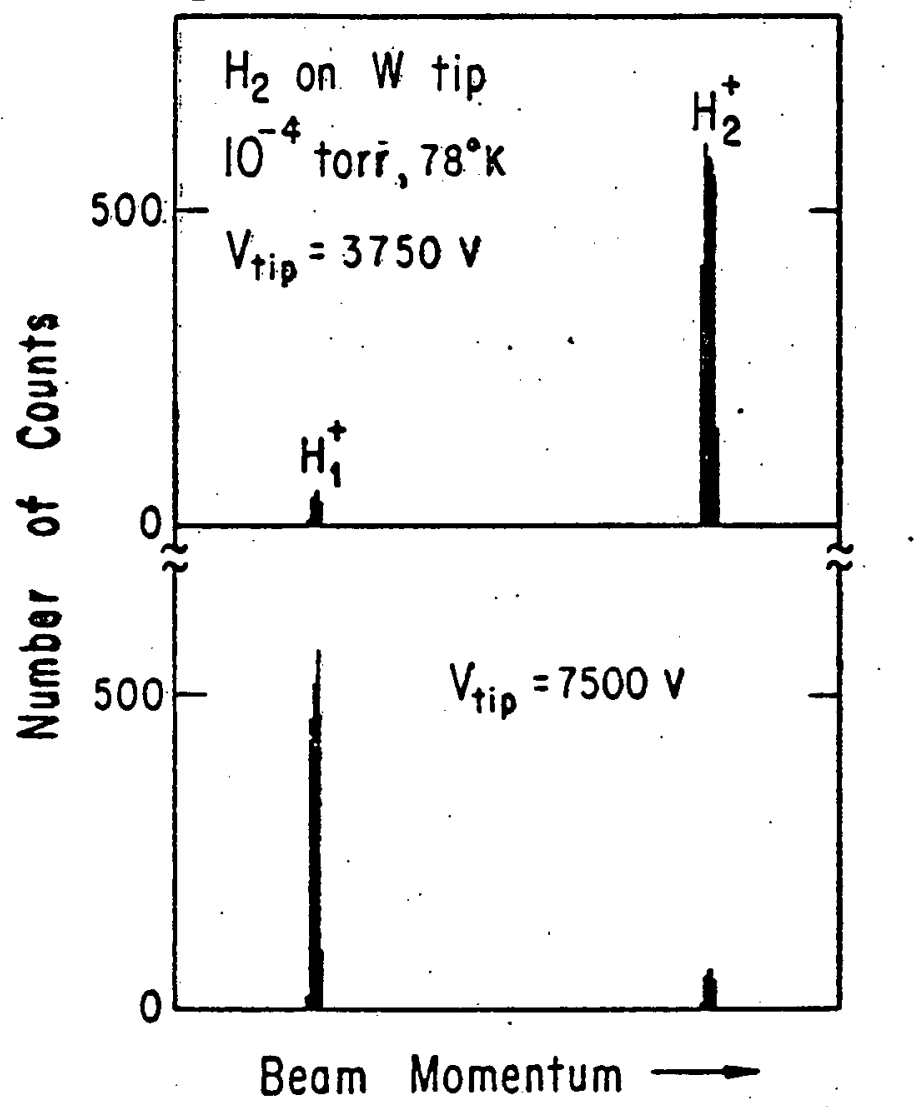

a)

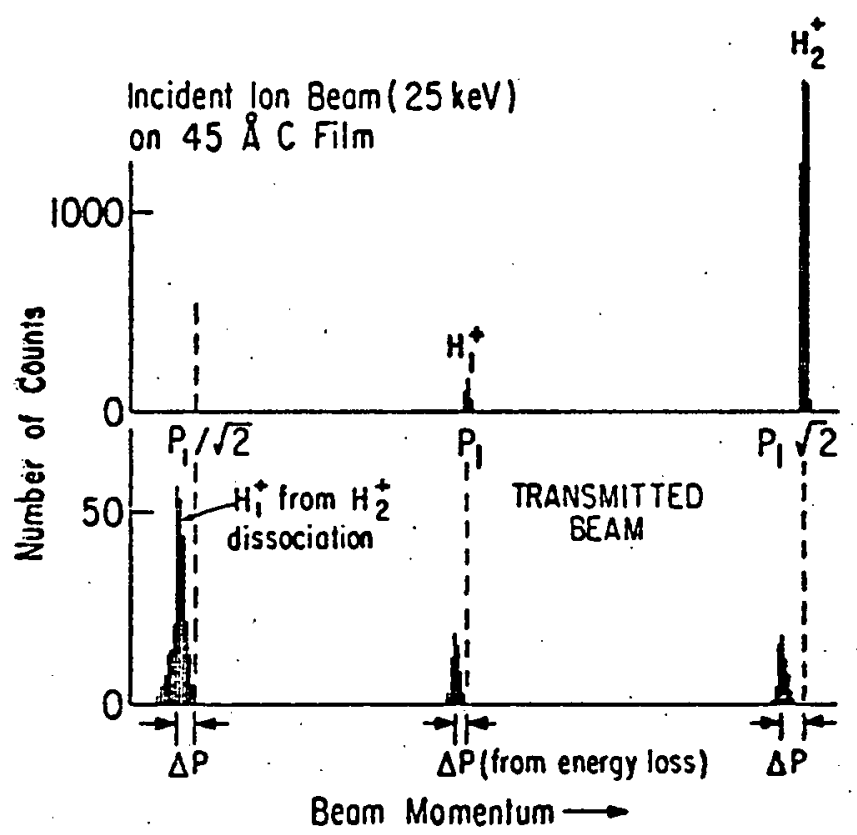

b)

Fig. 2 - Momentum analysis of incident and Transmitted beams. 
311

Summary of results $b_{n}$ Chicago group a) Physical framework

b) Statistical preamble (Table 2)

C) Experimental results at $v<v_{F}$

d) Phenomenology 


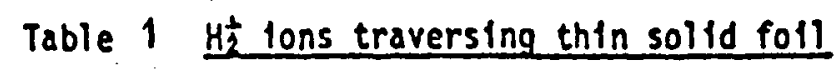

\begin{tabular}{|c|c|c|c|c|}
\hline & \multicolumn{2}{|c|}{$v<v_{F}$} & \multicolumn{2}{|c|}{$v>v_{F}$} \\
\hline & CONDUCTOR & INSULATOR & CONDUCTOR & INSULATOR \\
\hline Screenting in solid & statte. & static & dynamtc & dynamic \\
\hline Potentlal in solid & $\begin{array}{l}\text { Screened: } \\
\frac{e}{r} \exp \left(-\lambda_{s} r\right) \\
\dot{\lambda}_{s} \sim v_{F} / \omega_{p} \sqrt{3}\end{array}$ & $\begin{array}{l}\text { Attenuated: } \\
\because \frac{\mathrm{e}}{\mathrm{r}} \frac{1}{\varepsilon}\end{array}$ & $\begin{array}{l}\text { Screened: } \\
\frac{e}{r} \exp \left[-\lambda_{d}(v) r\right] \\
\lambda_{d} \sim v / \omega_{p} \\
\end{array}$ & $\begin{array}{l}\text { Unscreened for: } \\
r_{0}<<v / \omega_{p} \\
\text { Attenuated for: } \\
r_{0} \gg>v / \omega_{p}\end{array}$ \\
\hline $\begin{array}{l}t^{3 / 2} \text { growth of internuc- } \\
\text { lear separation due to } \\
\text { multiple Coulomb scatter- } \\
\text { ing. }\end{array}$ & Yes & Yes & Yes & Yes $\because{ }^{\prime}$ \\
\hline Coulomb explosion & No & Attenuated & Yes for $r_{0} \leqslant v / w_{p}$ & $\begin{array}{l}\text { Yes for } r_{0} \lesssim v / \omega_{p} \\
\text { Attenuated for } \\
r_{0}>v / \omega_{p}\end{array}$ \\
\hline Ion neutralization & Uncorrelated & Cluster effects & $\begin{array}{l}\text { Cluster effects for } \\
r_{0} \in \vee \omega_{p}\end{array}$ & (1) \\
\hline Energy loss & $\begin{array}{l}\text { Cluster effects } \\
\text { negilgible }\end{array}$ & Cluster effects (?) & $\begin{array}{l}\text { Cluster effects for } \\
\text { ro }\left\{\cdot v \omega_{p}\right.\end{array}$ & Cluster effects (?) \\
\hline Molecular recombination & Yes & . (2) & Yes. & (2). \\
\hline Convoy electrons & $\because \quad \ldots$ & $\cdots$ & $\begin{array}{l}\text { Cluster effects in } \\
\text { yeloctity and angular } \\
\text { distribution }\end{array}$ & $\therefore(1)$ \\
\hline
\end{tabular}


Table ${ }^{313}$

Statistical considerations relevant to

molecular ion dissociation/ recombination

in solids (Escovitz, 1979)(3)

Dissociation:

Recombination:
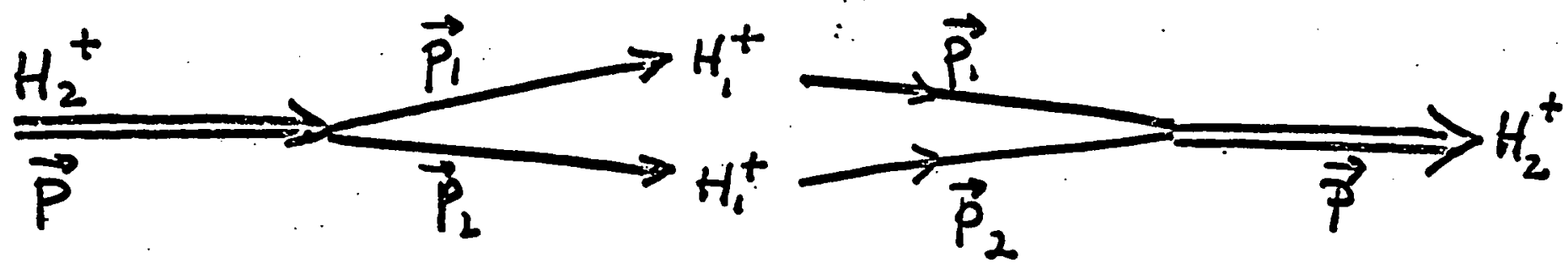

(1) Momentum spectra: $\quad \vec{p}=\vec{p}_{1}+\vec{p}_{2}$

$$
\begin{aligned}
& \langle\vec{p}\rangle=2\left\langle\vec{p}_{1}\right\rangle=2\left\langle\vec{p}_{2}\right\rangle \\
& \sigma(\vec{p})=\sqrt{2}\left[\sigma^{2}\left(\vec{p}_{1}\right)+\rho\left(\vec{p}_{1}, \vec{p}_{2}\right) \sigma\left(\vec{p}_{1}\right) \sigma\left(\vec{p}_{2}\right)\right]^{1 / 2}
\end{aligned}
$$

If $\vec{p}_{1}, \vec{p}_{2}$ statist. indep. $\rightarrow$ cove $=0 \rightarrow \sigma(\vec{p})=\sqrt{2} \sigma\left(\vec{p}_{1}\right)$

(2) Energy loss spectra: $\Delta T=\Delta T_{1}+\Delta T_{2}$ $\langle\Delta T\rangle=2\left\langle\Delta T_{1}\right\rangle=2\left\langle\Delta T_{2}\right\rangle$ (statistics only)

$$
\sigma(\Delta T)=\sqrt{2}\left[\sigma^{2}\left(\Delta T_{1}\right)+\rho\left(\Delta T_{1}, \Delta T_{2}\right) \sigma\left(\Delta T_{1}\right) \sigma\left(\Delta T_{2}\right)\right]^{1 / 2}
$$

if $\operatorname{cov}=0 \rightarrow \sigma(\Delta T)=\sqrt{2} \sigma\left(\Delta T_{0}\right)$

(3) Velocity spectra: $\vec{v}=\frac{1}{2}\left(\vec{v}_{1}+\vec{v}_{2}\right)$

$$
\sigma(\vec{v})=\frac{1}{\sqrt{2}}\left[\sigma^{2}\left(\vec{v}_{1}\right)+\rho\left(\vec{v}_{1}, \vec{v}_{2}\right) \sigma\left(\vec{v}_{1}\right) \sigma\left(\vec{v}_{2}\right)\right]^{1 / 2}
$$

If $\operatorname{cov}=0 \rightarrow \sigma(\vec{v})=\frac{1}{\sqrt{2}} \sigma\left(\vec{v}_{1}\right)$ 
Experiments performed:

a) Transmission/ recombination of $\mathrm{H}_{2}^{+}$

Beam : $25 \mathrm{keV} \mathrm{H}_{2}^{+}\left(v=0.5 v_{F}\right.$ in () $12.5,25 \mathrm{keV} \mathrm{H}^{*}$

Targets : $0.3-0.9 \mu \mathrm{g} / \mathrm{cm}^{2}$ holey $C$ films

Measured : Fractions of $\mathrm{H}_{2}^{+}$, energy loss spectra of $\mathrm{H}_{2}^{+}$, of $\mathrm{H}_{1}^{+}$of same velocity and of $\mathrm{H}_{2}^{+}$dissociation products, including $\mathrm{H}^{+}$in coincidence with $H^{\circ}$. $(1,3,4)$

b) Ion neutralization studies

Beam: $25 \mathrm{keV} \mathrm{H}+12.5 \mathrm{keV} \mathrm{H}$

Targets: $0.3-2 \mu \mathrm{g} / \mathrm{cm}^{2}$ holey $C$ and $\left(\mathrm{CH}_{2}\right)_{n}$ films

Measured: Nentral/changed ratios, energy loss spectra of all transmitted components. 
315

c) Experimental result at $v<v_{F}$

(1) $\mathrm{H}_{2}^{+} \longrightarrow \mathrm{H}_{2}^{+} ;$, of serve:

$\Delta T \approx 2 \Delta T_{1} \rightarrow N_{0}$ interference

$\sigma(\Delta T) \approx \sqrt{2} \sigma\left(\Delta T_{1}\right) \rightarrow N_{0}$ statistical

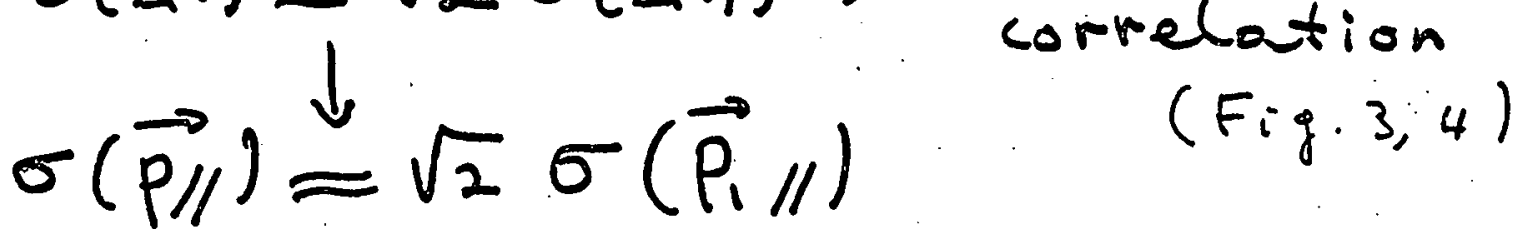

$\downarrow$ equivalent to

$\sigma\left(\vec{v}_{\| /}\right) \approx \frac{1}{\sqrt{2}} \sigma\left(\vec{v}_{1 / 1}\right) \rightarrow$ same as

See ret. $(3,4) \quad$ oblocityed for of convoy electrons at $v>0$ F

(2) (dE/dx)/amn agree (5) with Andersen 2 Ziegler (1977) $\Omega^{2} / a m a$ agree ${ }^{(s)}$ with

Bonderup \& Hoelplund $(1971)^{(7)}$ at 12.5, 25 keY. (Fig. 5) See ref. (5)

(3) $\mathrm{H}_{2}^{+} \rightarrow \mathrm{H}^{+}+\mathrm{H}^{(0,+)} ; \quad \mathrm{H}_{2}^{+} \rightarrow \mathrm{H}^{+}+\mathrm{H}^{0}$ (wince.) Lo similar energy at (Fr gl) Spectra Coulomb explosion Evidence for the formation of $15 \sigma_{g}$ and $2 p \sigma_{n}$ states with equal probability. See ref. $(3,4)$. 
ENERGY LOSS IN THE DISSOCIATION / RECOMBINATION OF H $\mathrm{H}_{2}^{+}$

IN THE TRAVERSAL OF THIN C FOILS FOR $v<v_{F}$

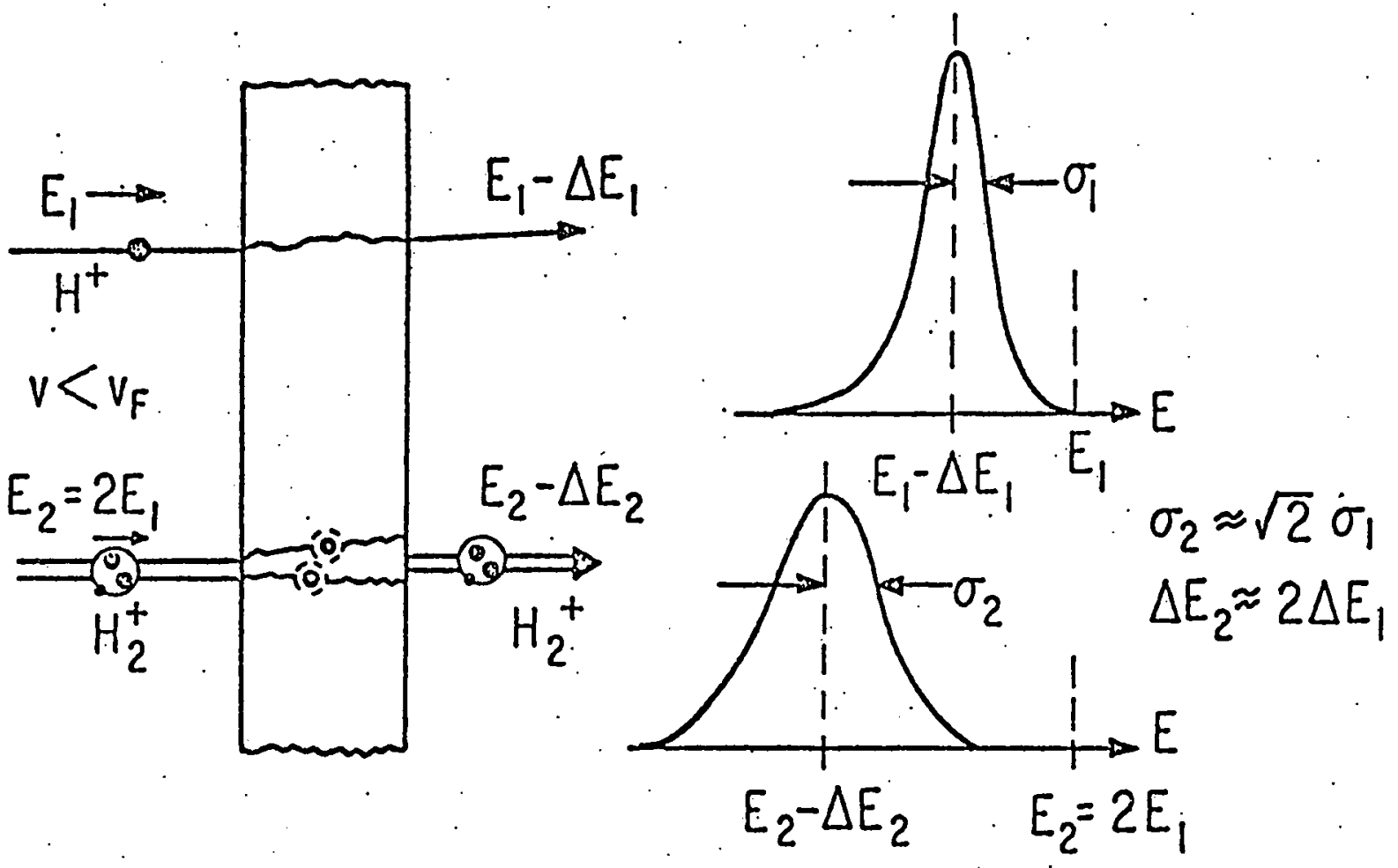

Fig. 3 


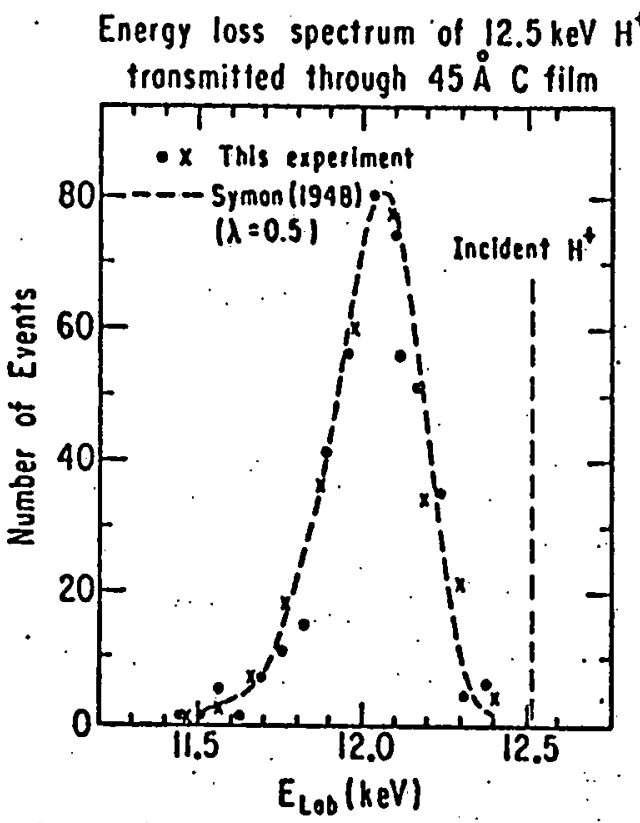

Energy loss spectrum of $12.5 \mathrm{keV} \mathrm{H}^{*}$
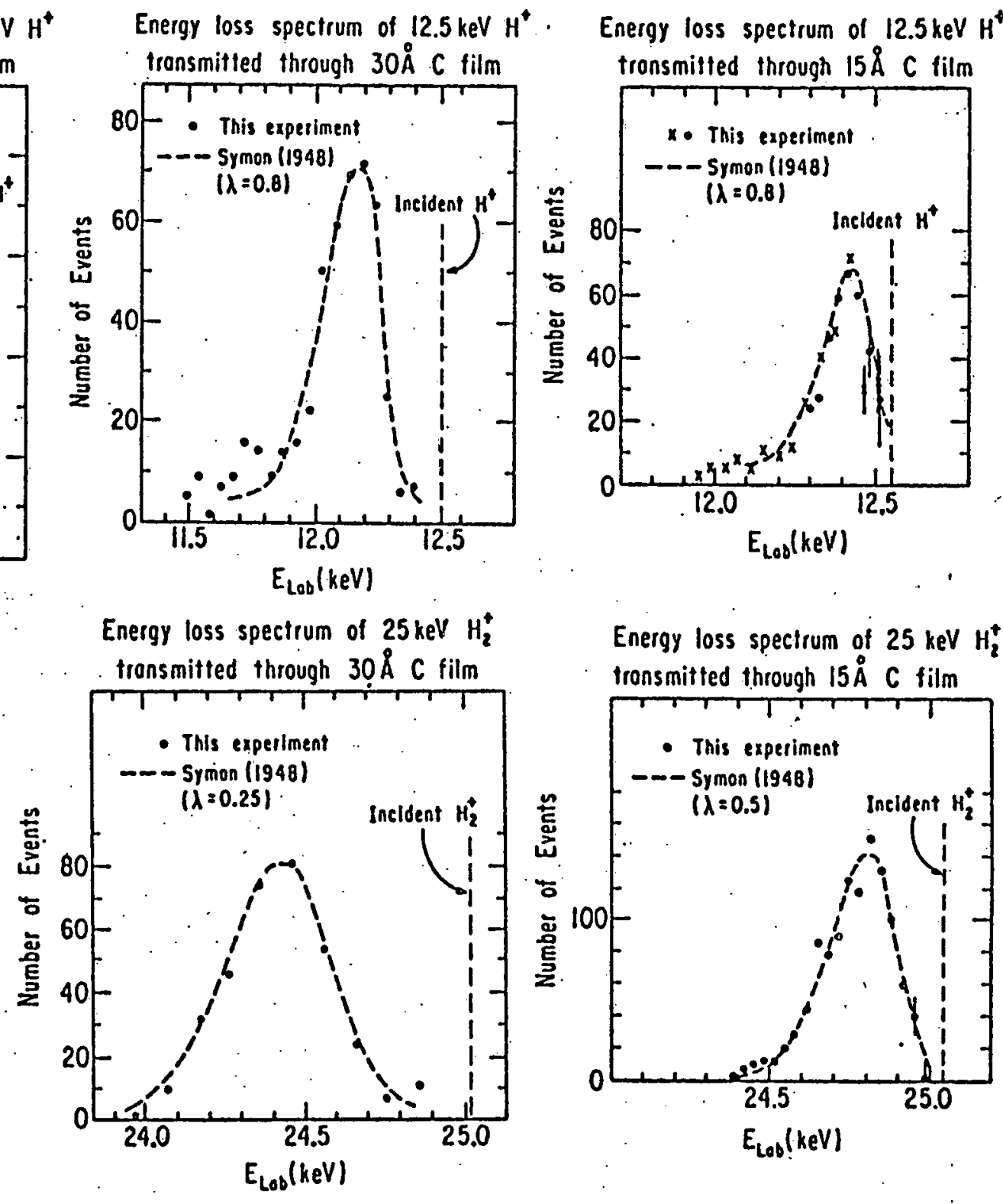

Energy loss spectrum of $25 \mathrm{keV} \mathrm{H}$ tronsmitted through $15 \AA \mathrm{C}$ tilm

Fig. 4

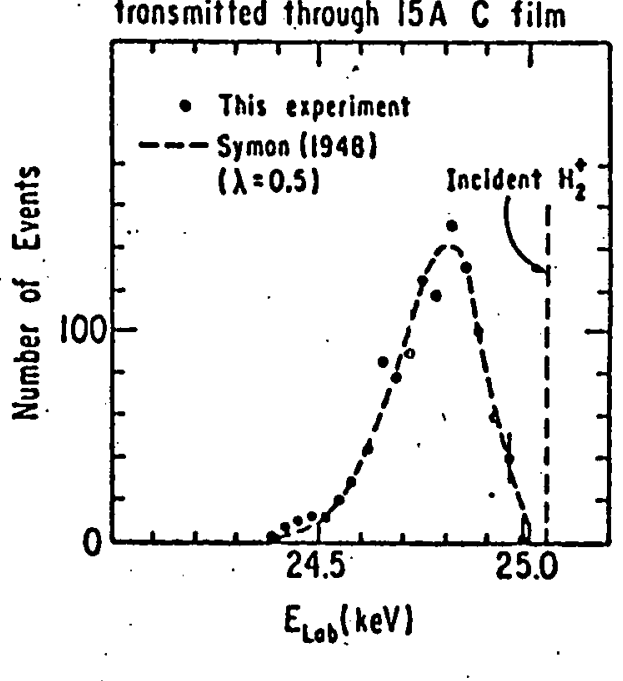



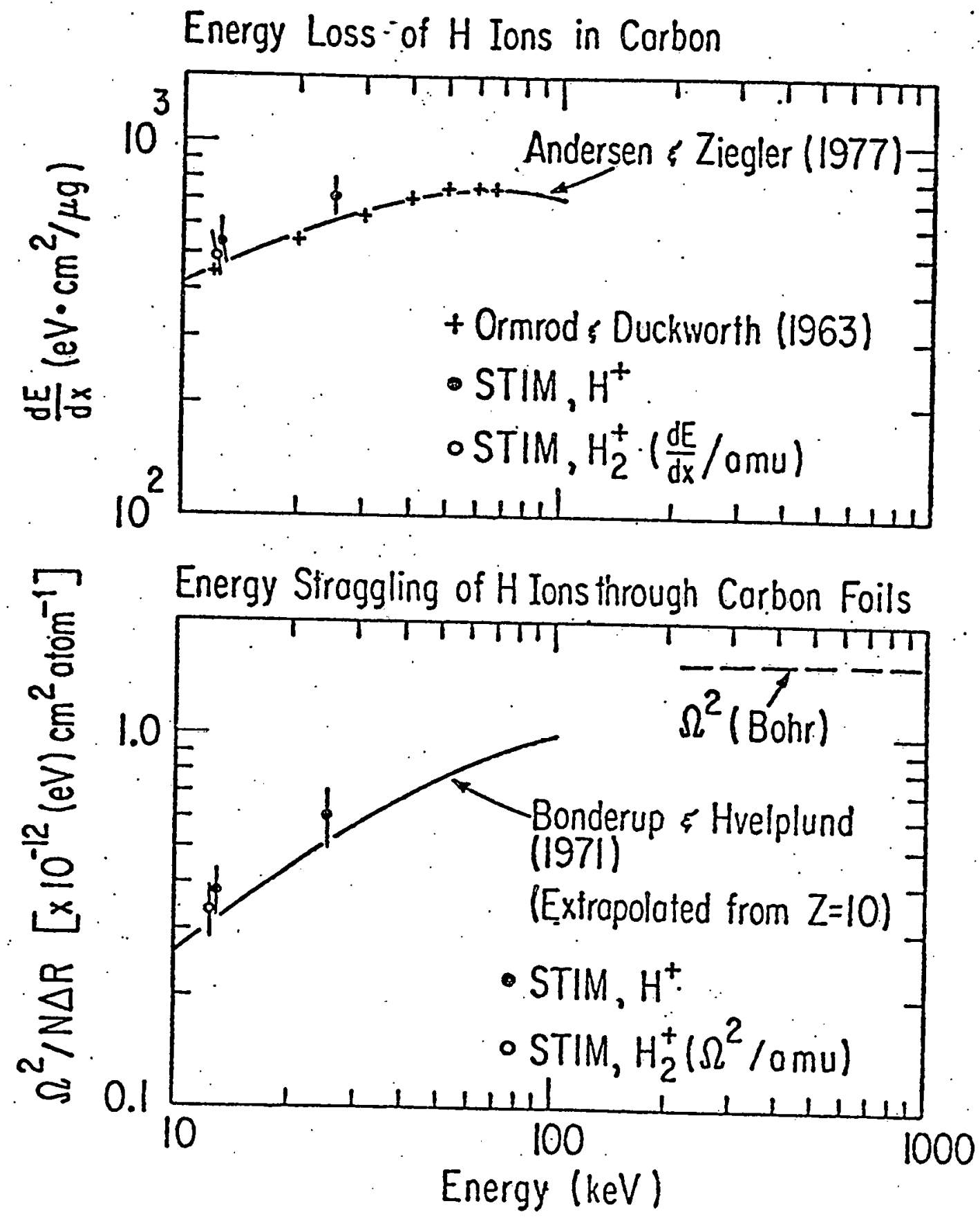

Fig. 5 

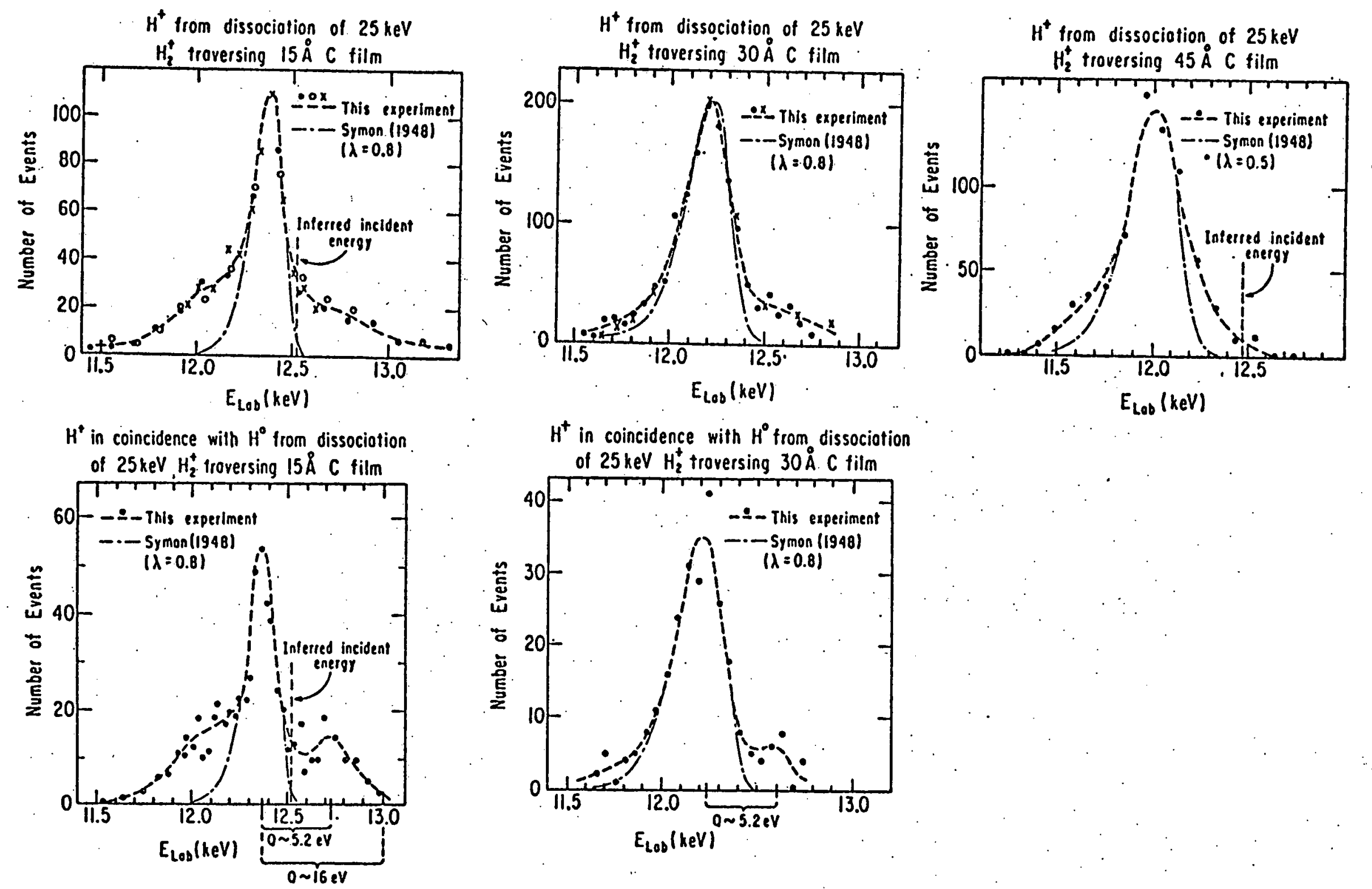

$F_{i g}: 6$ 
320

(14). Center peak in dissociation spectra (attributed to $15 \sigma_{g}$ state) has same width as spectra. of single incident protons of some velocity; $(3,4)$ Width increases with foil thickeners.

(5) Find evidence for cooperative effects in ion neutralization for insulator (poly ethylene): (8)

$$
\begin{aligned}
& \frac{\frac{N^{0}}{N^{+}}\left(H_{2}^{+}\right)}{\frac{N^{0}}{N^{+}}\left(H^{+}\right)} \approx 1.5 \text { for }\left(\mathrm{CH}_{e}\right)_{n} \rightarrow \begin{array}{l}
\rightarrow \text { analogy } \\
\text { with "long } \\
\text { dwell time }
\end{array} \\
& \frac{N_{0}}{N^{+}}\left(H_{2}^{+}\right) \approx 1.0 \text { lon } C \text { clustereffect" } \\
& \frac{N^{0}}{N^{+}}\left(H^{+}\right)=1.0 \text { for }
\end{aligned}
$$


321

d) Phenomenology

(1) Computations $(1,3)$ show that growth of internuclear separation following $\mathrm{H}_{2}{ }^{+}$. dissociation in solid is dominated by multiple Coulomb scattering, at all energies up to several MeV. Original internuclear separation dominates up to dwell times $\sim 2-3$ ts. (fig. 7 )

Consequence of multiple Coulomb scattering is the build-up of Com. transverse energy $\left\langle Q_{T \text {, scat }}\right\rangle \Rightarrow H_{2}^{+}$binding energy. $\mathrm{H}_{2}^{+}$recombination will occur either for proton pairs with $Q_{T \text {.scale }}<B$, or through energy dissipation to third body. ( see ref (3)).

(Fig. 8)

(2) Electron capture and rms internuclear separation at toil exit seem to play key role in $\mathrm{H}_{2}^{+}$recombination:

If $Y\left(\mathrm{H}_{2}^{+}\right) / \phi_{0}$ is plotted us $\left\langle S^{2}\right\rangle^{1 / 2}$, data at all energies collapse into unified functional dependence. $(1,3)$ This unification in terms of $\left\langle S^{2}\right\rangle^{1 / 2}$ is an improvement oven that as a function of dwell -time.

(Fig. 9, 10, 11) 
Growing Internuclear Separation Following Dissociation of $\mathrm{H}_{2}^{+}$Molecular Ion Traversing a Conductor

$V<V_{F}$

Stotic screening regime

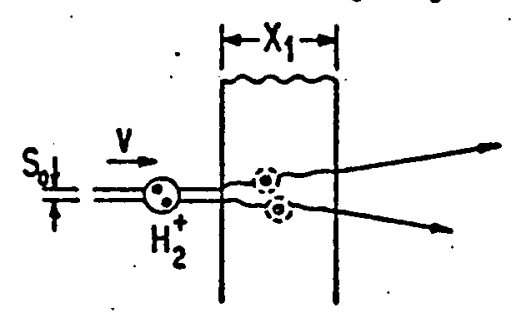

$$
=
$$<smiles>C1CCCC1</smiles>

$V>V_{F}$

Dynomic screening regime

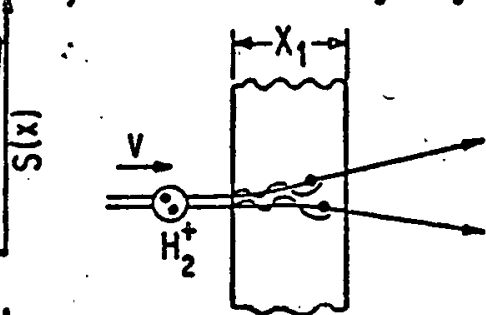

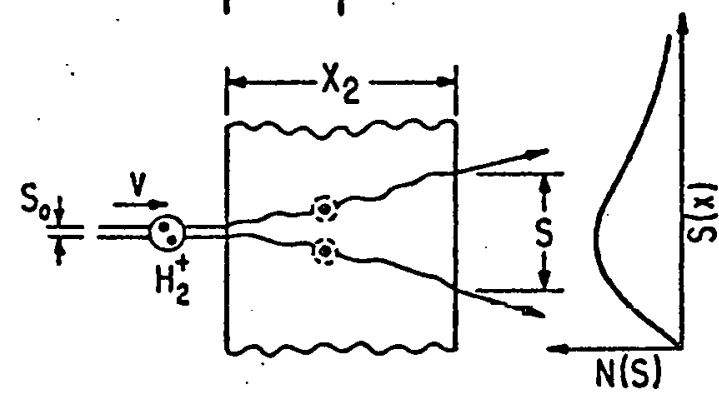

$\left\langle s^{2}\right\rangle=\langle$
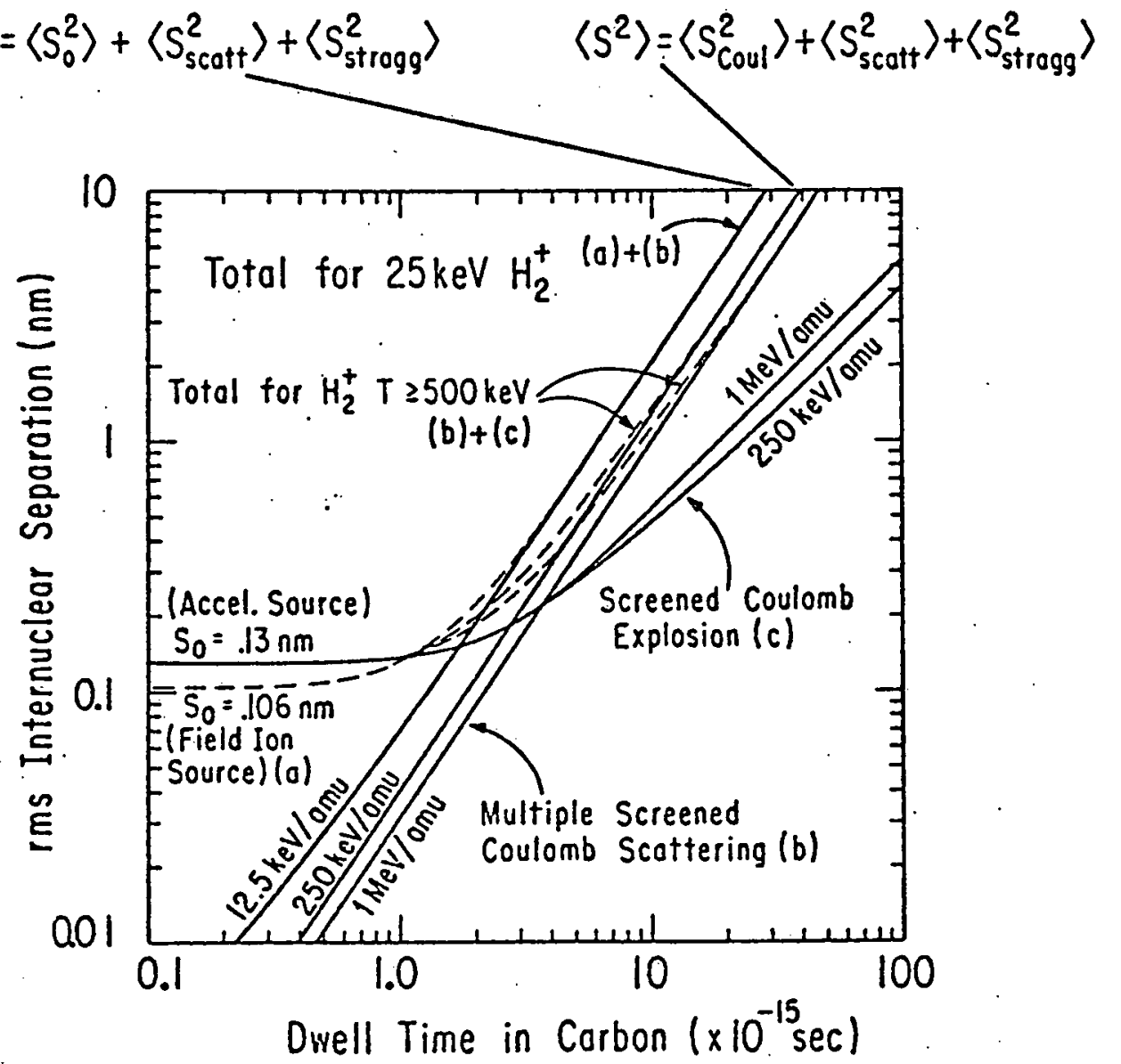

Fig. 7 (From net. (5)) 


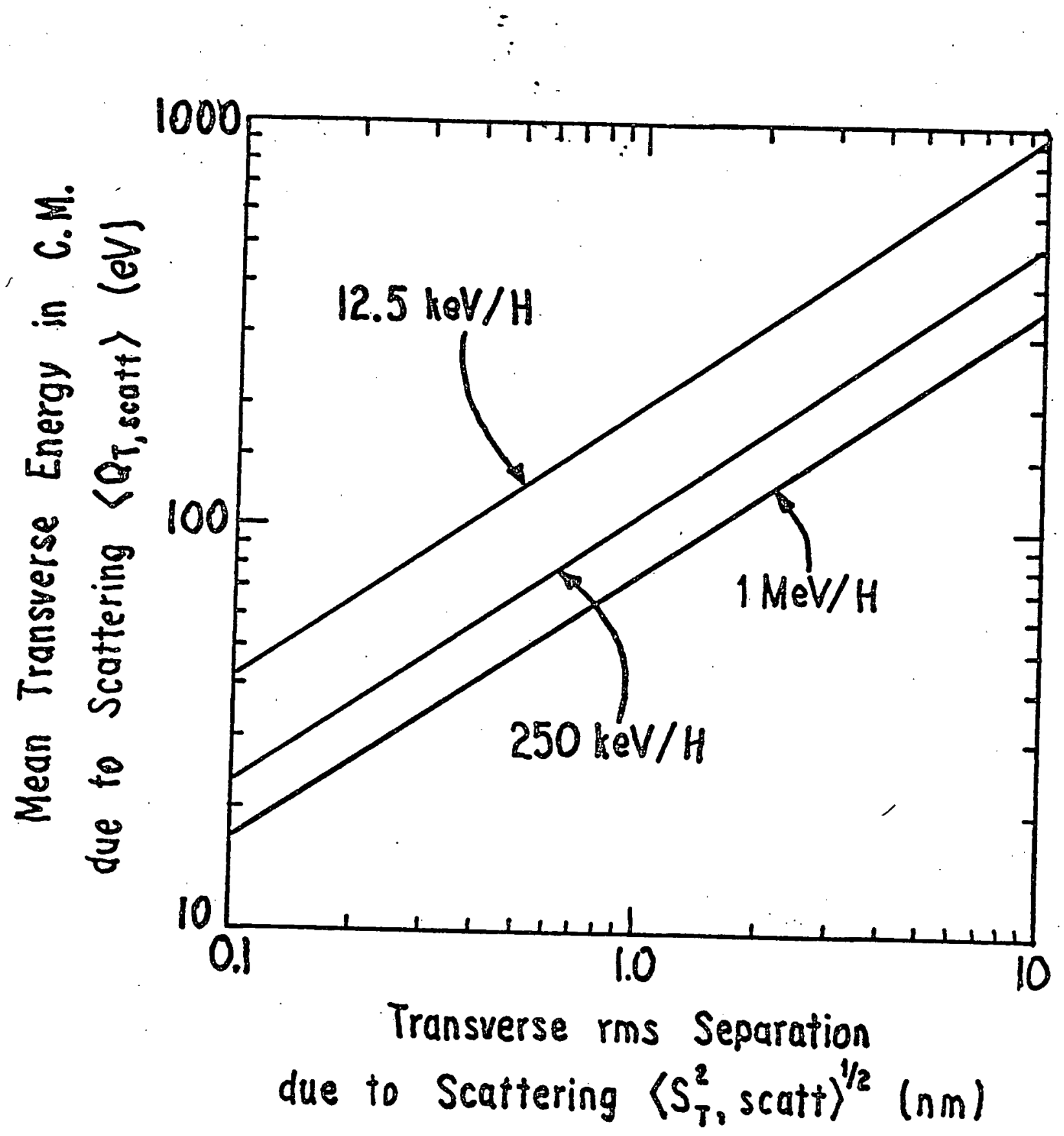

Fig. 8 (From ret ${ }^{(3)}$ ) 
324

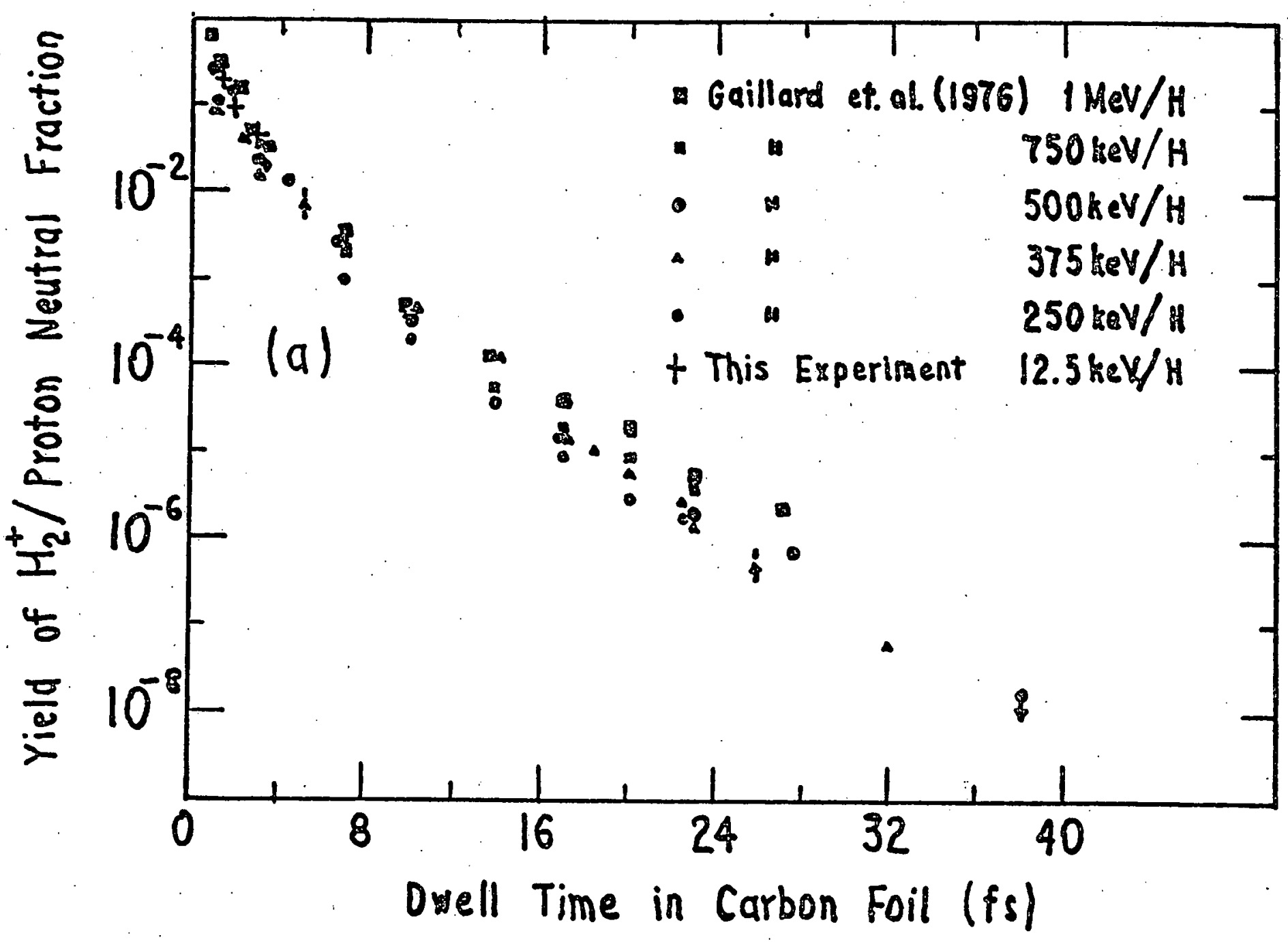

Data from ref. $(1,3)$ and ref. ${ }^{(10)}$.

Fig. 9 (From ref. (3)) 


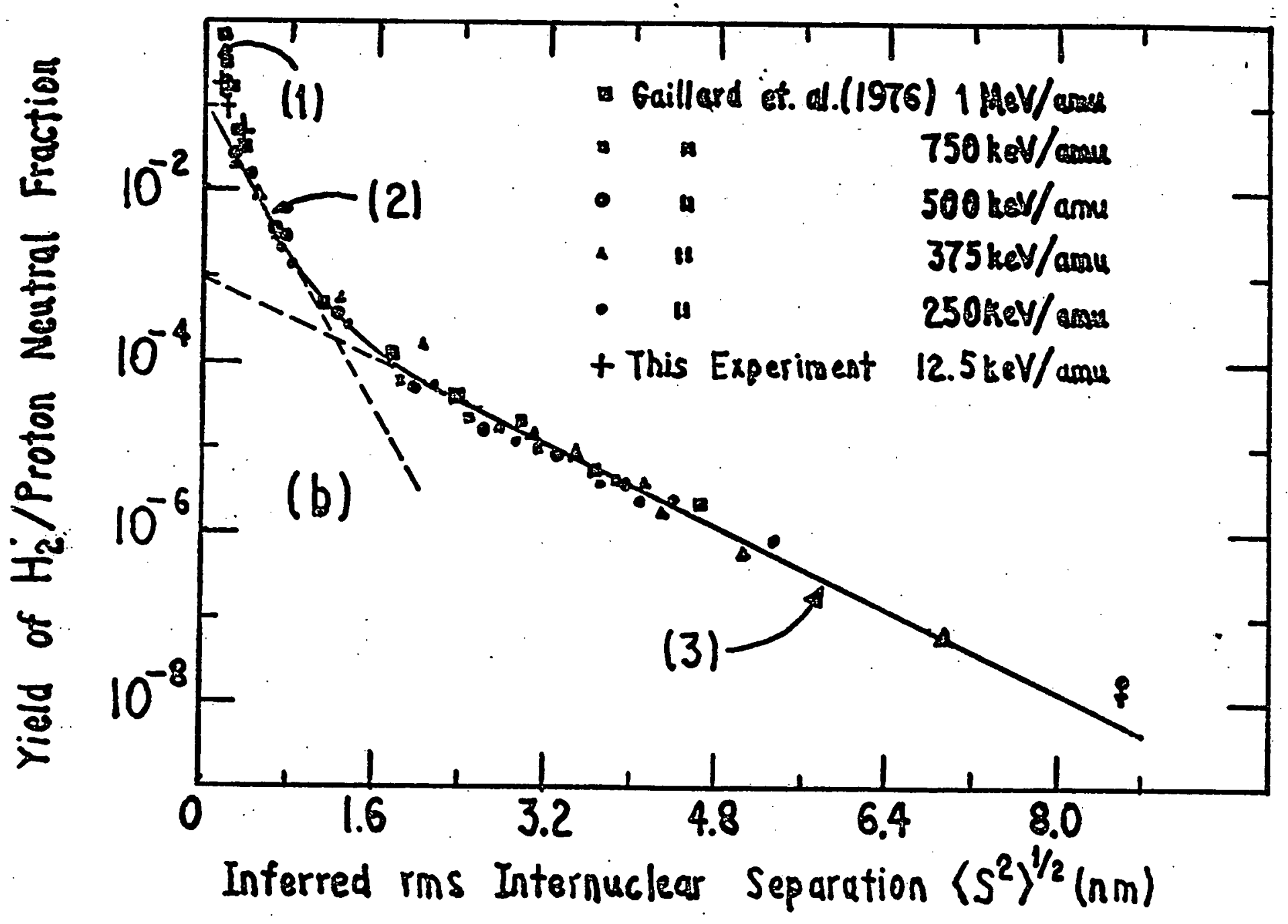

Data from ref. $(1,3)$ and ref. $(10)$.

$$
\text { Fig. } 10 \text { (From ret. (3)) }
$$


326

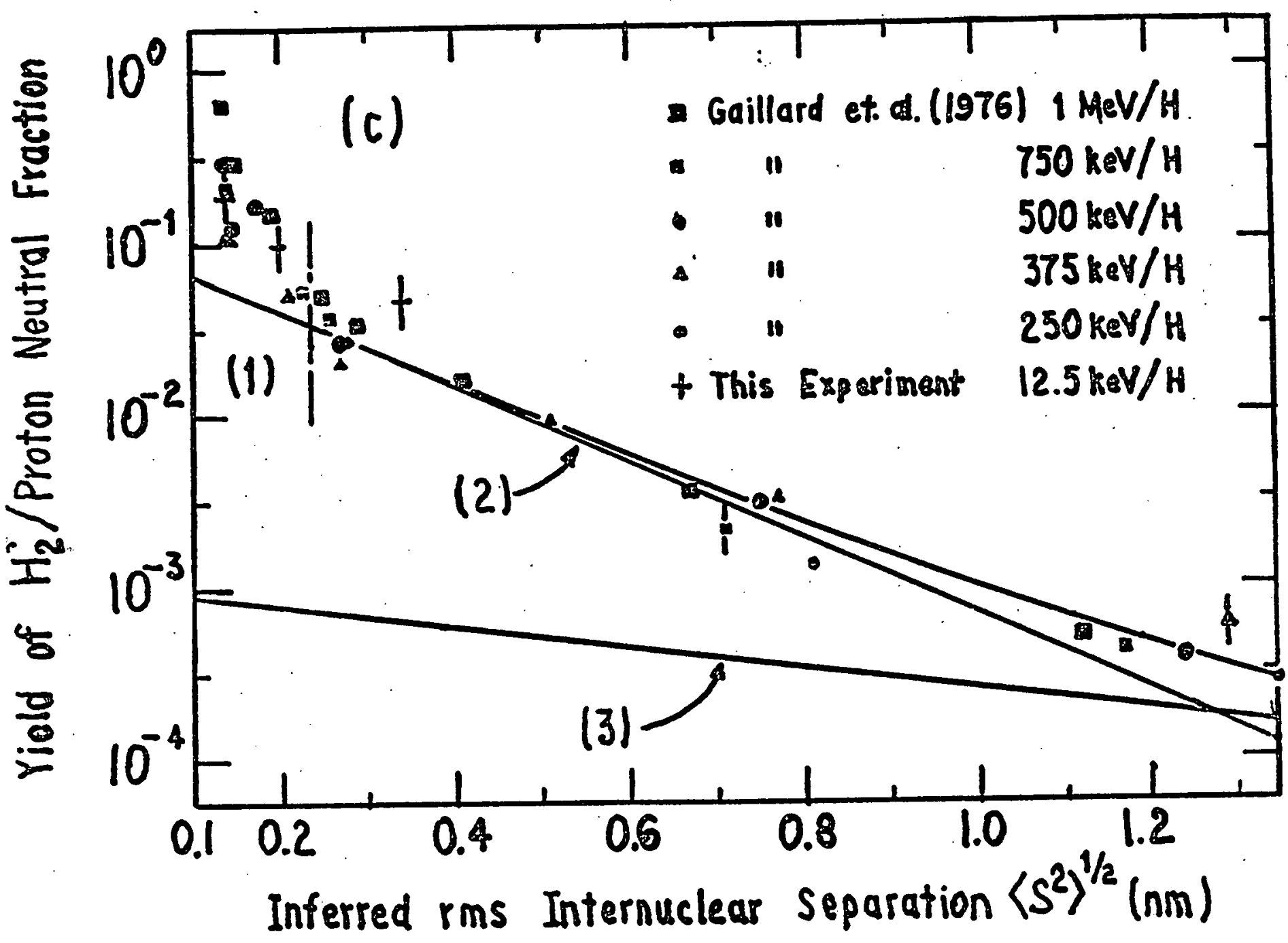

Data from ref. $(1,3)$ and ref. $(10)$

(3) 
327

(3) Evidence for wateriding states could, in priciciple, be obtainable from a statistical analysis of the energy loss spectra of recombined molecule: positive covariance $\rightarrow$ indication of wakeriding.

However, Coulomb explosion inside foil contributes negative covariance (anticorrclation).

This may cancel out any evidence of Wakeriding.

Experiment sensitive to wateriding (3)

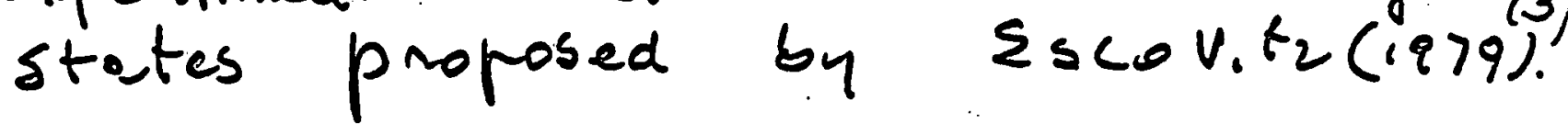

To be measured is the energy loss in $H^{+}$recombination. (F:g.12) 
328

(a)

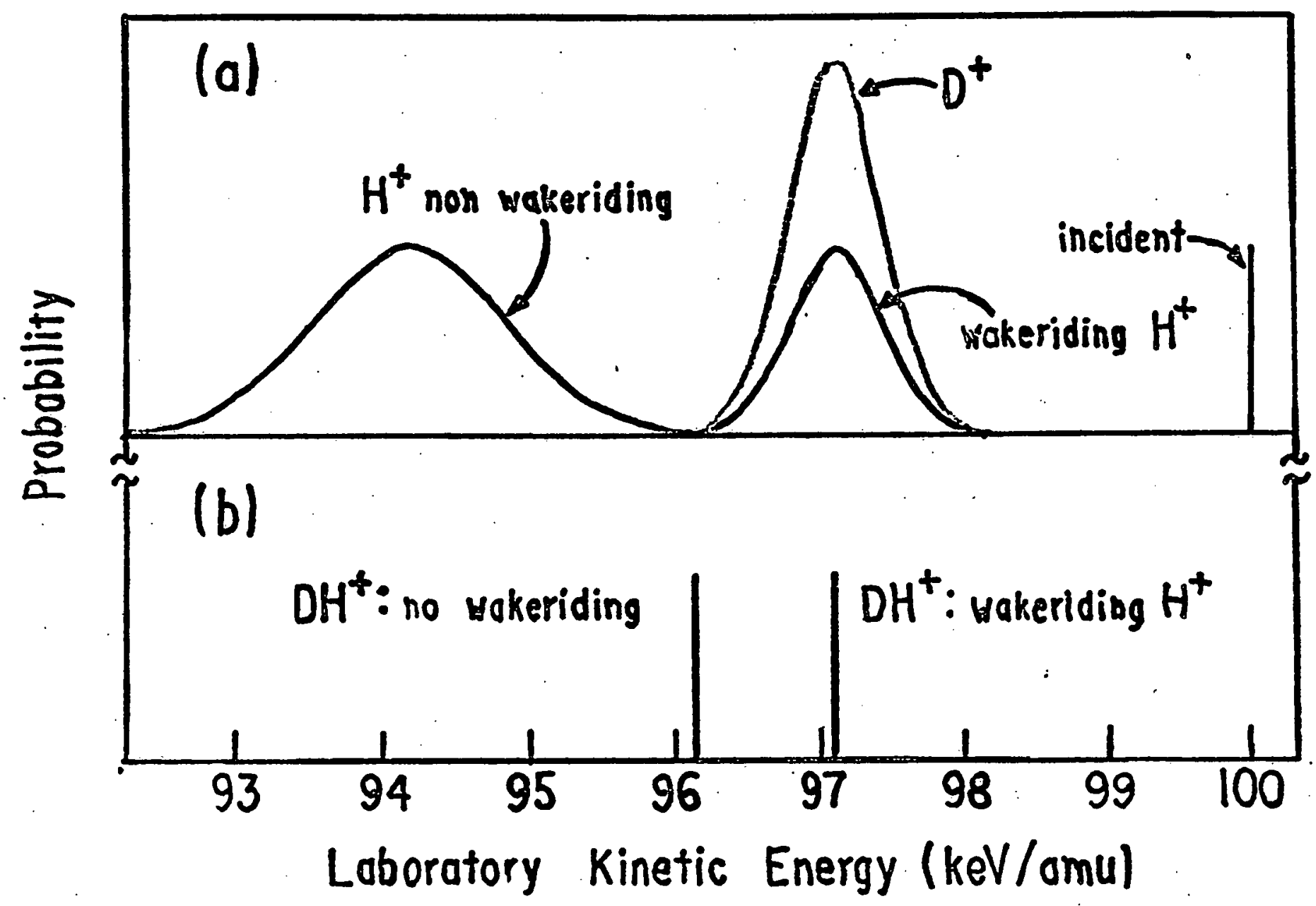

Fig. 12 a). Expected energy spectra for $D^{+}$and $\mathrm{H}^{+}$from $300 \mathrm{keV} \mathrm{DH}^{+}$traversing $6 \mu \mathrm{g} / \mathrm{cm}^{2}$ carbon foil

b) Mean energies of emerging $\mathrm{DH}^{+}$ with and without wakeriding $\mathrm{H}^{+}$ (From net. (3)) 
An ovinview.

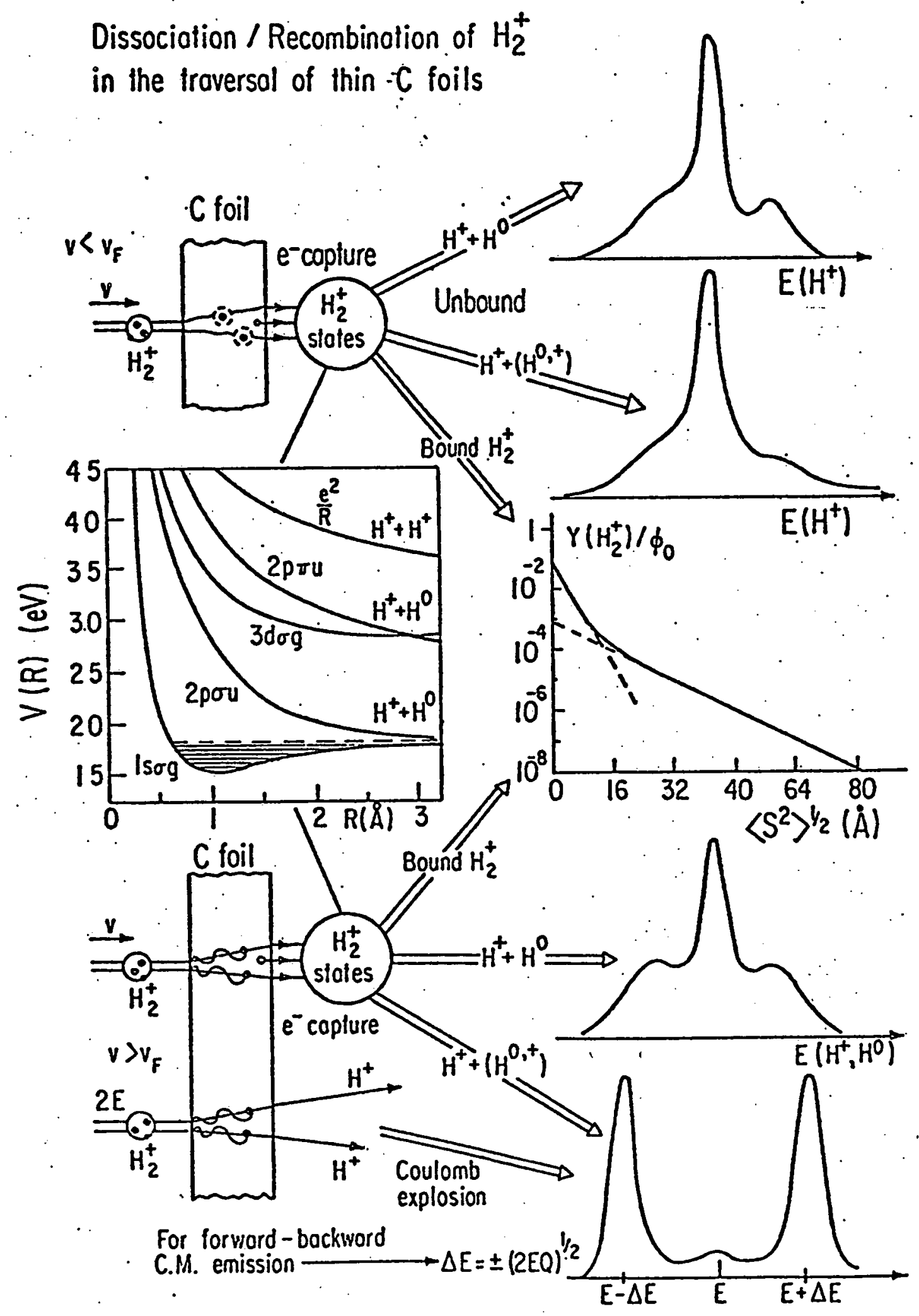

F:g. 13 (From ut. (5)) 
330

References.

(1) W. H. Escovitz: T.R. Fox and R. Levi-Setti, IEEE Transactions on Nudear Science, Ud. NS-26; $1395 \%(1979)$

(2) J.R. Hiskes. Phys. Res. 122,1207 (1961).

(3) W.H.Escovitz. Thesis, The University of Chicage, 1979.

(4) W. H. Escovitz, T.R. Fox and R. Levi-Setti, IEEE Trousactions on Nuclear Science. Sol. NS-26, 1147 (1979)

(5) R. Levi-Setti and T.R. Fox, Proc. II Int'l Couf on lon Beam Analysis, Aanthus, Deumark. Jure 197\%.

Nucl. Iustr. and Meth., in press.

(b) H. H. Andersen and J. F.Ziegler, Hydrogen Stopring Powens and Ranges in All Elements. Vol. 3 of the storring and Rouges of lous in Matter, ed. J.F.Ziegler (Pengamon. New Youk .1977). See Toble 3 and graphs for C[6].

Also: J.H. Ormrod and H.E. Duck worth, Can. J. Phys. 411.424 (1963)

(7) E. Bonderiup and P. Hodplumd. Phys.Rer. A 4,572 (1971) 
331

Ref. cout'd.

(8) T.R. Fox, wouk in progress.

(9) M.J. Gaillard, J.C. Poizat, A. Ratkowshi, $J$. Remillieux and M. Anzas, Phys. Rev. A16, 2323 (1972).

(10) M. J. Gaillard, J. C. Poizat, A. Ratkowski, and J.Remillienx, Nucl. Instr. and Meth. 132, 69 (1976) 
THIS PAGE

WAS INTENTIONALLY

LEFT BLANK 
R. A. Baragiola

Atomic Collisions Division

Centro Atomico Bariloche

8400 S. C. de Bariloche

Argentina

H. G. Berry

Physics Division

Argonne National Laboratory

Argonne, IL 60439

W. Brandt

Department of Physics

New York University

New York, N.Y. 10003

P. J. Cooney

Department of Physics

Middlebury College

Middlebury, VT 05753

N. Cue

Department of Physics

State University of New York

Albany, New York 12222

R. DeSerio

Department of Physics

University of Chicago

Chicago, IL . 60637

P. M. Echenique

Fisica del Estado Solido

Faculdad de Ciencas

Universidat de Barcelona

Barcelona - 14

Spain

A. K. Edwards

Department of Physics and Astronomy

University of Georgia

Athens, GA 30601

D. G. E11 is

Department of Engineering Physics

University of Toledo

Toledo, OH 43606
W. Escovitz

Department of Physics

University of Chicago

Chicago, IL 60637

A. Fatbis

Nuclear Physics Division

Weizmann Institute of Science

Rehovoth, Israel

T. R. Fox

Department of Physics

University of Chicago

Chicago, IL 60637

F. Fujimoto

College of General Education

University of Tokyo

Tokyo 153

Japan

M. Gaillard

Institut de Physique Nucleaire

Universite Lyon - 1

69621 Villeurbanne

France

T. J. Gay

Department of Physics

University of Chicago

Chicago, IL 60637

D. S。 Gemmel1

Physics Division

Argonne National Laboratory

Argonne, IL 60439

R。 Kaim

Physics Department

Weizmann Institute of Science

Rehovoth, Israel

E. P. Ranter

Department of Physics

Rutgers University

New Brunswick, N.Y. 02139 
M. Kiragawa

Department of Electronics

North Shore College

Atsugi 243

Japan

R. Laubert

Department of Physics

East Carolina University

Greenville, N.C. 27834

R. Levi-Setti

E. Fermi Institute

University of Chicago

Chicago, IL 60637

S. Levit

Physics Department

Weizmann Institute of Science

Rehovoth, Israel

M. W. Lucas

Department of Physics

University of Sussex

Sussex, ENGLAND

Y. H. Ohtsukt

Department of Physics

Waseda University

Tokyo 160

Japan

W. Pietsch

I. Physikalisches Institut

Universitat $\mathrm{zu}$ Koln

Universitatsstrasse 14

50.00 Koln 41

W. Germany

I. Plesser

Physics Division

Argonne National Laboratory

Argonne, IL 60439

P. Pronko

Materials Science Division Argonne National Laboratory Argonne, IL 60439
J。 Remillieux

Institut de Physique Nucleaire

Universite Lyon - 1

69621 Villeurbanne

France

R。 H。 RItchie

Health Physics Division

Oak Ridge National Laboratory

Oak RIdge, TN 37830

R. M。 Schectman

Department of Engineering Physics

University of Toledo

Toledo, OH 43696

P. Thleberger

Physics Department

Brookhaven National Laboratory

Upton, N. Y. 11973

P. B。 Treacy

Nuclear Physics Department

The Australian National University

P.0. Box 4, Canberra ACT 2600

Australia

Z. Vager

Nuclear Physics Department

Weizmann Institute of Science

Rehovoth, Israel

H. E. Wegner

Physics Department

Brookhaven National Laboratory

Upton, N.Y. 11973

B. J. Zabransky

Physics Division

Argonne National Laboratory

Argonne, IL 60439 